\title{
Development of Design Tools for Coining Process Using FEM
}

\author{
By \\ KAI GUO \\ M. A. Sc, B. Eng

\begin{abstract}
A dissertation submitted to
the Faculty of Graduate Studies and Research

in partial fulfillment of

the requirements for the degree of

Doctor of Philosophy
\end{abstract}

Department of Mechanical and Aerospace Engineering

Ottawa-Carleton Institute of

Mechanical and Aerospace Engineering

Carleton University

Ottawa, Ontario

November, 2009

(c) KAI GUO, 2009 


$\begin{array}{ll}\begin{array}{l}\text { Library and Archives } \\ \text { Canada }\end{array} & \begin{array}{l}\text { Bibliothèque et } \\ \text { Archives Canada }\end{array} \\ \begin{array}{l}\text { Published Heritage } \\ \text { Branch }\end{array} & \begin{array}{l}\text { Direction du } \\ \text { Patrimoine de l'édition }\end{array} \\ \begin{array}{l}\text { 395 Wellington Street } \\ \text { Ottawa ON K1A ON4 } \\ \text { Canada }\end{array} & \begin{array}{l}\text { 395, rue Wellington } \\ \text { Ottawa ON K1A ON4 } \\ \text { Canada }\end{array}\end{array}$

Your file Votre référence

ISBN: 978-0-494-63850-7

Ourfile Notro reference

ISBN: 978-0-494-63850-7

NOTICE:

AVIS:

The author has granted a nonexclusive license allowing Library and Archives Canada to reproduce, publish, archive, preserve, conserve, communicate to the public by telecommunication or on the Internet, loan, distribute and sell theses worldwide, for commercial or noncommercial purposes, in microform, paper, electronic and/or any other formats.

The author retains copyright ownership and moral rights in this thesis. Neither the thesis nor substantial extracts from it may be printed or otherwise reproduced without the author's permission.

L'auteur a accordé une licence non exclusive permettant à la Bibliothèque et Archives Canada de reproduire, publier, archiver, sauvegarder, conserver, transmettre au public par télécommunication ou par l'Internet, prêter, distribuer et vendre des thèses partout dans le monde, à des fins commerciales ou autres, sur support microforme, papier, électronique et/ou autres formats.

L'auteur conserve la propriété du droit d'auteur et des droits moraux qui protège cette thèse. $\mathrm{Ni}$ la thèse ni des extraits substantiels de celle-ci ne doivent être imprimés ou autrement reproduits sans son autorisation.
In compliance with the Canadian Privacy Act some supporting forms may have been removed from this thesis.

While these forms may be included in the document page count, their removal does not represent any loss of content from the thesis.
Conformément à la loi canadienne sur la protection de la vie privée, quelques formulaires secondaires ont été enlevés de cette thèse.

Bien que ces formulaires aient inclus dans la pagination, il n'y aura aucun contenu manquant.

\section{Canadä}




\section{Abstract}

Coining is a forging process in which a metallic disk, characterized by a low volumesurface ratio, is deformed with the aim to impress 3D images on its three surfaces. Due to the large production volumes and the high quality standards required for the final products in terms of final dimensions, tolerances and surface finishing, such cold forging process requires a careful evaluation and choice of the proper operation parameters. To facilitate the design process and understand the mechanism and metal flow during the minting process of a silver coin, a study was undertaken to develop the design tools for coining process.

The finite element method is found to be an effective tool to simulate the coining process. The FEM approach was validated by investigating the effects of some key numerical parameters through FEM simulation. The typical coining material properties and the coining interface friction conditions were investigated. The fine silver material properties and a two-parameter non-classical bilinear friction model were successfully obtained through experimental and numerical methods.

Using the material properties and frictional parameters obtained, a two-dimensional axisymmetric coining process was simulated with a FEM code, ABAQUS. The model consists of a blank, dies and collar that form the blank into a coin. The compression force, deformation pattern and stress, strain contours were predicted, which illustrates how the material flows as the deformation progresses. Through the FEM simulations, three major coining design parameters were investigated: die curvature, rim height and surface relief. By changing one of them at a time, coining loads, material flow, stress and strain developments were obtained. The effects of these parameters were analyzed and discussed. 
A series of three-dimensional FEM models were developed using the input models and the numerical approach established. A simplified three-dimensional FEM simulation was performed for the axisymmetric model to compare with the results of the two-dimensional axisymmetric coining simulation. Three-dimensional coining with the reduced features of industrial design and serrations were then simulated. The effects of three-dimensional serrations and reduced relief were analyzed and discussed. Through the simulations, the material flow, loading patterns, die cavity filling and other conditions like stress and strain in the coin can be obtained. Through this work, it is established that the detailed 2D and 3D finite element simulations can be used to predict the mint coining process reliably. These simulations can be used as design tools for the silver dollar coin manufacturing process. 


\section{Acknowledgments}

I would first like to express my great thanks to my supervisors, Professor Robert Bell and Professor Xin Wang, for their tremendous support, encouragement, patience and invaluable guidance.

I would like also to acknowledge and thank the support from Dr Xianyao Li and other supporting staff at the Royal Canadian Mint. Financial support from the Ontario Center of Excellences (OCE) and Natural Science and Engineering Research Council of Canada (NSERC) is gratefully acknowledged.

Finally, I would like to thank my family for all of their support through my pursuit of the $\mathrm{PhD}$ degree. My love is always with my parents, my brothers and my wife, without them none of this would have been possible. 


\section{Table of Contents}

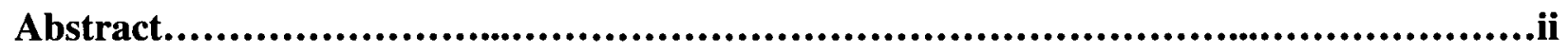

Acknowledgments.......................................................................iv

Table of Contents.................................................................................v

List of Tables..............................................................................ix

List of Figures...........................................................................xi

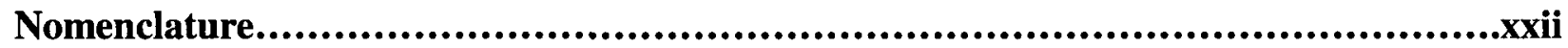

\section{CHAPTER 1}

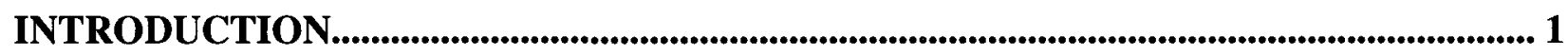

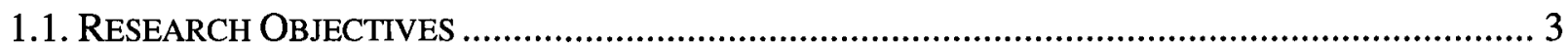

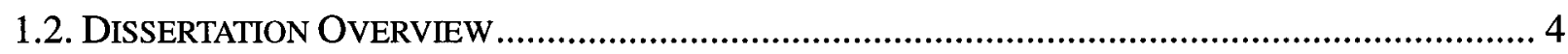

\section{CHAPTER 2}

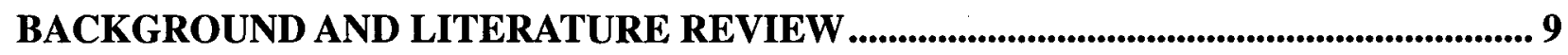

2.1 FORGING CONCEPTS AND CLASSIFICATIONS ............................................................ 9

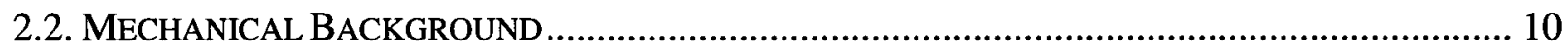

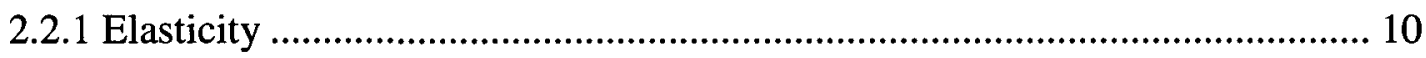

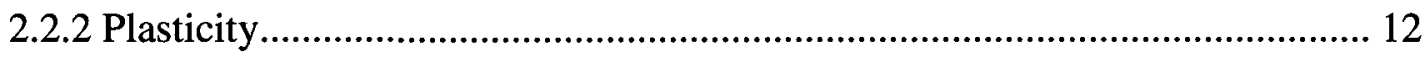

2.3 CoINING AND THE FINITE-ELEMENT METHOD ........................................................ 14

2.3.1 FEM Solution for Material Nonlinearity .......................................... 16

2.3.2 FEM Solution for Geometric Nonlinearity ........................................... 17

2.3.3. Elastic-Plastic Formulation and Rigid-Plastic Formulation ...................... 19 


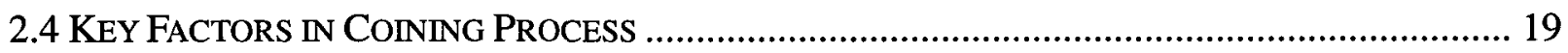

2.4.1 Material Properties .................................................................................... 20

2.4.2 Temperature in Metal Forming ………………......................................... 21

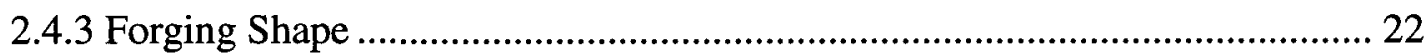

2.4.4 Friction in Metal Forming........................................................................... 23

2.5 RECENT DEVELOPMENTS IN METAL FoRgING AND COINING ........................................... 25

\section{CHAPTER 3}

NUMERICAL SIMULATION USING FEM ...................................................................... 30

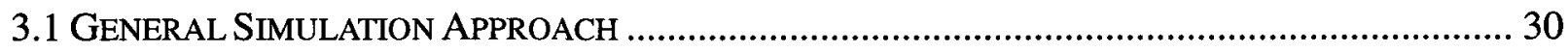

3.2 THE DOMINANT FACTORS AND THEIR EFFECTS IN FEM SIMULATIONS .................................. 31

3.2.1 Hourglass Controls in ABAQUS ................................................................ 31

3.2.2 Contact Simulation in ABAQUS/Explicit .................................................. 32

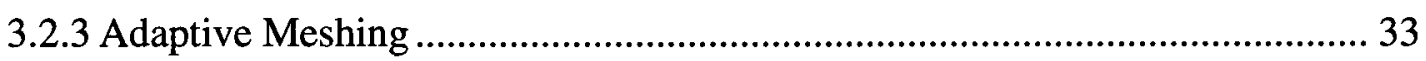

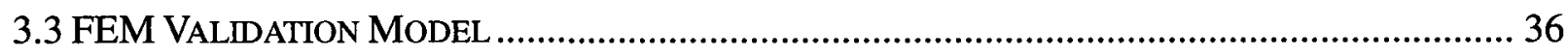

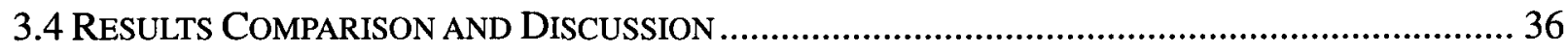

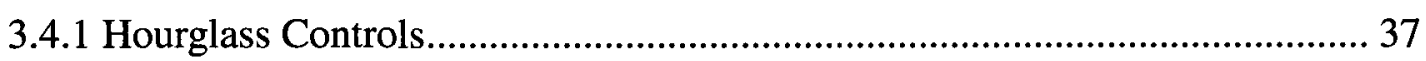

3.4.2 Contact Simulation......................................................................................... 37

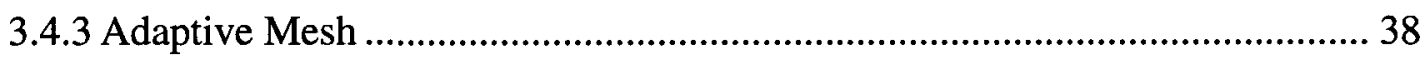

\section{CHAPTER 4}

COINING MATERIAL PROPERTY EVALUATIONS .......................................................... 46

4.1 MATERIAL PROPERTIES FOR COINING SIMULATIONS ................................................... 46

4.2 APPRoACHES To OBtAIN THE MATERIAL PROPERTIES .................................................... 47 


\section{CHAPTER 5}

FRICTION CONDITION EVALUATIONS OF COINING PROCESS.................................. 56

5.1 FrICTION BEHAVIOR IN COINING PROCESS ..................................................................... 56

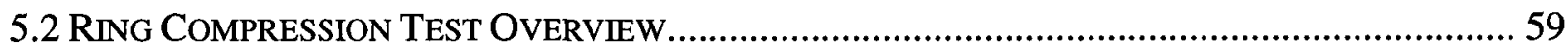

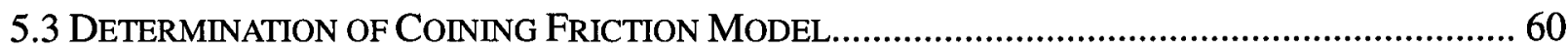

5.3.1 Ring Compression Procedures ........................................................................ 60

5.3.2 Finite Element Modeling of Ring Compression Test........................................ 62

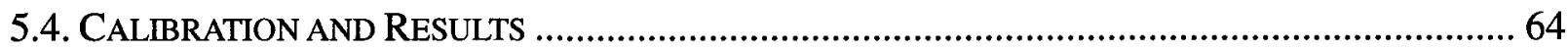

\section{CHAPTER 6}

TWO DIMENSIONAL AXISYMMETRIC COINING SIMULATION................................. 93

6.1 GEOMETRY OF Two DIMENSIONAL FEM COINING MODEL .................................................. 93

6.2 Two-DIMENSIONAL AXISYMMETRIC FEM MODEL .............................................................. 95

6.3 TWO-DIMENSIONAL FEM COINING MODEL VALIDATION ..................................................... 96

6.3.1 Mesh Convergence..................................................................................... 96

6.3.2 Validations of Numerical Parameter ............................................................... 98

6.3.3 Contact Pressure Development in Coining Process ....................................... 100

6.4 PARAMETRIC STUdY OF COINING PROCESS ................................................................. 100

6.4.1 Top Die Curvature Variations ........................................................................... 101

6.4.2 Bottom Die Curvature Variations.................................................................. 101

6.4.3 Rim Height Variations.................................................................................... 103

6.4.4 Relief Height Variations............................................................................ 105 


\section{CHAPTER 7}

THREE DIMENSIONAL COINING SIMULATION.................................................................... 135

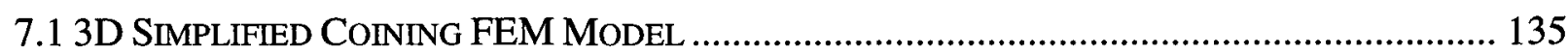

7.2 3D FEM SIMULATION OF COINING MODEL WITH SERRATIONS ON SIDE RING ..................... 137

7.3 3D FEM COINING SIMULATION WITH RELIEF ON ONE OR TWO SIDES ................................. 138

7.3.1 Coin Feature Scanning File Conversion ……............................................... 139

7.3.2 3D FEM Coining Process Simulation with Surface Designs......................... 140

\section{CHAPTER 8}

CONCLUSIONS AND RECOMMENDATIONS .......................................................... 172

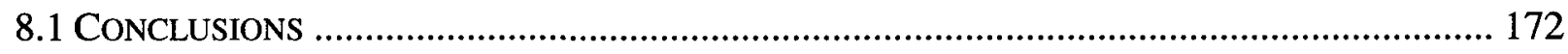

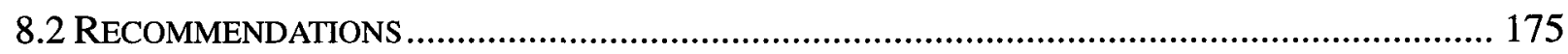

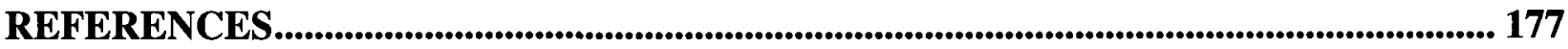

Appendix 


\section{List of Tables}

Table 4-1. Impurities in commercial fine silver...........................................................53

Table 4-2. Tensile test data of fine silver...............................................54

Table 4-3. The true stress-strain data of fine silver......................................55

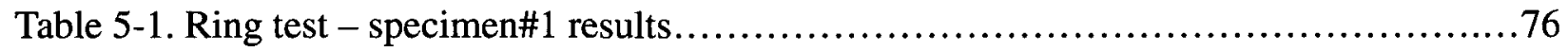

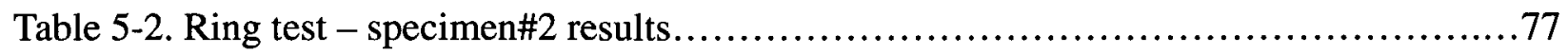

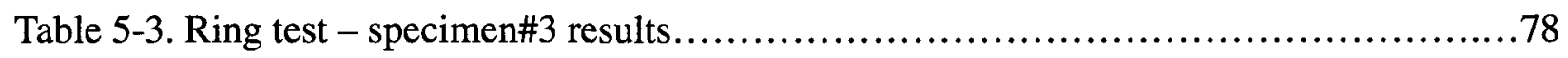

Table 5-4. Ring test - specimen\#4 results......................................... 79

Table 5-5. Ring test - specimen\#5 results........................................... 80

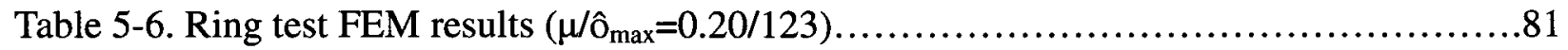

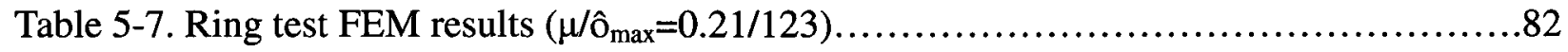

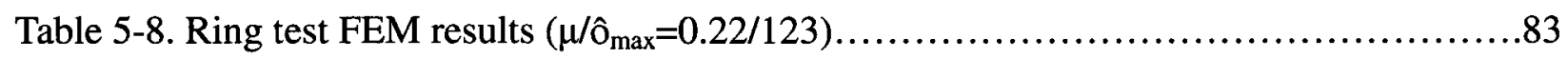

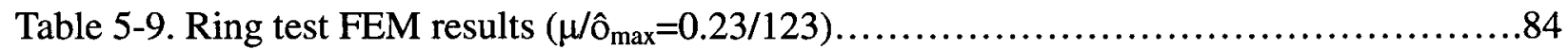

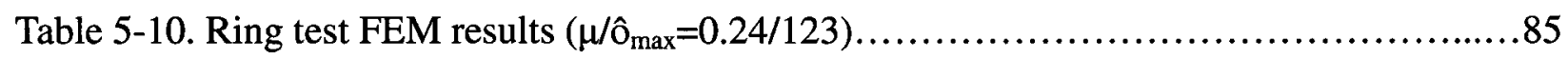

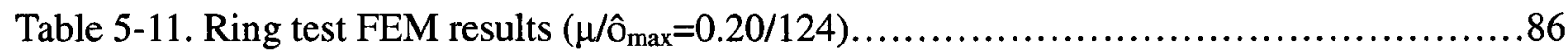

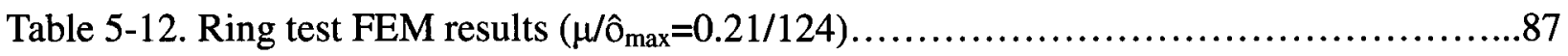

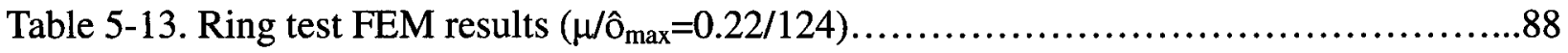

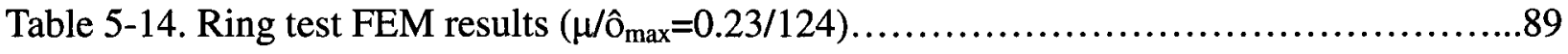

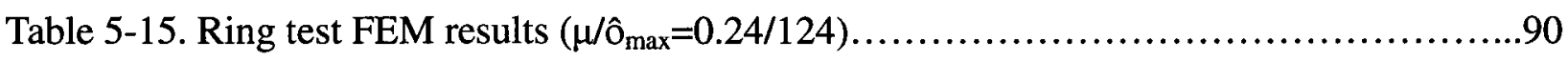

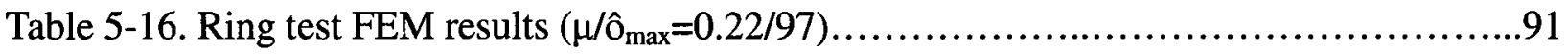

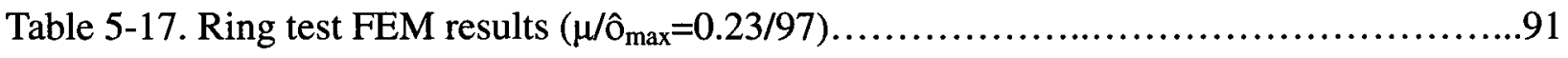

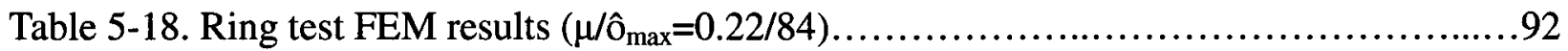




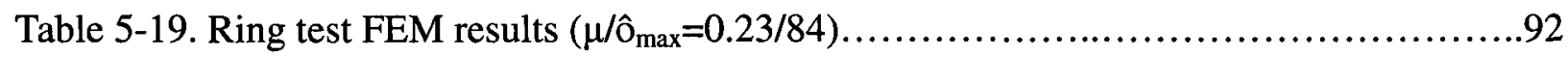

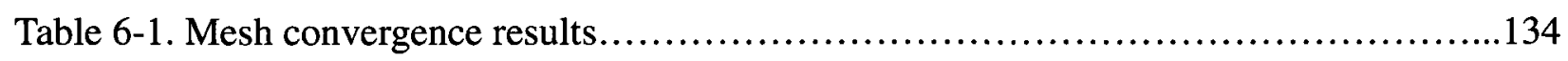

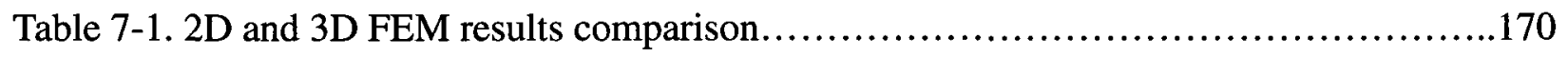

Table 7-2. 3D coining model comparison............................................. 170

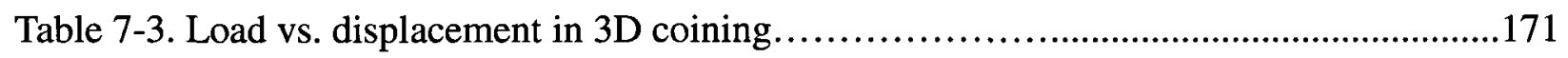

Table A-1. Load against displacement results in ring test..............................219

Table A-2a. Load Vs Displacement with varying top curvature...........................220

Table A-2b. Load Vs Displacement with varying top curvature............................221

Table A-3a. Load Vs Displacement with varying bottom curvature.........................222

Table A-3b. Load Vs Displacement with varying bottom curvature...........................223

Table A-4a. Load Vs Displacement with varying rim height............................224

Table A-4b. Load Vs Displacement with varying rim height..............................225

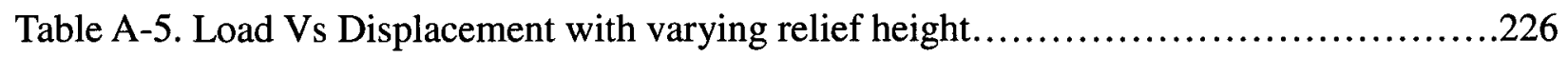




\section{List of Figures}

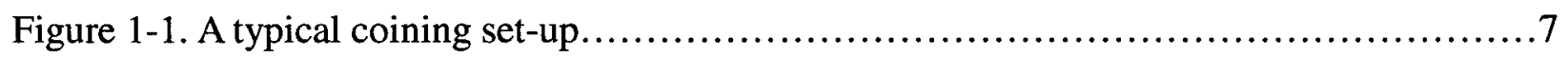

Figure 1-2. Development approach for design tools for coining process $\ldots \ldots \ldots \ldots \ldots \ldots \ldots \ldots . .8$

Figure 2-1. Schematic representation of the die set-up utilized for producing coins

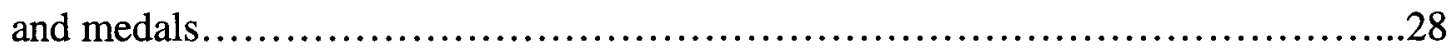

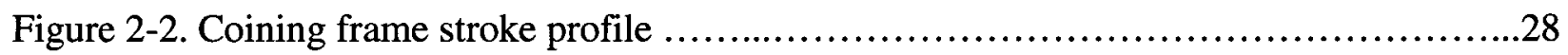

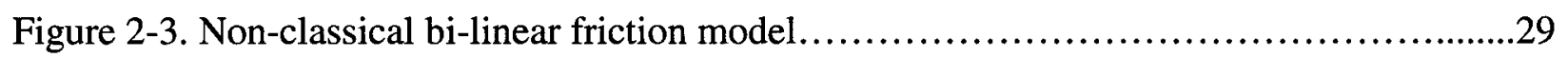

Figure 2-4. The two types of bimetallic coinage .......................................29

Figure 3-1. Comparison of implicit and explicit solution costs [ABAQUS, 2006].................39

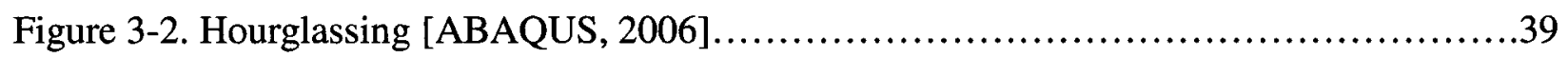

Figure 3-3. Adaptive remeshing process [ABAQUS, 2006] ................................40

Figure 3-4. Motion of mesh and material with various methods [ABAQUS, 2006] .............41

Figure 3-5. Comparison of non-adaptive meshing and adaptive meshing analysis

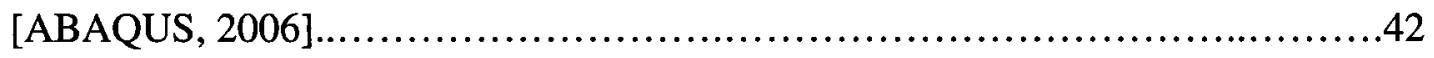

Figure 3-6. Deformed mesh in a ALE simulation [ABAQUS, 2006].........................42

Figure 3-7. 2D axisymmetric ring upsetting FEM model..................................43

Figure 3-8. Initial mesh of the simulation................................................43

Figure 3-9. Deformed mesh of the simulation................................................

Figure 3-10. FEM simulation results with different stiffness models..........................44

Figure 3-11. FEM simulation results with different hour-glass controls.....................44

Figure 3-12. FEM simulations results with different contact constraints.......................45

Figure 3-13. FEM simulation results with different adaptive meshing........................45 
Figure 4-1. Uni-axial tensile test specimen ..........................................50

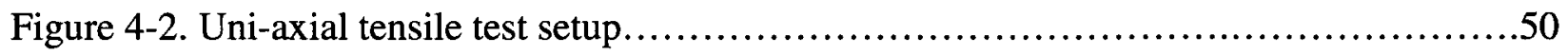

Figure 4-3. Extensometer setup on specimen.........................................51

Figure 4-4a. Experimental strain hardening model of fine silver..........................52

Figure 4-4b. The averages engineering stress-strain curve of fine silver.....................52

Figure 4-5. The averaged true stress-strain model of fine silver...........................53

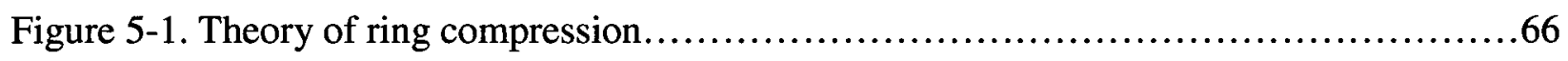

Figure 5-2. A typical calibration curve of ring compression test (copper)

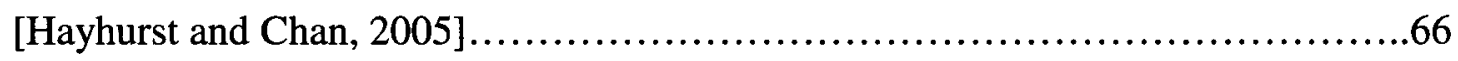

Figure 5-3. Two platens made of alloyed tool steel.........................................67

Figure 5-4. Ring positioned at the center of platens....................................67

Figure 5-5. a) Original and b) deformed silver ring....................................68

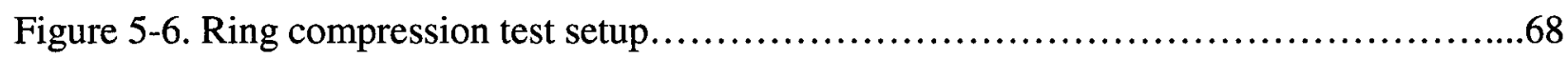

Figure 5-7. Experimental results [Guo. K. et. al., 2008]..................................69

Figure 5-8. FEM model of ring compression test....................................69

Figure 5-9. a) Original FEM mesh b) Deformed FEM mesh of ring compression test..................70

Figure 5-10. Calibration curves achieved by FEM method [Guo. K. et. al., 2008]................71

Figure 5-11. Experimental data of ring test and best fitting curve...........................71

Figure 5-12. The fitting curve of Experimental data against the calibration curves...............72

Figure 5-13. The fitting curve of Experimental data against the calibration curves...............72

Figure 5-14. The fitting curve of Experimental data against the calibration curves...............73

Figure 5-15. Experimental and FEM loads at the final step.................................73

Figure 5-16. Shear stress contour in ring test........................................... 74

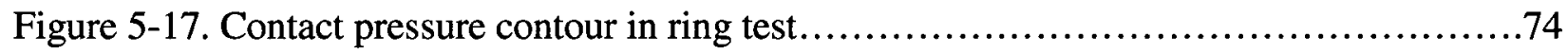


Figure 5-18. Nominal contact pressure development in experimental and FEM ring test.........75

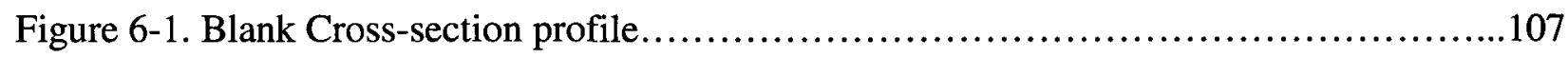

Figure $6-2$. The corss-section of a rimmed blank....................................... 107

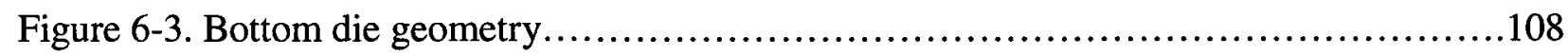

Figure 6-4. 2D axisymmetric coining FEM model in coarse mesh (2000 elements)............109

Figure 6-5. 2D axisymmetric coining FEM model in refined mesh (8000 elements)...............110

Figure 6-6. Load vs. displacement for mesh convergence test...........................111

Figure 6-7. Coining FEM results in different hourglass control approaches..................111

Figure 6-8. Coining FEM results in different hourglass scale factor combinations.............112

Figure 6-9. Coining FEM results in different contact controls............................112

Figure 6-10. Coining FEM results in different adaptive meshing controls....................113

Figure 6-11. The nominal contact pressure development in coining.........................113

Figure 6-12. Metal deformed mesh patterns showing outer $20 \%$ of blank

(Top die curvature $=0.3 \mathrm{~mm}$, Bottom die curvature $=0.19 \mathrm{mmm}$,

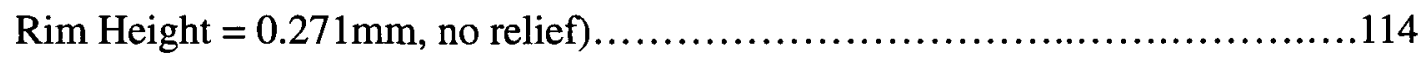

Figure 6-13. Material flow velocity patterns showing outer $20 \%$ of blank

(Top die curvature $=0.3 \mathrm{~mm}$, Bottom die curvature $=0.19 \mathrm{~mm}$,

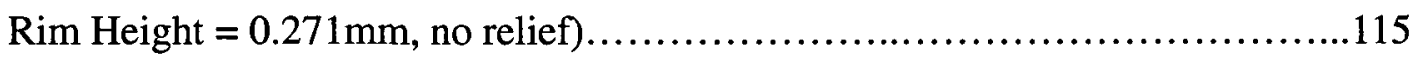

Figure 6-14. Von Mises stress contour (Top die curvature $=0.3 \mathrm{~mm}$, Bottom die

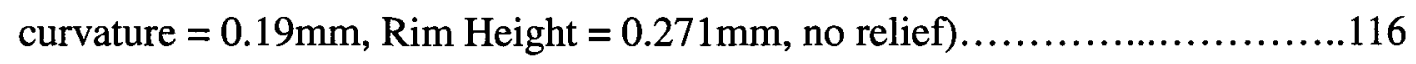

Figure 6-15. Equivalent plastic strain contour (Top die curvature $=0.3 \mathrm{~mm}$,

Bottom die curvature $=0.19 \mathrm{~mm}$, Rim Height $=0.271 \mathrm{~mm}$, no relief $) \ldots \ldots \ldots \ldots \ldots 117$ 
Figure 6-16. Metal deformed mesh patterns showing outer 20\% of blank

(Top die curvature $=0.3 \mathrm{~mm}$, Bottom die curvature $=0.3 \mathrm{~mm}$,

Rim Height $=0.271 \mathrm{~mm}$, no relief)

Figure 6-17. Material flow velocity patterns showing outer $20 \%$ blank

(Top die curvature $=0.3 \mathrm{~mm}$, Bottom die curvature $=0.3 \mathrm{~mm}$,

Rim Height $=0.271 \mathrm{~mm}$, no relief)

Figure 6-18. Von Mises stress contour $($ Top die curvature $=0.3 \mathrm{~mm}$, Bottom die

curvature $=0.3 \mathrm{~mm}$, Rim Height $=0.271 \mathrm{~mm}$, no relief)

Figure 6-19. Equivalent plastic strain contour (Top die curvature $=0.3 \mathrm{~mm}$, Bottom die curvature $=0.3 \mathrm{~mm}$, Rim Height $=0.271 \mathrm{~mm}$, no relief $)$

Figure 6-20. Metal deformed mesh patterns showing outer 20\% of blank

(Top die curvature $=0.3 \mathrm{~mm}$, Bottom die curvature $=0.19 \mathrm{~mm}$,

Rim Height $=0.3 \mathrm{~mm}$, no relief) .

Figure 6-21. Material flow velocity patterns showing outer $20 \%$ of blank

(Top die curvature $=0.3 \mathrm{~mm}$, Bottom die curvature $=0.19 \mathrm{~mm}$,

Rim Height $=0.3 \mathrm{~mm}$, no relief)

Figure 6-22. Von Mises stress contour (Top die curvature $=0.3 \mathrm{~mm}$, Bottom die curvature $=0.19 \mathrm{~mm}$, Rim Height $=0.3 \mathrm{~mm}$, no relief)

Figure 6-23. Equivalent plastic strain contour (Top die curvature $=0.3 \mathrm{~mm}$, Bottom die curvature $=0.19 \mathrm{~mm}$, Rim Height $=0.3 \mathrm{~mm}$, no relief)

Figure 6-24. Metal deformed mesh patterns showing outer 55\% of blank

(Top die curvature $=0.3 \mathrm{~mm}$, Bottom die curvature $=0.19 \mathrm{~mm}$,

Rim Height $=0.271 \mathrm{~mm}$, relief $=0.10 \mathrm{~mm}$ ) 
Figure 6-25. Material flow velocity patterns showing outer 55\% of blank

(Top die curvature $=0.3 \mathrm{~mm}$, Bottom die curvature $=0.19 \mathrm{~mm}$,

Rim Height $=0.271 \mathrm{~mm}$, relief $=0.10 \mathrm{~mm})$

Figure 6-26. Von Mises stress contour (Top die curvature $=0.3 \mathrm{~mm}$, Bottom die curvature $=0.19 \mathrm{~mm}$, Rim Height $=0.271 \mathrm{~mm}$, relief $=0.10 \mathrm{~mm}$ )

Figure 6-27. Equivalent plastic strain contour (Top die curvature $=0.3 \mathrm{~mm}$, Bottom die curvature $=0.19 \mathrm{~mm}$, Rim Height $=0.271 \mathrm{~mm}$, relief $=0.10 \mathrm{~mm}$.

Figure $6-28$. Loading paths with varying top die curvatures............................... 130

Figure 6-28b. Loads at $100 \%$ axial stroke with varying top die curvatures.....................130

Figure 6-29a. Loading paths with varying bottom die curvatures.........................131

Figure 6-29b. Lads at $100 \%$ axial stroke with varying bottom die curvatures..................131

Figure 6-30a. Loading paths with varying rim heights................................... 132

Figure 6-30b. Loads at $100 \%$ axial stroke with varying rim heights......................... 132

Figure 6-31a. Loading paths with varying relief heights....................................

Figure 6-31b. Loads at $100 \%$ axial stroke with varying relief heights........................133

Figure 7-1. Three-dimensional silver blank mesh created with brick elements.................144

Figure 7-2. Simplified 3D finite element coining model................................ 144

Figure 7-3. Deformed blank in 3D coining simulation.................................. 145

Figure 7-4. 2D and 3D FEM simulation results comparison...............................146

Figure 7-5a. Serration geometry [Royal Canadian Mint, 2007] ..........................147

Figure 7-5b. FEM Coining configuration with serrations on side ring ......................147

Figure 7-6. Deformed 3D coining blank with serrations..................................148

Figure 7-7. Von Mises stress contour on the simple coin with serrations......................149

Figure 7-8. Equivalent plastic strain contour on the simple coin with serrations................149 
Figure 7-9. 3D coining model comparison ............................................ 150

Figure 7-10. Design features of a coin by scanning ....................................... 151

Figure 7-11. Solid coin profile FEM model........................................... 152

Figure 7-12. A silver dollar coin design at RCM.....................................153

Figure 7-13a. Reverse die and its cross-section of the coining die with one-side relief.........154

Figure 7-13b. Obverse die and its cross-section of the coining die with one-side relief........155

Figure 7-14a. Reverse die and its cross-section of the coining die with two-side relief..........156

Figure 7-14b. Obverse die and its cross-section of the coining die with two-side relief........157

Figure 7-15. Rigid die mesh of one-side relief model.................................158

Figure 7-16. Rigid die mesh of two-side relief model..................................159

Figure 7-17. The side ring inner surface mesh...........................................

Figure $7-18$. The coining simulation assembly sketch..................................160

Figure 7-19. Deformed mesh of the one-side relief model...................................161

Figure 7-20. The Von Mises stress contour of the one-side relief model.......................162

Figure 7-21. The equivalent plastic strain contour of the one-side relief model...............163

Figure 7-22. The Von Mises stress contour on the cross-section of one-side relief model.......164

Figure 7-23. The equivalent plastic strain contour on the cross-section of one-side relief model....................................................164

Figure 7-24. Deformed mesh of the two-side relief model................................165

Figure 7-25. The Von Mises stress contour of the two-side relief model........................166

Figure 7-26. The equivalent plastic strain contour of the two-side relief model................167

Figure 7-27. The Von Mises stress contour on the cross-section of two-side relief model.......168

Figure 7-28. The equivalent plastic strain contour on the cross-section of two-side relief model 168 
Figure 7-29. Loading path in 3D coining.

Figure A-1. Metal deformed mesh patterns showing outer $20 \%$ of blank

(Top die curvature $=0.05 \mathrm{~mm}$, Bottom die curvature $=0.19 \mathrm{mmm}$,

Rim Height $=0.271 \mathrm{~mm}$, no relief)

Figure A-2. Metal deformed mesh patterns showing outer $20 \%$ of blank

(Top die curvature $=0.1 \mathrm{~mm}$, Bottom die curvature $=0.19 \mathrm{~mm}$,

Rim Height $=0.271 \mathrm{~mm}$, no relief $)$

Figure A-3. Metal deformed mesh patterns showing outer $20 \%$ of blank

(Top die curvature $=0.15 \mathrm{~mm}$, Bottom die curvature $=0.19 \mathrm{~mm}$,

Rim Height $=0.271 \mathrm{~mm}$, no relief $)$

Figure A-4. Metal deformed mesh patterns showing outer $20 \%$ of blank

(Top die curvature $=0.2 \mathrm{~mm}$, Bottom die curvature $=0.19 \mathrm{~mm}$,

Rim Height $=0.271 \mathrm{~mm}$, no relief)

Figure A-5. Metal deformed mesh patterns showing outer 20\% of blank

(Top die curvature $=0.25 \mathrm{~mm}$, Bottom die curvature $=0.19 \mathrm{~mm}$,

Rim Height $=0.271 \mathrm{~mm}$, no relief $)$.

Figure A-6. Material flow velocity patterns showing outer $20 \%$ of blank

(Top die curvature $=0.05 \mathrm{~mm}$, Bottom die curvature $=0.19 \mathrm{~mm}$,

Rim Height $=0.271 \mathrm{~mm}$, no relief)

Figure A-7. Material flow velocity patterns showing outer $20 \%$ of blank

(Top die curvature $=0.1 \mathrm{~mm}$, Bottom die curvature $=0.19 \mathrm{~mm}$,

Rim Height $=0.271 \mathrm{~mm}$, no relief) 
Figure A-8. Material flow velocity patterns showing outer $20 \%$ of blank

(Top die curvature $=0.15 \mathrm{~mm}$, Bottom die curvature $=0.19 \mathrm{~mm}$,

Rim Height $=0.271 \mathrm{~mm}$, no relief .

Figure A-9. Material flow velocity patterns showing outer $20 \%$ of blank

(Top die curvature $=0.2 \mathrm{~mm}$, Bottom die curvature $=0.19 \mathrm{~mm}$,

Rim Height $=0.271 \mathrm{~mm}$, no relief)

Figure A-10. Material flow velocity patterns showing outer $20 \%$ of blank

(Top die curvature $=0.25 \mathrm{~mm}$, Bottom die curvature $=0.19 \mathrm{~mm}$,

Rim Height $=0.271 \mathrm{~mm}$, no relief)

Figure A-11. Metal deformed mesh patterns showing outer $20 \%$ of blank

(Top die curvature $=0.3 \mathrm{~mm}$, Bottom die curvature $=0.05 \mathrm{~mm}$,

Rim Height $=0.271 \mathrm{~mm}$, no relief $)$

Figure A-12. Metal deformed mesh patterns showing outer $20 \%$ of blank

(Top die curvature $=0.3 \mathrm{~mm}$, Bottom die curvature $=0.1 \mathrm{~mm}$,

Rim Height $=0.271 \mathrm{~mm}$, no relief)

Figure A-13. Metal deformed mesh patterns showing outer $20 \%$ of blank

(Top die curvature $=0.3 \mathrm{~mm}$, Bottom die curvature $=0.15 \mathrm{~mm}$,

Rim Height $=0.271 \mathrm{~mm}$, no relief)

Figure A-14. Metal deformed mesh patterns showing outer 20\% of blank

(Top die curvature $=0.3 \mathrm{~mm}$, Bottom die curvature $=0.2 \mathrm{~mm}$,

Rim Height $=0.271 \mathrm{~mm}$, no relief)

Figure A-15. Metal deformed mesh patterns showing outer 20\% of blank

(Top die curvature $=0.3 \mathrm{~mm}$, Bottom die curvature $=0.25 \mathrm{~mm}$,

Rim Height $=0.271 \mathrm{~mm}$, no relief) 
Figure A-16. Material flow velocity patterns showing outer $20 \%$ of blank (Top die curvature $=0.3 \mathrm{~mm}$, Bottom die curvature $=0.05 \mathrm{~mm}$, Rim Height $=0.271 \mathrm{~mm}$, no relief)

Figure A-17. Material flow velocity patterns showing outer $20 \%$ of blank (Top die curvature $=0.3 \mathrm{~mm}$, Bottom die curvature $=0.1 \mathrm{~mm}$, Rim Height $=0.271 \mathrm{~mm}$, no relief) 199

Figure A-18. Material flow velocity patterns showing outer $20 \%$ of blank

(Top die curvature $=0.3 \mathrm{~mm}$, Bottom die curvature $=0.15 \mathrm{~mm}$,

Rim Height $=0.271 \mathrm{~mm}$, no relief) .200

Figure A-19. Material flow velocity patterns showing outer $20 \%$ of blank

(Top die curvature $=0.3 \mathrm{~mm}$, Bottom die curvature $=0.2 \mathrm{~mm}$,

Rim Height $=0.271 \mathrm{~mm}$, no relief) .201

Figure A-20. Material flow velocity patterns showing outer $20 \%$ of blank

(Top die curvature $=0.3 \mathrm{~mm}$, Bottom die curvature $=0.25 \mathrm{~mm}$,

Rim Height $=0.271 \mathrm{~mm}$, no relief) .202

Figure A-21. Metal deformed mesh patterns showing outer $20 \%$ of blank

(Top die curvature $=0.3 \mathrm{~mm}$, Bottom die curvature $=0.19 \mathrm{~mm}$,

Rim Height $=0.18 \mathrm{~mm}$, no relief) .203

Figure A-22. Metal deformed mesh patterns showing outer $20 \%$ of blank

(Top die curvature $=0.3 \mathrm{~mm}$, Bottom die curvature $=0.19 \mathrm{~mm}$,

Rim Height $=0.2 \mathrm{~mm}$, no relief) .204

Figure A-23. Metal deformed mesh patterns showing outer $20 \%$ of blank

(Top die curvature $=0.3 \mathrm{~mm}$, Bottom die curvature $=0.19 \mathrm{~mm}$,

Rim Height $=0.22 \mathrm{~mm}$, no relief) .205

xix 
Figure A-24. Metal deformed mesh patterns showing outer $20 \%$ of blank (Top die curvature $=0.3 \mathrm{~mm}$, Bottom die curvature $=0.19 \mathrm{~mm}$, Rim Height $=0.24 \mathrm{~mm}$, no relief $)$ .206

Figure A-25. Metal deformed mesh patterns showing outer $20 \%$ of blank (Top die curvature $=0.3 \mathrm{~mm}$, Bottom die curvature $=0.19 \mathrm{~mm}$, Rim Height $=0.26 \mathrm{~mm}$, no relief) .207

Figure A-26. Metal deformed mesh patterns showing outer $20 \%$ of blank (Top die curvature $=0.3 \mathrm{~mm}$, Bottom die curvature $=0.19 \mathrm{~mm}$,

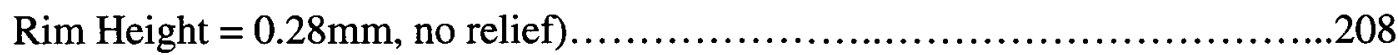

Figure A-27. Material flow velocity patterns showing outer $20 \%$ of blank

(Top die curvature $=0.3 \mathrm{~mm}$, Bottom die curvature $=0.19 \mathrm{~mm}$, Rim Height $=0.18 \mathrm{~mm}$, no relief) .209

Figure A-28. Material flow velocity patterns showing outer $20 \%$ of blank (Top die curvature $=0.3 \mathrm{~mm}$, Bottom die curvature $=0.19 \mathrm{~mm}$, Rim Height $=0.2 \mathrm{~mm}$, no relief $)$ .210

Figure A-29. Material flow velocity patterns showing outer $20 \%$ of blank (Top die curvature $=0.3 \mathrm{~mm}$, Bottom die curvature $=0.19 \mathrm{~mm}$, Rim Height $=0.22 \mathrm{~mm}$, no relief)

Figure A-30. Material flow velocity patterns showing outer $20 \%$ of blank (Top die curvature $=0.3 \mathrm{~mm}$, Bottom die curvature $=0.19 \mathrm{~mm}$, Rim Height $=0.24 \mathrm{~mm}$, no relief) .212

Figure A-31. Material flow velocity patterns showing outer $20 \%$ of blank (Top die curvature $=0.3 \mathrm{~mm}$, Bottom die curvature $=0.19 \mathrm{~mm}$, Rim Height $=0.26 \mathrm{~mm}$, no relief) .213 
Figure A-32. Material flow velocity patterns showing outer $20 \%$ of blank (Top die curvature $=0.3 \mathrm{~mm}$, Bottom die curvature $=0.19 \mathrm{~mm}$, Rim Height $=0.28 \mathrm{~mm}$, no relief) .214

Figure A-33. Metal deformed mesh patterns showing outer 55\% of blank (Top die curvature $=0.3 \mathrm{~mm}$, Bottom die curvature $=0.19 \mathrm{~mm}$, Rim Height $=0.271 \mathrm{~mm}$, relief $=0.08 \mathrm{~mm})$ 215

Figure A-34. Metal deformed mesh patterns showing outer 55\% of blank

(Top die curvature $=0.3 \mathrm{~mm}$, Bottom die curvature $=0.19 \mathrm{~mm}$,

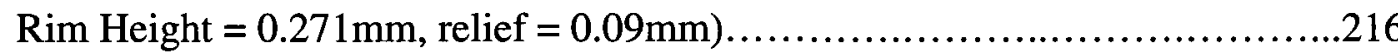

Figure A-35. Material flow velocity patterns showing outer 55\% of blank (Top die curvature $=0.3 \mathrm{~mm}$, Bottom die curvature $=0.19 \mathrm{~mm}$, Rim Height $=0.271 \mathrm{~mm}$, relief $=0.08 \mathrm{~mm})$ 217

Figure A-36. Material flow velocity patterns showing outer 55\% of blank (Top die curvature $=0.3 \mathrm{~mm}$, Bottom die curvature $=0.19 \mathrm{~mm}$, Rim Height $=0.271 \mathrm{~mm}$, relief $=0.08 \mathrm{~mm})$ .218 


\section{Nomenclature}

\section{Greek Symbols}

\begin{tabular}{|c|c|}
\hline$\sigma$ & Stress tensor \\
\hline$\sigma_{\mathrm{V}}$ & Volumetric stress \\
\hline$\sigma_{\mathrm{D}}$ & Deviatoric deviators \\
\hline$\sigma_{\mathrm{f}}$ & Flow stress \\
\hline$\sigma_{\mathrm{u}}$ & Ultimate strength \\
\hline$\sigma_{\mathrm{y}}$ & Yield stress \\
\hline$\varepsilon$ & Strain tensor \\
\hline $\bar{\varepsilon}$ & Axial strain \\
\hline$\varepsilon_{\mathrm{v}}$ & Volumetric strain \\
\hline$\varepsilon_{\mathrm{D}}$ & Deviatoric strain \\
\hline$\varepsilon_{\text {norm }}$ & Normal strain \\
\hline$\varepsilon_{\ln }$ & Logarithmic total strain \\
\hline$\varepsilon_{\mathrm{ln}}^{p l}$ & Logarithmic (true) strain \\
\hline $\mathrm{d} \varepsilon_{\mathrm{ij}}$ & Total strain increment \\
\hline$\varepsilon^{\mathrm{p}}$ & Plastic strain \\
\hline$\varepsilon^{\mathrm{e}}$ & Elastic strain \\
\hline$\mu$ & Coulomb's coefficient of friction \\
\hline$v_{\mathrm{T}}$ & Tangent Poisson's ratio \\
\hline$\sigma_{\mathrm{y}}$ & Yield strength in pure shear \\
\hline$\sigma_{0}$ & Yield stress in tension \\
\hline$\sigma_{i}(i=1,2,3)$ & Principle values of the stress tensor \\
\hline $\bar{\sigma}$ & Axial/effective stress \\
\hline$\sigma_{\text {norm }}$ & Normal stress \\
\hline$\sigma_{\text {true }}$ & True stress \\
\hline$\Delta$ & A finite increment \\
\hline
\end{tabular}




\section{Latin Symbols}

ALE

[B]

$\mathrm{C}$

[C( $\sigma)]$

$\left[\mathrm{C}^{\mathrm{e}}\right]$

$\left[\mathrm{C}^{\mathrm{p}}\right]$

$\left[\mathrm{C}^{\mathrm{ep}}\right]$

[D]

$\mathrm{E}_{\mathrm{T}}$

$f$

$f(\sigma)$

FEM

G

$\mathrm{I}_{\mathrm{i}}(\mathrm{i}=1,2,3)$

$\mathrm{J}_{\mathrm{i}}(\mathrm{i}=1,2,3)$

$\mathrm{J}_{\mathrm{D} 2}$

$\mathrm{K}$

k

[k]

$\left[\mathrm{k}_{\mathrm{G}}\right]$

$\mathrm{m}$

M

$\mathrm{p}$

[qi]

$\left[\mathrm{Q}_{\mathrm{i}}\right]$

$\mathrm{s}^{\mathrm{s}}, \mathrm{s}^{\mathrm{r}}, \mathrm{s}^{\mathrm{w}}$

$\mathrm{V}$

Y
Arbitrary Lagrangian Eulerian

Transformation matrix

Constant

Tangent stiffness matrix

Elasticity matrix

Plasticity matrix

Elastic-plastic matrix

Constitutive matrix

Tangent modulus

Friction shear stress

Yield function

Finite element method

Shear modulus

Strain invariant

Stress invariant

Second deviatoric stress invariant

Bulk modulus

Shear strength

Stiffness matrix

Geometric stiffness

Friction factor

Total number of elements

Compressive normal stress

Displacements matrix

Loads matrix

Hourglass scaling factors

Element volume

Yield stress in uniaxial tension 


\section{Chapter 1}

\section{Introduction}

During the last few years, the interest of the industries in innovative applications based on the miniaturization technology has been continuously growing [Engel and Eckstein, 2003] [Geiger et. al, 2001]. Due to the requirement to produce highly integrated and compact components, the industries have been demanding a reliable technology able to ensure large production volumes and, at the same time, the high quality standards required to the final products in terms of final dimensions, tolerances and surface finish. To achieve such high standards, it is necessary to determine the optimum means of producing sound products. The optimization criteria may vary, depending on product requirements, but establishing an appropriate criterion requires a thorough understanding of the manufacturing process. In metal-forming technology, proper design and control require, among other things, the determination of deformation mechanics involved in the processes. Without the knowledge of the influences of variables such as friction conditions, material properties, and workpiece geometry on the process mechanics, it would not be possible to design the dies and the equipment adequately, or to predict and prevent the occurrence of defects.

In the last thousand years, coining has been the major process of producing coins. In such process, a billet of material is plastically deformed by dies into another, more complex shape in a short time with the aim to impress three-dimensional image on its three surfaces, as shown in Figure 1-1. Such cold forging process is characterized by two main features that differentiate it from the traditional closed-die forging, namely the small ratio between the final surface geometry pattern and the workpiece diameter, and the small ratio between the obtained protrusion height - or cavity depth - and the workpiece thickness [Ike and Plancak, 1998]. The dies are used to determine the final geometric configuration of the coining. The ideal coining die design will produce a near net-shape coining. It has to be noted that despite coining being one of 
the most ancient metal forming technologies, and it is used all over the world for a very long time, technological research publications on the coining process are somewhat limited. Coining is in fact widely used for the currency production, developed by the governments, and the available know-how is usually kept secret. Among the limited research published, Wang [Wang et. al., 2007] performed experimental coining investigation on pure aluminum billets to study the effects of die cavity dimension on the microforming ability. Buffa [Buffa et. al., 2007] investigated the relationships between the obtained geometry and the starting preform shape and highlighted the significance of the preform design in order to obtain a final product that satisfies the strict geometrical tolerances required. Choi [Choi et. al., 1997] developed the rigid-plastic finite-element method for process design in three-dimensional plastic deformation and applied it to a precision coining process. Leitao investigated the production of bimetallic coin [Leitao et al, 1997]. More related background review will be shown in Chapter 2.

For a long time, die and process design in the Royal Canadian Mint has been following the conventional method which is based largely on empirical formulas, intuition and past experience. There has been no systematic analysis. Thus, the development of these dies has been part art and part science. If a design is an entirely new configuration, it may take several attempts to determine an acceptable die and billet configuration, which may or may not be optimized to minimize flash, internal voids, or other material irregularities. This current conventional process is expensive and time-consuming due to the hours required and the material waste created from attempts to manufacture an acceptable coin. In addition, the empirical approach does not predict internal material flows for complex coin designs, which are important for the quality control in the coining process. To get a maximum die life and proper die-workpiece contact surface profile design, understanding the coining process mechanics and metal flow becomes a key issue. The metal flow and process mechanics are controlled by both material properties (strain-hardenability, anisotropy, etc.) and the boundary conditions (die geometry, blank size, friction condition, etc.). They can be investigated using numerical simulation approaches.

During the last decade, research has been performed in the area using finite element 
analysis (FEA) for simulation of metal forming operations, including forging [Rowe et al, 1991]. Recent advances in finite element analysis methods, computer hardware and software developments make it feasible to use finite element analysis to simulate the coining process. The application of quantitative numerical simulations of the coining process will be necessary and common practice in the coining process design in the near future. The finite element approach for die design provides a means of predicting load, material flow, stress and strain conditions, without the material and labour costs associated with a trial and error manufacturing technique. It also helps to identify and correct potential coining problems prior to die fabrication, cut time and costs associated with tooling rework, reduce overall effort needed for prototyping and decrease the number of trials and re-designs required. Two-dimensional analysis can be used to study the material flow for the axisymmetric coining process and three-dimensional analysis allows the prediction of material flow in all directions. Parameters such as die geometry, friction conditions and material properties are easily varied to determine the sensitivities associated with these parameters, thus producing an optimized, rather than just marginal design. Due to the high loads required for the process and the strict tolerances usually needed in the obtained part, a careful evaluation and choice of the proper operation parameters is needed [Ike, 2003][Bohm et. al., 2001][Kim et. al., 2006].

\subsection{Research Objectives}

This research focuses on the design tool development for the Canadian Silver Dollar coining process by the finite element method (FEM) and the issues associated with this simulation, such as obtaining the material properties and coining interface friction models though numerical and experimental approaches, as shown in Figure 1-2. Through FEM simulations, the initial design can be evaluated if the design targets are achieved, like uniform material flow, proper cavity filling and appropriate coining load. The results of the simulations will provide guidelines for the further modifications if the design targets were not achieved. The objective in this research is to develop a finite element design platform which not only generates simulation 
results for the silver dollar coining processes currently used at Royal Canadian Mint, but does so in a way which would be appropriate for any new configuration designs in the minting industry. The research is set to give comprehensive investigations on the effects of the design features in the tooling design process, and the results will help to reduce the design cycle of each new set of coins. The research was broken into five main thrusts:

(1) Development of the finite element procedure suitable for the simulation of coining process. This part of the investigation will provide a suitable numerical formulation and approach for the coining process simulation.

(2) Development of a more accurate material property model of fine silver.

(3) Development of a friction model between die and fine silver blank.

(4) Simulation of simplified 2D coining processes using finite element approaches. This part will verify the chosen numerical approach and document the variations and effects of the key design parameters.

(5) Simulation of 3D coining processes using finite element approaches. The final part will investigate the effects of serrations and relief.

Through these developments, the necessary components for a new coin design will be established, and can be applied in the coining process analysis.

\subsection{Dissertation Overview}

This dissertation documents the FEM analysis of the silver dollar coining process. The order of chapters in this document is organized to build on initial research and work up to chapters dealing with the research objectives. A brief summary of each chapter is provided here as part of this overview.

Chapter 1 is the introduction of the dissertation.

Chapter 2 provides the background information for a better understanding of the materials presented in the rest of the dissertation. The background material includes the detailed information on the mechanical background, finite element method and key assumptions of the 
coining process and their justifications. The recent coining related developments are presented at the end of the chapter.

Chapter 3 is the preliminary step to establish the finite element model for coining process. This chapter will examine the general finite element approaches and formulations, and will introduce and verify some key numerical parameters using an upsetting process FEM simulation. The application of the numerical parameter combination in the coining finite element simulation will be set up. With the fundamental modeling concerns addressed, it was possible to move on to the coining FEM modeling.

Chapter 4 addresses the mechanical properties of a typical material used at Royal Canadian Mint (RCM) - fine silver. The specifications of the mechanical properties of fine silver will be presented and the approaches to obtain such properties will be discussed. The resulting material properties are then available for use in the numerical modeling of the silver dollar coining process.

Chapter 5 discusses the principle friction behavior and its evaluation in coining operations. A nontraditional bilinear friction model will be obtained through numerical and experimental approaches, which is used as an input boundary conditions for the coining process simulations.

Chapter 6 details the development of a two-dimensional finite element model of silver dollar coining process. It starts with the 2D FEM model convergence discussion by using the results of mesh convergence and numerical parameter verification. Several key design parameters were investigated and their effects were discussed.

Chapter 7 starts by simulating a simplified three-dimensional coining process and the results from the simplified three-dimensional coining will be used to compare to the results of two-dimensional axisymmetric coining. The comparison was performed to show that three-dimensional models can be used as a coining process design tool. And a full-size three-dimensional coining with reduced industrial design features were investigated with the verified design tool using finite element approach. The results of the 3D features, like serrations and relief, were presented and discussed. 
Chapter 8 presents the general conclusions on the research followed by the recommendations for the possible future work on this topic. 


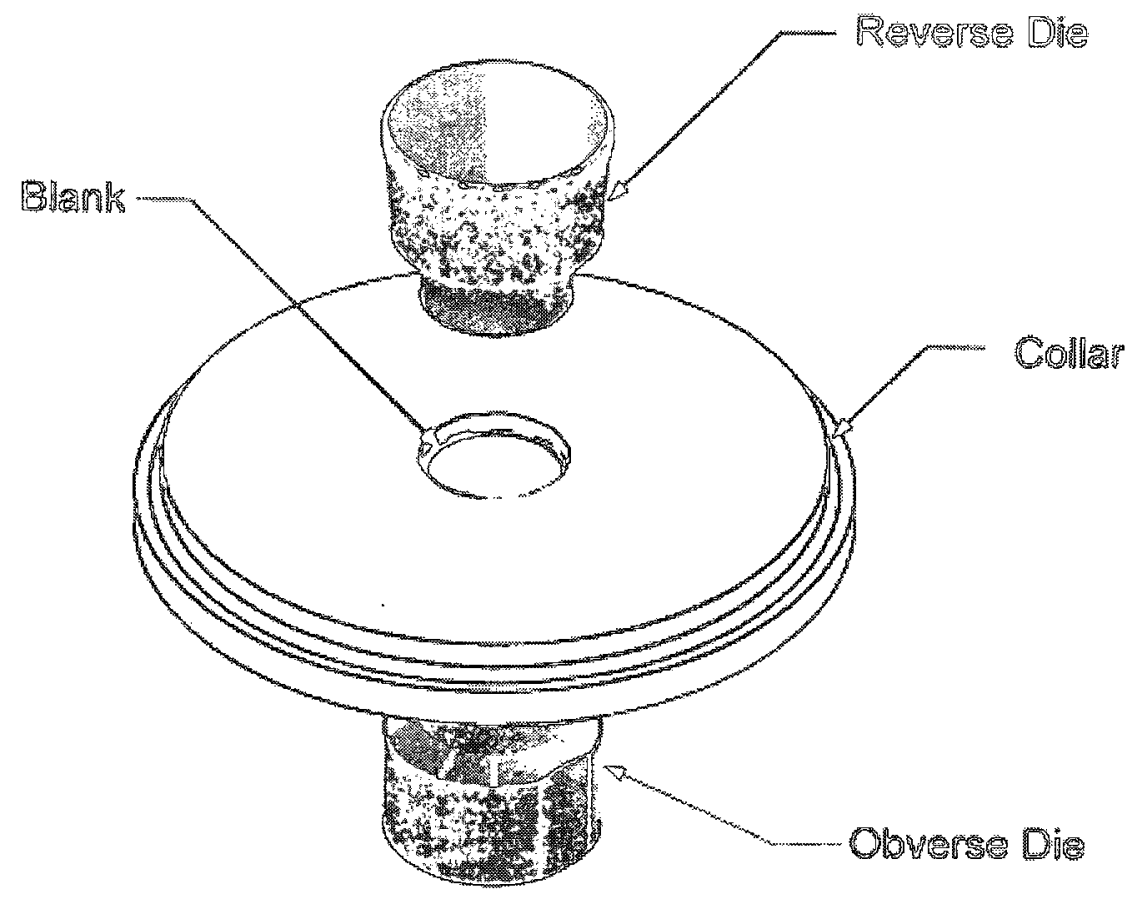

Figure 1-1. A typical coining set-up 


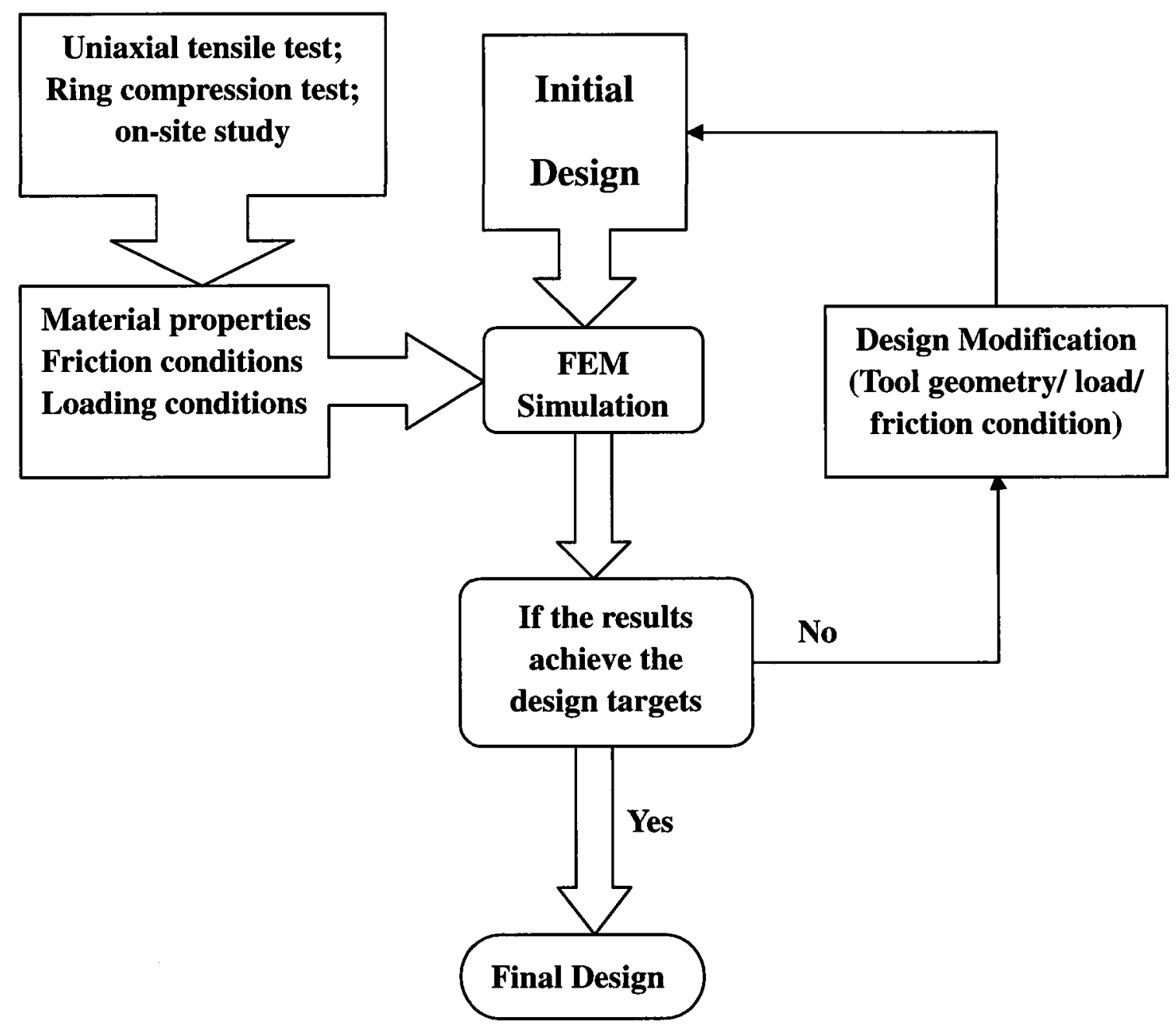

Figure 1-2. Development approach for design tools for coining process 


\section{Chapter 2}

\section{Background and Literature Review}

The design, control, and optimization of a forming process requires sound analytical knowledge regarding metal flow, load, stresses and strain as well as technological information related to die design, experimental and numerical methods. In this Chapter, the general material behavior mechanics and the numerical approach used for the research will be discussed. Some operational features in coining and the key assumptions used in the analysis will be discussed. And the general comments about the prior work and the recent developments related to metal forming and coining are also presented.

\subsection{Forging concepts and classifications}

Forging as a method of metalworking processes is broadly used in various industries. It is defined as a bulk, plastic deformation process of a metallic material, either hot or cold, to some predetermined shape primarily by compressive forces usually exerted by a hammer or press. It is called a bulk deformation process because a significant change in the thickness of the workpiece usually occurs as compared to a sheetmetal process [Edward, 1991]. Coining is a forging operation, in which a prepared blank is compressed between the dies whilst it is being retained and positioned by a collar. The result of a coining operation is a well-defined imprint of the dies on the disk (Fig. 2.1) [Leitao, 1997]. Coining is a very complex forging process from the point of view of the mechanics of deformation or of metal flow. It is difficult to analyze, because of such factors as material nonlinearity, large deformation and the variable interface friction between the material being deformed and the dies, all of which present a real challenge to evaluation and analysis. 


\subsection{Mechanical Background}

The purpose of this analysis in coining is to investigate the mechanics of the deformation process. The deformation mechanics provides the means of determining how the metal flows, how the desired geometry can be obtained, and what the expected loads of the coining process are. In coining process, the elasticity and plasticity will occur in the beginning and later stage of the process respectively. Thus, it is necessary to go through the mechanical background involved in the metal forming process.

There are many alternate, but equivalent forms to describe the mechanical governing equations. The equations prescribed in the following use the conventions in [Desai and Abel, 1982].

\subsubsection{Elasticity}

The basic idea of elasticity is: the deformation of a body depends only on its current loading, and is independent of history, rate, time, path, and other variables. Extensions of the idea can be made to include thermal strains (thermoelasticity) or time-dependent effects (viscoelasticity, anelasticity). That is, an unloaded elastic body always returns to its original shape, no matter what happens to it between the two unloaded times. The relationship between stress $(\sigma)$ and strain $(\varepsilon)$ can be presented as:

$$
\sigma=f(\varepsilon) \quad \text { or } \quad \varepsilon=f(\sigma)
$$

Within the proportional elastic limit, the simplest stress-strain equation, Hooke's law, can be used. For nonlinear elastic behavior the material parameters depend upon the state of stress. An incremental approach often used for nonlinear analysis approximates the nonlinear behavior as piecewise linear. Hence, the principles developed for linear elastic behavior become applicable in the range of each small increment. It is generally possible to obtain a relationship between small increments of stress and strain in the form

$$
\mathrm{E}_{\mathrm{T}}=\frac{\mathrm{d} \bar{\sigma}}{\mathrm{d} \bar{\varepsilon}}
$$

Where, $\mathrm{E}_{\mathrm{T}}$ is Tangent modulus at any point, $\bar{\sigma}$ and $\bar{\varepsilon}$ represent the axial stress and strain at this 
point. $\mathrm{E}_{\mathrm{T}}$ can be approximately evaluated by an expression such as

$$
E_{T} \cong \frac{\Delta \bar{\sigma}}{\Delta \bar{\varepsilon}}
$$

Where $\Delta$ denotes a finite increment.

If the experimental behavior is obtained from tests, the elastic parameters, such as tangent modulus and tangent Poisson's ratio $\mathrm{E}_{\mathrm{T}}$ and $v_{\mathrm{T}}$, can be appropriately defined. Then, the shear modulus

$$
G=\frac{E_{T}}{2\left(1+v_{T}\right)}
$$

And the bulk modulus

$$
K=\frac{E_{T}}{3\left(1-2 v_{T}\right)}
$$

The strain at a point can be decomposed into its volumetric $\left(\varepsilon_{\mathrm{v}}\right)$ and deviatoric $\left(\varepsilon_{\mathrm{D}}\right)$ components

$$
\begin{aligned}
& \{\varepsilon\}^{T}=\left\{\varepsilon_{v}\right\}+\left\{\varepsilon_{D}\right\} \\
& \left\{\varepsilon_{v}\right\}^{T}=\left[\begin{array}{llllll}
I_{1} / 3 & I_{1} / 3 & I_{1} / 3 & 0 & 0 & 0
\end{array}\right] \\
& \left\{\varepsilon_{D}\right\}^{T}=\left[\varepsilon_{x}-I_{1} / 3, \varepsilon_{y}-I_{1} / 3, \varepsilon_{z}-I_{1} / 3, \gamma_{x y}, \gamma_{y z}, \gamma_{z x}\right]=\left[\varepsilon_{D x} \varepsilon_{D y} \varepsilon_{D z} \gamma_{x y} \gamma_{y z} \gamma_{z x}\right]
\end{aligned}
$$

where $I_{1}$ is the strain invariant

$$
I_{1}=\varepsilon_{x}+\varepsilon_{y}+\varepsilon_{z}=\varepsilon_{1}+\varepsilon_{2}+\varepsilon_{3}
$$

where, $\varepsilon_{\mathrm{i}}(\mathrm{i}=1,2,3)$ are the principal strain

Similarly, the state of stress is the sum of the volumetric $\sigma_{v}$ and deviatoric stresses $\sigma_{\mathrm{D}}$

$$
\begin{aligned}
& \{\sigma\}^{\mathrm{T}}=\left\{\sigma_{v}\right\}^{\mathrm{T}}+\left\{\sigma_{\mathrm{D}}\right\}^{\mathrm{T}} \\
& \left\{\sigma_{v}\right\}^{\mathrm{T}}=\left[\begin{array}{llllll}
\mathrm{J}_{1} / 3 & \mathrm{~J}_{1} / 3 & \mathrm{~J}_{1} / 3 & 0 & 0 & 0
\end{array}\right] \\
& \left\{\sigma_{\mathrm{D}}\right\}^{\mathrm{T}}=\left[\sigma_{\mathrm{x}}-\mathrm{J}_{1} / 3, \sigma_{\mathrm{y}}-\mathrm{J}_{1} / 3, \sigma_{\mathrm{z}}-\mathrm{J}_{1} / 3, \tau_{\mathrm{xy}}, \tau_{\mathrm{yz}}, \tau_{\mathrm{zx}}\right] \\
& =\left[\sigma_{\mathrm{Dx}}, \sigma_{\mathrm{Dy}}, \sigma_{\mathrm{Dz}}, \tau_{\mathrm{xy}}, \tau_{\mathrm{yz}}, \tau_{\mathrm{zx}}\right]
\end{aligned}
$$

And the invariants of the stress

$$
\begin{aligned}
& \mathrm{J}_{1}=\sigma_{\mathrm{x}}+\sigma_{\mathrm{y}}+\sigma_{\mathrm{z}}=\sigma_{1}+\sigma_{2}+\sigma_{3} \\
& \mathrm{~J}_{2}=\sigma_{\mathrm{x}} \sigma_{\mathrm{y}}+\sigma_{\mathrm{y}} \sigma_{\mathrm{z}}+\sigma_{\mathrm{z}} \sigma_{\mathrm{x}}-\tau_{\mathrm{xy}}^{2}-\tau_{\mathrm{yz}}^{2}-\tau_{\mathrm{zx}}^{2}=\sigma_{1}+\sigma_{2}+\sigma_{3} \\
& \mathrm{~J}_{2}=\sigma_{\mathrm{x}} \sigma_{\mathrm{y}} \sigma_{\mathrm{z}}+2 \tau_{\mathrm{xy}} \tau_{\mathrm{yz}} \tau_{\mathrm{zx}}-\sigma_{\mathrm{x}} \tau_{\mathrm{yz}}^{2}-\sigma_{\mathrm{y}} \tau_{\mathrm{zx}}^{2}-\sigma_{\mathrm{z}} \tau_{\mathrm{xy}}^{2}=\sigma_{1} \sigma_{2} \sigma_{3}
\end{aligned}
$$

where, $\sigma_{i}(i=1,2,3)$ are the principal stress 
For general three-dimensional states of stress, the constitutive relation can be expressed as:

$$
\{\sigma\}=\left\{\sigma_{v}\right\}+\left\{\sigma_{D}\right\}=3 K\left\{\varepsilon_{v}\right\}+2 G\left\{\varepsilon_{D}\right\}
$$

\subsubsection{Plasticity}

In an elastic-plastic material, the linear elastic formulations can be used for the behavior prior to the proportional limit. In the zone between the proportional limit and the yield point, the nonlinear elastic behavior can be approximated by piecewise linear. However, the plastic behavior beyond the yield point may require a different approach.

To specify the critical or yield stress at which the plastic deformations initiate, a criterion is expressed through an equation of a yield surface

$$
f(\{\sigma\})=f\left(J_{1}, J_{2}, J_{3}\right)=0
$$

where, $\mathrm{J}_{1}, \mathrm{~J}_{2}, \mathrm{~J}_{3}$ are the stress invariants in Eq (2.9)

This is the equation of a surface in the three-dimensional space with coordinates $\sigma_{1}, \sigma_{2}, \sigma_{3}$, the principal stresses. If the state of stress is such that $\mathrm{f}<0$, the material is in the elastic range. When $\mathrm{f}=0$, a plastic state is attained and the plasticity theory must be used to determine subsequent plastic behavior under increasing stress or strain. Two simple criteria that are most generally used for the analysis of isotropic metal deformation are von Mises criterion and Tresca's criterion.

The von Mises yield criterion is one form of equation (2.11) and presumes that yielding is caused by the maximum distortion energy. The yield surface is

$$
f=J_{D 2}-\bar{\sigma}_{0}=0
$$

where $\bar{\sigma}_{0}$ indicates an experimentally determined yield stress and $J_{D 2}$ is the second deviatoric stress invariant. In terms of stresses, the von Mises criterion is

$$
f=\frac{1}{2}\left|\left(\sigma_{1}-\sigma_{2}\right)^{2}+\left(\sigma_{2}-\sigma_{3}\right)^{2}+\left(\sigma_{3}-\sigma_{1}\right)^{2}\right|-\bar{\sigma}_{0}^{2}=0
$$

Where, $\sigma_{i}(i=1,2,3)$ are the principal values of the stress tensor and $\sigma_{0}$ is the yield stress in tension.

- Uniaxial tension when 


$$
\sigma_{1}=\sigma_{0}, \quad \sigma_{2}=\sigma_{3}=0
$$

- Pure shear when

$$
\sigma_{1}=-\sigma_{3}=\tau_{Y}
$$

The Tresca criterion can be represented as

$$
f=4 J_{D 2}^{3}-27 J_{D 3}^{2}-36 C^{2} J_{D 2}^{2}+96 C^{4} J_{D 2}-64 C^{6}=0
$$

The yield surface is determined by the maximum shearing stress

$$
\sigma_{\max }-\sigma_{\min }=2 C \text { or } \sigma_{1}-\sigma_{3}=2 C \text { with } \sigma_{1} \geq \sigma_{2} \geq \sigma_{3}
$$

when Tresca criterion applies, the constant $\mathrm{C}$ can be readily obtained from the following simple, standard tests:

- For uniaxial tension, yielding will occur when $\sigma_{1}$ reaches the yield strength in tension, $\sigma_{0}$, i.e., when $\sigma_{1}=\sigma_{0}$ and $\tau_{\max }=\frac{1}{2} \sigma_{0}$, or $\left|\sigma_{1}-0\right|=2 C=\sigma_{0}$

- For pure shear, yielding will occur when

$$
\begin{gathered}
\tau_{\max }=\sigma_{1}=-\sigma_{3} \quad \text { and } \quad \sigma_{2}=0 \quad \text { or } \\
\left|\sigma_{1}-\left(-\sigma_{1}\right)\right|=2 C=2 \sigma_{1}=2 \tau_{Y}
\end{gathered}
$$

when the criterion is observed, then $\tau_{Y}=\frac{1}{2} \sigma_{0}$ or the yield strength in pure shear is predicted to be equal to one-half the yield strength in tension.

According to the Von Mises criterion, the tensile and shear yield stresses are related as follows:

$$
\sigma_{0}=\sqrt{3} \tau_{Y} \quad \text { or } \quad \tau_{Y}=\frac{\sigma_{0}}{\sqrt{3}}
$$

There are two major theories of plastic behavior beyond the yield point, the deformation theory and incremental or flow theory. In the deformation theory, the plastic strains are uniquely defined by the state of stress, whereas in the incremental theory the plastic strains depend upon a combination of factors, such as the increments of stress and strain and the state of stress. In this research, the incremental theory is used.

In the incremental theory, the total strain vector $\{\varepsilon\}$ can be decomposed into elastic $\left\{\varepsilon^{\mathrm{e}}\right\}$ and plastic $\left\{\varepsilon^{\mathrm{P}}\right\}$ components as follows:

$$
\{\varepsilon\}=\left\{\varepsilon^{\mathrm{e}}\right\}+\left\{\varepsilon^{\mathrm{P}}\right\}
$$


The increments of plastic strain $\left\{d \varepsilon^{P}\right\}$ can be expressed as the function of the current stress, the strain increments, and the stress increments:

$$
\left\{d \varepsilon^{P}\right\}=\left\{d \varepsilon^{P}\right\}(\{\sigma\},\{d \varepsilon\},\{d \sigma\})
$$

Here, $d$ denotes an increment. Then, equation (2.20) can be written as

$$
\{\mathrm{d} \varepsilon\}=\left\{\mathrm{d} \varepsilon^{\mathrm{e}}\right\}+\left\{\mathrm{d} \varepsilon^{\mathrm{P}}\right\}
$$

In which

$$
\left\{d \varepsilon^{\mathrm{e}}\right\}=[D]\{d \sigma\}=\left[C^{\mathrm{e}}\right]^{-1}\{d \sigma\}
$$

Where $[D]$ is constitutive strain-stress matrix, $\left[C^{e}\right]$ is elasticity matrix From Eq (2.21b) and Eq (2.21c),

$$
\{\mathrm{d} \sigma\}=\left[\mathrm{C}^{\mathrm{e}}\right]\left(\{\mathrm{d} \varepsilon\}-\left\{\mathrm{d} \varepsilon^{\mathrm{P}}\right\}\right)
$$

which can be rewritten as

$$
\{\mathrm{d} \sigma\}=\left[\mathrm{C}^{\mathrm{ep}}\right]\{\mathrm{d} \varepsilon\}
$$

where $\left[\mathrm{C}^{\mathrm{ep}}\right]$ is called the elastic-plastic matrix, and is expressed as

$$
\left[\mathrm{C}^{\mathrm{ep}}\right]=\left[\mathrm{C}^{\mathrm{e}}\right]-\left[\mathrm{C}^{\mathrm{p}}\right]
$$

where $\left[\mathrm{C}^{\mathrm{p}}\right]$ is plasticity matrix and is given by

$$
\left[C^{\mathrm{p}}\right]=\frac{2 \mathrm{G}}{\frac{2}{3} \bar{\sigma}^{2}\left(1+\frac{H^{\prime}}{3 \mathrm{G}}\right)}\left[\begin{array}{cccccc}
\sigma_{\mathrm{Dx}}^{2} & \sigma_{\mathrm{Dx}} \sigma_{\mathrm{Dy}} & \sigma_{\mathrm{Dx}} \sigma_{\mathrm{Dz}} & \sigma_{\mathrm{Dx}} \tau_{\mathrm{xy}} & \sigma_{\mathrm{Dx}} \tau_{\mathrm{yz}} & \sigma_{\mathrm{Dx}} \tau_{\mathrm{zx}} \\
& \sigma_{\mathrm{Dy}}^{2} & \sigma_{\mathrm{Dy}} \sigma_{\mathrm{Dz}} & \sigma_{\mathrm{Dy}} \tau_{\mathrm{xy}} & \sigma_{\mathrm{Dy}} \tau_{\mathrm{yz}} & \sigma_{\mathrm{Dy}} \tau_{\mathrm{zx}} \\
& & \sigma_{\mathrm{Dz}}^{2} & \sigma_{\mathrm{Dz}} \tau_{\mathrm{xy}} & \sigma_{\mathrm{Dz}} \tau_{\mathrm{yz}} & \sigma_{\mathrm{Dz}} \tau_{\mathrm{zx}} \\
& & & \tau_{\mathrm{xy}}^{2} & \tau_{\mathrm{xy}} \tau_{\mathrm{yz}} & \tau_{\mathrm{xy}} \tau_{\mathrm{zx}} \\
& \text { Symmetrical } & & & \tau_{\mathrm{yz}}^{2} & \tau_{\mathrm{yz}} \tau_{\mathrm{zx}} \\
& & & & & \tau_{\mathrm{zx}}^{2}
\end{array}\right]
$$

$\sigma_{\mathrm{Dx}}$ etc. are given in $\mathrm{Eq}(2.8), \bar{\sigma}$ is called the equivalent or effective stress which is related to the equivalent plastic strain $\bar{\varepsilon}^{P}$ by

$$
H^{\prime}=d \bar{\sigma} / d \bar{\varepsilon}^{P}
$$

$H^{\prime}$ is the slope of the curve relating effective stress, $\bar{\sigma}$, and effective strain, $\int d \bar{\varepsilon}^{P}$.

\subsection{Coining and the Finite-Element Method}

The analytical methods such as the slab method, bounding methods and slip-line field method have been useful in predicting forming loads, overall geometry changes of simple deforming workpieces, qualitative modes of metal flow, and the determination of approximate 
optimum process conditions. However, accurate determination of the effects of various process parameters on the detailed metal flow became possible only recently, when the finite-element method was developed for the analyses. The finite-element technique, whose engineering birth and boom in the 1960s was due to the application of computers to structure analysis, has spread to a variety of engineering and physical science disciplines. The basic concept of the finite-element method is one of discretization. The finite-element model is constructed in the following manner [Mote, 1980]. A number of finite points are identified in the domain of the function, and the values of the function and its derivatives, when appropriate, are specified at these points. The points are called nodal points. The domain of the function is represented approximately by a finite collection of subdomains called finite elements. The domain is then an assemblage of elements connected together appropriately on their boundaries. The function is approximated locally within each element by continuous functions that are uniquely described in terms of the nodal-point values associated with the particular element. The path to the solution of a finite-element problem consists of five specific steps: (1) identification of the problem; (2) definition of the element; (3) establishment of the element equation; (4) the assemblage of element equations; and (5) the numerical solution of the global equations. The main advantages of the finite-element method are: (a) the capability of obtaining detailed solutions of the mechanics in a deforming body, namely, loads, shapes, material flows, strains and stresses distributions; and (b) the fact that a commercial computer code, like ABAQUS and ANSYS, can be used for a large variety of problems by simply changing the input data. The FEM approach has been widely used in the simulations of coining processes [Leitao et. al., 1997] [Marques and Martins, 1991] [Choi et. al., 1997], as described in section 2.5.

As a complex process, coining process involves both material nonlinearity and large deformation and the FEM method will be used to simulate the coining process. Therefore, in the following section, the general finite element solution of nonlinear problems will be presented by referring to [Desai and Abel, 1982] 


\subsubsection{FEM Solution for Material Nonlinearity}

The solution of nonlinear problems by the finite element method is usually attempted by the incremental procedures. The basis of the incremental procedure is the subdivision of the load $\{Q\}$ into many small partial loads or increments. The load is applied one increment at a time, and during the application of each increment the equations are assumed to be linear. In other words, the stiffness matrix $[\mathrm{k}]$ is assumed to a fixed value throughout each increment, but it may take different values during different load increments. The solution for each step of loading is obtained as an increment of the displacement $\{\mathrm{q}\}$.These displacement increments are accumulated to give the total displacement at any loading stage, and the incremental process is repeated until the total load has been reached.

Let the initial or reference state of a body given by the initial loads and displacements, $\left\{Q_{0}\right\}$ and $\left\{q_{0}\right\}$. The total load is divided into $M$ increments, thus the total effective load is

$$
\{Q\}=\left\{Q_{0}\right\}+\sum_{j=1}^{M}\left\{\Delta Q_{j}\right\}
$$

where the $\Delta$ notation is used to indicate a finite increment. Hence, after the application of the $i^{\text {th }}$ increment, the load is given by

$$
\left\{Q_{i}\right\}=\left\{Q_{0}\right\}+\sum_{j=1}^{i}\left\{\Delta Q_{j}\right\}
$$

where, $\left\{Q_{M}\right\}=\{Q\}$.

similarly, after the $\mathrm{i}^{\text {th }}$ step, the displacements are

$$
\left\{q_{i}\right\}=\left\{q_{0}\right\}+\sum_{j=1}^{i}\left\{\Delta q_{j}\right\}
$$

To calculate the increment of displacements, a fixed value of the stiffness is used, which is evaluated at the end of the previous increment

$$
\left[k_{i-1}\right]\left\{\Delta q_{i}\right\}=\left\{\Delta Q_{i}\right\} \text { for } \mathrm{i}=1,2,3, \ldots, \mathrm{M}
$$

where,

$$
\left[k_{i-1}\right]=\left[k_{i-1}\left(\left\{q_{i-1}\right\},\left\{Q_{i-1}\right\}\right)\right]
$$

where, $\left[k_{0}\right]$ is the initial value of the stiffness, which can be calculated from material constants derived from the given stress-strain curves at the start of the loading. 
In the incremental procedure the tangent moduli are used to formulate the tangent stiffness matrix $[\mathrm{C}(\sigma)]$ and compute the stiffness matrix $[\mathrm{k}]$ in equation (2.28). The accuracy of the incremental procedure can be improved by taking smaller increments of the loads.

In applying the incremental procedure, the elastic-plastic constitutive law, equation (2.22b), can be expressed in terms of finite increments as

$$
\{\Delta \sigma\}=\left[C^{\mathrm{ep}}\right]\{\Delta \varepsilon\}
$$

The matrix $\left[\mathrm{C}^{\mathrm{ep}}\right]$ is updated for each increment of load by calculating the tangent stiffness at the end of each increment and used for the succeeding increment, equation (2.28b). The increments of plastic strain $\left\{\Delta \varepsilon^{\mathrm{p}}\right\}$ required for the modification of $\left[\mathrm{C}^{\mathrm{ep}}\right]$ are calculated from equation (2.21a). Once $\left[\mathrm{C}^{\mathrm{ep}}\right]$ is obtained, the element stiffness can be calculated by the equation

$$
[k]=\iiint_{V}[B]^{T}\left[C^{e p}\right][B] d V
$$

where, $[\mathrm{B}]$ is transformation matrix, $\mathrm{V}$ is the element volume.

\subsubsection{FEM Solution for Geometric Nonlinearity}

In a geometric nonlinear problem, the displacements are large but the strains are small. The principal effect of large displacements is that the changes of geometry brought about by the displacements can no longer be neglected. This nonlinear problem can be solved through approximating it by a sequence of linearized subproblems. For the displacement vector [q], stiffness matrix [k] and load vector [Q] in the global system, these in the local system are related by the transformations [T]

$$
\begin{gathered}
\left\{q_{g}\right\}=[T]^{T}\left\{q_{l}\right\} \\
{\left[k_{g}\right]=[T]^{T}\left[k_{l}\right][T]} \\
\left\{Q_{g}\right\}=[T]^{T}\left\{Q_{l}\right\}
\end{gathered}
$$

For the large displacement problem, the direction cosines in the transformation matrix [T] become functions of the displacement state as well as of the initial geometry, therefore 
nonlinearities are introduced into the equilibrium equations. Symbolically, this can be denoted by

$$
[\mathrm{T}]=[\mathrm{T}(\{\mathrm{q}\})]
$$

The stiffness matrix in local coordinates $\left[k_{l}\right]$ is linear for small strains, that is, it is not a function of the displacements. Hence it remains the same for all states of deformation. However, because of the nonlinearity of the transformation equation (2.32), the element stiffness in global coordinates $\left[k_{g}\right]$ varies with the displacement state.

For an increment of load $\{\Delta Q\}$, the equation (2.33) can be used to expressed the change in the load vector as

$$
\left\{Q_{g}\right\}+\left\{\Delta Q_{g}\right\}=\left([T]^{T}+[\Delta T]^{T}\right)\left(\left\{Q_{l}\right\}+\left\{\Delta Q_{l}\right\}\right)
$$

Here, $\left\{Q_{\mathrm{g}}\right\},\left\{\mathrm{Q}_{1}\right\}$ and $[\mathrm{T}]$ are the known loads and the transformation at the beginning of the increment. Performing the matrix multiplication and neglect the higher order term for a small increment, an equation can be obtained by substituting the standard incremental equilibrium equation as

$$
\left\{\Delta Q_{g}\right\}=\left[k_{g}\right]\left\{\Delta q_{g}\right\}+[\Delta T]^{T}\left\{Q_{l}\right\}
$$

The second term on the right represents the effect of the change of geometry on the global equilibrium equation of the element. The equation can be rewritten as

$$
\left[k_{g}\right]\left\{\Delta q_{g}\right\}+\left(\sum_{i=1}^{n} Q_{l i}\left[G_{i}\right]\right)\left\{\Delta q_{g}\right\}=\left\{\Delta Q_{g}\right\}
$$

where, $i$ denotes the increment of the $i^{\text {th }}$ column and matrix $\left[G_{i}\right]$ is symmetrical

$$
\left[G_{i}\right]=\frac{\partial\{T\}_{i}}{\partial\left\{q_{g}\right\}}=\left[\frac{\partial\{T\}_{i}}{\partial\left\{q_{g 1}\right\}} \frac{\partial\{T\}_{i}}{\partial\left\{q_{g 2}\right\}} \ldots \frac{\partial\{T\}_{i}}{\partial\left\{q_{g n}\right\}}\right]
$$

The quantity in parentheses is called the geometric stiffness $\left[k_{G}\right]$, thus equation (2.37) can be written as

$$
\begin{gathered}
\left([k]+\left[k_{G}\right]\right)\{\Delta q\}=\{\Delta Q\} \\
{\left[k_{G}\right]=\sum_{i=1}^{n} Q_{l i}\left[G_{i}\right]}
\end{gathered}
$$

where the subscript $g$ has been dropped. The above formulation follows [Desai and Abel, 1972]. The incremental form of equation (2.38a) is

$$
\left([k]+\left[k_{G}\right]\right)_{i-1}\left\{\Delta q_{i}\right\}=\left\{\Delta Q_{i}\right\}
$$


where the subscript $i$-1 indicates that the stiffness matrices are evaluated for the state of displacement at the beginning of the increment. Clearly, the increment method for large displacements requires more effort than that for material nonlinearity, because two stiffnesses must be calculated at each increment. However, the effort to recompute $[k]$ is minimized by noting that the matrix $\left[k_{l}\right]$ is invariant and only the transformation in equation (2.32) changes.

\subsubsection{Elastic-Plastic Formulation and Rigid-Plastic Formulation}

The application of the finite-element method to metal-forming problems began as an extension of structural analysis techniques to the plastic deformation regime. In the analysis of metal forming, plastic strains outweigh elastic strains and the idealization of rigid-plastic or rigid-viscoplastic material behavior is acceptable. The resulting analysis based on this assumption is known as the rigid-plastic formulation [Zienkiewicz, 1984]. In other applications, phenomena associated with elasticity cannot be neglected. In the elastic-plastic formulation [Nagtegaal and Veldpaus, 1984], the material is considered to behave as an elastic-plastic or elastic-viscoplastic solid. In the rigid-plastic approach, at a certain stage in the process of quasi-static distortion, the shape of the body, the state of inhomogeneity, and the current values of material parameters are supposed to be given or to have been determined already. Solutions to this problem are the stress and velocity distributions that satisfy the governing equations and the boundary conditions. In the elastic-plastic approach, in addition to the current states of the body, the internal distribution of the stress also is supposed to be known and the boundary conditions are prescribed in terms of velocity and traction-rate. Distributions of velocity and stress-rate (or displacement and stress-increment) are the solutions to the problem. In the coining process, both elastic and plastic effects are to be investigated. Thus the elastic-plastic formulation will be used in the FEM analysis.

\subsection{Key Factors in Coining Process}

Coning is a very complex deformation process with many factors involved. It would be 
expensive and time-consuming for the numerical analysis to take very factor into account. It would be helpful and necessary to identify those factors which may have large effects in the coining process. Some of these key factors and the related assumptions for the coining process will be discussed in this section.

\subsubsection{Material Properties}

For a given material composition and deformation/heat-treatment history, the flow stress (or effective stress) and the workability (or formability) in various directions (anisotropy), are the most important material variables in the analysis of a metal-forming process. For a given microstructure, the flow stress is expressed as a function of strain, strain-rate, and temperature. It is influenced by (1) factors unrelated to the deformation process, such as chemical composition, metallurgical structure, phase, grain size, segregation and prior strain history, and (2) factors related to the process, such as temperature, degree of deformation and rate of deformation. Workability or formability is the capability of a material to deform without failure; it depends on (1) conditions existing during deformation processing, such as temperature, rate of deformation,

stresses and strain history, and (2) material variables, such as composition, voids, inclusions, and initial microstructure.

In hot forming of metals at temperatures above the recrystallization temperature, the influence of strain on flow stress is insignificant, and the influence of strain-rate becomes increasingly important. Conversely, at room temperature (i.e., in cold forming), the effect of strain-rate on flow stress is negligible, and the effect of strain on flow stress (i.e., strain hardening) is most important. Material nonlinearity poses another analytical problem, in which the relationship between stresses and strains or other material properties are complicated functions. To be useful in metal-forming analyses, the flow stresses of metals must be determined experimentally for the strain, strain-rate, and temperature conditions that exist in metal-forming processes. The methods most commonly used for obtaining flow stress data are uniaxial tensile, uniform compression, and torsion tests. 
To investigate the strain-rate effect in coining process, a simple analysis is conducted. Figure 2-2, provided by the Royal Canadian Mint [Royal Canadian Mint, 2007], illustrates the coining frame stroke profile of the coining press machine at the Royal Canadian Mint. The press machine provides a type of mechanical striking with a flying wheel. It has been observed that the contact between die and blank will only occur in the last 25 degree range of crank angle, which corresponds to about $0.4 \mathrm{~mm}$ displacement of the die. Given the stroke rate at 45 stroke per minute [Royal Canadian Mint, 2007], the contact time for $0.4 \mathrm{~mm}$ displacement is around 0.1 second. Taking a typical silver blank thickness -- $2.5 \mathrm{~mm}$, provided by Royal Canadian Mint [Royal Canadian Mint, 2007], the nominal strain is around 16\% and the nominal strain rate is about $1.6 \mathrm{~s}^{-1}$. According to Haddad [Haddad, 2000], the range of strain rates from $10^{-1}$ to $10^{2} \mathrm{~s}^{-1}$ is generally referred to as the intermediate or medium strain-rate regime. Within this regime, strain-rate effects may become a consideration in metals, but the magnitude of such effects could be quite small [Haddad, 2000]. The inertia and wave propagation effects become important when the strain-rate is $10^{3} \mathrm{~s}^{-1}$ or higher (dynamic loading) and at strain-rates of $10^{5} \mathrm{~s}^{-1}$ or higher, a transition from nominally isothermal conditions to adiabatic conditions happens [Haddad, 2000]. Since the coining nominal strain-rate falls at the lower end of medium strain-rate regime and it is far different from impact loading conditions, it is reasonably expected that the strain-rate effects are small. And to take the advantage of the simple and standard quasi-static test, the material properties under quasi-static loading condition will be used to approximate the material response during coining process. Therefore, in this research, the quasi-static uniaxial tension test of fine silver will be conducted to obtain the stress-strain behavior.

\subsubsection{Temperature in Metal Forming}

In metal-forming processes, both plastic deformation and friction contribute to heat generation. The temperatures developed in the process influence lubrication conditions, tool life, and the properties of the final product, and most significantly, determine the maximum deformation speed that can be used for producing sound products without excessive tool damage. 
Thus, temperatures generated during plastic deformation greatly influence the productivity of metal-forming processes [Altan, Oh and Gegel, 1983]. The magnitudes and distribution of temperatures depend mainly on:

- The initial material and die temperatures

- Heat generation due to plastic deformation and friction at the die-material interface

- Heat transfer between the deforming material and the dies and between the material and the environment (air or coolant)

The influence of temperatures in metal-forming operations is most dramatic in hot forming operations, where the contact time under pressure between the deforming material and the dies is the most significant factor influencing temperature conditions. As has been mentioned in Chapter one, the coining process is characterized by two main features that differentiate it from the traditional closed die forging, namely the small ratio between the final surface geometry pattern and the workpiece diameter, and the small ratio between the obtained protrusion height - or cavity depth - and the workpiece thickness [Ike and Plancak, 1998]. The nominal strain of $16 \%$ and strain-rate of $1.6 \mathrm{~s}^{-1}$ in a typical silver coining process discussed in the last section also shows that the strain level and loading rate is not high enough to cause significant temperature variations. The thermal effects in the coin are further reduced by the fact that the size of a typical silver dollar is very small relative to the size of die and the high heat transfer between silver coin and metal dies. This fact can also be verified by the on-site coining process observations at the Royal Canadian Mint, in which the temperature variation of the coin right after the coining process is negligible. Therefore thermal softening effects will not be included in the coining process simulation. In this research, the coining process is performed at the room temperature and isothermal forming condition, it does not consider temperature-dependent materials and heat generated by plastic work.

\subsubsection{Forging Shape}

The main objective of coining process design is to ensure adequate flow of the metal in the 
dies so that the desired finished part geometry can be obtained without defects and with appropriate loads. Often several operations (preforming or blocking) are needed to achieve gradual flow of metal from an initially simple shape (cylinder or round-cornered square billet) into the more complex shape of the final coining. The geometric nonlinearity, also known as large strain or large displacement analysis, directly influences material flow, and therefore it affects the distribution of the effective strain and flow stress in the deformation zone. In cold metal forging, complex forging geometry will result in more forging steps and higher loading to obtain adequate metal flow and final sound product, but on the other hand, higher loading and more forging steps will lower the die life and productivity.

To reduce the computational size and time of the simulation, using symmetry conditions of the forging shape to simplify the model becomes necessary. A simplified model also improves the numerical stability of the numerical simulations. To prevent any violation of symmetry conditions of the coining model, it will be of crucial importance to have axisymmetry in coining geometry, loading and material properties. From [Altan, Oh and Gegel, 1983], it is known that in case of forming loads that are applied out of the symmetry plane of the die, the whole die may distort leading to the generation of moments. For coining or generally net-shape forming, this

kind of non-symmetric load can affect the process considerably. Another critical geometrical issue is the mathematical description of die surfaces. In most of the commercial finite element codes, the surfaces of the dies are digitized by a surface mesh during the transfer of the CAD-data into the FEM codes. This will exert a significant effect in the case of small fillet radii because it will require great detailed CAD data and a much refined mesh to capture the small geometrical details.

\subsubsection{Friction in Metal Forming}

In metal forming processes, frictional forces are generated at the interface between the tools and deformation materials. These frictional forces have the following effects:

- The total deformation loads are increased; 
- The internal structure and surface characteristics (surface finish and surface defects) of the products are influenced;

- Wear is produced on the tooling material, thus reducing its useful life;

- Dimensional variations are produced in the processed material;

Because of these effects, friction is considered as a major variable in coining operations and must be adequately understood to optimize processing procedures for economically producing coins with the desired geometry and surface characteristics.

There are presently two different parameters that attempt to define quantitatively the amount of friction existing between two contacting surfaces:

(i) Using Coulomb's coefficient of friction $\mu$, the friction shear stress, $f_{s}$ is most commonly expressed by [Von Karman, 1925] [ Schey, 1983] as

$$
f_{s}=\mu p
$$

where, $p$ being a compressive normal stress to the interface,

(ii) Using friction factor $m$, the friction shear stress, $f_{s}$ expressed by [Siebel,1930, Schey, 1983] as

$$
f_{s}=m k
$$

where, $k$ being the shear strength of the deforming material, where $0 \leq m \leq 1$.

Studies in forming mechanics indicate that Eq. (2.41) adequately represents the friction condition in high normal interface stress processes while Eq. (2.40) is commonly used for representation of friction in low normal interface stress processes. For a complete coining process cycle, the process will experience a low normal stress at beginning to the high normal stress when the metal fills up the dies and to the low normal stress again when unloading. Therefore, it would be ideal to find a friction model which is capable of modeling behavior at both low and high normal interface stress. With the similar objectives in mind, Oden and Pires [Oden and Pires, 1983] and Zhong [Zhong, 1989] have developed a mathematical analysis for modeling realistic friction contact conditions with non-linear characteristics, this approach is 
available for use in the finite element analysis code ABAQUS [ABAQUS, 2000] as a bi-linear approximation to the non-linear case. This bi-linear friction model combines Coulomb friction and the friction factor techniques as shown schematically in Figure 2-3. Although the combined model exhibits a more realistic condition in coining processes, the key is how to obtain the friction coefficient $\mu$ and the friction factor $\mathrm{m}$ through experiments. The process to evaluate the interface friction condition in coining will be discussed in detail in the Chapter 5.

\subsection{Recent Developments in Metal Forging and Coining}

Recently, to handle more complicated forming processes, three-dimensional finite-element analysis has been developed. Park and Kobayashi [Park and Kobayashi, 1984] analyzed the three-dimensional deformation of the open-die forging of blocks, whilst Mori and Osakada [Mori and Osakada, 1984] simulated the three-dimensional rolling process. Davey [Davey et al, 2001] developed adaptive preconditioning solution schemes to enhance the ring rolling process simulations. Surdon and Chenot [Surdon and Chenot, 1987] analyzed the hot forging of blocks between flat dies. Shiau [Shiau, 1989] performed the sequence design of open-die forging. Kim [Kim, 1989] developed a three-dimensional finite-element code for both ring rolling and shape rolling. Choi [Choi et al, 1997] developed the rigid-plastic finite-element method for process design in three-dimensional plastic deformation and applied it to a precision coining process. The method was carried out to find the problems involved in the coining process and successfully determined the amount of piercing. Jorge [Jorge et al, 1994] utilized the penalty constrained method and rigid-plastic flow formulation to model large three-dimensional metal forming deformations and they developed a three-dimensional contact algorithm suitable for metal forming and extended the "arc tangent" friction model [Chen and Kobayashi, 1978] to the three-dimensional hexahedral element. Zhao [Zhao et al, 2002] used the FEM-based inverse die contact tracking method to design the preform shapes for a track link blocker forging to reduce the material lost due to flash. Victor and Taylan [Victor and Taylan, 2000] investigated the preform design for flashless forging of a connecting rod and introduced a new tooling concept 
for forging of complex parts with a controlled amount of flash. Sedighi and Tokmechi [Sedighi and Tokmechi, 2007] proposed a new algorithm of a suitable method for practical designation of performs for complex parts through preliminary preform selection and improvement of the preform geometry to reduce the material waste and forging loads.

In particular, a new type of bimetallic coin is getting more interest from the Mints round the world. The combination of different materials with distinctly different material properties, especially the deformation behavior, makes the adaption of new production parameters and manufacturing philosophy necessary. Leitao [Leitao. et. al., 1997] utilized 2D axisymmetric FEM model to characterize the metal flow and design an appropriate geometry for the mechanical joint in the bimetallic coin. The first bimetallic coin produced on a large scale was introduced by the Italian Government Mint in 1982 (500 Lire), and signaled an important achievement. The success of the 500 Lire bimetallic coin in the field of coinage stimulated other countries to develop their own bimetallic coins (e.g., France in 1988 (10 Francs) and Portugal in 1989 and 1991 (100 and 200 Escudos)). Bimetallic coins are presently used widely in many other countries around the world, as well as the new type of coin made from three components (20 Francs trimetallic coin produced by France). More recently, France and Austria proposed bimetallic collection coins in gold and silver. In 2003, a special 25 Euro bi-metallic coin "700 Jahre Stadt Hall" was issued by the Austrian Mint, which used the colored niobium as inserts. The possibility to color the surface by anodic oxidation was used for the first time for coining manufacturing [Grill and Gnadenberger, 2006]. The series was continued in 2004 with the second edition of "150 Jahre Semmeringbahn" with green colored niobium inserts, and in 2005 with the third edition of "50 Jahre Fernsehen" with a purple colored niobium insert. So far, the technology utilized for producing bimetallic coins, hereafter named bimetallic ring technology, has consisted of the utilization of an inner disk (center) and an outside ring of different materials, generally with different colors (Figure 2-4a). The coin is produced in two stages: firstly, the center is placed inside the outer ring with a little clearance, and secondly, the two parts are assembled together with the impartion of the surface details by the minting dies. 
Despite the efforts made to produce bimetallic coins on a large scale going back to the beginning of the 1980s, there is general absence of technical information about this technology. The first studies in this field were published by Marques and Martins [Marques and Martins, 1991] in collaboration with the Portuguese Government Mint (Imprensa Nacional Casa de Moeda - INCM) in 1991. They used a 2D FEM program based on a rigid-plastic formulation to simulate the joint of a bi-metal coin. The new bimetallic technology proposed is bimetallic foil technology, based on a concept entirely different to that of bimetallic ring technology. Bimetallic foil technology employs two disks of different materials, one being very thin (foil) (see Figure $2-4 b)$, assembled together by mechanical means, during the impartion of the surface details by the minting dies. The coin is produced in a multistage sequence consisting of three metal forming operations (preforming, rimming and coining) and one intermediate annealing before the coining operation. Coinage of bicolor coins using the new technology is an alternative to conventional bimetallic ring technology currently utilized worldwide. The technology allows the coining of gold - silver specimens with larger diameters and lower costs than those that would arise from the utilization of monometallic blanks.

The above works carried out are primarily focusing on the new numerical approaches applied in the 2D and 3D metal forming simulations, new tooling and performing concept for forging, the new technologies to produce bimetallic coin. But none of these works gave a thorough investigation on the suitable simulation approach for practical coining and the effects of various process parameters.

The work discussed above has shown that there is a need for a more systematic design tool for determining the optimum coining condition. Therefore, a finite element simulation approach for coining process will be developed in this research, which will consider the practical design work practices at Royal Canadian Mint and includes the effects of typical material properties, quasi-static state and nonuniform material flow, the coining interface friction and detailed die designs. The FEM design tool development will begin, as described in the dissertation overview, with the validation of FEM parameters and the modeling methodology to be used. 


\begin{tabular}{|l|l|}
\hline 1 & UPPER DIE \\
\hline 2 & DISK \\
\hline 3 & COLLAR \\
\hline 4 & PRE-STRESSING COLLAR \\
\hline 5 & LOWER DIE \\
\hline
\end{tabular}

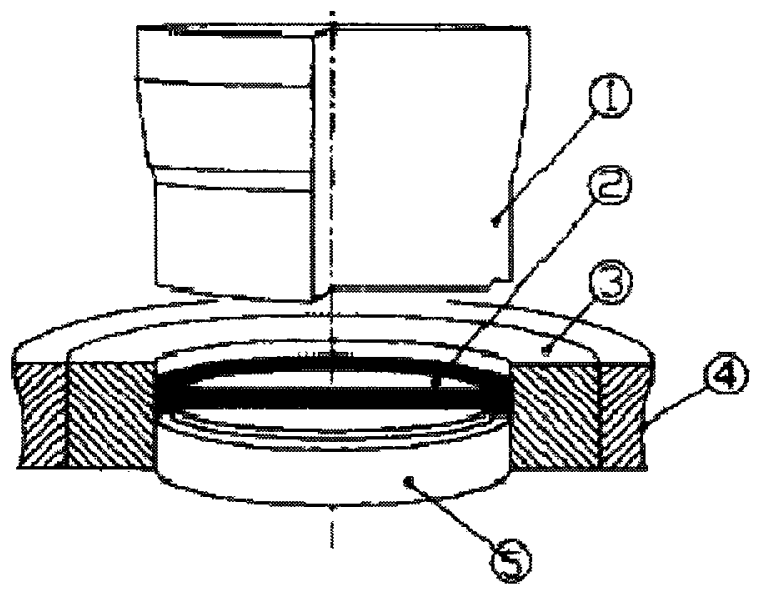

Figure 2-1. Schematic representation of the die set-up utilized for producing coins and medals [Leitao. et. al., 1997]

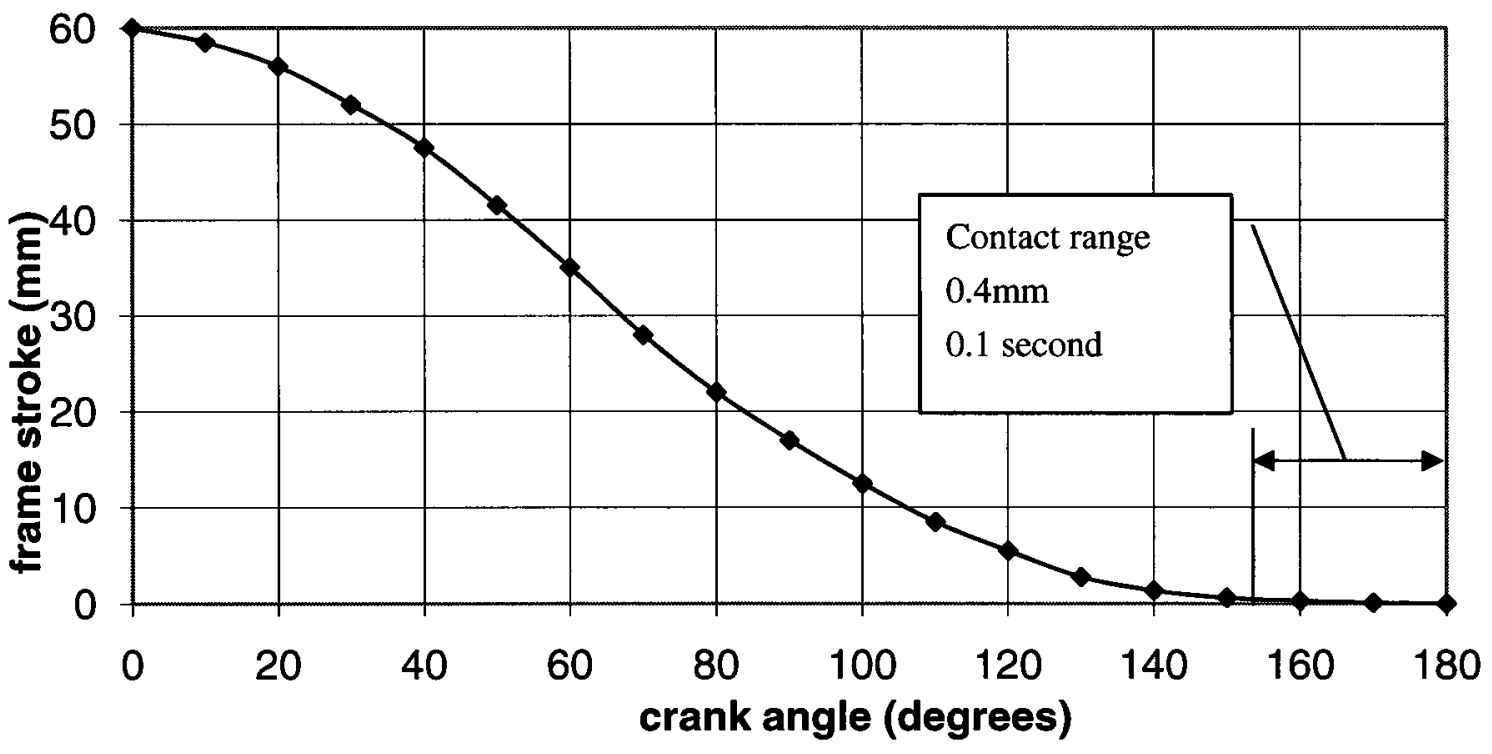

Figure 2-2. Coining frame stroke profile [Royal Canadian Mint, 2007] 


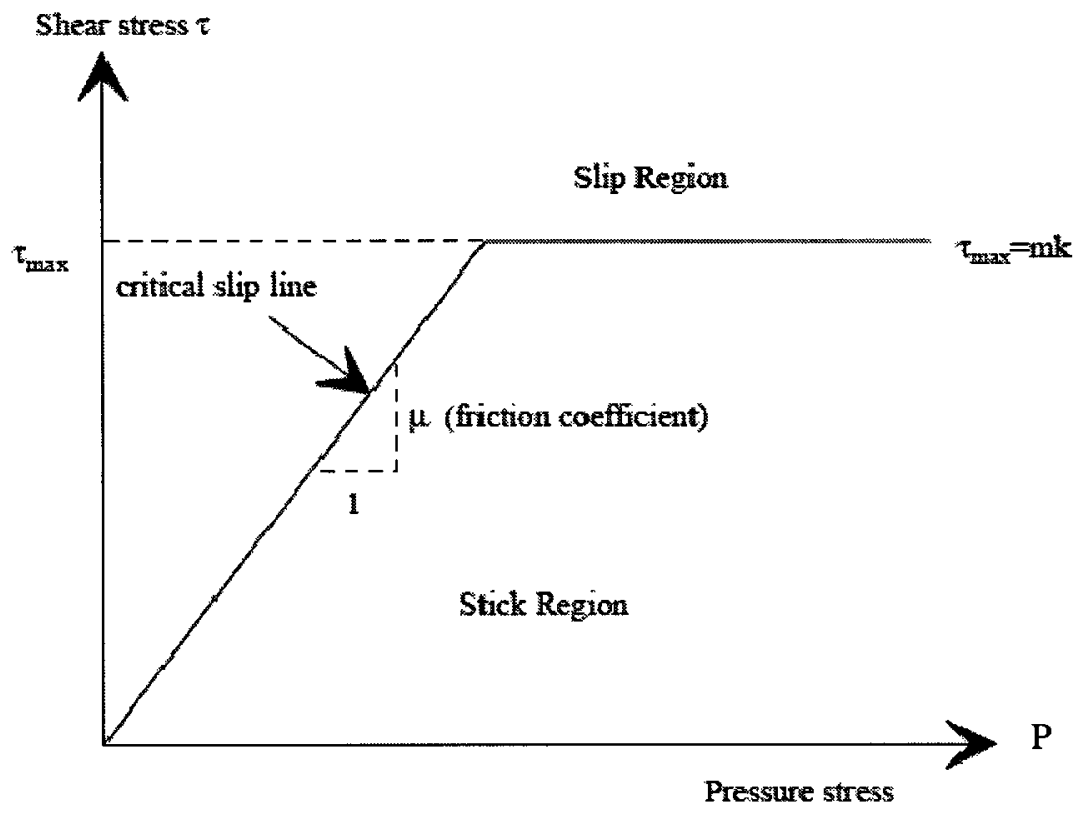

Figure 2-3. Non-classical bi-linear friction model

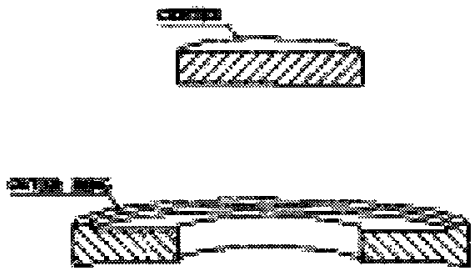

(a)

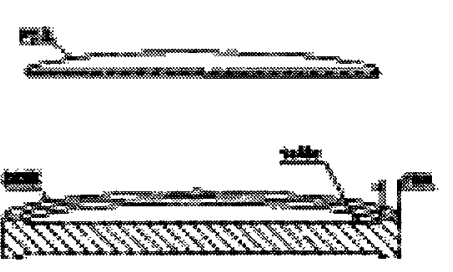

(b)

Figure 2-4. The two types of bimetallic coinage [Leitao. et. al., 1997]

(a) bimetallic ring technology; (b) bimetallic foil technology 


\section{Chapter 3}

\section{Numerical Simulation Using FEM}

This section will examine the general finite element approaches and formulations using ABAQUS, some key numerical parameters will be illustrated and investigated using an upsetting process FEM simulation.

\subsection{General Simulation Approach}

As have been discussed in Chapter 2, coining processes are generally considered quasi-static in nature, therefore inertial and thermal effects are assumed to be negligible. To model such processes, two basic solution procedures in ABAQUS can be used: Implicit method and Explicit method. The implicit procedure is available in ABAQUS/Standard and the explicit procedure is available in ABAQUS/Explicit. In both methods, the nonlinear response is obtained incrementally. Because the coining process is considered to be quasi-static, it would appear that the implicit static procedure is an appropriate choice for modeling purpose. However, as the model size increases, solution cost for the implicit procedure increases more steeply than for the explicit procedure, as shown in Figure 3-1 [ABAQUS, 2006]. Therefore, considering the model size we might construct for the coining processes, the explicit method is generally more efficient computationally.

In addition, coining processes are dominated by contact boundary conditions and the implicit procedure in ABAQUS/Standard uses constraints to enforce these conditions. The solver must iterate to satisfy all contact conditions, therefore cutback of the time increment will occur if contact conditions cannot be satisfied in a reasonable number of iterations. That is to say that when the models get complex and have a large number of possible contact points and changing contact conditions can result in extremely small time increments or convergence difficulties. In the explicit procedure, no iterations are required to enforce contact conditions and a kinematic or 
penalty method can be used to enforce the contact constraints. Thus, the explicit procedure in ABAQUS/Explicit offers a general contact capability that allows easy definition of multiple contact interactions. Alternatively, a contact pair approach is available which only requires the specification of contact interaction pairs.

To take advantage of the benefits that the ABAQUS/Explicit offers, the numerical analysis approach in this research will use ABAQUS/Explicit method. The investigation on the effects of several dominant numerical parameters in the FEM analysis using ABAQUS/Explicit becomes essential before it can be used to establish the numerical models for the coining process. They are discussed in the next section.

\subsection{The Dominant Factors and Their Effects in FEM Simulations}

Considering the coining process to be simulated, three ABAQUS parameters were chosen as the dominant factors, namely Hourglass Controls, Contact Simulation and Adaptive Meshing. These factors are illustrated in the following sections.

\subsubsection{Hourglass Controls in ABAQUS}

In ABAQUS/Explicit the default formulation for solid, shell, and membrane elements can be chosen to perform satisfactorily on a wide class of quasi-static and explicit dynamic simulations. However, certain formulations give rise to some trade-off between accuracy and performance. ABAQUS/Explicit provides section controls to modify these element formulations so that one can optimize these objectives for a specific application.

The formulation for reduced-integration elements considers only the linearly varying part of the incremental displacement field in the element for the calculation of the increment of physical strain. The remaining part of the nodal incremental displacement field is the hourglass field and can be expressed in terms of hourglass modes. The hourglass mode is a spurious zero energy

mode in that no energy is required to deform the element, as shown in Figure 3-2. Excitation of these modes may lead to severe mesh distortion, with no stresses resisting the deformation. 
Hourglass control attempts to minimize this problem without introducing excessive constraints on the element's physical response.

The following three methods [ABAQUS, 2006] in ABAQUS for suppressing the hourglass modes will be investigated using simple model FEM simulations.

\section{Integral Viscoelastic Approach in ABAQUS/Explicit}

The integral viscoelastic approach in ABAQUS/Explicit generates more resistance to hourglass forces early in the analysis step where sudden dynamic loading is more probable. The integral viscoelastic form of hourglass control is available for all reduced-integration elements and is the default form in ABAQUS/Explicit. It is the most computationally intensive hourglass control method.

\section{Kelvin Viscoelastic Approach In ABAQUS/Explicit}

The Kelvin viscoelastic hourglass control in ABAQUS/Explicit is a linear combination of stiffness and viscous hourglass control

\section{Enhanced Hourglass Control Approach In ABAQUS/Explicit}

The enhanced hourglass control approach in ABAQUS/Explicit represents a refinement of the pure stiffness method in which the stiffness coefficients are based on the enhanced assumed strain method.

In addition, the Integral and Kelvin Viscoelastic approach for suppressing the hourglass mode can also be affected by the combinations of three scaling factors which are dimensionless and control the specific displacement degrees of freedom within the element, like rotation and transverse displacement. The effects of these scaling factors will also be investigated.

\subsubsection{Contact Simulation in ABAQUS/Explicit}

ABAQUS/Explicit provides two algorithms for modeling contact interactions. The general contact algorithm allows very simple definitions of contact with very few restrictions on the types of surfaces involved. The contact pair algorithm has more restrictions on the types of surfaces involved and often requires more careful definition of contact, however, it allows for 
some interaction behaviors that are not available with the general contact algorithm. The two contact algorithms combine to provide the capacities in ABAQUS/Explicit, such as contact between rigid and/or deformable bodies, contact of a body with itself, finite-sliding or small-sliding contact and so on. Although the general contact algorithm is more powerful and allows for simpler contact definitions, the contact pair algorithm must be used in certain cases where more specialized contact features are desired, such as two-dimensional surfaces, kinematically enforced contact, small-sliding contact, exponential and no separation contact pressure-overclosure models [ABAQUS, 2006]. In addition, the general contact algorithm places more restrictions on adaptive meshing than the contact pair algorithm. Contact output is more complete for a contact pair analysis.

Due to the contact situations in the coining process, the contact pair algorithm was selected in the FEM simulation. The contact formulation for the contact pair algorithm in ABAQUS/Explicit [ABAQUS, 2006] includes kinematic or penalty constraint enforcement methods. By default, all contact pairs in an ABAQUS/Explicit simulation use a kinematic contact algorithm to strictly enforce contact constraints. Alternatively a penalty contact algorithm can be chosen, which has a weaker enforcement of contact constraints but allows for treatment of more general types of contact. The penalty contact algorithm can model some types of contact that the kinematic contact algorithm cannot. Another difference between kinematic and penalty contact is that the critical time increment is unaffected by kinematic contact but can be affected by penalty contact. More detailed explanations can be found in ABAQUS theory manual [ABAQUS, 2006].

\subsubsection{Adaptive Meshing}

The availability of the adaptive meshing techniques in ABAQUS can help one optimize a mesh and, therefore, obtain quality solutions while controlling the cost of the analysis. The technique uses the adaptive, or solution-dependent, processes to adapt a mesh to meet the analysis goals, as shown in Figure 3-3 [ABAQUS, 2006]. Three adaptive meshing techniques are available 
in ABAQUS: Arbitrary Lagrangian Eulerian (ALE) adaptive meshing, varying topology adaptive remeshing and mesh-to-mesh solution mapping, to enable rezoning analysis. Only the ALE adaptive meshing technique is available for use in ABAQUS/Explicit.

\section{ALE Adaptive Meshing}

Arbitrary Lagrangian Eulerian (ALE) adaptive meshing provides control of mesh distortion. ALE adaptive meshing uses a single mesh definition that is gradually smoothed within analysis steps. The term ALE implies a broad range of analysis approaches, from purely Lagrangian analysis, in which the node motion corresponds to material motion, to purely Eulerian analysis, in which the nodes remain fixed in space and material "flows" through the elements. Typically ALE analyses use an approach between these two extremes, mesh motion is somewhat independent of the material motion, as shown in Figure 3-4 [ABAQUS, 2006].

One can use adaptive meshing to control element distortion in cases where large deformation or loss of material occurs. Figure 3-5 illustrates a case where adaptive meshing limits mesh distortion in a bulk forming simulation [ABAQUS, 2006]. Unlike other adaptivity techniques, adaptive meshing operates on the original mesh definition and is, therefore, useful only when a single mesh can be effective for the duration of a simulation. The mesh is adapted through smoothing of the mesh nodes. This smoothing is typically applied frequently within analysis steps. ALE adaptive meshing is a tool that makes it possible to maintain a high-quality mesh throughout an analysis, even when large deformation or loss of material occurs, by allowing the mesh to move independently of the material. Figure 3-6 shows the node movements in the adaptive domain. ALE adaptive meshing in ABAQUS does not alter the topology (elements and connectivity) of the mesh, which implies some limitations on the ability of this method to maintain a high-quality mesh upon extreme deformation.

Because the final shape of the product in metal forming can be drastically different from the original shape, a mesh that is optimal for the original product geometry can become unsuitable in 
later stages of the process when large material deformation leads to severe element distortion and entanglement. Element aspect ratios can also degrade in zones with high strain concentrations. Both of these factors can lead to a loss of accuracy, a reduction in the size of the stable time increment, or even termination of the problem. Therefore, adaptive meshing is particularly effective for simulations of metal forming processes such as forging, extrusion, and rolling.

\section{Controlling the Frequency of ALE Adaptive Meshing}

In most cases the frequency of adaptive meshing is the parameter that most affects the mesh quality and the computational efficiency of adaptive meshing. A typical adaptive mesh application without Eulerian boundaries will require adaptive meshing every 5-100 increments [ABAQUS, 2006].

\section{Controlling the Intensity of ALE Adaptive Meshing}

During each adaptive meshing increment, the new mesh is created by performing one or more mesh sweeps and then advecting the solution variables to the new mesh. In an adaptive meshing increment, a new, smoother mesh is created by sweeping iteratively over the adaptive mesh domain. During each mesh sweep, nodes in the domain are relocated—based on the current positions of neighboring nodes and elements - to reduce element distortion. In a typical sweep a node is moved a fraction of the characteristic length of any element surrounding the node. Increasing the number of sweeps increases the intensity of adaptive meshing in each adaptive meshing increment [ABAQUS, 2006]. Ideally, an advection sweep will be performed only once, after all mesh sweeps for the increment are complete. However, numerical stability of the advection sweep is maintained only if the difference between the old mesh and the new mesh is small. Therefore, if after a mesh sweep the total accumulated movement of any node in the domain is greater than $50 \%$ of the characteristic length of any adjacent element, an advection sweep is performed to remap the solution variables from the old mesh to the intermediate mesh. Mesh sweeps will continue until the specified number is reached or until the movement of any node 
again exceeds the 50\% threshold. At this time an advection sweep is performed again to map variables from the last intermediate mesh to the new intermediate mesh. The cycle will continue until the number of mesh sweeps reaches the specified number [ABAQUS, 2006]. In the later coining process simulations, the adaptive mesh technique will be used as a key mesh control method in the finite element simulations.

\subsection{FEM Validation Model}

In order to investigate the validity and numerical convergence of the non-linear FEM approach, an axisymmetric two dimensional ring compression finite element model generated in ABAQUS was used for the evaluation. The elastic-plastic formulation and Lagrangian reference system is used, where subsequent deformation is referred to the original undeformed configuration and the incremental solution method for large plastic deformation is used. The evaluation uses a basic open die forging model and due to the facts that have been discussed in Chapter 2, the simulation does not consider temperature-dependent materials, heat generated by plastic work, rate effects or the residual effects. The initial mesh and die configuration for the open die forging process is shown in Figure 3-7. In view of the symmetrical geometry of the circular ring, a quarter of it is chosen for the numerical analysis. CAX4R elements (4 node bilinear axisymmetric quad with reduced integration) were used in the mesh of the blank, as

shown in Figure 3-8. During the process, the thickness of the blank is reduced by about $50 \%-60 \%$ as the rigid punch presses down, as shown in Figure 3-9.

\subsection{Results Comparison and Discussion}

The most important numerical parameters which have been identified for affecting the final quality were Hourglass Controls, Contact Simulation and Adaptive Meshing. As a consequence,

several simulations with different numerical parameters have been carried out using finite-element code ABAQUS/Explicit and the results are shown and discussed in the following paragraphs. 


\subsubsection{Hourglass Controls}

Figure 3-10 shows the load against height reduction results from the three models with different hourglass control approaches. The three FEM models have the same default contact control, hourglass control scaling factors and adaptive meshing parameters. The three models give very similar results, which show the models are insensitive to different hourglass control approaches in the case of ring compression simulations. Due to the insensitivity of different models, the default mode in ABAQUS/Explicit, integral viscoelastic approach will be used in the later coining simulations.

The load against height reduction results from the four models with different hourglass scaling factors are shown in Figure 3-11. The four different models have the same hourglass approach, contact control and adaptive meshing paramters. Figure 3-11 gives very similar results in the height reduction range of $0 \%-50 \%$, but shows a small sensitivity to different scaling factor combination in the final stage of the height reduction (50\%-60\%). The model with 4/1/0 scaling factor combination has slightly higher load reading. The model with equally balanced factor ratio has lowest load reading. Since the results converges, the default scale factor combination $1 / 1 / 0$ will be used for the later FEM coining simulations.

\subsubsection{Contact Simulation}

The effects of different contact simulations are shown in Figure 3-12. No significant difference was observed. Two approaches present quite close and convergent solutions for the simulation, but the model with penalty contact simulation needs about $40 \%$ more time for the simulation than the model with kinematic contact simulation. In the later $2 \mathrm{D}$ and $3 \mathrm{D}$ coining simulations, the default mode in ABAQUS/Explicit, kinematic contact simulation will be used. 


\subsubsection{Adaptive Mesh}

Figure 3-13 shows the results in load against height reduction from the five models with different adaptive meshing paramter combinations in frequency/sweep numbers. These models having the same hourglass controls, hourglass scaling factor combination and contact simulation, show a sensitivity to the combinations of adaptive meshing parameters. When the sweep number is 10 , the loading pattern presents fluctuating abnormal behavior. A smoother loading path is achieved when the sweep number is 1 . The greatest fluctuation happens on the lower frequency adaptivity model (frequency=20). It shows an appropriate high frequency (Frequency $=200$ ) in adaptivive meshing control can help to achieve better convergent results, but at the expense of longer simulation time. Also, it should be noted that once the parameter combinations fall into a certain range, a slight change of the parameters would not significantly affect the results. This indicates the importance of having a good estimation and careful determination of adaptive meshing parameters combination range for the simulation.

Through the analysis, it was shown that the different approaches of hourglass controls and contact simulation would not cause any significant divergence to the results of the FEM ring compression simulations. Adaptive mesh control is a case depending parameter, some trial-and-error and experience will be helpful to determine the appropriate values. It has been noted that due to the simple geometry and contact in the ring compression simulation, the process may not reflect the real conditions in the coining process. It was also realized that the ring deformation process may not trigger the hourglass instability or require adaptive meshing during the simulation. These initial test results will be further verified using $2 \mathrm{D}$ asymmetric coining models in Chapter 6 , which are considered to be closer to the real coining conditions. 


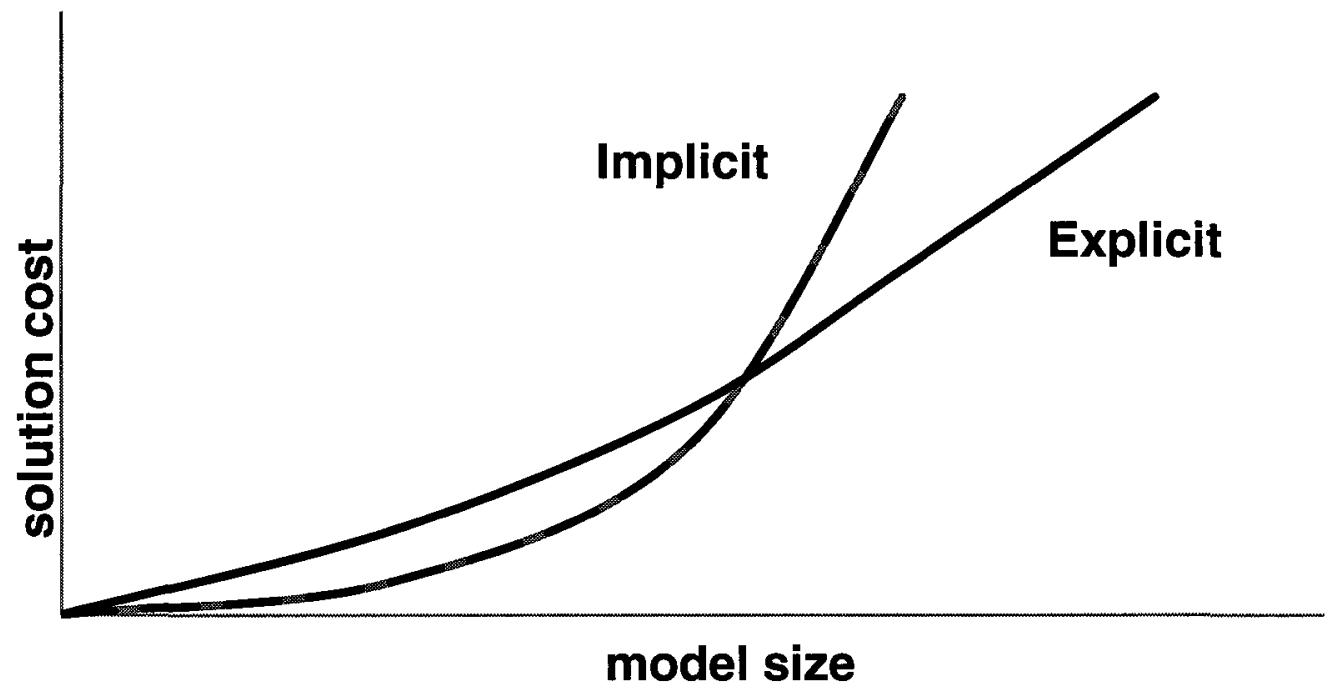

Figure 3-1. Comparison of implicit and explicit solution costs [ABAQUS, 2006]

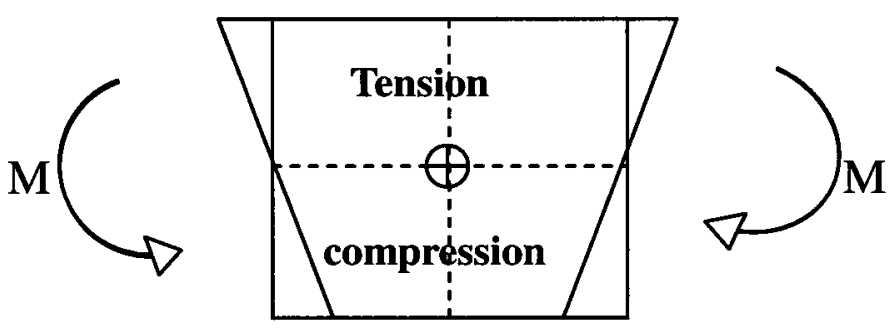

Figure 3-2. Hourglassing [ABAQUS, 2006] 


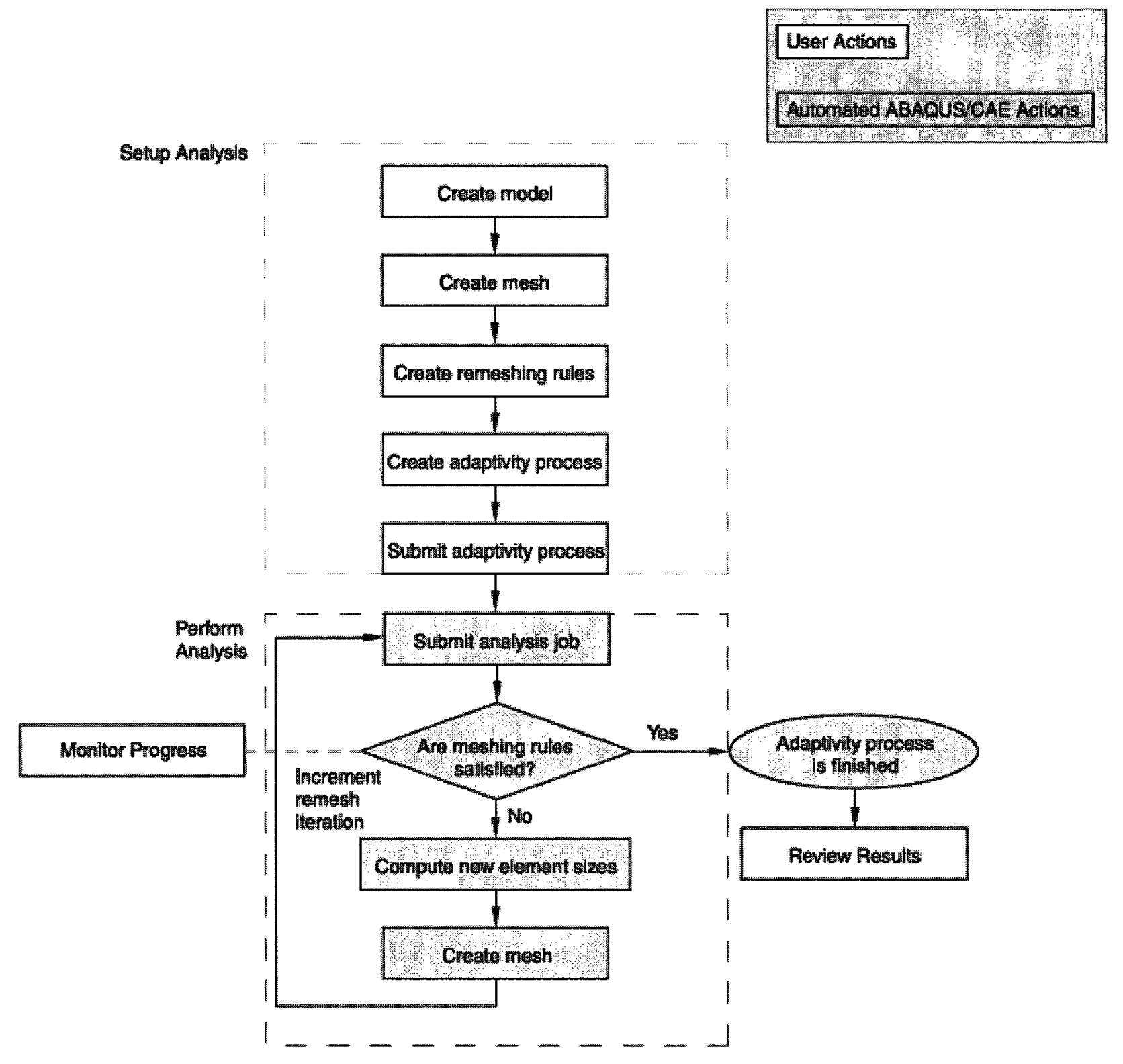

Figure 3-3. Adaptive remeshing process [ABAQUS, 2006] 


\section{Lagrangian}
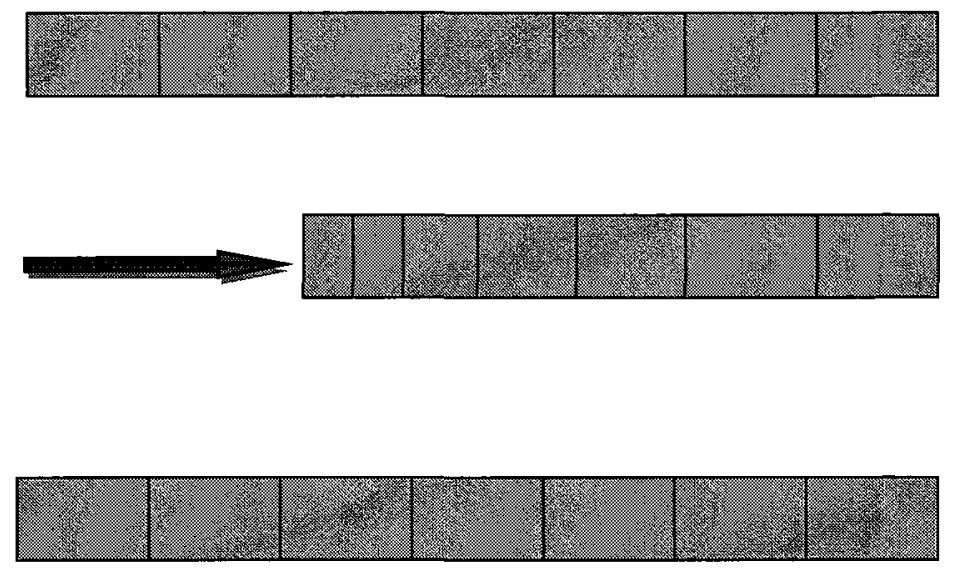

\section{ALE}
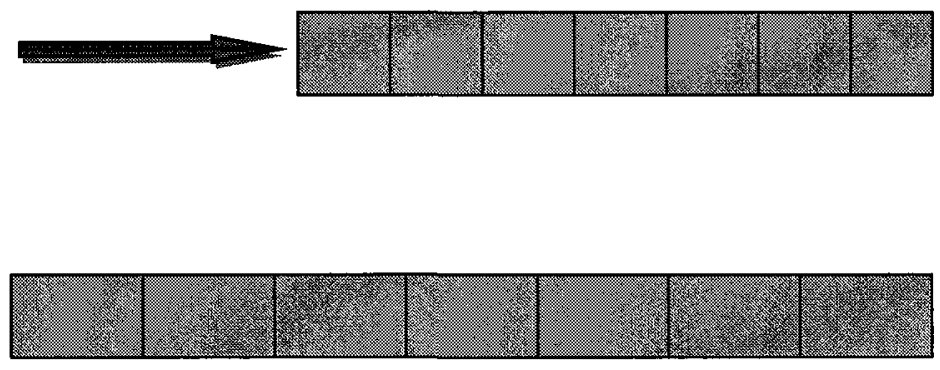

\section{Eulerian}

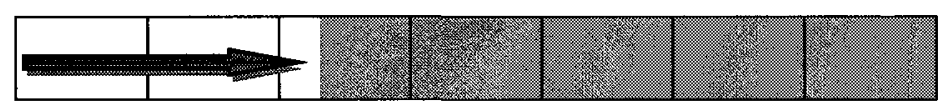

Figure 3-4. Motion of mesh and material with various methods [ABAQUS, 2006] 


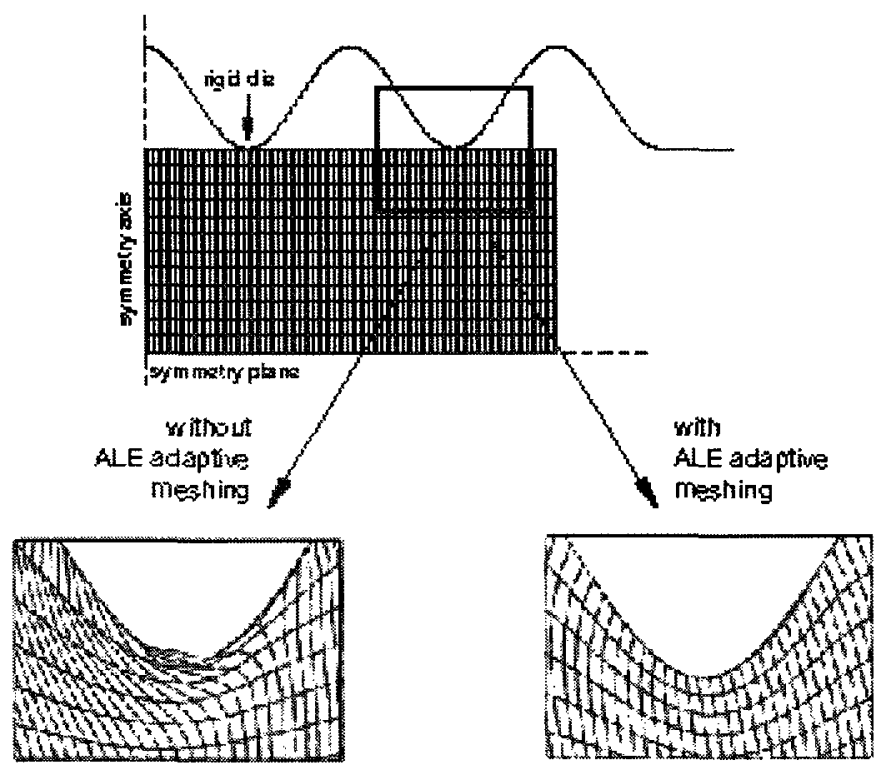

Figure 3-5. Comparison of non-adaptive meshing and adaptive meshing analysis [ABAQUS, 2006]

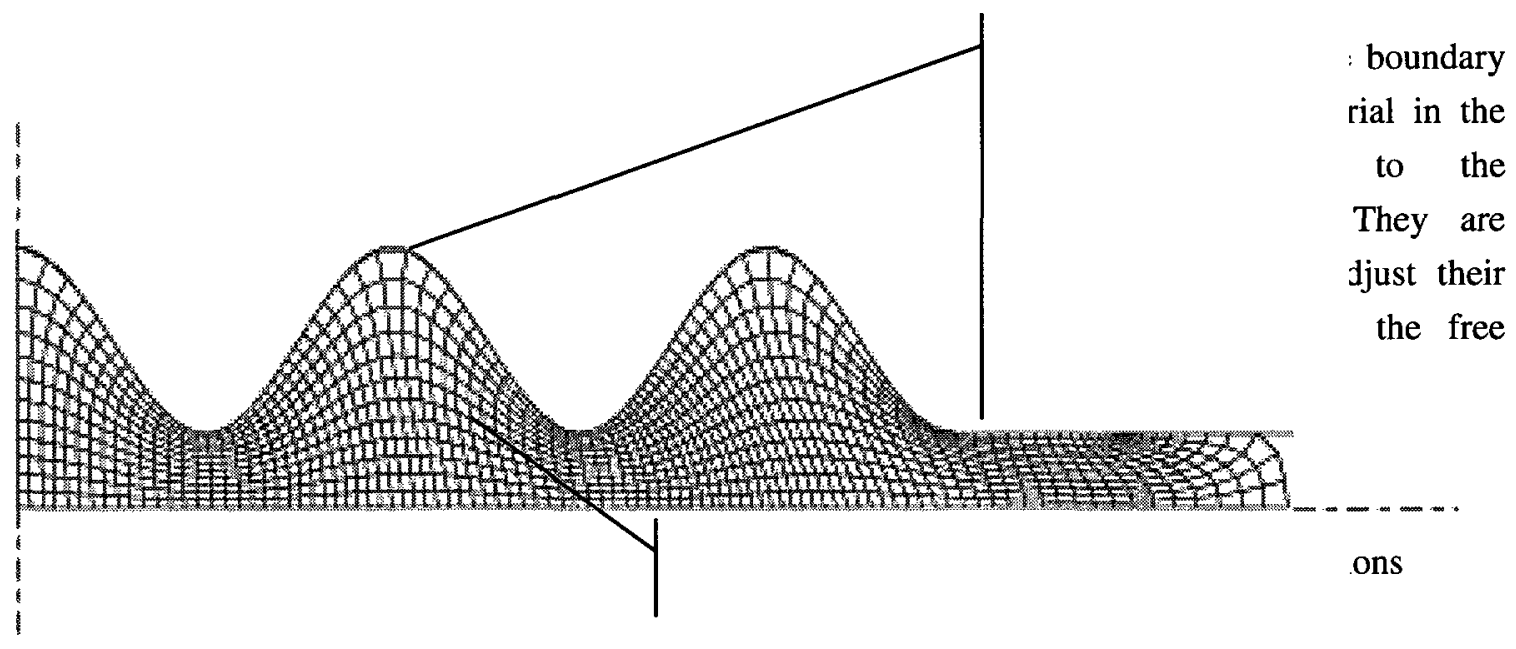

Figure 3-6. Deformed mesh in a ALE simulation [ABAQUS, 2006] 


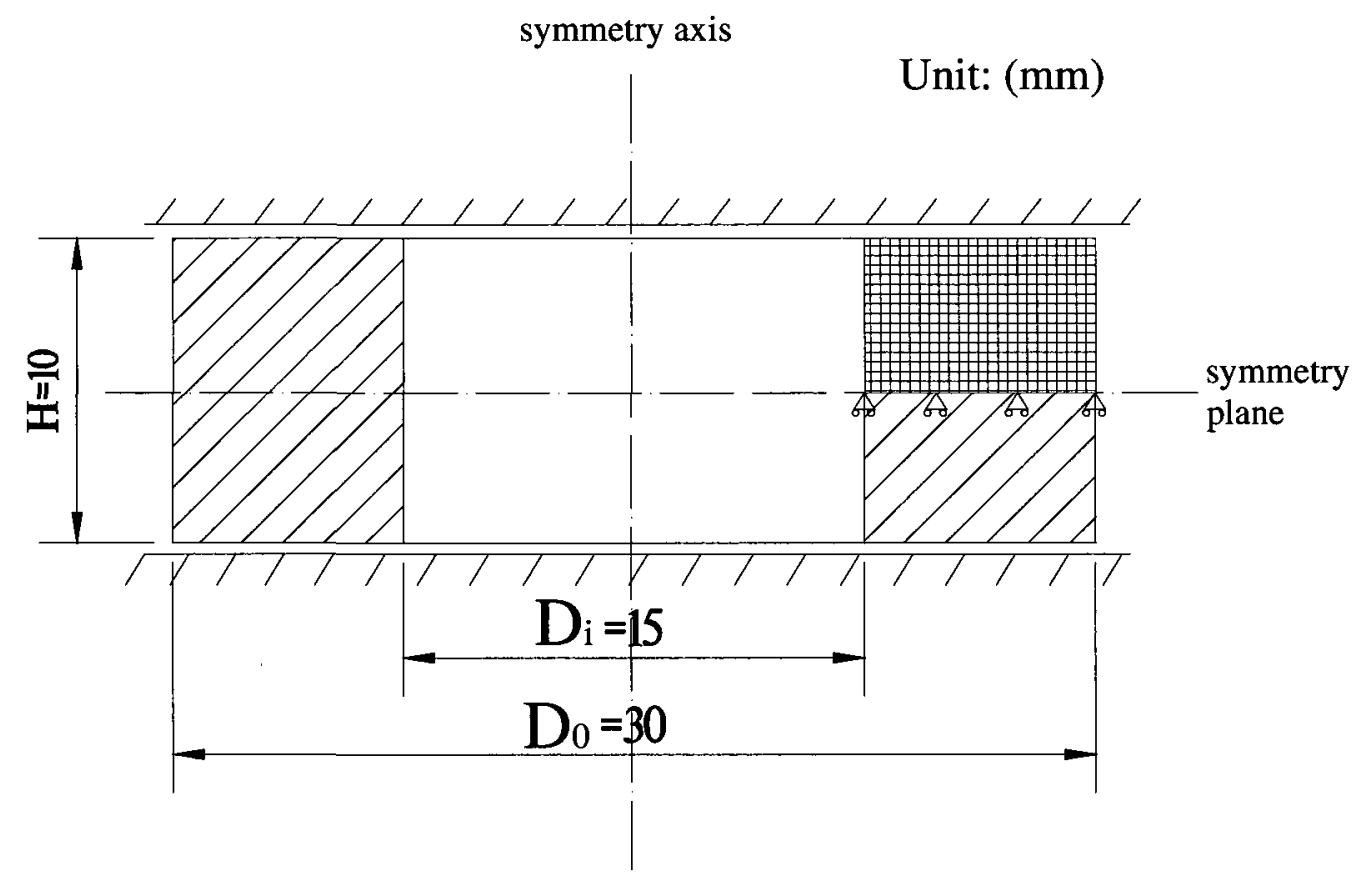

Figure 3-7. 2D axisymmetric ring upsetting FEM model

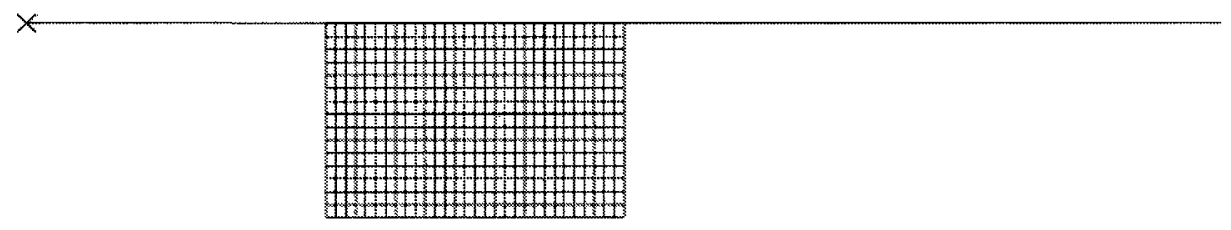

Figure 3-8. Initial mesh of the simulation

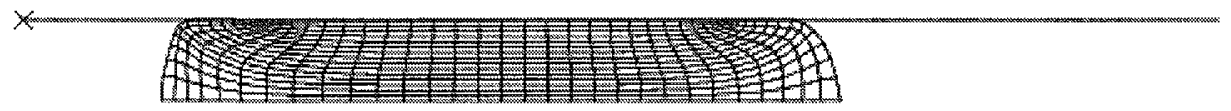

Figure 3-9. Deformed mesh of the simulation 


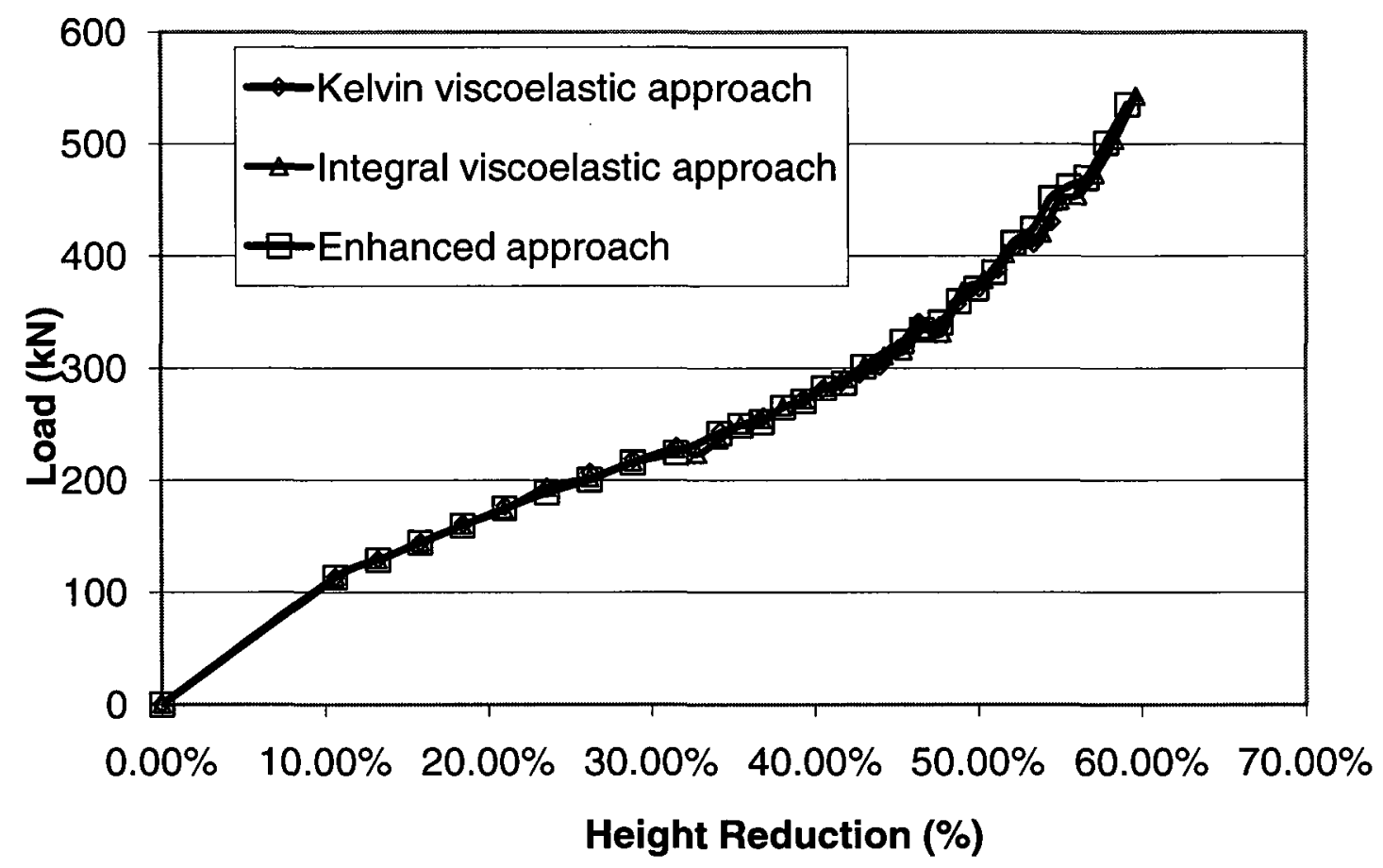

Figure 3-10. FEM simulation results with different hourglass control approachs

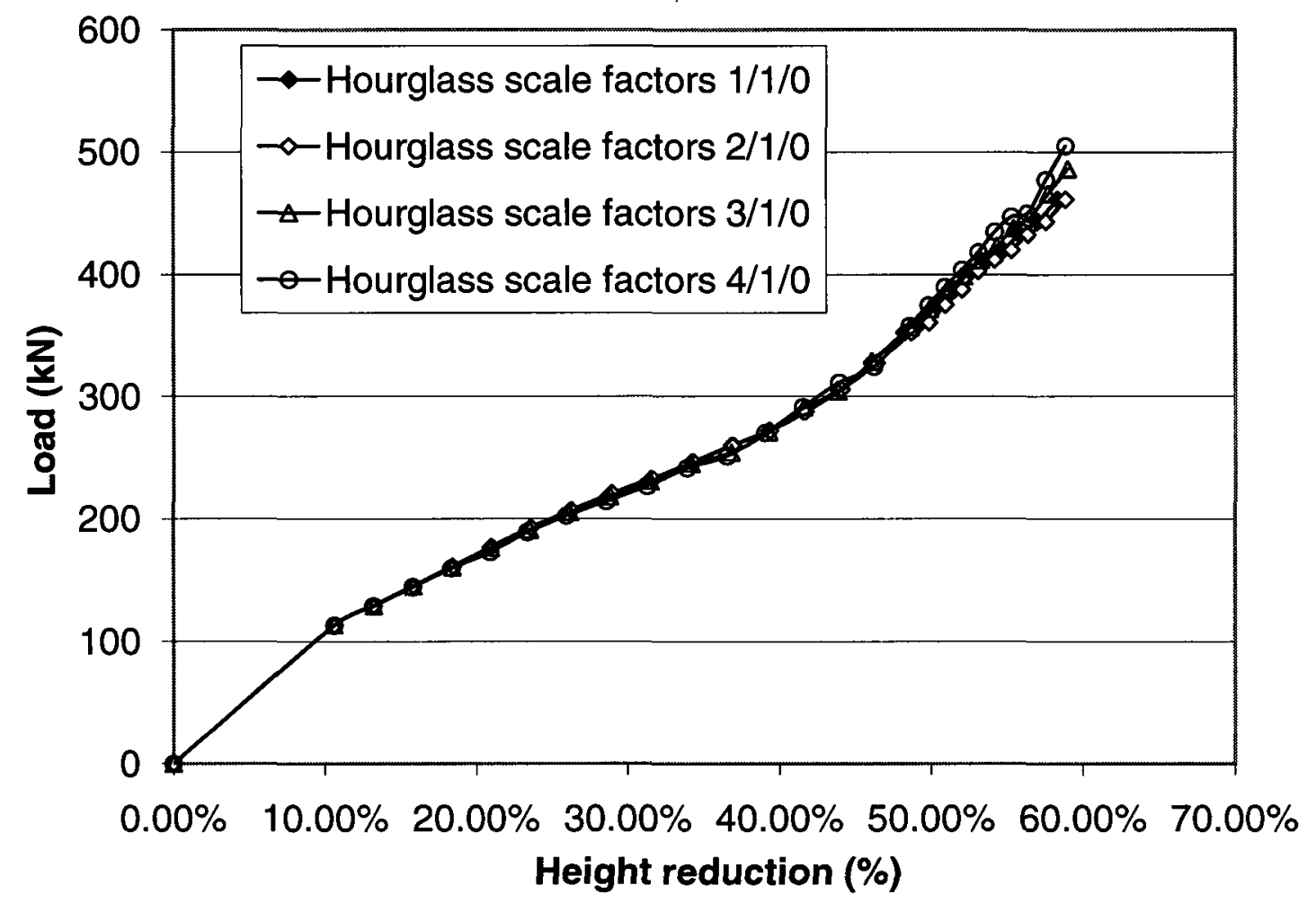

Figure 3-11. FEM simulation results with different hourglass scale factors 


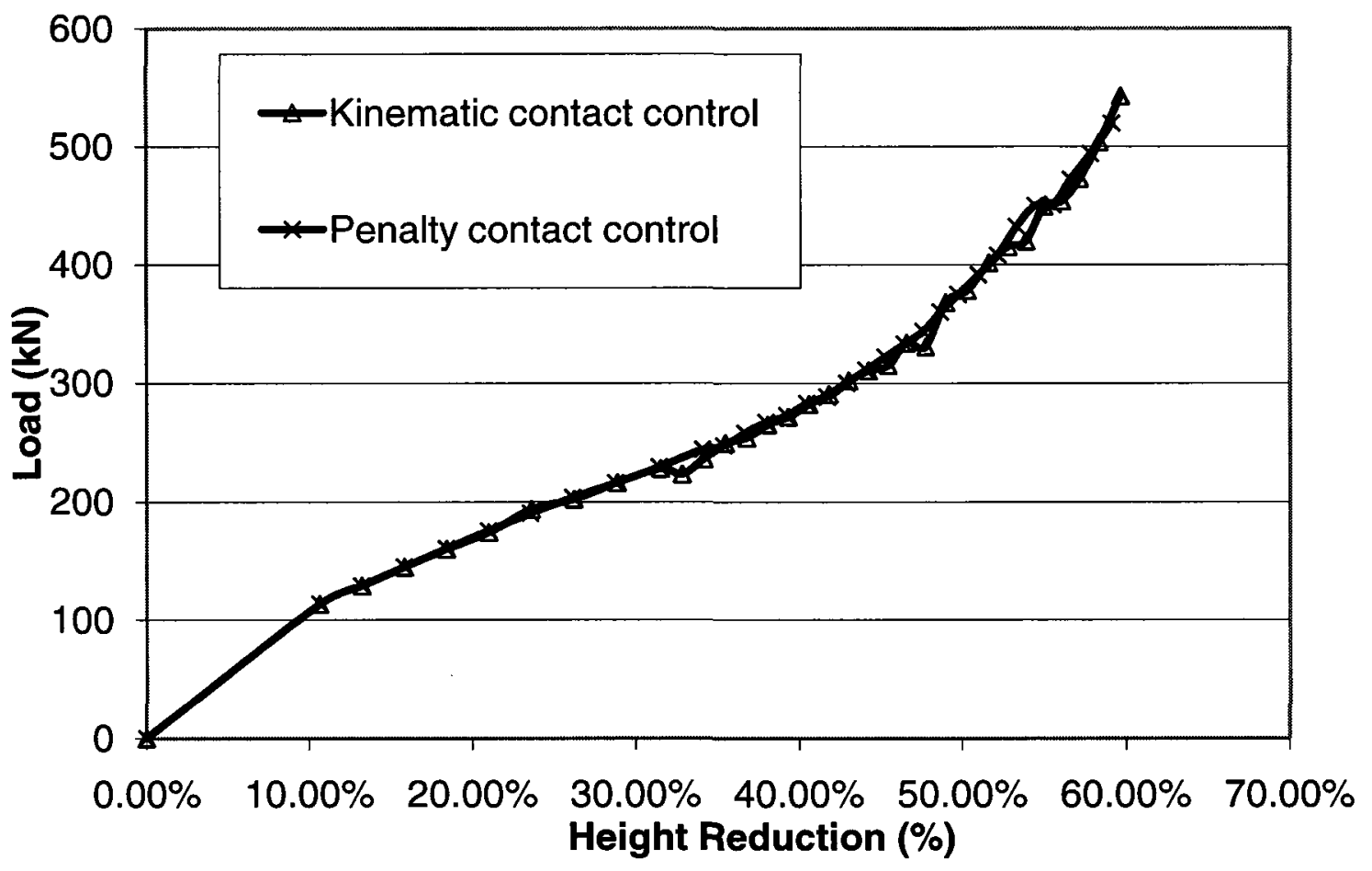

Figure 3-12. FEM simulation results with different contact controls

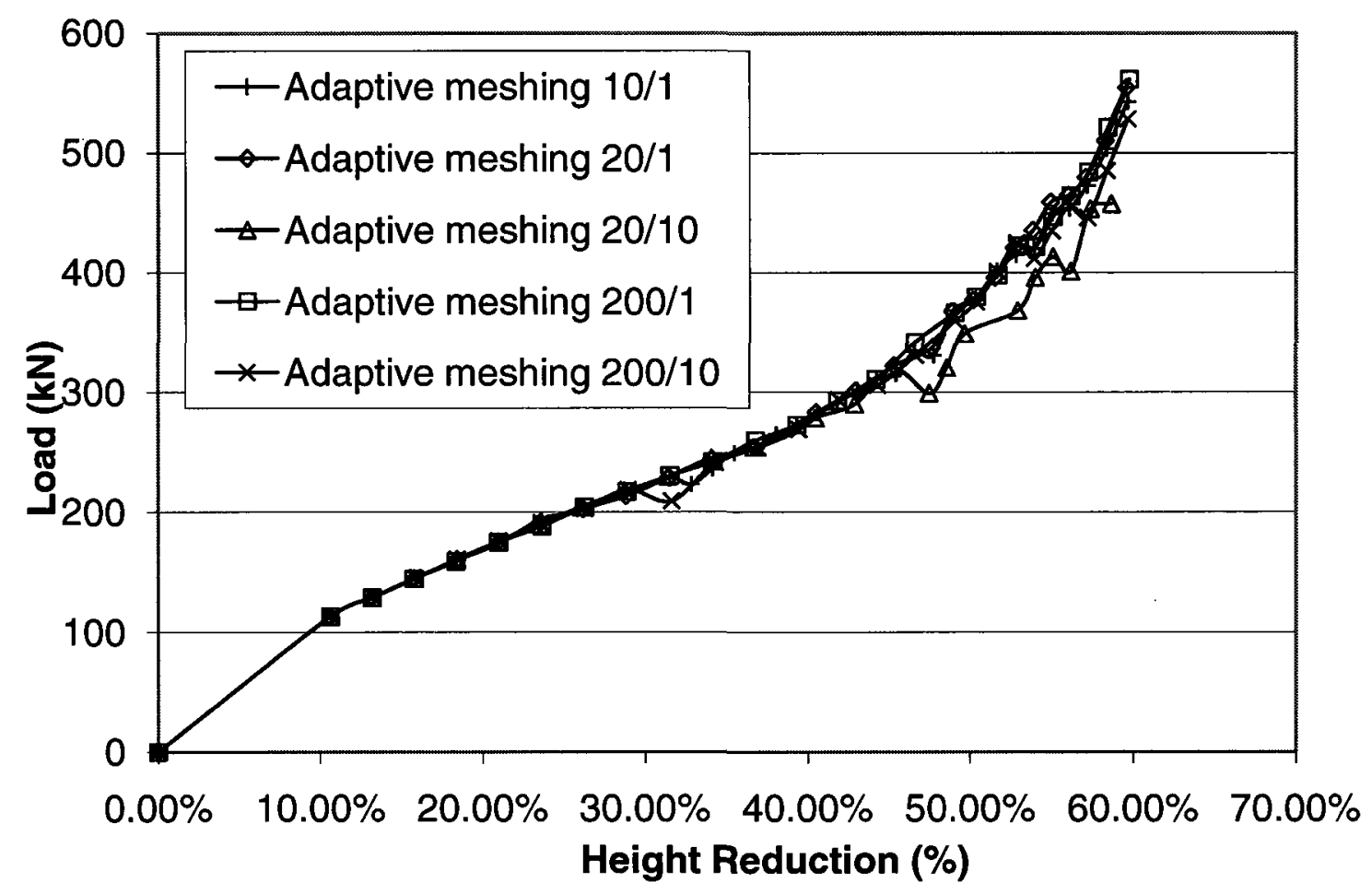

Figure 3-13. FEM simulation results with different adaptive meshing 


\section{Chapter 4}

\section{Coining Material Property Evaluations}

In this chapter, the mechanical properties of a typical material used at Royal Canadian Mint (RCM) will be addressed. Fine silver, as a basic material for a majority of collection coins produced by RCM, will be used as a typical material for the coining process simulations. The validity and accuracy of such simulations will depend on the validity and accuracy of the material property model incorporated into the numerical simulations. In the following sections, the specifications of the mechanical properties of fine silver will be obtained and the procedures to obtain such properties will be discussed. The resulting material properties are then available for use in the numerical modeling of silver dollar coining process.

\subsection{Material Properties for Coining Simulations}

In discussing the properties of silver it is of paramount importance that the level of purity be specified. "Fine" silver, by definition, contains at least 99.9 per cent Ag. Common impurities and the usual amounts of each are listed in the Table 4-1 [Industrial Products Bulletin, 1959]. A tentative ASTM specification [ASTM, 1970] is also presented.

"High" fine silver contains at least 99.95 per cent $\mathrm{Ag}$, and laboratory refined or "spectroscopically pure" silver contains not more than 0.001 per cent total identifiable impurities. Gas content, in particular oxygen, is not specified in any purity specification. Unless otherwise noted, the following discussion will be limited to commercial "fine" silver.

\section{Young's Modulus, Density, Poisson Ratio}

In the literature there are often various values for a given property. Where multiple values were found, a representative published value [Journal of R.N.I.S.T, 1995] was chosen for use in the simulations.

Young's Modulus $\quad 82.5 \mathrm{GPa}$ (at 293K) 
Density $\left(20^{\circ} \mathrm{C}\right) \quad 10492 \mathrm{~kg} / \mathrm{m}^{3}$

Poisson Ratio $\quad 0.364$ (at 293K)

Tensile Yield Strength

Various values have been reported for the yield strength of fine silver. Unfortunately, the purity levels in the test material, the complete mechanical working history, the degree of anisotropy, and the grain size are not available in any of these reports. To have a valid value for the practical simulation, an experimental value was measured using the specimens in the same fine silver material as the silver dollar produced by Royal Canadian Mint.

\section{Material Strain-Hardening Behavior}

The strain-hardening properties of fine silver are sensitive to history, impurities, and grain size as the other properties already enumerated. An experiment was conducted, which is discussed in the following section, to evaluate the strain-hardening properties using the specimens in the same fine silver material as the Royal Canadian Mint silver dollar.

The following section will address the methods used in the experiments to obtain the material hardening properties and yield strength of the fine silver being used at Royal Canadian Mint.

\subsection{Approaches to Obtain the Material Properties}

Fine silver is a very ductile material, therefore a standard uni-axial tensile test was performed to evaluate the material strain hardening behavior. The uni-axial tensile test is one of the most widely performed mechanical property tests. Standardized tension testing methods have been developed under the auspices of ASTM and are published in the Annual Book of ASTM standards [ASTM, 1982]. ASTM E8 standard methods specify important details about test specimen selection, size, and shape, test setup and procedures, and data analysis. Average strain rates for most tension tests range between $10^{-2}$ and $10^{-5} \mathrm{~s}^{-1}$.

\section{Test Specimen Preparation}

Four specimens were cut from a fine silver blank at the Royal Canadian Mint. Then the specimens were annealed per RCM's standard annealing procedure for fine silver. The specimens 
were made according to the size and shape defined by the standards of ASTM E8. The specimen has a minimum section gauge width of $12.5 \mathrm{~mm}$, gauge length of $50 \mathrm{~mm}$ and overall length of $200 \mathrm{~mm}$ as shown in Figure 4-1. The surface finish of test specimens received from a machine shop is not always smooth, and detailed surface examination was used to make sure the surface is free of deep scratches, gouges, or edge tears and any discontinuities were minimized by polishing or by further machining.

\section{Test Setup and Procedures}

The specimens were manufactured to minimize the design tolerances that can unfavorably affect the positioning of specimens in a test setup and introduce unwanted bending stresses. The test specimen was installed in the grips with a minimum of distortion. And careful alignment measures were taken to minimize the displacements from the intended loading axis and unwanted bending stresses.

The fine silver specimens were tested using MTS 810 material testing system, as shown in Figure 4-2. An extensometer device was calibrated and properly fastened to the specimen. The strain was measured by a MTS extensometer across the central parallel sided gauge length of $20 \mathrm{~mm}$, as shown in Figure 4-3. The uni-axial test was conducted by controlling the rate of straining, in which the allowable limits for rate of straining was specified in millimeters per millimeter per second. In this test, four silver specimens were tested at room temperature at two constant strain rates of $0.83 \%$ per second and $0.25 \%$ per second.

\subsection{Fine Silver Strain Hardening Behavior Obtained}

The experimental load-strain results and averaged data are presented in Table 4-2. The fine silver strain hardening curves were plotted in Figure 4-4a. The four curves in the figure are very close, which indicates that as long as the testing remains in the quasi-static range, the strain rate difference has no influence on the results. The engineering stress-strain curve was obtained by averaging the load-strain results and using the cross section at the minimum uniform section of the specimens, presented in Figure 4-4b. 
The yield strength of fine silver was determined by the "offset method". From the engineering stress-strain curve, the $0.2 \%$ offset criterion was used to obtain the yield stress $\sigma_{y}$ of $61 \mathrm{MPa}$, Also, The ultimate strength $\sigma_{\mathrm{u}}$ of fine silver was found to be $168.3 \mathrm{MPa}$. Therefore, the flow stress $\sigma_{\mathrm{f}}$ was obtained to be $115 \mathrm{MPa}$ by using equation (4.1)

$$
\sigma_{\mathrm{f}}=\left(\sigma_{\mathrm{y}}+\sigma_{\mathrm{u}}\right) / 2
$$

The true stress-strain behavior of fine silver was needed as the input for the FEM analysis. A simple conversion of nominal engineering stress-strain to true stress and logarithmic strain was carried out by using the following equations:

$$
\begin{gathered}
\sigma_{\text {true }}=\sigma_{\text {norm }}\left(1+\varepsilon_{\text {norm }}\right) \\
\varepsilon_{\text {ln }}=\ln \left(1+\varepsilon_{\text {norm }}\right)
\end{gathered}
$$

where: $\sigma_{\text {norm }}$ : Normal Stress

$\varepsilon_{\text {norm }}:$ Normal strain

$\sigma_{\text {true }}:$ True stress

$\varepsilon_{\ln } \quad:$ Logarithmic total strain

$$
\varepsilon_{l n}^{p l}=\varepsilon_{l n}-\frac{\sigma_{\text {true }}}{E}
$$

where $\varepsilon_{\ln }^{p l}$ is logarithmic (true) strain

The true stress-strain curve of the fine silver was calculated and presented in Figure 4-5. It is known that for cold bulk forming processes, usually the stress-strain curve at room temperature suffices, and should be available until an equivalent plastic strain of about 3 . However, the tensile test can only produce curves up to a strain of 0.3 to 0.4 . Hence, standard tests give only $10 \%-15 \%$ of the required range of the hardening data. The usual practice is to extrapolate the remaining $85 \%-90 \%$ of the flow curve from the available experimental data. Since the flow curve is flat after a strain of unit magnitude, for bulk forming processing it is recommended to take a constant flow stress after this strain [Tekkaya, 2005]. Thus, the fine silver strain hardening curve was obtained, in Figure 4-5 and Table 4-3, by extrapolating the remaining part of the curve up to 1 from the experimental data and remaining constant after the unit strain. These mechanical and true stress-true strain data were used as inputs in the later FEM coining simulations. 


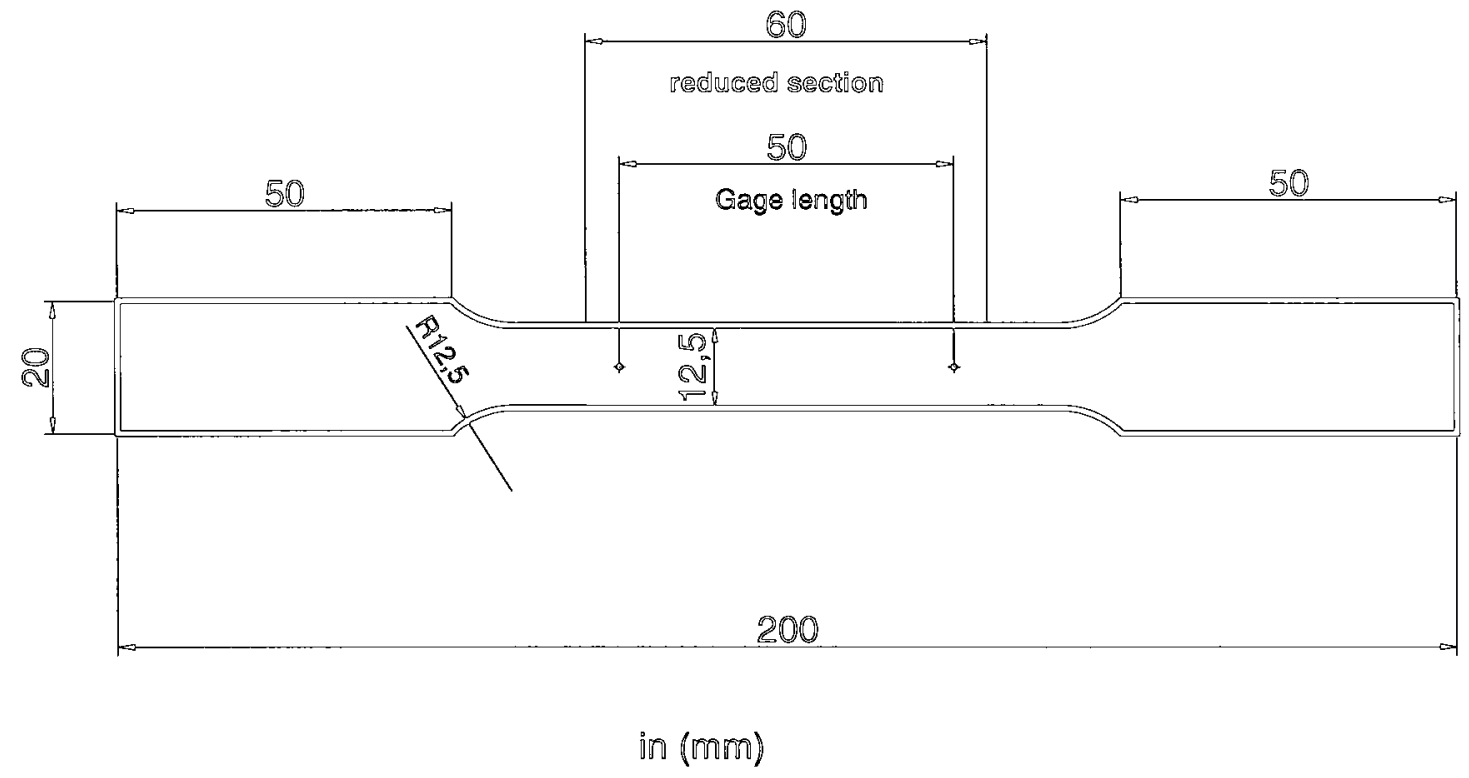

Figure 4-1. Uni-axial tensile test specimen (thickmess $=3 \mathrm{~mm}$ )

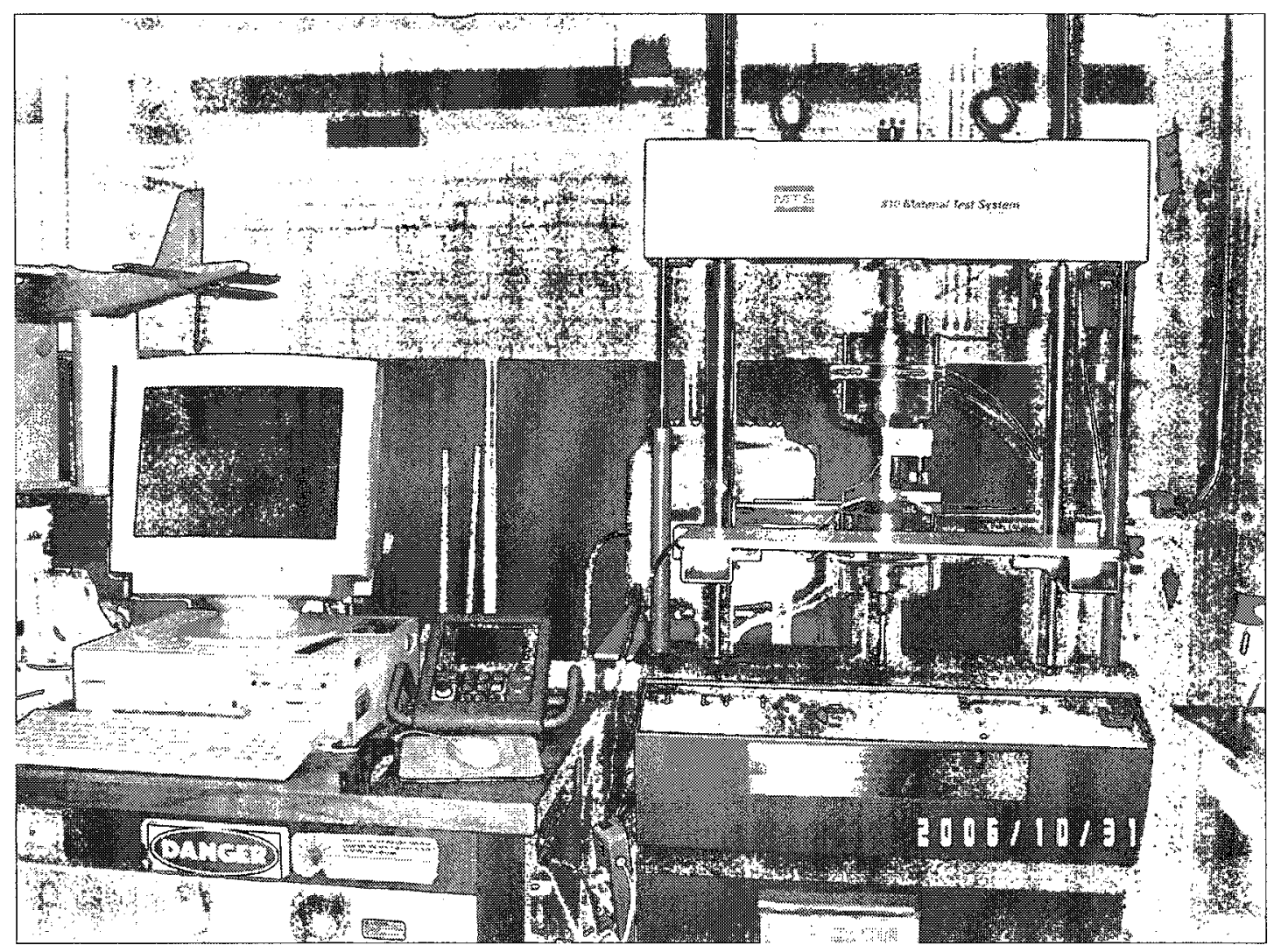

Figure 4-2. Uni-axial tensile test setup 


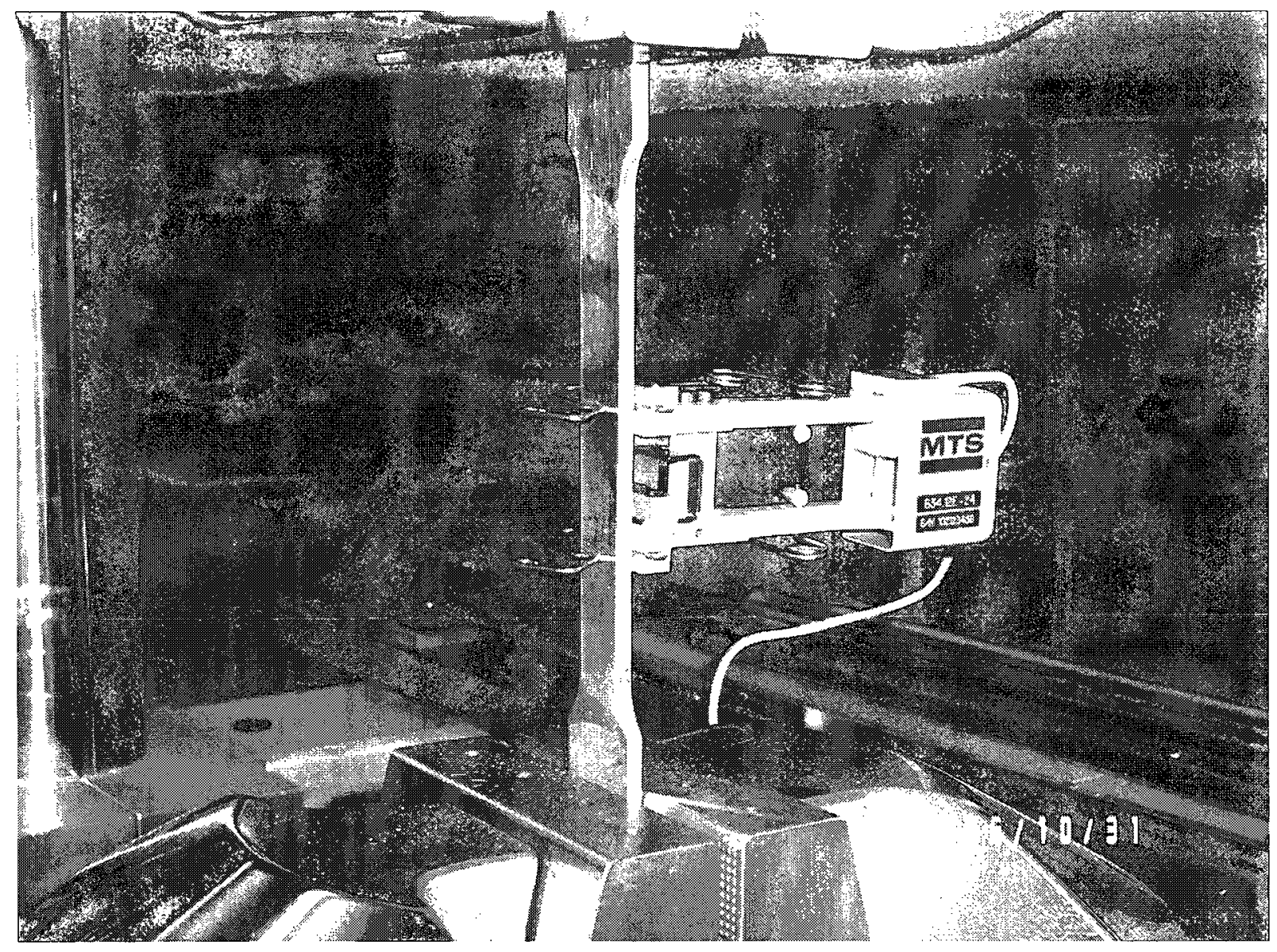

Figure 4-3. Extensometer setup on specimen 


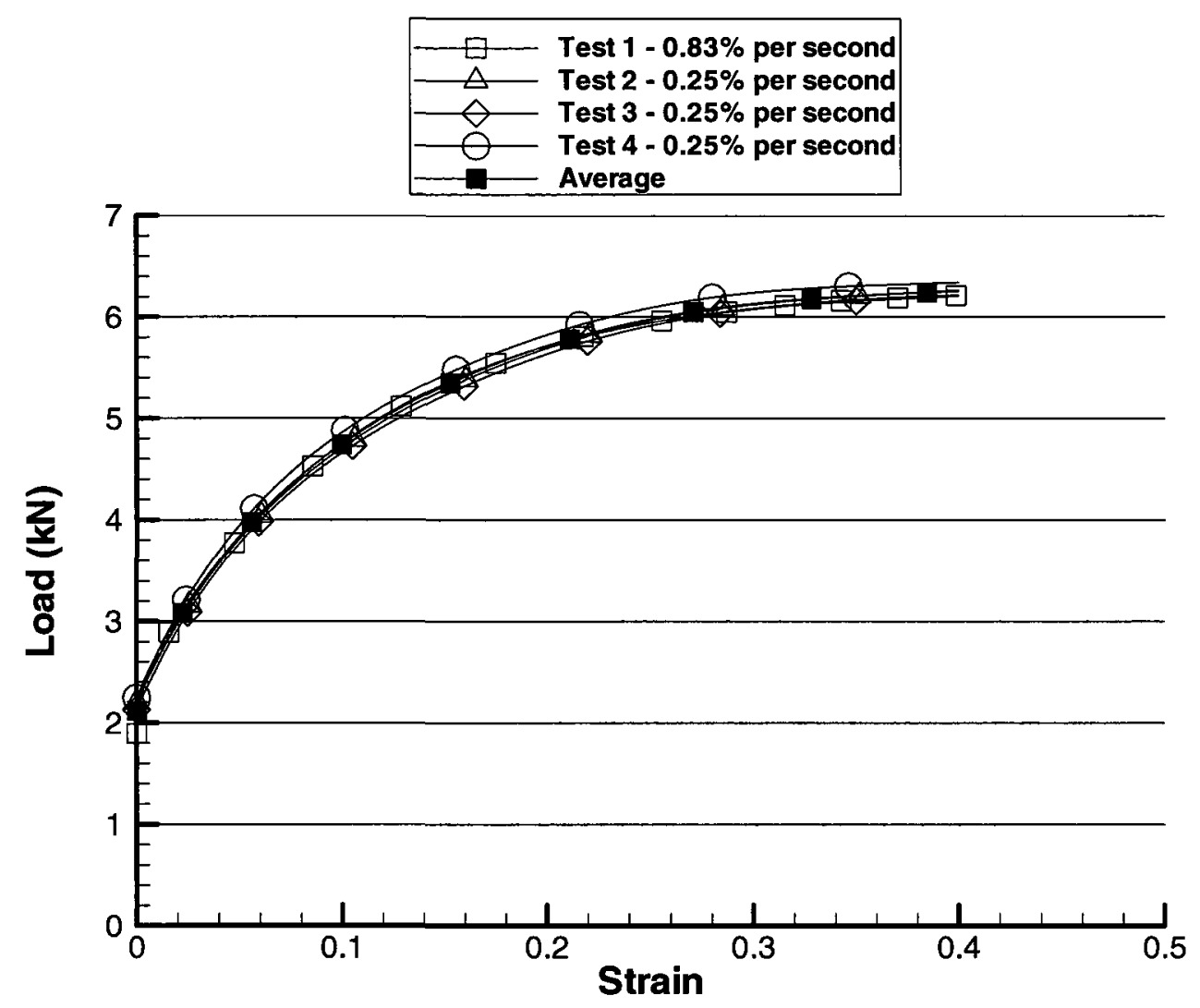

Figure 4-4a Experimental strain hardening model of fine silver

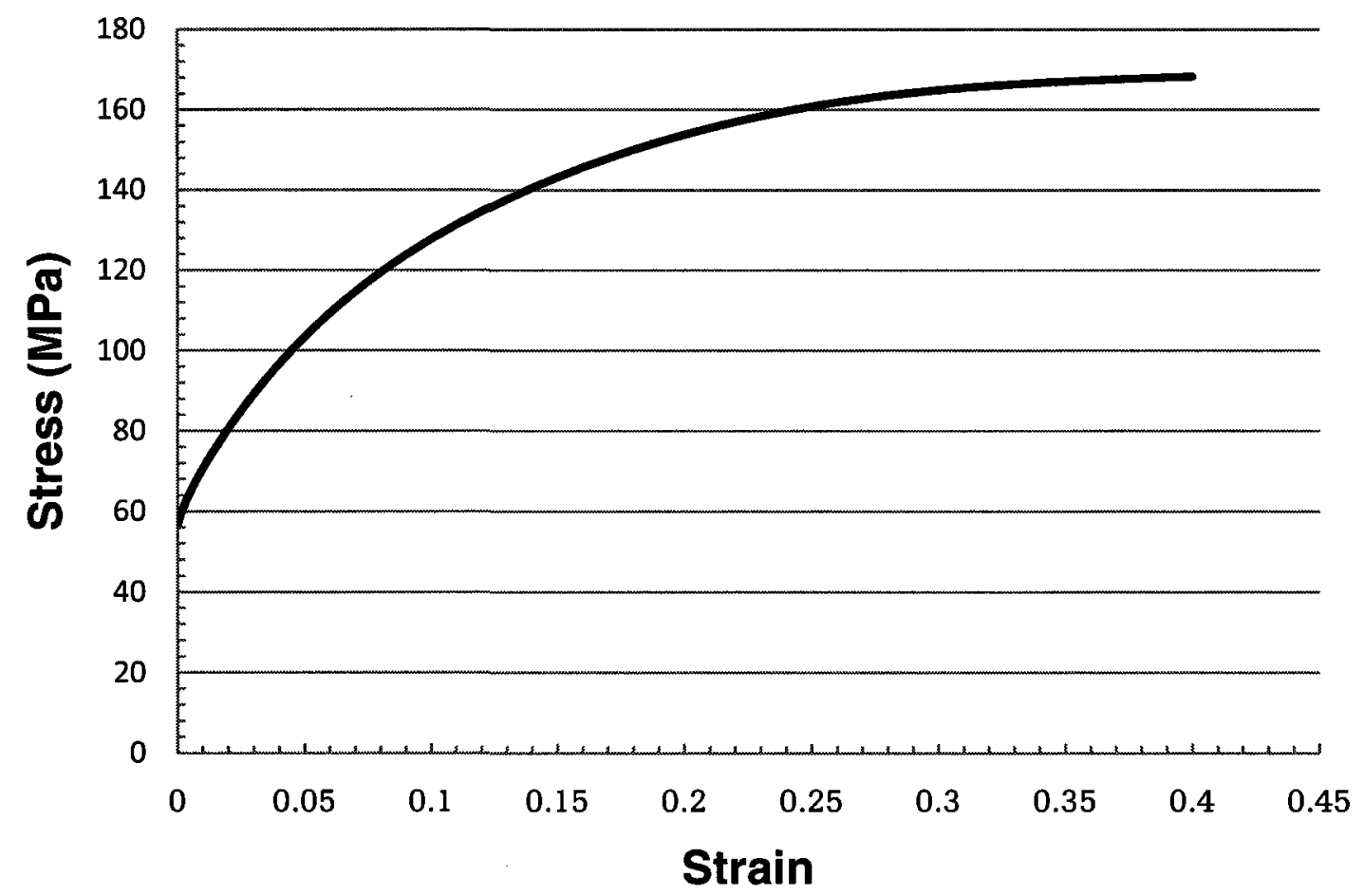

Figure 4-4b. The averaged engineering stress-strain curve of fine silver 


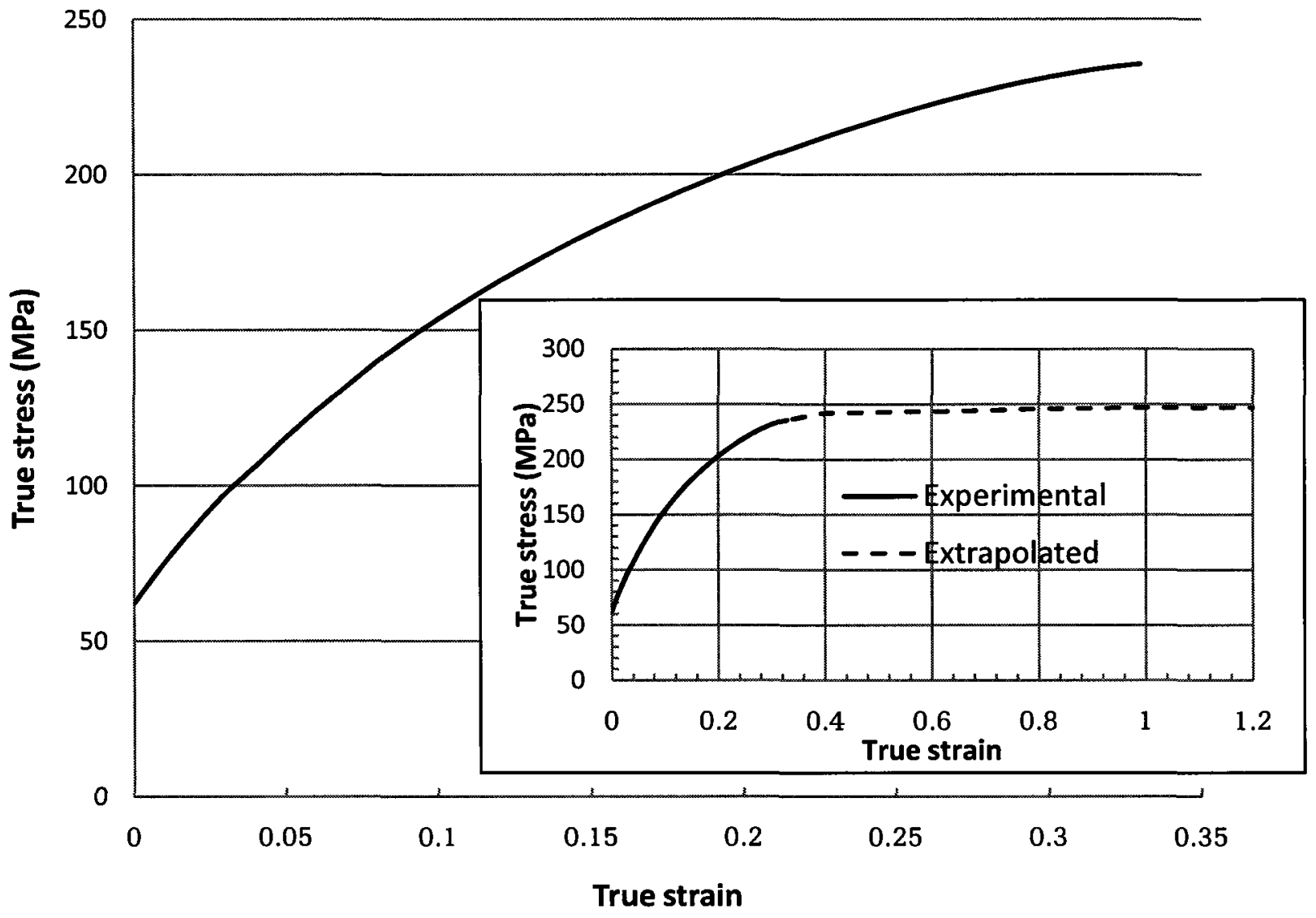

Figure 4-5 The true stress-strain model of fine silver

\begin{tabular}{|ccc|}
\hline Element & $\begin{array}{c}\text { Amount, } \\
\text { per cent }\end{array}$ & $\begin{array}{c}\text { ASTM } \\
\text { per Tent Max. } \\
\text { per cent }\end{array}$ \\
\hline Silver + copper & $\ldots$ & $\mathbf{9 9 . 9 5 ~}$ min \\
\hline Copper & $\mathbf{0 . 0 5}$ & $\mathbf{0 . 0 8}$ \\
\hline Lead & $\mathbf{0 . 0 0 4}$ & $\mathbf{0 . 0 2 5}$ \\
\hline Iron & $\mathbf{0 . 0 0 3}$ & $\mathbf{0 . 0 0 2}$ \\
\hline Nickel & $<\mathbf{0 . 0 0 1}$ & $\ldots$ \\
\hline Manganese & $<\mathbf{0 . 0 0 1}$ & $\ldots$ \\
\hline Magnesium & $<\mathbf{0 . 0 0 1}$ & $\ldots$ \\
\hline Silicon & $<\mathbf{0 . 0 0 1}$ & $\ldots$ \\
\hline Bismuth & $<\mathbf{0 . 0 0 1}$ & $\mathbf{0 . 0 0 1}$ \\
\hline
\end{tabular}

Table 4-1. Impurities in commercial fine silver 


\begin{tabular}{|c|c|c|c|c|c|}
\hline \multirow{2}{*}{ Strain } & \multicolumn{5}{|c|}{ Load (kN) } \\
\hline & Test 1 & Test 2 & Test 3 & Test 4 & Average \\
\hline 0.0002 & 1.89487077 & 2.205547 & 2.128991 & 2.245989 & 2.11884935 \\
\hline 0.01 & 2.6820438 & 2.61828 & 2.556297 & 2.687646 & 2.63606681 \\
\hline 0.03 & 3.3296912 & 3.291238 & 3.245708 & 3.401872 & 3.31712734 \\
\hline 0.05 & 3.8353636 & 3.815963 & 3.775444 & 3.951065 & 3.84445894 \\
\hline 0.07 & 4.2536445 & 4.231201 & 4.189044 & 4.378523 & 4.2631032 \\
\hline 0.09 & 4.6050334 & 4.56812 & 4.521251 & 4.719348 & 4.60343817 \\
\hline 0.11 & 4.8940468 & 4.847992 & 4.795818 & 4.997969 & 4.88395632 \\
\hline 0.13 & 5.1362729 & 5.085501 & 5.028976 & 5.231514 & 5.12056579 \\
\hline 0.15 & 5.3414321 & 5.289125 & 5.229989 & 5.430243 & 5.32269733 \\
\hline 0.17 & 5.5118413 & 5.469519 & 5.409677 & 5.605801 & 5.49920974 \\
\hline 0.18 & 5.585927 & 5.549837 & 5.490323 & 5.683983 & 5.5775174 \\
\hline 0.19 & 5.6545553 & 5.625621 & 5.566832 & 5.757824 & 5.65120822 \\
\hline 0.21 & 5.7688789 & 5.759322 & 5.702862 & 5.888351 & 5.77985338 \\
\hline 0.23 & 5.8669477 & 5.873064 & 5.819646 & 5.999606 & 5.88981598 \\
\hline 0.24 & 5.9051929 & 5.92253 & 5.870702 & 6.047965 & 5.93659743 \\
\hline 0.25 & 5.9416399 & 5.96647 & 5.916148 & 6.090819 & 5.97876911 \\
\hline 0.26 & 5.9809775 & 6.006271 & 5.957345 & 6.129455 & 6.01851205 \\
\hline 0.27 & 6.0025048 & 6.041591 & 5.993867 & 6.163466 & 6.05035718 \\
\hline 0.28 & 6.0315976 & 6.072745 & 6.02598 & 6.193095 & 6.0808544 \\
\hline 0.29 & 6.0590348 & 6.100016 & 6.053928 & 6.218563 & 6.10788541 \\
\hline 0.30 & 6.0852447 & 6.123902 & 6.078187 & 6.240303 & 6.13190904 \\
\hline 0.31 & 6.1033101 & 6.144756 & 6.099096 & 6.258624 & 6.15144642 \\
\hline 0.32 & 6.1209126 & 6.163029 & 6.11711 & 6.27395 & 6.1687503 \\
\hline 0.33 & 6.1411123 & 6.179177 & 6.132705 & 6.286728 & 6.18493073 \\
\hline 0.35 & 6.1696596 & 6.206662 & 6.158371 & 6.306359 & 6.21026301 \\
\hline 0.36 & 6.1820817 & 6.218792 & 6.169362 & 6.314145 & 6.22109511 \\
\hline 0.37 & 6.1920986 & 6.230336 & 6.179713 & 6.321186 & 6.23083316 \\
\hline 0.38 & 6.2014446 & 6.241472 & 6.189701 & 6.32782 & 6.24010918 \\
\hline 0.39 & 6.2135887 & 6.252105 & 6.19936 & 6.334218 & 6.24981796 \\
\hline 0.40 & 6.2109685 & 6.262078 & 6.208659 & 6.340487 & 6.25554791 \\
\hline 0.403 & 6.2177086 & 6.254058 & 6.209522 & 6.348944 & 6.2543081 \\
\hline
\end{tabular}

Table 4-2. Tensile test data of fine silver 


\begin{tabular}{|c|c|c|c|}
\hline True strain & True stress $(\mathrm{MPa})$ & True strain & True stress (MPa) \\
\hline 0 & 62.09 & 0.31 & 233.04 \\
\hline 0.01 & 75.06 & 0.32 & 234.48 \\
\hline 0.02 & 86.66 & 0.33 & 235.53 \\
\hline 0.03 & 97.3 & 0.34 & 236.51 \\
\hline 0.04 & 106.09 & 0.35 & 237.49 \\
\hline 0.05 & 115.26 & 0.36 & 238.36 \\
\hline 0.06 & 123.81 & 0.37 & 239.23 \\
\hline 0.07 & 131.83 & 0.38 & 240.18 \\
\hline 0.08 & 139.88 & 0.39 & 241.12 \\
\hline 0.09 & 146.91 & 0.4 & 241.82 \\
\hline 0.1 & 153.55 & 0.5 & 242.73 \\
\hline 0.11 & 159.79 & 0.6 & 243.75 \\
\hline 0.12 & 165.66 & 0.7 & 244.83 \\
\hline 0.13 & 171.19 & 0.8 & 245.84 \\
\hline 0.14 & 176.41 & 0.9 & 246.21 \\
\hline 0.15 & 181.35 & 1 & 246.80 \\
\hline 0.16 & 186.04 & 2 & 246.80 \\
\hline 0.17 & 190.48 & 3 & 246.80 \\
\hline 0.18 & 194.71 & & \\
\hline 0.19 & 198.74 & & \\
\hline 0.2 & 202.58 & & \\
\hline 0.21 & 206.25 & & \\
\hline 0.22 & 209.74 & & \\
\hline 0.23 & 213.06 & & \\
\hline 0.24 & 216.22 & & \\
\hline 0.25 & 219.21 & & \\
\hline 0.26 & 222.03 & & \\
\hline 0.27 & 224.66 & & \\
\hline 0.28 & 227.10 & & \\
\hline 0.29 & 229.32 & & \\
\hline 0.3 & 231.31 & & \\
\hline
\end{tabular}

Table 4-3. The true stress-strain data of fine silver 


\section{Chapter 5}

\section{Friction Condition Evaluations of Coining Process}

Friction between a workpiece and dies or tools dominates the deformation patterns and performance of many forming operations. Friction depends sensitively on a host of variables that are not usually well characterized and are quite diverse for various forming operations. Until recently, forming analysis was a trial and error process. Thus friction is often the least quantified of all phenomena involved in forming. In this Chapter, the principal friction behavior and its evaluation in the coining operations and how it is applied to the numerical analysis will be presented.

\subsection{Friction Behavior in Coining Process}

In a coining process, forming occurs between solid metals and the dies which are very hard and abrasion-resistant in order to reduce wear between the surfaces of the die and the silver blank. They are not only in contact with the blank but invariably must slide over it during the forming operation. The process is designed to be lubricant free to maintain a shiny surface quality, which is considered to be critical for a collection coin. Metal-to-metal contact occurs in the coining process and the surfaces of workpiece and die are separated by a boundary layer of molecular dimensions. In such cases so-called boundary lubrication occurs which is well recognized in practice but whose basic laws are not so well known. From the study with radioactive metals [Thomsen. et. al., 1965], it has been found that the boundary layer can be punctured by local high spots in the surfaces and that wear appears to be the result of alternate forming and breaking of metallic junctions. Thus in boundary lubrication the nature and condition of the contacting surfaces becomes extremely important. Many factors can change the nature and condition on the contacting surfaces [Edward, 1991].

- Contact pressure, simple experiments show that the friction force increases as the 
contact pressure or normal force increases, at least at low pressures.

- Materials. The elastic and plastic properties of the materials in contact will certainly affect how difficult for the materials to slide across one another. At low contact pressures, the asperities are more likely to interact elastically. Also, the materials may have different fracture characteristics that lead to changes in the surface topology and debris on the contacting surface.

- Surface roughness. The shape and density of asperities may have a major effect on friction. However, even the measurement of surface roughness is difficult and its qualification is not standard, except for a few simple parameters.

- Debris. The presence of other material at the contact surfaces is important. The distribution of the debris depends intimately on surface conditions, pressure, and sliding speed, and knowledge of all of these depends on the forming operation analysis.

- Sliding speed. It is shown that the dynamic friction force is less than the static friction force.

- Concurrent deformation. This effect is particularly germane for metal forming because the workpiece is usually deforming plastically while the friction forces are operating. The deformation changes the surface roughness, eliminates most elastic effects in the workpiece, and opens up a new surface by the action of dislocation slip.

With all of these variables influencing friction, it is apparent why it is necessary to make measurements under conditions as close as possible to the real ones in the forming operations. For purposes of simulation, it is necessary to adopt simple laws that allow measurement of a limited number of coefficients.

One of the friction laws is known as Coulomb's law. It states that the magnitude of the friction force is proportional to the magnitude of the normal force, as shown in Equation (2.40) [Von Karman, 1925, Schey, 1983]

$$
f_{s}=\mu p
$$

where, $\mathrm{p}$ is normal force and $\mu$ is the friction coefficient with the basic idea that it is a constant 
with respect to contact pressure for a given situation.

Coulomb's law, Eq (2.40), is conceptually correct at small pressures, in that the limiting tangential force approaches zero as the pressure tends toward zero. The law is usually found applicable at low contact pressures relative to material strength before there is a great deal of deformation. While Coulomb's law has the proper form for lightly loaded bodies, it drastically overestimates friction at high contact pressures.

A simple experiment shows that friction at the interface cannot exceed the shear strength of the material because the material can slide at this shear strength even if the interface is bound tightly (welded). This condition is called friction factor law, which is proper for a high contact pressure regime where the friction is only weakly dependent on pressure. The friction law may be represented generally in equation (2.41) [Siebel, 1930, Schey, 1983]

$$
f_{s}=m k
$$

where, $\mathrm{k}$ is the shear strength of the material, which can be determined from Tresca or Von Mises yield functions. For a Tresca material, the shear strength is equal to one-half of the material strength in uniaxial tension:

$$
\text { Tresca Yield Criterion: } \quad k=\frac{\sigma_{0}}{2}
$$

where, $\sigma_{0}$ is the tensile strength of the material

whereas for a Von Mises material the shear strength can be found:

$$
\text { Von Mises Yield Criterion: } \quad k=\frac{\sigma_{0}}{\sqrt{3}}
$$

The friction conditions in coining introduce very nonlinear behaviors that make it difficult for numerical simulations. For example, a surface point on a workpiece experiences variations from low contact pressure at the beginning of the coining process to high pressures friction near the shear strength of the material at the finishing stage of coining, and back to low contact pressure when the die is unloaded and removed from the material surface. The problem is then to select a friction model which is capable of modeling behavior at both low and high normal contact pressure. A bi-linear friction model is available for use in the finite element analysis code 
ABAQUS as an approximation to the non-linear case. This bi-linear friction model, as shown in Figure 2-3, combines the Coulomb friction and the friction factor technique [Guo. et. al., 2008]. Although the combined model exhibits a more realistic condition in coining processes, the difficulty is how to obtain the friction coefficient $\mu$, and the friction factor value $\mathrm{m}$, through experiments in the presence of workpiece strain hardening. The next section will present a computational-experimental approach to determine the parameters in the combined Coulomb-friction factor model by using the ring test.

\subsection{Ring Compression Test Overview}

Of the many techniques, the ring compression test technique has been widely used to measure the coefficient of friction or frictional shear factor at the die/workpiece interface in forging process. During a typical silver coining process, the process proceeds with a condition similar to the open-die forging at least up to $70-80 \%$ of the process before the material starts filling the die cavities at the corners. Therefore, the ring compression test was chosen as the means to evaluate the contact friction conditions in the silver coining process. In fact, many researchers have used the technique in the various investigations of closed-die forging processes. For instance, Leitao [Leitao. et. al., 1997] used the technique to evaluate the friction conditions in the coining process. While others such as Kwan [Kwan,C.T, 2002], Sedighi \& Tokmechi [Sedighi and Tokmechi, 2007], Altinbalik [Altinbalik. Et. al, 2007], Fereshtch \& Hosseini [Fereshtch and Hosseini, 2006] and Alves [Alves. et. al. 2003] used the technique to evaluate the friction conditions in the closed-die forging processes.

The technique was established by Kunogi [Kunoqi, 1956] and further developed by the works of Kudo [Kudo, 1960], Male \& Cockcroft [Male and Cockcroft, 1964] and Hawkyard \& Johnson [Hawkyard and Johnson, 1967]. The technique relates dimensional changes of a workpiece to the coefficient of friction. When a short, hollow cylinder is compressed axially between flat, parallel, rigid platens the diameter of the hole may either increase, decrease, or remain constant according to the amount of frictional constraint imposed by the platens. Under 
frictionless conditions the hole size increases proportionately to the outer diameter and, the cylinder compresses as would the corresponding portion of a compressed solid cylinder. With increasing frictional constraint, the rate of expansion of the hole decreases and eventually the compressive hoop stress developed at the hole is sufficient to cause the hole to contract. In a compression process involving constant friction at the platens, the change in geometry, as the cylinder shortens, causes the friction constraint to progressively increase. This has the effect that the hole may initially increase in diameter and then contract. By properly choosing the initial proportions of the cylinder, dimensional changes of the hole can thus provide a sensitive indication of the platen friction. As shown in Figure 5-1, a ring with outer radius $R_{o}$, inner radius $R_{i}$, neutral radius $R_{n}$ and height $2 \mathrm{~h}$ is compressed between two parallel plates. The inner radius of the ring increases $\left(R_{i} \geq R_{n}\right)$ for the case of lower friction and decreases $\left(R_{i}<R_{n}\right)$ for the case of higher friction. To establish quantitative correlations between the friction coefficient and geometric changes of a ring specimen, the friction calibration curves, shown in Figure 5-2, can be generated by relating the percentage reduction in internal diameter to the percentage reduction in height of the test specimen, from which either the coefficient of friction, the friction shear factor or both can be derived. The ring test has an advantage when applied to the study of friction, in that no direct measurement of force is required. Specimens in different size gave the same behavior provided that geometric similarity was maintained. Other specimen geometries gave different behavior but did not affect the nature of the results [Male and Cockcroft, 1964].

\subsection{Determination of Coining Friction Model}

This section will present the experimental and numerical approach to determine the friction coefficients in a bi-linear combined model by using the ring test and finite element method.

\subsubsection{Ring Compression Procedures}

Five compression test rings of fine silver were made with the same material as those in the uniaxial tensile tests, as shown in Figure 5-5a. The ring specimen ratio of 6:3:2 was used, where 
the first number denotes the external diameter and the second number indicates the internal diameter of the ring specimen while the last number represents the thickness of the ring specimen. The outer and inner diameters of the ring are $D_{O}=30 \mathrm{~mm}$ and $D_{i}=15 \mathrm{~mm}$ respectively, and the height is $\mathrm{H}=10 \mathrm{~mm}$. The ring surface was ground and polished by the Royal Canadian Mint to give the same surface condition as that of a typical silver blank. To identify diameters to be measured during the test, three lines were marked, at equal angular divisions on the inner surface of ring testpieces. To simulate the actual coining conditions, two platens (one top die, one bottom die) were made by the Royal Canadian Mint using the alloyed tool steel currently being used at the Royal Canadian Mint [Royal Canadian Mint, 2007], as shown in Figure 5-3.

The platens were heat treated and the platen surfaces were coated with TiCrN coating by using Magnetron Sputtering Coating which is used for real dies at the Royal Canadian Mint. The platens have the thickness of $10 \mathrm{~mm}$ and a $5 \mathrm{~mm}$ diameter hole in the center. On the back surface of one platen, a $5 \mathrm{~mm}$ diameter groove was designed from the center to the edge to allow the air to escape during the compression test. A locating device was designed to ensure the rings were positioned at the center of the platen which will ensure an even distribution of the force over the face of the ring during the compression test, as shown in Figure 5-4.

The test pieces were compressed with dry surface in the MTS material test system, as shown in Figure 5-6, at room temperature. During the test, the top platen was displaced about $5 \mathrm{~mm}$ at a constant rate. Following each $3 \%$ increment of axial strain, the load was reduced to zero and the platens and testpiece were removed from the machine. The diameters were measured at the marked positions. The platen and testpiece surfaces were cleaned with alcohol liquid and dried, and the platens and testpiece were replaced in its original position in the MTS machine and the process repeated until the total displacement was achieved. Five rings were compressed under the same conditions. Figure 5-5 shows one of the rings at its original size (Figure 5-5a) and after deformation (Figure 5-5b). Five sets of experimental results, presented in Table 5-1 through Table 5-5, were obtained and plotted in the change of inside diameter against the height reduction for the five fine silver rings, as shown in Figure 5-7. 


\subsubsection{Finite Element Modeling of Ring Compression Test}

In order to determine the frictional calibration curves of fine silver coining, the finite element code $\mathrm{ABAQUS}$ was used to simulate the compression of a cylindrical ring between two rigid platens. The nonlinear strain hardening material model obtained in Chapter 4 was used as the input for the finite element analysis. The dimensions of the ring model are the same as those for the ring compression testpieces used in the experiments, $30 \mathrm{~mm}$ in outside diameter, $15 \mathrm{~mm}$ in inside diameter and $10 \mathrm{~mm}$ in height. Due to the axisymmetric nature of the problem, as well as the existence of two axes of symmetry within each plane, a two-dimensional model representing one quarter of the cylindrical ring cross-section was constructed, as shown in Figure 5-8.

The geometry of the ring was modeled with a total of 450 quadrilateral elements as shown in Figure 5-9a. The 4-node bilinear, reduced integration with 'hourglass' control element was chosen. The 'hourglass' control was selected to avoid unwanted deformation modes. The reduced integration formulation was employed to avoid shear and volumetric 'locking' which can occur with the full integration formulation.

The testpiece and the rigid compression platens were modeled as the contact pair. The top and bottom compression platens were modeled as the rigid platens. When the upper and lower dies approach each other and compresses the ring, it is anticipated that material originally on the lateral surface of the ring moving in a radial direction may ultimately appear at the ring-platen interfaces. It is thus important to include the elements on the lateral surface in the surface element group. Due to the fact that the ring is loaded far above the yield strength of the blank material, sticking-friction conditions were imposed at the interface between the ring and the die. This process will depend on the value of the friction coefficient and maximum shear stress value of the interface. The non-classical ABAQUS [ABAQUS, 1989] friction model, shown in Figure 2-4, was used to model the interfacial friction conditions between platen and ring in compression process. When surfaces are in contact, they transmit shear as well as normal stress. The contacting surfaces do not slide over each other as long as the shear stress $\tau$ is less than the 
coefficient of friction times the normal pressure, $\mu p$, and also less than a critical value of shear stress $\tau_{\max }$ as shown in Figure 2-4. Sliding occurs when $\tau>\mu \sigma_{N}$, for $\tau<\tau_{\max }$; or when $\tau=m k$, for $\tau=\tau_{\max }$. The friction coefficient and the maximum frictional shear stress value are specified for the interface elements. For each ring compression simulation, the value of $\tau_{\max }$ is set to be a constant, determined in terms of the shear yield stress of the testpiece. The maximum shear stress at the ring/platen interface is not allowed to exceed $\tau_{\max }$. The deformation was controlled by a rigid body reference node defined at the rigid surface along the vertical axis of the ring model. The reference node defining the platens was constrained to have no rotation and no radial displacement and the axial displacement was defined to move about $2.5 \mathrm{~mm}$ down the axis. To improve the deformed mesh quality, adaptive meshing technique for the non-linear analysis was utilized. Figure 5-9b indicates the deformed shape of the ring model obtained from the FEM analysis.

To generate friction calibration curves, the model was simulated for different coefficients of friction $\mu$ and $\tau_{\max }$. The friction calibration curves were then generated by plotting the percentage change of inner diameter against the percentage reduction of the height for different friction parameters. In the simulations, four maximum shear stresses $\tau_{\max }=84 \mathrm{MPa}$ and $\tau_{\max }=97$ $\mathrm{MPa}, \tau_{\max }=123 \mathrm{MPa}$ and $\tau_{\max }=142 \mathrm{MPa}$ were tested. The first set of simulation results were obtained by fixing the value of $\tau_{\max }=142 \mathrm{MPa}$ and varying the value of $\mu=0.20,0.21,0.22$, $0.23,0.24$. The second set of results were obtained by fixing the value of $\tau_{\max }=123 \mathrm{MPa}$ and varying the value of $\mu=0.20,0.21,0.22,0.23,0.24$. The last two sets of results were obtained by fixing $\tau_{\max }=97 \mathrm{MPa}$ and $\tau_{\max }=84 \mathrm{MPa}$ and varying $\mu=0.22,0.23$ respectively. The four sets of simulation results for $\mu / \tau_{\max }=\mu / 142, \mu / 123, \mu / 97, \mu / 84$ were presented in Table 5-6 through Table 5-19 and plotted as the friction calibration curves of $\Delta \mathrm{D} / \mathrm{D}$ against $\Delta \mathrm{H} / \mathrm{H}$, as shown in Figure 5-10 [Guo. et. al., 2008]. 


\subsection{Calibration and Results}

The friction calibration curves obtained from the FEM analysis were used to compare to the experimental ring compression results. To overcome the scattering of the experimental results, a best fitting polynomial curve was generated for the experimental results of five silver rings, as shown in Figure 5-11. The five degree polynomial fitting curve presents a good fit quality with a $\mathrm{R}$-square value of 0.98 . The calibration processes were performed in two steps. In the first step, the value of $\mu$ was evaluated by calibrating the experimental data using each of the calibration curves. The value of $\mu$ can be determined, which provides the best match with the fitting curve of experimental data. For the clearness and brevity of the calibration process, only two pairs of the calibration curves were chosen as the examples of calibrating the experimental data, shown in Figure 5-12 and 5-13. Through the calibrating process, it is found that when $\mu=0.22$, the calibration curves give the best match to the experimental results [Guo. et. al., 2008].

In the second step, the chosen value of $\mu$ was fixed, and the value of $\tau_{\max }$ varied with small perturbations for a better match. This process has been repeated until a value of $\tau_{\max }$ has been found, which provides a close agreement with the experimental results. Figure 5-14 shows four values of maximum shear stress giving very close simulation results for $\mu=0.22$, which indicates the friction calibration curve is not sensitive to $\tau_{\max }$ for $\mu=0.22$. Since the flow stress of fine silver obtained in Chapter 4 is $115 \mathrm{MPa}, \tau_{\max }=123 \mathrm{MPa}$ was chosen as the result for the calibration [Guo. et. al, 2008].

To compare the results obtained for the non-classical two-parameter friction model and the fine silver material property model, the experimental and FEM results of loads at about 55\% height reduction of five rings were compared in Figure 5-15 [Guo, et. al, 2008]. The differences are between $1 \%$ and $7 \%$, which shows the results from the FEM model has a good agreement with the experimental results.

As a further comparison, the shear stress developed in the FEM simulation of ring compression was plotted in Figure 5-16. The figure shows that the maximum shear stress on the 
contact layer of FEM simulation of ring compression at about $60 \%$ height reduction is $123.7 \mathrm{MPa}$, which is very close to the chosen $\tau_{\max }(123 \mathrm{MPa})$.

Finally, the contact pressure developed in the FEM simulation of ring compression at about $60 \%$ height reduction was plotted in Figure 5-17 and the nominal averaged contact pressure development in the experiment and FEM was plotted in Figure 5-18. Due to the buckling effects in the ring experiment, the contact area was calculated by using averaged diameter, which causes the experimental results to be somewhat lower than the FEM results. But in general, as shown in the figures, the experimental and FEM results are quite consistent and similar. Both of them present the maximum contact pressure at around $0.6 \mathrm{GPa}$.

All the above comparisons are quite consistent and therefore give added confidence in the two-parameter friction model and the key assumptions used in this coining simulation work.

In conclusion, the proposed ring compression technique [Kunoqi, 1956], friction calibration curves [Male and Cockcroft, 1964] and non-classical friction model [Hayhurst and Chan, 2005] methods were successfully applied to evaluate the friction conditions of a silver coining process. A combined two parameter friction model was derived to describe the frictional behavior between the testpiece and platen for a silver coining process. It has been shown that through careful calibrations, the friction model can accurately predict the deformation of silver ring compression process. The agreements between FEM results and experimental results show this approach provides an effective and repeatable means to evaluate the frictional behavior under different friction conditions of different silver coining process [Guo. et. al., 2008]. The friction values for fine silver, obtained from these tests, are $\mu=0.22$ and $\tau_{\max }=123 \mathrm{MPa}$. The derived two parameter combined friction model will be used in all the subsequent finite element simulation of fine silver coining process. 

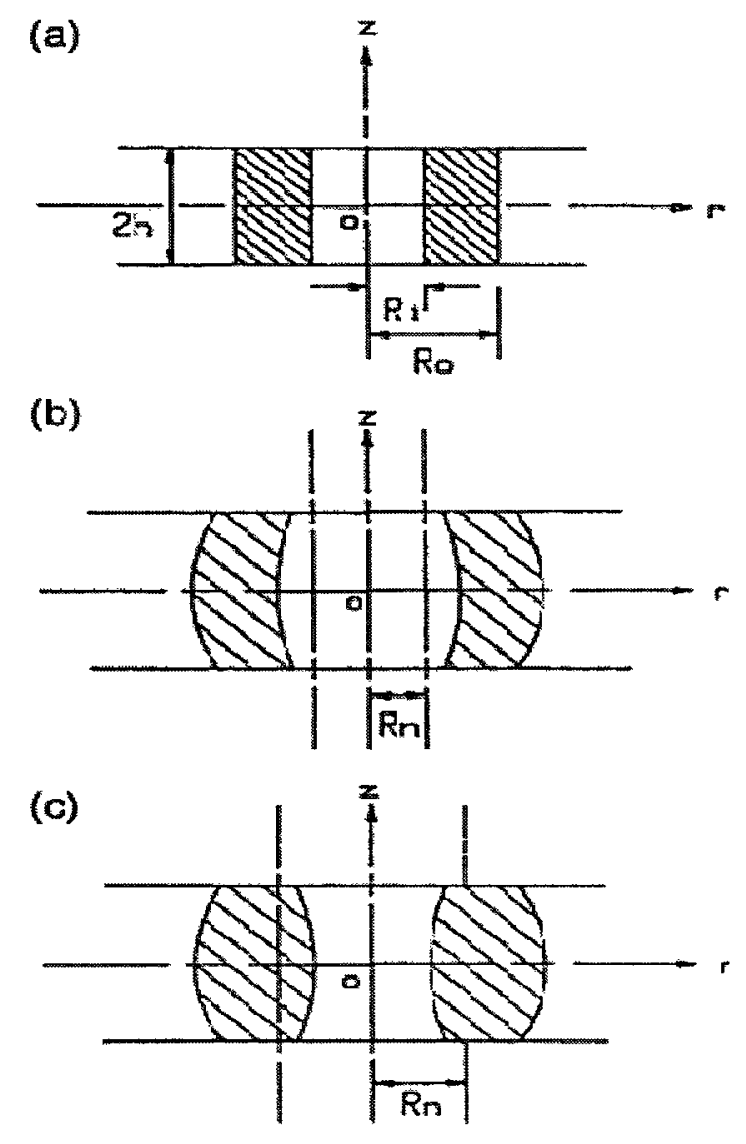

Figure 5-1. Theory of ring compression

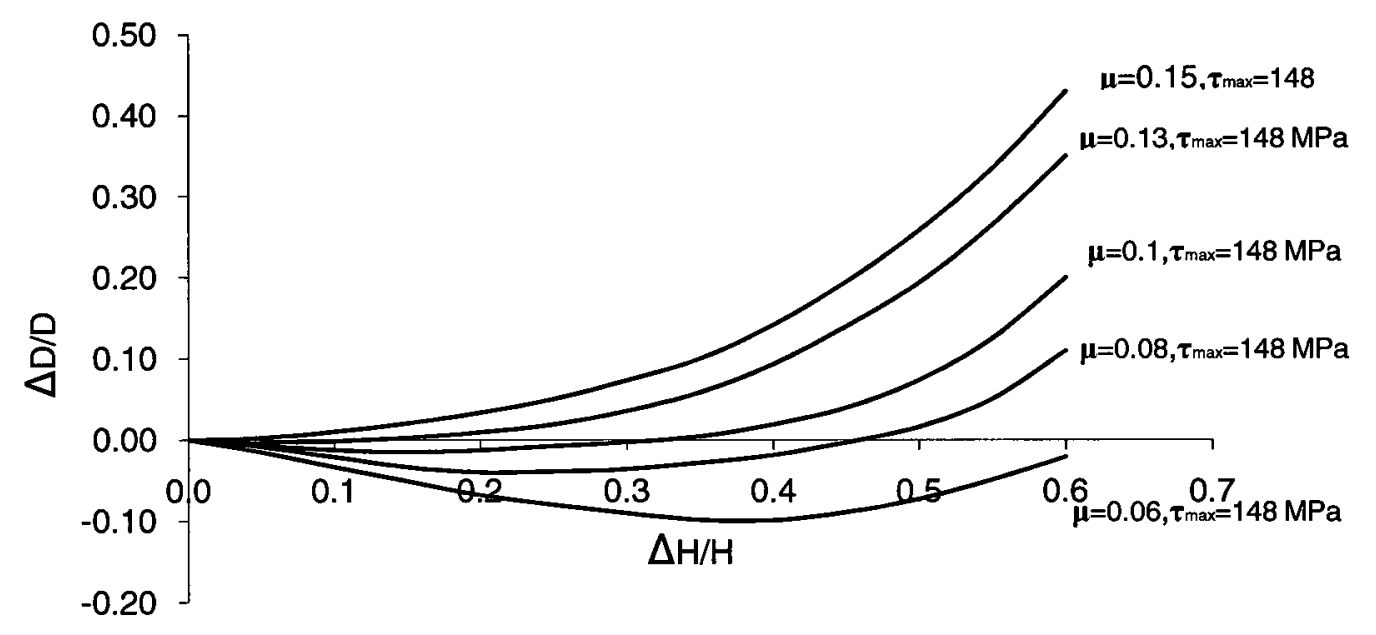

Figure 5-2. A typical calibration curve of ring compression test (copper) [Hayhurst and Chan, 2005] 


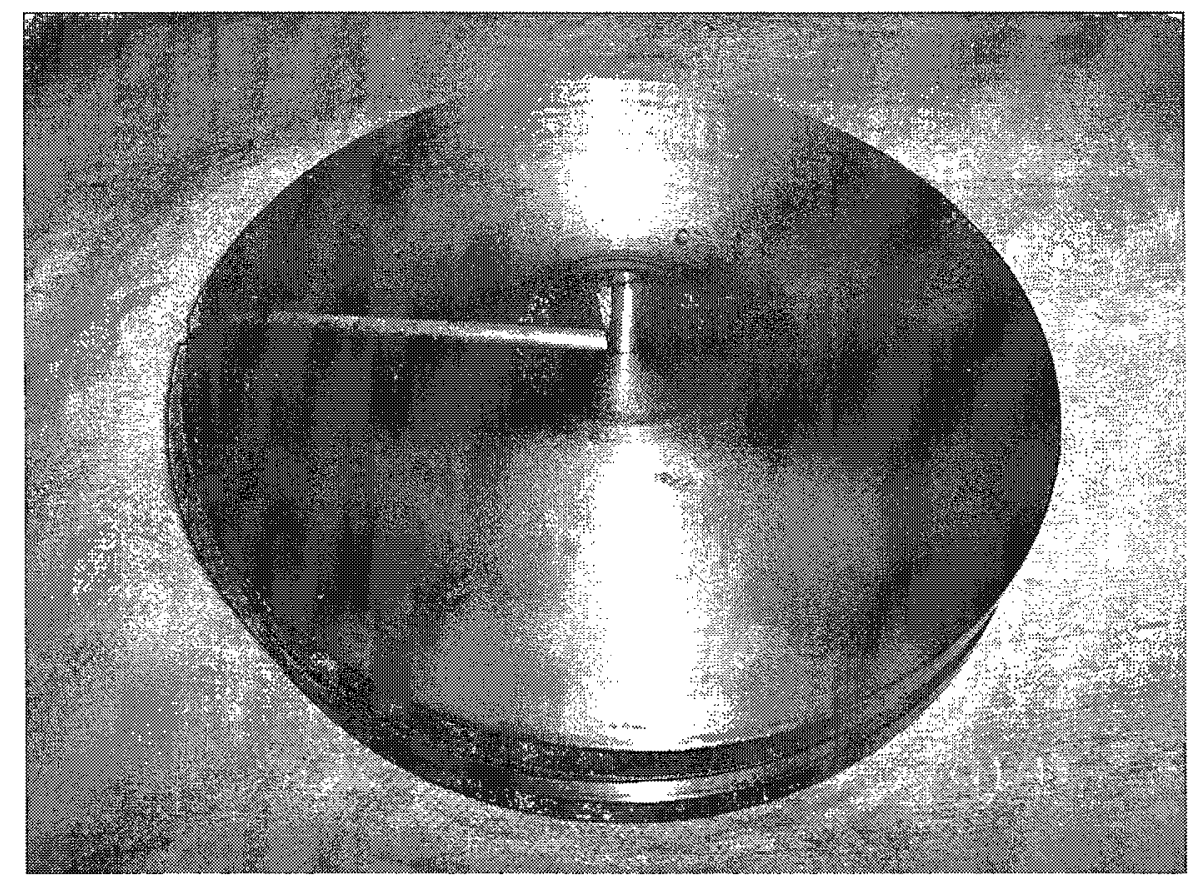

Figure 5-3. Two platens made of alloyed tool steel

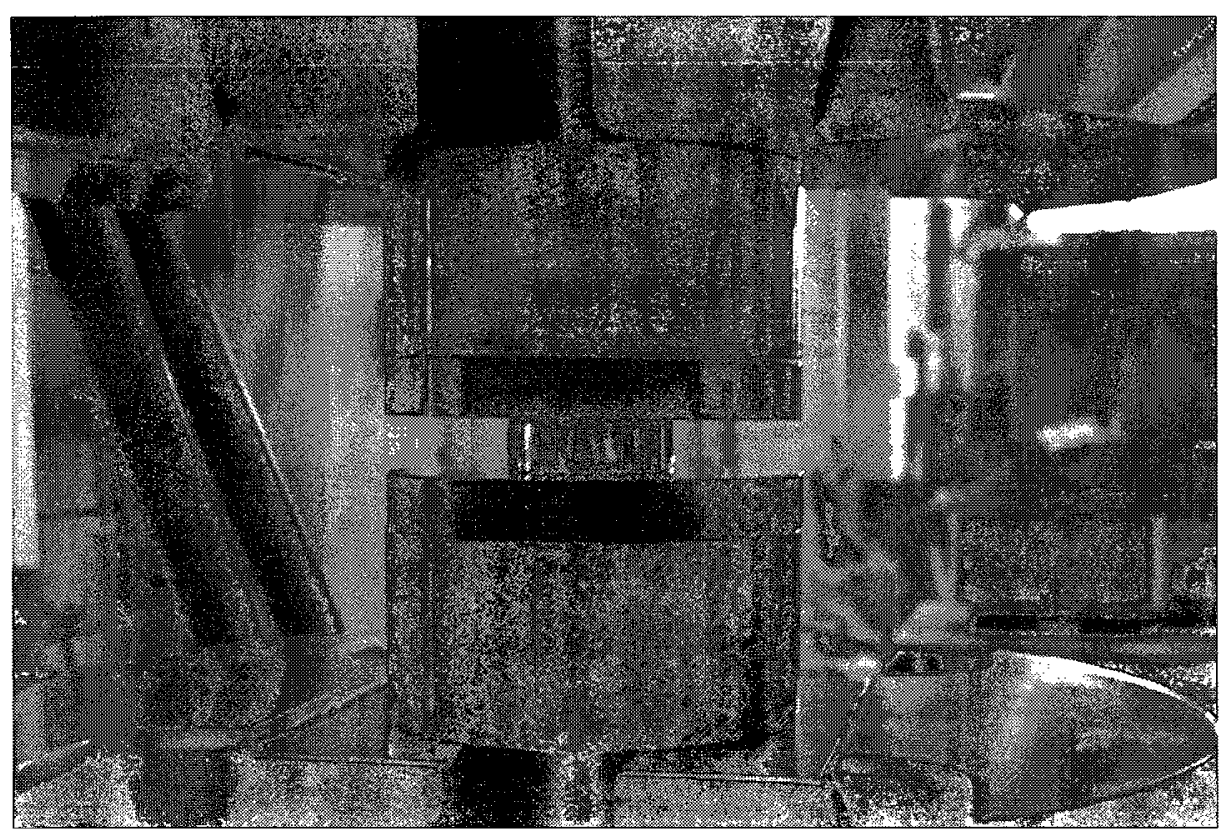

Figure 5-4. Ring positioned at the center of platens 



Figure 5-5. a) Original and b) deformed silver ring

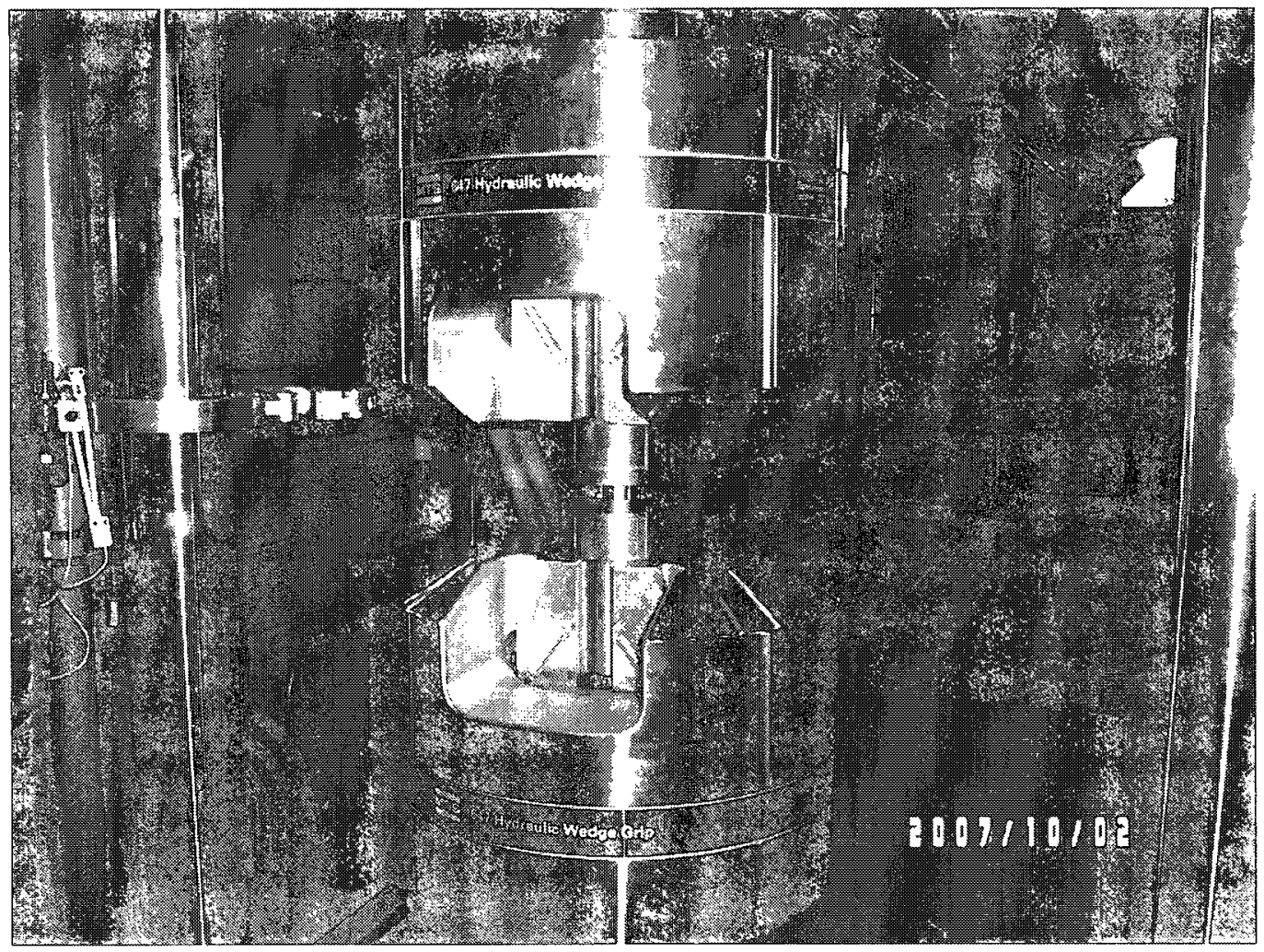

Figure 5-6. Ring compression test setup 


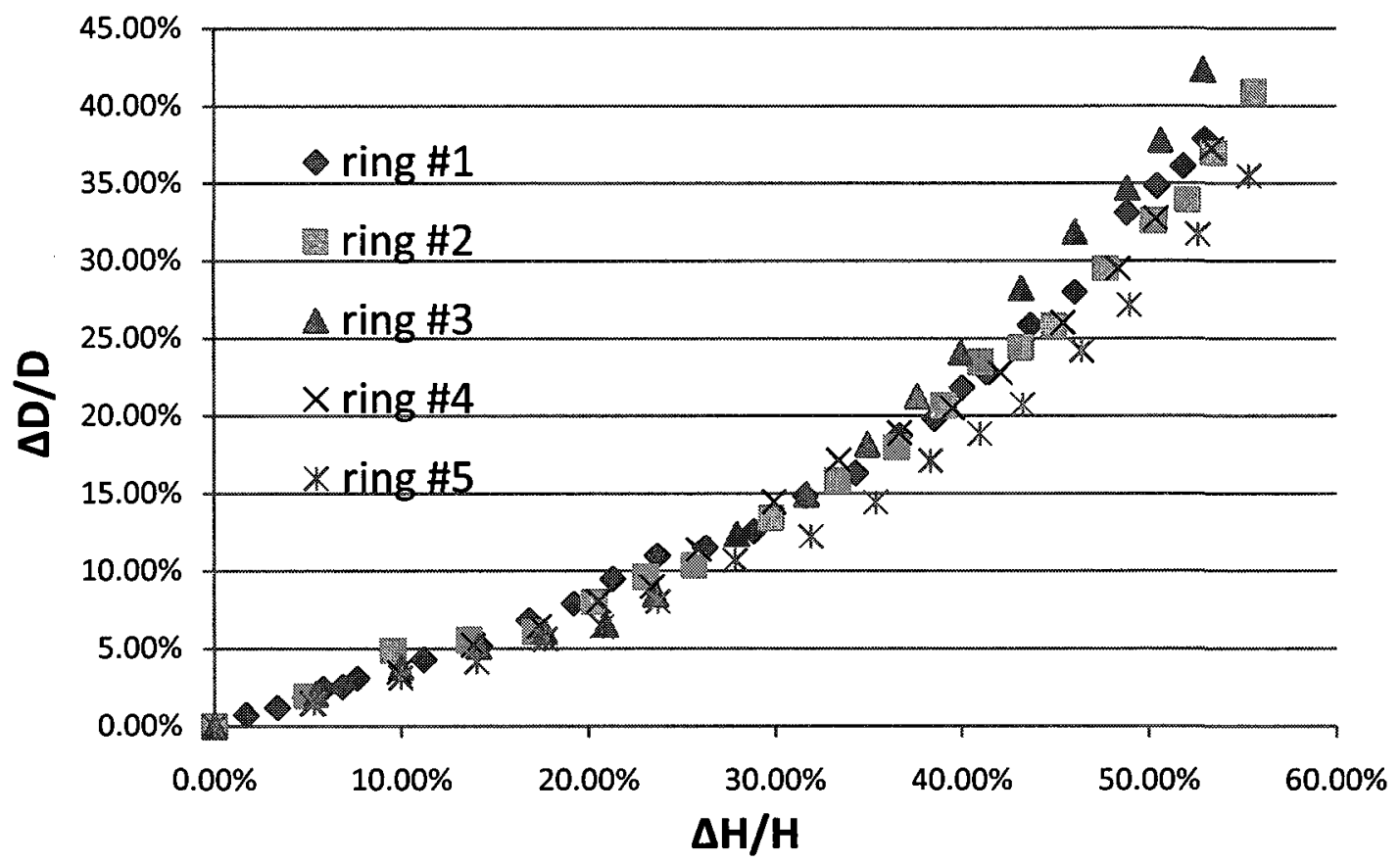

Figure 5-7. Experimental results [Guo. K. et. al., 2008]

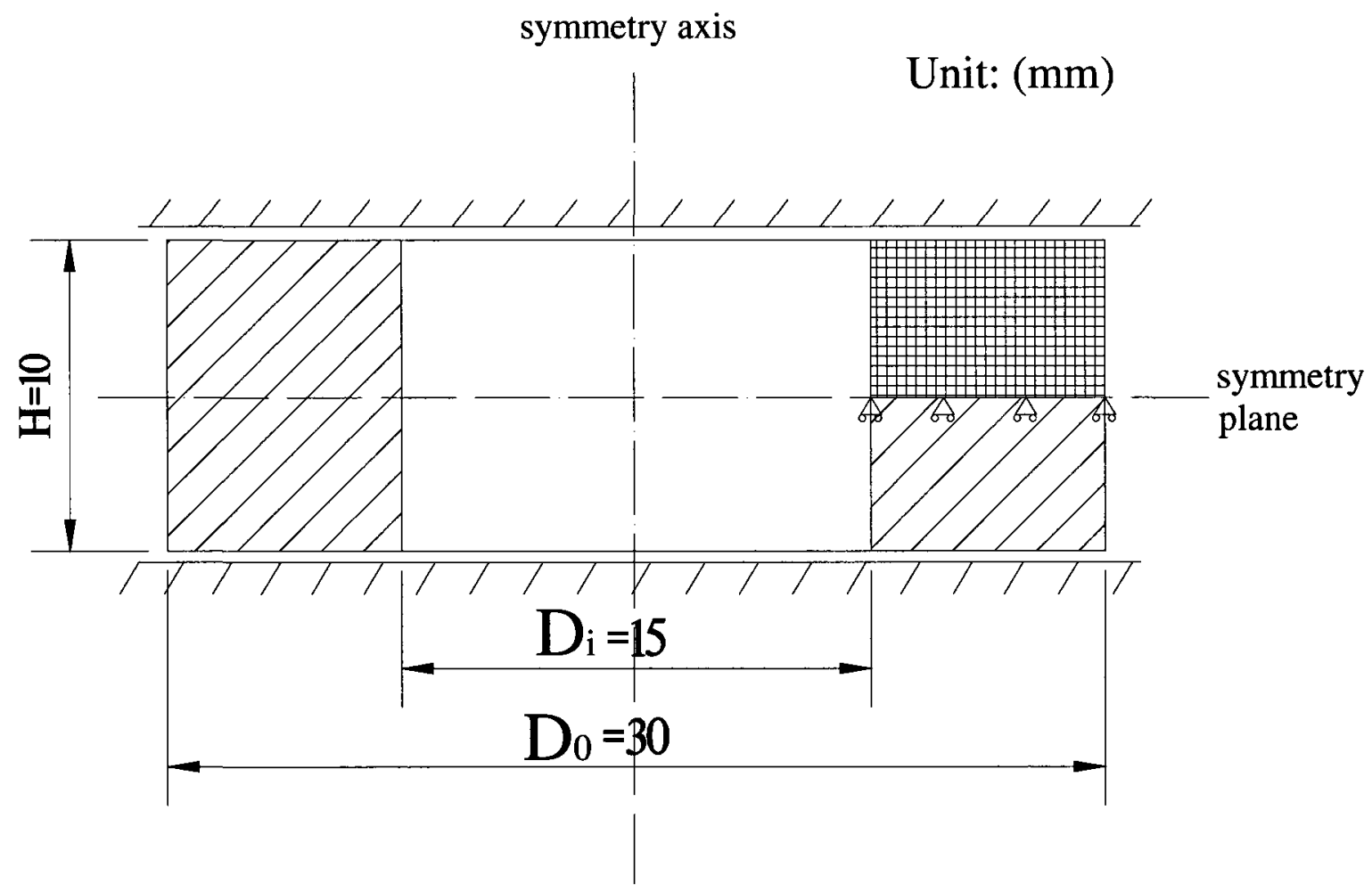

Figure 5-8. FEM model of ring compression test 


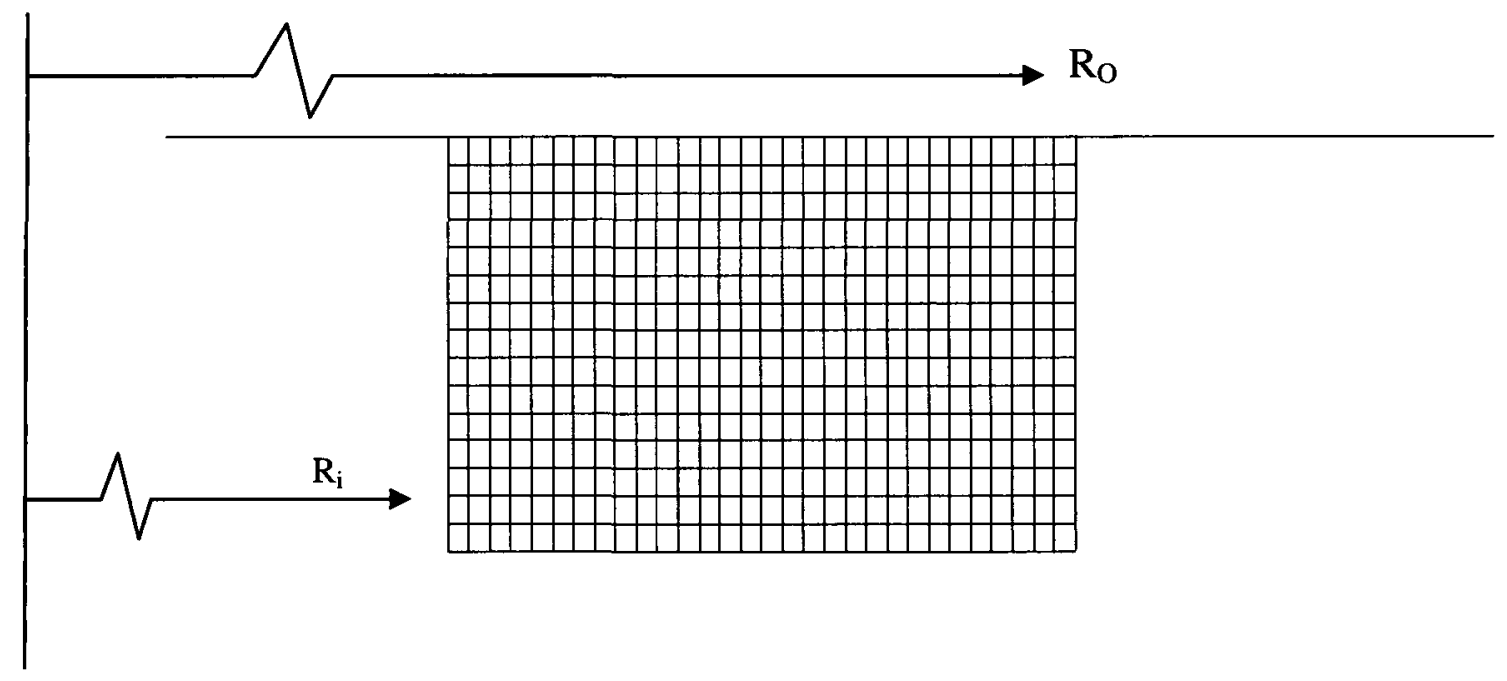

(a)

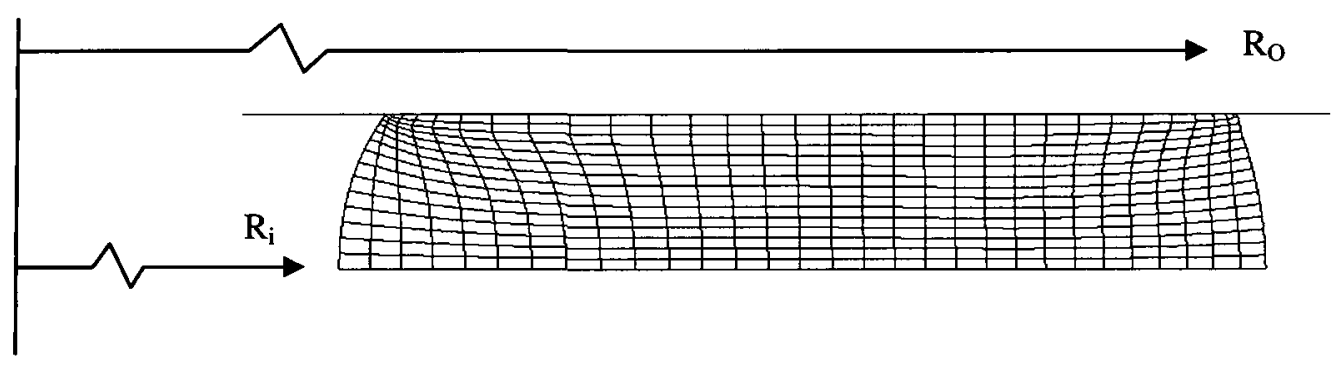

( b )

Figure 5-9. a) Original FEM mesh b) Deformed FEM mesh of ring compression test 


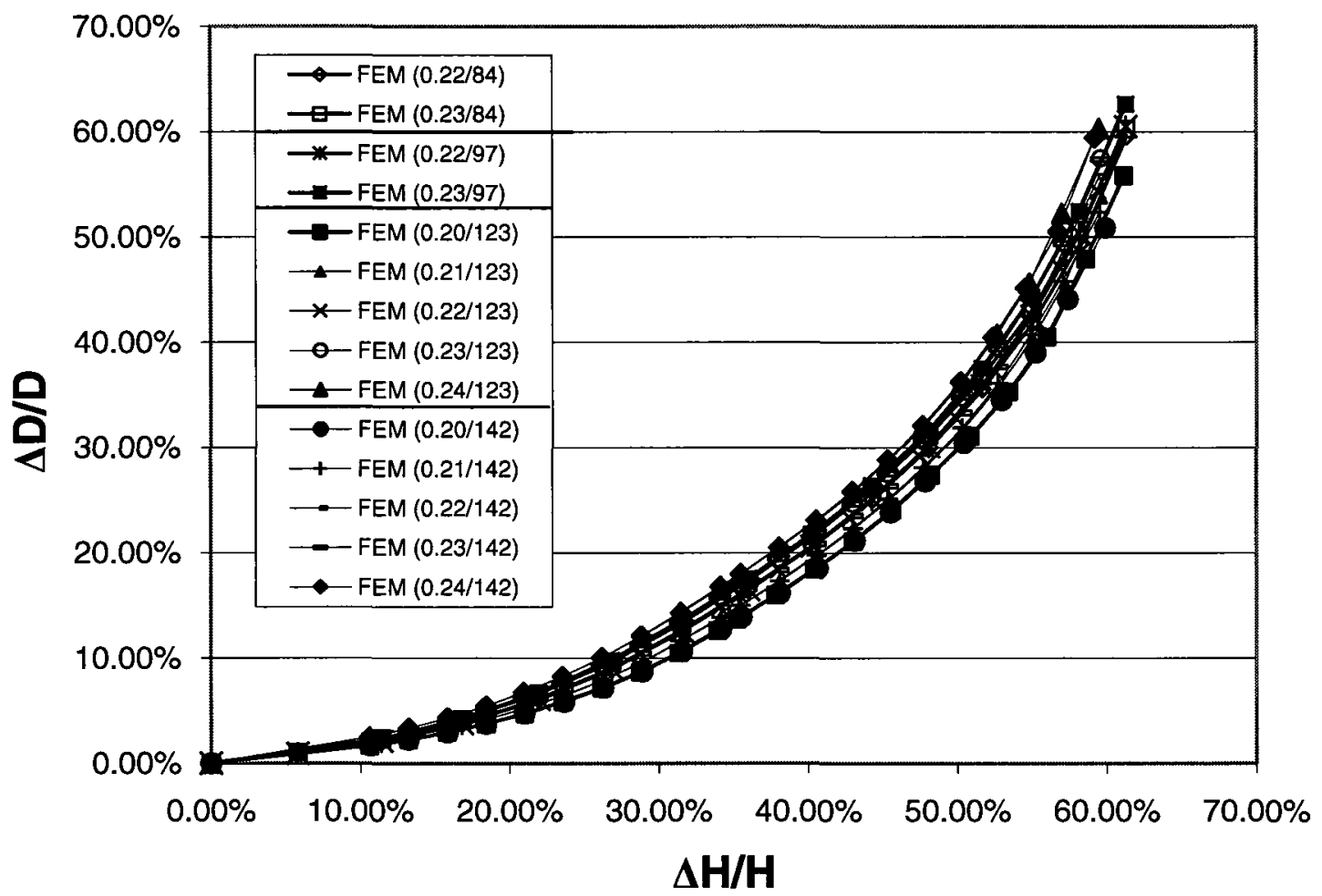

Figure 5-10. Calibration curves achieved by FEM method [Guo. K. et. al., 2008]

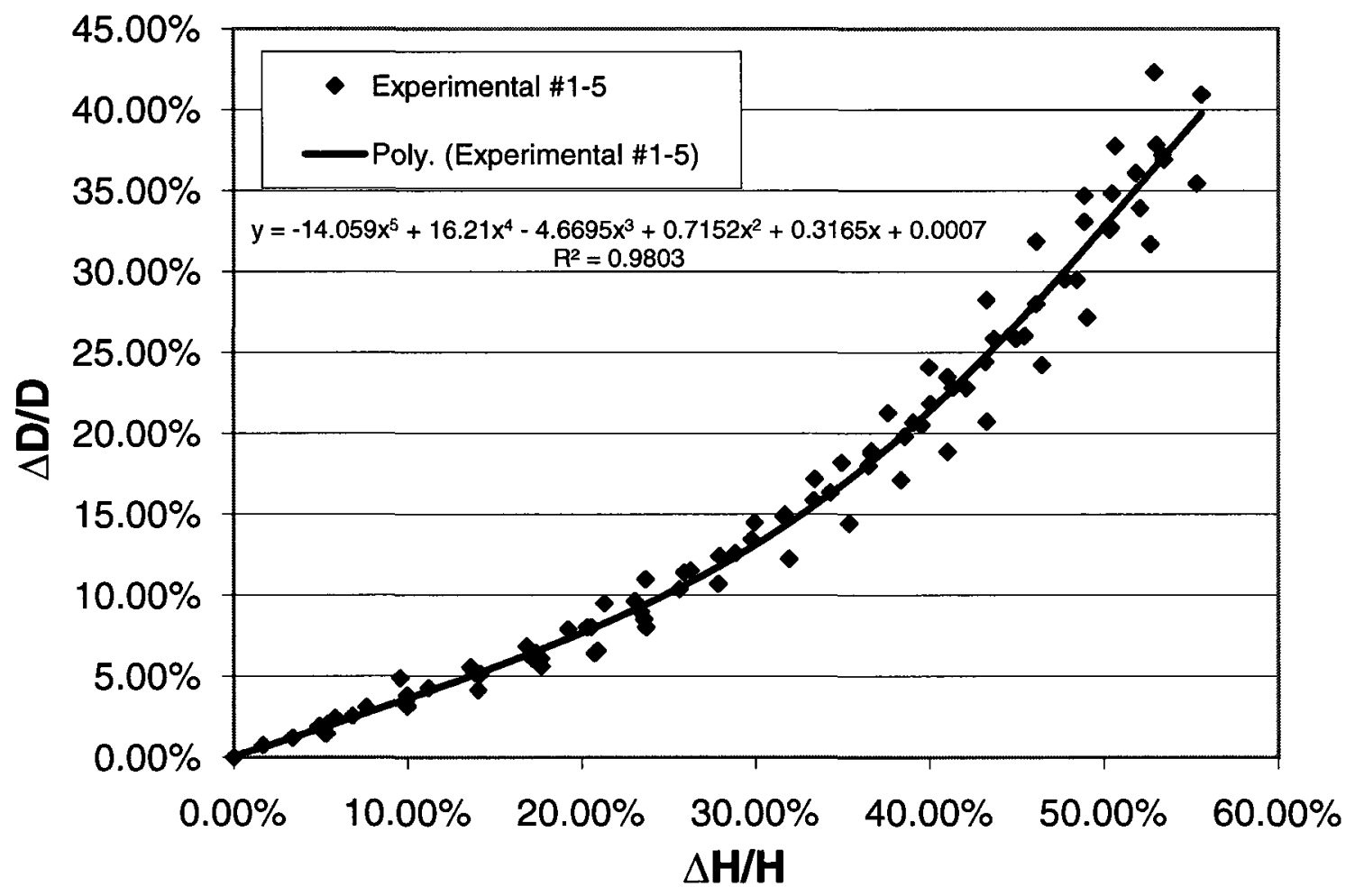

Figure 5-11. Experimental data of ring test and best fitting curve 


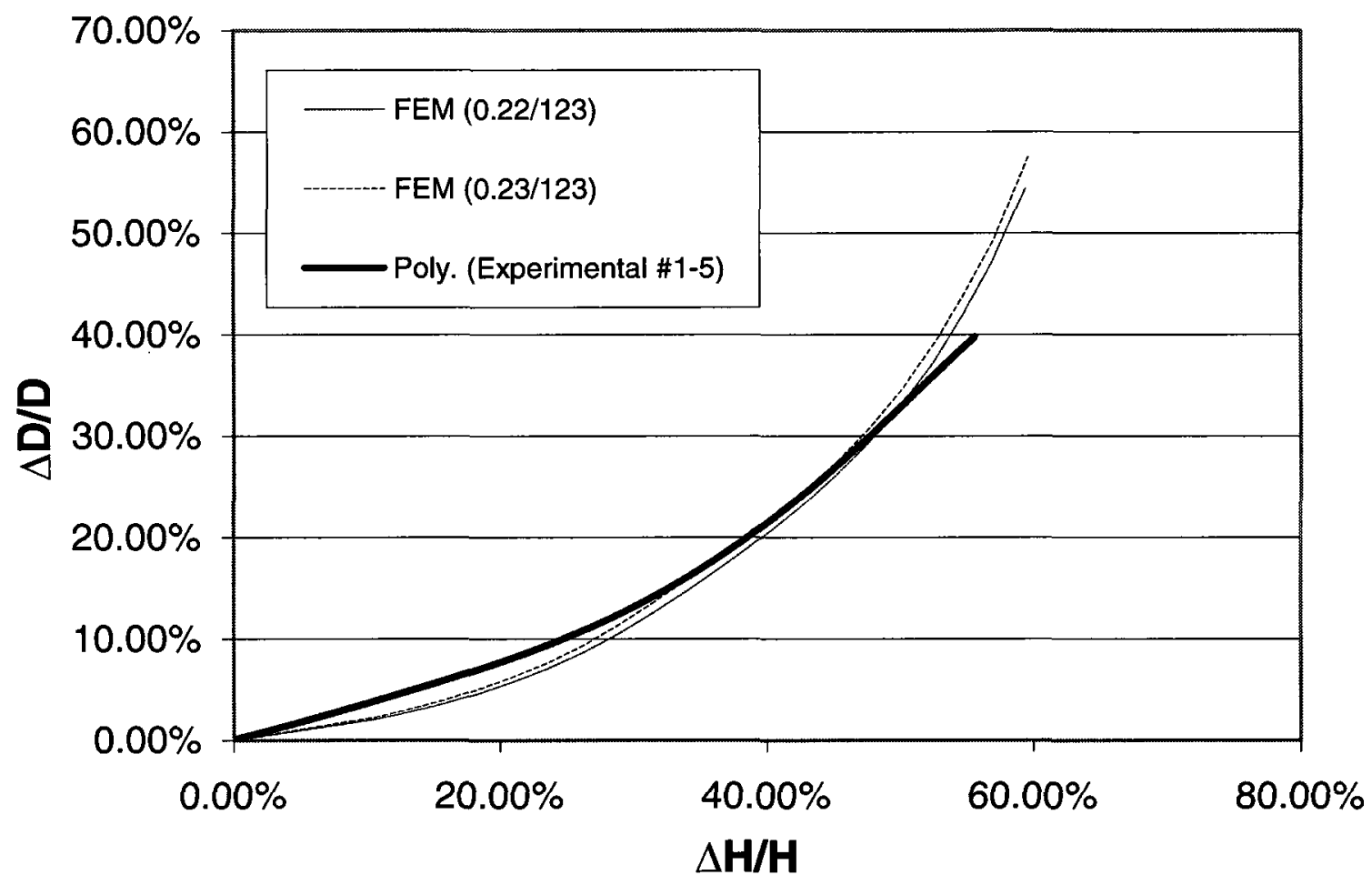

Figure 5-12. The fitting curve of experimental data against the calibration curves

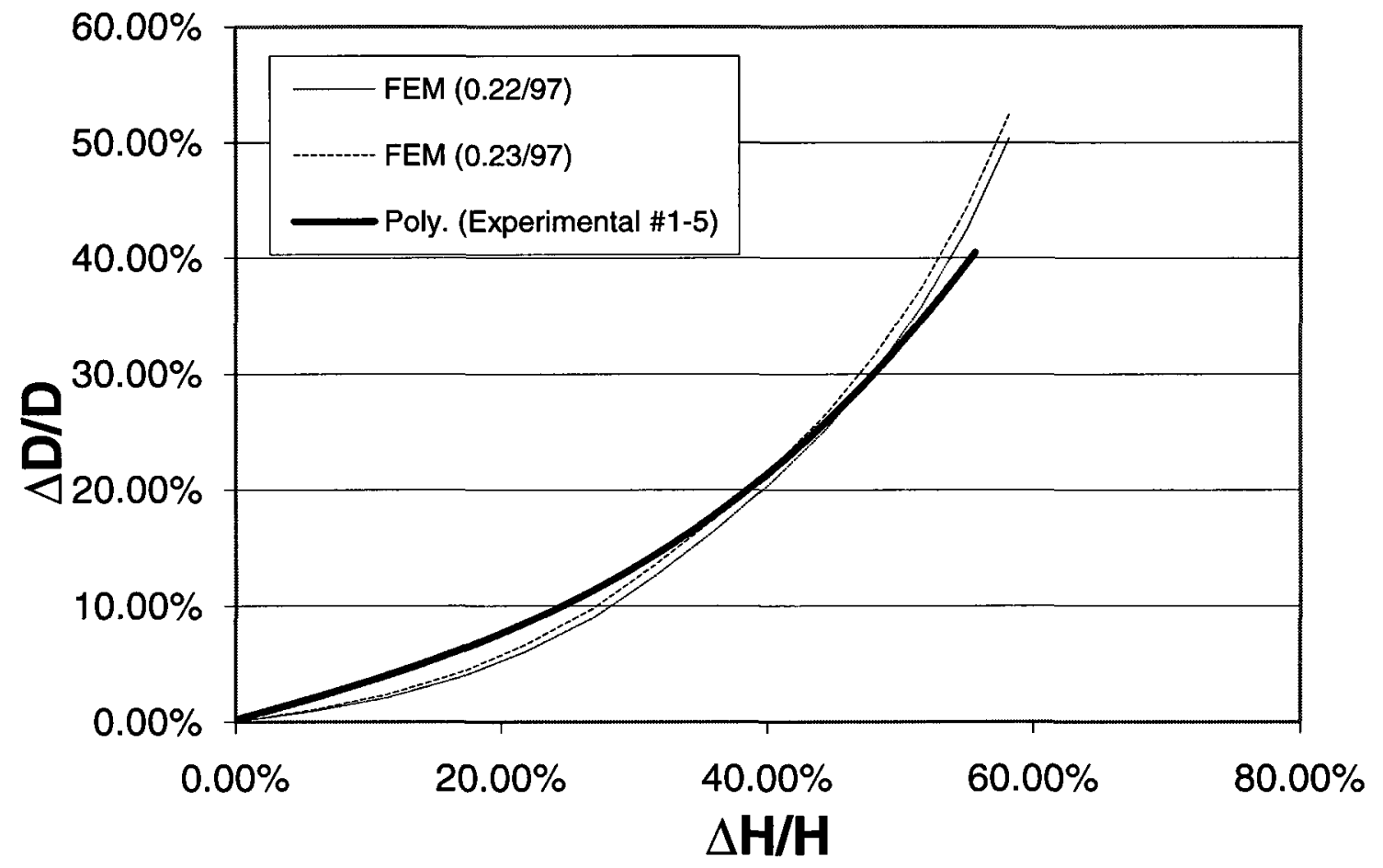

Figure 5-13. The fitting curve of experimental data against the calibration curves 


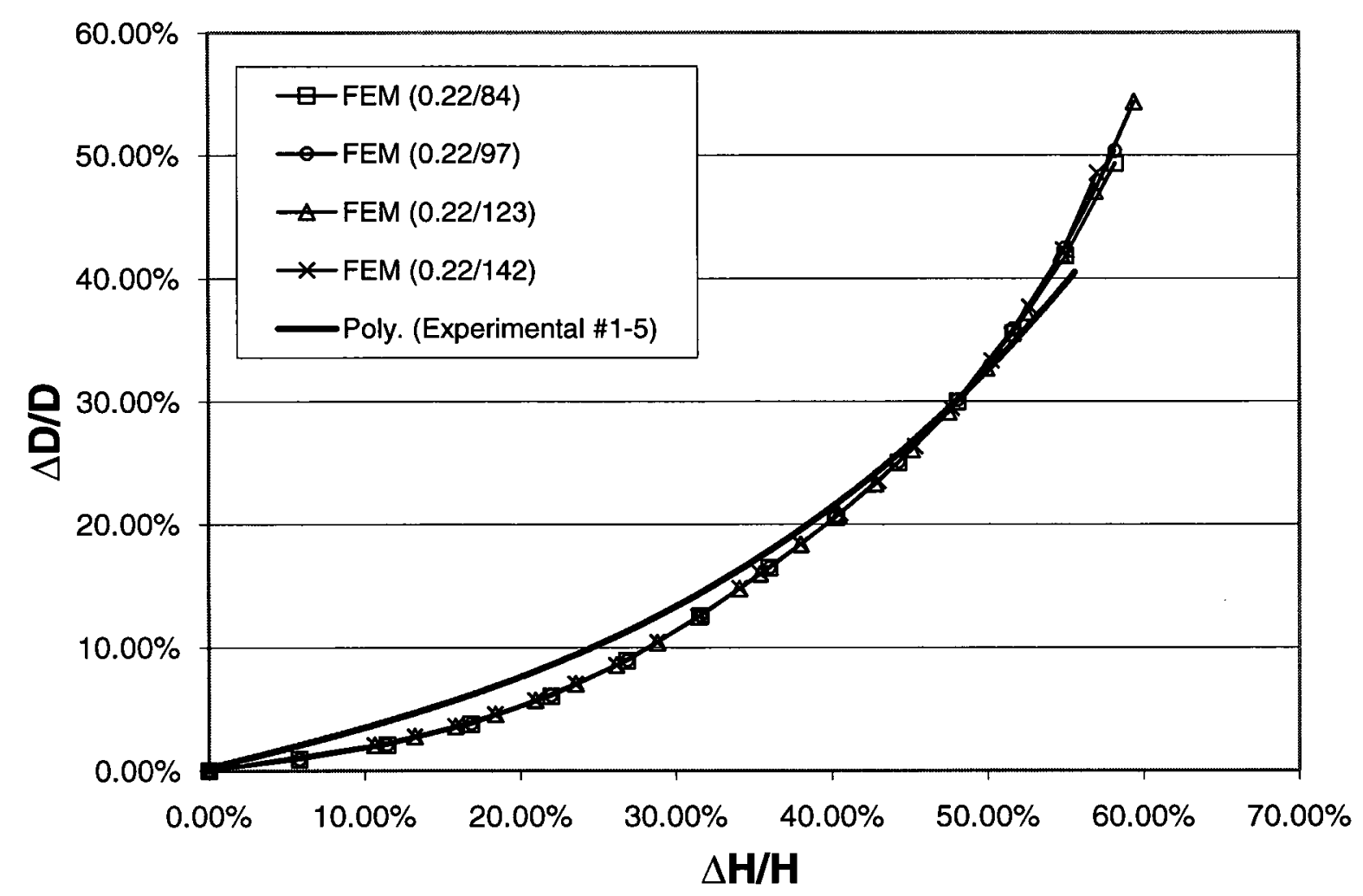

Figure 5-14. The fitting curve of experimental data against the calibration curves

\section{Load at last step}

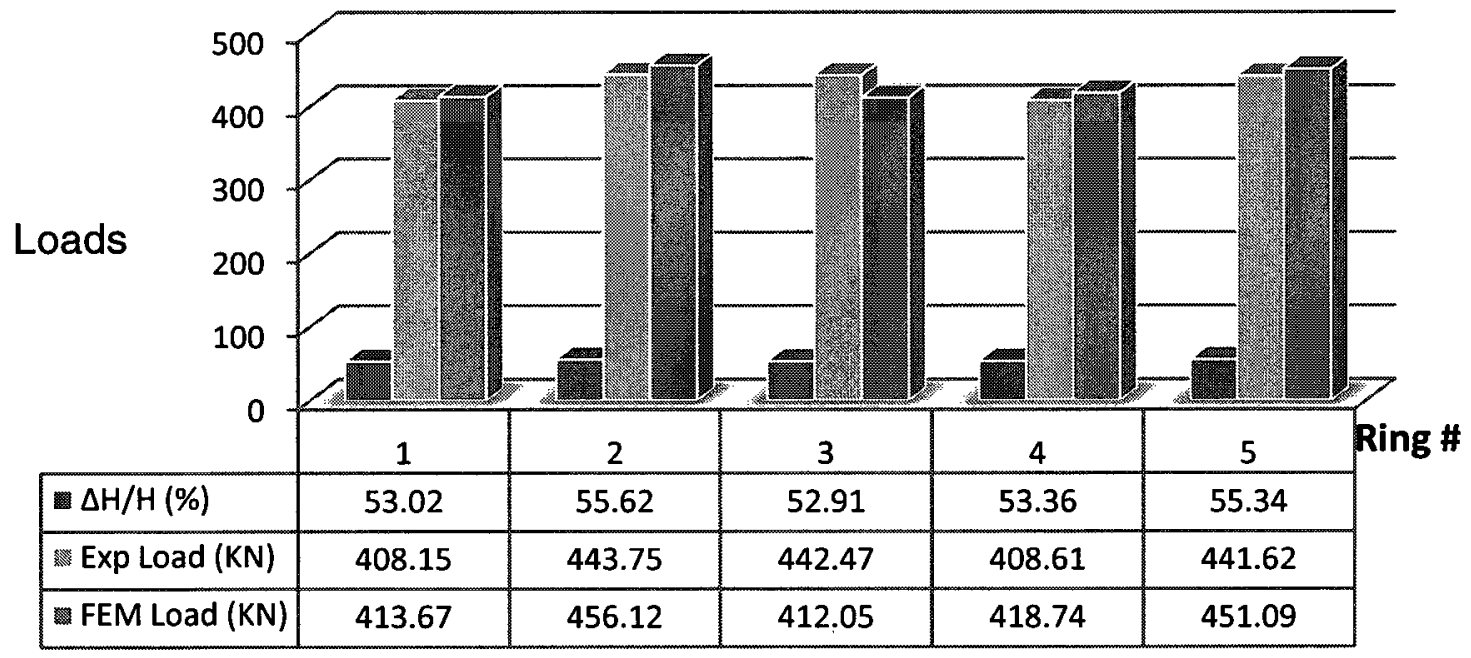

Figure 5-15. Experimental and FEM loads at the final step 


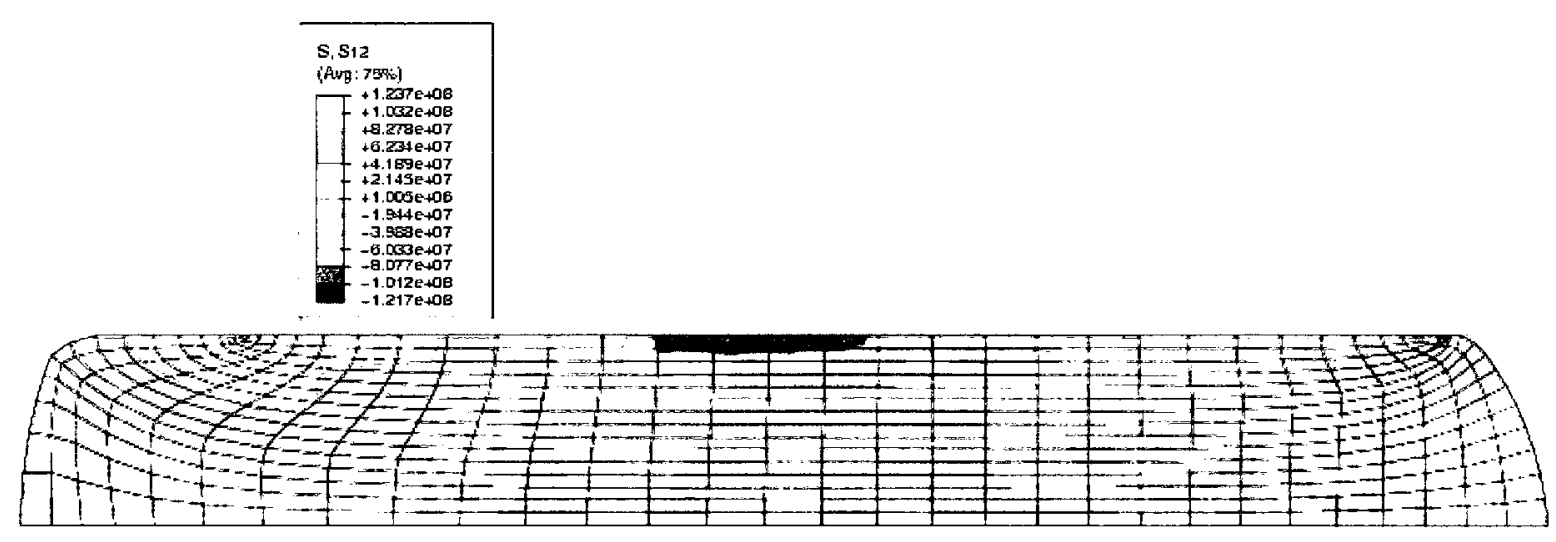

Figure 5-16. Shear stress contour in ring test

Figure 5-17. Contact pressure contour in ring test 


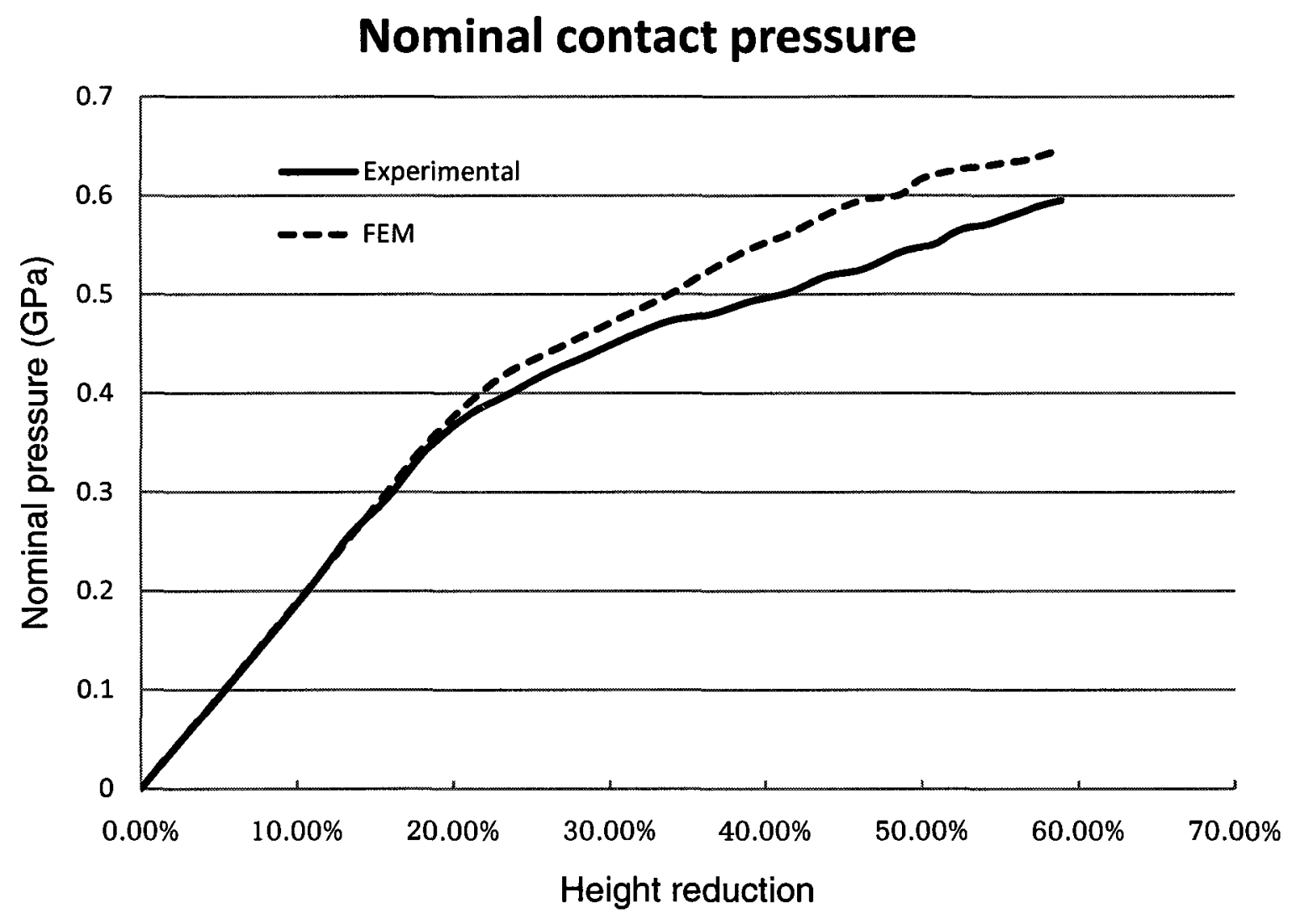

Figure 5-18. Nominal contact pressure development in experimental and FEM ring test 


\begin{tabular}{|c|c|c|c|c|c|}
\hline \multicolumn{5}{|c|}{$\begin{array}{c}\text { Experiment-specimen\#1 } \\
\left(\mathrm{H}_{0}=10.09, \mathrm{D}_{0}=14.94\right)\end{array}$} & \multirow{2}{*}{$\begin{array}{c}\text { Unit: mm } \\
\qquad \mathrm{D} / \mathrm{D}\end{array}$} \\
\hline $\mathrm{H}$ & $\begin{array}{c}\Delta \mathrm{H} \\
\left(\mathrm{H}_{0}-\mathrm{H}\right)\end{array}$ & $D$ & $\begin{array}{c}\Delta \mathrm{D} \\
\left(\mathrm{D}-\mathrm{D}_{0}\right)\end{array}$ & $\Delta \mathrm{H} / \mathrm{H}$ & \\
\hline 10.09 & 0 & 14.94 & 0 & $0.00 \%$ & $0.00 \%$ \\
\hline 9.92 & 0.17 & 14.83 & 0.11 & $1.68 \%$ & $0.74 \%$ \\
\hline 9.75 & 0.34 & 14.76 & 0.18 & $3.37 \%$ & $1.20 \%$ \\
\hline 9.6 & 0.49 & 14.66 & 0.28 & $4.86 \%$ & $1.87 \%$ \\
\hline 9.5 & 0.59 & 14.58 & 0.36 & $5.85 \%$ & $2.41 \%$ \\
\hline 9.4 & 0.69 & 14.56 & 0.38 & $6.84 \%$ & $2.54 \%$ \\
\hline 9.32 & 0.77 & 14.48 & 0.46 & $7.63 \%$ & $3.08 \%$ \\
\hline 8.96 & 1.13 & 14.3 & 0.64 & $11.20 \%$ & $4.28 \%$ \\
\hline 8.66 & 1.43 & 14.17 & 0.77 & $14.17 \%$ & $5.15 \%$ \\
\hline 8.39 & 1.7 & 13.92 & 1.02 & $16.85 \%$ & $6.83 \%$ \\
\hline 8.15 & 1.94 & 13.76 & 1.18 & $19.23 \%$ & $7.90 \%$ \\
\hline 7.94 & 2.15 & 13.52 & 1.42 & $21.31 \%$ & $9.50 \%$ \\
\hline 7.7 & 2.39 & 13.3 & 1.64 & $23.69 \%$ & $10.98 \%$ \\
\hline 7.44 & 2.65 & 13.22 & 1.72 & $26.26 \%$ & $11.51 \%$ \\
\hline 7.18 & 2.91 & 13.06 & 1.88 & $28.84 \%$ & $12.58 \%$ \\
\hline 6.9 & 3.19 & 12.72 & 2.22 & $31.62 \%$ & $14.86 \%$ \\
\hline 6.63 & 3.46 & 12.5 & 2.44 & $34.29 \%$ & $16.33 \%$ \\
\hline 6.39 & 3.7 & 12.14 & 2.8 & $36.67 \%$ & $18.74 \%$ \\
\hline 6.2 & 3.89 & 11.98 & 2.96 & $38.55 \%$ & $19.81 \%$ \\
\hline 6.05 & 4.04 & 11.68 & 3.26 & $40.04 \%$ & $21.82 \%$ \\
\hline 5.92 & 4.17 & 11.53 & 3.41 & $41.33 \%$ & $22.82 \%$ \\
\hline 5.68 & 4.41 & 11.08 & 3.86 & $43.71 \%$ & $25.84 \%$ \\
\hline 5.44 & 4.65 & 10.76 & 4.18 & $46.09 \%$ & $27.98 \%$ \\
\hline 5.16 & 4.93 & 10 & 4.94 & $48.86 \%$ & $33.07 \%$ \\
\hline 5 & 5.09 & 9.74 & 5.2 & $50.45 \%$ & $34.81 \%$ \\
\hline 4.86 & 5.23 & 9.55 & 5.39 & $51.83 \%$ & $36.08 \%$ \\
\hline 4.74 & 5.35 & 9.29 & 5.65 & $53.02 \%$ & $37.82 \%$ \\
\hline
\end{tabular}

Table 5-1. Ring test - specimen\#1 results 


\begin{tabular}{|c|c|c|c|c|c|}
\hline \multicolumn{5}{|c|}{$\begin{array}{c}\text { Experiment-specimen\#2 } \\
\left(\mathrm{H}_{0}=10.14, \mathrm{D}_{0}=14.95\right)\end{array}$} & \multirow{2}{*}{$\frac{\text { Unit: } \mathrm{mm}}{\Delta \mathrm{D} / \mathrm{D}}$} \\
\hline $\mathrm{H}$ & $\begin{array}{c}\Delta \mathrm{H} \\
\left(\mathrm{H}_{0}-\mathrm{H}\right)\end{array}$ & D & $\begin{array}{c}\Delta D \\
\left(D-D_{0}\right)\end{array}$ & $\Delta \mathrm{H} / \mathrm{H}$ & \\
\hline 10.14 & 0 & 14.95 & 0 & $0.00 \%$ & $0.00 \%$ \\
\hline 9.64 & 0.5 & 14.66 & 0.29 & $4.93 \%$ & $1.94 \%$ \\
\hline 9.17 & 0.97 & 14.22 & 0.73 & $9.57 \%$ & $4.88 \%$ \\
\hline 8.76 & 1.38 & 14.12 & 0.83 & $13.61 \%$ & $5.55 \%$ \\
\hline 8.4 & 1.74 & 14.04 & 0.91 & $17.16 \%$ & $6.09 \%$ \\
\hline 8.08 & 2.06 & 13.75 & 1.2 & $20.32 \%$ & $8.03 \%$ \\
\hline 7.8 & 2.34 & 13.51 & 1.44 & $23.08 \%$ & $9.63 \%$ \\
\hline 7.54 & 2.6 & 13.4 & 1.55 & $25.64 \%$ & $10.37 \%$ \\
\hline 7.12 & 3.02 & 12.94 & 2.01 & $29.78 \%$ & $13.44 \%$ \\
\hline 6.76 & 3.38 & 12.58 & 2.37 & $33.33 \%$ & $15.85 \%$ \\
\hline 6.44 & 3.7 & 12.26 & 2.69 & $36.49 \%$ & $17.99 \%$ \\
\hline 6.18 & 3.96 & 11.86 & 3.09 & $39.05 \%$ & $20.67 \%$ \\
\hline 5.98 & 4.16 & 11.44 & 3.51 & $41.03 \%$ & $23.48 \%$ \\
\hline 5.76 & 4.38 & 11.3 & 3.65 & $43.20 \%$ & $24.41 \%$ \\
\hline 5.58 & 4.56 & 11.09 & 3.86 & $44.97 \%$ & $25.82 \%$ \\
\hline 5.3 & 4.84 & 10.54 & 4.41 & $47.73 \%$ & $29.50 \%$ \\
\hline 5.04 & 5.1 & 10.08 & 4.87 & $50.30 \%$ & $32.58 \%$ \\
\hline 4.86 & 5.28 & 9.88 & 5.07 & $52.07 \%$ & $33.91 \%$ \\
\hline 4.72 & 5.42 & 9.43 & 5.52 & $53.45 \%$ & $36.92 \%$ \\
\hline 4.5 & 5.64 & 8.83 & 6.12 & $55.62 \%$ & $40.94 \%$ \\
\hline
\end{tabular}

Table 5-2. Ring test - specimen\#2 results 


\begin{tabular}{|c|c|c|c|c|c|}
\hline \multicolumn{7}{|c|}{$\begin{array}{c}\text { Experiment-specimen\#3 } \\
\left(\mathrm{H}_{0}=10.13, \mathrm{D}_{0}=14.91\right)\end{array}$} \\
\hline $\mathrm{H}$ & $\begin{array}{c}\Delta \mathrm{H} \\
\left(\mathrm{H}_{0}-\mathrm{H}\right)\end{array}$ & $\mathrm{D}$ & $\begin{array}{c}\Delta \mathrm{D} \\
\left(\mathrm{D}-\mathrm{D}_{0}\right)\end{array}$ & $\Delta \mathrm{H} / \mathrm{H}$ & $\Delta \mathrm{D} / \mathrm{D}$ \\
\hline 10.13 & 0 & 14.91 & 0 & $0.00 \%$ & $0.00 \%$ \\
\hline 9.58 & 0.55 & 14.6 & 0.31 & $5.43 \%$ & $2.08 \%$ \\
\hline 9.12 & 1.01 & 14.34 & 0.57 & $9.97 \%$ & $3.82 \%$ \\
\hline 8.7 & 1.43 & 14.14 & 0.77 & $14.12 \%$ & $5.16 \%$ \\
\hline 8.34 & 1.79 & 14 & 0.91 & $17.67 \%$ & $6.10 \%$ \\
\hline 8.01 & 2.12 & 13.93 & 0.98 & $20.93 \%$ & $6.57 \%$ \\
\hline 7.74 & 2.39 & 13.64 & 1.27 & $23.59 \%$ & $8.52 \%$ \\
\hline 7.3 & 2.83 & 13.06 & 1.85 & $27.94 \%$ & $12.41 \%$ \\
\hline 6.92 & 3.21 & 12.68 & 2.23 & $31.69 \%$ & $14.96 \%$ \\
\hline 6.59 & 3.54 & 12.2 & 2.71 & $34.95 \%$ & $18.18 \%$ \\
\hline 6.32 & 3.81 & 11.74 & 3.17 & $37.61 \%$ & $21.26 \%$ \\
\hline 6.08 & 4.05 & 11.32 & 3.59 & $39.98 \%$ & $24.08 \%$ \\
\hline 5.75 & 4.38 & 10.7 & 4.21 & $43.24 \%$ & $28.24 \%$ \\
\hline 5.46 & 4.67 & 10.16 & 4.75 & $46.10 \%$ & $31.86 \%$ \\
\hline 5.18 & 4.95 & 9.74 & 5.17 & $48.86 \%$ & $34.67 \%$ \\
\hline 5 & 5.13 & 9.28 & 5.63 & $50.64 \%$ & $37.76 \%$ \\
\hline 4.77 & 5.36 & 8.6 & 6.31 & $52.91 \%$ & $42.32 \%$ \\
\hline
\end{tabular}

Table 5-3. Ring test - specimen\#3 results 


\begin{tabular}{|c|c|c|c|c|c|}
\hline \multicolumn{5}{|c|}{$\begin{array}{c}\text { Experiment-specimen\#4 } \\
\left(\mathrm{H}_{0}=10.12, \mathrm{D}_{0}=14.92\right)\end{array}$} & Unit: mm \\
\hline $\mathrm{H}$ & $\begin{array}{c}\Delta \mathrm{H} \\
\left(\mathrm{H}_{0}-\mathrm{H}\right)\end{array}$ & D & $\begin{array}{c}\Delta D \\
\left(D-D_{0}\right)\end{array}$ & $\Delta \mathrm{H} / \mathrm{H}$ & $\Delta \mathrm{D} / \mathrm{D}$ \\
\hline 10.12 & 0 & 14.92 & 0 & $0.00 \%$ & $0.00 \%$ \\
\hline 9.59 & 0.53 & 14.7 & 0.22 & $5.24 \%$ & $1.47 \%$ \\
\hline 9.12 & 1 & 14.4 & 0.52 & $9.88 \%$ & $3.49 \%$ \\
\hline 8.72 & 1.4 & 14.14 & 0.78 & $13.83 \%$ & $5.23 \%$ \\
\hline 8.36 & 1.76 & 13.96 & 0.96 & $17.39 \%$ & $6.43 \%$ \\
\hline 8.04 & 2.08 & 13.72 & 1.2 & $20.55 \%$ & $8.04 \%$ \\
\hline 7.75 & 2.37 & 13.58 & 1.34 & $23.42 \%$ & $8.98 \%$ \\
\hline 7.5 & 2.62 & 13.22 & 1.7 & $25.89 \%$ & $11.39 \%$ \\
\hline 7.09 & 3.03 & 12.76 & 2.16 & $29.94 \%$ & $14.48 \%$ \\
\hline 6.74 & 3.38 & 12.36 & 2.56 & $33.40 \%$ & $17.16 \%$ \\
\hline 6.41 & 3.71 & 12.1 & 2.82 & $36.66 \%$ & $18.90 \%$ \\
\hline 6.12 & 4 & 11.86 & 3.06 & $39.53 \%$ & $20.51 \%$ \\
\hline 5.86 & 4.26 & 11.52 & 3.4 & $42.09 \%$ & $22.79 \%$ \\
\hline 5.52 & 4.6 & 11.04 & 3.88 & $45.45 \%$ & $26.01 \%$ \\
\hline 5.22 & 4.9 & 10.52 & 4.4 & $48.42 \%$ & $29.49 \%$ \\
\hline 5.02 & 5.1 & 10.04 & 4.88 & $50.40 \%$ & $32.71 \%$ \\
\hline 4.72 & 5.4 & 9.37 & 5.55 & $53.36 \%$ & $37.20 \%$ \\
\hline
\end{tabular}

Table 5-4. Ring test - specimen\#4 results 


\begin{tabular}{|c|c|c|c|c|c|}
\hline \multicolumn{5}{|c|}{$\begin{array}{c}\text { Experiment-specimen\#5 } \\
\left(\mathrm{H}_{0}=10.12, \mathrm{D}_{0}=14.96\right)\end{array}$} & \multirow{2}{*}{$\begin{array}{r}\text { Unit: } \mathrm{mm} \\
\Delta \mathrm{D} / \mathrm{D}\end{array}$} \\
\hline $\mathrm{H}$ & $\begin{array}{c}\Delta \mathrm{H} \\
\left(\mathrm{H}_{0}-\mathrm{H}\right)\end{array}$ & $D$ & $\begin{array}{c}\Delta \mathrm{D} \\
\left(\mathrm{D}-\mathrm{D}_{0}\right)\end{array}$ & $\Delta \mathrm{H} / \mathrm{H}$ & \\
\hline 10.12 & 0 & 14.96 & 0 & $0.00 \%$ & $0.00 \%$ \\
\hline 9.58 & 0.54 & 14.74 & 0.22 & $5.34 \%$ & $1.47 \%$ \\
\hline 9.11 & 1.01 & 14.49 & 0.47 & $9.98 \%$ & $3.14 \%$ \\
\hline 8.7 & 1.42 & 14.34 & 0.62 & $14.03 \%$ & $4.14 \%$ \\
\hline 8.33 & 1.79 & 14.12 & 0.84 & $17.69 \%$ & $5.61 \%$ \\
\hline 8.02 & 2.1 & 14 & 0.96 & $20.75 \%$ & $6.42 \%$ \\
\hline 7.72 & 2.4 & 13.76 & 1.2 & $23.72 \%$ & $8.02 \%$ \\
\hline 7.3 & 2.82 & 13.36 & 1.6 & $27.87 \%$ & $10.70 \%$ \\
\hline 6.89 & 3.23 & 13.13 & 1.83 & $31.92 \%$ & $12.23 \%$ \\
\hline 6.54 & 3.58 & 12.8 & 2.16 & $35.38 \%$ & $14.44 \%$ \\
\hline 6.24 & 3.88 & 12.4 & 2.56 & $38.34 \%$ & $17.11 \%$ \\
\hline 5.97 & 4.15 & 12.14 & 2.82 & $41.01 \%$ & $18.85 \%$ \\
\hline 5.74 & 4.38 & 11.86 & 3.1 & $43.28 \%$ & $20.72 \%$ \\
\hline 5.42 & 4.7 & 11.34 & 3.62 & $46.44 \%$ & $24.20 \%$ \\
\hline 5.16 & 4.96 & 10.9 & 4.06 & $49.01 \%$ & $27.14 \%$ \\
\hline 4.79 & 5.33 & 10.22 & 4.74 & $52.67 \%$ & $31.68 \%$ \\
\hline 4.52 & 5.6 & 9.66 & 5.3 & $55.34 \%$ & $35.43 \%$ \\
\hline
\end{tabular}

Table 5-5. Ring test - specimen\#5 results 


\begin{tabular}{|c|c|c|c|c|c|}
\hline \multicolumn{6}{|c|}{ FEM Simulation $\# 1\left(\mu=0.20, \tau_{\max }=123.41 \mathrm{MPa}\right)$} \\
\hline & & $\left(\mathrm{H}_{0}=1\right.$ & $\left.\rho_{0}=15\right)$ & & Unit: mm \\
\hline $\mathrm{H} / 2$ & $\begin{array}{c}\Delta \mathrm{H} / 2 \\
\left(\mathrm{H}_{0}-\mathrm{H}\right) / 2\end{array}$ & $\mathrm{D} / 2$ & $\begin{array}{c}\Delta \mathrm{D} / 2 \\
\left(\mathrm{D}-\mathrm{D}_{0}\right) / 2\end{array}$ & $\Delta \mathrm{H} / \mathrm{H}$ & $\Delta \mathrm{D} / \mathrm{D}$ \\
\hline 5 & 0 & 7.5 & 0 & $0.00 \%$ & $0.00 \%$ \\
\hline 4.469 & 0.531 & 7.375 & 0.125 & $10.62 \%$ & $1.67 \%$ \\
\hline 4.339 & 0.661 & 7.331 & 0.169 & $13.22 \%$ & $2.25 \%$ \\
\hline 4.21 & 0.79 & 7.279 & 0.221 & $15.80 \%$ & $2.95 \%$ \\
\hline 4.08 & 0.92 & 7.218 & 0.282 & $18.40 \%$ & $3.76 \%$ \\
\hline 3.95 & 1.05 & 7.146 & 0.354 & $21.00 \%$ & $4.72 \%$ \\
\hline 3.821 & 1.179 & 7.061 & 0.439 & $23.58 \%$ & $5.85 \%$ \\
\hline 3.691 & 1.309 & 6.964 & 0.536 & $26.18 \%$ & $7.15 \%$ \\
\hline 3.562 & 1.438 & 6.851 & 0.649 & $28.76 \%$ & $8.65 \%$ \\
\hline 3.432 & 1.568 & 6.713 & 0.787 & $31.36 \%$ & $10.49 \%$ \\
\hline 3.302 & 1.698 & 6.556 & 0.944 & $33.96 \%$ & $12.59 \%$ \\
\hline 3.238 & 1.762 & 6.474 & 1.026 & $35.24 \%$ & $13.68 \%$ \\
\hline 3.108 & 1.892 & 6.303 & 1.197 & $37.84 \%$ & $15.96 \%$ \\
\hline 2.978 & 2.022 & 6.118 & 1.382 & $40.44 \%$ & $18.43 \%$ \\
\hline 2.849 & 2.151 & 5.917 & 1.583 & $43.02 \%$ & $21.11 \%$ \\
\hline 2.719 & 2.281 & 5.694 & 1.806 & $45.62 \%$ & $24.08 \%$ \\
\hline 2.59 & 2.41 & 5.446 & 2.054 & $48.20 \%$ & $27.39 \%$ \\
\hline 2.46 & 2.54 & 5.171 & 2.329 & $50.80 \%$ & $31.05 \%$ \\
\hline 2.33 & 2.67 & 4.852 & 2.648 & $53.40 \%$ & $35.31 \%$ \\
\hline 2.201 & 2.799 & 4.462 & 3.038 & $55.98 \%$ & $40.51 \%$ \\
\hline 2.071 & 2.929 & 3.908 & 3.592 & $58.58 \%$ & $47.89 \%$ \\
\hline 1.942 & 3.058 & 3.314 & 4.186 & $61.16 \%$ & $55.81 \%$ \\
\hline
\end{tabular}

Table 5-6. Ring test FEM results $\left(\mu / \tau_{\max }=0.20 / 123\right)$ 


\begin{tabular}{|c|c|c|c|c|c|}
\hline \multicolumn{6}{|c|}{ FEM Simulation $\# 2\left(\mu=0.21, \tau_{\max }=123.41 \mathrm{MPa}\right)$} \\
\hline \multicolumn{5}{|c|}{$\left(H_{0}=10, D_{0}=15\right)$} & \multirow{2}{*}{$\frac{\text { Unit: } \mathrm{mm}}{\Delta \mathrm{D} / \mathrm{D}}$} \\
\hline $\mathrm{H} / 2$ & $\begin{array}{c}\Delta H / 2 \\
\left(H_{0}-H\right) / 2\end{array}$ & $\mathrm{D} / 2$ & $\begin{array}{c}\Delta \mathrm{D} / 2 \\
\left(\mathrm{D}-\mathrm{D}_{0}\right) / 2\end{array}$ & $\Delta \mathrm{H} / \mathrm{H}$ & \\
\hline 5 & 0 & 7.5 & 0 & $0.00 \%$ & $0.00 \%$ \\
\hline 4.469 & 0.531 & 7.36 & 0.14 & $10.62 \%$ & $1.87 \%$ \\
\hline 4.34 & 0.66 & 7.312 & 0.188 & $13.20 \%$ & $2.51 \%$ \\
\hline 4.209 & 0.791 & 7.254 & 0.246 & $15.82 \%$ & $3.28 \%$ \\
\hline 4.08 & 0.92 & 7.187 & 0.313 & $18.40 \%$ & $4.17 \%$ \\
\hline 3.95 & 1.05 & 7.109 & 0.391 & $21.00 \%$ & $5.21 \%$ \\
\hline 3.819 & 1.181 & 7.017 & 0.483 & $23.62 \%$ & $6.44 \%$ \\
\hline 3.687 & 1.313 & 6.908 & 0.592 & $26.26 \%$ & $7.89 \%$ \\
\hline 3.555 & 1.445 & 6.78 & 0.72 & $28.90 \%$ & $9.60 \%$ \\
\hline 3.423 & 1.577 & 6.627 & 0.873 & $31.54 \%$ & $11.64 \%$ \\
\hline 3.291 & 1.709 & 6.462 & 1.038 & $34.18 \%$ & $13.84 \%$ \\
\hline 3.225 & 1.775 & 6.376 & 1.124 & $35.50 \%$ & $14.99 \%$ \\
\hline 3.095 & 1.905 & 6.202 & 1.298 & $38.10 \%$ & $17.31 \%$ \\
\hline 2.97 & 2.03 & 6.019 & 1.481 & $40.60 \%$ & $19.75 \%$ \\
\hline 2.849 & 2.151 & 5.828 & 1.672 & $43.02 \%$ & $22.29 \%$ \\
\hline 2.731 & 2.269 & 5.624 & 1.876 & $45.38 \%$ & $25.01 \%$ \\
\hline 2.613 & 2.387 & 5.401 & 2.099 & $47.74 \%$ & $27.99 \%$ \\
\hline 2.485 & 2.515 & 5.124 & 2.376 & $50.30 \%$ & $31.68 \%$ \\
\hline 2.362 & 2.638 & 4.814 & 2.686 & $52.76 \%$ & $35.81 \%$ \\
\hline 2.249 & 2.751 & 4.47 & 3.03 & $55.02 \%$ & $40.40 \%$ \\
\hline 2.141 & 2.859 & 4.078 & 3.422 & $57.18 \%$ & $45.63 \%$ \\
\hline 2.016 & 2.984 & 3.475 & 4.025 & $59.68 \%$ & $53.67 \%$ \\
\hline
\end{tabular}

Table 5-7. Ring test FEM results $\left(\mu / \tau_{\max }=0.21 / 123\right)$ 


\begin{tabular}{|c|c|c|c|c|c|}
\hline \multicolumn{6}{|c|}{ FEM Simulation $\# 3\left(\mu=0.22, \tau_{\max }=123.41 \mathrm{MPa}\right)$} \\
\hline $\mathrm{H} / 2$ & $\begin{array}{c}\Delta H / 2 \\
\left(H_{0}-H\right) / 2\end{array}$ & $\mathrm{D} / 2$ & $\begin{array}{c}\Delta \mathrm{D} / 2 \\
\left(\mathrm{D}-\mathrm{D}_{0}\right) / 2\end{array}$ & $\Delta \mathrm{H} / \mathrm{H}$ & $\Delta \mathrm{D} / \mathrm{D}$ \\
\hline 5 & 0 & 7.5 & 0 & $0.00 \%$ & $0.00 \%$ \\
\hline 4.469 & 0.531 & 7.345 & 0.155 & $10.62 \%$ & $2.07 \%$ \\
\hline 4.34 & 0.66 & 7.292 & 0.208 & $13.20 \%$ & $2.77 \%$ \\
\hline 4.21 & 0.79 & 7.23 & 0.27 & $15.80 \%$ & $3.60 \%$ \\
\hline 4.082 & 0.918 & 7.157 & 0.343 & $18.36 \%$ & $4.57 \%$ \\
\hline 3.953 & 1.047 & 7.072 & 0.428 & $20.94 \%$ & $5.71 \%$ \\
\hline 3.823 & 1.177 & 6.972 & 0.528 & $23.54 \%$ & $7.04 \%$ \\
\hline 3.692 & 1.308 & 6.857 & 0.643 & $26.16 \%$ & $8.57 \%$ \\
\hline 3.56 & 1.44 & 6.72 & 0.78 & $28.80 \%$ & $10.40 \%$ \\
\hline 3.428 & 1.572 & 6.562 & 0.938 & $31.44 \%$ & $12.51 \%$ \\
\hline 3.296 & 1.704 & 6.392 & 1.108 & $34.08 \%$ & $14.77 \%$ \\
\hline 3.23 & 1.77 & 6.303 & 1.197 & $35.40 \%$ & $15.96 \%$ \\
\hline 3.1 & 1.9 & 6.123 & 1.377 & $38.00 \%$ & $18.36 \%$ \\
\hline 2.979 & 2.021 & 5.942 & 1.558 & $40.42 \%$ & $20.77 \%$ \\
\hline 2.861 & 2.139 & 5.752 & 1.748 & $42.78 \%$ & $23.31 \%$ \\
\hline 2.743 & 2.257 & 5.543 & 1.957 & $45.14 \%$ & $26.09 \%$ \\
\hline 2.626 & 2.374 & 5.315 & 2.185 & $47.48 \%$ & $29.13 \%$ \\
\hline 2.504 & 2.496 & 5.047 & 2.453 & $49.92 \%$ & $32.71 \%$ \\
\hline 2.375 & 2.625 & 4.711 & 2.789 & $52.50 \%$ & $37.19 \%$ \\
\hline 2.263 & 2.737 & 4.355 & 3.145 & $54.74 \%$ & $41.93 \%$ \\
\hline 2.155 & 2.845 & 3.971 & 3.529 & $56.90 \%$ & $47.05 \%$ \\
\hline 2.03 & 2.97 & 3.423 & 4.077 & $59.40 \%$ & $54.36 \%$ \\
\hline
\end{tabular}

Table 5-8. Ring test FEM results $\left(\mu / \tau_{\max }=0.22 / 123\right)$ 


\begin{tabular}{|c|c|c|c|c|c|}
\hline \multicolumn{6}{|c|}{ FEM Simulation $\# 4\left(\mu=0.23, \tau_{\max }=123.41 \mathrm{MPa}\right)$} \\
\hline & & $\left(\mathrm{H}_{0}=1\right.$ & $\left.0_{0}=15\right)$ & & Unit: mm \\
\hline $\mathrm{H} / 2$ & $\begin{array}{c}\Delta \mathrm{H} / 2 \\
\left(\mathrm{H}_{0}-\mathrm{H}\right) / 2\end{array}$ & $\mathrm{D} / 2$ & $\begin{array}{c}\Delta \mathrm{D} / 2 \\
\left(\mathrm{D}-\mathrm{D}_{0}\right) / 2\end{array}$ & $\Delta \mathbf{H} / \mathbf{H}$ & $\Delta \mathrm{D} / \mathrm{D}$ \\
\hline 5 & 0 & 7.5 & 0 & $0.00 \%$ & $0.00 \%$ \\
\hline 4.469 & 0.531 & 7.33 & 0.17 & $10.62 \%$ & $2.27 \%$ \\
\hline 4.339 & 0.661 & 7.272 & 0.228 & $13.22 \%$ & $3.04 \%$ \\
\hline 4.209 & 0.791 & 7.204 & 0.296 & $15.82 \%$ & $3.95 \%$ \\
\hline 4.081 & 0.919 & 7.124 & 0.376 & $18.38 \%$ & $5.01 \%$ \\
\hline 3.953 & 1.047 & 7.034 & 0.466 & $20.94 \%$ & $6.21 \%$ \\
\hline 3.824 & 1.176 & 6.93 & 0.57 & $23.52 \%$ & $7.60 \%$ \\
\hline 3.692 & 1.308 & 6.805 & 0.695 & $26.16 \%$ & $9.27 \%$ \\
\hline 3.56 & 1.44 & 6.656 & 0.844 & $28.80 \%$ & $11.25 \%$ \\
\hline 3.428 & 1.572 & 6.488 & 1.012 & $31.44 \%$ & $13.49 \%$ \\
\hline 3.295 & 1.705 & 6.31 & 1.19 & $34.10 \%$ & $15.87 \%$ \\
\hline 3.229 & 1.771 & 6.222 & 1.278 & $35.42 \%$ & $17.04 \%$ \\
\hline 3.1 & 1.9 & 6.041 & 1.459 & $38.00 \%$ & $19.45 \%$ \\
\hline 2.974 & 2.026 & 5.85 & 1.65 & $40.52 \%$ & $22.00 \%$ \\
\hline 2.851 & 2.149 & 5.649 & 1.851 & $42.98 \%$ & $24.68 \%$ \\
\hline 2.733 & 2.267 & 5.434 & 2.066 & $45.34 \%$ & $27.55 \%$ \\
\hline 2.615 & 2.385 & 5.193 & 2.307 & $47.70 \%$ & $30.76 \%$ \\
\hline 2.487 & 2.513 & 4.893 & 2.607 & $50.26 \%$ & $34.76 \%$ \\
\hline 2.362 & 2.638 & 4.542 & 2.958 & $52.76 \%$ & $39.44 \%$ \\
\hline 2.253 & 2.747 & 4.184 & 3.316 & $54.94 \%$ & $44.21 \%$ \\
\hline 2.144 & 2.856 & 3.792 & 3.708 & $57.12 \%$ & $49.44 \%$ \\
\hline 2.02 & 2.98 & 3.188 & 4.312 & $59.60 \%$ & $57.49 \%$ \\
\hline
\end{tabular}

Table 5-9. Ring test FEM results $\left(\mu / \tau_{\max }=0.23 / 123\right)$ 


\begin{tabular}{|c|c|c|c|c|c|}
\hline \multicolumn{6}{|c|}{ FEM Simulation $\# 5\left(\mu=0.24, \tau_{\max }=123.41 \mathrm{MPa}\right)$} \\
\hline & & $\left(\mathrm{H}_{0}=1\right.$ & $\left.O_{0}=15\right)$ & & Unit: mm \\
\hline$H / 2$ & $\begin{array}{c}\Delta \mathrm{H} / 2 \\
\left(\mathrm{H}_{0}-\mathrm{H}\right) / 2\end{array}$ & $\mathrm{D} / 2$ & $\begin{array}{c}\Delta \mathrm{D} / 2 \\
\left(\mathrm{D}-\mathrm{D}_{0}\right) / 2\end{array}$ & $\Delta \mathrm{H} / \mathrm{H}$ & $\Delta \mathrm{D} / \mathrm{D}$ \\
\hline 5 & 0 & 7.5 & 0 & $0.00 \%$ & $0.00 \%$ \\
\hline 4.469 & 0.531 & 7.316 & 0.184 & $10.62 \%$ & $2.45 \%$ \\
\hline 4.339 & 0.661 & 7.252 & 0.248 & $13.22 \%$ & $3.31 \%$ \\
\hline 4.209 & 0.791 & 7.178 & 0.322 & $15.82 \%$ & $4.29 \%$ \\
\hline 4.08 & 0.92 & 7.093 & 0.407 & $18.40 \%$ & $5.43 \%$ \\
\hline 3.953 & 1.047 & 6.995 & 0.505 & $20.94 \%$ & $6.73 \%$ \\
\hline 3.823 & 1.177 & 6.882 & 0.618 & $23.54 \%$ & $8.24 \%$ \\
\hline 3.691 & 1.309 & 6.749 & 0.751 & $26.18 \%$ & $10.01 \%$ \\
\hline 3.559 & 1.441 & 6.596 & 0.904 & $28.82 \%$ & $12.05 \%$ \\
\hline 3.427 & 1.573 & 6.426 & 1.074 & $31.46 \%$ & $14.32 \%$ \\
\hline 3.293 & 1.707 & 6.243 & 1.257 & $34.14 \%$ & $16.76 \%$ \\
\hline 3.226 & 1.774 & 6.151 & 1.349 & $35.48 \%$ & $17.99 \%$ \\
\hline 3.097 & 1.903 & 5.963 & 1.537 & $38.06 \%$ & $20.49 \%$ \\
\hline 2.972 & 2.028 & 5.772 & 1.728 & $40.56 \%$ & $23.04 \%$ \\
\hline 2.851 & 2.149 & 5.568 & 1.932 & $42.98 \%$ & $25.76 \%$ \\
\hline 2.733 & 2.267 & 5.347 & 2.153 & $45.34 \%$ & $28.71 \%$ \\
\hline 2.616 & 2.384 & 5.1 & 2.4 & $47.68 \%$ & $32.00 \%$ \\
\hline 2.487 & 2.513 & 4.788 & 2.712 & $50.26 \%$ & $36.16 \%$ \\
\hline 2.368 & 2.632 & 4.444 & 3.056 & $52.64 \%$ & $40.75 \%$ \\
\hline 2.26 & 2.74 & 4.081 & 3.419 & $54.80 \%$ & $45.59 \%$ \\
\hline 2.151 & 2.849 & 3.583 & 3.917 & $56.98 \%$ & $52.23 \%$ \\
\hline 2.026 & 2.974 & 2.977 & 4.523 & $59.48 \%$ & $60.31 \%$ \\
\hline
\end{tabular}

Table 5-10. Ring test FEM results $\left(\mu / \tau_{\max }=0.24 / 123\right)$ 


\begin{tabular}{|c|c|c|c|c|c|}
\hline \multicolumn{6}{|c|}{ FEM Simulation $\# 6\left(\mu=0.20, \tau_{\max }=142.5 \mathrm{MPa}\right)$} \\
\hline \multicolumn{5}{|c|}{$\left(\mathrm{H}_{0}=10, \mathrm{D}_{0}=15\right)$} & \multirow{2}{*}{$\frac{\text { Unit: } \mathrm{mm}}{\Delta \mathrm{D} / \mathrm{D}}$} \\
\hline $\mathrm{H} / 2$ & $\begin{array}{c}\Delta \mathrm{H} / 2 \\
\left(\mathrm{H}_{0}-\mathrm{H}\right) / 2\end{array}$ & $\mathrm{D} / 2$ & $\begin{array}{c}\Delta D / 2 \\
\left(D-D_{0}\right) / 2\end{array}$ & $\Delta \mathrm{H} / \mathrm{H}$ & \\
\hline 5 & 0 & 7.5 & 0 & $0.00 \%$ & $0.00 \%$ \\
\hline 4.469 & 0.531 & 7.375 & 0.125 & $10.62 \%$ & $1.67 \%$ \\
\hline 4.34 & 0.66 & 7.331 & 0.169 & $13.20 \%$ & $2.25 \%$ \\
\hline 4.209 & 0.791 & 7.28 & 0.22 & $15.82 \%$ & $2.93 \%$ \\
\hline 4.08 & 0.92 & 7.219 & 0.281 & $18.40 \%$ & $3.75 \%$ \\
\hline 3.95 & 1.05 & 7.146 & 0.354 & $21.00 \%$ & $4.72 \%$ \\
\hline 3.818 & 1.182 & 7.063 & 0.437 & $23.64 \%$ & $5.83 \%$ \\
\hline 3.686 & 1.314 & 6.962 & 0.538 & $26.28 \%$ & $7.17 \%$ \\
\hline 3.554 & 1.446 & 6.846 & 0.654 & $28.92 \%$ & $8.72 \%$ \\
\hline 3.422 & 1.578 & 6.704 & 0.796 & $31.56 \%$ & $10.61 \%$ \\
\hline 3.29 & 1.71 & 6.544 & 0.956 & $34.20 \%$ & $12.75 \%$ \\
\hline 3.223 & 1.777 & 6.459 & 1.041 & $35.54 \%$ & $13.88 \%$ \\
\hline 3.094 & 1.906 & 6.288 & 1.212 & $38.12 \%$ & $16.16 \%$ \\
\hline 2.968 & 2.032 & 6.107 & 1.393 & $40.64 \%$ & $18.57 \%$ \\
\hline 2.844 & 2.156 & 5.915 & 1.585 & $43.12 \%$ & $21.13 \%$ \\
\hline 2.725 & 2.275 & 5.711 & 1.789 & $45.50 \%$ & $23.85 \%$ \\
\hline 2.608 & 2.392 & 5.491 & 2.009 & $47.84 \%$ & $26.79 \%$ \\
\hline 2.479 & 2.521 & 5.22 & 2.28 & $50.42 \%$ & $30.40 \%$ \\
\hline 2.354 & 2.646 & 4.913 & 2.587 & $52.92 \%$ & $34.49 \%$ \\
\hline 2.239 & 2.761 & 4.574 & 2.926 & $55.22 \%$ & $39.01 \%$ \\
\hline 2.131 & 2.869 & 4.196 & 3.304 & $57.38 \%$ & $44.05 \%$ \\
\hline 2.006 & 2.994 & 3.685 & 3.815 & $59.88 \%$ & $50.87 \%$ \\
\hline
\end{tabular}

Table 5-11. Ring test FEM results $\left(\mu / \tau_{\max }=0.20 / 142\right)$ 


\begin{tabular}{|c|c|c|c|c|c|}
\hline \multicolumn{6}{|c|}{ FEM Simulation $\# 7\left(\mu=0.21, \tau_{\max }=142.5 \mathrm{MPa}\right)$} \\
\hline $\mathrm{H} / 2$ & $\begin{array}{c}\Delta H / 2 \\
\left(H_{0}-H\right) / 2\end{array}$ & $D / 2$ & $\begin{array}{c}\Delta D / 2 \\
\left(D-D_{0}\right) / 2\end{array}$ & $\Delta \mathrm{H} / \mathrm{H}$ & $\Delta \mathrm{D} / \mathrm{D}$ \\
\hline 5 & 0 & 7.5 & 0 & $0.00 \%$ & $0.00 \%$ \\
\hline 4.469 & 0.531 & 7.36 & 0.14 & $10.62 \%$ & $1.87 \%$ \\
\hline 4.34 & 0.66 & 7.312 & 0.188 & $13.20 \%$ & $2.51 \%$ \\
\hline 4.209 & 0.791 & 7.254 & 0.246 & $15.82 \%$ & $3.28 \%$ \\
\hline 4.08 & 0.92 & 7.187 & 0.313 & $18.40 \%$ & $4.17 \%$ \\
\hline 3.95 & 1.05 & 7.109 & 0.391 & $21.00 \%$ & $5.21 \%$ \\
\hline 3.819 & 1.181 & 7.018 & 0.482 & $23.62 \%$ & $6.43 \%$ \\
\hline 3.687 & 1.313 & 6.908 & 0.592 & $26.26 \%$ & $7.89 \%$ \\
\hline 3.555 & 1.445 & 6.779 & 0.721 & $28.90 \%$ & $9.61 \%$ \\
\hline 3.423 & 1.577 & 6.627 & 0.873 & $31.54 \%$ & $11.64 \%$ \\
\hline 3.29 & 1.71 & 6.462 & 1.038 & $34.20 \%$ & $13.84 \%$ \\
\hline 3.225 & 1.775 & 6.376 & 1.124 & $35.50 \%$ & $14.99 \%$ \\
\hline 3.095 & 1.905 & 6.199 & 1.301 & $38.10 \%$ & $17.35 \%$ \\
\hline 2.971 & 2.029 & 6.016 & 1.484 & $40.58 \%$ & $19.79 \%$ \\
\hline 2.85 & 2.15 & 5.824 & 1.676 & $43.00 \%$ & $22.35 \%$ \\
\hline 2.732 & 2.268 & 5.616 & 1.884 & $45.36 \%$ & $25.12 \%$ \\
\hline 2.615 & 2.385 & 5.391 & 2.109 & $47.70 \%$ & $28.12 \%$ \\
\hline 2.486 & 2.514 & 5.108 & 2.392 & $50.28 \%$ & $31.89 \%$ \\
\hline 2.365 & 2.635 & 4.793 & 2.707 & $52.70 \%$ & $36.09 \%$ \\
\hline 2.256 & 2.744 & 4.446 & 3.054 & $54.88 \%$ & $40.72 \%$ \\
\hline 2.148 & 2.852 & 4.066 & 3.434 & $57.04 \%$ & $45.79 \%$ \\
\hline 2.023 & 2.977 & 3.571 & 3.929 & $59.54 \%$ & $52.39 \%$ \\
\hline
\end{tabular}

Table 5-12. Ring test FEM results $\left(\mu / \tau_{\max }=0.21 / 142\right)$ 


\begin{tabular}{|c|c|c|c|c|c|}
\hline \multicolumn{6}{|c|}{ FEM Simulation $\# 8\left(\mu=0.22, \tau_{\max }=142.5 \mathrm{MPa}\right)$} \\
\hline $\mathrm{H} / 2$ & $\begin{array}{c}\Delta \mathrm{H} / 2 \\
\left(\mathrm{H}_{0}-\mathrm{H}\right) / 2\end{array}$ & $\mathrm{D} / 2$ & $\begin{array}{c}\Delta \mathrm{D} / 2 \\
\left(\mathrm{D}-\mathrm{D}_{0}\right) / 2\end{array}$ & $\Delta \mathrm{H} / \mathrm{H}$ & $\Delta \mathrm{D} / \mathrm{D}$ \\
\hline 5 & 0 & 7.5 & 0 & $0.00 \%$ & $0.00 \%$ \\
\hline 4.469 & 0.531 & 7.345 & 0.155 & $10.62 \%$ & $2.07 \%$ \\
\hline 4.34 & 0.66 & 7.292 & 0.208 & $13.20 \%$ & $2.77 \%$ \\
\hline 4.21 & 0.79 & 7.23 & 0.27 & $15.80 \%$ & $3.60 \%$ \\
\hline 4.082 & 0.918 & 7.157 & 0.343 & $18.36 \%$ & $4.57 \%$ \\
\hline 3.953 & 1.047 & 7.072 & 0.428 & $20.94 \%$ & $5.71 \%$ \\
\hline 3.823 & 1.177 & 6.972 & 0.528 & $23.54 \%$ & $7.04 \%$ \\
\hline 3.692 & 1.308 & 6.857 & 0.643 & $26.16 \%$ & $8.57 \%$ \\
\hline 3.56 & 1.44 & 6.718 & 0.782 & $28.80 \%$ & $10.43 \%$ \\
\hline 3.428 & 1.572 & 6.561 & 0.939 & $31.44 \%$ & $12.52 \%$ \\
\hline 3.295 & 1.705 & 6.389 & 1.111 & $34.10 \%$ & $14.81 \%$ \\
\hline 3.229 & 1.771 & 6.3 & 1.2 & $35.42 \%$ & $16.00 \%$ \\
\hline 3.1 & 1.9 & 6.121 & 1.379 & $38.00 \%$ & $18.39 \%$ \\
\hline 2.974 & 2.026 & 5.934 & 1.566 & $40.52 \%$ & $20.88 \%$ \\
\hline 2.851 & 2.149 & 5.735 & 1.765 & $42.98 \%$ & $23.53 \%$ \\
\hline 2.733 & 2.267 & 5.523 & 1.977 & $45.34 \%$ & $26.36 \%$ \\
\hline 2.616 & 2.384 & 5.292 & 2.208 & $47.68 \%$ & $29.44 \%$ \\
\hline 2.488 & 2.512 & 5.003 & 2.497 & $50.24 \%$ & $33.29 \%$ \\
\hline 2.366 & 2.634 & 4.675 & 2.825 & $52.68 \%$ & $37.67 \%$ \\
\hline 2.257 & 2.743 & 4.327 & 3.173 & $54.86 \%$ & $42.31 \%$ \\
\hline 2.148 & 2.852 & 3.859 & 3.641 & $57.04 \%$ & $48.55 \%$ \\
\hline 2.024 & 2.976 & 3.318 & 4.182 & $59.52 \%$ & $55.76 \%$ \\
\hline
\end{tabular}

Table 5-13. Ring test FEM results $\left(\mu / \tau_{\max }=0.22 / 142\right)$ 


\begin{tabular}{|c|c|c|c|c|c|}
\hline \multicolumn{6}{|c|}{ FEM Simulation $\# 9\left(\mu=0.23, \tau_{\max }=142.5 \mathrm{MPa}\right)$} \\
\hline $\mathrm{H} / 2$ & $\begin{array}{c}\Delta \mathrm{H} / 2 \\
\left(\mathrm{H}_{0}-\mathrm{H}\right) / 2\end{array}$ & $\mathrm{D} / 2$ & $\begin{array}{c}\Delta \mathrm{D} / 2 \\
\left(\mathrm{D}-\mathrm{D}_{0}\right) / 2\end{array}$ & $\Delta \mathrm{H} / \mathrm{H}$ & $\Delta \mathrm{D} / \mathrm{D}$ \\
\hline 5 & 0 & 7.5 & 0 & $0.00 \%$ & $0.00 \%$ \\
\hline 4.469 & 0.531 & 7.33 & 0.17 & $10.62 \%$ & $2.27 \%$ \\
\hline 4.339 & 0.661 & 7.272 & 0.228 & $13.22 \%$ & $3.04 \%$ \\
\hline 4.209 & 0.791 & 7.204 & 0.296 & $15.82 \%$ & $3.95 \%$ \\
\hline 4.081 & 0.919 & 7.124 & 0.376 & $18.38 \%$ & $5.01 \%$ \\
\hline 3.953 & 1.047 & 7.034 & 0.466 & $20.94 \%$ & $6.21 \%$ \\
\hline 3.824 & 1.176 & 6.929 & 0.571 & $23.52 \%$ & $7.61 \%$ \\
\hline 3.692 & 1.308 & 6.805 & 0.695 & $26.16 \%$ & $9.27 \%$ \\
\hline 3.56 & 1.44 & 6.656 & 0.844 & $28.80 \%$ & $11.25 \%$ \\
\hline 3.428 & 1.572 & 6.495 & 1.005 & $31.44 \%$ & $13.40 \%$ \\
\hline 3.295 & 1.705 & 6.317 & 1.183 & $34.10 \%$ & $15.77 \%$ \\
\hline 3.229 & 1.771 & 6.226 & 1.274 & $35.42 \%$ & $16.99 \%$ \\
\hline 3.099 & 1.901 & 6.043 & 1.457 & $38.02 \%$ & $19.43 \%$ \\
\hline 2.974 & 2.026 & 5.853 & 1.647 & $40.52 \%$ & $21.96 \%$ \\
\hline 2.851 & 2.149 & 5.651 & 1.849 & $42.98 \%$ & $24.65 \%$ \\
\hline 2.733 & 2.267 & 5.435 & 2.065 & $45.34 \%$ & $27.53 \%$ \\
\hline 2.615 & 2.385 & 5.194 & 2.306 & $47.70 \%$ & $30.75 \%$ \\
\hline 2.486 & 2.514 & 4.892 & 2.608 & $50.28 \%$ & $34.77 \%$ \\
\hline 2.363 & 2.637 & 4.549 & 2.951 & $52.74 \%$ & $39.35 \%$ \\
\hline 2.254 & 2.746 & 4.188 & 3.312 & $54.92 \%$ & $44.16 \%$ \\
\hline 2.145 & 2.855 & 3.795 & 3.705 & $57.10 \%$ & $49.40 \%$ \\
\hline 2.02 & 2.98 & 3.197 & 4.303 & $59.60 \%$ & $57.37 \%$ \\
\hline
\end{tabular}

Table 5-14. Ring test FEM results $\left(\mu / \tau_{\max }=0.23 / 142\right)$ 


\begin{tabular}{|c|c|c|c|c|c|}
\hline \multicolumn{6}{|c|}{ FEM Simulation $\# 10\left(\mu=0.24, \tau_{\max }=142.5 \mathrm{MPa}\right)$} \\
\hline $\mathrm{H} / 2$ & $\begin{array}{c}\Delta \mathrm{H} / 2 \\
\left(\mathrm{H}_{0}-\mathrm{H}\right) / 2\end{array}$ & $D / 2$ & $\begin{array}{c}\Delta D / 2 \\
\left(D-D_{0}\right) / 2\end{array}$ & $\Delta \mathrm{H} / \mathrm{H}$ & $\Delta \mathrm{D} / \mathrm{D}$ \\
\hline 5 & 0 & 7.5 & 0 & $0.00 \%$ & $0.00 \%$ \\
\hline 4.469 & 0.531 & 7.316 & 0.184 & $10.62 \%$ & $2.45 \%$ \\
\hline 4.339 & 0.661 & 7.252 & 0.248 & $13.22 \%$ & $3.31 \%$ \\
\hline 4.209 & 0.791 & 7.178 & 0.322 & $15.82 \%$ & $4.29 \%$ \\
\hline 4.08 & 0.92 & 7.093 & 0.407 & $18.40 \%$ & $5.43 \%$ \\
\hline 3.953 & 1.047 & 6.995 & 0.505 & $20.94 \%$ & $6.73 \%$ \\
\hline 3.823 & 1.177 & 6.882 & 0.618 & $23.54 \%$ & $8.24 \%$ \\
\hline 3.691 & 1.309 & 6.749 & 0.751 & $26.18 \%$ & $10.01 \%$ \\
\hline 3.558 & 1.442 & 6.595 & 0.905 & $28.84 \%$ & $12.07 \%$ \\
\hline 3.426 & 1.574 & 6.43 & 1.07 & $31.48 \%$ & $14.27 \%$ \\
\hline 3.293 & 1.707 & 6.245 & 1.255 & $34.14 \%$ & $16.73 \%$ \\
\hline 3.226 & 1.774 & 6.151 & 1.349 & $35.48 \%$ & $17.99 \%$ \\
\hline 3.097 & 1.903 & 5.963 & 1.537 & $38.06 \%$ & $20.49 \%$ \\
\hline 2.973 & 2.027 & 5.77 & 1.73 & $40.54 \%$ & $23.07 \%$ \\
\hline 2.852 & 2.148 & 5.564 & 1.936 & $42.96 \%$ & $25.81 \%$ \\
\hline 2.734 & 2.266 & 5.34 & 2.16 & $45.32 \%$ & $28.80 \%$ \\
\hline 2.618 & 2.382 & 5.095 & 2.405 & $47.64 \%$ & $32.07 \%$ \\
\hline 2.491 & 2.509 & 4.786 & 2.714 & $50.18 \%$ & $36.19 \%$ \\
\hline 2.382 & 2.618 & 4.467 & 3.033 & $52.36 \%$ & $40.44 \%$ \\
\hline 2.273 & 2.727 & 4.113 & 3.387 & $54.54 \%$ & $45.16 \%$ \\
\hline 2.164 & 2.836 & 3.712 & 3.788 & $56.72 \%$ & $50.51 \%$ \\
\hline 2.04 & 2.96 & 3.043 & 4.457 & $59.20 \%$ & $59.43 \%$ \\
\hline
\end{tabular}

Table 5-15. Ring test FEM results $\left(\mu / \tau_{\max }=0.24 / 142\right)$ 


\begin{tabular}{|c|c|c|c|c|c|}
\hline \multicolumn{6}{|c|}{$\begin{array}{l}\text { FEM Simulation \#11 }\left(\mu=0.22, \tau_{\max }=97 \mathrm{MPa}\right) \\
\qquad\left(\mathrm{H}_{0}=10, \mathrm{D}_{0}=15\right)\end{array}$} \\
\hline $\mathrm{H} / 2$ & $\begin{array}{c}\Delta \mathrm{H} / 2 \\
\left(\mathrm{H}_{0}-\mathrm{H}\right) / 2\end{array}$ & $D / 2$ & $\begin{array}{c}\Delta \mathrm{D} / 2 \\
\left(\mathrm{D}-\mathrm{D}_{0}\right) / 2 \\
\end{array}$ & $\Delta \mathrm{H} / \mathrm{H}$ & $\Delta \mathrm{D} / \mathrm{D}$ \\
\hline 5 & 0 & 7.5 & 0 & $0.00 \%$ & $0.00 \%$ \\
\hline 4.709 & 0.291 & 7.431 & 0.069 & $5.82 \%$ & $0.92 \%$ \\
\hline 4.429 & 0.571 & 7.343 & 0.157 & $11.42 \%$ & $2.09 \%$ \\
\hline 4.16 & 0.84 & 7.216 & 0.284 & $16.80 \%$ & $3.79 \%$ \\
\hline 3.902 & 1.098 & 7.042 & 0.458 & $21.96 \%$ & $6.11 \%$ \\
\hline 3.657 & 1.343 & 6.828 & 0.672 & $26.86 \%$ & $8.96 \%$ \\
\hline 3.422 & 1.578 & 6.555 & 0.945 & $31.56 \%$ & $12.60 \%$ \\
\hline 3.199 & 1.801 & 6.263 & 1.237 & $36.02 \%$ & $16.49 \%$ \\
\hline 2.987 & 2.013 & 5.954 & 1.546 & $40.26 \%$ & $20.61 \%$ \\
\hline 2.786 & 2.214 & 5.618 & 1.882 & $44.28 \%$ & $25.09 \%$ \\
\hline 2.597 & 2.403 & 5.242 & 2.258 & $48.06 \%$ & $30.11 \%$ \\
\hline 2.419 & 2.581 & 4.809 & 2.691 & $51.62 \%$ & $35.88 \%$ \\
\hline 2.251 & 2.749 & 4.317 & 3.183 & $54.98 \%$ & $42.44 \%$ \\
\hline 2.091 & 2.909 & 3.725 & 3.775 & $58.18 \%$ & $50.33 \%$ \\
\hline
\end{tabular}

Table 5-16. Ring test FEM results $\left(\mu / \tau_{\max }=0.22 / 97\right)$

\begin{tabular}{|c|c|c|c|c|c|}
\hline \multicolumn{6}{|c|}{$\begin{array}{l}\text { FEM Simulation } \# 12\left(\mu=0.23, \tau_{\max }=97 \mathrm{MPa}\right) \\
\qquad\left(\mathrm{H}_{0}=10, \mathrm{D}_{0}=15\right) \quad \text { Unit: } \mathrm{mm}\end{array}$} \\
\hline $\mathrm{H} / 2$ & $\begin{array}{c}\Delta \mathrm{H} / 2 \\
\left(\mathrm{H}_{0}-\mathrm{H}\right) / 2\end{array}$ & $\mathrm{D} / 2$ & $\begin{array}{c}\Delta \mathrm{D} / 2 \\
\left(\mathrm{D}-\mathrm{D}_{0}\right) / 2\end{array}$ & $\Delta \mathrm{H} / \mathrm{H}$ & $\Delta \mathrm{D} / \mathrm{D}$ \\
\hline 5 & 0 & 7.5 & 0 & $0.00 \%$ & $0.00 \%$ \\
\hline 4.709 & 0.291 & 7.422 & 0.078 & $5.82 \%$ & $1.04 \%$ \\
\hline 4.429 & 0.571 & 7.324 & 0.176 & $11.42 \%$ & $2.35 \%$ \\
\hline 4.16 & 0.84 & 7.184 & 0.316 & $16.80 \%$ & $4.21 \%$ \\
\hline 3.903 & 1.097 & 6.998 & 0.502 & $21.94 \%$ & $6.69 \%$ \\
\hline 3.657 & 1.343 & 6.767 & 0.733 & $26.86 \%$ & $9.77 \%$ \\
\hline 3.422 & 1.578 & 6.484 & 1.016 & $31.56 \%$ & $13.55 \%$ \\
\hline 3.199 & 1.801 & 6.183 & 1.317 & $36.02 \%$ & $17.56 \%$ \\
\hline 2.987 & 2.013 & 5.867 & 1.633 & $40.26 \%$ & $21.77 \%$ \\
\hline 2.787 & 2.213 & 5.521 & 1.979 & $44.26 \%$ & $26.39 \%$ \\
\hline 2.598 & 2.402 & 5.131 & 2.369 & $48.04 \%$ & $31.59 \%$ \\
\hline 2.419 & 2.581 & 4.687 & 2.813 & $51.62 \%$ & $37.51 \%$ \\
\hline 2.252 & 2.748 & 4.18 & 3.32 & $54.96 \%$ & $44.27 \%$ \\
\hline 2.091 & 2.909 & 3.57 & 3.93 & $58.18 \%$ & $52.40 \%$ \\
\hline
\end{tabular}

Table 5-17. Ring test FEM results $\left(\mu / \tau_{\max }=0.23 / 97\right)$ 
FEM Simulation $\# 11\left(\mu=0.22, \tau_{\max }=84 \mathrm{MPa}\right)$

\begin{tabular}{|c|c|c|c|c|c|}
\hline \multicolumn{7}{|c|}{$\left(\mathrm{H}_{0}=10, \mathrm{D}_{0}=15\right)$} & \multicolumn{2}{c|}{ Unit: $\mathrm{mm}$} \\
\hline $\mathrm{H} / 2$ & $\begin{array}{c}\Delta \mathrm{H} / 2 \\
\left(\mathrm{H}_{0}-\mathrm{H}\right) / 2\end{array}$ & $\mathrm{D} / 2$ & $\begin{array}{c}\Delta \mathrm{D} / 2 \\
\left(\mathrm{D}-\mathrm{D}_{0}\right) / 2\end{array}$ & $\Delta \mathrm{H} / \mathrm{H}$ & $\Delta \mathrm{D} / \mathrm{D}$ \\
\hline 5 & 0 & 7.5 & 0 & $0.00 \%$ & $0.00 \%$ \\
\hline 4.709 & 0.291 & 7.431 & 0.069 & $5.82 \%$ & $0.92 \%$ \\
\hline 4.429 & 0.571 & 7.343 & 0.157 & $11.42 \%$ & $2.09 \%$ \\
\hline 4.16 & 0.84 & 7.217 & 0.283 & $16.80 \%$ & $3.77 \%$ \\
\hline 3.902 & 1.098 & 7.047 & 0.453 & $21.96 \%$ & $6.04 \%$ \\
\hline 3.657 & 1.343 & 6.829 & 0.671 & $26.86 \%$ & $8.95 \%$ \\
\hline 3.422 & 1.578 & 6.56 & 0.94 & $31.56 \%$ & $12.53 \%$ \\
\hline 3.199 & 1.801 & 6.266 & 1.234 & $36.02 \%$ & $16.45 \%$ \\
\hline 2.987 & 2.013 & 5.958 & 1.542 & $40.26 \%$ & $20.56 \%$ \\
\hline 2.786 & 2.214 & 5.622 & 1.878 & $44.28 \%$ & $25.04 \%$ \\
\hline 2.597 & 2.403 & 5.251 & 2.249 & $48.06 \%$ & $29.99 \%$ \\
\hline 2.419 & 2.581 & 4.835 & 2.665 & $51.62 \%$ & $35.53 \%$ \\
\hline 2.25 & 2.75 & 4.364 & 3.136 & $55.00 \%$ & $41.81 \%$ \\
\hline 2.09 & 2.91 & 3.801 & 3.699 & $58.20 \%$ & $49.32 \%$ \\
\hline
\end{tabular}

Table 5-18. Ring test FEM results $\left(\mu / \tau_{\max }=0.22 / 84\right)$

\begin{tabular}{|c|c|c|c|c|c|}
\hline \multicolumn{6}{|c|}{$\begin{array}{l}\text { FEM Simulation } \# 12\left(\mu=0.23, \tau_{\max }=84 \mathrm{MPa}\right) \\
\qquad\left(\mathrm{H}_{0}=10, \mathrm{D}_{0}=15\right) \quad \text { Unit: } \mathrm{mm}\end{array}$} \\
\hline $\mathrm{H} / 2$ & $\begin{array}{c}\Delta \mathrm{H} / 2 \\
\left(\mathrm{H}_{0}-\mathrm{H}\right) / 2\end{array}$ & $\mathrm{D} / 2$ & $\begin{array}{c}\Delta \mathrm{D} / 2 \\
\left(\mathrm{D}-\mathrm{D}_{0}\right) / 2\end{array}$ & $\Delta \mathrm{H} / \mathrm{H}$ & $\Delta \mathrm{D} / \mathrm{D}$ \\
\hline 5 & 0 & 7.5 & 0 & $0.00 \%$ & $0.00 \%$ \\
\hline 4.709 & 0.291 & 7.422 & 0.078 & $5.82 \%$ & $1.04 \%$ \\
\hline 4.429 & 0.571 & 7.324 & 0.176 & $11.42 \%$ & $2.35 \%$ \\
\hline 4.16 & 0.84 & 7.186 & 0.314 & $16.80 \%$ & $4.19 \%$ \\
\hline 3.903 & 1.097 & 7.003 & 0.497 & $21.94 \%$ & $6.63 \%$ \\
\hline 3.657 & 1.343 & 6.776 & 0.724 & $26.86 \%$ & $9.65 \%$ \\
\hline 3.422 & 1.578 & 6.499 & 1.001 & $31.56 \%$ & $13.35 \%$ \\
\hline 3.199 & 1.801 & 6.199 & 1.301 & $36.02 \%$ & $17.35 \%$ \\
\hline 2.987 & 2.013 & 5.883 & 1.617 & $40.26 \%$ & $21.56 \%$ \\
\hline 2.786 & 2.214 & 5.54 & 1.96 & $44.28 \%$ & $26.13 \%$ \\
\hline 2.597 & 2.403 & 5.159 & 2.341 & $48.06 \%$ & $31.21 \%$ \\
\hline 2.418 & 2.582 & 4.741 & 2.759 & $51.64 \%$ & $36.79 \%$ \\
\hline 2.25 & 2.75 & 4.273 & 3.227 & $55.00 \%$ & $43.03 \%$ \\
\hline 2.09 & 2.91 & 3.699 & 3.801 & $58.20 \%$ & $50.68 \%$ \\
\hline
\end{tabular}

Table 5-19. Ring test FEM results $\left(\mu / \tau_{\max }=0.23 / 84\right)$ 


\section{Chapter 6}

\section{Two Dimensional Axisymmetric Coining Simulation}

The coining process is too complex and computationally expensive to study the variation and interaction of the numerous parameters. Instead, an understanding of the mechanics during a coining process is often obtained through the study of idealized parameters in simpler geometries. In this chapter, the effects of geometrical design parameters will be investigated by finite element simulation using the material properties obtained in Chapter 4, the friction model developed in Chapter 5 and the numerical parameters discussed in Chapter 3. The description of the finite element model for two dimensional simplified coining will be presented here and the effects of each parameter will be presented and discussed. The chapter will start by discussing the geometry for two dimensional axisymmetric coining.

\subsection{Geometry of Two Dimensional FEM Coining Model}

In this section, a two-dimensional axisymmetric coining process will be investigated. The basic geometrical parameters of the blank and die will be defined. The coinage production starts with a blank, which is a piece metal being prepared for coinage. The blank is cut from a strip of metal. To improve the formability and metal flow for the following coining process, a preliminary rimming process is conducted on the blank. In this operation, a raised edge is added on the circumference of the blank, shown in Figure 6-1. The thickness of the blank is called the Gauge and the outside diameter of rimmed blank is the Rimmed Diameter. The shape of raised edge after rimming step is called the Rimming Profile. The thickness of rimmed edge is also identified on the figure. The actual blank dimension was measured from the cross-section of a real rimmed blank supplied by Royal Canadian Mint and shown in Figure 6-2:

- Gauge: $2.486 \mathrm{~mm}$

- Rimmed Diameter: $35.64 \mathrm{~mm}$ 
- Rimmed Edge Thickness: $2.64 \mathrm{~mm}$

- Rimming Profile: by measuring the rimmed blank cross-section

A two dimensional idealized die is shown in Figure 6-3. On the die, the outside diameter of the field area (Inner diameter at the base of the rim) is called Flat-to-Flat Diameter. The curvature is the average dimension of the profile on the surface of the die. The edge depth on the die is the Rim Height. A severest $0.05 \mathrm{~mm}$ fillet radius was selected for the corner transition on the die. The die diameter is defined to be the diameter on the outside circumference of the die. Based on the past coining die design experience, the following typical dimension range of the parameters of the die will be investigated:

- Top Die

- Flat-to-Flat Diameter: $34.09 \mathrm{~mm}$

- Diameter: $35.57 \mathrm{~mm}$

- Fillet Radius: $0.05 \mathrm{~mm}$

- Curvature: $0.05 \mathrm{~mm}-0.3 \mathrm{~mm}$

- Rim Height: $0.18 \mathrm{~mm}-0.3 \mathrm{~mm}$

- Relief Height: $0.05 \mathrm{~mm}-0.08 \mathrm{~mm}$

- Relief Width: $17 \mathrm{~mm}$

- Bottom Die

- Flat-to-Flat Diameter: $33.91 \mathrm{~mm}$

- Diameter: $35.54 \mathrm{~mm}$

- Fillet Radius: $0.05 \mathrm{~mm}$

- Curvature: $0.05 \mathrm{~mm}-0.3 \mathrm{~mm}$

a Rim Height: $0.18 \mathrm{~mm}-0.3 \mathrm{~mm}$

- Relief Height: $0.05 \mathrm{~mm}-0.08 \mathrm{~mm}$

- Relief Width: $17 \mathrm{~mm}$ 


\subsection{Two-Dimensional Axisymmetric FEM Model}

The axisymmetric FEM model made use of elastic-plastic formulation and CAX4R elements, shown in Figure 6-4a, which are the four-node axisymmetric bilinear elements with reduced integration and hourglass control. The hourglass control associated with first-order elements was selected to avoid unwanted deformation modes. The reduced integration formulation was employed to avoid shear and volumetric "locking" which can occur with the full integration formulation, as discussed in Chapter 3. The integral viscoelastic hourglass control approach with 1/1/0 scale factors is applied. Adaptive meshing technology was used in the entire blank domain to improve the mesh quality during the coining process.

The imposed boundary conditions were those of symmetry on the axis at $r=0$. The top die, bottom die and collar were modeled by analytical rigid surfaces. The bottom die and collar was fixed with no movement. A rigid body reference node assigned for the rigid surface that defines the top die movement was constrained to have no rotation and no radial displacement and the axial displacement was prescribed to move down the axis. For the die-workpiece interface interactions, it is necessary to specify a friction condition. The non-traditional bi-linear two-parameter combined friction model obtained in Chapter 5 was applied in the FEM simulation. The kinematic contact algorithm and contact pairs between top die/blank, bottom die/blank and blank/collar were defined to enforce the contact interactions in the coining process. The friction coefficient, 0.22 , is specified and the maximum frictional shear stress is set to be 123.4 MPa, as discussed in Chapter 5. Furthermore, it was assumed that the annealing performed before the coining operation would totally remove all the effects of cold working from the previous preforming and rimming stages. Consequently, the metal blank recovers its original softness before the coining operation, and no residual strains or stresses are therefore to be taken into account.

The finite element simulation used the isotropic straining hardening property and other mechanical properties obtained in Chapter 4.

- Friction Coefficient: 0.22 
- Shear Stress: $123.4 \mathrm{MPa}$

- Density: $10492 \mathrm{~kg} / \mathrm{m}^{3}$

- Poisson's ratio: 0.364

- Young's modulus: $82.5 \mathrm{GPa}$

The finite element solver of ABAQUS V6.7 was used to perform the simulation. The results of the simulation are presented in the following section.

\subsection{Two-Dimensional FEM coining model validation}

In this section, the mesh refinement convergence will be discussed first. Then the numerical parameter validations of FEM and the contact pressure in the coining process will be addressed.

\subsubsection{Mesh Convergence}

As a common approach for a FEM simulation, it is necessary to examine the mesh convergence before a 2D FEM coining model is set up. Since the surface through the center is a plane of symmetry, the finite element model is axisymmetric, including a half of the blank, top die and bottom die. The die geometry of the FEM model for mesh converging test is as following.

- Top Die

- Flat-to-Flat Diameter: $34.09 \mathrm{~mm}$

- Diameter: $35.57 \mathrm{~mm}$

- Fillet Radius: $0.05 \mathrm{~mm}$

- Curvature: $0.3 \mathrm{~mm}$

- Rim Height: $0.27 \mathrm{~mm}$

- Relief Height: no

- Relief Width: no

- Bottom Die 
- Flat-to-Flat Diameter: $33.91 \mathrm{~mm}$

- Diameter: $35.54 \mathrm{~mm}$

- Fillet Radius: $0.05 \mathrm{~mm}$

- Curvature: $0.2 \mathrm{~mm}$

- Rim Height: $0.27 \mathrm{~mm}$

- Relief Height: no

- Relief Width: no

The silver blank geometry is as the same as discussed in section 6.1. Two different mesh density models were used, one coarse model contains about 2000 axisymmetric elements, shown in Figure 6-4a, and one refined model has about 8000 axisymmetric elements, shown in Figure 6-5a. In the rimmed edge section, the element size in the coarse mesh is about $0.05 \mathrm{~mm}$, the element size in the refined mesh is about $0.025 \mathrm{~mm}$. Other than the mesh density, all of the conditions were kept the same between two FEM models. Figure 6-4b and Figure 6-5b show the deformed mesh at the end of the simulation. From the deformed figures, both of the models can simulate the coining process well.

The Von Mises stress contours from the two models were presented in Figure 6-4c and Figure 6-5c. The two figures show the similar stress distributions, in which the high stress areas are located at the coin surface and rim edge sections with the same maximum stress at $247 \mathrm{MPa}$.

In addition, the results of load vs. displacement from the two models were plotted in Figure 6-6 and tabulated in Table 6-1. The loads at $100 \%$ axial stroke were compared. The $100 \%$ axial stroke was defined as the distance when the flash is about to form, more specifically, when $0.01 \mathrm{~mm}$ height flash enters the gap between die and collar. The coarse mesh predicted the load at $100 \%$ axial stroke to be $1716 \mathrm{kN}$ and the refined mesh gave $1689 \mathrm{kN}$. The difference between the models is only about $2 \%$.

Figure 6-4d and 6-5d show the local deformed mesh at the rim corner from the two models. It has been noted that the mesh doesn't fill up the corner perfectly although the refined mesh does present a better quality. However, the objective of this research is to obtain the overall 
loading and material flow behavior of silver coining process, not the local stress and strain magnitudes at the vicinities of the corners. Given that the severest situation for the fillet radius was chosen and the agreements between the two models in terms of load and stress distribution, the two models provide a reasonable converged approximation of the overall behavior of coining process.

Therefore, it is reasonable to say that the results of the two models are converged. The refined mesh will be used for the following coining FEM simulation.

\subsubsection{Validations of Numerical Parameters}

As have been discussed in Chapter 3, coining process can involve more complicated geometry and contact situation than the ring compression simulation, which will require more care to decide which parameter is suitable for the coining simulation. Therefore, to establish a more exhaustive test of the numerical parameters, the effects of the three dominant factors discussed in Chapter 3, namely Hourglass controls, Contact simulation and Adaptive meshing, which are available in ABAQUS, will be further investigated using the two dimensional axisymmetric coining models.

- Three different hourglass control approaches were studied first, namely Kelvin viscoelastic approach, Integral viscoelastic approach and Enhanced approach. The results in Load vs. Displacement were plotted in Figure 6-7.

- Four different combinations of hourglass scaling factors $(1 / 1 / 0,2 / 1 / 0,3 / 1 / 0,4 / 1 / 0)$ were investigated, and the Load vs. Displacement results were presented in Figure 6-8.

- Two contact simulation methods, Kinematic and Penalty, were tested and shown in Figure 6-9

- Two adaptive meshing control model, $200 / 1$ and $10 / 1$, were investigated and shown in Figure 6-10.

For the hourglass control approach in Figure 6-7, the model with an enhanced approach 
predicts a little higher load, the model with a Kelvin viscoelastic approach gives a relatively lower load and the integral viscoelastic model is in the middle. But in general, the three hourglass control approaches show very close loading path, which indicates all of the three models can perform satisfactorily in simulating the coining process. The default hourglass control approach in ABAQUS/Explicit, the integral viscoelastic approach, will be applied in the coining process simulation.

Different hourglass scaling factor combinations didn't show any significant difference in Figure 6-8, the results are almost identical for the displacement from 0 to $3.2 \mathrm{~mm}$, even though some minor differences start to show in the final stage. Therefore this range of combinations is considered not sensitive to the coining simulation and the default combination, therefore $1 / 1 / 0$, will be used in the coining process simulation.

For contact simulation and adaptive meshing controls, both of the parameters were shown to be not very sensitive in the $2 \mathrm{D}$ coining simulations. Different contact control models give very similar loading paths and show small differences at the end of the simulation. But it is worthy to be noted that the simulation time of the model with the penalty contact control almost doubles the time needed for the kinematic contact control. And the simulation with adaptive mesh 200/1 needs around $40 \%$ more time than the simulation with 10/1 adaptive mesh. The time difference may be not quite significant in the $2 \mathrm{D}$ axisymmetric simulations, but it definitely make a lot differences in larger models, like 3D simulations. The kinematic contact control and adaptive meshing parameter combination of $10 / 1$ will be used in the $2 \mathrm{D}$ coining process simulations

Through the investigation, it has been validated that various hourglass and contact control combinations in the test range would not cause any significant differences in the results of $2 \mathrm{D}$ coining simulation other than the computing time. Adaptive meshing is a case by case parameter. In the following $2 \mathrm{D}$ axisymmetric coining simulations, the default hourglass control approach the integral viscoelastic approach with $1 / 1 / 0$ scaling factors, kinematic contact simulation and adaptive meshing 10/1 will be applied. And in the later 3D coining simulations, a case dependent adaptive meshing parameter will be used. 


\subsubsection{Contact Pressure Development in Coining Process}

The maximum shear stress $\tau_{\max }$ becomes important when high contact pressures are generated since a Coulomb friction model will overestimate the friction shear stresses. The contact pressure generated in coining process may not be in the similar range of the pressure in ring test, which is used to obtain the friction model for the coining simulation. Therefore, it is worthy to take a look at the pressure development during a typical coining process and compare it to the ring test.

The nominal contact pressures were calculated for the coining process with the same die and blank geometry as in section 6.3.1. The results are plotted in Figure 6-11, which indicates the maximum contact pressure at $100 \%$ axial stroke is about $1.7 \mathrm{GPa}$. Comparing with Figure 5-18, the maximum contact pressure in the ring compression test is about $0.7 \mathrm{GPa}$. The results indicate that the contact pressure in the ring test is in the similar range of the coining process. In fact, up to $0.32 \mathrm{~mm}$ displacement in Figure 6-11, which accounts for $85 \%$ of the whole coining process, the contact pressures in the coining process match very well with the ring test. Even though in the last $15 \%$ of the coining process, the contact pressure increases rapidly, it still can be considered to be of the same order as the ring test. Therefore it is reasonably expected that ring compression test can be used to evaluate the friction conditions in the silver coining process and that the non-classical two-parameter friction model obtained from the ring test can be applied to the silver coining FEM simulation.

\subsection{Parametric Study of Coining Process}

In this section, four key die parameter variations will be investigated using the finite element method. The effect of each parameter will be investigated by varying it as shown below while keeping the other three parameters constant, which will take the default values.

Top Die Curvature (mm): 0.05, 0.1, 0.15, 0.2, 0.25, 0.3 (default)

Bottom Die Curvature (mm): 0.05, 0.1, 0.15, 0.2 (default), 0.25, 0.3

Rim Height (mm): 0.18, 0.2, 0.22, 0.24, 0.26, 0.271 (default), 0.28, 0.3 
Relief Height (mm): 0.08, 0.09, 0.1 (default)

Relief Width (mm): 17 (fixed)

\subsubsection{Top Die Curvature Variations}

In this section, the effects of varying the top die curvature were evaluated. The relief is not included in the model. The metal deformed mesh patterns of a typical model with $0.3 \mathrm{~mm}$ top die curvature at $50 \%, 75 \%, 90 \%$ and $100 \%$ of top die axial stroke were presented in the Figure 6-12. The material flow velocity patterns at the above moments were plotted in Figure 6-13, in which the length of arrows shows the velocity magnitude. The Von Mises stress and equivalent plastic strain contours were plotted in Figure 6-14 and Figure 6-15 respectively. For the brevity and clearness, the plots for the remaining study of the top die curvature variations can be found in Figure A-1 through A-10 in the Appendix.

\subsubsection{Bottom Die Curvature Variations}

In this section, the effects of bottom die curvature variations were evaluated. The metal deformed mesh patterns of a typical model with $0.3 \mathrm{~mm}$ bottom die curvature at $50 \%, 75 \%, 90 \%$ and $100 \%$ of top die axial stroke were presented in the Figure 6-16. The material flow patterns at the above moments were plotted in the Figure 6-17. The Von Mises stress and equivalent plastic strain contours were plotted in Figure 6-18 and Figure 6-19 respectively. The plots of the remaining study of the bottom die curvature variations can be found in Figure A-11 through A-20 in the Appendix.

From the observations of FEM simulation results, the top die curvatures and bottom die curvatures have the similar effects. When bottom die curvature is fixed, the top die with bigger curvature was observed to need larger displacements to establish the full contact along the curvature with the blank. This can be explained due to the facts that the bigger die curvature will provide more empty space for the blank to fill up. Once the full contact along the curvature is established, the blank will start to form the rimmed edge following the similar path. From the 
mesh deforming patterns in Figure 6-12 and 6-16, it can be seen that for the part of $75 \%$ of the process, the major deformation happens between the die curvature and blank. In the last $10 \%$ of the process, the material starts to fill the cavities at the rim corners. The material flow patterns in Figure 6-13 and 6-17 of the two models also show that the material flows radially outwards before $75 \%$ axial stroke. The major material flows at $90 \%$ axial stroke are in the rim edge section. At $100 \%$ axial stroke, the material flows only happen at the rim corner and in the flash. The stress and strain developments in the two models are also quite similar.

As the process progresses, the stresses gradually develop from the inner radius to the outer radius, shown in Figure 6-14 and 6-18. The stress perturbations arise when surface elements deform to make contact with the die surface, which lead to the change from the high stress level at the blank surface to the relatively low stress level at mid-thickness. At the end of the coining, the maximum stress located at the mid-curvature surface and the rim section, about $247 \mathrm{MPa}$, are higher than those in remaining parts of the coin. The strain developments in the two models are shown in Figure 6-15 and 6-19 and the figures show similar trends as the stress development. The strain gradient (from high to low) is from the curvature surface to the mid thickness and from the inner part to the outer part and becomes less when the coining process is approaching its end with the exception at the rim corner, where the maximum equivalent plastic strain is between 2.5 and 2.9 .

The curvature effects can be further understood by studying the loading paths with varying die curvatures, as shown in Figure 6-28a and Figure 6-29a based on the data presented in Table A-2 and Table A-3. Both figures show that the models with smaller die curvatures will have steeper loading paths. It is interesting to note that the models with larger die curvatures have higher final loads at $100 \%$ axial stroke although the difference is small (about $2 \%$ ), as shown in Figure 6-28b and Figure 6-29b, under which the die is fully filled with a maximum $0.01 \mathrm{~mm}$ flash. Due to the facts that the flash size of each model can vary from 0 to $0.01 \mathrm{~mm}$ and the load results from each model are very close, some discrepancy can be seen from the loads at $100 \%$ axial stroke. But the discrepancy will not affect the general trend observed above. Since 
the rim edge section is the last part of the die to be filled up and all the models with varying die curvatures have the same rim edge sections, it demonstrates that the final filling in the rim edge section of the die determines the final section of the loading path. That also explains why all the simulation paths have the parallel loading path at their final stages, as shown in Figure 6-28 and Figure 6-29.

To summarize the varying die curvatures effects, it is reasonable to say that: First, the die curvature will only determine the point where the full contact along the curvature between the die and blank can be established. Second, the model with bigger die curvature will have larger die displacement to fully filled die and higher load at $100 \%$ axial stroke. Third, with the same rim edge section, the smaller die curvature model will have a steeper loading path. Fourth, the different models with the same rim edge section will experience the similar load around the $100 \%$ axial stroke (between $1640 \mathrm{kN}$ and $1760 \mathrm{kN}$ ).

\subsubsection{Rim Height Variations}

In this section, the effects of rim height variations were evaluated. No relief was generated in the model. The metal deformed mesh patterns of a typical model with $0.3 \mathrm{~mm}$ rim height at $50 \%, 75 \%, 90 \%$ and $100 \%$ of top die axial stroke were presented in the Figure 6-20. The material flow patterns of the model at the above moments were plotted in the Figure 6-21. The Von Mises stress and equivalent plastic strain contours were plotted in Figure 6-22 and Figure 6-23 respectively. The plots of the remaining study of the rim height variations can be found in Figure A-21 through A-32 in the Appendix.

In these simulations, the models have the same die curvature and only differ in the rim edge section. From the point of view of deforming mesh and material flow, it is hard to observe any significant difference. The similar trend observed in the curvature study can also be seen here. The major outward radial material flowing happened until 75\% axial stroke. Rim edge section filling occurred at $90 \%$ axial stroke. At $100 \%$ axial stroke, the major material flows are at rim corner and in the flash, as seen in Figure 6-20 and 6-21. 
The Von Mises stress and equivalent plastic strain contours, shown in Figure 6-22 and 6-23, show a similar stress and strain distributions in curvature study. The stresses at the mid-curvature surface and the rim section are higher and the strain gradient (from high to low) is from the curvature surface to the mid thickness and from the inner part to the outer part except the rim edge section. The contours show a somewhat bigger band area which denotes high stress with a maximum of $247 \mathrm{MPa}$ and high strain with a maximum of 2.2 , at the $100 \%$ die axial stroke. This indicates the process has gone through severe deformation and may require higher loads due to the change of the rim section.

The loading behavior was further summarized in Figure 6-30a, also presented in Table A-4. The effects of rim height can be seen by comparing the loading paths of the different models. All the models follow almost identical loading path before the full contact along the curvature was established. The loading behavior shows that the rim height variations would not affect the deforming patterns until the full contact along the curvature between die and blank was established. The only differences among the models are caused by the filling process of rim edge sections with different rim heights. And in Figure 6-30b, it can be seen that bigger rim height leads to bigger displacement and $2 \%-5 \%$ higher load (between $1530 \mathrm{kN}$ and $1630 \mathrm{kN}$ ) at $100 \%$ axial stroke instant due to the more empty space in the die. In the Figure 6-30a, this effect was shown by the parallel paths around the $100 \%$ axial stroke. In other words, different rim height models will have the identical loading paths before they show variations during the rim filling phase (from $75 \%$ to $100 \%$ of the axial stroke), as shown in Figure 6-30a.

Therefore, the effects of rim height variation can be summarized as following: First, bigger rim height will result in larger displacement and higher finishing loads at $100 \%$ axial stroke. Second, different rim height will make no difference in the loading paths before full contact along the curvature between die and blank was established. Third, it verifies one conclusion given in the above section, that it is the rim edge section that determines the finishing load at $100 \%$ axial stroke. 


\subsubsection{Relief Height Variations}

In this section, the effects of relief height variations were evaluated. For the clarity of this thesis, the relief width is fixed at $17 \mathrm{~mm}$, as defined in section 6.1 . The metal deformed mesh patterns of a typical model with a relief height $0.1 \mathrm{~mm}$ at $50 \%, 75 \%, 90 \%$ and $100 \%$ of top die axial stroke were presented in the Figure 6-24. The material flow patterns of the model at the above moments were plotted in the Figure 6-25. The Von Mises stress and equivalent plastic strain contours were plotted in Figure 6-26 and Figure 6-27 respectively. The plots of the remaining study of the relief height variations can be found in Figure A-33 through A-36 in the Appendix.

To investigate the effects of relief heights, FEM models were defined to have the same curvature and rim edge section, but different relief heights. The value varies from $0.08 \mathrm{~mm}$ to $0.1 \mathrm{~mm}$ based on the experience of practical silver coin design. From the mesh deformation and material flow Figures 6-24 and 6-25, the most significant difference in this model comparing to the models in the above section is the existence of an axial plane near the relief edge, which causes the material at one side of the plane to flow inwards and the material at the other side of the plane to flow outwards until about $50-60 \%$ of axial stroke when the relief cavities are filled. From material flow patterns shown in Figure 6-25, at 75\% axial stroke the material already starts to the fill the rim edge sections, which is earlier than the models in curvature study and rim height study. This can be further verified by the material flow pattern at the $90 \%$ axial stroke, which shows that the rim edge section has been almost filled up. At $100 \%$ axial stroke, the major material flows are at rim corners and in the flash, which is similar to the curvature study and rim height study at $100 \%$ axial stroke.

The relief also causes a difference to the stress and strain developments. As shown in Figure 6-26 and 6-27, the stress and strain perturbations always arise from the relief corner and spread to the rest of the blank. In most of the process, up to $90 \%$ of axial stroke, the vicinity of the relief corner always has the largest stress and strain gradients. And at $100 \%$ axial stroke, the sections of relief and rim edge predict a maximum stress level at about $247 \mathrm{MPa}$ and a maximum 
strain level at about 3 . The relief and rim edge become equally critical in terms of stress and strain.

The loading paths with varying relief height were presented in Figure 6-31a and Table A-5. These results show there is no major loading pattern difference caused by varying the relief heights in the range defined in this chapter. All three models follow very similar loading paths before and after the full contact along the curvature is established, as shown in Figure 6-31a. Also from Figure 6-31b, it can be seen that the load at $100 \%$ axial stroke identified on each model falls in the similar range of $1480-1640 \mathrm{kN}$. The FEM simulations show that the relief section was always filled before rim edge section. From the analysis of curvature and rim height, it is observed that it is the rim section filling that determines the final load at $100 \%$ axial stroke and it is the curvature that determines the loading path shape. Given the same rim edge section and curvature of each model, it is reasonable to expect that all the models follow very similar loading paths and give similar loads at $100 \%$ axial stroke. Therefore, in general, at least in this small variation range $(0.02 \mathrm{~mm})$ of relief height, the relief height effects on load are not significant as compared to that of curvature and rim height. With the simple relief design in the model, it is still the curvature and rim edge section that define the loading shape and final load. However, the relief will significantly affect the stress and strain developments and distributions, which may affect the final coin shape and surface quality.

Through the investigation of curvature, rim height and relief in a two dimensional domain, we have obtained a clear understanding of the effects of these three key design parameters in an idealized two dimensional coining design. The average loads obtained at the $100 \%$ axial stroke in each case were compared to the actual coining load of about $1500 \mathrm{kN}$ [Royal Canadian Mint, 2007], the tonnage used in forming of silver dollar at the Royal Canadian Mint. The simulation and actual loads fall into the same range, which gives added confidence in the 2D axisymmetric coining simulation. To better understand the practical coining process, three dimensional coining simulation and investigation using the practical silver coin design will be conducted in the next Chapter. 


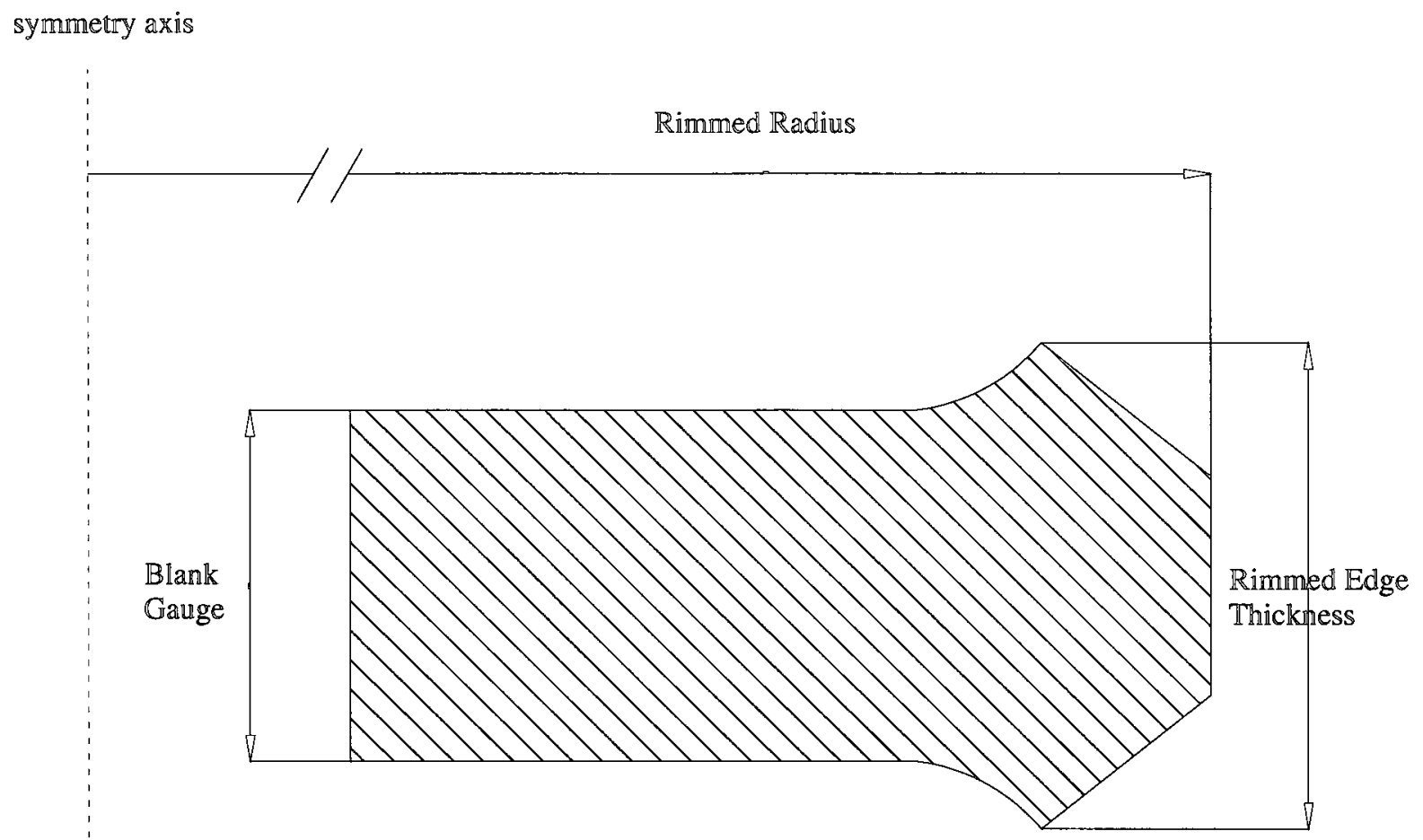

Figure 6-1. Blank Cross-section profile

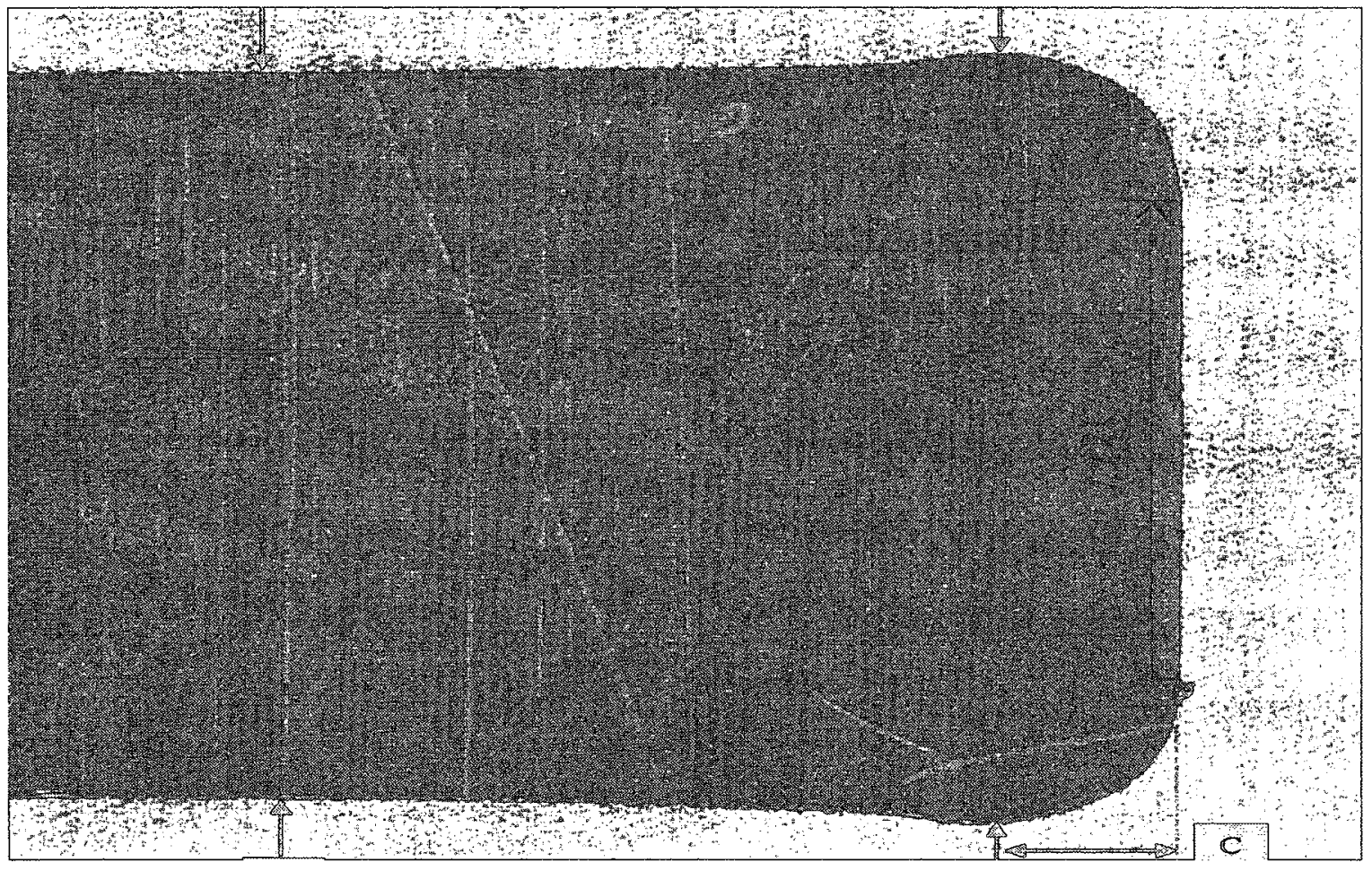

Figure 6-2. The cross-section of a rimmed blank 


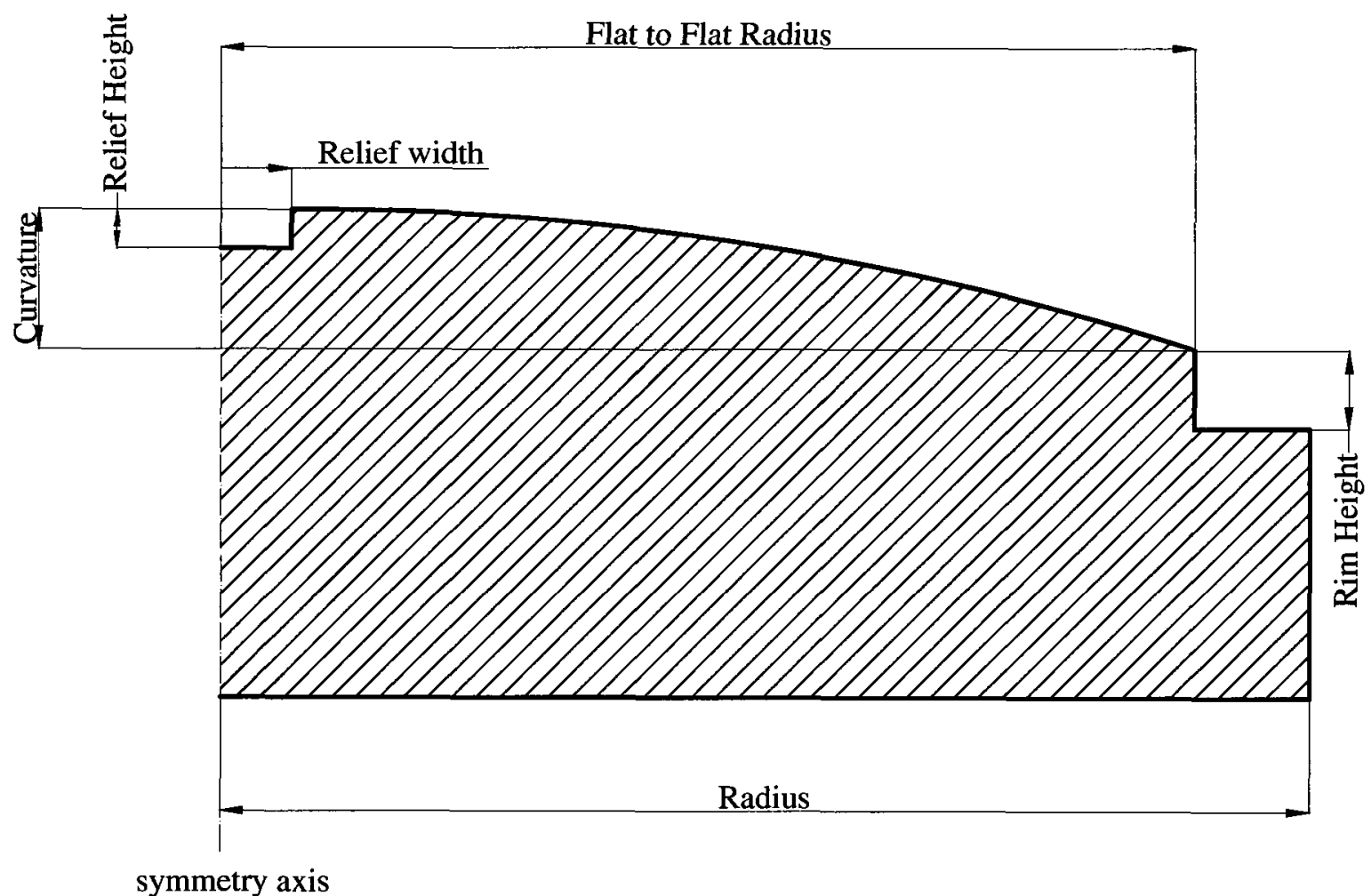

Figure 6-3. Bottom die geometry 


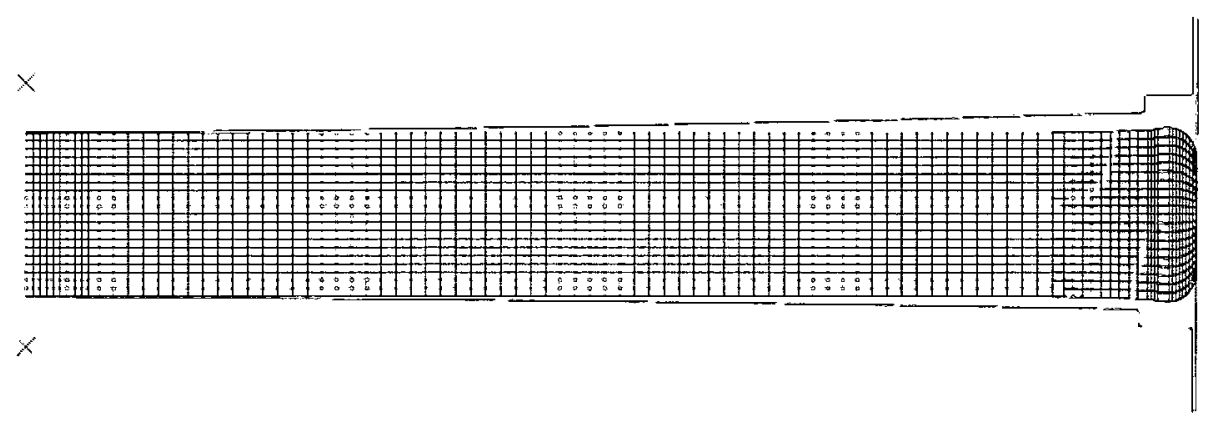

(a) Initial mesh

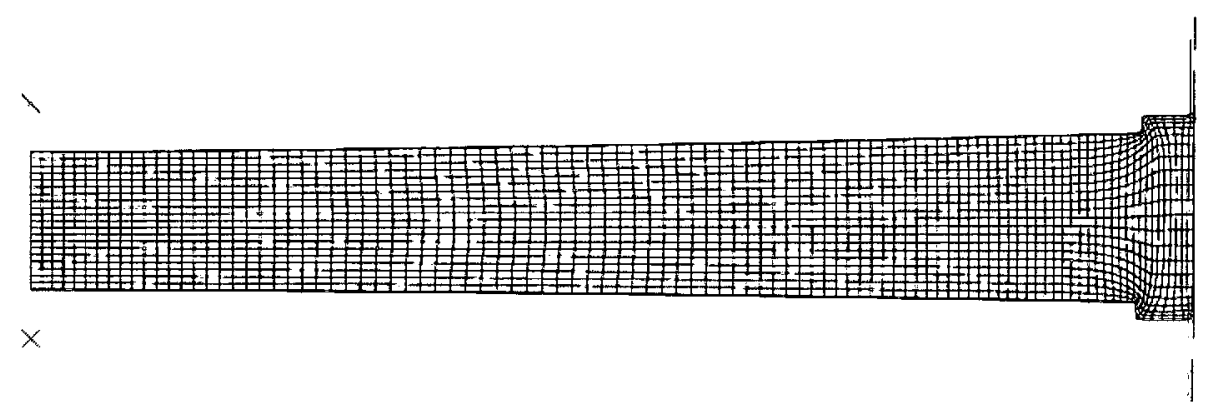

(b) Final mesh

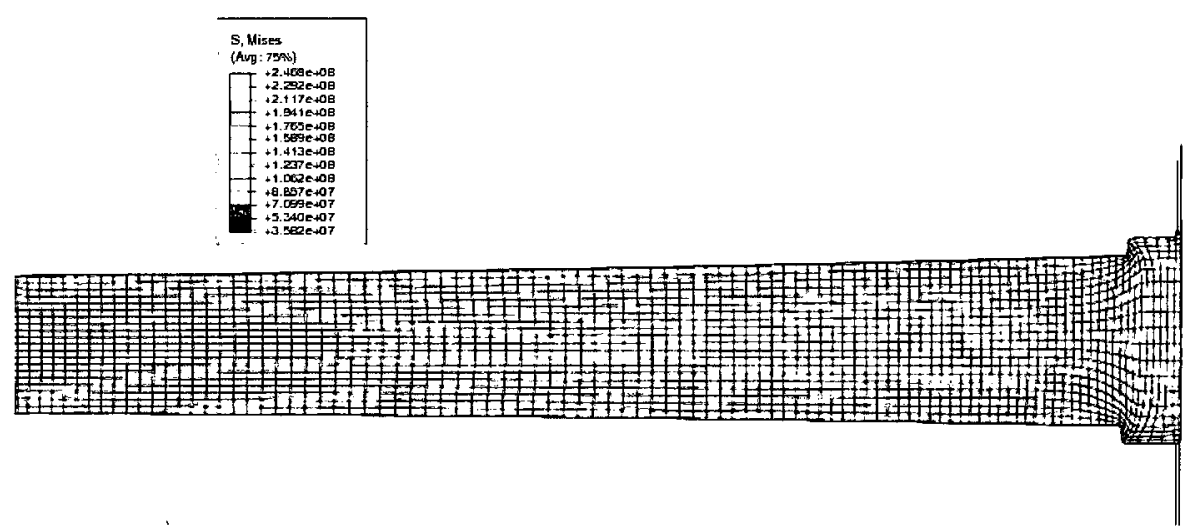

(c) Von Mises stress contour

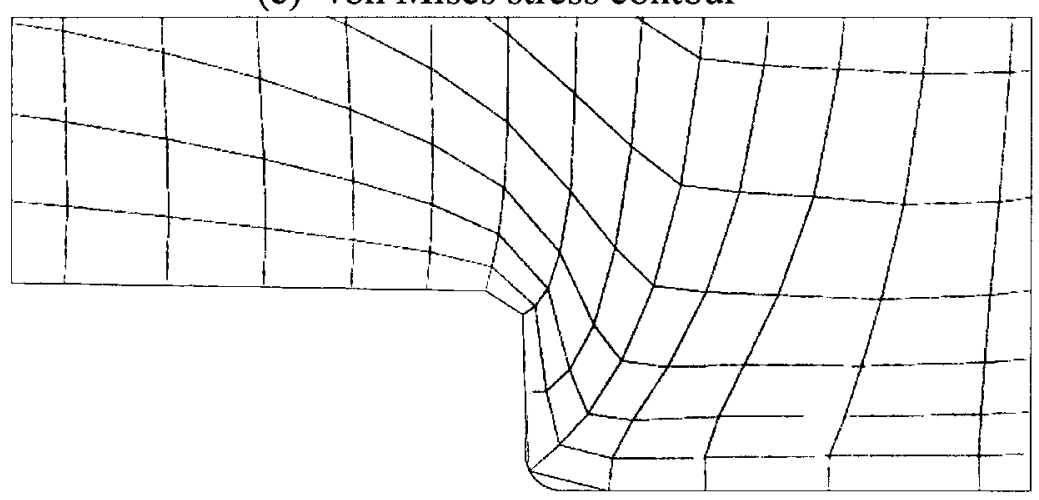

(d) Local mesh at rim corner

Figure 6-4. 2D axisymmetric coining FEM model in coarse mesh (2000 elements) 


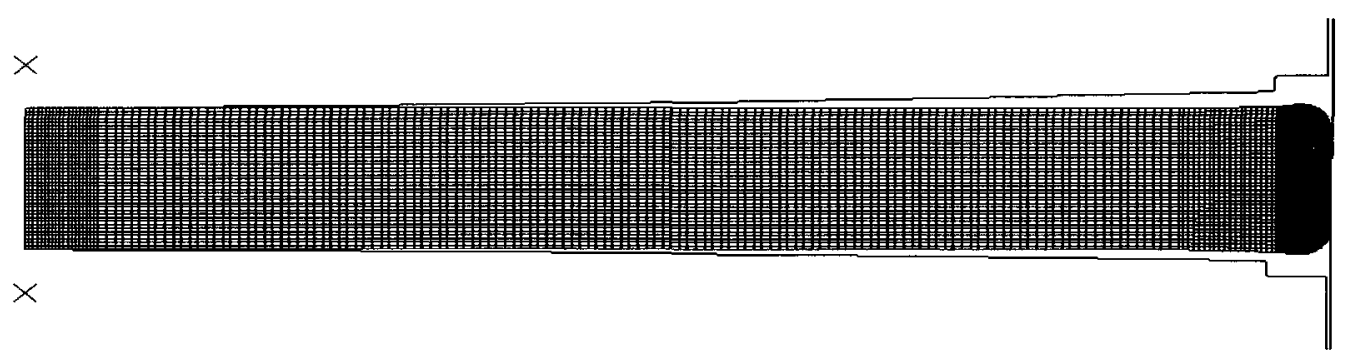

(a) Initial mesh

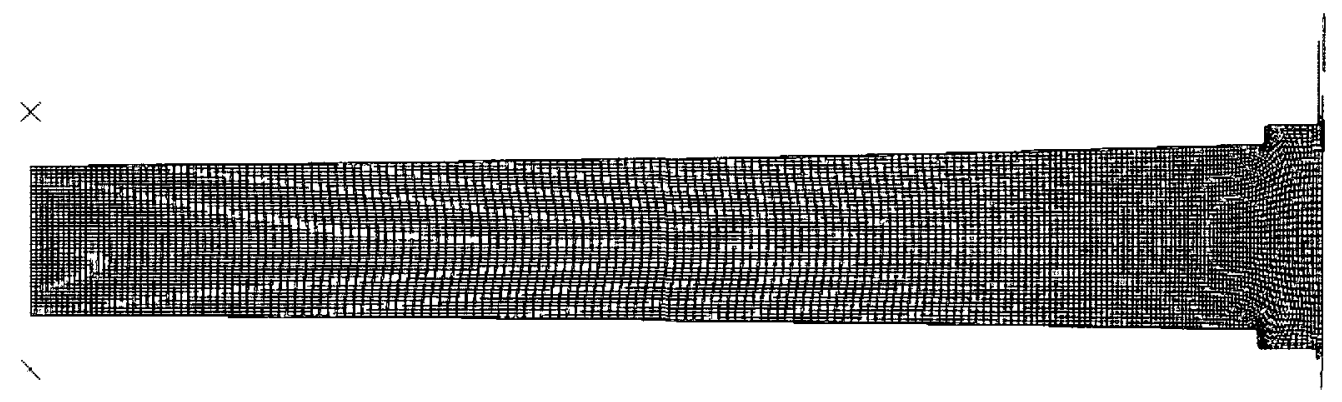

(b) Final mesh

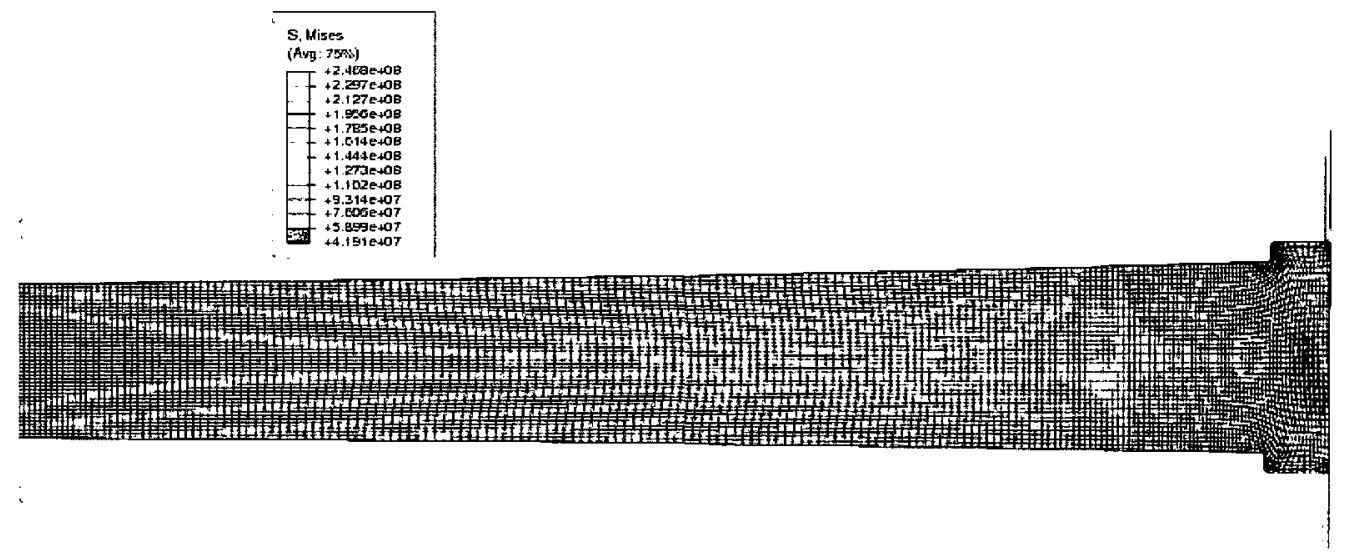

(c) Von Mises stress contour

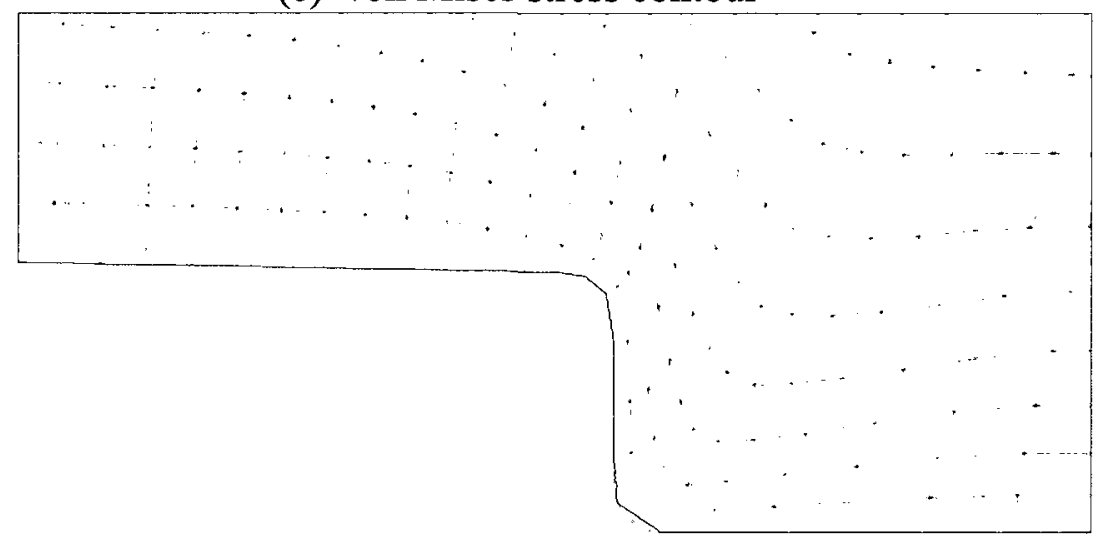

(d) Local mesh at rim corner

Figure 6-5. 2D axisymmetric coining FEM model in refined mesh (8000 elements) 


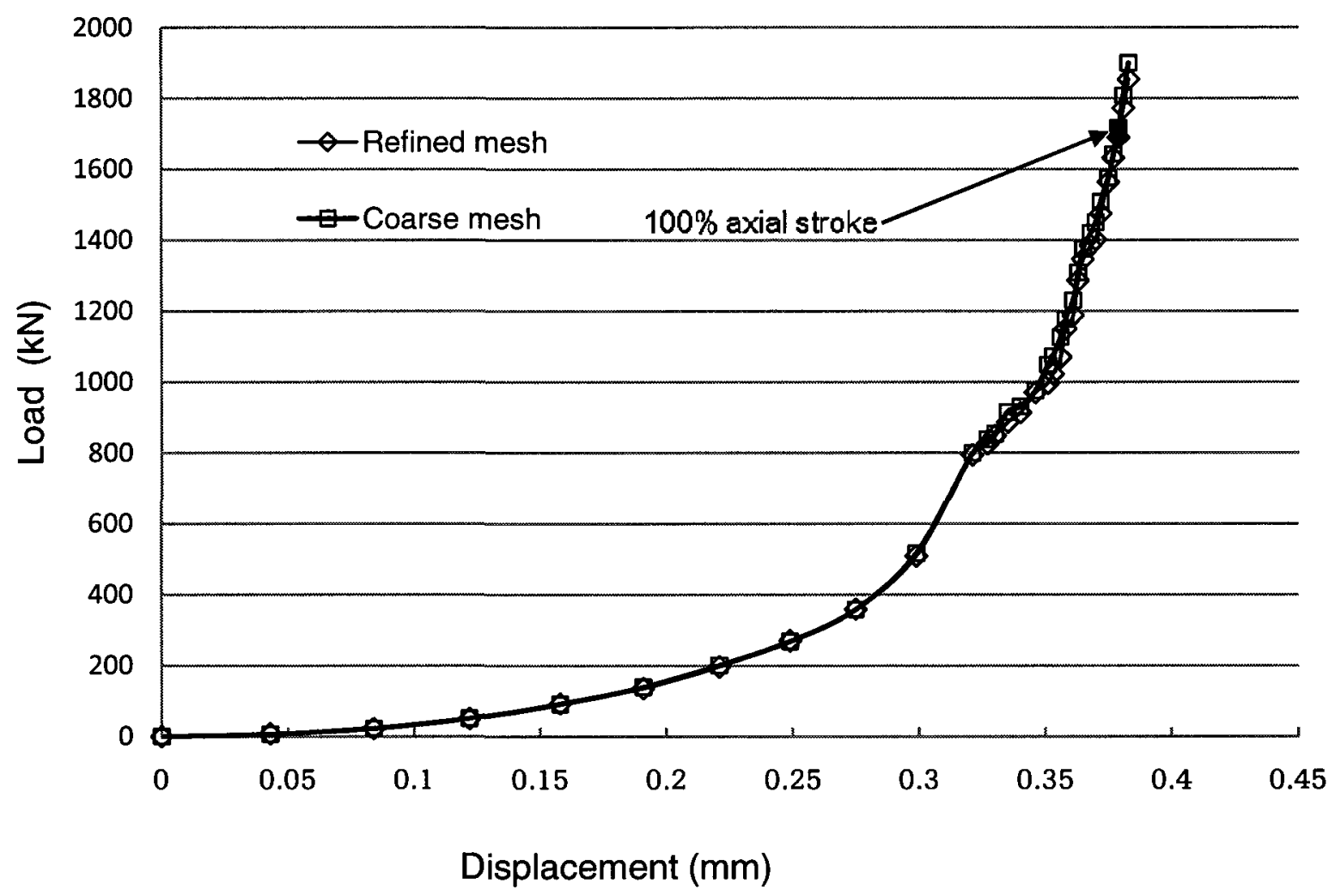

Figure 6-6. Load vs. displacement for mesh convergence test

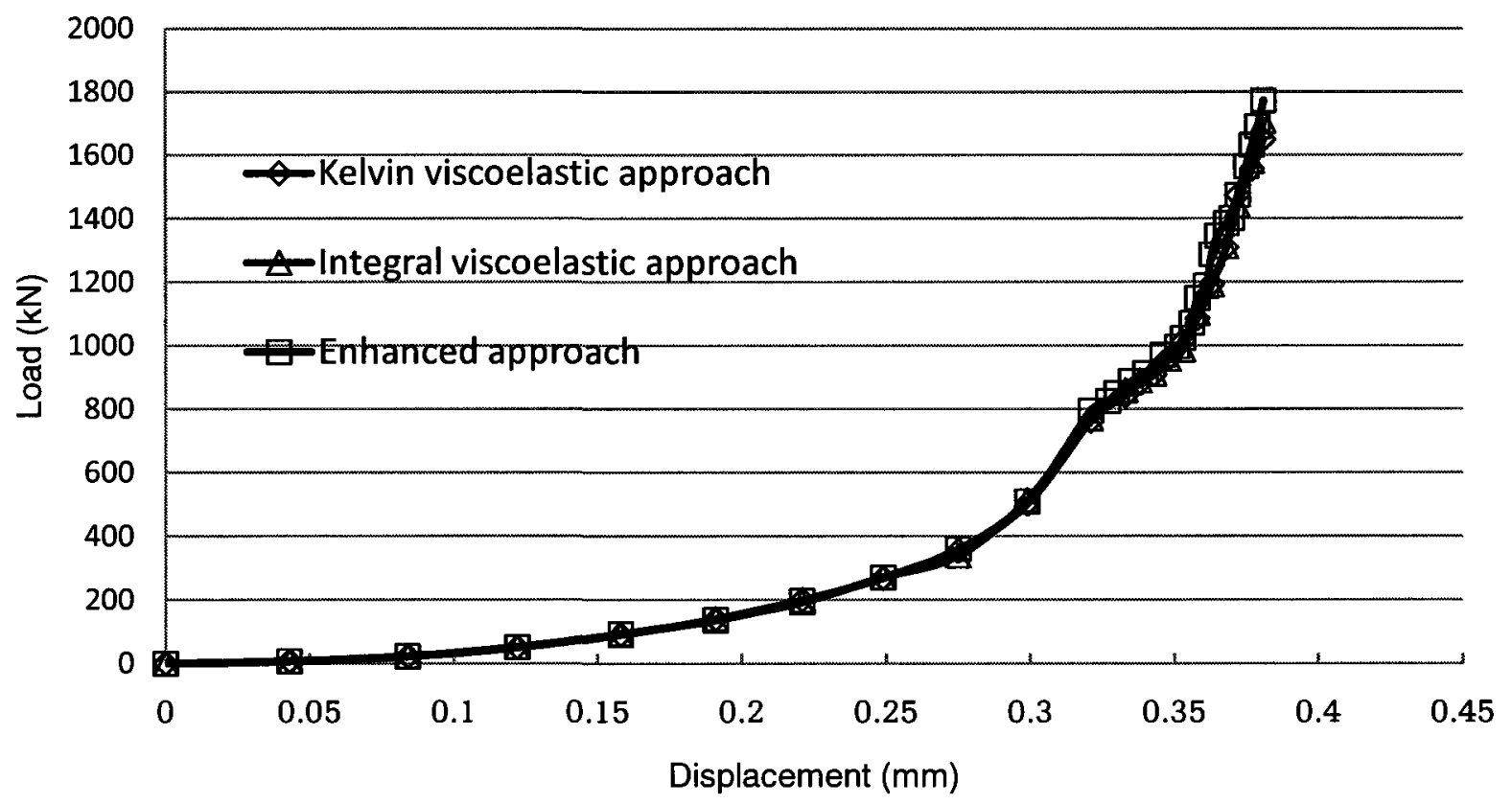

Figure 6-7. Coining FEM results in different hourglass control approaches 


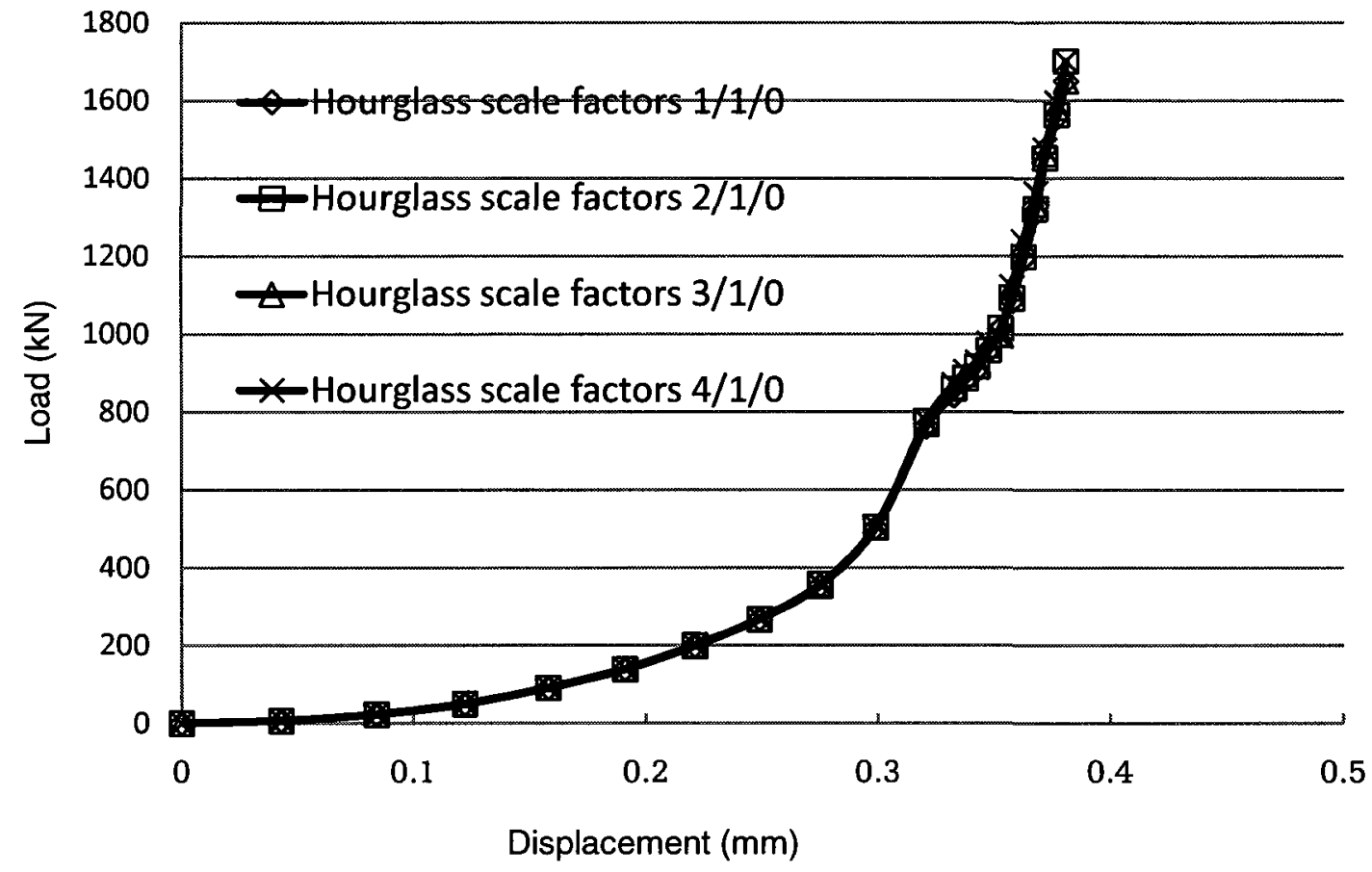

Figure 6-8. Coining FEM results in different hourglass scale factor combinations

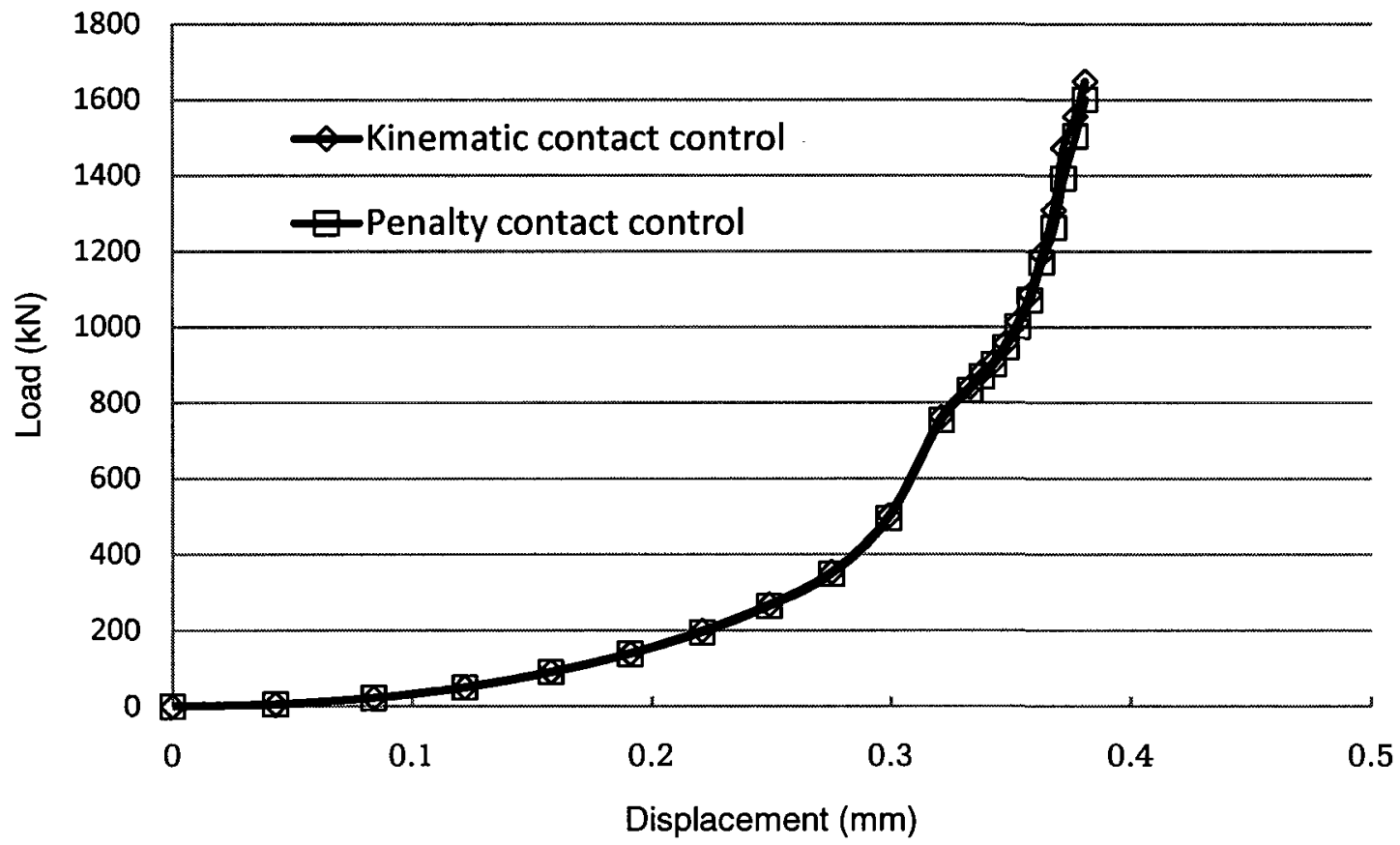

Figure 6-9. Coining FEM results in different contact controls 


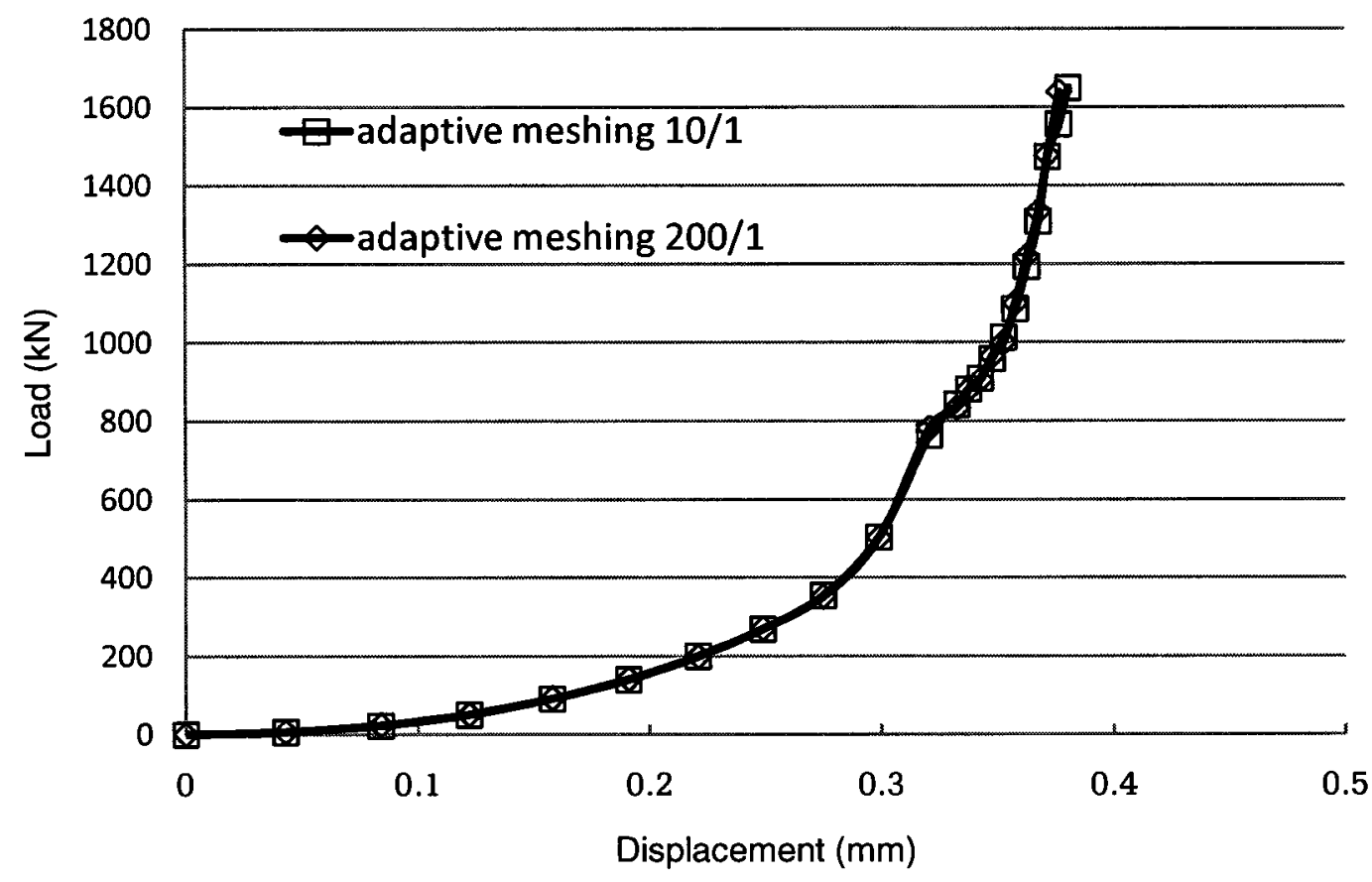

Figure 6-10. Coining FEM results in different adaptive meshing controls

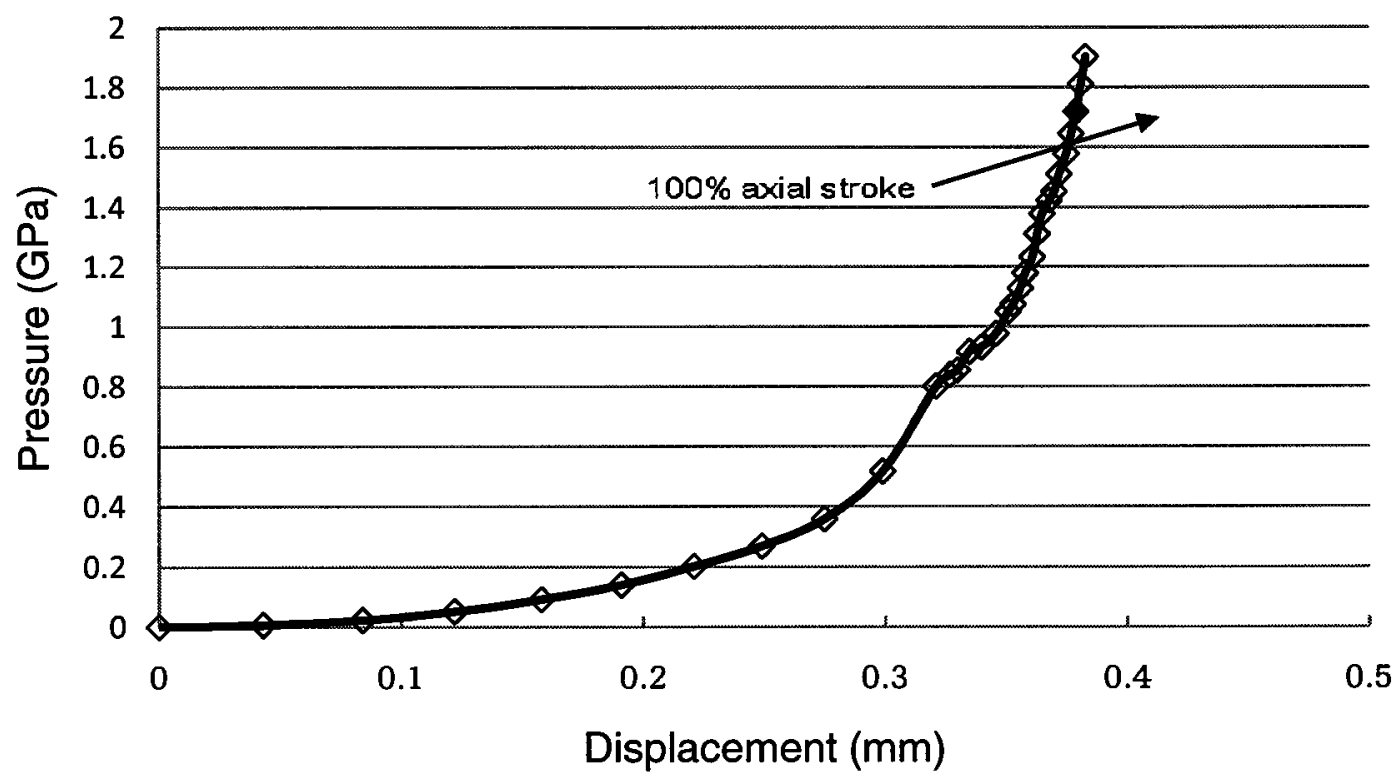

Figure 6-11. The nominal contact pressure development in coining 


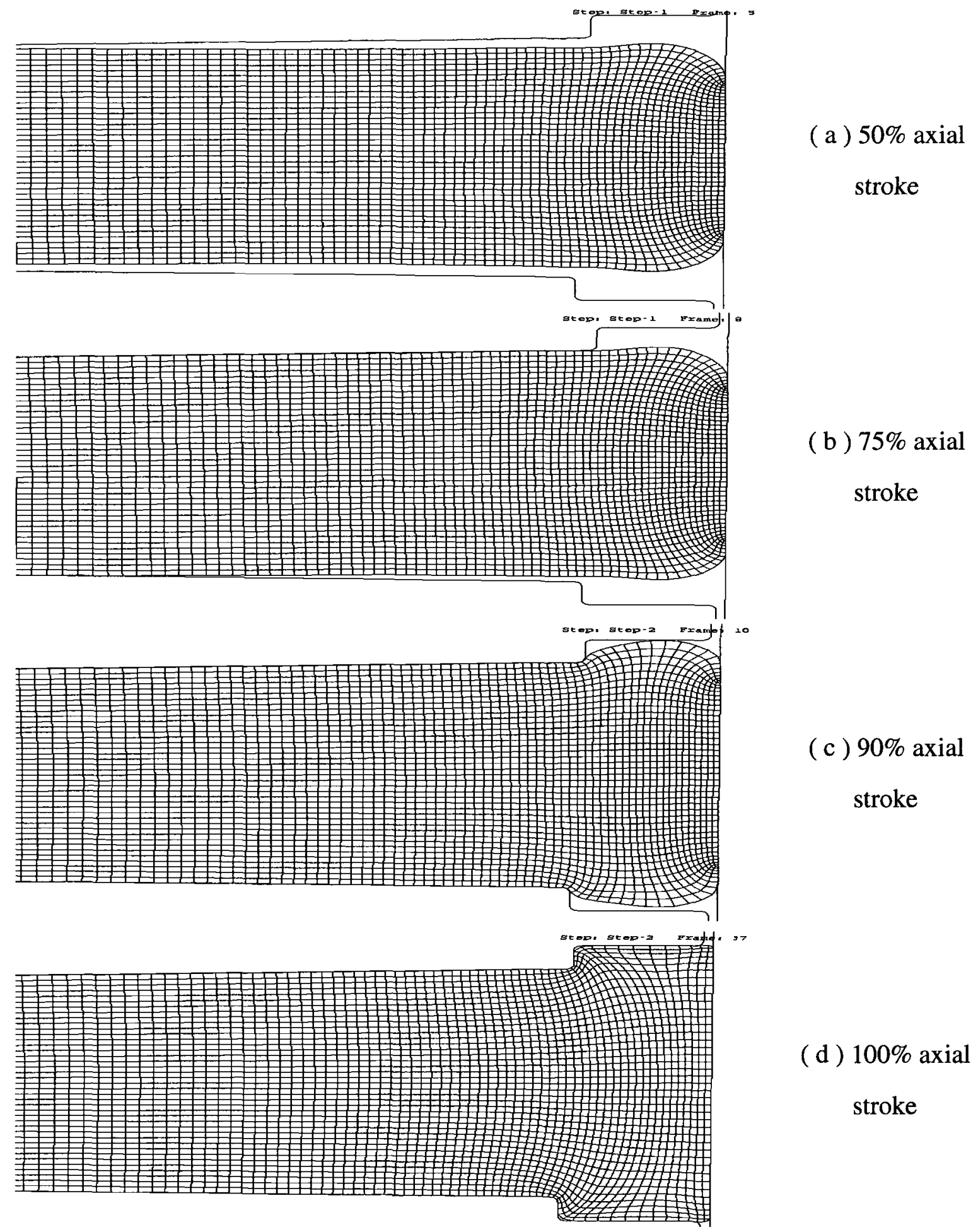

Top die curvature study

Figure 6-12. Metal deformed mesh patterns showing outer 20\% of blank

(Top die curvature $=0.3 \mathrm{~mm}$, Bottom die curvature $=0.19 \mathrm{~mm}$, Rim Height $=0.271 \mathrm{~mm}$, no relief) 

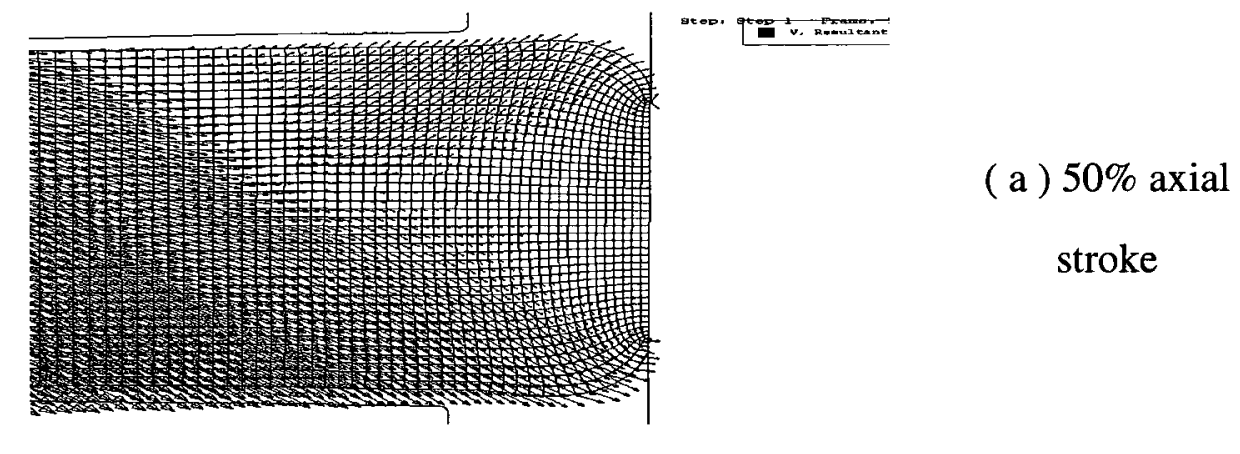

(a ) $50 \%$ axial stroke
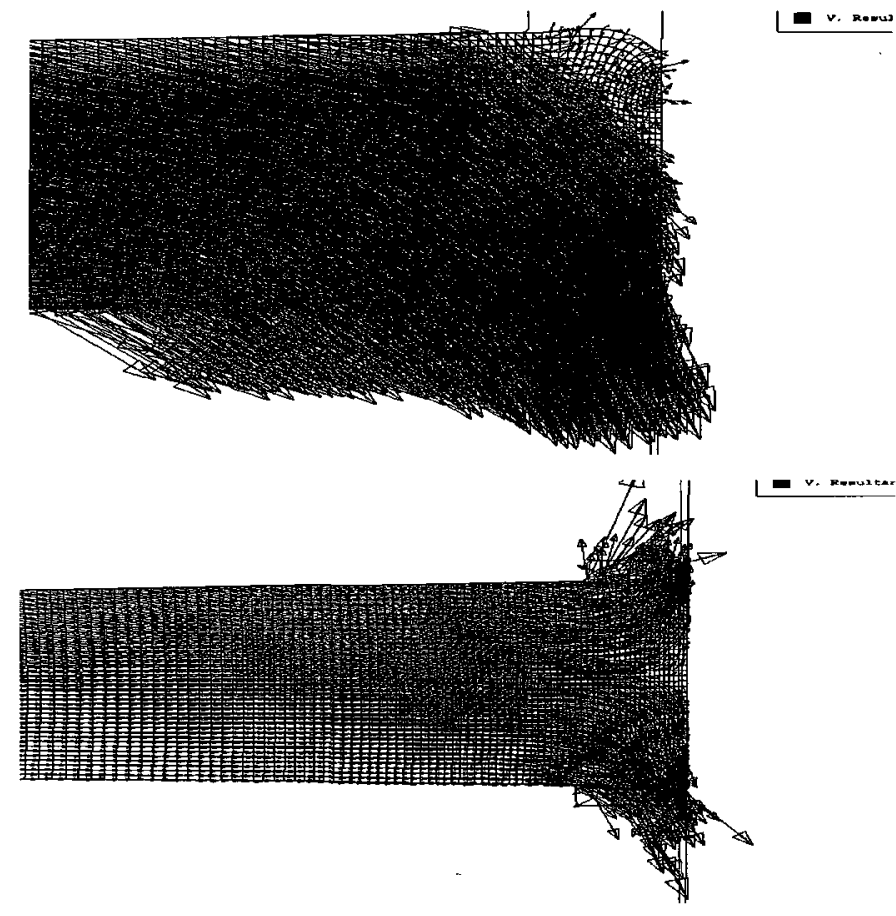

(b ) $75 \%$ axial stroke

(c ) $90 \%$ axial stroke

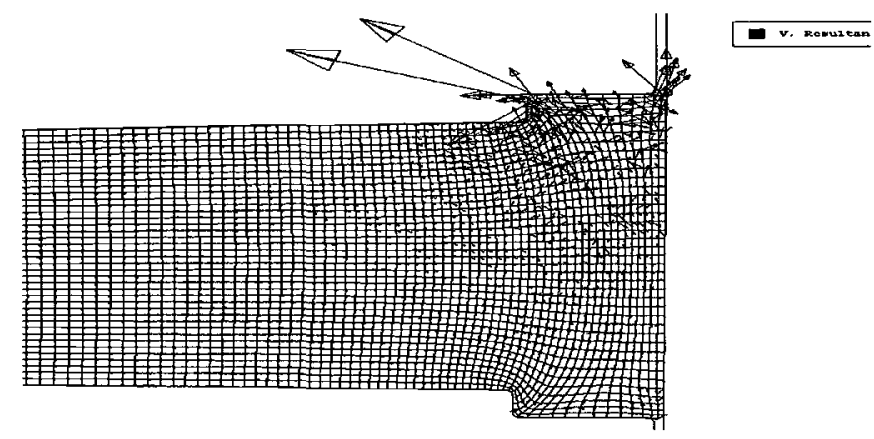

(d) $100 \%$ axial stroke

Top die curvature study

Figure 6-13. Material flow velocity patterns showing outer $20 \%$ of blank

(Top die curvature $=0.3 \mathrm{~mm}$, Bottom die curvature $=0.19 \mathrm{~mm}$, Rim Height $=0.271 \mathrm{~mm}$, no relief) 


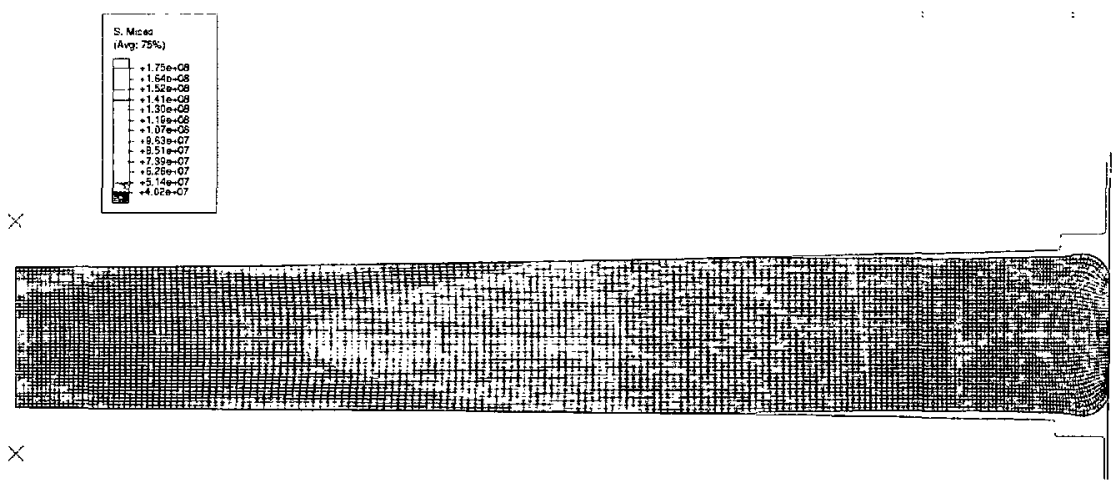

(a) $50 \%$

axial stroke

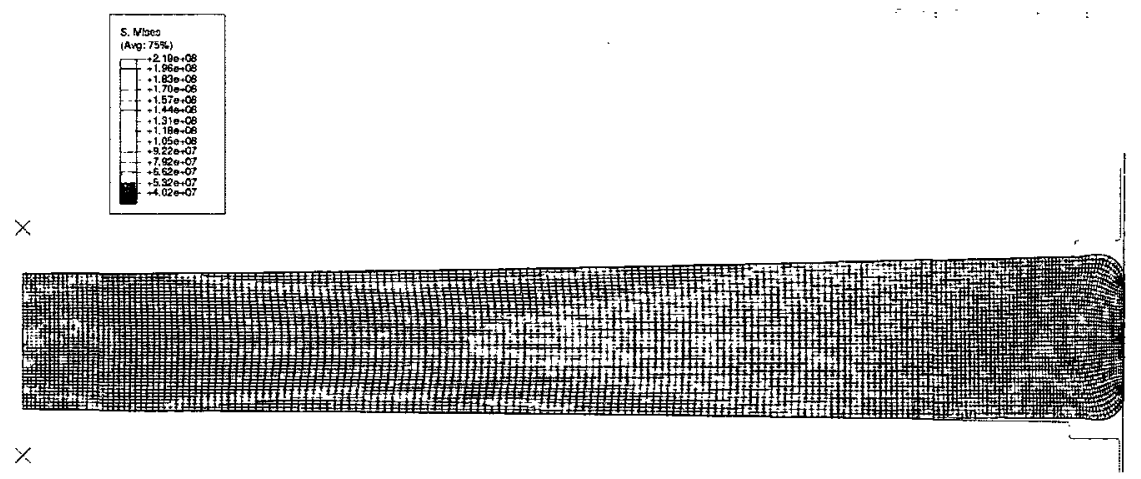

(b) $75 \%$

axial stroke
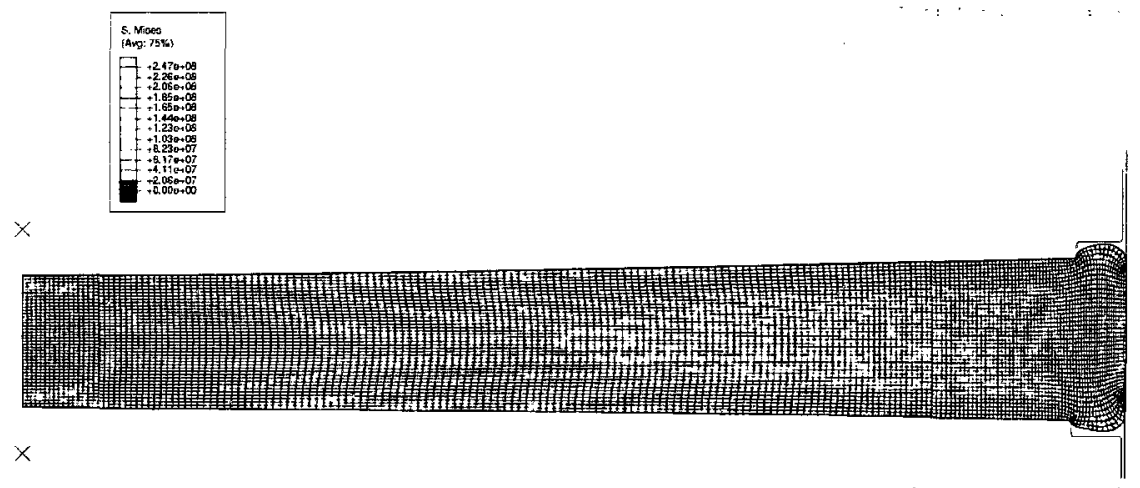

(c) $90 \%$

axial stroke

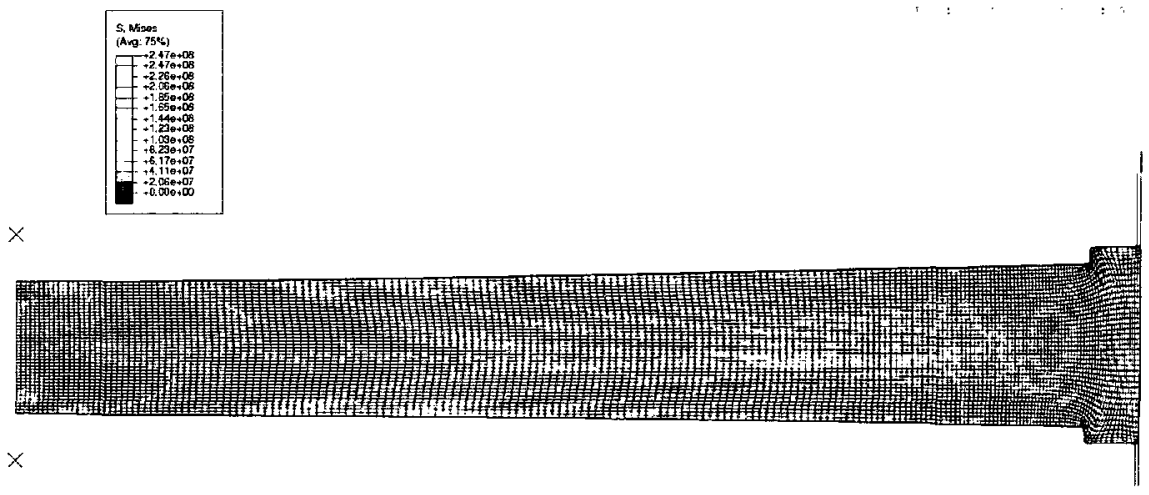

Top die curvature study

Figure 6-14. Von Mises stress contour

(Top die curvature $=0.3 \mathrm{~mm}$, Bottom die curvature $=0.19 \mathrm{~mm}$, Rim Height $=0.271 \mathrm{~mm}$, no relief) 


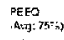
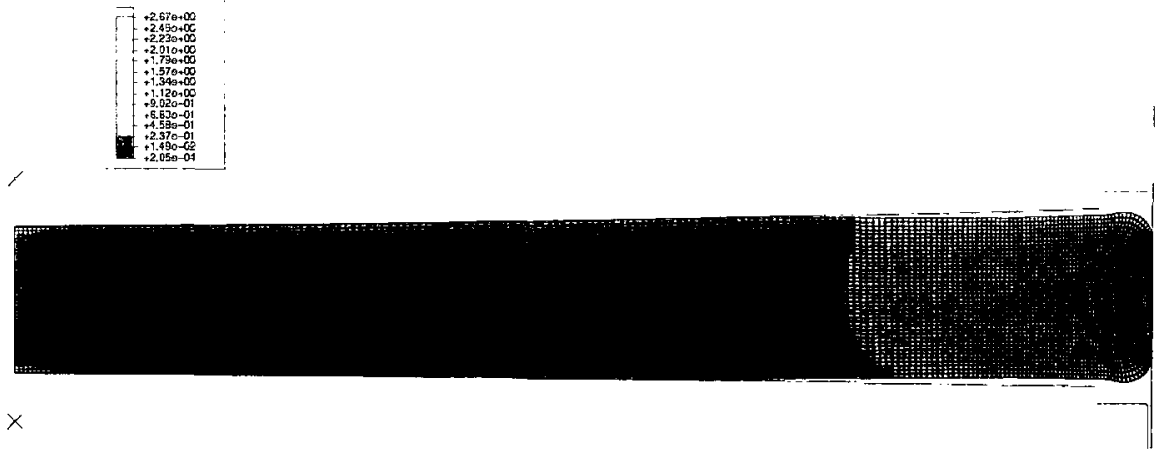

(a) $50 \%$

axial stroke

(b) $75 \%$

axial stroke
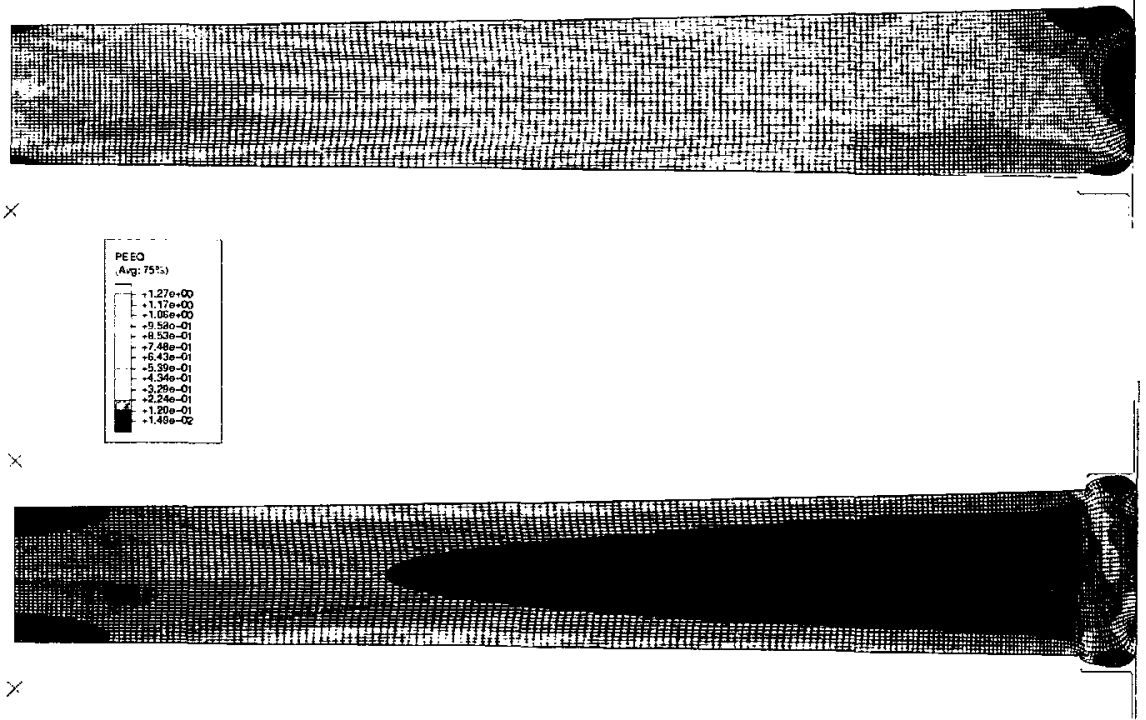

(c) $90 \%$

axial stroke

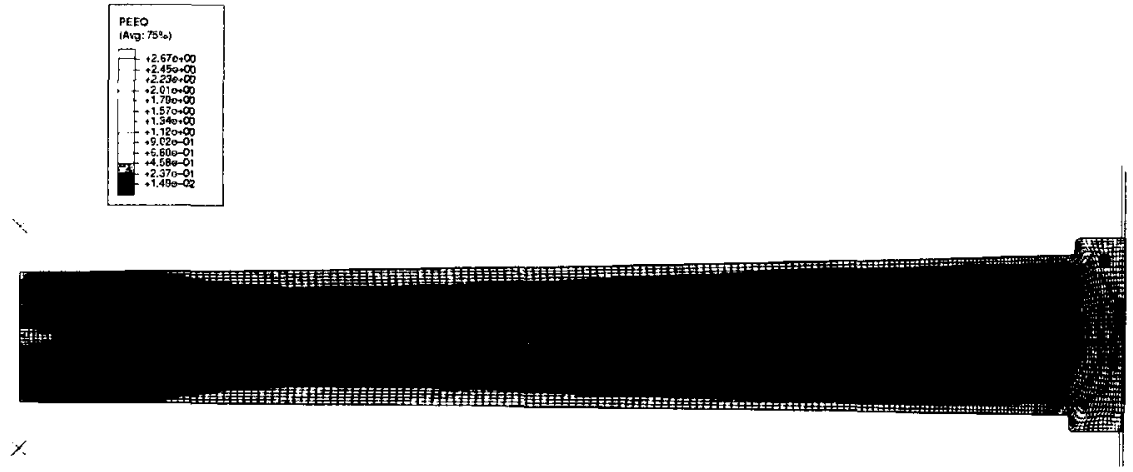

(d) $100 \%$

axial stroke

Top die curvature study

Figure 6-15. Equivalent plastic strain contour

(Top die curvature $=0.3 \mathrm{~mm}$, Bottom die curvature $=0.19 \mathrm{~mm}$, Rim Height $=0.271 \mathrm{~mm}$, no relief) 


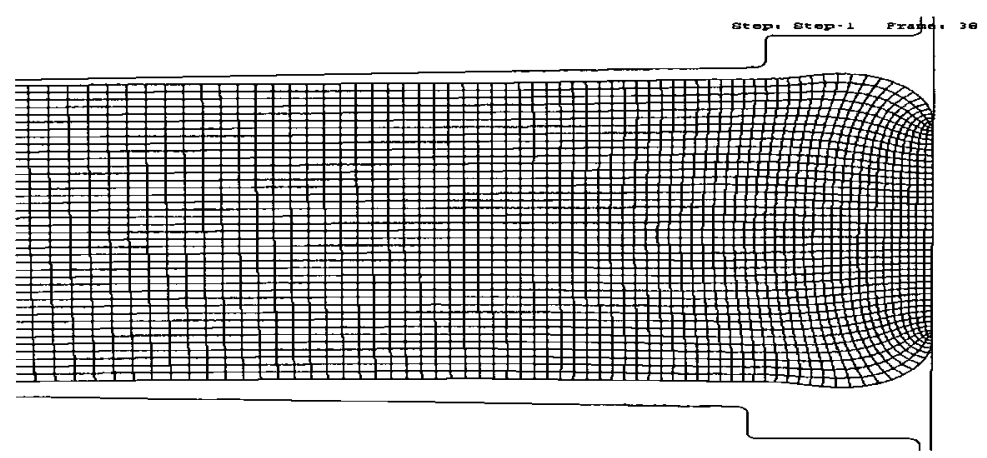

(a) $50 \%$ axial stroke

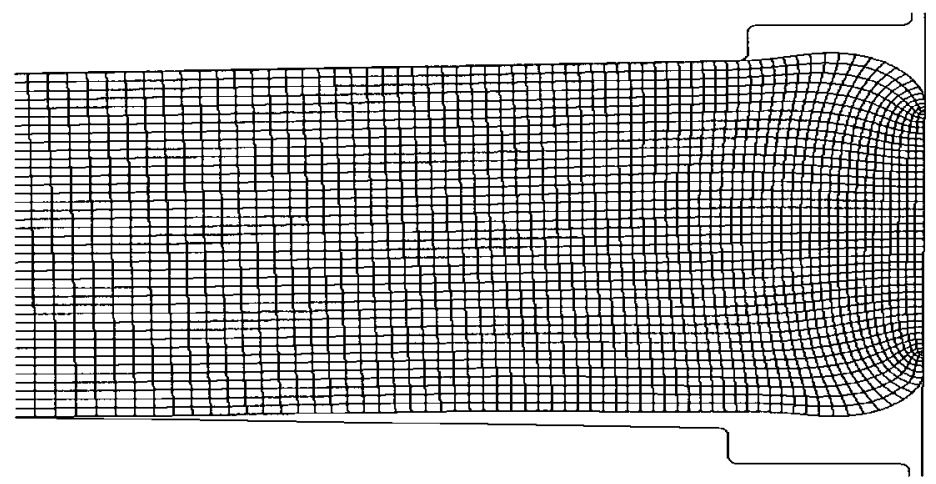

(b) $75 \%$ axial stroke

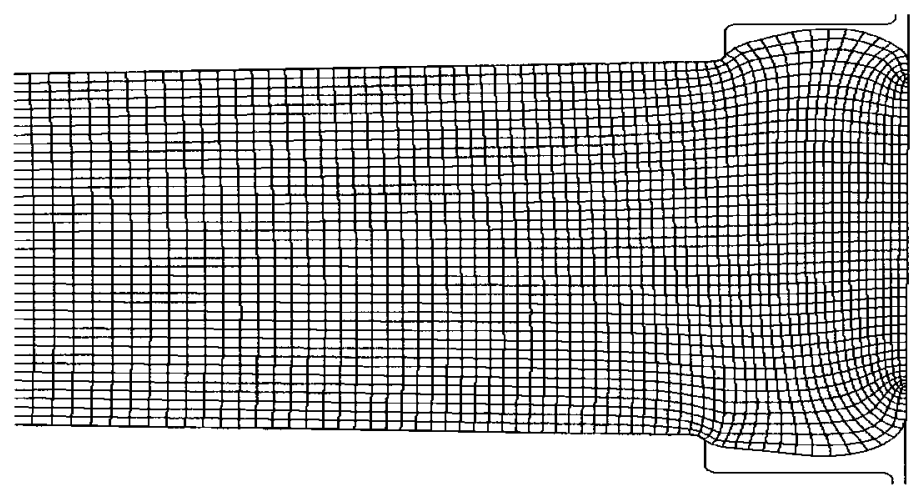

(c) $90 \%$ axial stroke

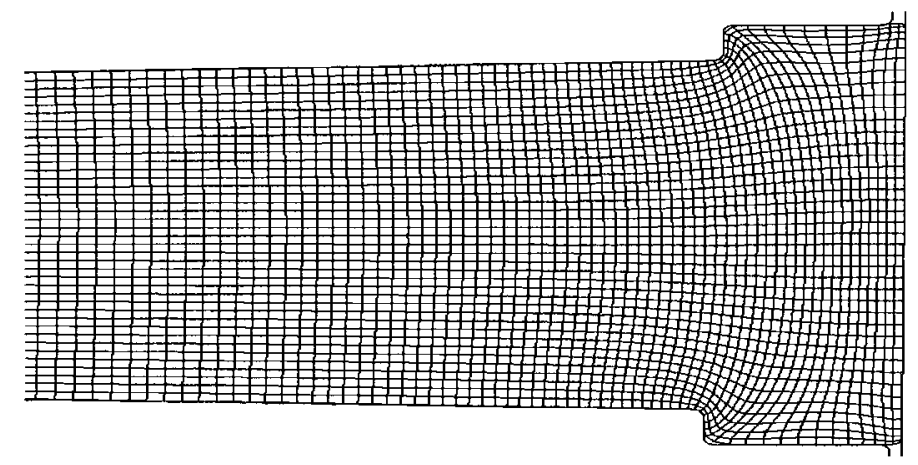

(d) $100 \%$ axial stroke

\section{Bottom die curvature study}

Figure 6-16. Metal deformed mesh patterns showing outer $20 \%$ of blank (Top die curvature $=0.3 \mathrm{~mm}$, Bottom die curvature $=0.3 \mathrm{~mm}$, Rim Height $=0.271 \mathrm{~mm}$, no relief) 


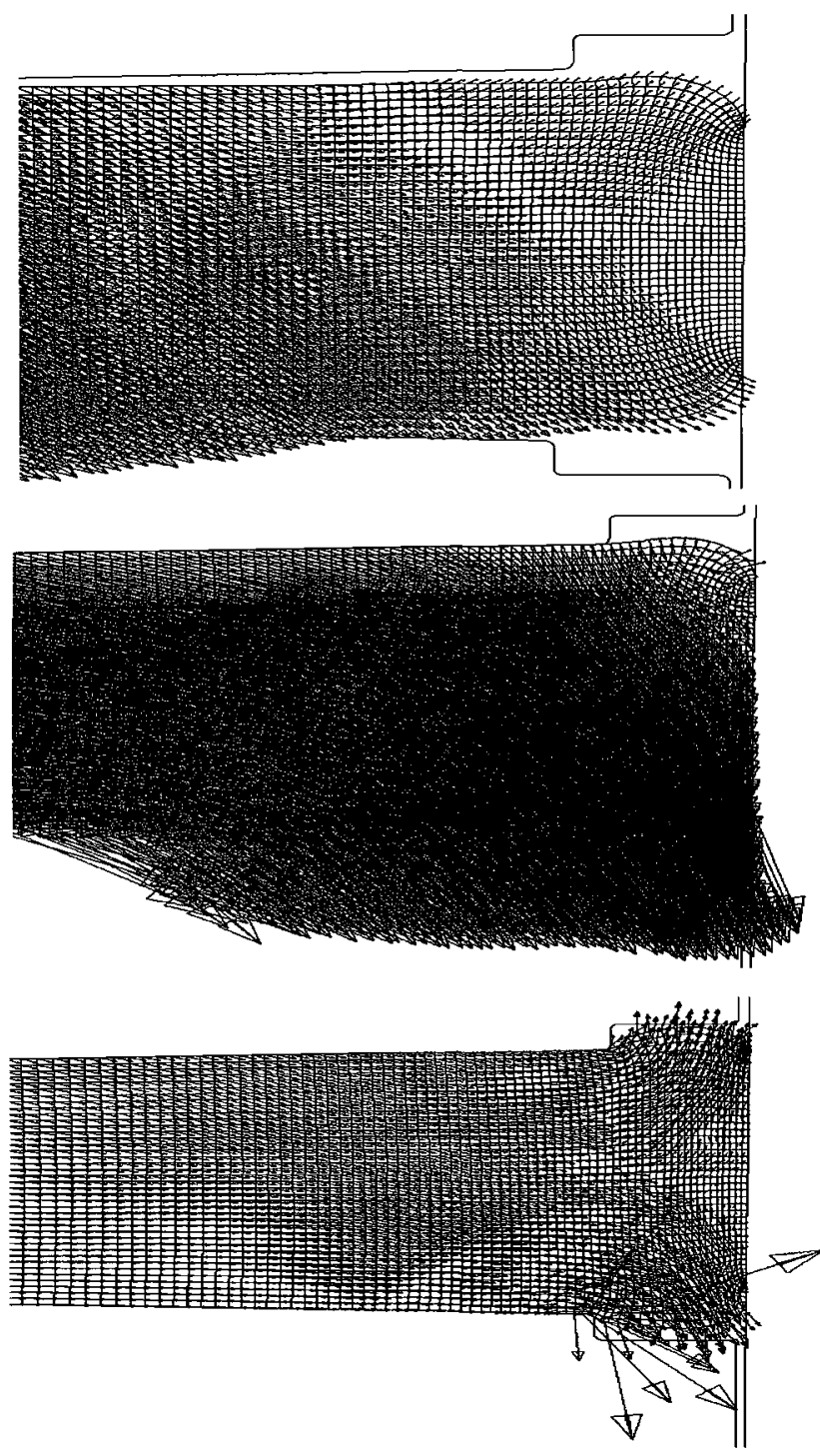

(a) $50 \%$ axial stroke

(b) $75 \%$ axial stroke

(c) $90 \%$ axial stroke

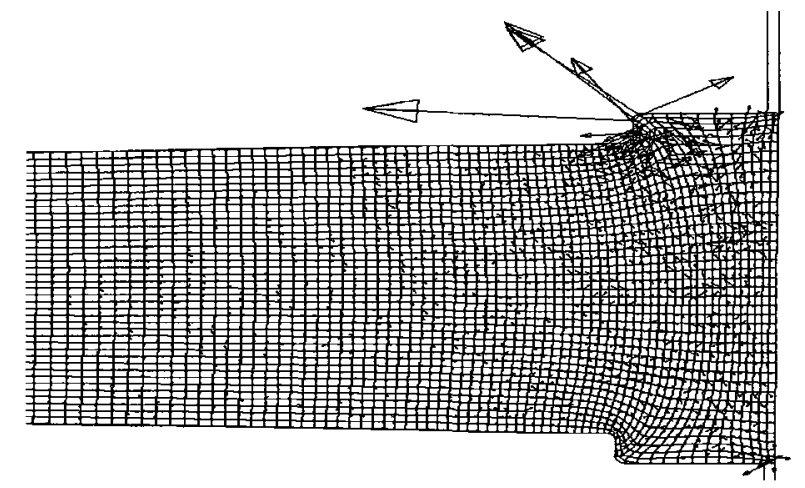

(d) $100 \%$ axial stroke

Bottom die curvature study

Figure 6-17. Material flow velocity patterns showing outer $20 \%$ of blank

(Top die curvature $=0.3 \mathrm{~mm}$, Bottom die curvature $=0.3 \mathrm{~mm}$, Rim Height $=0.271 \mathrm{~mm}$, no relief) 

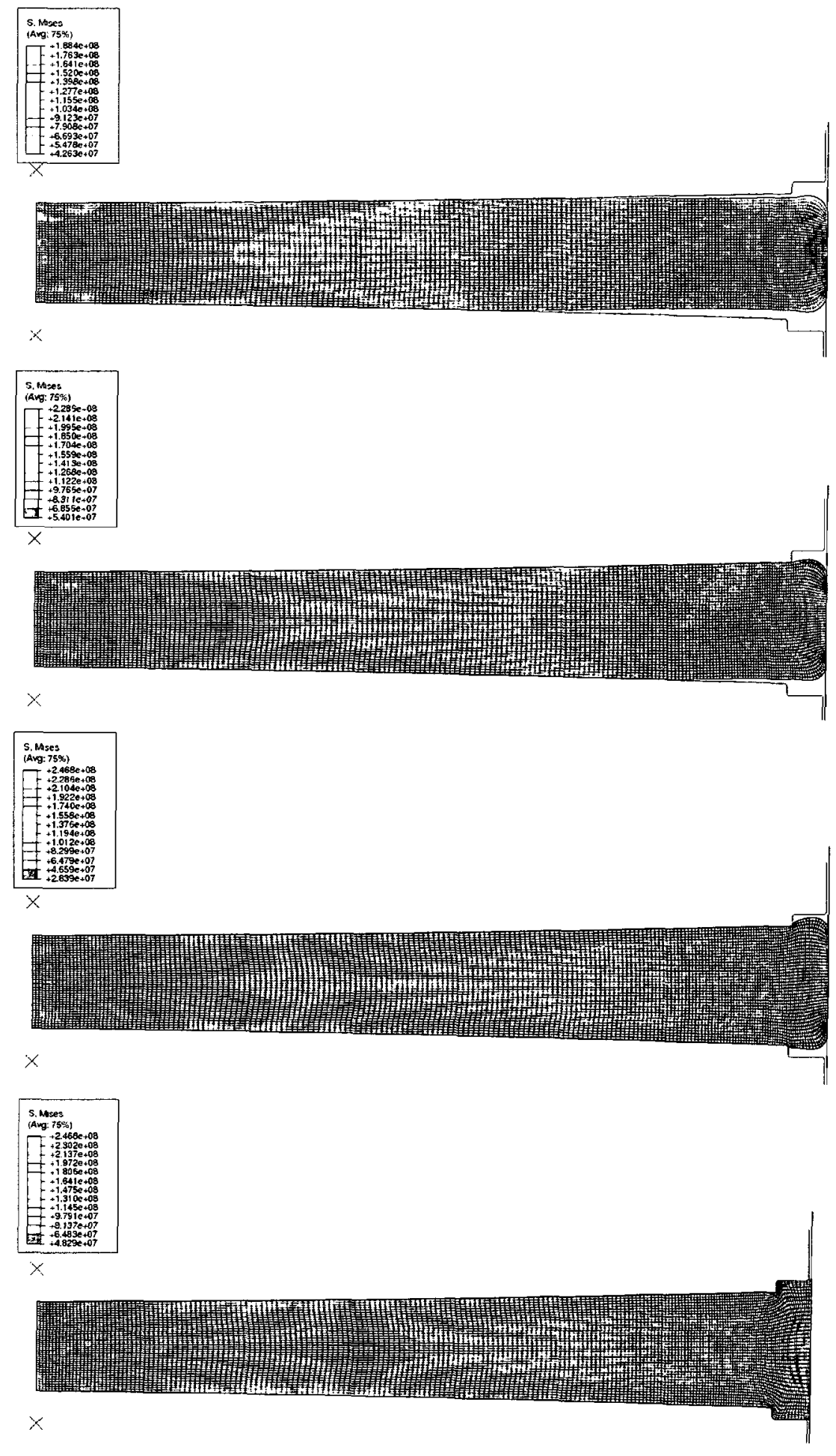

Bottom die curvature study (a) $50 \%$

axial stroke

(b) $75 \%$

axial stroke

(c) $90 \%$

axial stroke

(d) $100 \%$

axial stroke

Figure 6-18. Von Mises stress contour

(Top die curvature $=0.3 \mathrm{~mm}$, Bottom die curvature $=0.3 \mathrm{~mm}$, Rim Height $=0.271 \mathrm{~mm}$, no relief) 


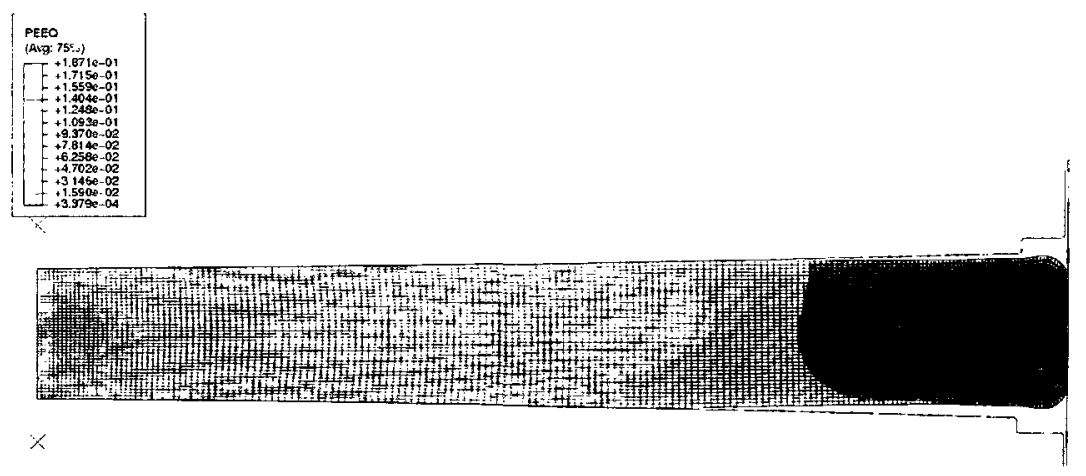

(a) $50 \%$

axial stroke

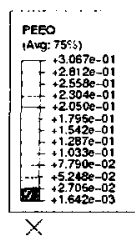

(b) $75 \%$

axial stroke
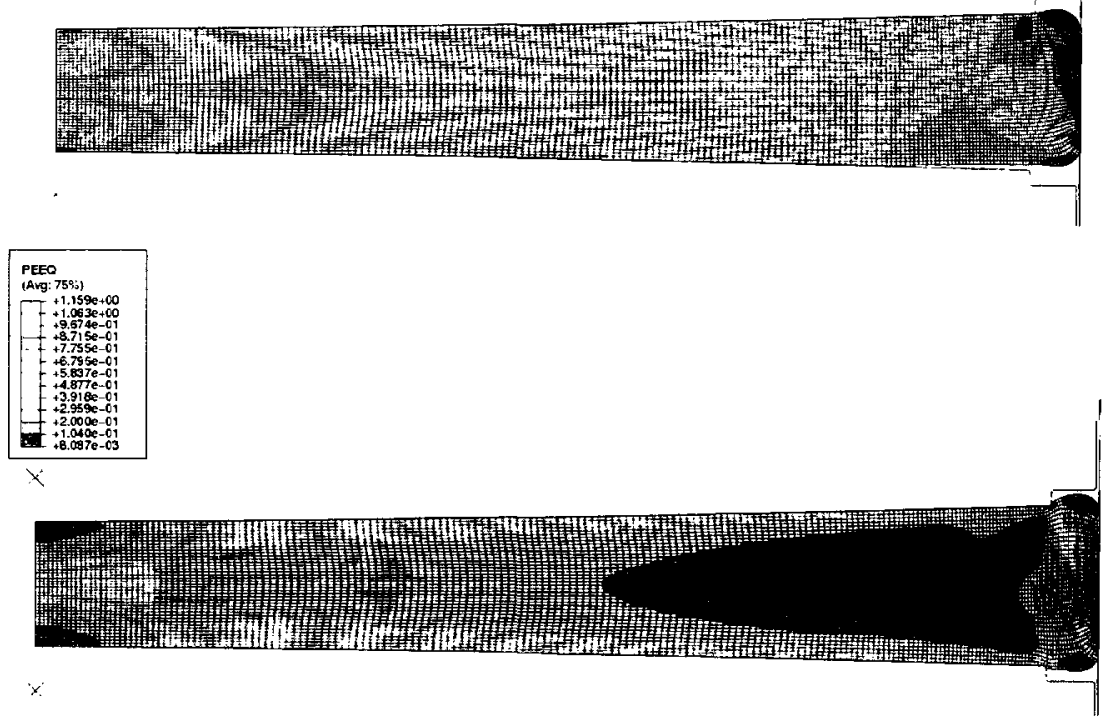

(c) $90 \%$

axial stroke

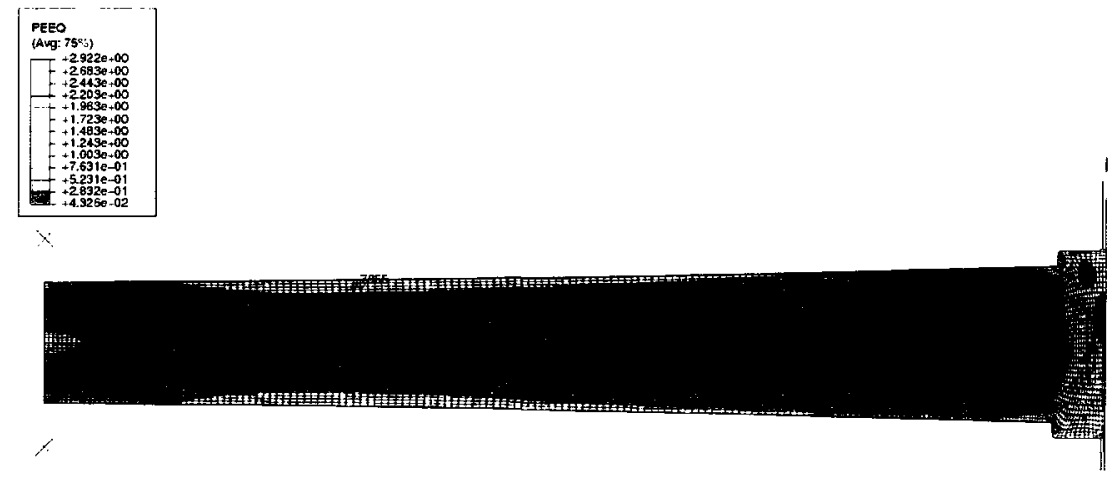

(d) $100 \%$

axial stroke

Bottom die curvature study

Figure 6-19. Equivalent plastic strain contour

(Top die curvature $=0.3 \mathrm{~mm}$, Bottom die curvature $=0.3 \mathrm{~mm}$, Rim Height $=0.271 \mathrm{~mm}$, no relief) 


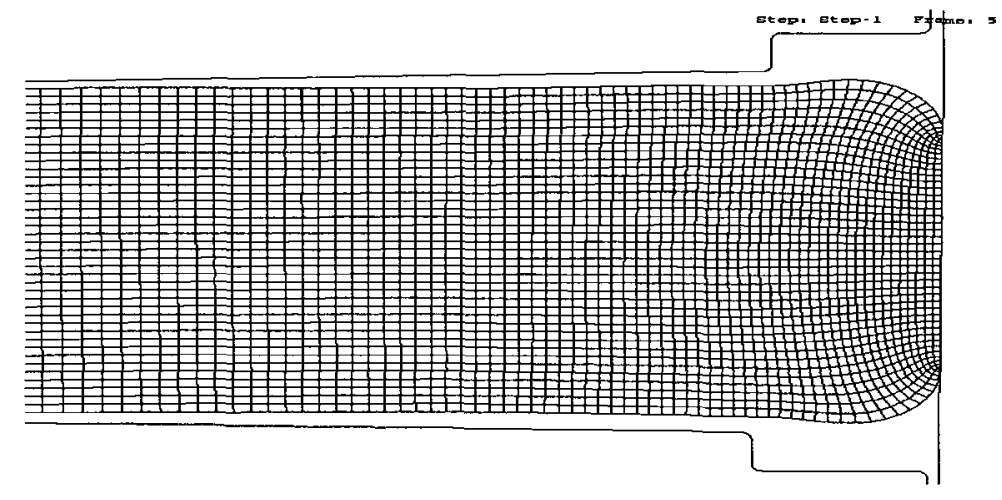

( a ) $50 \%$ axial

stroke

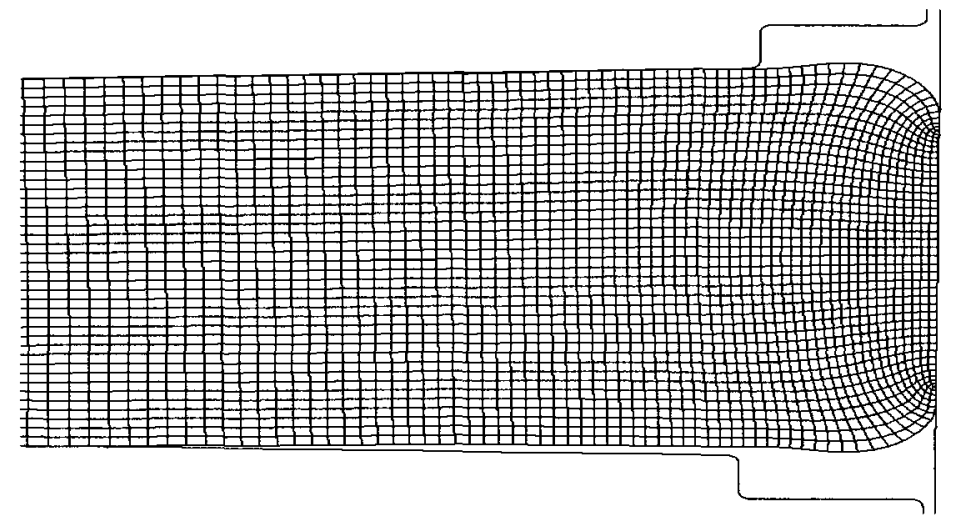

(b ) $75 \%$ axial stroke

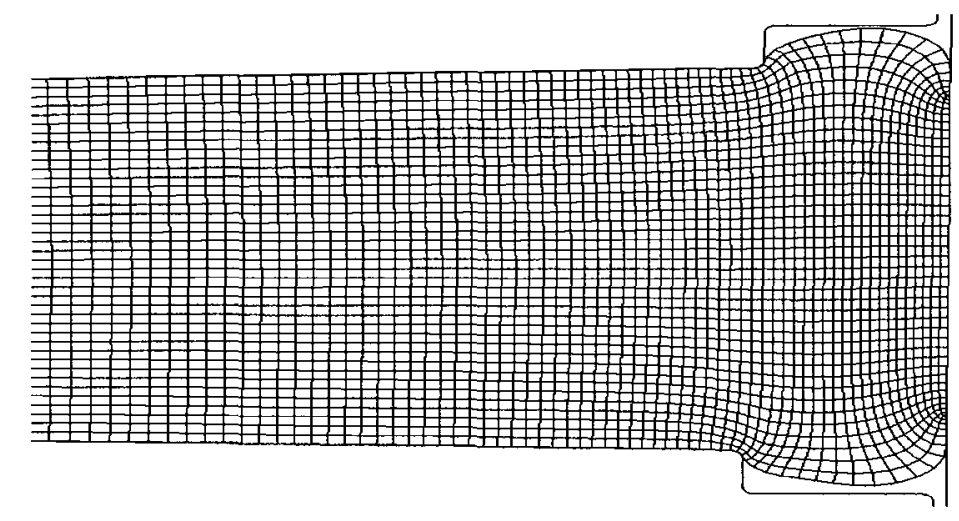

(c) $90 \%$ axial stroke

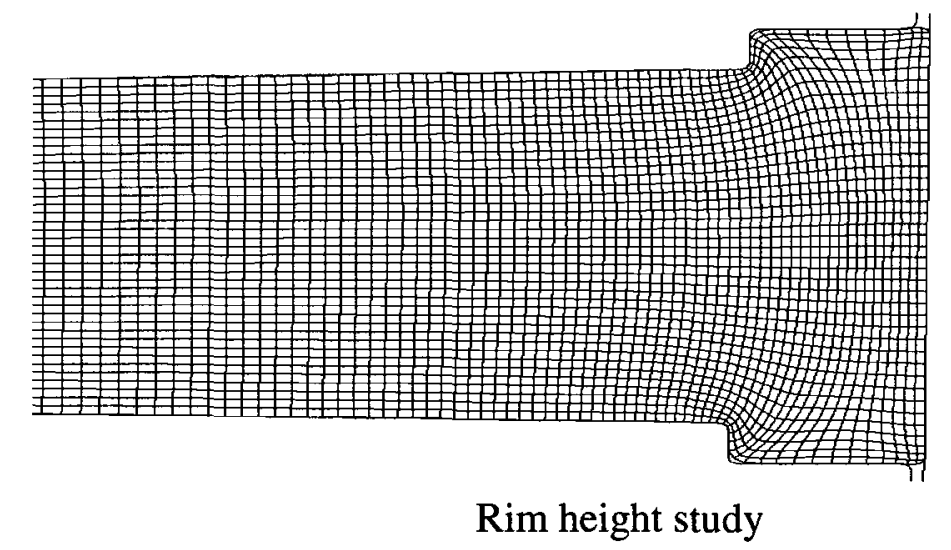

(d ) $100 \%$ axial stroke

Figure 6-20. Metal deformed mesh patterns showing outer $20 \%$ of blank (Top die curvature $=0.3 \mathrm{~mm}$, Bottom die curvature $=0.19 \mathrm{~mm}$, Rim Height $=0.3 \mathrm{~mm}$, no relief) 


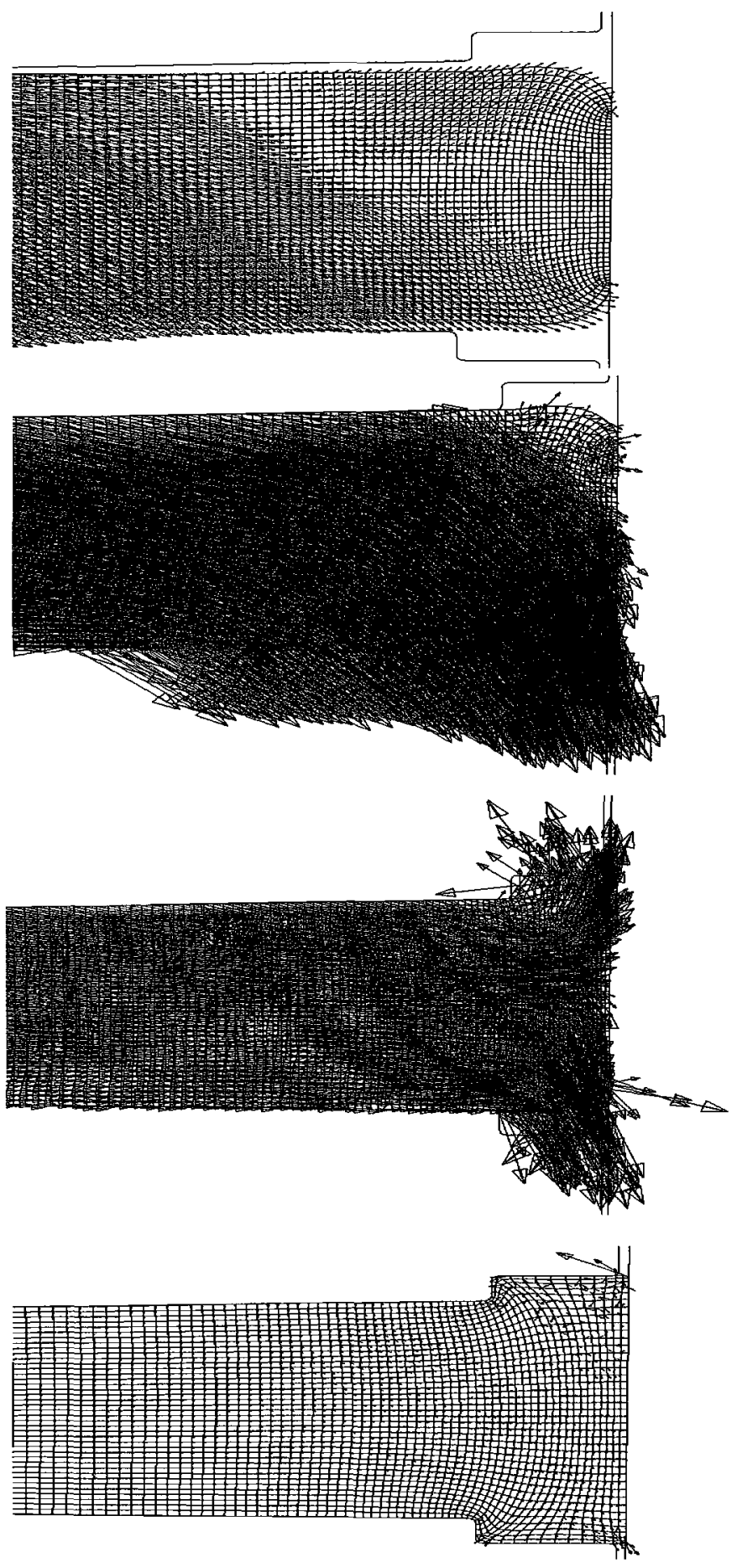

(a) $50 \%$ axial

stroke

(b) $75 \%$ axial stroke

(c) $90 \%$ axial stroke

(d) $100 \%$ axial stroke

\section{Rim height study}

Figure 6-21. Material flow velocity patterns showing outer $20 \%$ of blank (Top die curvature $=0.3 \mathrm{~mm}$, Bottom die curvature $=0.19 \mathrm{~mm}$, Rim Height $=0.3 \mathrm{~mm}$, no relief) 


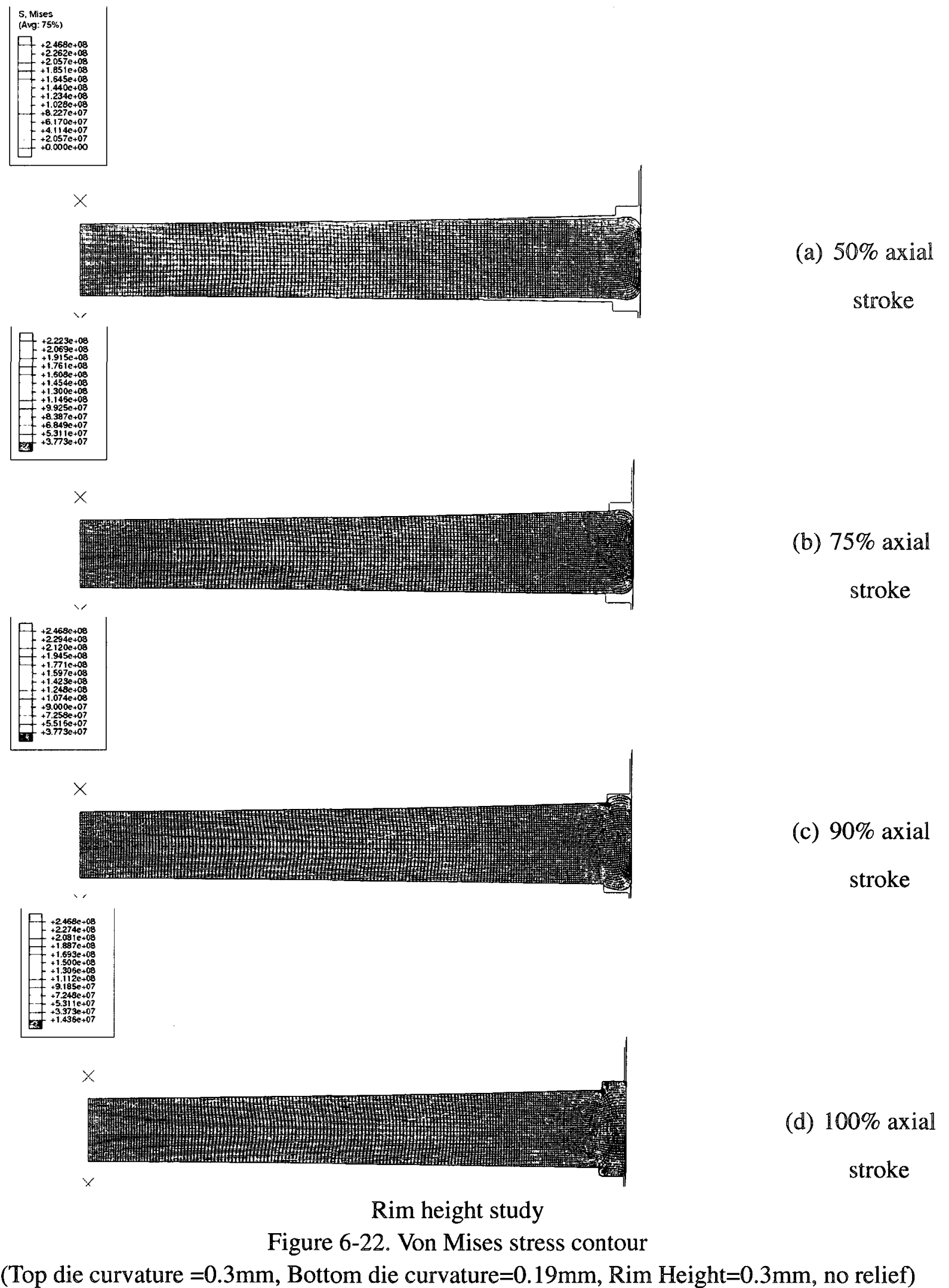

(Top die curvature $=0.3 \mathrm{~mm}$, Bottom die curvature $=0.19 \mathrm{~mm}$, Rim Height $=0.3 \mathrm{~mm}$, no relief) 


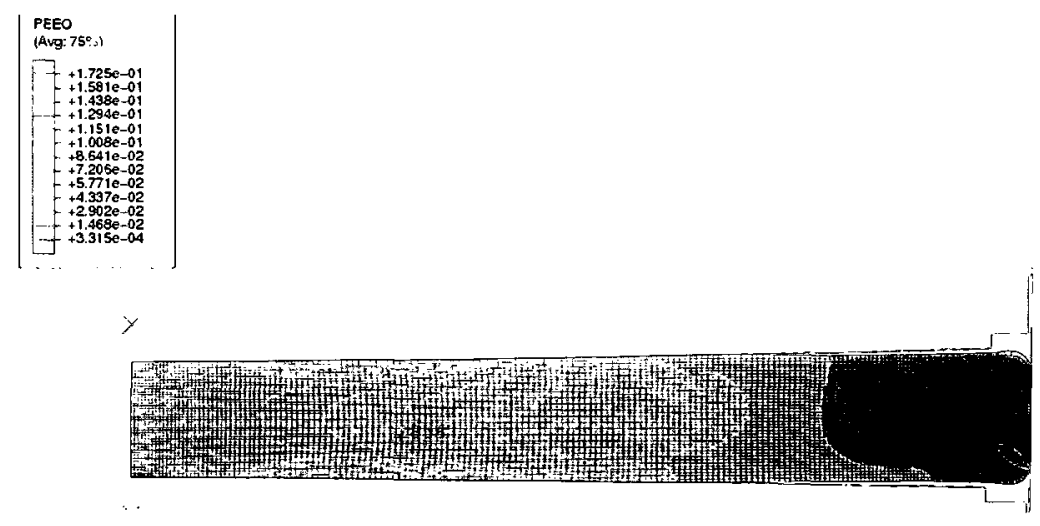

(a) 50\% axial stroke
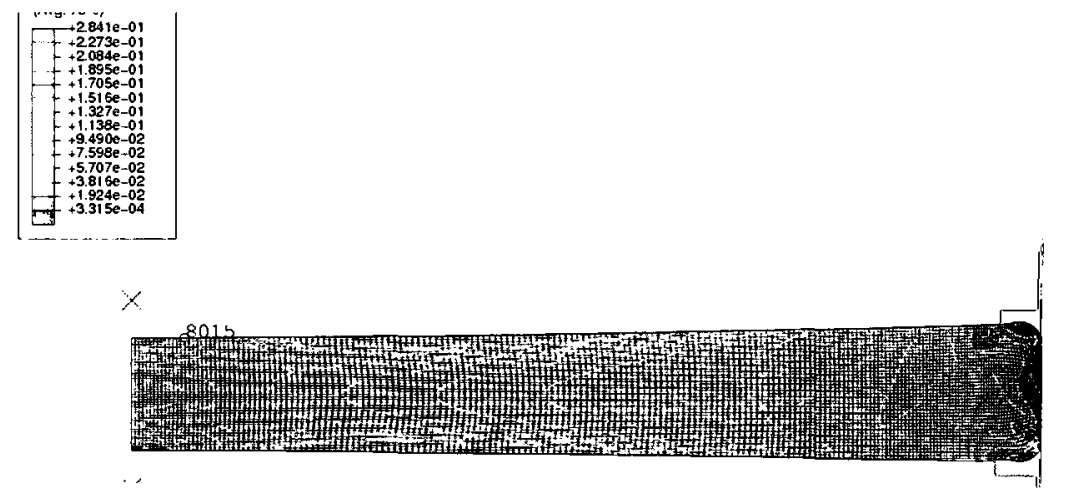

(b) $75 \%$ axial stroke
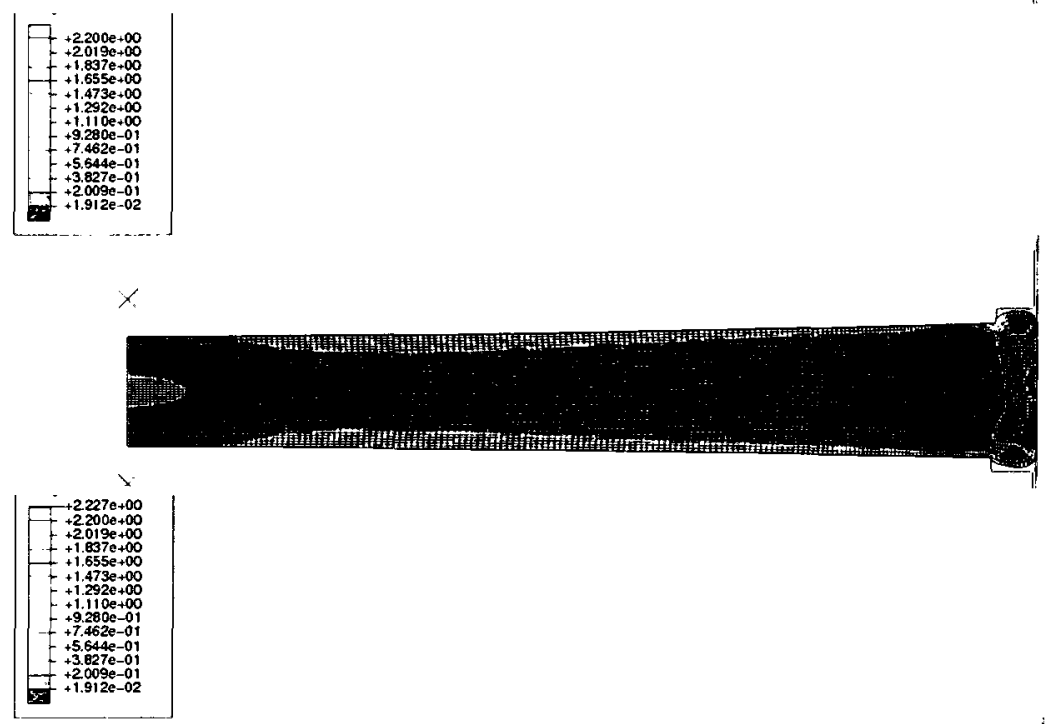

(c) $90 \%$ axial stroke

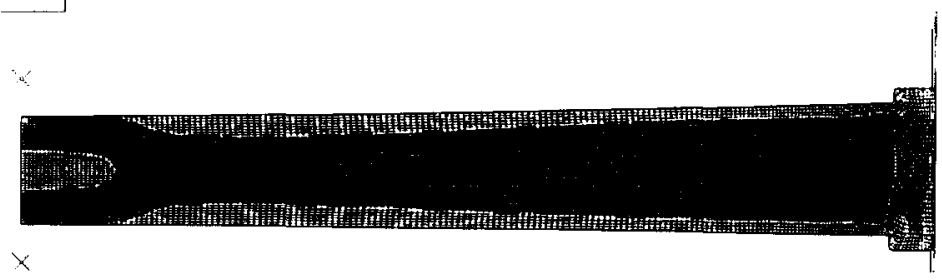

Rim height study

Figure 6-23. Equivalent plastic strain contour

(Top die curvature $=0.3 \mathrm{~mm}$, Bottom die curvature $=0.19 \mathrm{~mm}$, Rim Height $=0.3 \mathrm{~mm}$, no relief) 


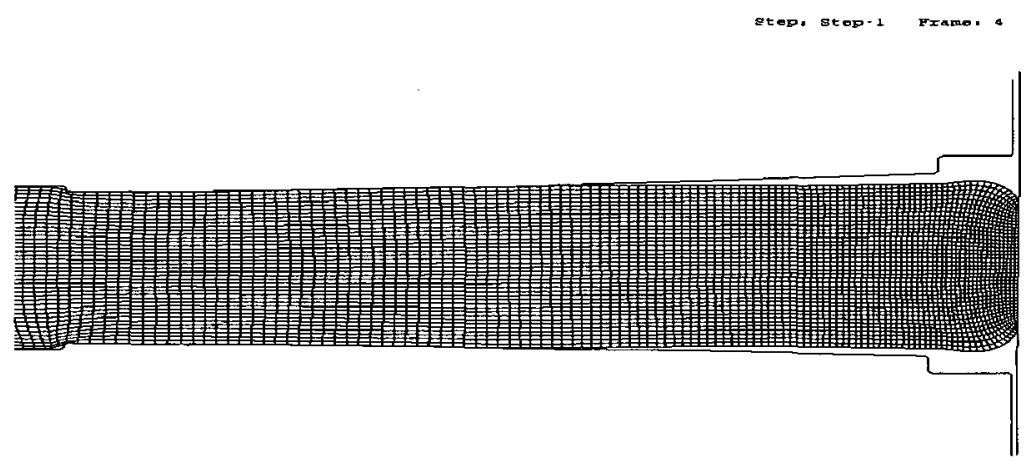

( a ) $50 \%$ axial stroke

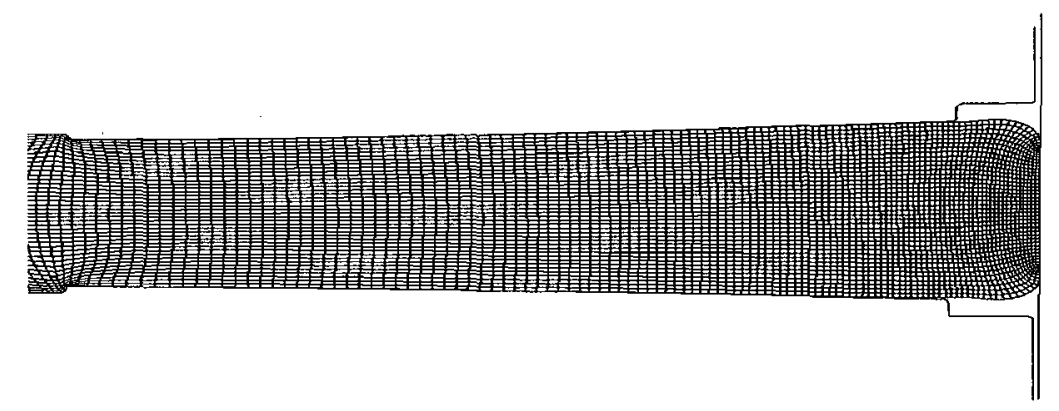

(b ) $75 \%$ axial stroke

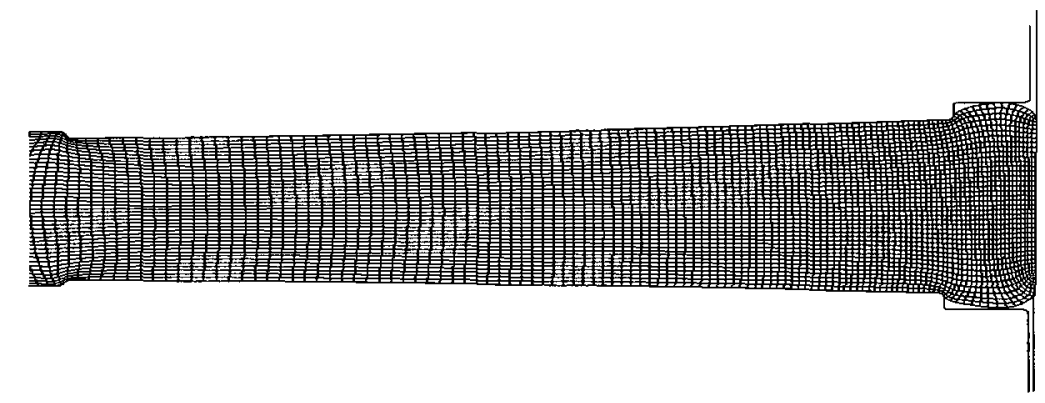

(c) $90 \%$ axial stroke

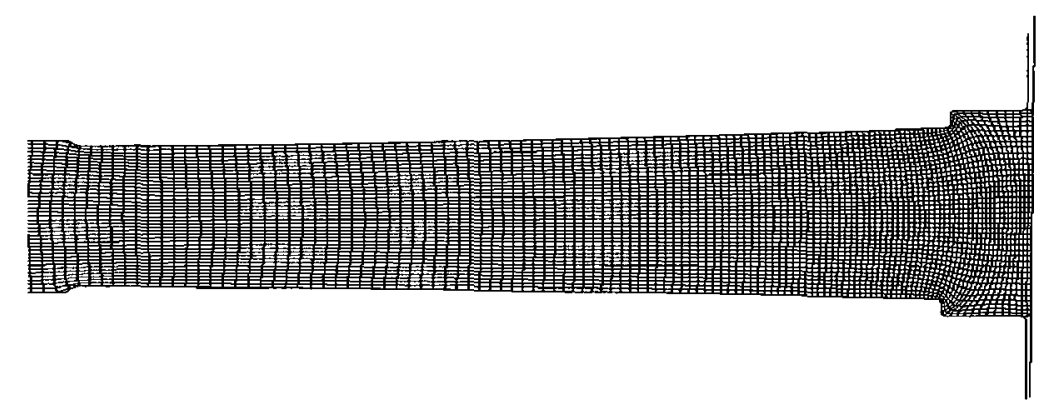

(d) $100 \%$ axial stroke

\section{Relief height study}

Figure 6-24. Metal deformed mesh patterns showing outer 55\% of blank (Top die curvature $=0.3 \mathrm{~mm}$, Bottom die curvature $=0.19 \mathrm{~mm}$, Rim Height $=0.271 \mathrm{~mm}$, relief $=0.1 \mathrm{~mm}$ ) 


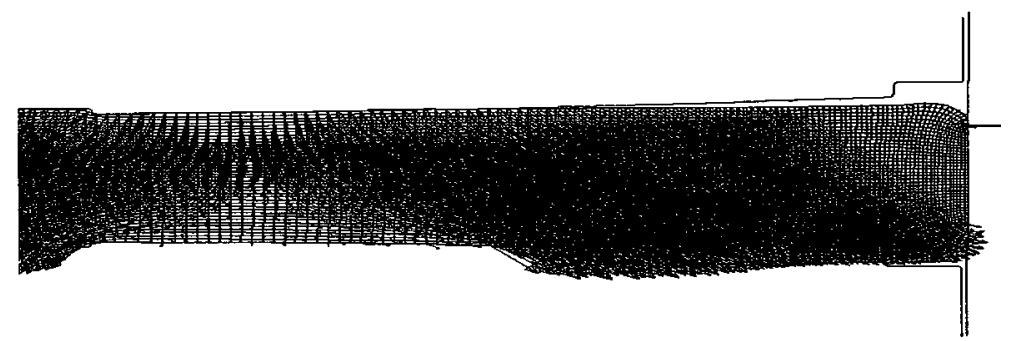

(a) $50 \%$ axial stroke

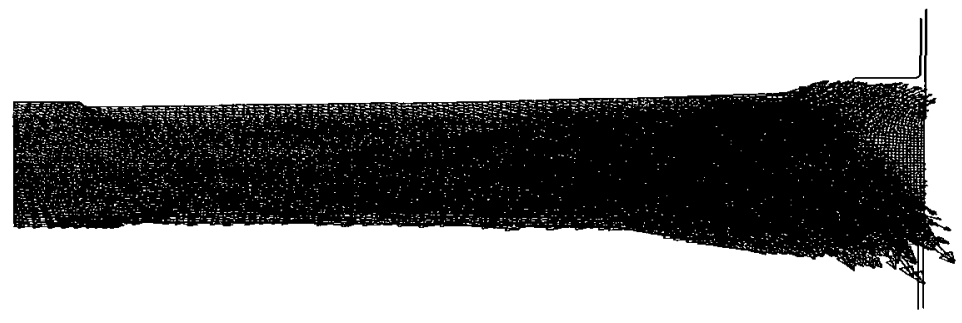

(b ) $75 \%$ axial stroke

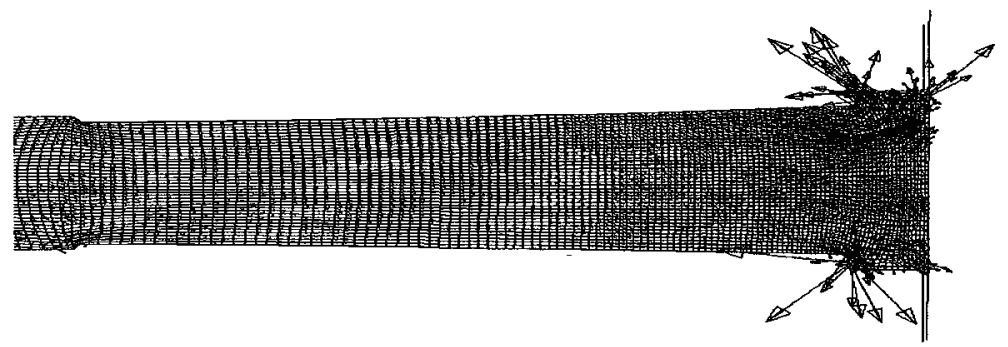

(c) $90 \%$ axial stroke

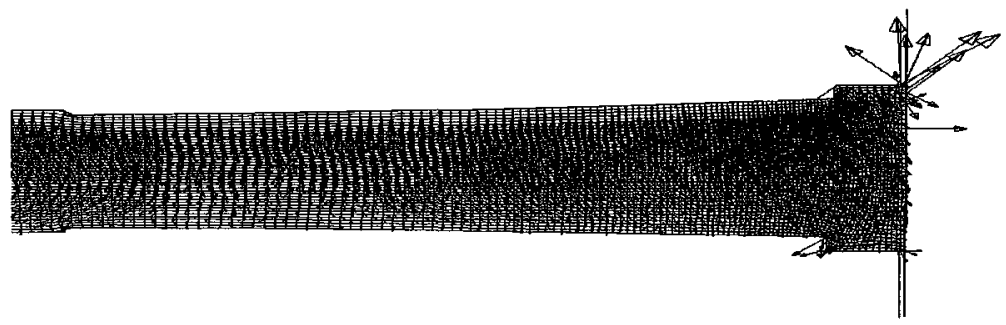

(d) $100 \%$ axial stroke

Relief height study

Figure 6-25. Material flow velocity patterns showing outer 55\% of blank (Top die curvature $=0.3 \mathrm{~mm}$, Bottom die curvature $=0.19 \mathrm{~mm}$, Rim Height $=0.271 \mathrm{~mm}$, relief $=0.1 \mathrm{~mm}$ ) 


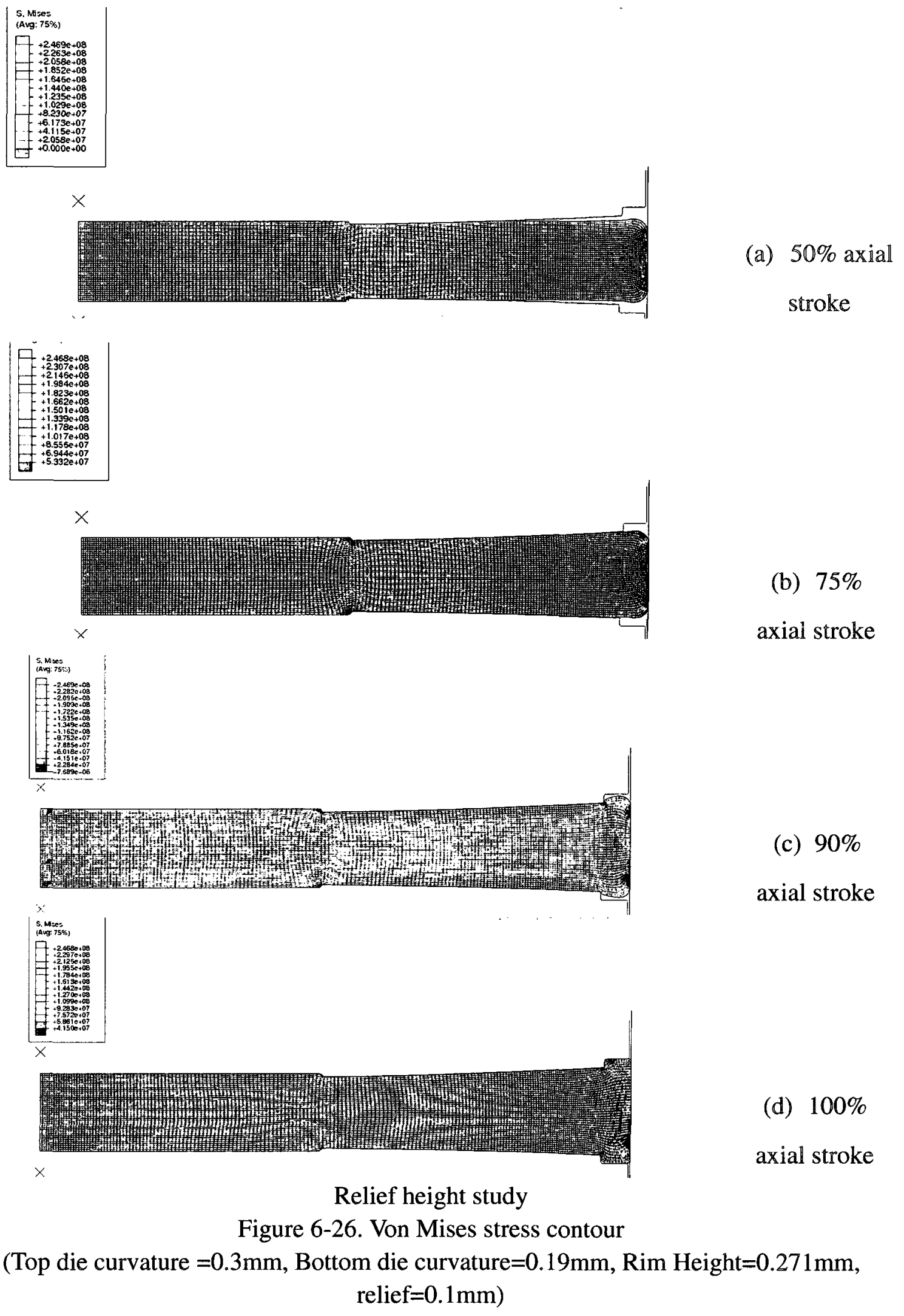




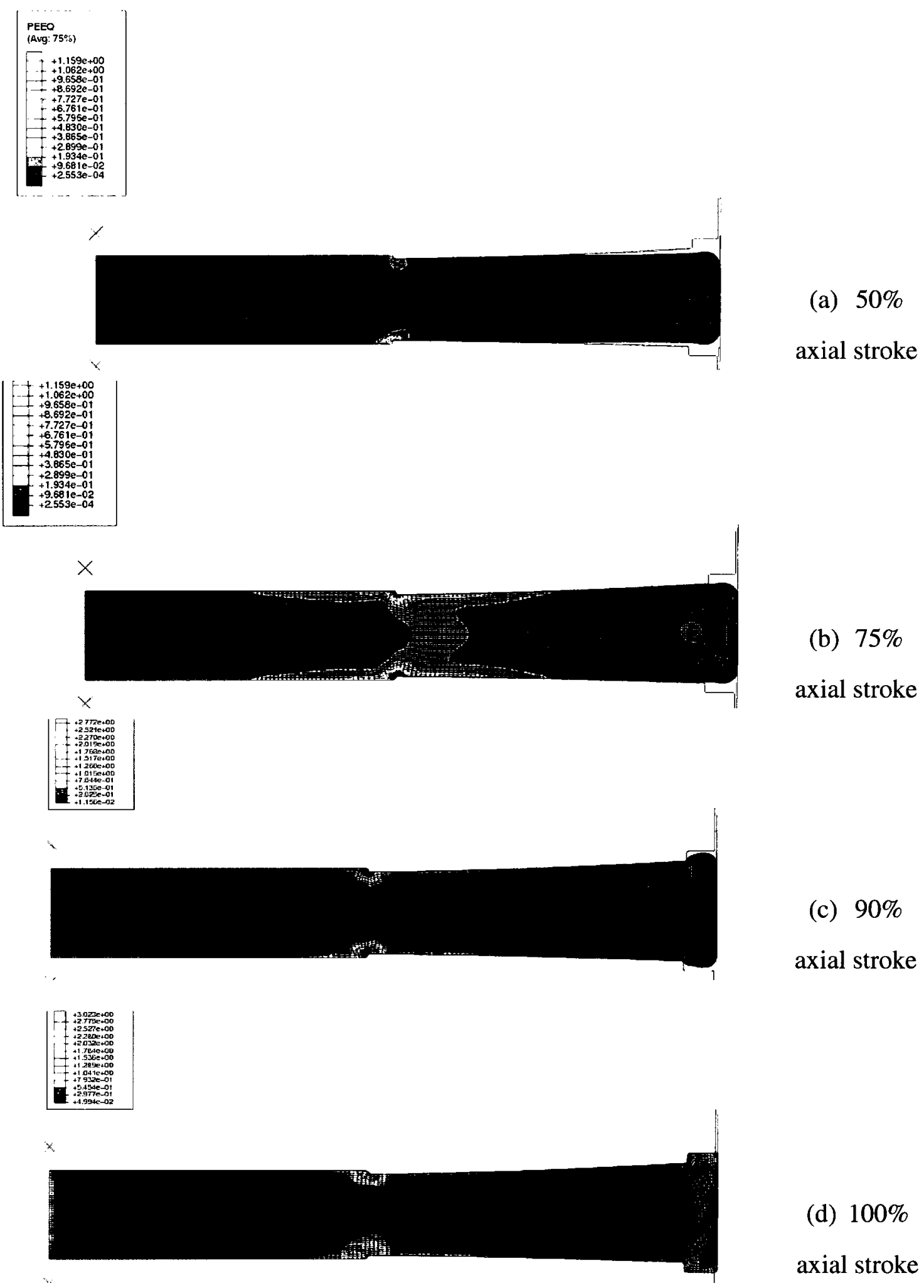

Figure 6-27. Equivalent plastic strain contour

(Top die curvature $=0.3 \mathrm{~mm}$, Bottom die curvature $=0.19 \mathrm{~mm}$, Rim Height $=0.271 \mathrm{~mm}$, relief $=0.1 \mathrm{~mm}$ ) 


\section{top die curvature variations}

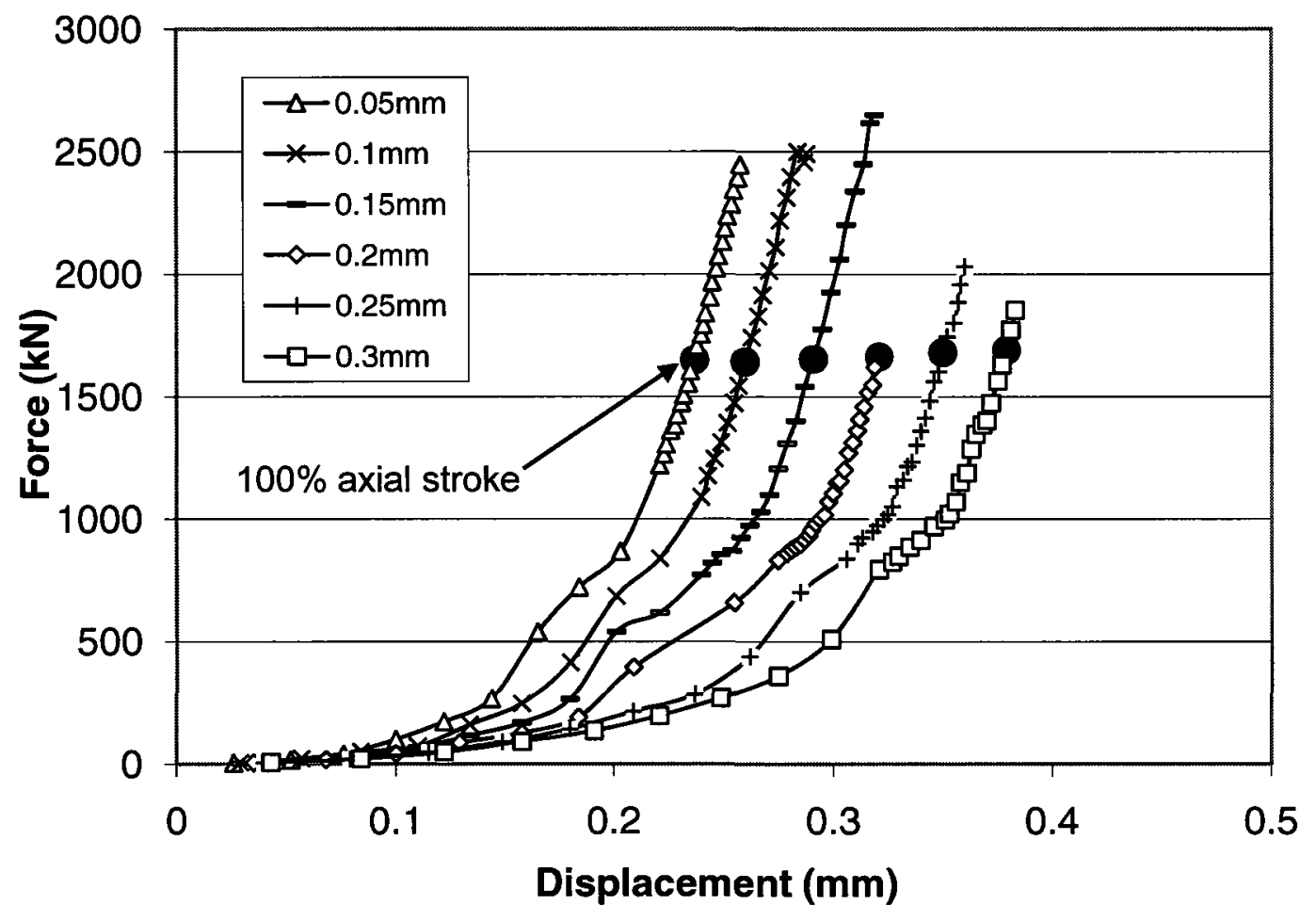

Figure 6-28a. Loading paths with varying top die curvatures

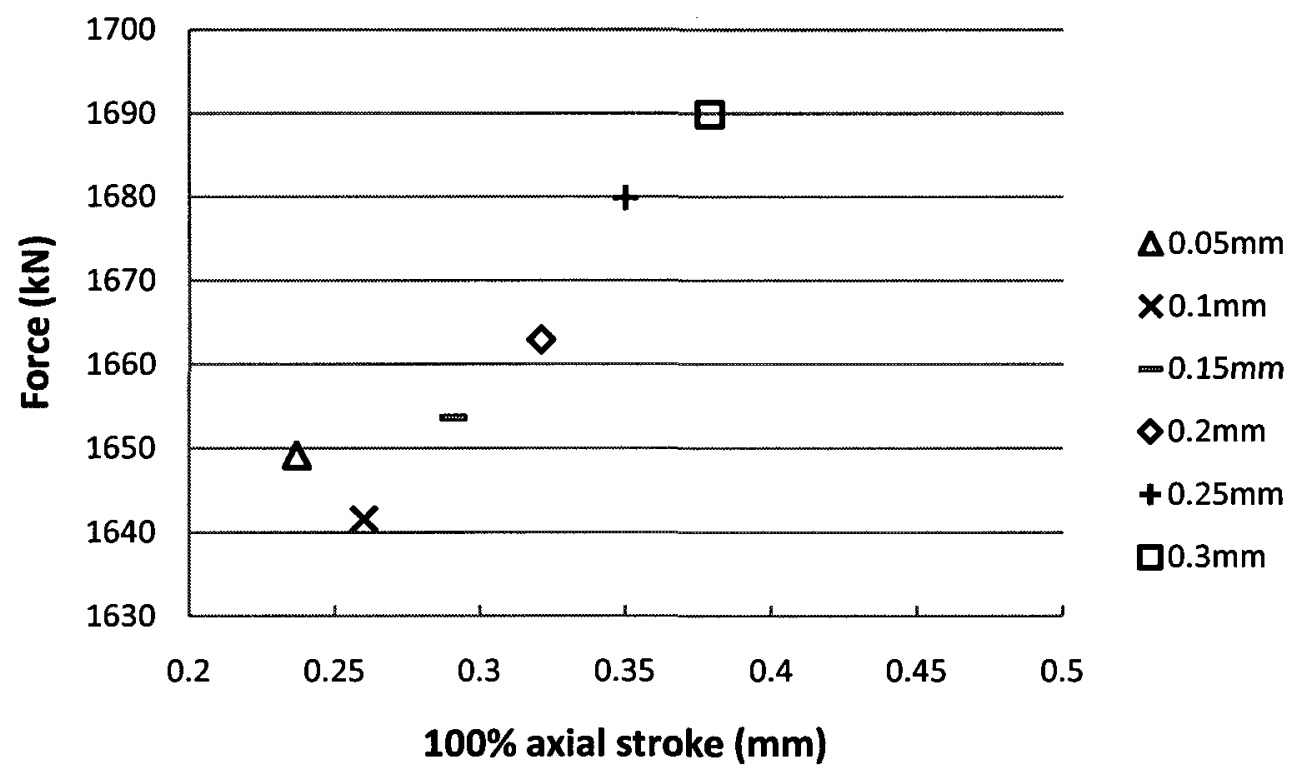

Figure 6-28b. Loads at $100 \%$ axial stroke with varying top die curvatures 


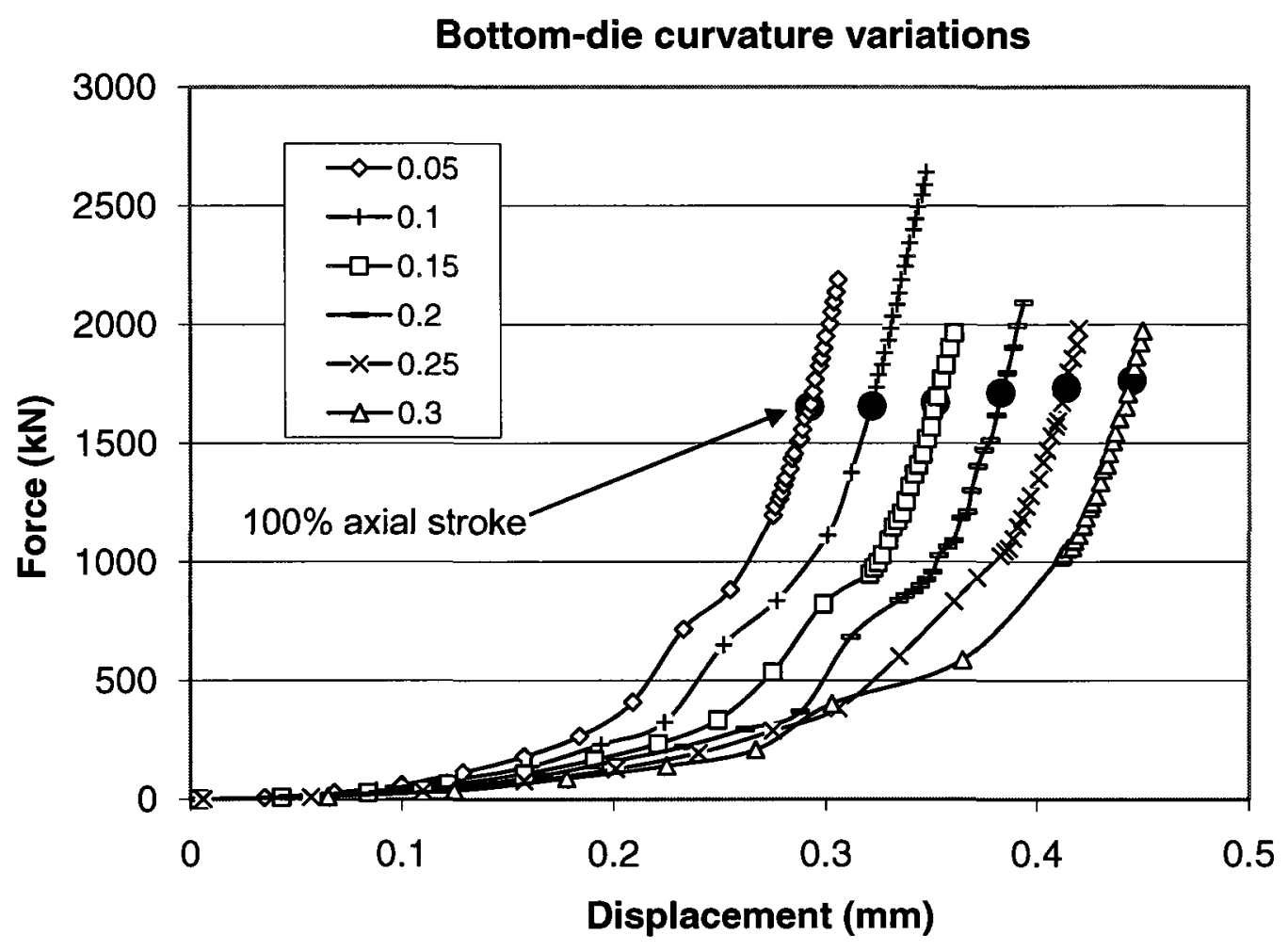

Figure 6-29a. Loading paths with varying bottom die curvatures

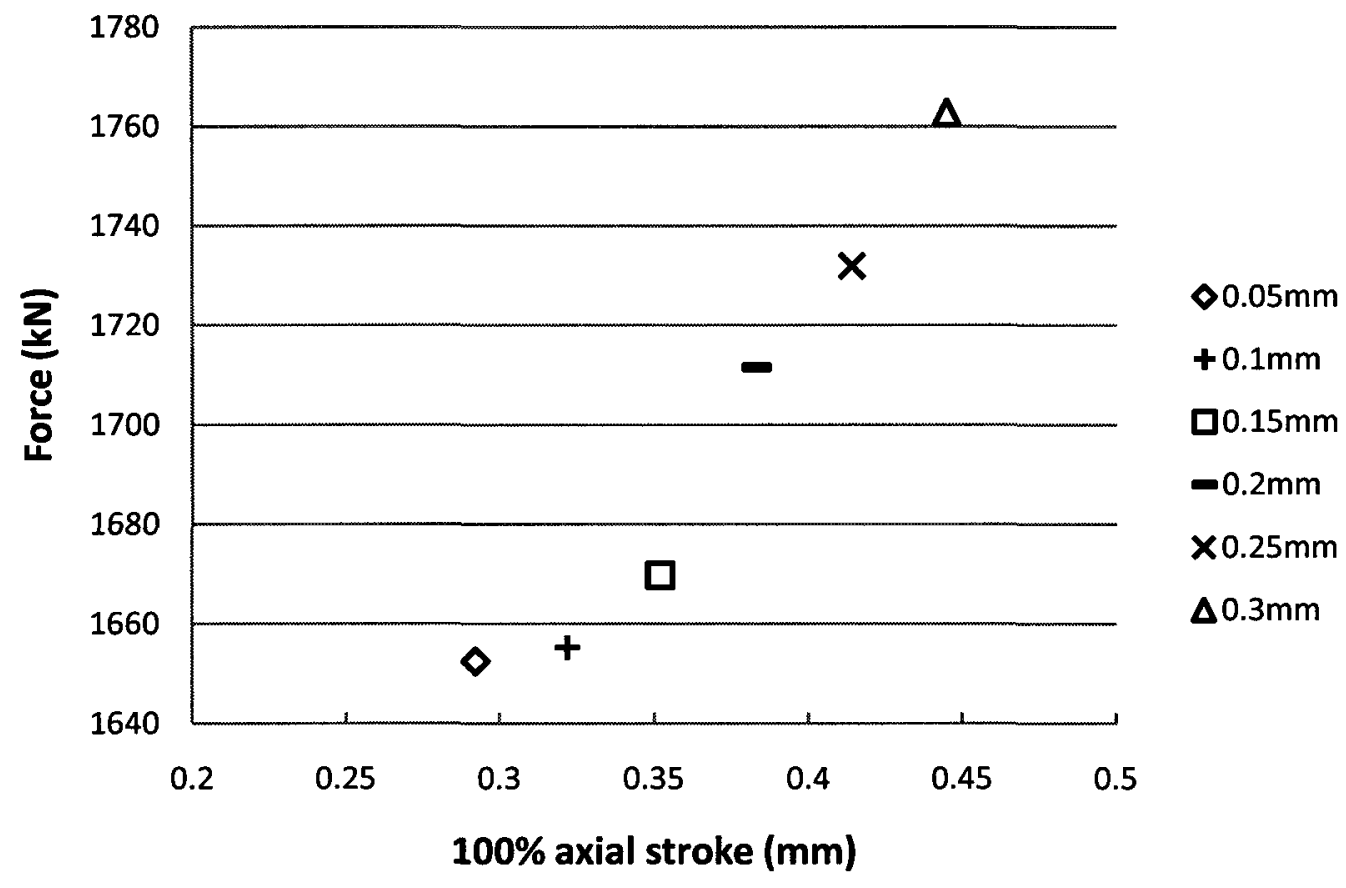

Figure 6-29b. Loads at $100 \%$ axial stroke with varying bottom die curvatures 


\section{rim height variations}

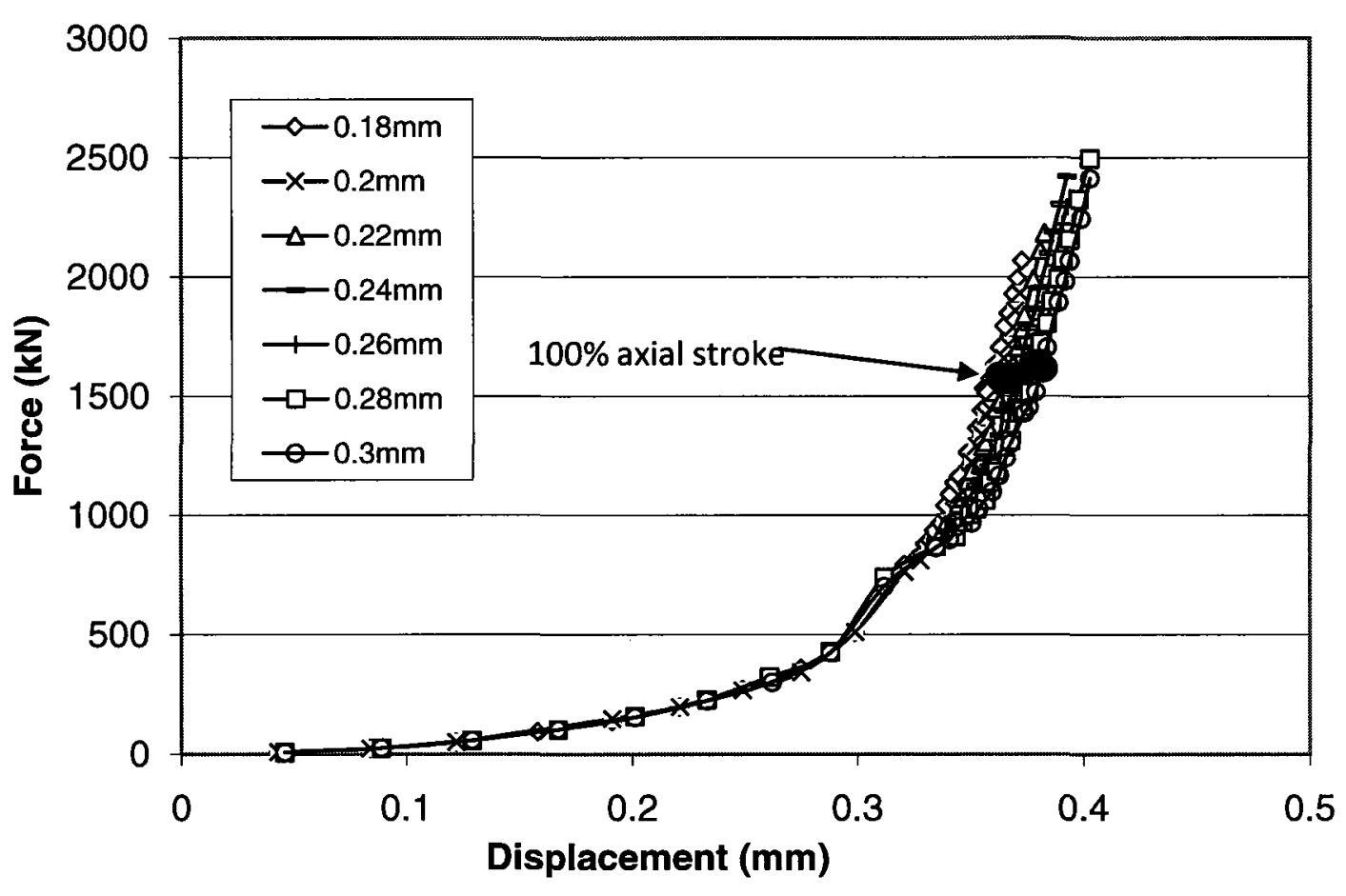

Figure 6-30a. Loading paths with varying rim heights

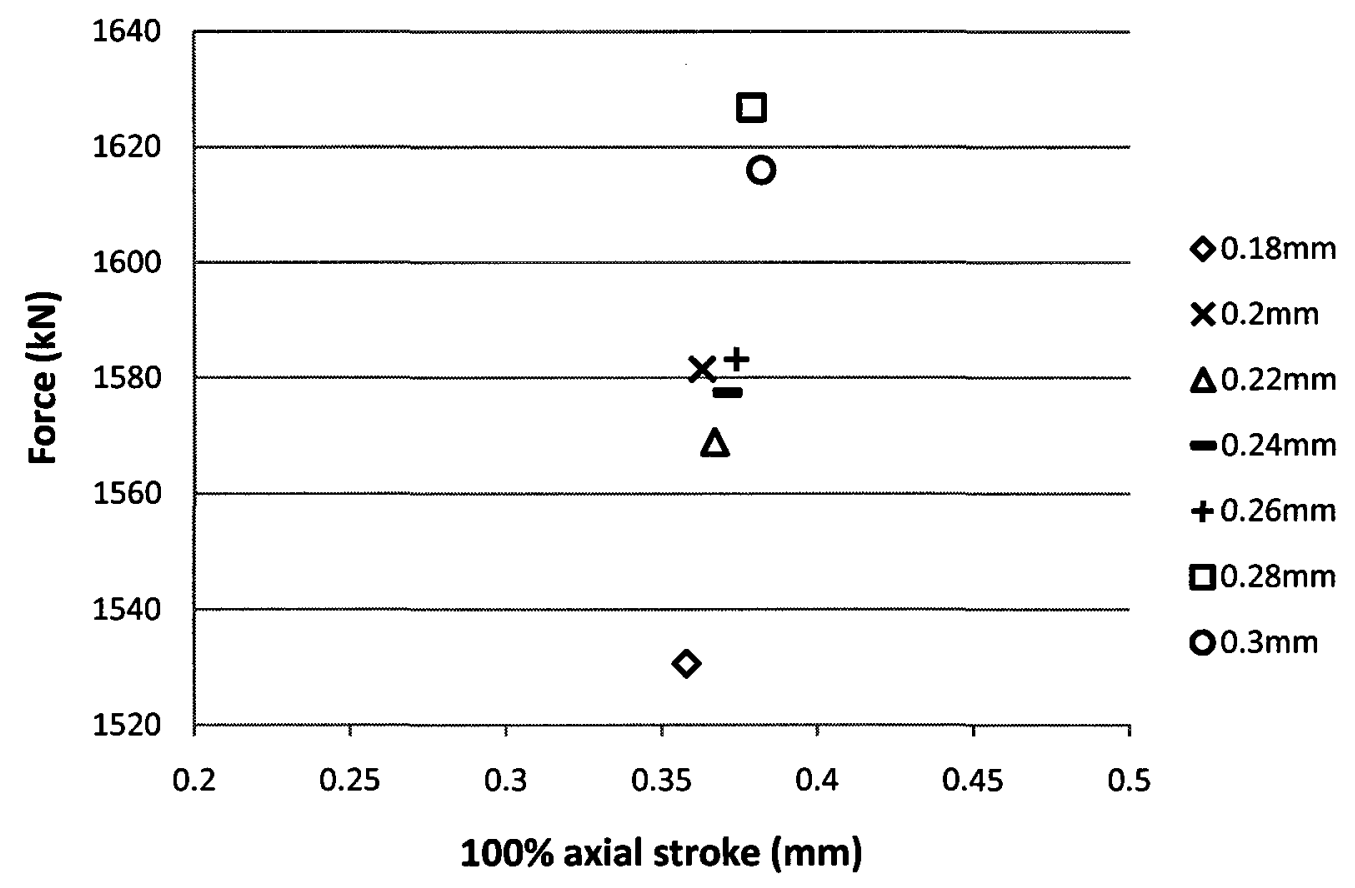

Figure 6-30b. Load at $100 \%$ axial stroke with varying rim heights 


\section{Relief variations}

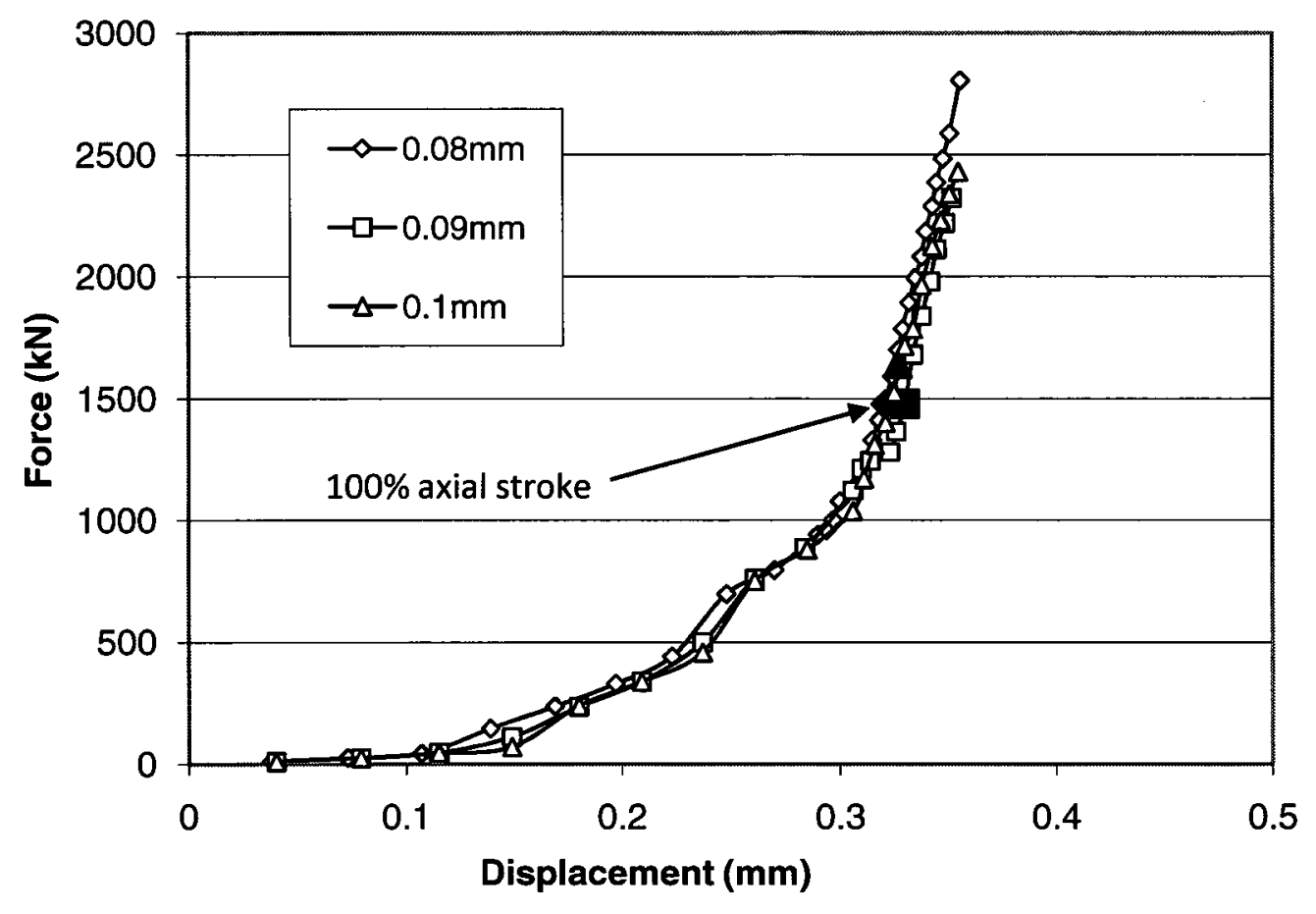

Figure 6-31a. Loading paths with varying relief heights

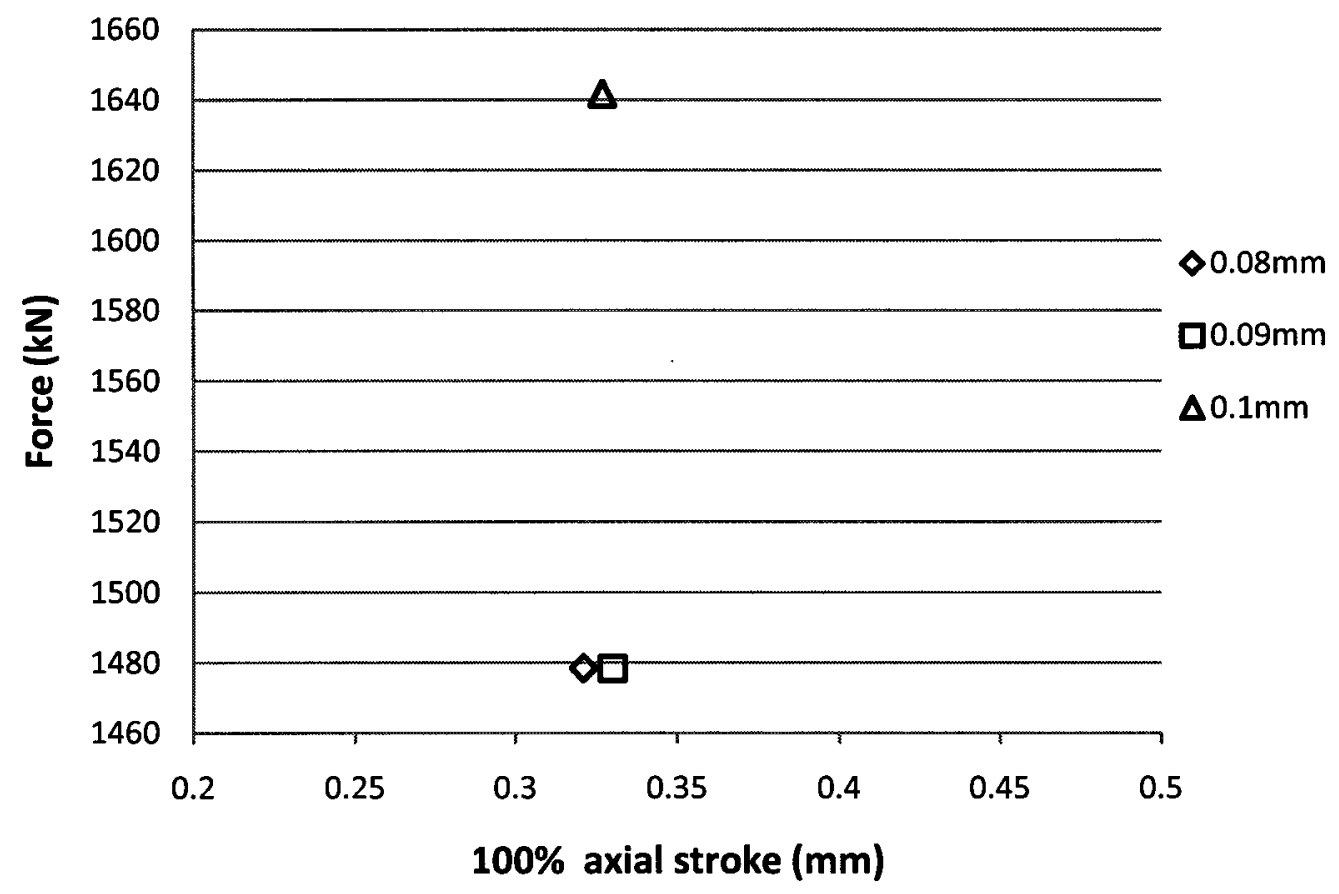

Figure 6-31b. Load at $100 \%$ axial stroke with varying relief heights 


\begin{tabular}{|c|c|c|c|}
\hline \multicolumn{4}{|c|}{ Mesh convergence } \\
\hline \multicolumn{2}{|c|}{ Coarse mesh } & \multicolumn{2}{|c|}{ Refined mesh } \\
\hline $\begin{array}{c}\text { Displacement } \\
(\mathrm{mm})\end{array}$ & $\begin{array}{l}\text { Load } \\
(\mathrm{kN})\end{array}$ & $\begin{array}{c}\text { Displacement } \\
(\mathrm{mm})\end{array}$ & $\begin{array}{l}\text { Load } \\
(\mathrm{kN})\end{array}$ \\
\hline 0 & 0 & 0 & 0 \\
\hline 0.043 & 6.16 & 0.043 & 7.18 \\
\hline 0.084 & 22.36 & 0.084 & 21.96 \\
\hline 0.122 & 50.83 & 0.122 & 49.55 \\
\hline 0.158 & 91.18 & 0.158 & 92.39 \\
\hline 0.191 & 139.35 & 0.191 & 135.86 \\
\hline 0.221 & 200.87 & 0.221 & 196.53 \\
\hline 0.249 & 268.09 & 0.249 & 269.96 \\
\hline 0.275 & 358.34 & 0.275 & 358.8 \\
\hline 0.299 & 516.87 & 0.299 & 508.88 \\
\hline 0.321 & 799.82 & 0.321 & 794.12 \\
\hline 0.327 & 838.71 & 0.327 & 824.56 \\
\hline 0.33 & 853.72 & 0.33 & 848.44 \\
\hline 0.335 & 915.28 & 0.335 & 886 \\
\hline 0.34 & 930.69 & 0.34 & 912.93 \\
\hline 0.346 & 975.2 & 0.346 & 969.26 \\
\hline 0.351 & 1048.37 & 0.351 & 996.02 \\
\hline 0.353 & 1072.54 & 0.353 & 1021.75 \\
\hline 0.356 & 1126.3 & 0.356 & 1069.58 \\
\hline 0.358 & 1177.03 & 0.358 & 1149.12 \\
\hline 0.361 & 1230.14 & 0.361 & 1187.41 \\
\hline 0.363 & 1308.65 & 0.363 & 1285.74 \\
\hline 0.365 & 1375.47 & 0.365 & 1345.51 \\
\hline 0.368 & 1420.65 & 0.368 & 1384.58 \\
\hline 0.37 & 1450.43 & 0.37 & 1400.81 \\
\hline 0.372 & 1508.18 & 0.372 & 1474.3 \\
\hline 0.375 & 1575.9 & 0.375 & 1563.26 \\
\hline 0.377 & 1642.91 & 0.377 & 1631.71 \\
\hline 0.379 & 1716.72 & 0.379 & 1689.85 \\
\hline 0.381 & 1808.12 & 0.381 & 1772.13 \\
\hline 0.383 & 1899.63 & 0.383 & 1853.33 \\
\hline
\end{tabular}

Table 6-1. Mesh convergence results 


\section{Chapter 7}

\section{Three Dimensional Coining Simulation}

The real silver coins made by coining are geometrically complex and not axisymmetric due to the various surface profile designs on both sides of the coin. The metal flow involved during the coining process is of a three-dimensional nature. Thus the FEM approach would become more practical and useful if it is capable of solving a three-dimensional coining process. In this Chapter, a series of three-dimensional models were used to simulate the 3D coining process. First, a 3D axisymmetric model is used to simulate the same geometry as the 2D model analyzed in Chapter 6. Then the effects of serrations and 3D relief were investigated using the corresponding 3D models.

\subsection{D Simplified Coining FEM Model}

A 2D axisymmetric finite element model of the coining process was presented in the previous chapter. A 3D FEM model of the coining process was created by extending the basic geometry of the die and silver blank used in the 2D simulation to 3D. Due to the axisymmetric nature of the 3D simplified model, a 20 degree slice of the blank was generated with about 300,000 C3D8R brick elements (8-node linear brick with reduced integration and hourglass control), as shown in Figure 7-1. The mesh density is much higher than the 2D refined mesh discussed in Chapter 6. The analytical rigid surfaces were created as the top, bottom dies and side ring. The material model and the contact friction model remained the same as that used in the 2D simulation described in Chapter 6. The integral viscoelastic hourglass control approach, kinematic contact simulation and adaptive meshing techniques described in Chapter 3 were applied in the 3D simulation. The displacement boundary conditions were imposed in the axial direction on the reference node of the rigid bodies. The rigid body reference node for the rigid surface that defines the dies and the side ring movement was constrained to have no rotation and 
no radial displacement, and the axial displacement of the punch (top die) was prescribed to move down the axis, while no movement was assigned to the bottom die. The finite element solver of ABAQUS V6.7 was used to perform the simulation.

The 3D simplified finite element model, shown in Figure 7-2, has the following geometric dimensions:

- Top die:

- Flat-to-Flat Diameter: $34.09 \mathrm{~mm}$

- Diameter: $35.57 \mathrm{~mm}$

- Fillet Radius: $0.05 \mathrm{~mm}$

- Curvature: $0.3 \mathrm{~mm}$

- Rim Height: $0.27 \mathrm{~mm}$

- Relief: no relief

- Bottom die:

- Flat-to-Flat Diameter: $33.91 \mathrm{~mm}$

口 Diameter: $35.54 \mathrm{~mm}$

- Fillet Radius: $0.05 \mathrm{~mm}$

- Curvature: $0.2 \mathrm{~mm}$

- Rim Height: $0.27 \mathrm{~mm}$

- Relief: no relief

Figure 7-3 shows the blank deformation at the end of the $3 \mathrm{D}$ coining simulation when the flash already forms in the gap between dies and side ring. The Load Vs Displacement solutions for the simplified coining process obtained from 2D and 3D FEM model are presented in Table 7-1 and Figure 7-4. The Load results obtained from 3D FEM simulation were adjusted by multiplying a factor 18 due to only a 20 degree fraction of blank was used in the $3 \mathrm{D}$ coining simulation. The results in Figure 7-4 and Table 7-1 show that 2D FEM simulation and 3D FEM simulation gave very close results for the same coining conditions. Around the point of $100 \%$ axial stroke as described in Chapter 6, the loads from 2D and 3D simulations are $1689 \mathrm{kN}$ and 
$1654 \mathrm{kN}$ respectively. The predicted load difference between the two models is only about $2 \%$. This shows a good agreement between the 2D and $3 \mathrm{D}$ simulation solutions and the two results fall in the same range of the actual silver coining load (about 1500kN) at Mint [Royal Canadian Mint, 2007]. In this way, it is shown that the 3D FEM approach is a reliable and repeatable method to predict a convergent loading in the coining process study where analytical or experimental results are not available.

\subsection{D FEM Simulation of Coining Model with Serrations on Side Ring}

In this section, the effects of serration on the side ring are to be investigated using the 3D simplified FEM coining model. The serration has a depth of $0.18 \mathrm{~mm}$ and a plug gauge of $35.67 \mathrm{~mm}$ [Royal Canadian Mint, 2007], the detailed geometry is shown in Figure 7-5a. The serrations are added on the inner surface of the side ring, as shown in Figure 7-5b.

The material flow was analyzed and Figure 7-6 shows the deformed blank obtained in the last step of 3D FEM coining process simulation. Figure 7-7 shows the Von Mises stress contour and the equivalent plastic strain contour was plotted in Figure 7-8. From the distributions of stress and strain on the cross-section plane of the coin, it can be seen that the distributions are quite consistent and similar to the results from 2D axisymmetric models in Figure 6-14, 6-15 and Figure 6-18, 6-19. The high stress bands (indicated by red) with a maximum of about $247 \mathrm{MPa}$ and high strain bands (indicated by light blue) with a maximum of about 1.8 are located at the curvature-blank interface area and the rim section. The maximum stress is very close to the maximum stress (about 247MPa) observed in the 2D axisymmetric coining simulations. The maximum strain is somewhat lower than the values (2.2-3) obtained in the 2D simulations, but the overall distribution is similar. The only significant difference is the high strain and stress distributions at the outer surface of the coin caused by the serrations.

The Load vs. Displacement data was compared to the results of flat side ring model simulation and presented in Table 7-2 and Figure 7-9. For the two curves in Figure 7-9, two breakpoints can be identified on each curve, where the curve slope dramatically changes. 
Specifically, Point A or A' corresponds to the beginning of the contact between the blank and the side ring. Point $\mathrm{B}$ or $\mathrm{B}^{\prime}$ corresponds to the moment when the external rim projection comes into contact with the die. At the same displacement, the load from the model with serration is observed to be lower than the load from the model without serration. There are a few factors to explain the effects: First, the initial contact of blank material with the side ring starts later, note the shifting of point $A$ to $A^{\prime}$, from $0.29 \mathrm{~mm}$ to $0.32 \mathrm{~mm}$. Second, the rim projection formation and contact with die were also delayed from B to B'. Finally, due to the larger effective side ring inner diameter induced by the addition of serrations on the inner surface of side ring, the radial material flow of the blank was increased. All of these reasons account for the similar trend but lower force observed at the same displacement for the simulation of 3D FEM model with serrations. But at the same time, it should be noted that in terms of the load at $100 \%$ axial stroke, the model with serration predicting $1734 \mathrm{kN}$, which is $4.8 \%$ higher than the load ( $1654 \mathrm{kN})$ from the model without serration.

\subsection{D FEM Coining Simulation with Relief on One or Two Sides}

The real coin production process at the Mint starts with the design by the artists. The coin surface features designed by the artists are then digitized by scanning and converted into the CAD data for the engineering department. Figure 7-10 shows the two side features of a typical silver coin after scanning.

However, with the exception of some special FEM codes, most common commercial FEM codes, such as ABAQUS and ANSYS, don't recognized the scanning file and can't take the scanning file as the input data for a FEM model to do the simulation. This will require some pre-work to be done to convert the scanning file into FEM workable file, like ACIS or STEP file. Therefore in order to facilitate the application of the 3D FEM approach in the real coining process, a brief description of data conversion will be presented in the next section. 


\subsubsection{Coin Feature Scanning File Conversion}

The Royal Canadian Mint generates coin surface designs in Stereolithography (STL) file format by ArtCAM software [Delcam, 2009] as shown in Figure 7-10. For the purposes of creating surfaces for finite element analysis, solid models of the surfaces must be generated. A research assistant, Christine Smith conducted some work to exam the STL file, reduce the file size and finally import the file into 3D CAD modeling program [Smith, 2009].

In scanning, STL files are used to build physical 3D CAD models for rapid prototyping (RP). STL files are created using a mesh made from triangles to represent the 3D surface geometry of the physical part. From the available PC memory, it was determined that a file size of 2.2 MB could be accommodated [Smith, 2009]. To create a manageable file to work with and produce an appropriate prototype, a reasonable resolution must be chosen. When the resolution is too low, the flat spots will show up when the part is produced. Increasing the resolution excessively does not improve the quality of the produced part and high resolution only slows the prototyping process.

From examination of the file formats compatibility and using trial and error, it was determined that the ACIS (.sat) or STEP (.step) files provide the best format for importing and exporting 3D geometry to/from CAD and finite element analysis [Smith, 2009].

From the examination of the scanning file, it is evident that the original Queen and Indian Head designs, shown in Figure 7-10, are highly complex with extremely large file sizes (70MB in total). The file size and complexity make it difficult to import into and manipulate in solid modeling computer aided design (CAD) software. The files were reduced to about $2 \mathrm{MB}$ in size to allow importation into CAD software.

The results of importing an STL file into 3D modeling and the finite element software were shown in Figure 7-11. The models contain the profiles of the Queen and Indian Head without complex depth curves [Smith, 2009]. 


\subsubsection{D FEM Coining Process Simulation with Surface Designs}

It has been found that the complexity and the file size make it very difficult to create a manageable model to simulate the coining process for a real silver dollar coin, shown in Figure 7-12. It was determined that some simplified models with the key design features will be used instead. To reduce the complexity of the coin surface relief, the letters in the design were removed and the surface relief of the figure was replaced by the $0.1 \mathrm{~mm}$ stepped cavities along the feature of the figure.

Two FEM models were created by converting and simplifying the STL scanning files provided by the Royal Canadian Mint. The two models have the same corresponding geometries, i.e. the diameters, curvatures, rim height and serrations. The only difference is that the first model (one-side relief model) has one die with $0.1 \mathrm{~mm}$ reduced Queen profile relief, the other blank die with no relief, as shown in Figure 7-13, and the second model (two-side relief model) has one die with the same Queen profile relief as in the first model, the other die with $0.1 \mathrm{~mm}$ reduced Indian-chief profile relief, as shown in Figure 7-14.

The solid die models were meshed by the rigid elements. Figure 7-15 and 7-16 shows the die mesh used in the simulation of the one-side relief model and two-side relief model respectively. The serrations were grooved on the inner surface of the side ring. Since only the serration surface has contact with the coining blank in the simulation, it will be sufficient to model the inner surface of the side ring by rigid element, shown in Figure 7-17. A circular blank with $35.63 \mathrm{~mm}$ diameter and $2.486 \mathrm{~mm}$ gauge was created and meshed with C3D8R brick elements. Considering the PC memory, result file size and computation time, about 1,600,000 elements were used in the model. The material model obtained in Chapter 4 , the friction model obtained in Chapter 5 and the numerical techniques discussed in Chapter 3 were applied in the 3D FEM coining process simulation and the simulation assembly sketch is shown in Figure 7-18.

The deformed mesh of the coining blank of the one-side relief model was presented in Figure 7-19. The stepped relief of Queen's head was formed on the reverse side of the coin, shown in Figure 7-19a. Figure 7-19b shows the blank obverse side (no relief) of the coin. Figure 
7-20 and 7-21 show the Von Mises stress and equivalent plastic strain contours on the coin surface in the one-side relief model. It can be seen that the high stress and strain bands are formed along the edge of the stepped cavities and serrations. The stress levels in the area covering the Queen's head relief on reverse sides and the corresponding area on obverse side are higher than the stress levels in the surrounding area. Figure 7-22 and Figure 7-23 show the Von Mises stress contour and equivalent plastic strain contour on a typical cross section of the deformed coin with one-side relief. In general, the stress and strain distributions on the cross-section are similar to the stress and strain distributions on the cross-section of 2D model in the relief study. The stress gradient (from high to low) is from contact surface to mid-thickness and from inner part to outer part. The high stress bands are at the vicinity of the stepped relief and in the rim section. The similar trend can be found in the strain distribution on the cross-section. The maximum stress in the coin is about 247MPa, which is very consistent with the maximum stress (about 247MPa) observed in the 2D simulations. The maximum strain is about 2.4, which is in the similar range of the values observed in $2 \mathrm{D}$ simulations.

The deformed mesh of the two-side relief model was presented in Figure 7-24. The Indian's head relief was formed on the obverse side of the coin, shown in Figure 7-24a. Figure 7-24b shows the Queen's head relief formed on the Reverse side of the coin. Figure 7-25 and 7-26 show the Von Mises stress and equivalent plastic strain contours respectively on the coin surface in the two-side relief model. On both sides of the coin, it can be seen that the high stress and strain bands are formed along the edge of the stepped cavities and serrations. It also can be observed that the stress and strain in the center areas covering the relief on both sides are somewhat higher than the surrounding area. Figure 7-27 and Figure 7-28 show the Von Mises stress contour and equivalent plastic strain contour on a typical cross section of the deformed coin with two-side relief. In general, the stress and strain distributions follow the same patterns as those in the one-side relief model except that the high stress and strain bands are bigger due to the added relief on the obverse side. The 3D two-side relief simulation predicted the maximum stress at about 247MPa, shown in Figure 7-25 and Figure 7-27, which is consistent with the 3D 
one-side relief simulation and 2D simulations, both of them giving the maximum stress at about 247MPa. The maximum strain in the 3D two-side relief simulation, around 2.7 , is in the similar range of the maximum strains in the one-side relief simulation and $2 \mathrm{D}$ simulations. The agreements in stress and strain among the 2D and 3D simulations show that the 2D simulations can capture the key features during the coining process.

The load-displacement results from the 3D FEM coining process simulations are presented in Table 7-3. And the results from the four different 3D models of no relief and no serrations, no relief, one-side relief and two-side relief were compared in Figure 7-29. As shown in Figure 7-29, the four loading curves show the effects of the factors in three-dimensional coining process. From Curve A to Curve B, it shows the effects of the serrations on side ring, as discussed in previous section. The effects of the reduced relief in three-dimensional coining were shown from the differences between curve $\mathrm{B}$ and $\mathrm{C}$ and the differences between curve $\mathrm{C}$ and $\mathrm{D}$. The effects are mainly due to the larger die cavities introduced by the relief added on the surfaces of the dies, which result in the formation of protrusion and the later contact between the coin blank and the side ring and between external border projection and the die as the material in the die cavities can't flow radially. The load at $100 \%$ axial stroke gradually increases from A to D as more features were included in the models, as shown in Figure 7-29.

In conclusion, the results of 3D axisymmetric models have been used to compare with the 2D axisymmetric results shown in Chapter 6. The effects of 3D features such as serrations and 3D surface designs were also investigated. All the materials and die designs in this research use the dimensions and characteristics of the "Silver Dollar" designs provided by Royal Canadian Mint. The loads at $100 \%$ axial stroke $(1654-1770 \mathrm{kN})$ from the 3D FEM simulations are consistent with the Mint's tonnage (about 1500kN) [Royal Canadian Mint, 2007] used in the real coining of the "silver dollar" and the difference is between $10 \%$ and $18 \%$, as shown in Figure 7-29, which gave added confidence in the simulations carried out in this research.

Overall, the 3D finite element coining simulation is shown to be an effective method to study the coining process. The key benefit of using finite element simulation is the ability to 
simulate the complex contact and the plastic deformation inexpensively. By starting with the material property evaluations for the precious metal and alloys used in the product at the Mint, a pool of material property models can be established like the fine silver material property model. The coining contact conditions can then be characterized by the friction model based on the die and blank materials, which represent other key input data. The initial coin profile designs can be converted into workable solid engineering models through the commercial codes. The effects of the initial designs can then be evaluated by studying the key design factors like curvature, rim height and relief height. And finally, the actual coining process can be simulated using all the various input data and the initial engineering design. Through the simulation, the material flow, load requirement, die cavity filling and other conditions like stress and strain in the coin can be obtained. And all of the results will provide indications in the design modifications. Most importantly, the effects of design modifications can be investigated by use of the simulation tool before they are fabricated.

It can also be concluded that during the silver dollar coining design process, it is reasonable to use the 2D FEM analysis to perform the preliminary evaluations of some typical coining parameters such as curvatures, rim height and relief height. Considering the higher computation costs of 3D FEM simulations, this level of analysis should be applied in the late prototype design and modifications stage. 


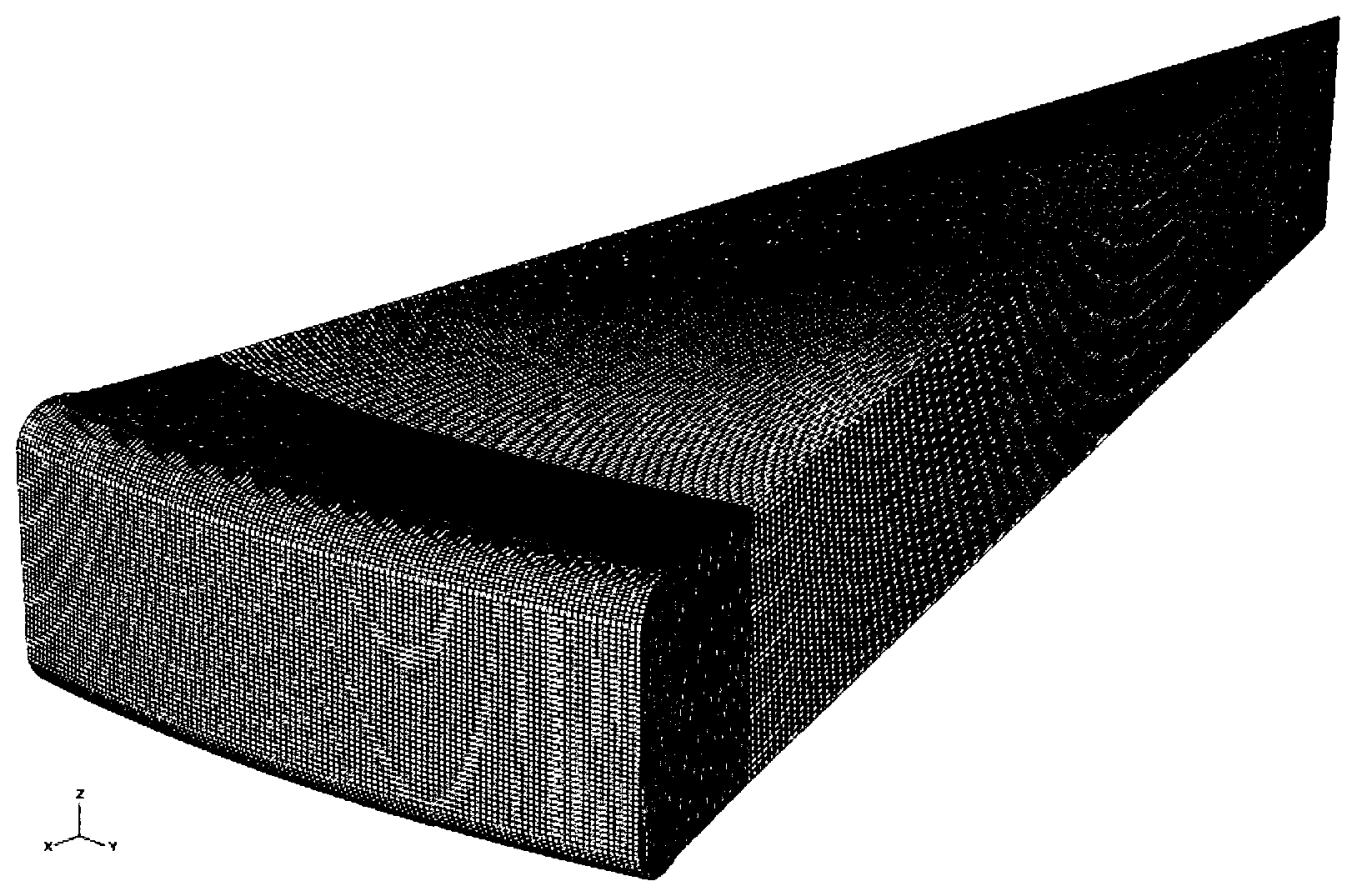

Figure 7-1. Three-dimensional silver blank mesh created with brick elements

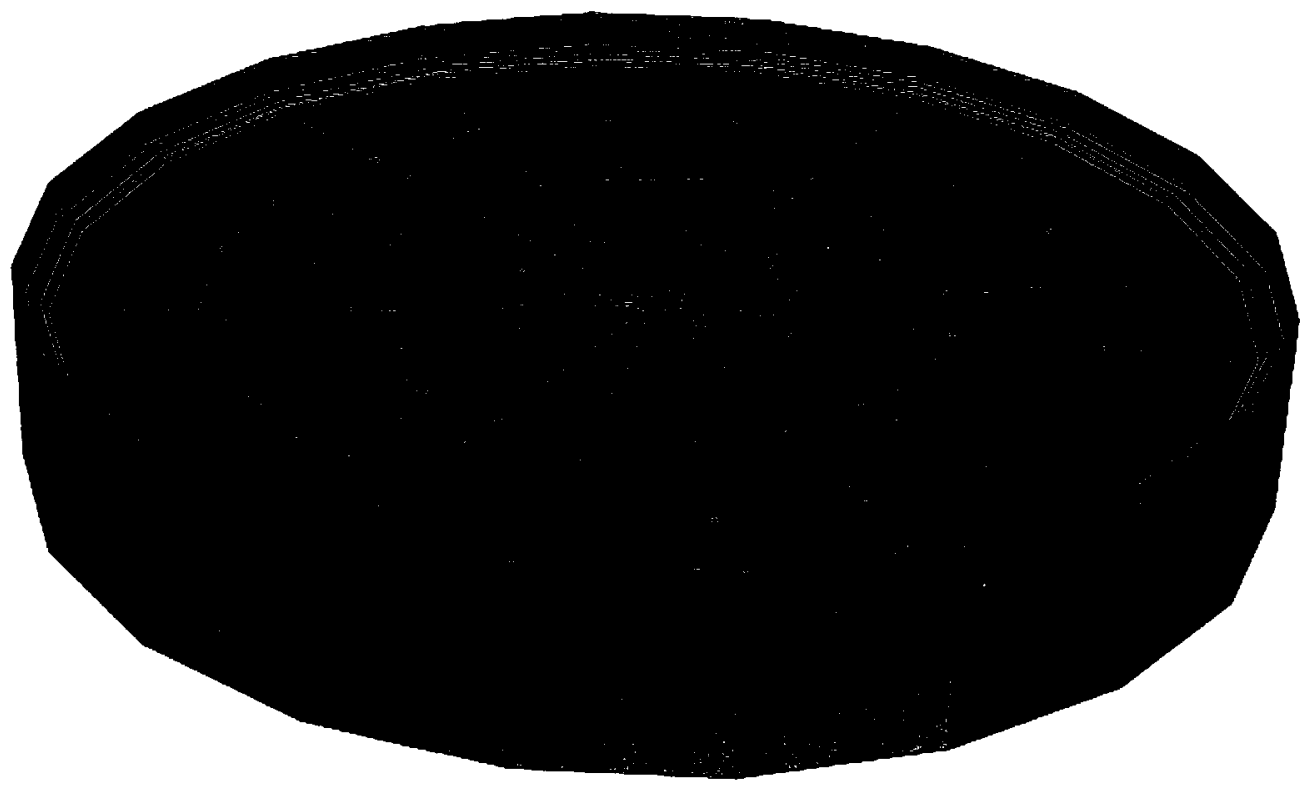

Figure 7-2. Simplified 3D finite element coining model 


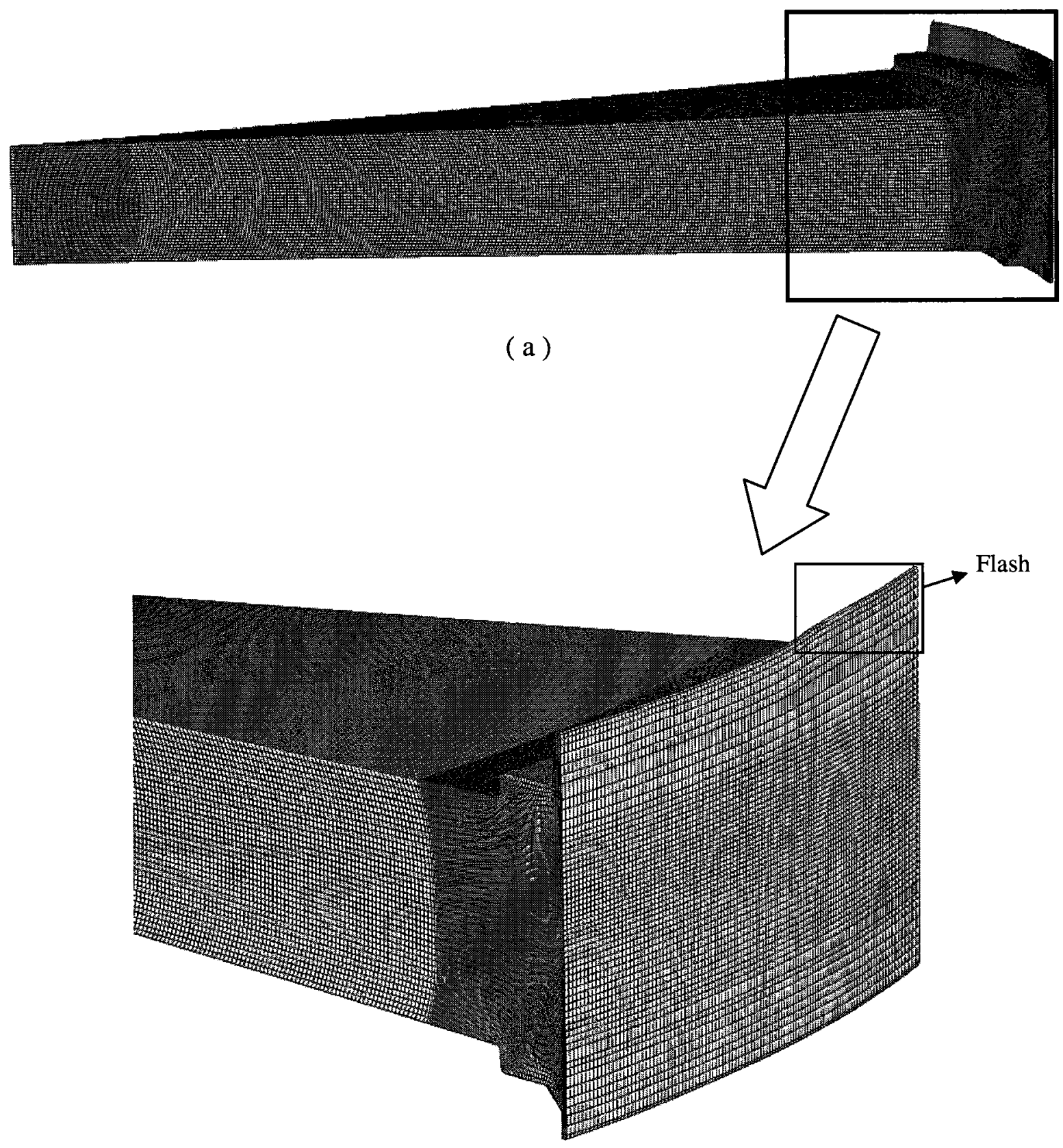

(b)

Figure 7-3. Deformed blank in 3D coining simulation 


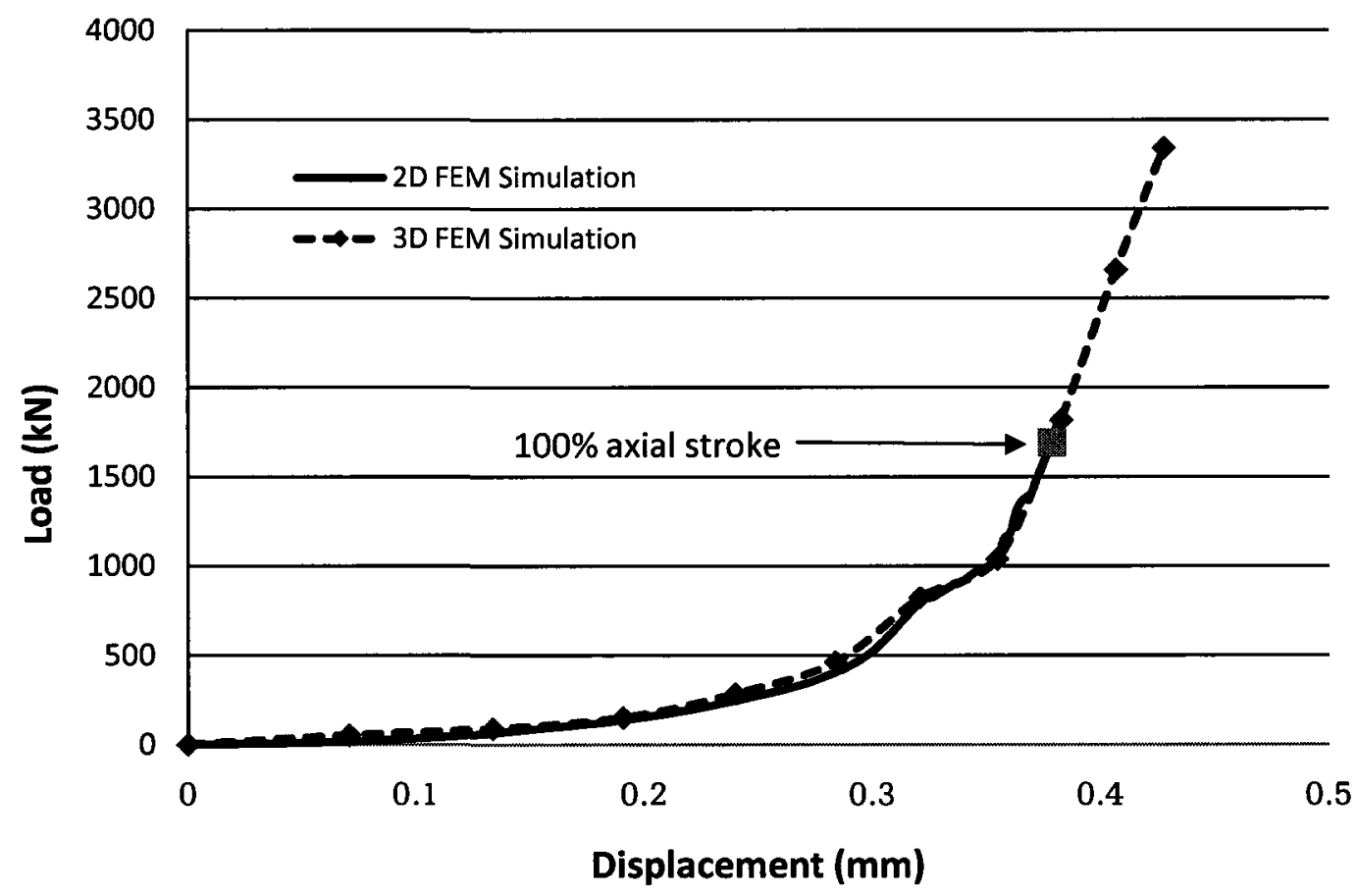

Figure 7-4. 2D and 3D FEM simulation results comparison 
(in $\mathrm{mm}$ )

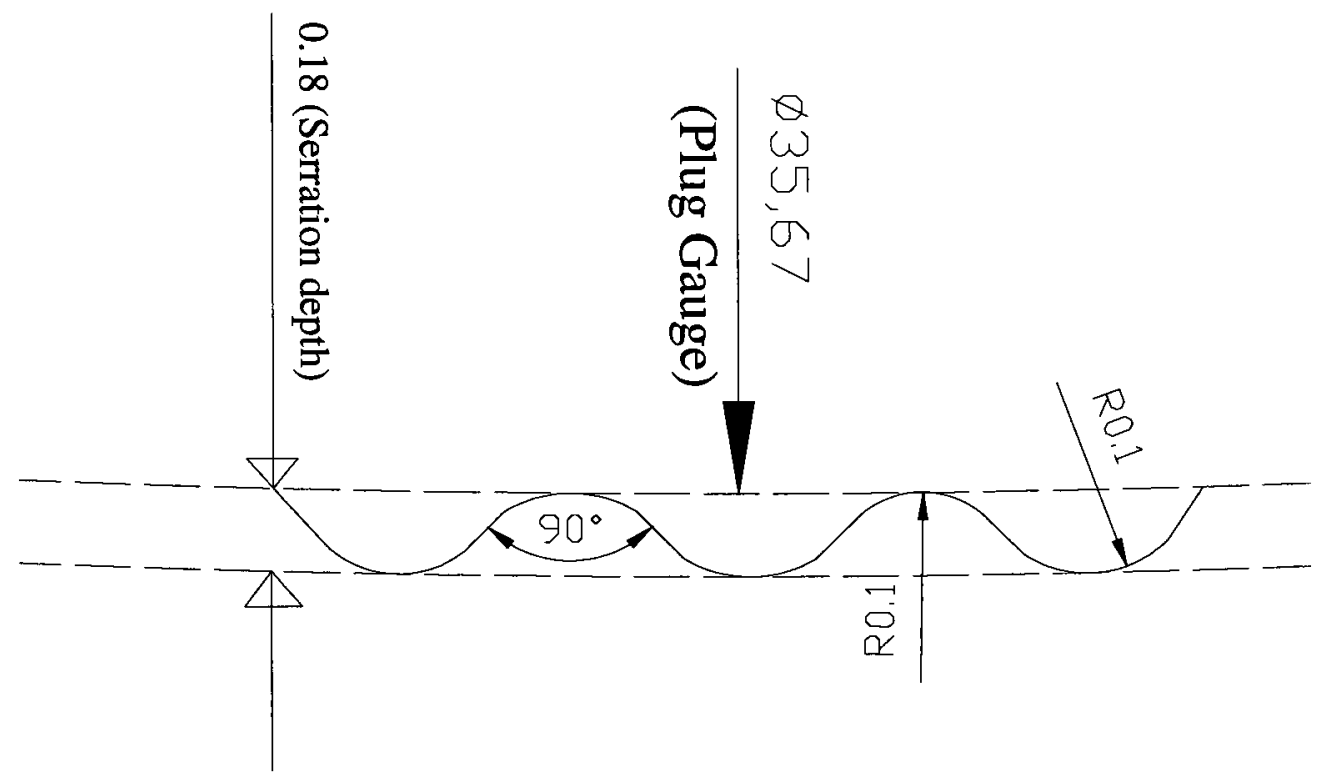

Figure 7-5a. Serration geometry [Royal Canadian Mint, 2007]

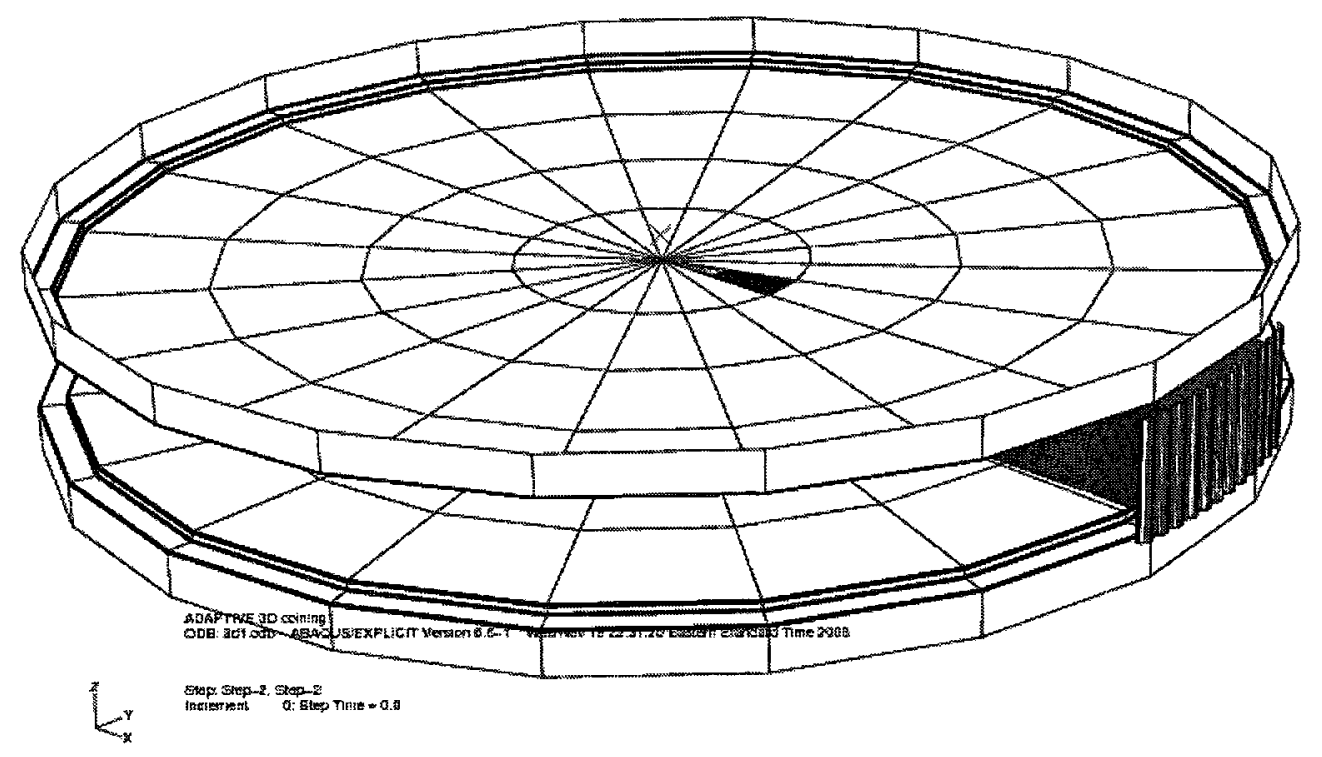

Figure 7-5b. FEM Coining configuration with serrations on side ring 


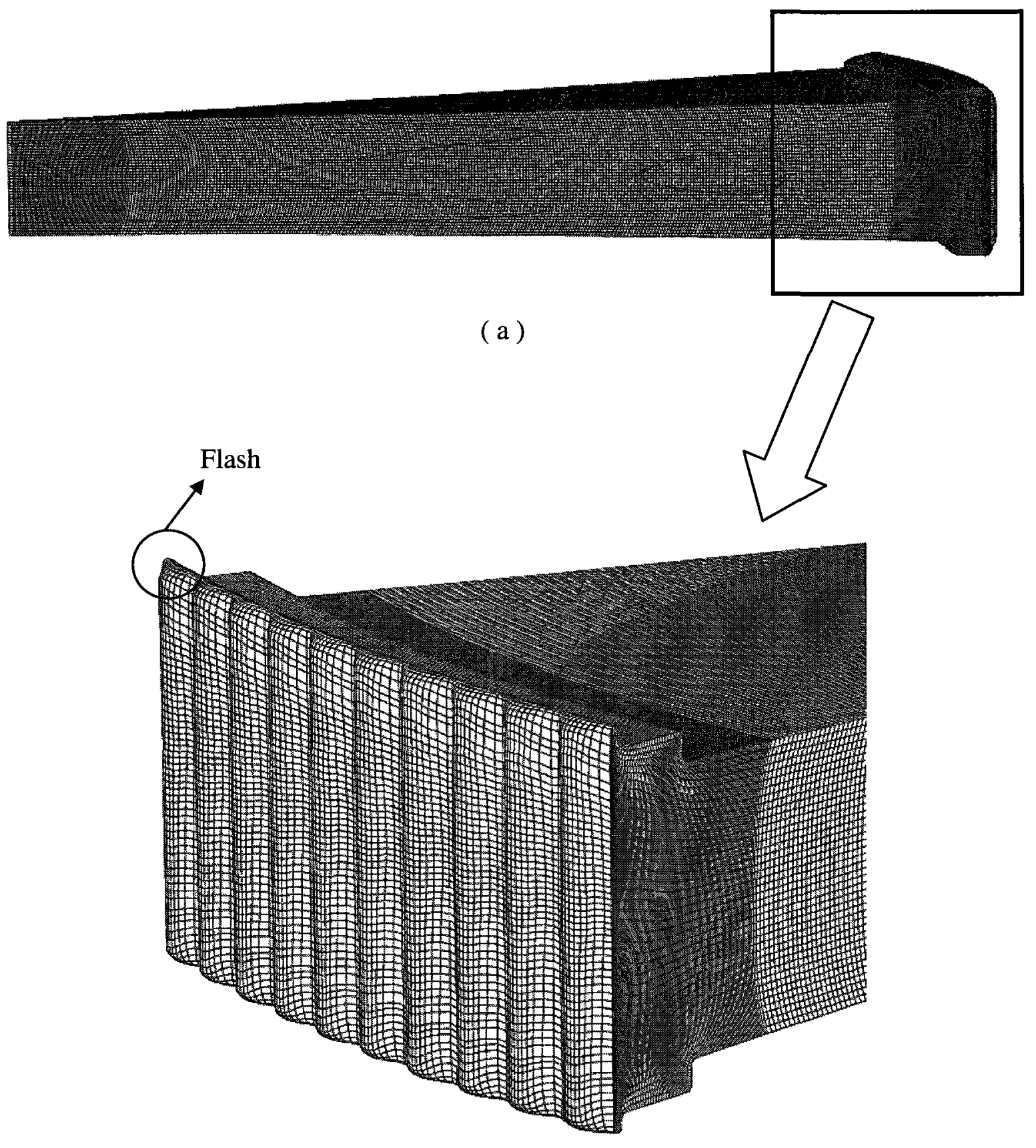

(b)

Figure 7-6. Deformed 3D coining blank with serrations 

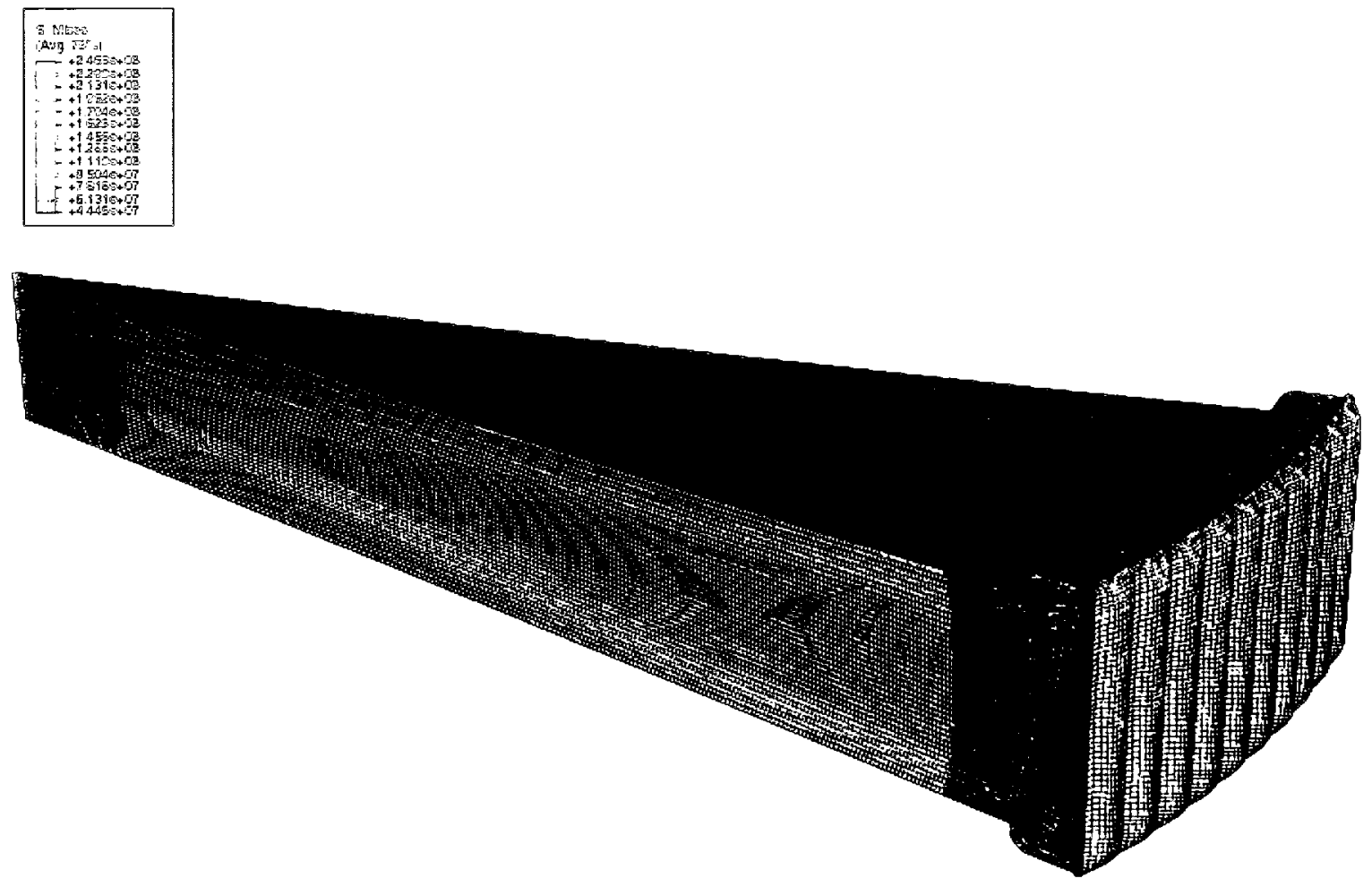

Figure 7-7. Von Mises stress contour on the simple coin with serrations
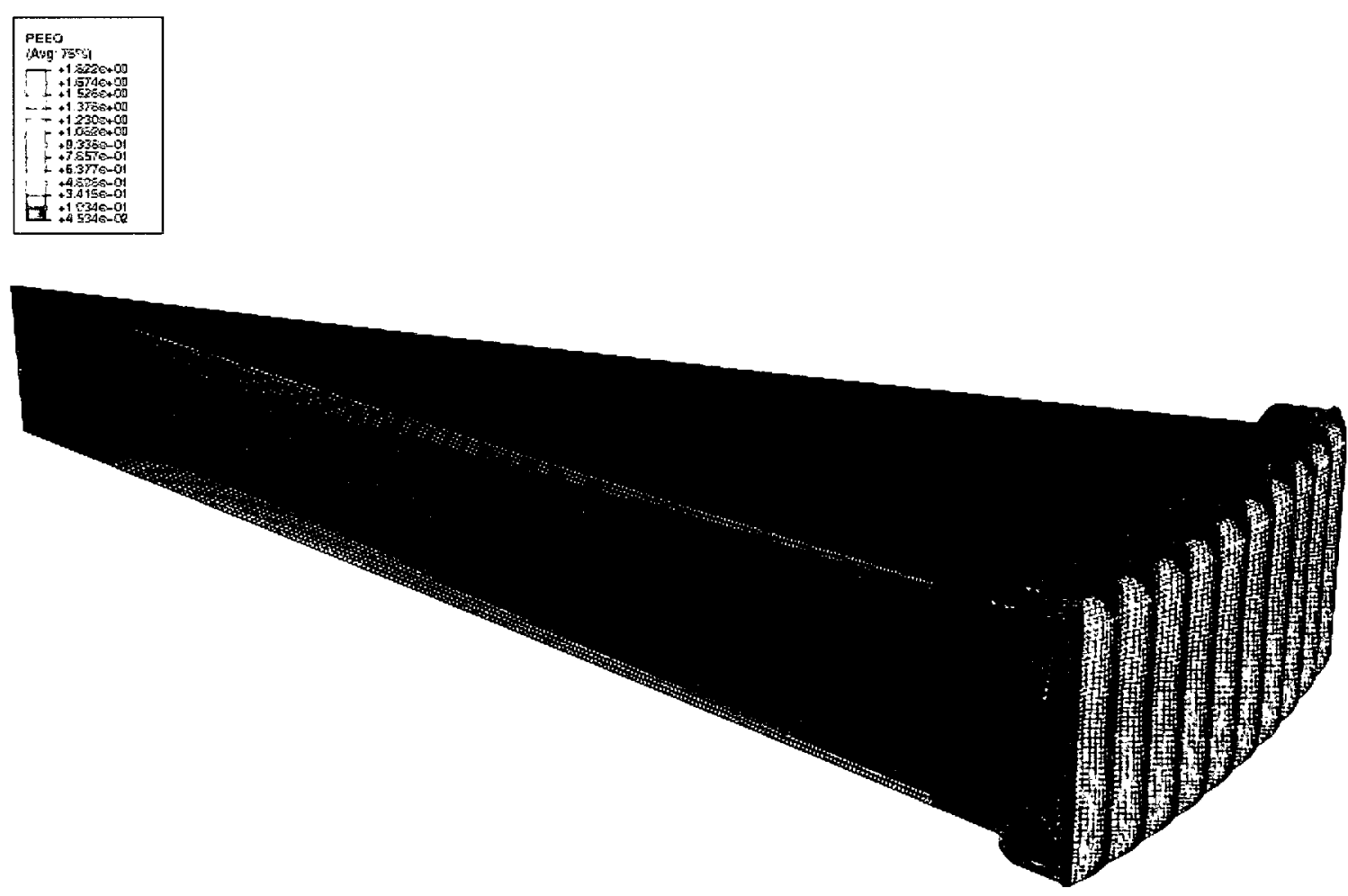

Figure 7-8. Equivalent plastic strain contour on the simple coin with serrations 


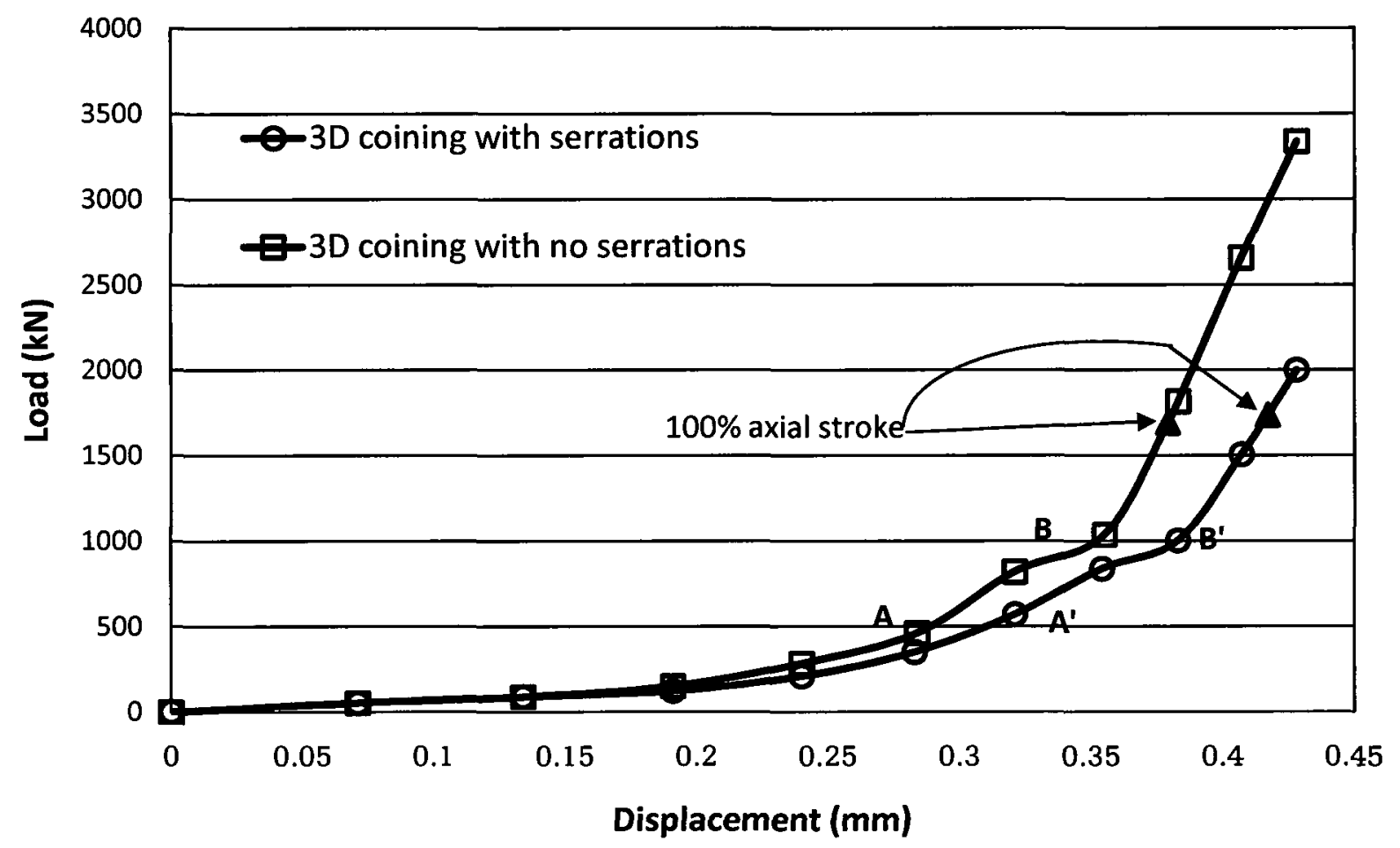

Figure 7-9. 3D coining model comparison 


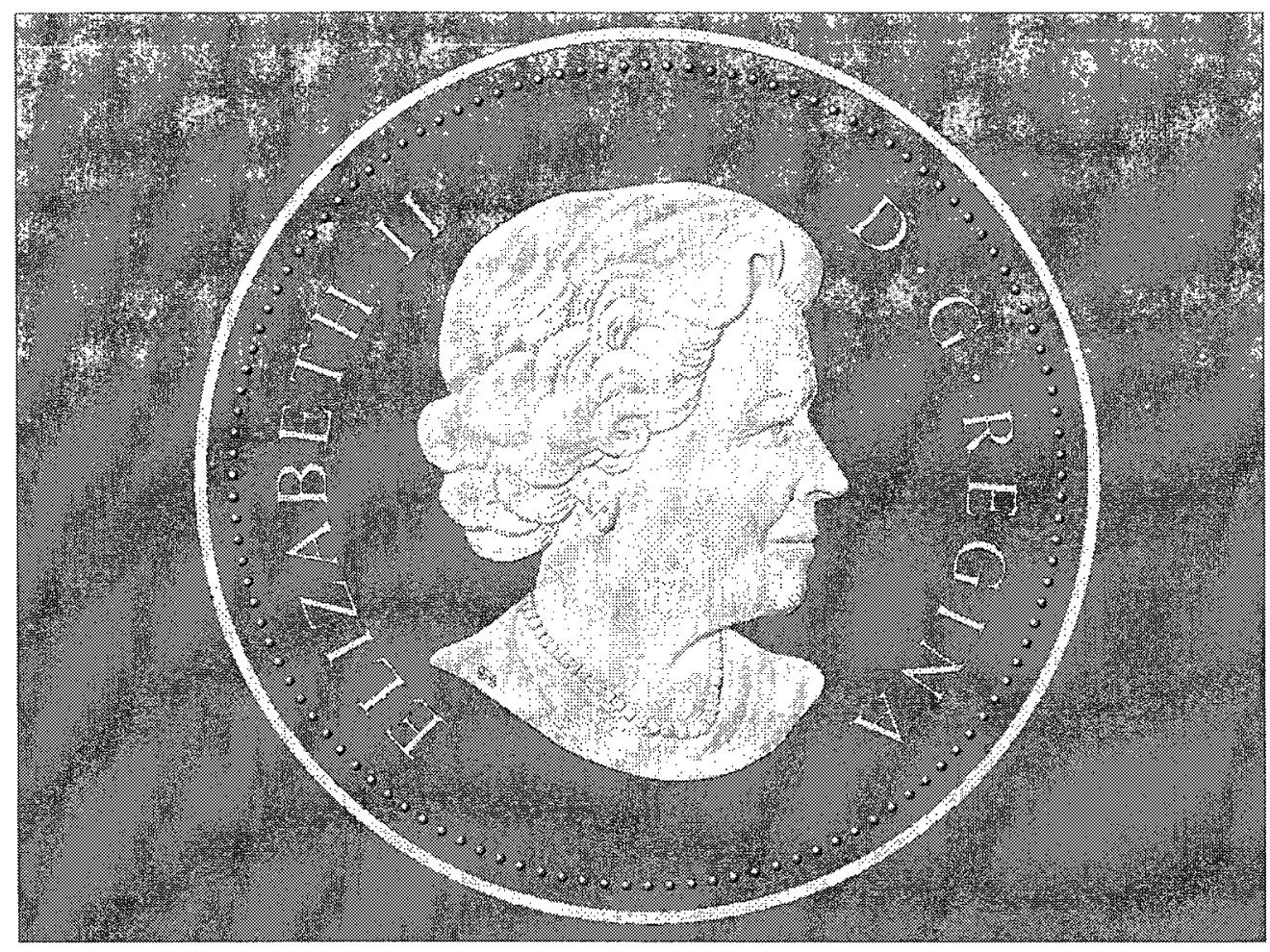

(a) Reverse side



(b) Obverse side

Figure 7-10. Design features of a coin by scanning 


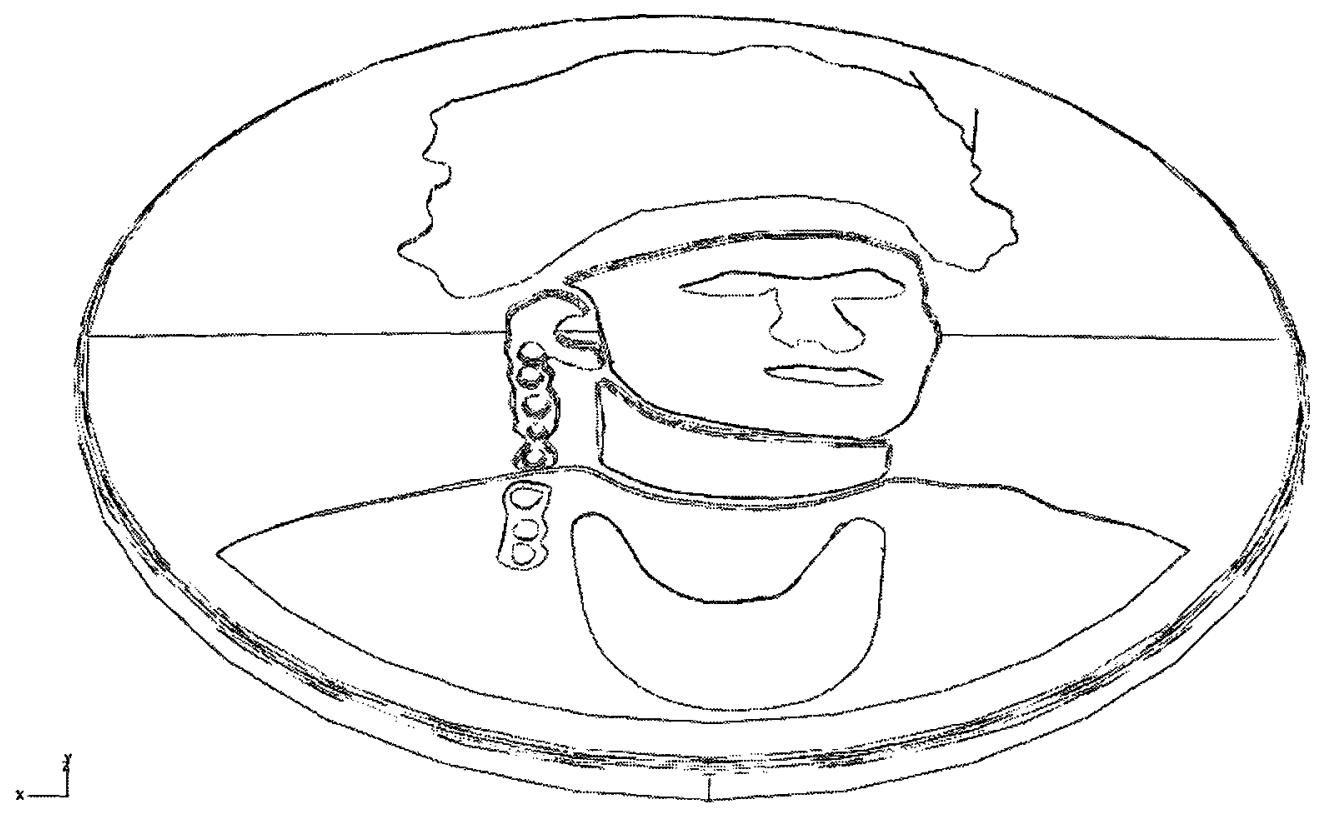

(a ) Obverse die

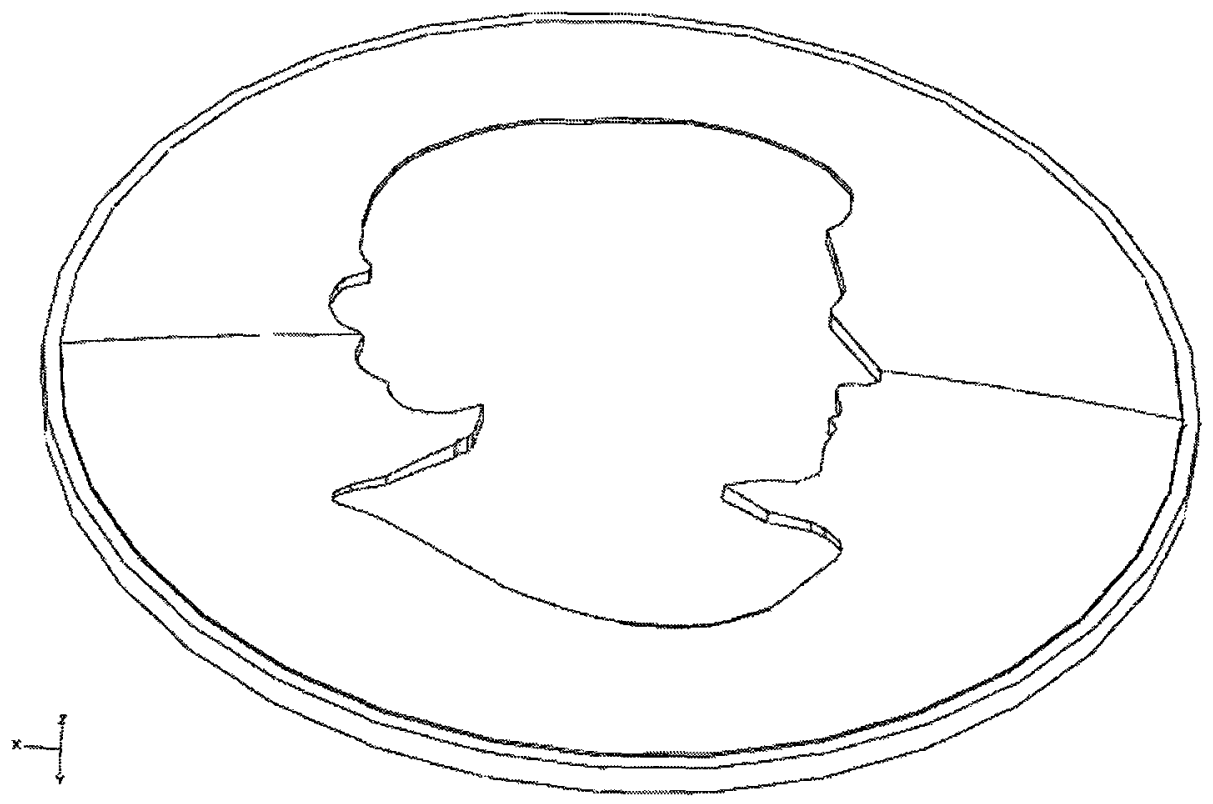

(b) Reverse die

Figure 7-11. Solid coin profile FEM model 




Figure 7-12. A silver dollar coin design at RCM 

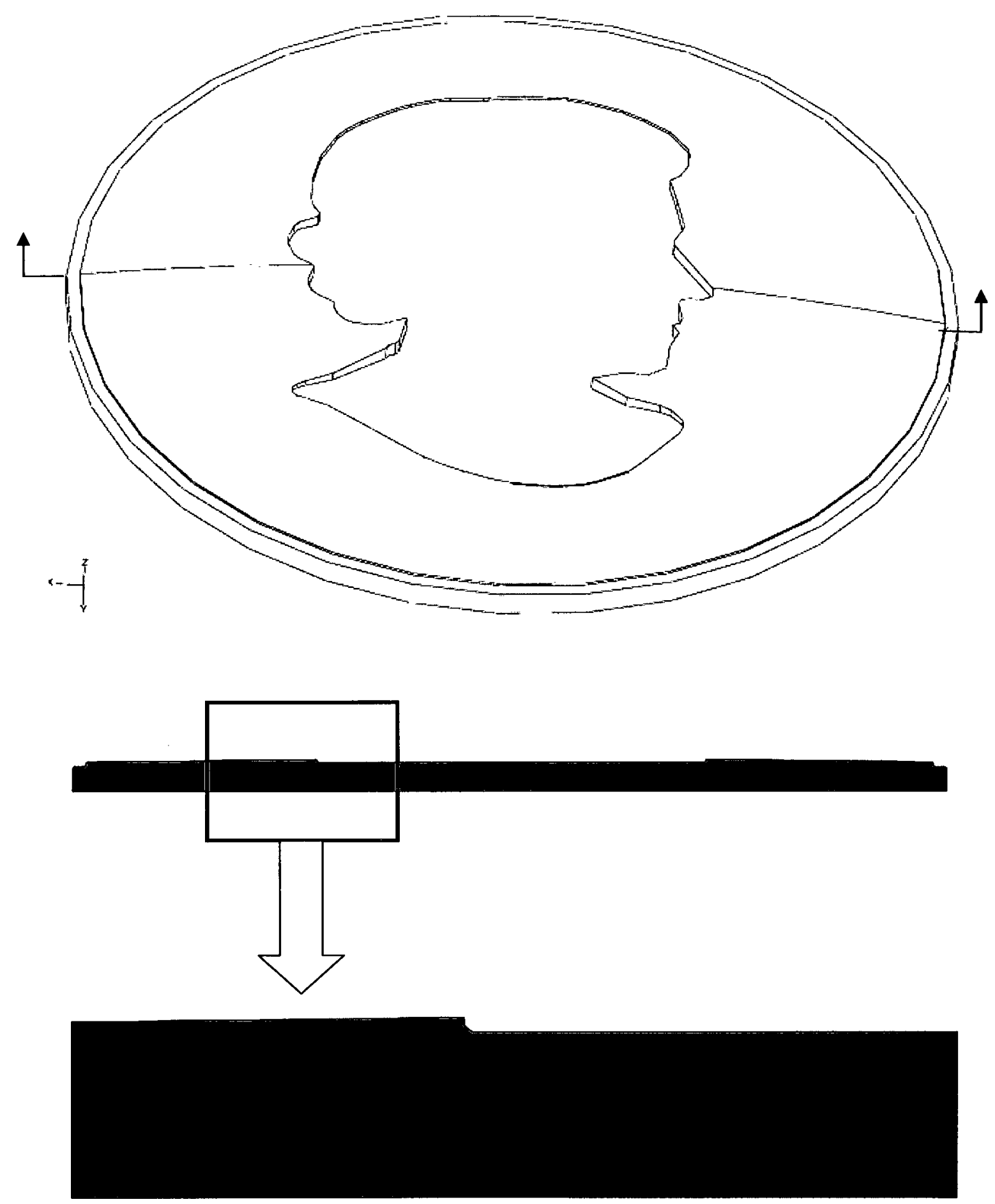

(a) Reverse Die and its cross-section

Figure 7-13. The coining dies with one-side relief 

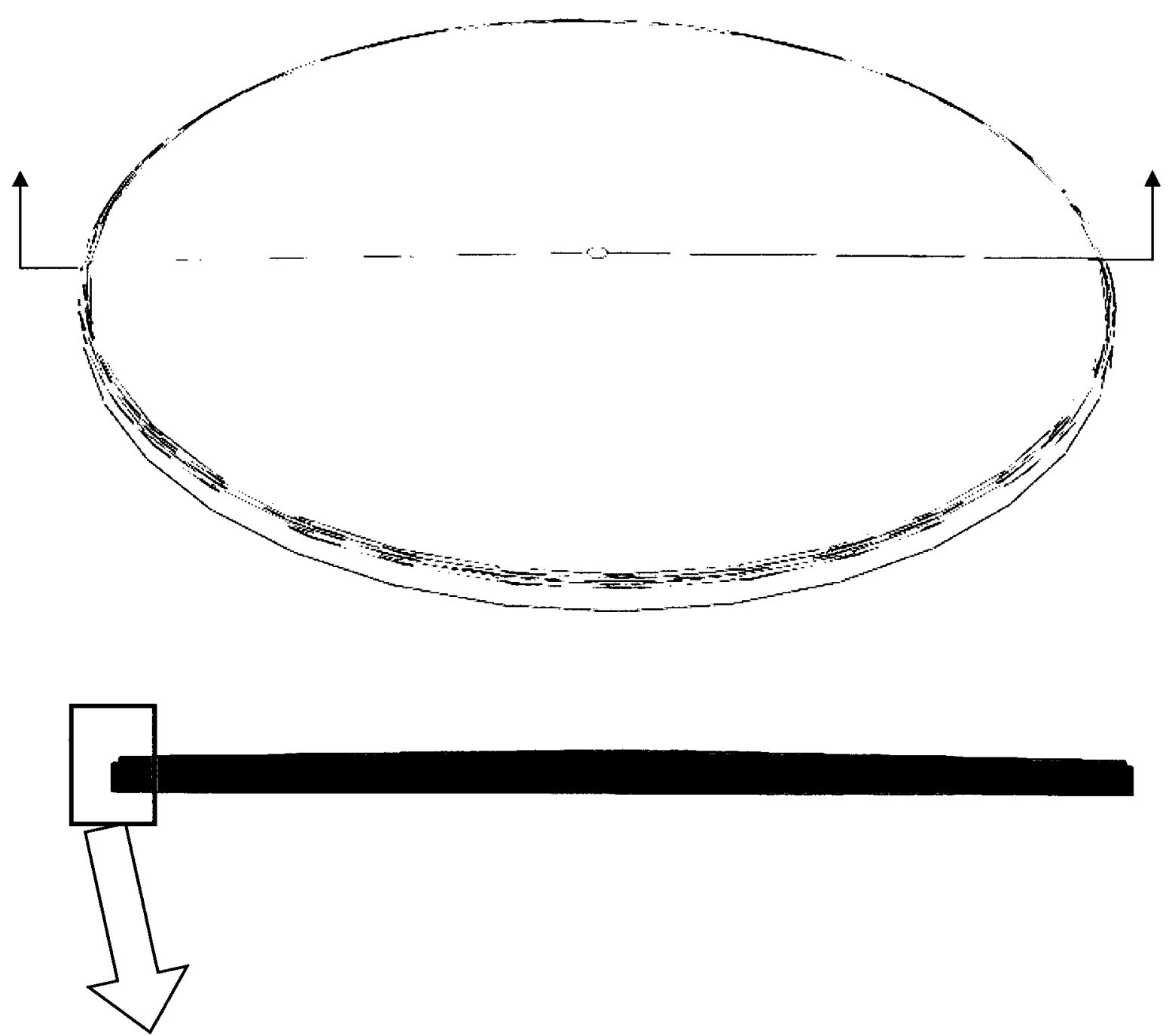

(b) Obverse Die and its cross-section

Figure 7-13. The coining dies with one-side relief 

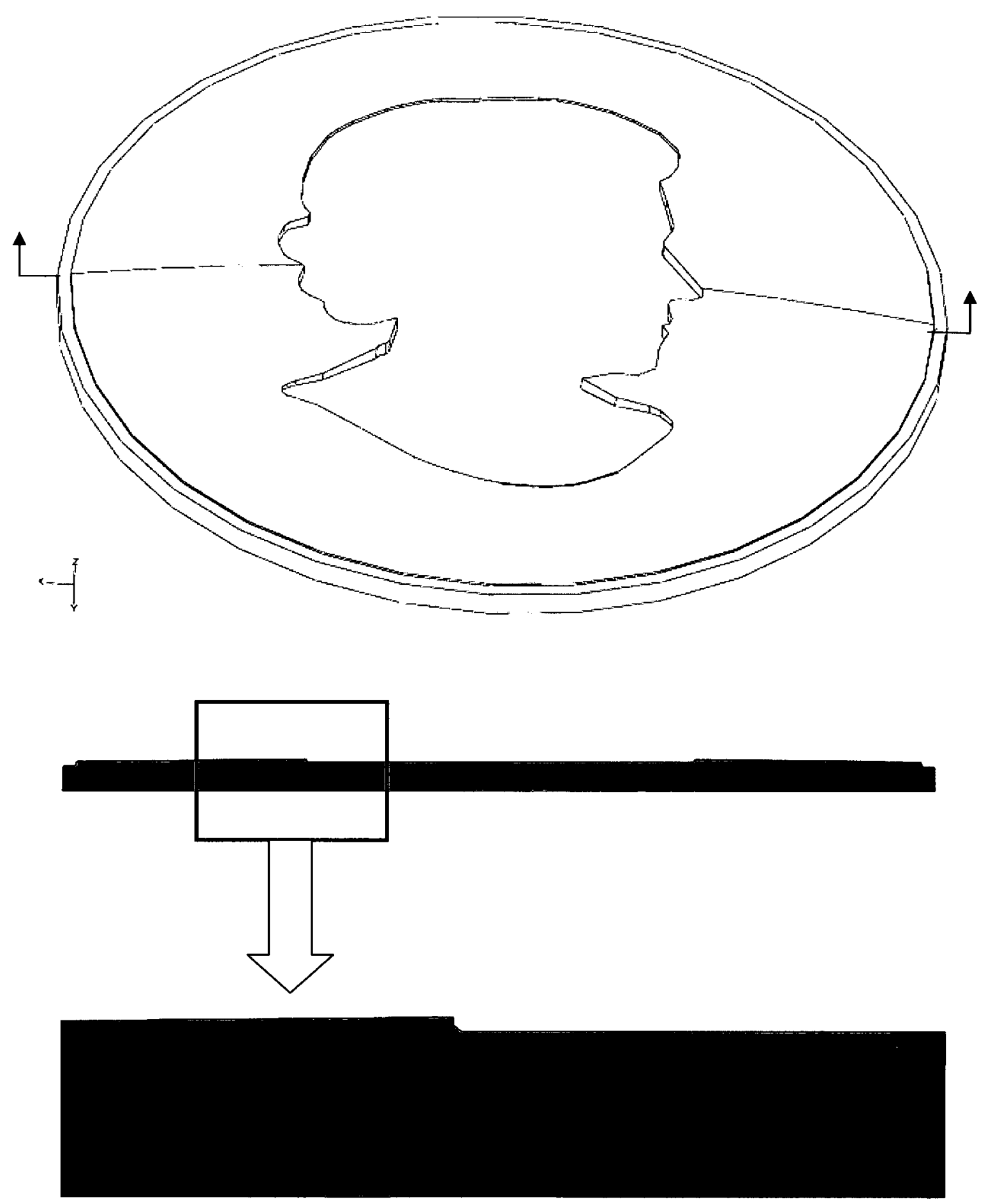

(a) Reverse Die and its cross-section

Figure 7-14. The coining dies with two-side relief 

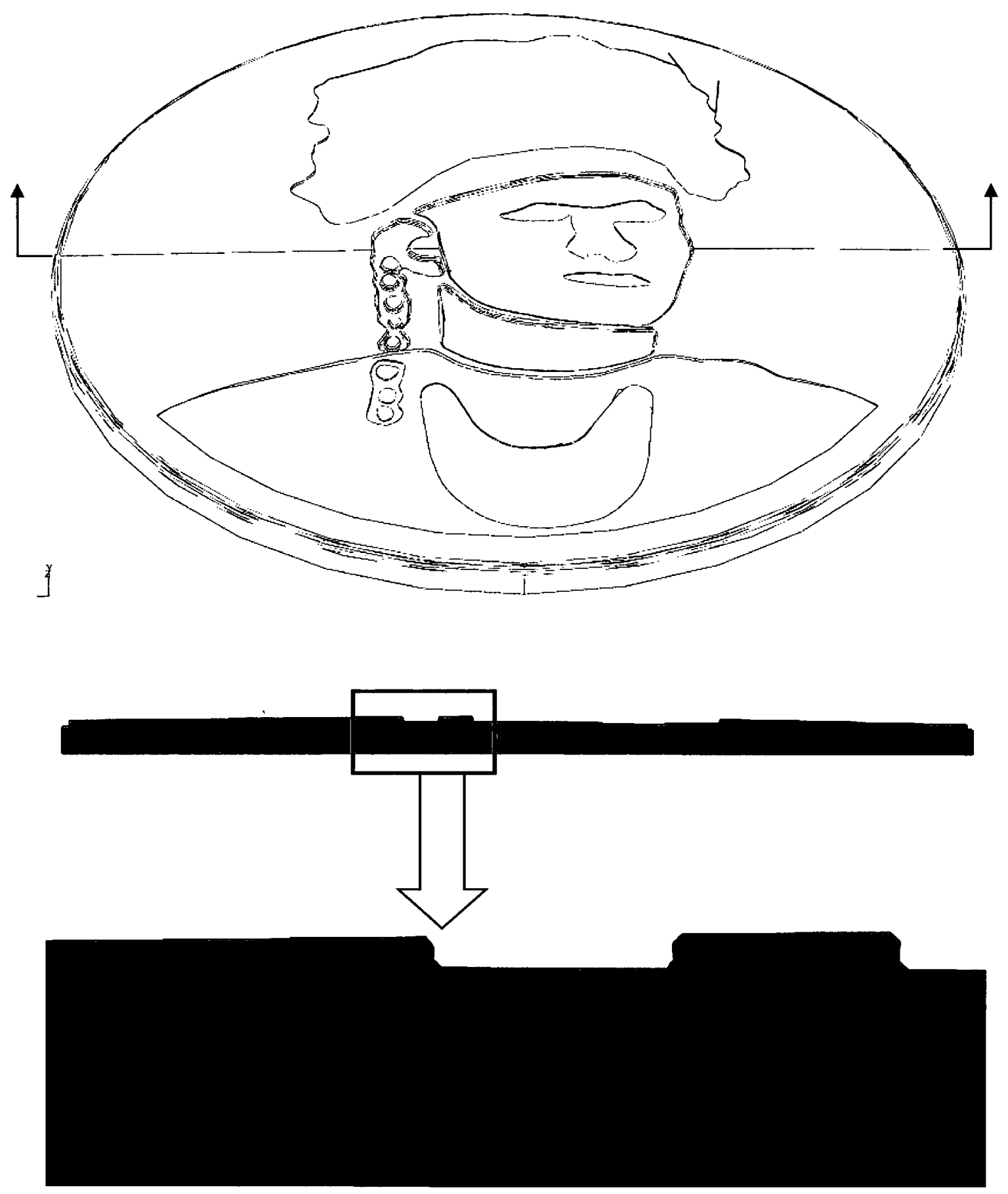

(b) Obverse Die and its cross-section

Figure 7-14. The coining dies with two-side relief 




(a) Reverse die mesh

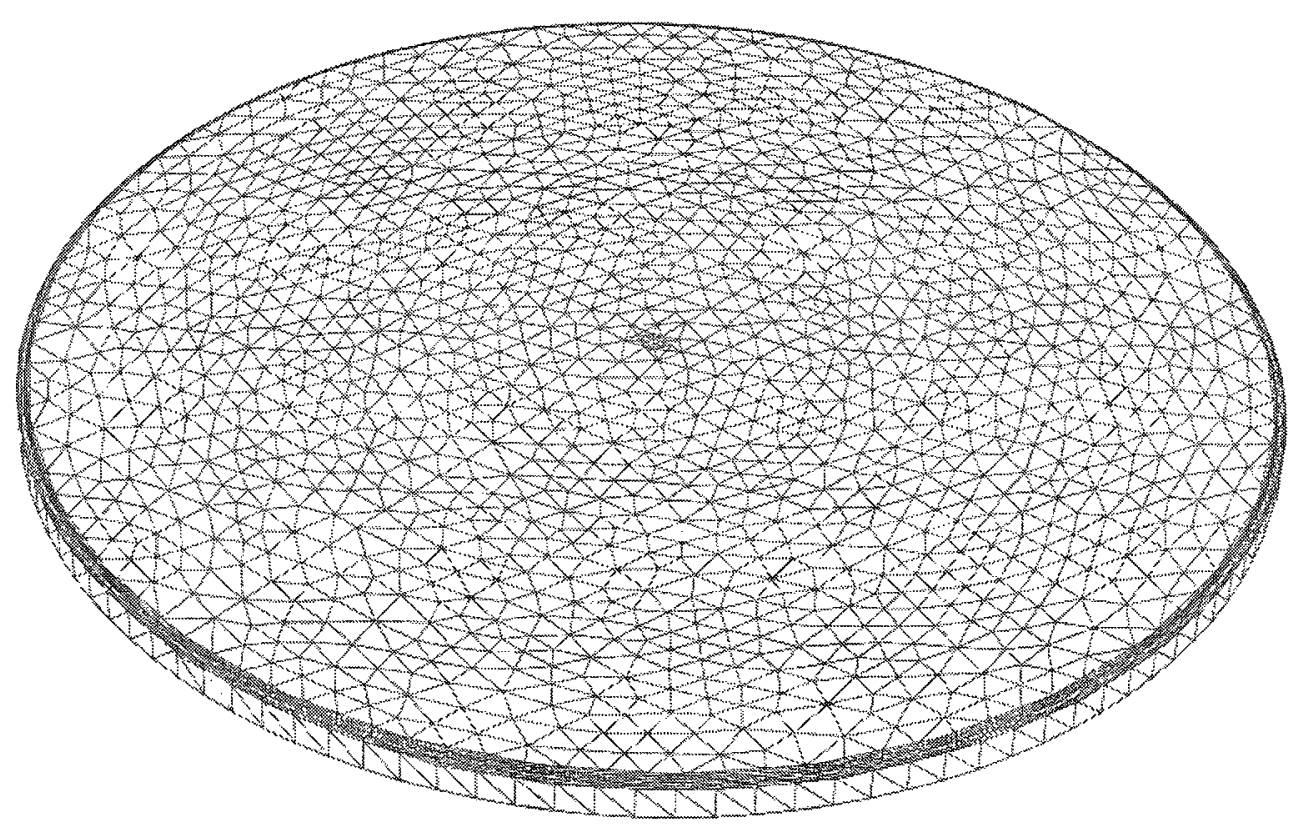

(b) Obverse die mesh

Figure 7-15. Rigid die mesh of one-side relief model 


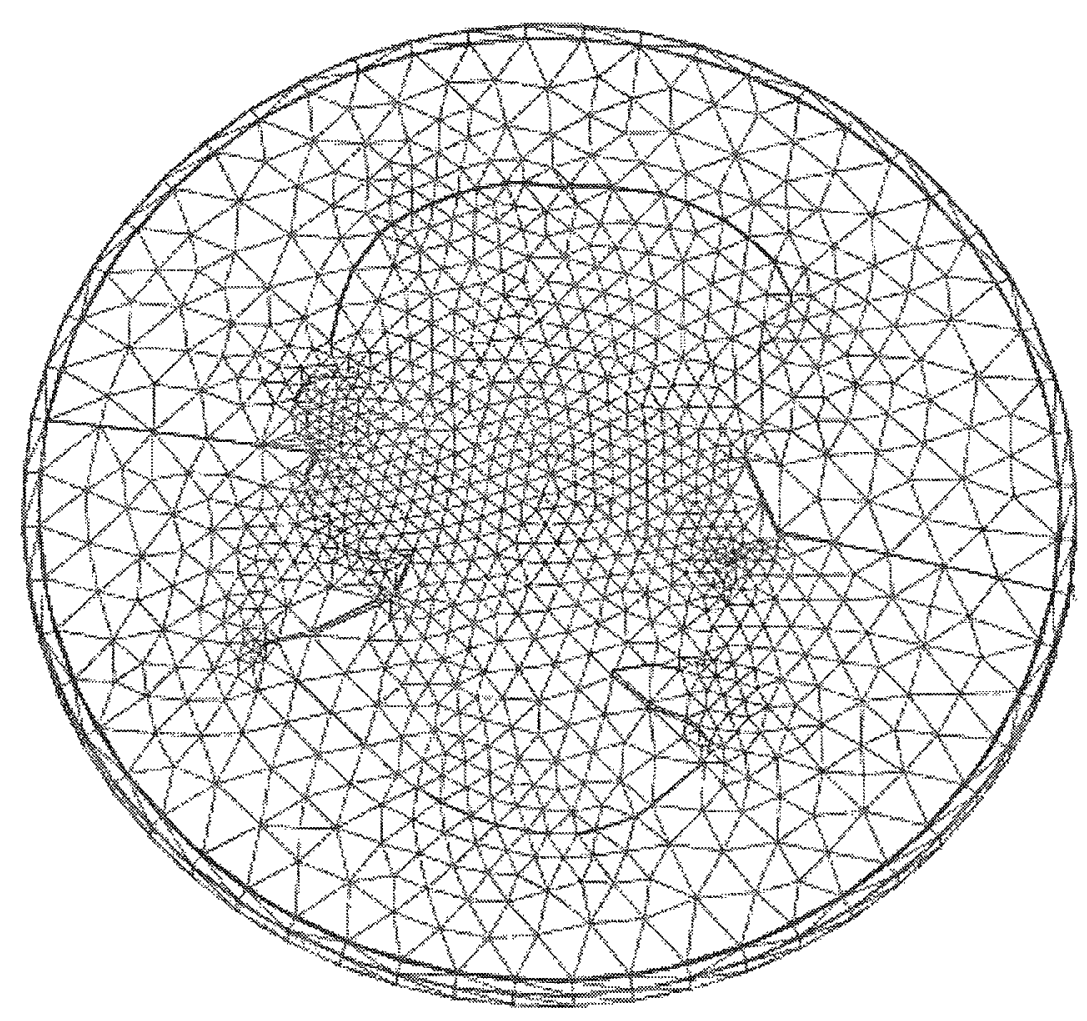

(a) Reverse die mesh

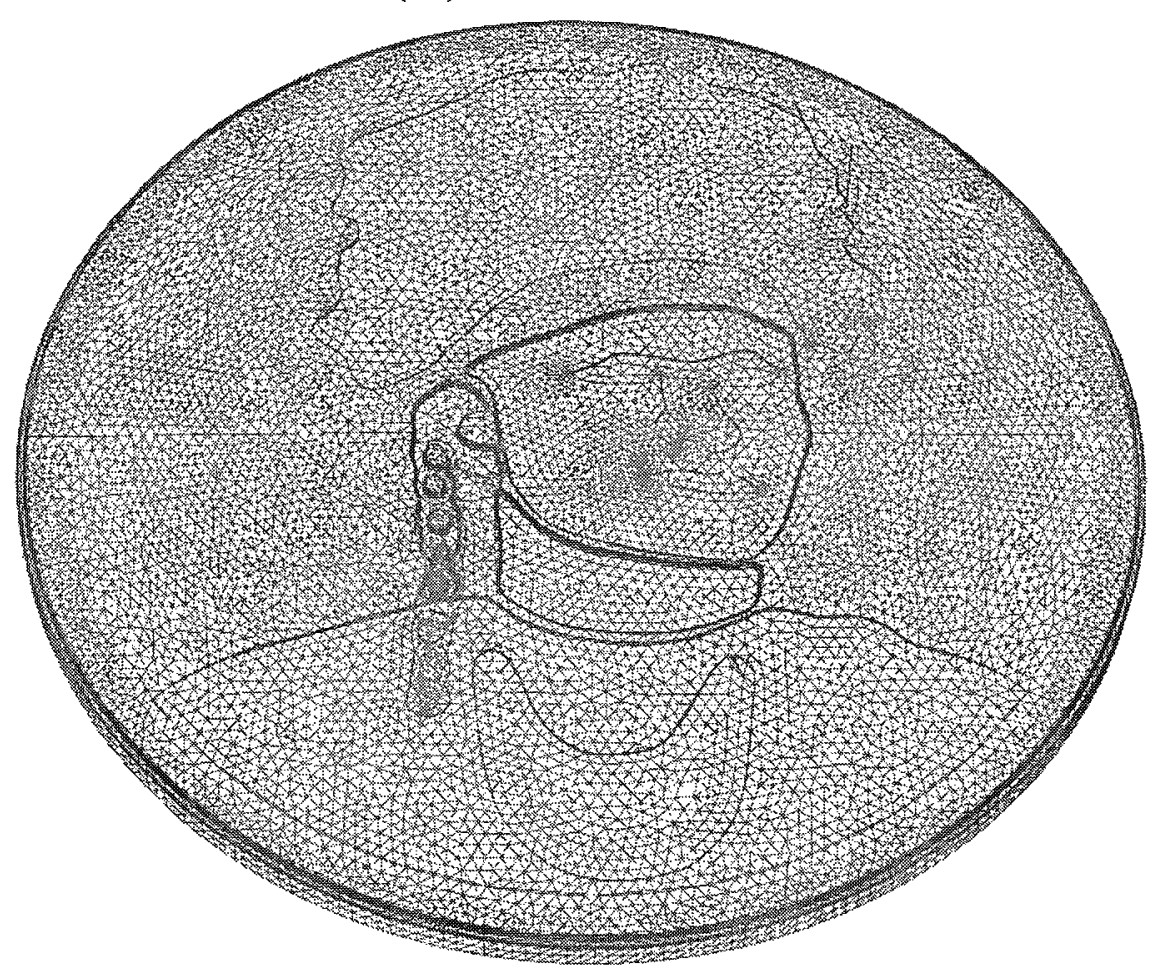

( b ) Obverse die mesh

Figure 7-16. Rigid die mesh of two-side relief model 


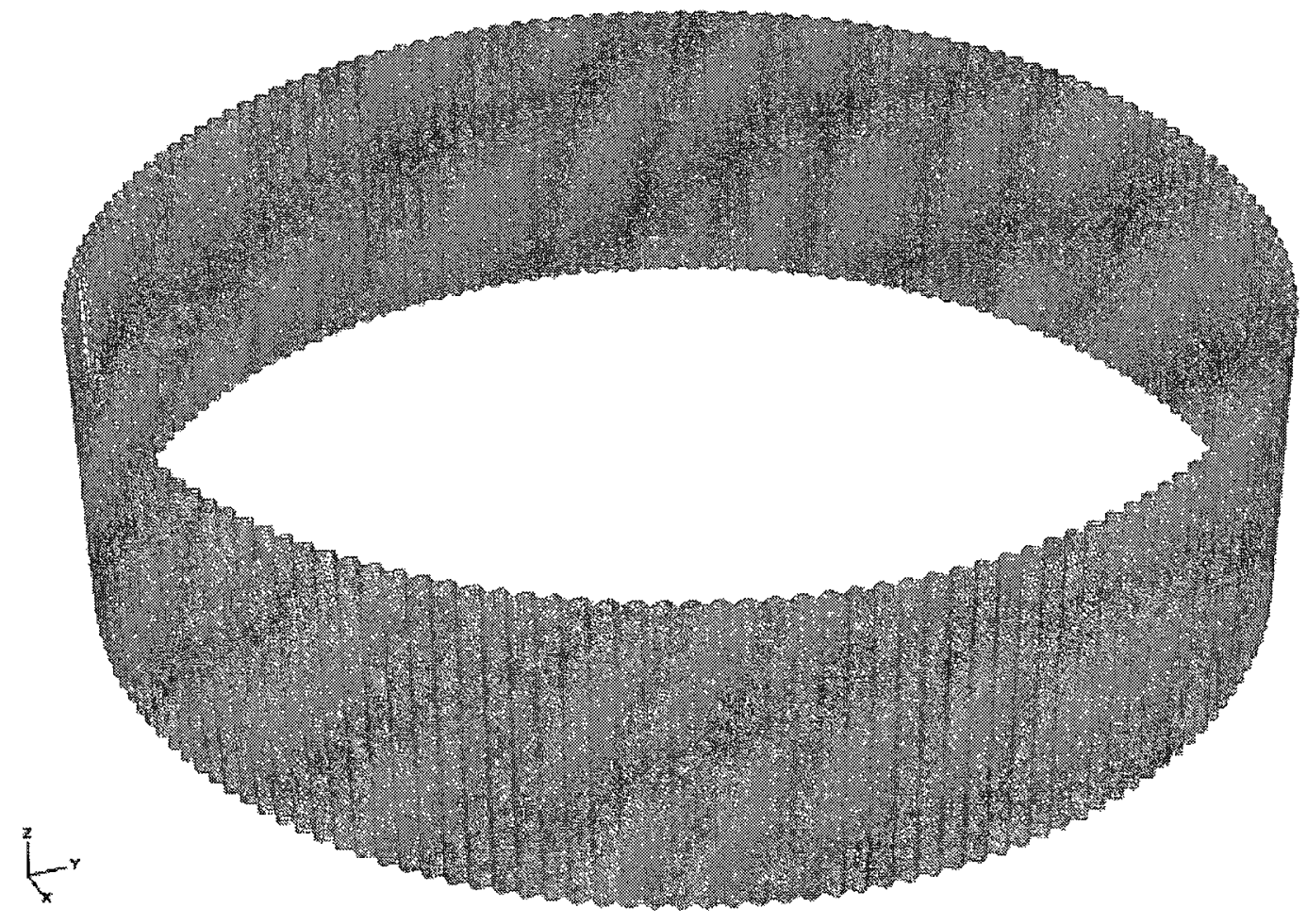

Figure 7-17. The side ring inner surface mesh

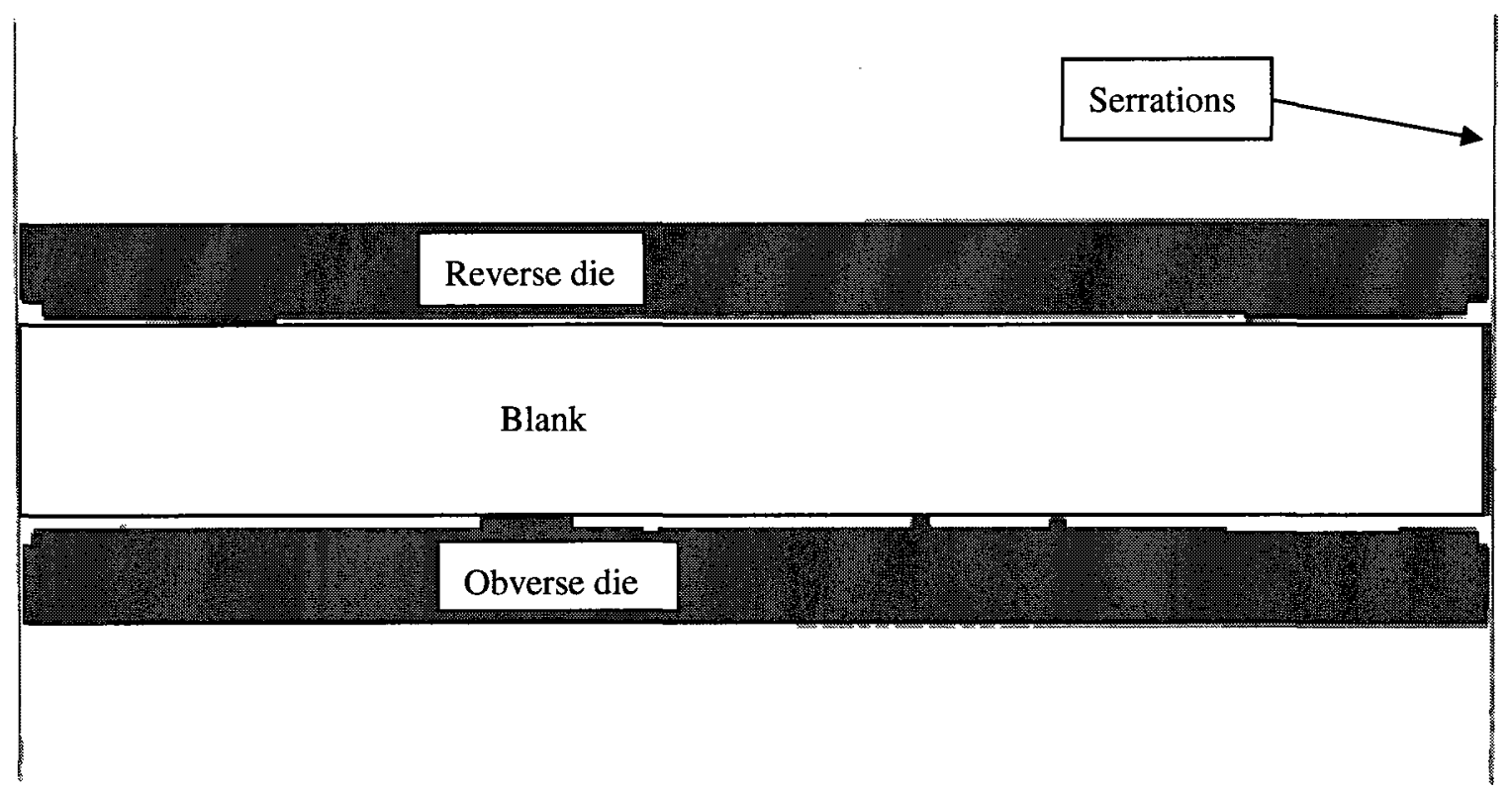

Figure 7-18. The coining simulation assembly sketch 


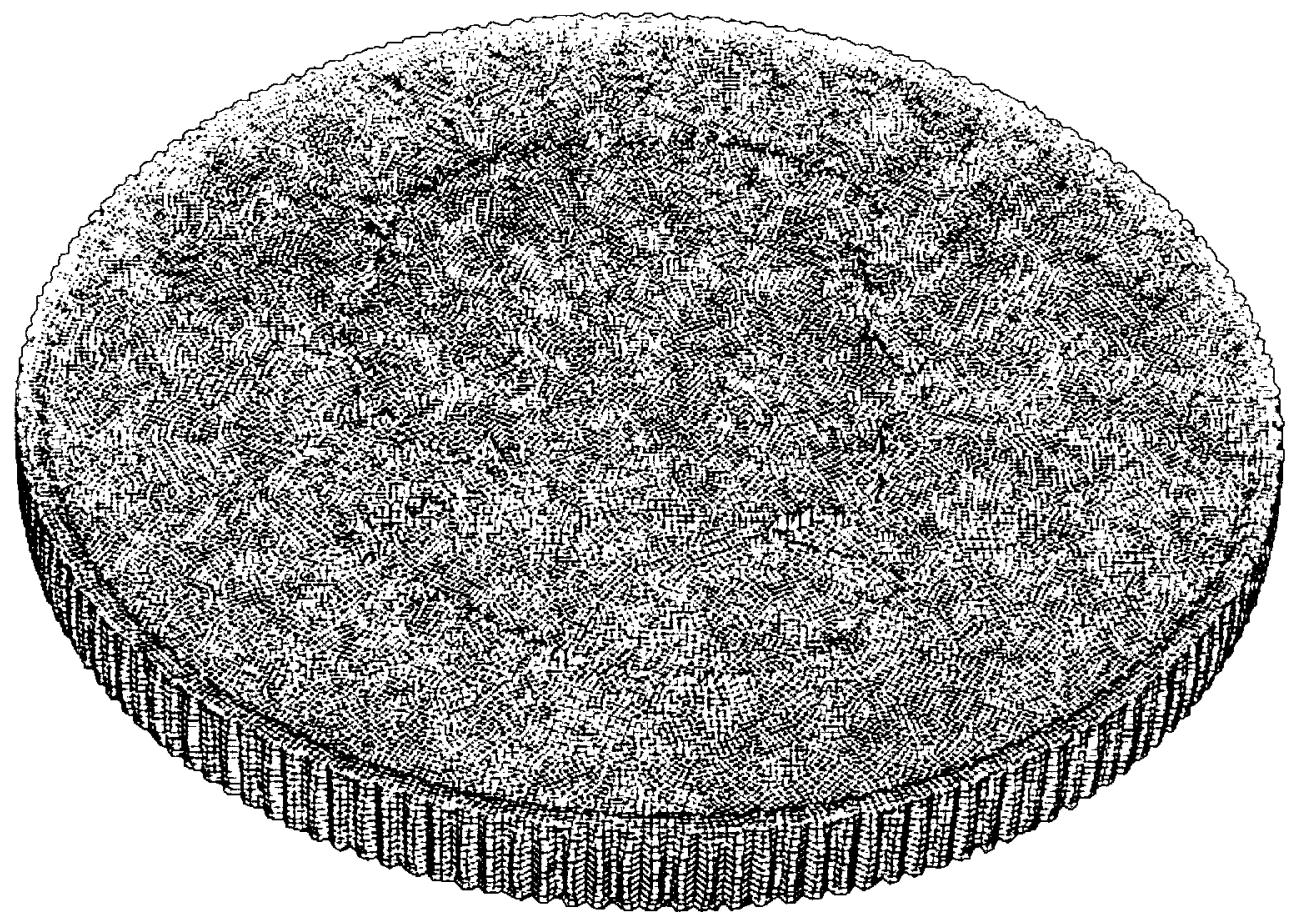

(a) Reverse side of deformed coin mesh

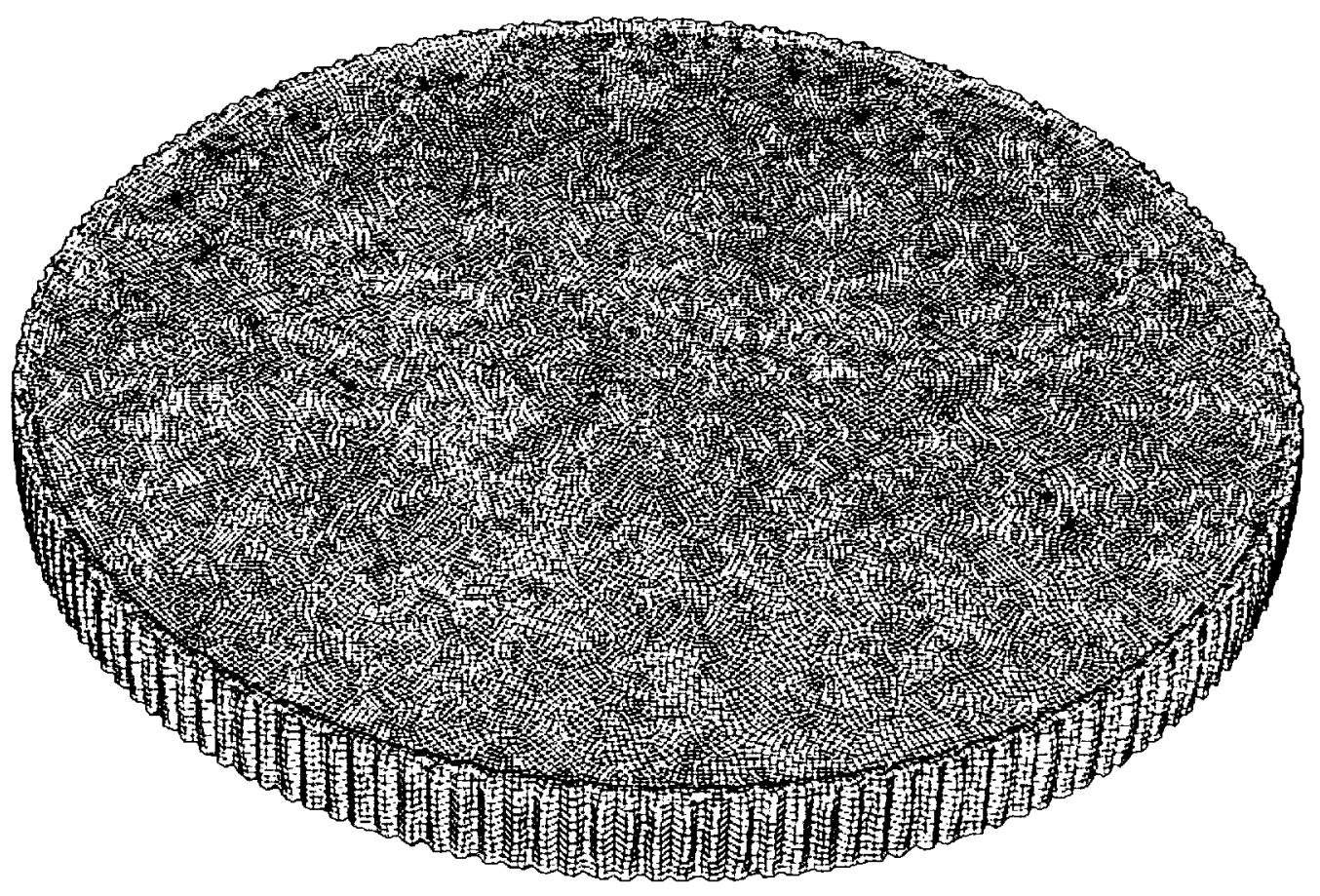

(b) Obverse side of deformed coin mesh

Figure 7-19. Deformed mesh of the one-side relief model 


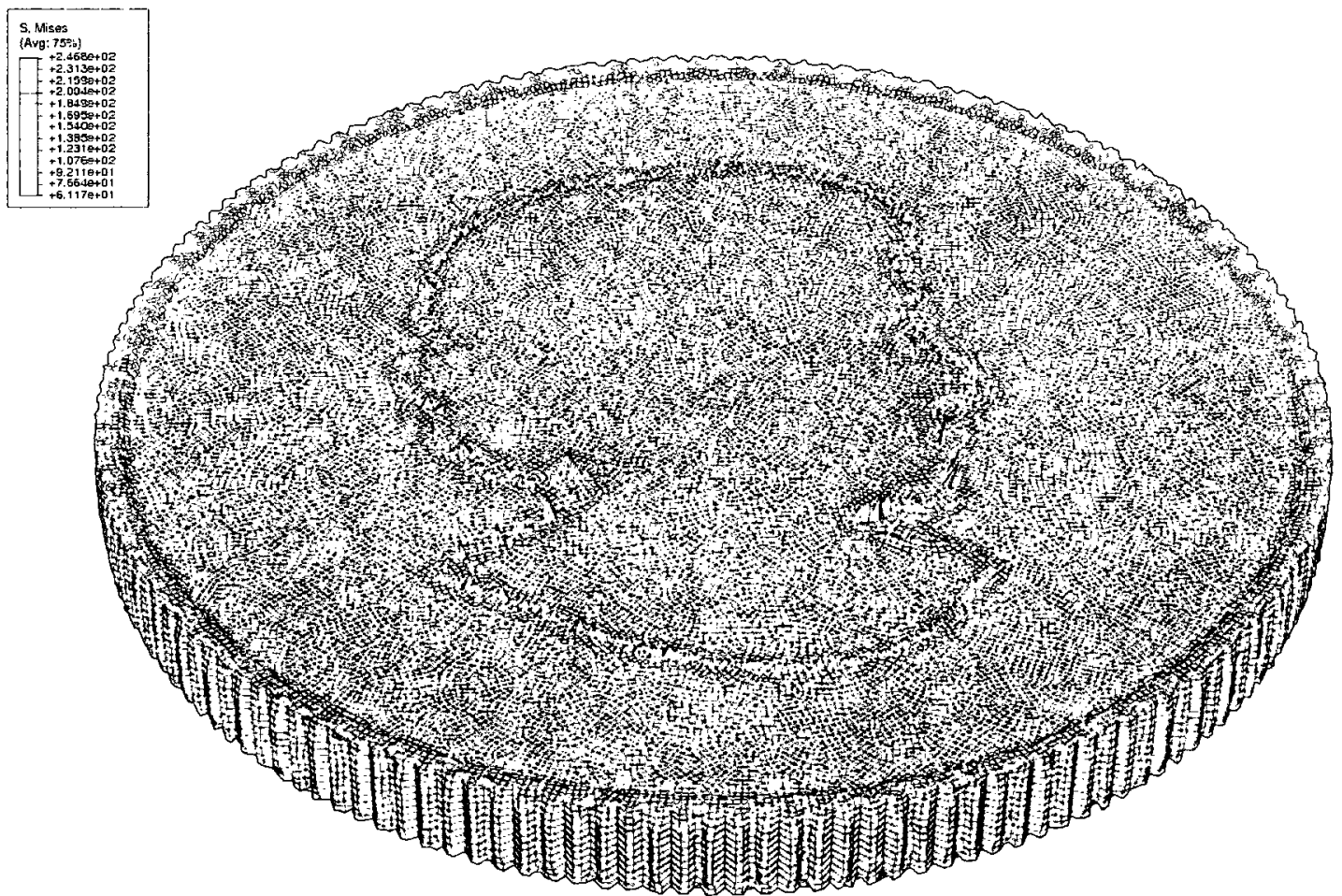

(a) Von Mises stress contour on reverse side

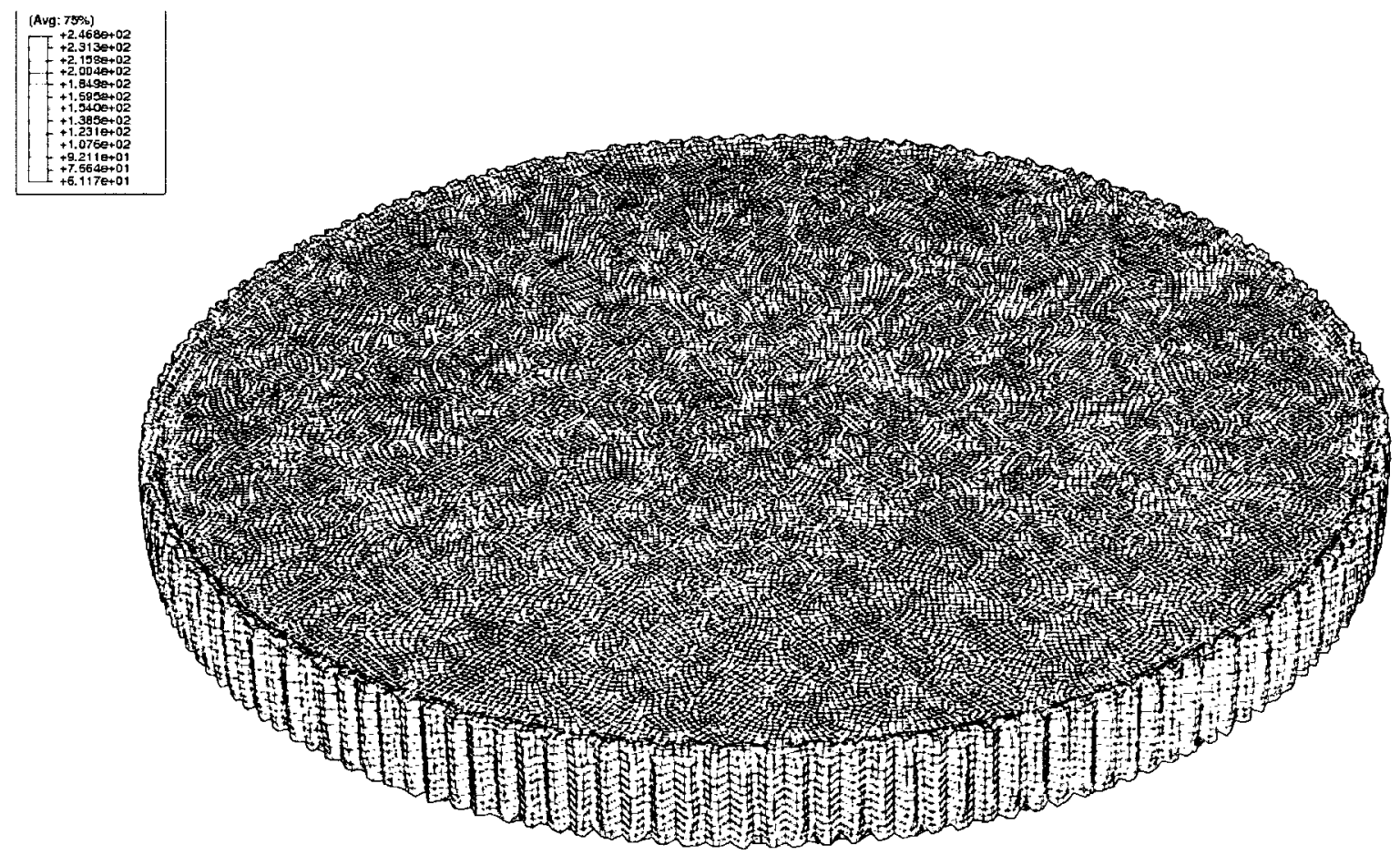

(b) Von Mises stress contour on obverse side

Figure 7-20. The Von Mises stress contour of one-side relief model 


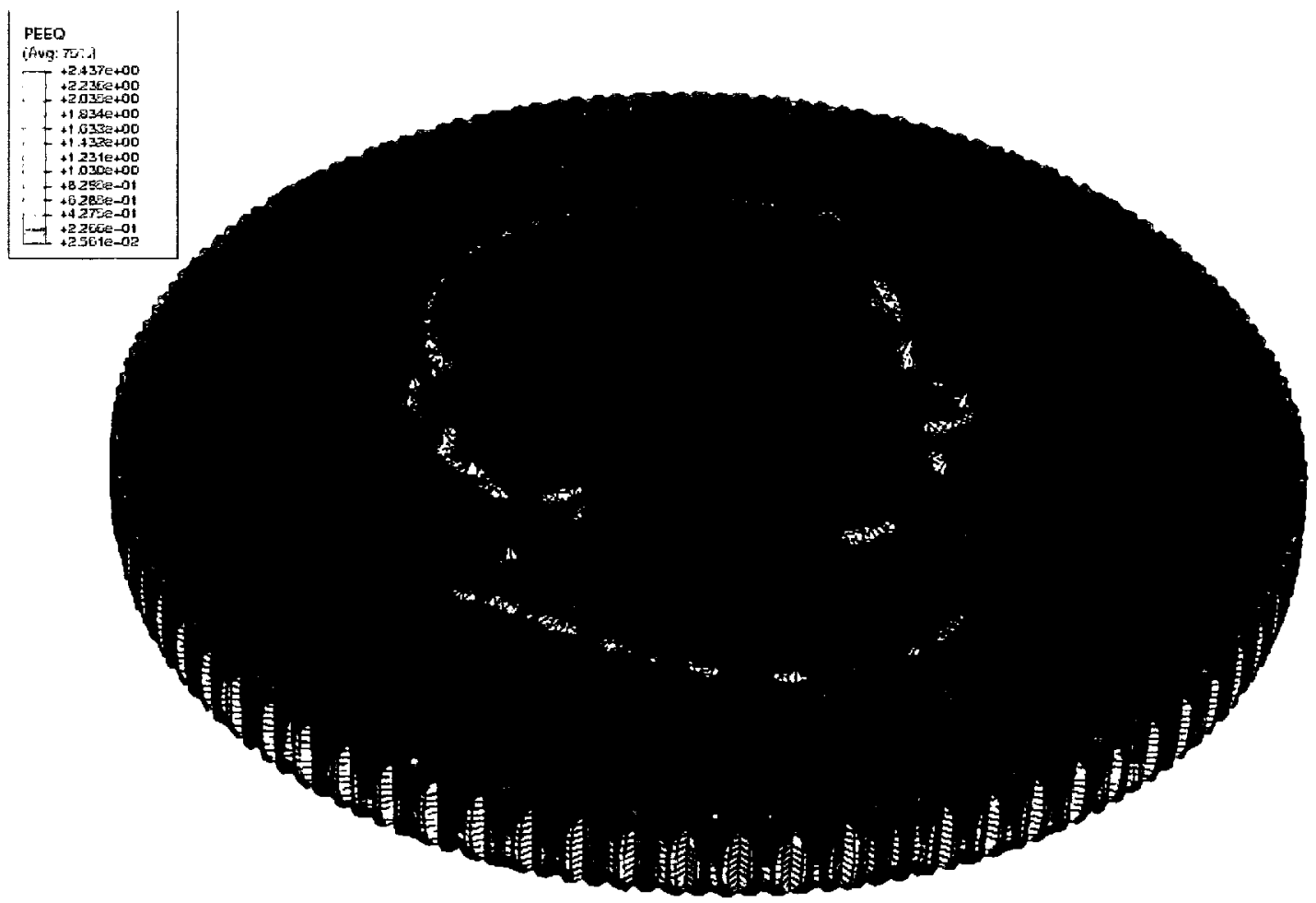

(a) Equivalent plastic strain contour on reverse side

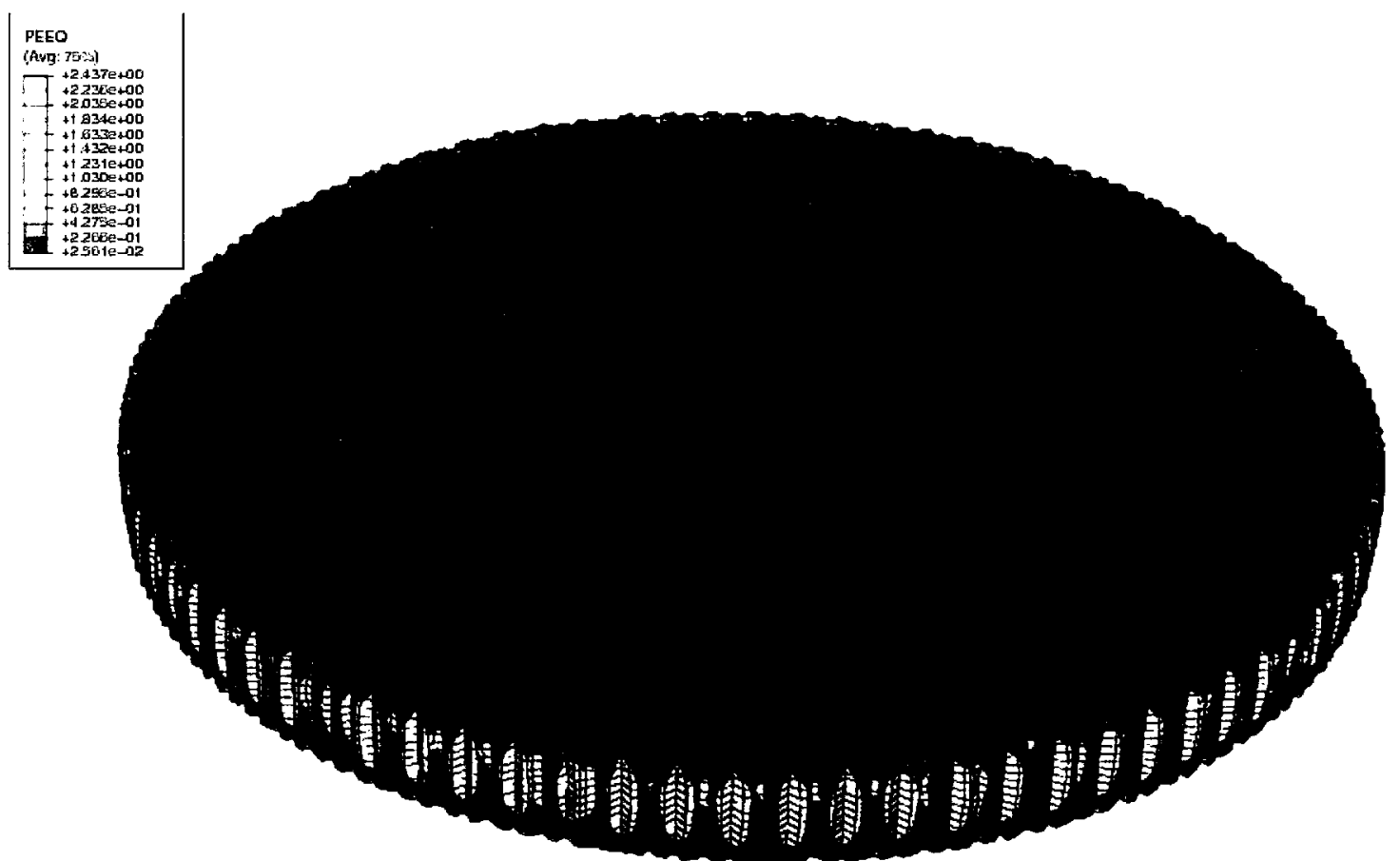

(b) Equivalent plastic strain contour on obverse side

Figure 7-21. The equivalent plastic strain contour of one-side relief model 


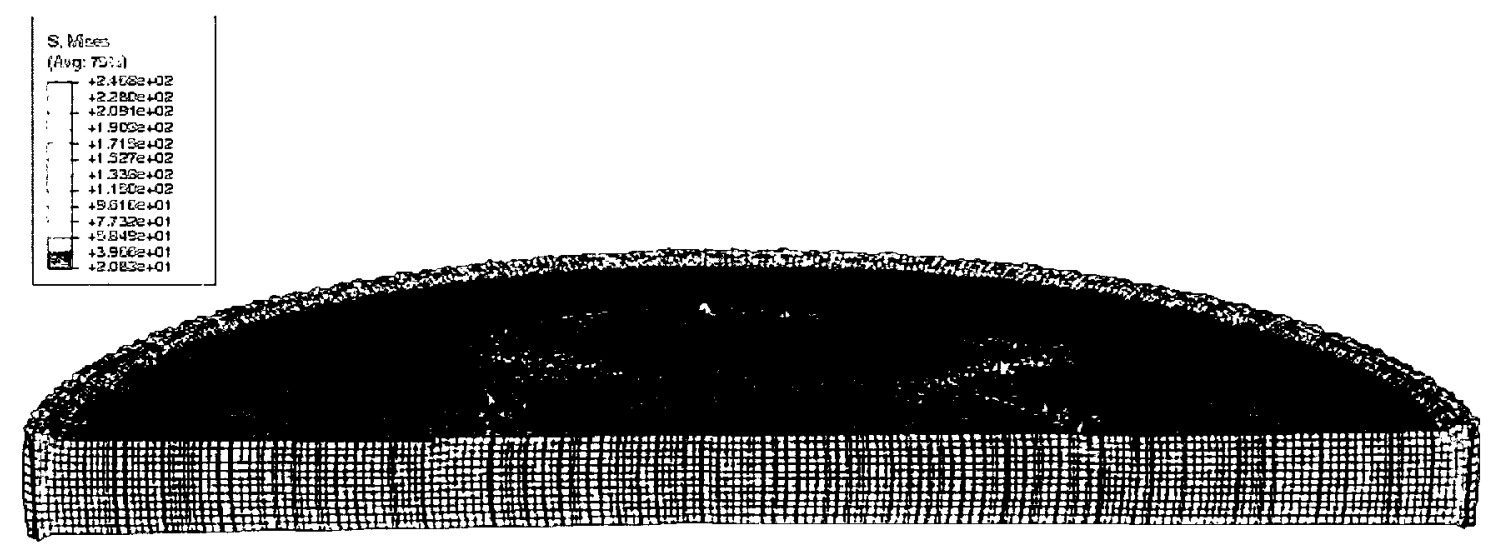

Figure 7-22. The Von Mises stress contour on the cross-section of one-side relief model

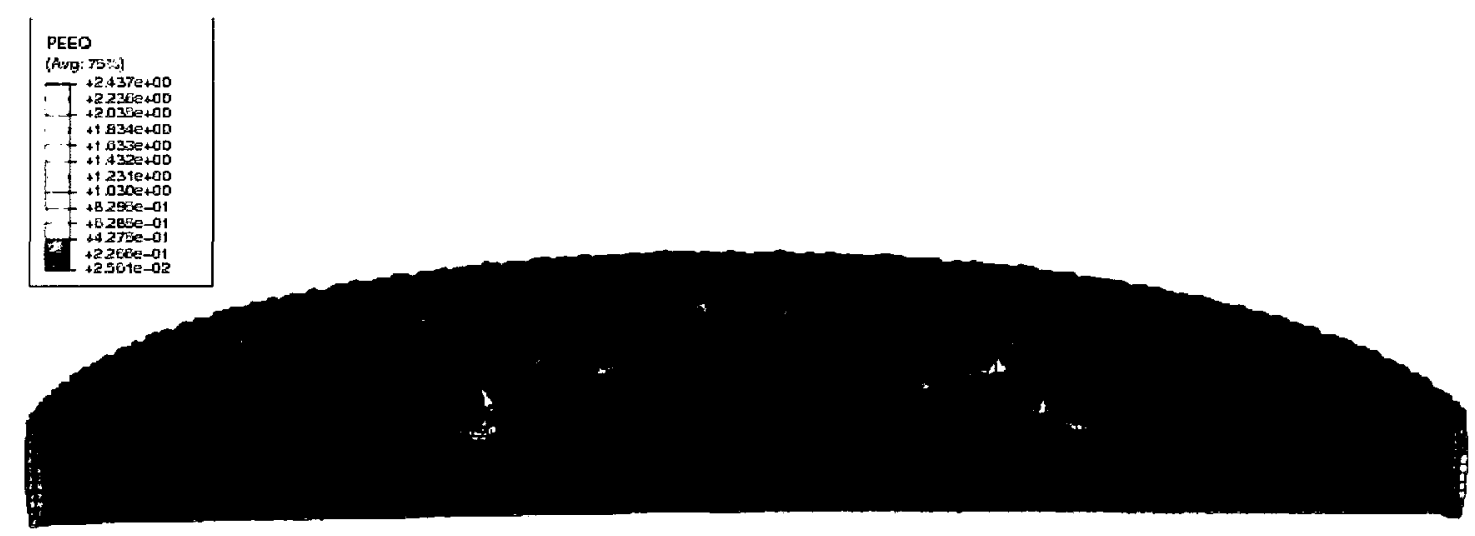

Figure 7-23. The equivalent plastic strain contour on the cross-section of one-side relief model 


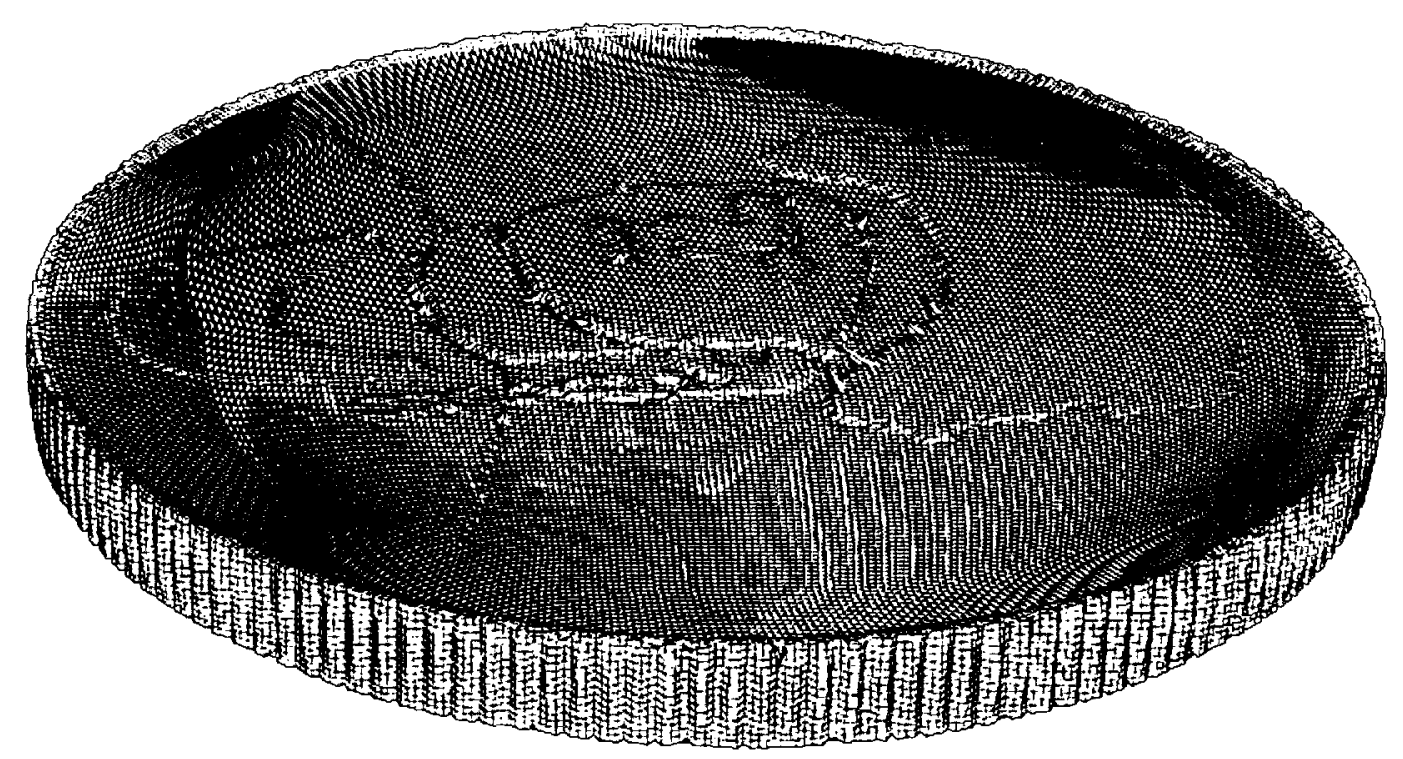

( a ) Obverse side of deformed coin mesh

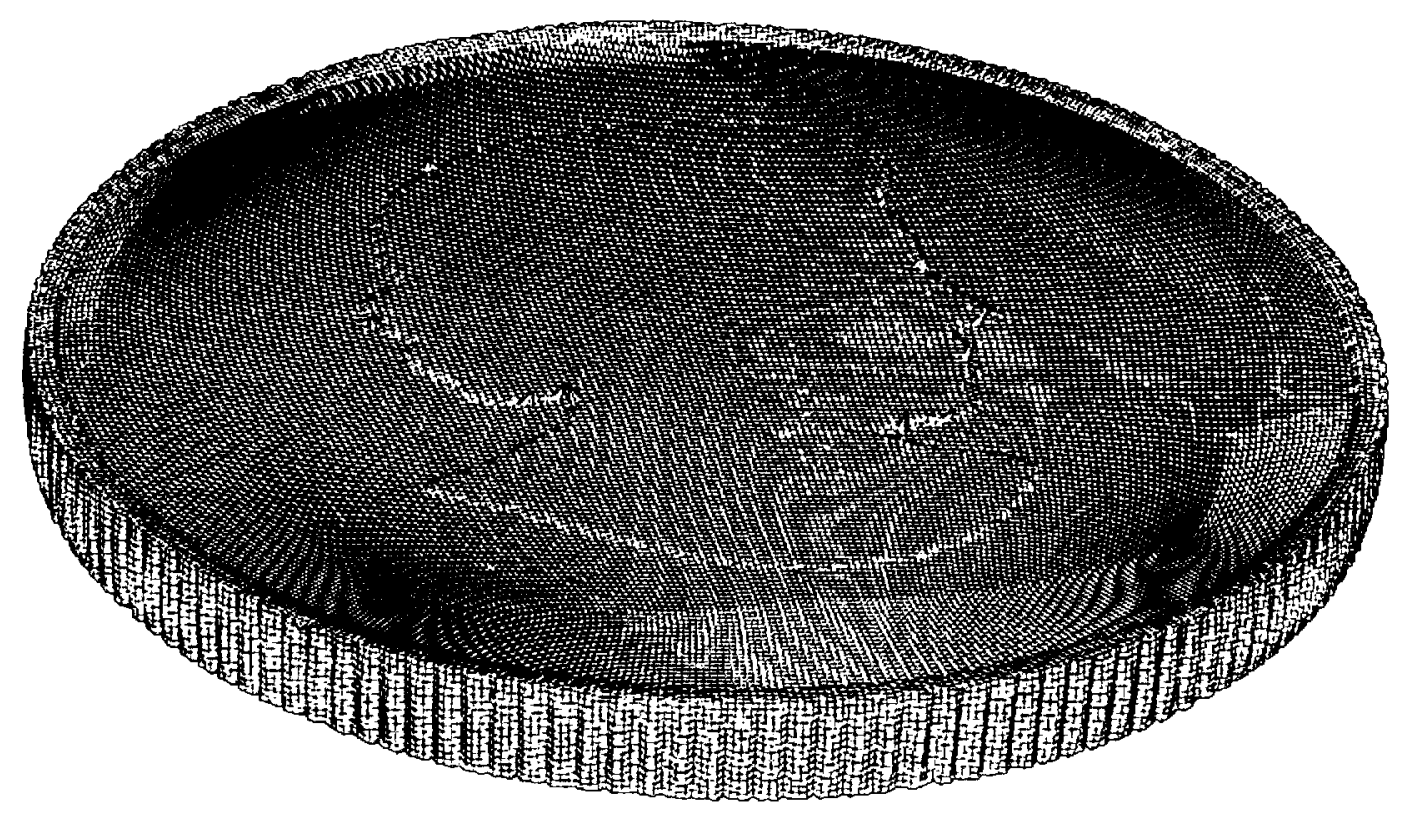

(b) Reverse side of deformed coin mesh

Figure 7-24. Deformed mesh of the two-side relief model 


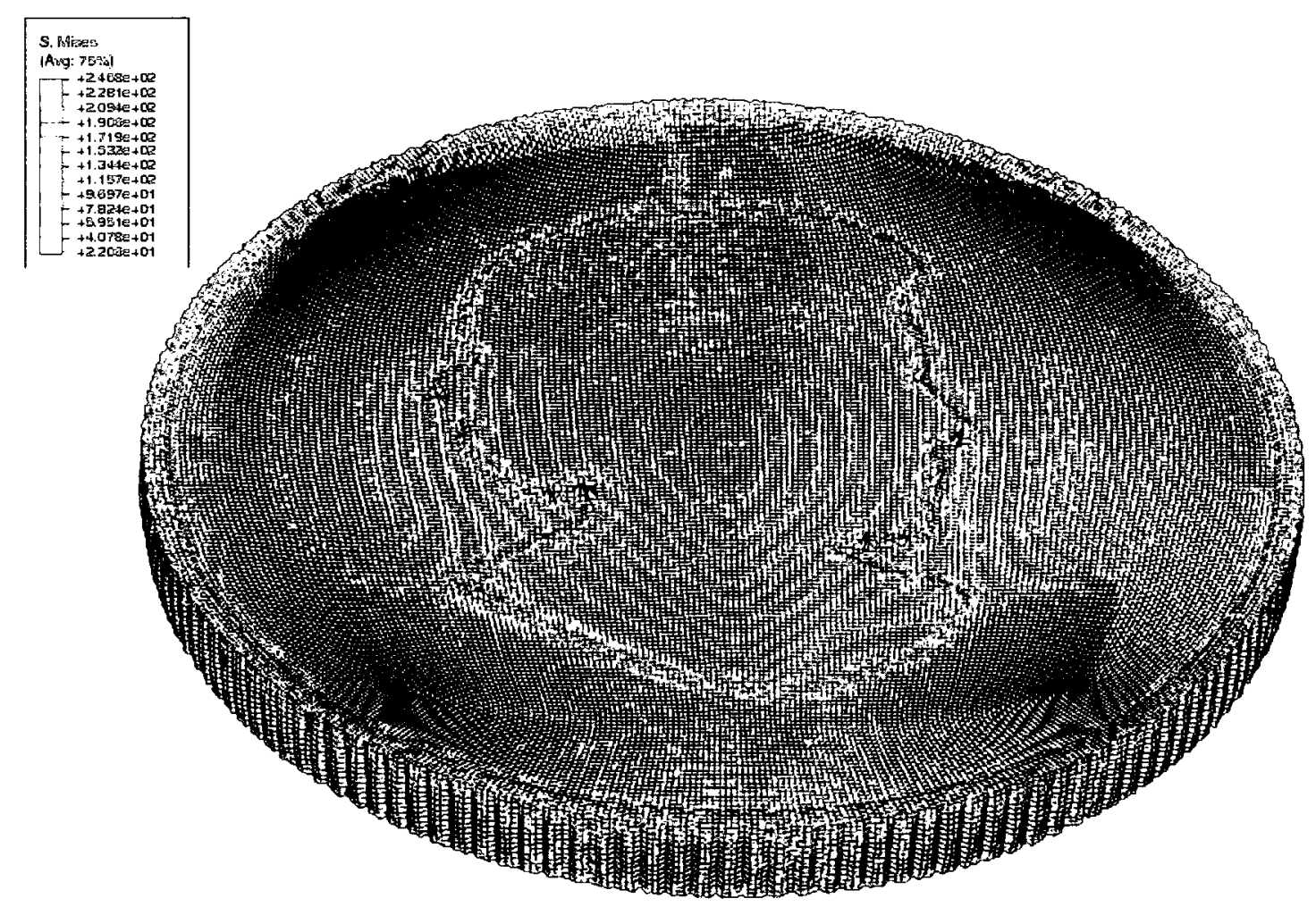

(a) Von Mises stress contour on reverse side

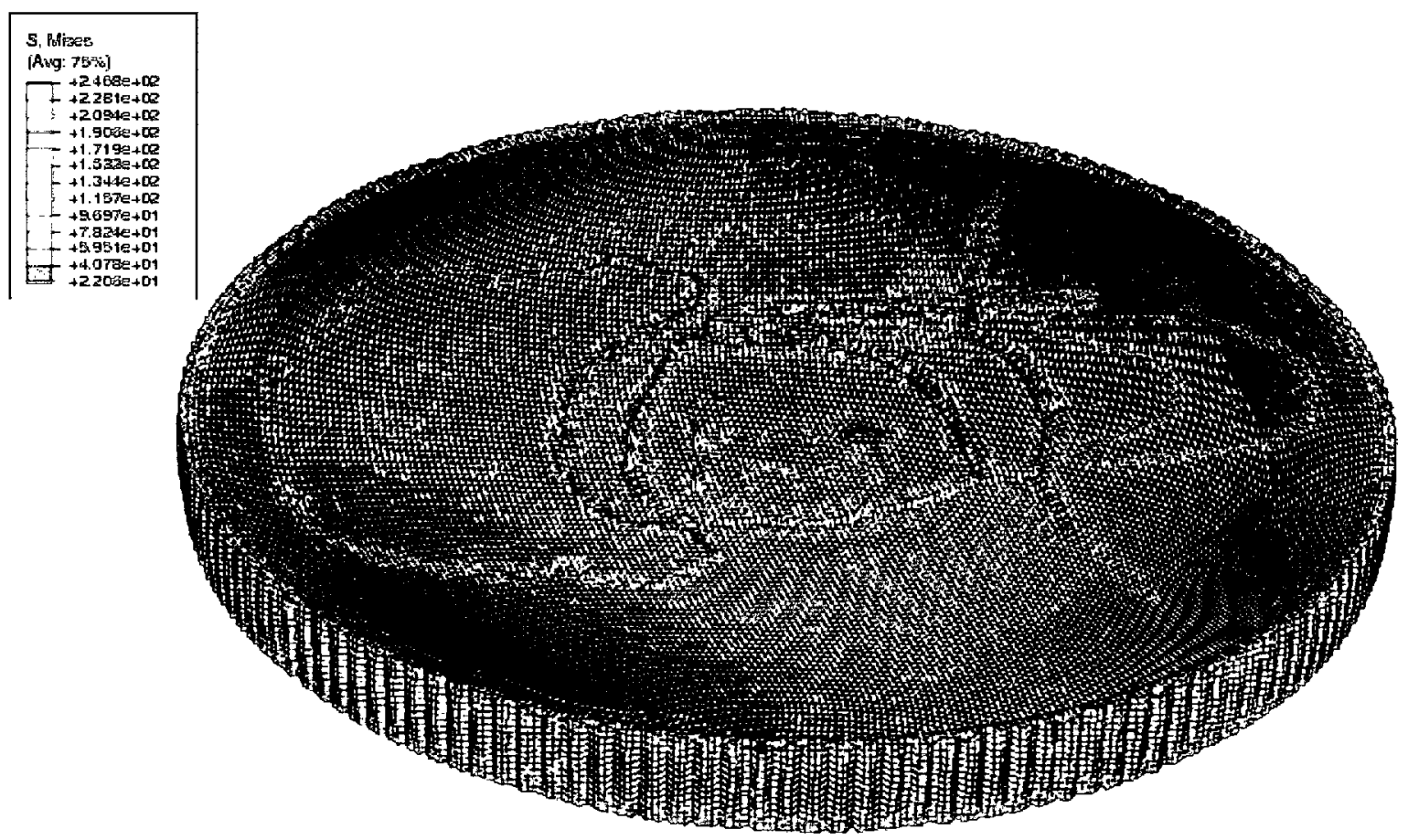

(b) Von Mises stress contour on obverse side

Figure 7-25. The Von Mises stress contour of two-side relief model 


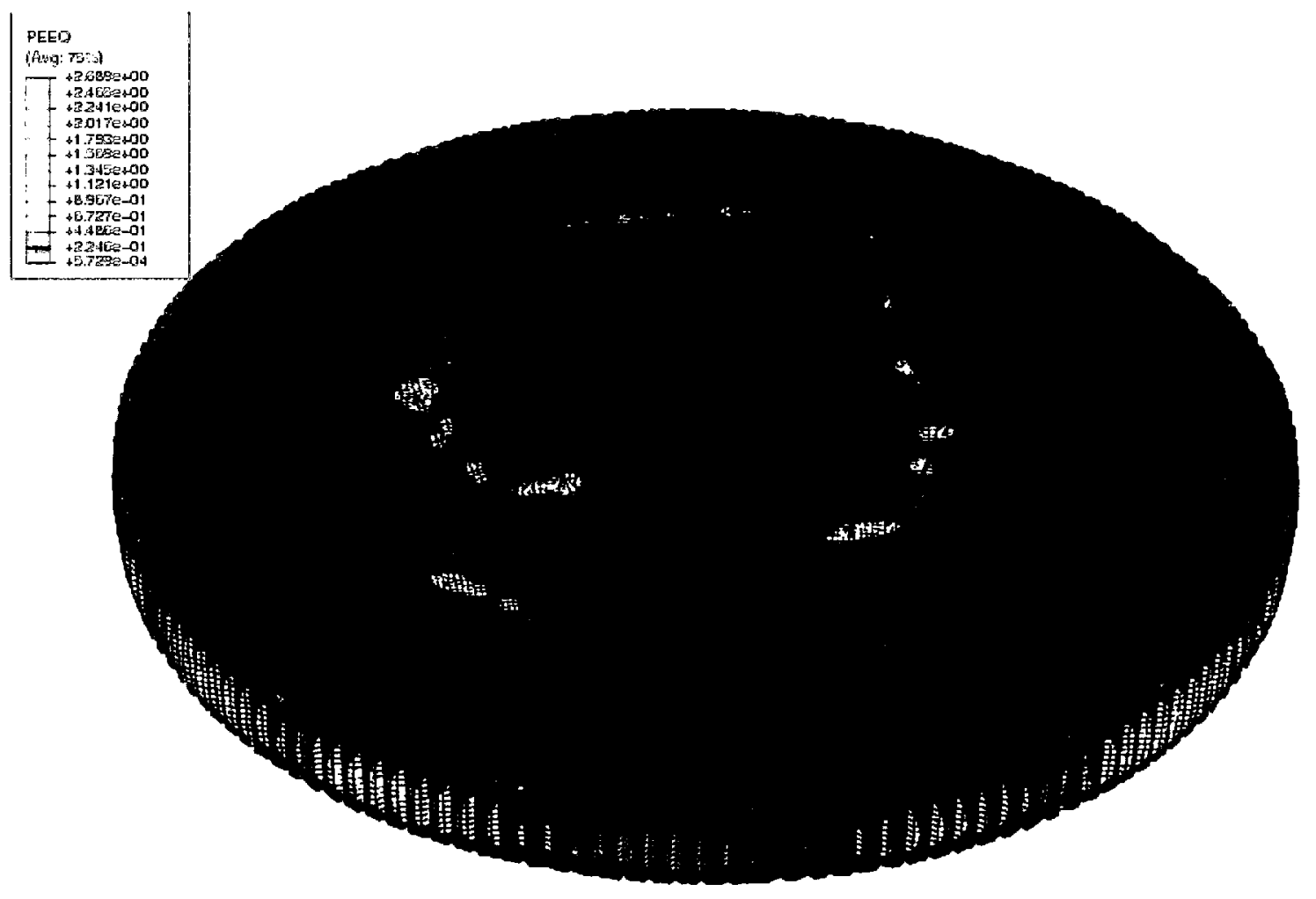

(a) Equivalent plastic strain contour on reverse side

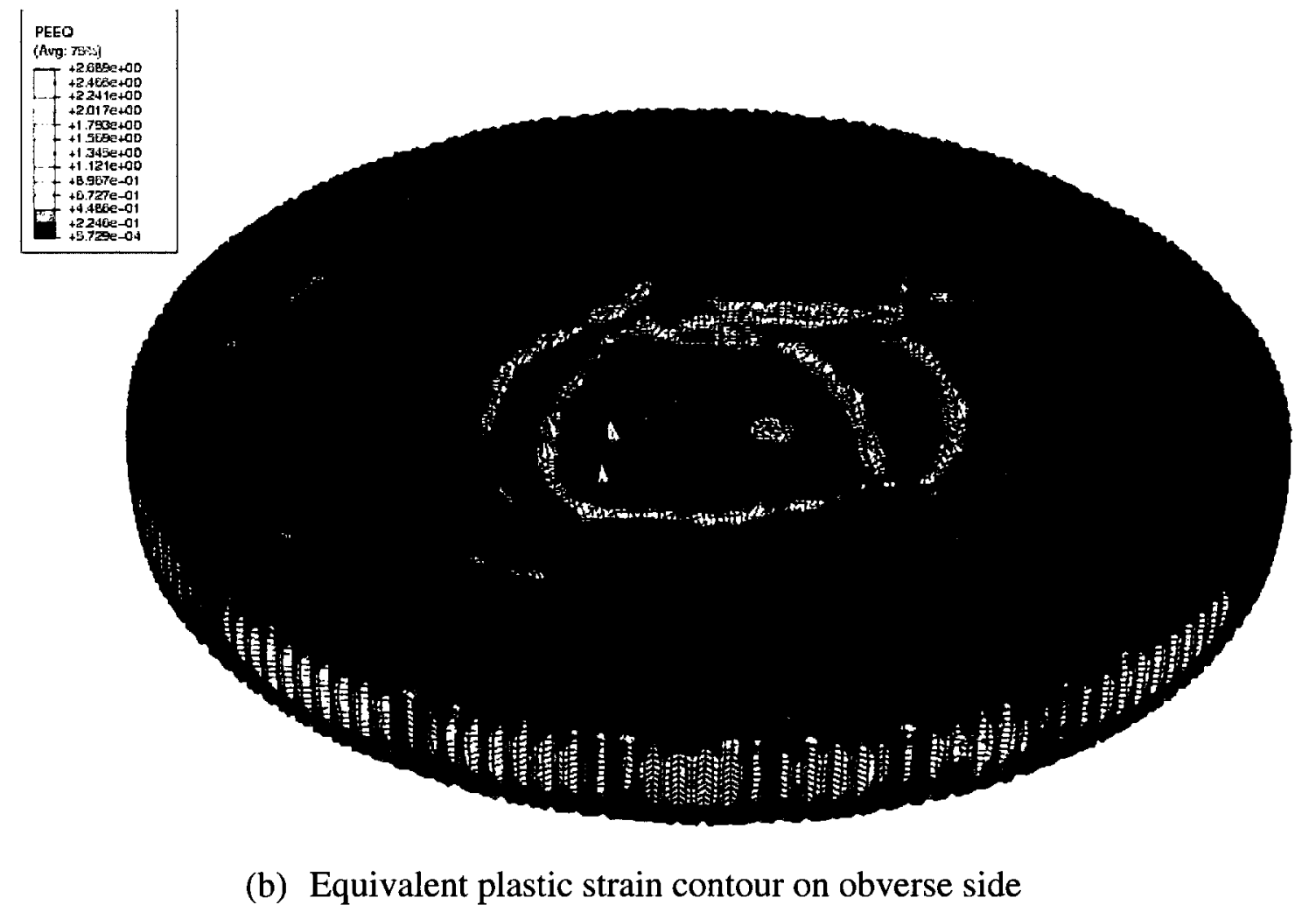

Figure 7-26. The equivalent plastic strain contour of two-side relief model 


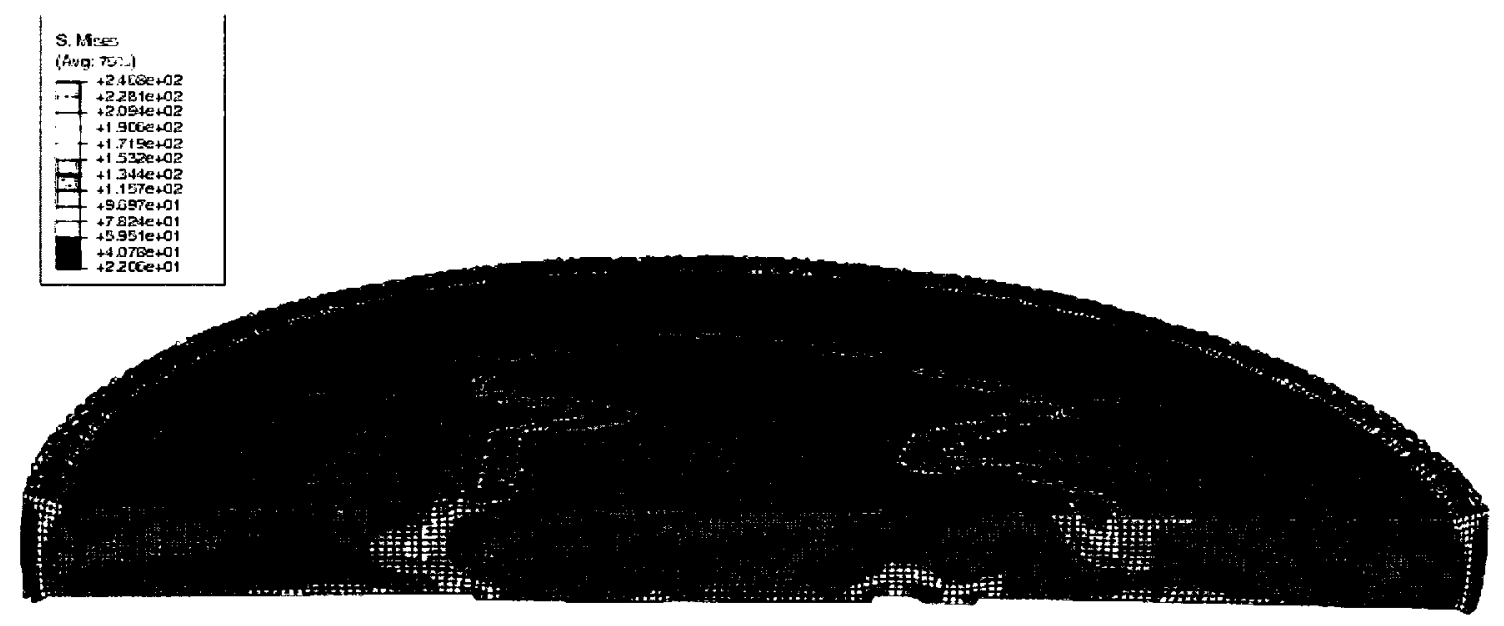

Figure 7-27. The Von Mises stress contour on the cross-section of two-side relief model

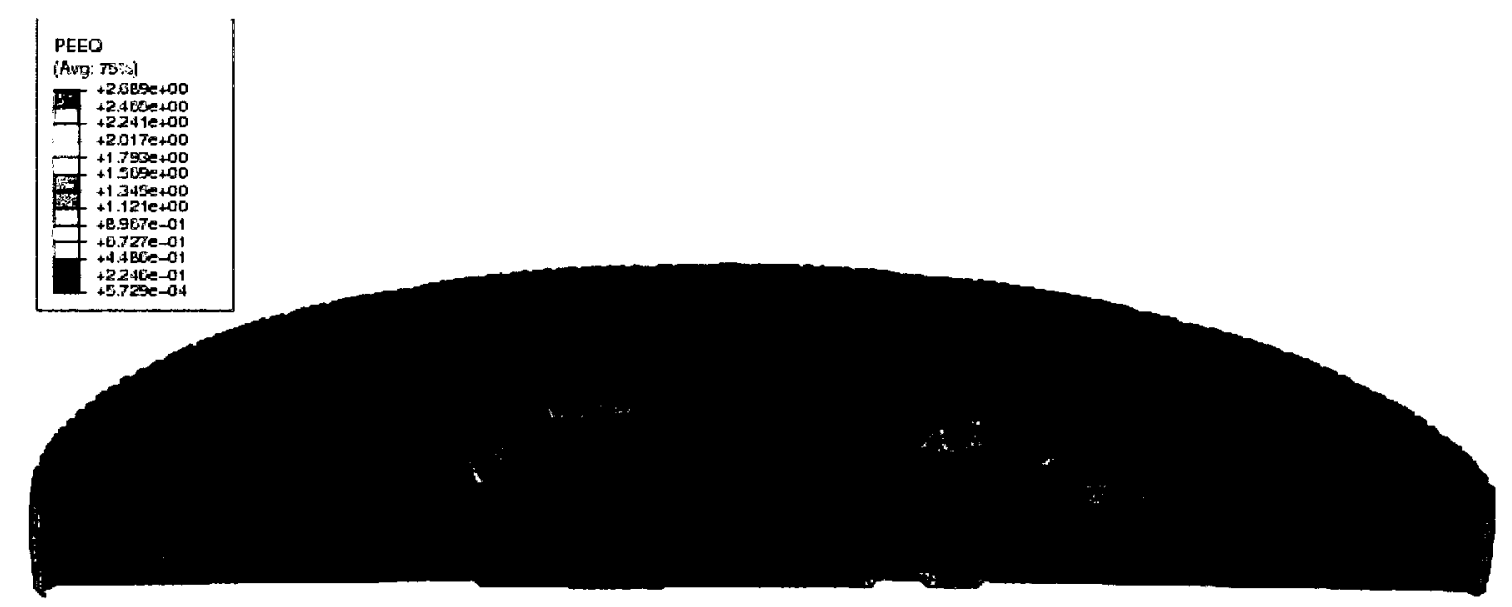

Figure 7-28. The equivalent plastic strain contour on the cross-section of two-side relief model 


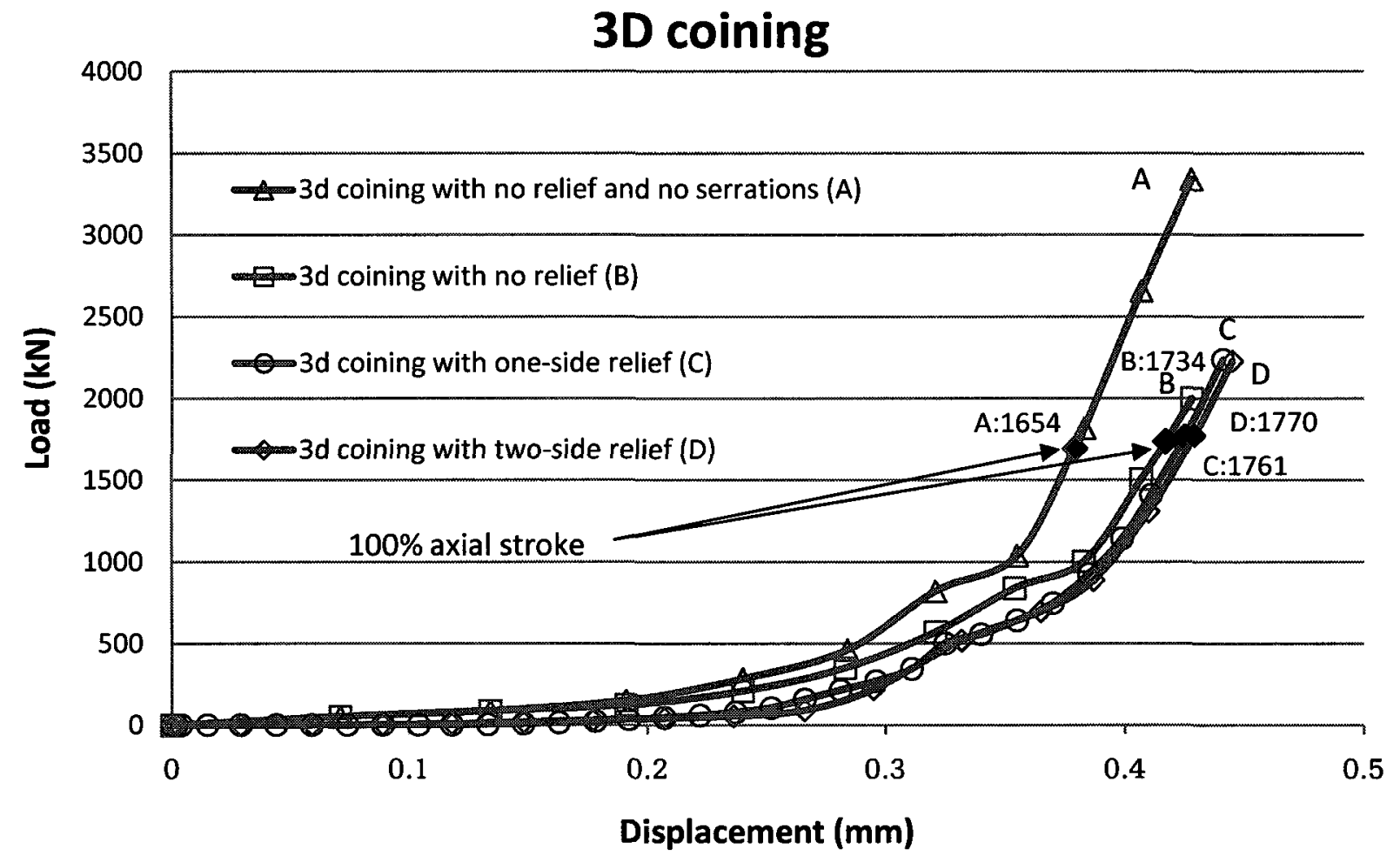

\begin{tabular}{|c|c|c|}
\hline Simulation case & Load at $100 \%$ axial stroke & $\begin{array}{c}\text { Difference (compare with } \\
\text { real coining load) }\end{array}$ \\
\hline A & $1654 \mathrm{kN}$ & $10.2 \%$ \\
\hline B & $1734 \mathrm{kN}$ & $15.6 \%$ \\
\hline C & $1761 \mathrm{kN}$ & $17.4 \%$ \\
\hline D & $1770 \mathrm{kN}$ & $18.0 \%$ \\
\hline Real coining at Mint & $1500 \mathrm{kN}$ & N/A \\
\hline
\end{tabular}

Figure 7-29. Loading path in 3D coining 


\begin{tabular}{|c|c||c|c|}
\hline \multicolumn{3}{|c|}{ Load Vs Die Displacement } \\
\hline \multicolumn{3}{|c|}{ 2D FEM Simulation } & \multicolumn{2}{|c|}{ 3D FEM Simulation } \\
\hline $\begin{array}{c}\text { Load } \\
(\mathrm{kN})\end{array}$ & $\begin{array}{c}\text { Displacement } \\
(\mathrm{mm})\end{array}$ & $\begin{array}{c}\text { Load } \\
(\mathrm{kN})\end{array}$ & $\begin{array}{c}\text { Displacement } \\
(\mathrm{mm})\end{array}$ \\
\hline 0 & 0 & 0 & 0 \\
\hline 21.96 & 0.084 & 51.63 & 0.071 \\
\hline 49.55 & 0.122 & 87.57 & 0.134 \\
\hline 135.86 & 0.191 & 149.63 & 0.191 \\
\hline 269.96 & 0.249 & 283.33 & 0.24 \\
\hline 358.8 & 0.275 & 460.50 & 0.284 \\
\hline 794.12 & 0.321 & 818.88 & 0.321 \\
\hline 1069.58 & 0.356 & 1036.17 & 0.355 \\
\hline 1345.51 & 0.365 & 1654.36 & 0.379 \\
\hline 1631.71 & 0.377 & 1815.23 & 0.383 \\
\hline 1689.85 & 0.379 & 2655.91 & 0.407 \\
\hline 1853.33 & 0.383 & 3337.40 & 0.428 \\
\hline
\end{tabular}

Table 7-1. 2D and 3D FEM results comparison

\begin{tabular}{|c|c||c|c|}
\hline \multicolumn{4}{|c|}{ Load Vs Die Displacement } \\
\hline 3D FEM Coining with no serrations & 3D FEM Coining with serrations \\
\hline $\begin{array}{c}\text { Load } \\
(\mathrm{kN})\end{array}$ & $\begin{array}{c}\text { Displacement } \\
(\mathrm{mm})\end{array}$ & $\begin{array}{c}\text { Load } \\
(\mathrm{kN})\end{array}$ & $\begin{array}{c}\text { Displacement } \\
(\mathrm{mm})\end{array}$ \\
\hline 0 & 0 & 0 & 0 \\
\hline 51.63 & 0.071 & 51.63 & 0.071 \\
\hline 87.57 & 0.134 & 87.53 & 0.134 \\
\hline 149.63 & 0.191 & 122.89 & 0.191 \\
\hline 283.33 & 0.24 & 208.66 & 0.24 \\
\hline 460.50 & 0.284 & 349.87 & 0.283 \\
\hline 818.88 & 0.321 & 569.52 & 0.321 \\
\hline 1036.17 & 0.355 & 837.20 & 0.354 \\
\hline 1815.23 & 0.383 & 1002.24 & 0.383 \\
\hline 2655.91 & 0.407 & 1504.82 & 0.407 \\
\hline 3337.40 & 0.428 & 1998.62 & 0.428 \\
\hline
\end{tabular}

Table 7-2. 3D coining model comparison 


\begin{tabular}{|c|c|c|c|}
\hline \multicolumn{4}{|c|}{ Load Vs Die Displacement } \\
\hline \multicolumn{2}{|c|}{$\begin{array}{l}\text { 3D FEM Coining } \\
\text { with one-side relief }\end{array}$} & \multicolumn{2}{|c|}{$\begin{array}{l}\text { 3D FEM Coining } \\
\text { with two-side relief }\end{array}$} \\
\hline $\begin{array}{l}\text { Load } \\
(\mathrm{kN})\end{array}$ & $\begin{array}{c}\text { Displacement } \\
(\mathrm{mm})\end{array}$ & $\begin{array}{l}\text { Load } \\
(\mathrm{kN})\end{array}$ & $\begin{array}{c}\text { Displacement } \\
(\mathrm{mm})\end{array}$ \\
\hline 0 & 0 & 0 & 0 \\
\hline 0.37 & 0.004 & 0.26 & 0.002 \\
\hline 0.50 & 0.029 & 0.76 & 0.029 \\
\hline 0.75 & 0.059 & 1.33 & 0.059 \\
\hline 1.22 & 0.089 & 3.56 & 0.089 \\
\hline 2.01 & 0.118 & 7.74 & 0.118 \\
\hline 8.95 & 0.148 & 17.58 & 0.148 \\
\hline 23.73 & 0.178 & 29.17 & 0.177 \\
\hline 46.43 & 0.207 & 41.44 & 0.207 \\
\hline 79.31 & 0.237 & 55.56 & 0.236 \\
\hline 157.09 & 0.266 & 89.77 & 0.266 \\
\hline 270.04 & 0.296 & 220.20 & 0.295 \\
\hline 554.73 & 0.340 & 517.91 & 0.332 \\
\hline 640.43 & 0.355 & 695.41 & 0.365 \\
\hline 923.96 & 0.385 & 886.63 & 0.387 \\
\hline 1407.97 & 0.411 & 1305.62 & 0.41 \\
\hline 2235.47 & 0.441 & 2227.54 & 0.445 \\
\hline
\end{tabular}

Table 7-3. Load vs. displacement in 3D coining 


\section{Chapter 8}

\section{Conclusions and Recommendations}

From the analysis in the prior chapters, the achievements obtained are summarized and some recommendations for the future work are given in the following.

\subsection{Conclusions}

Through the parametric study, general FEM analysis approaches (explicit FEM approach and implicit FEM approach) and several key numerical parameters, namely hourglass control, contact simulation and adaptive meshing - were investigated through the 2D axisymmetric FEM simulations of a ring compression and a coining process. The results of finite element simulations show that the explicit FEM approach using integral viscoelastic hourglass control, kinematic contact simulation and adaptive meshing technique is suitable and capable to simulate the coining process.

A typical material used for coining process - fine silver - was chosen as the material for all the FEM coining simulations. The quasi-static approximation of real coining process and the uniaxial tensile test were presented to evaluate the mechanical behavior of fine silver. The strain hardening curve (Figure 4-5, Table 4-3) and major mechanical behavior parameters were obtained with yield stress of $61 \mathrm{MPa}$, ultimate strength of $168 \mathrm{MPa}$ and flow stress of $115 \mathrm{MPa}$ and they are then converted into the material property models and used as the input data for the finite element coining process simulations.

As a contact dominant process, the critical coining interface friction conditions were investigated. The ring compression test which provides a particularly sensitive means for measuring forging friction was verified by the contact pressures. The experimental and numerical

combined approach was conducted to evaluate the friction behaviour of the silver coining process. A two dimensional axisymmetric FEM simulation of the ring compression was carried 
out to calibrate the experimental results of a fine silver ring. Through careful measurement of the geometrical changes of the ring, the calibration curves were obtained for different friction conditions. The parameters in the friction model for the fine silver coining process were obtained by calibrating the experimental results. The ring compression technique, friction calibration curves and non-classical friction model methods were applied to evaluate the friction conditions in the silver coining process. A two parameter friction model which combines the Coulomb friction model and the friction factor model was derived, with $\mu=0.22$ and $\tau_{\max }=123 \mathrm{MPa}$, to describe the frictional behavior between the blank and platen for a silver coining process. It has been shown that through careful calibrations, the finite element analysis using this friction model can accurately predict the deformation of silver ring compression process. The good agreement between FEM results and experimental results shows the approach provides an effective and repeatable means to evaluate the frictional behavior of coining process.

The two dimensional FEM coining process simulations were conducted using the material property model and two parameter friction model obtained. Three key profile design parameters were investigated: curvature, rim height and relief height. The deformed mesh and material flows patterns, stress and strain contours were plotted and discussed for models with different combination of curvature, rim height and relief height. The effects of the parameters were further illustrated and discussed by comparing the load paths of different models. It has been found that as the curvature, rim height and relief height increase, die displacement will also increase and the load at $100 \%$ axial stroke will increase 2\%-10\% (Figure 6-28 through Figure 6-31). The effects of the idealized parameters can be used as the fundamental guides or references for the future design work.

Finally, the three dimensional FEM simulations of the coining process were conducted. The 3D simulation was first used to compare with the simplified $2 \mathrm{D}$ axisymmetric analysis. A good agreement was achieved between the two-dimensional model and three-dimensional model with the same design parameters. Then the three dimensional FEM simulation of coining with serrations on the side ring was conducted. The effects of serrations were found to increase the die 
displacement and load at $100 \%$ axial stroke by $4.8 \%$ (Figure $7-9$ ). Then, the real coining process with features of serrations and reduced relief was simulated by applying the three-dimensional FEM approach and the input models obtained such as material property and friction model. The deformed mesh, stress and strain contours were plotted and discussed. The effects of coin surface reduced profile design (relief) and loading patterns were obtained. The die displacement and the load at $100 \%$ axial stroke gradually increases $10 \%-18 \%$ as more features were included in the model (Figure 7-29). In this research, all the materials and die designs used the "Silver Dollar" designs provided by Royal Canadian Mint. The coining loads at 100\% axial stroke obtained from the FEM simulations are very consistent with the Mint's tonnage used in the real forming of the "silver dollar". This agreement supports the 3D FEM coining simulation which can therefore be used to predict the material flow patterns, the loads, the stresses and strains for various coining processes. It can also be concluded that during the silver dollar coining design process, it is reasonable to apply the 2D FEM analysis to perform the preliminary coining evaluations of some typical parameters such as curvatures, rim height and relief height. Considering the higher computation costs of 3D FEM simulations, this level of analysis should be applied in the late prototype design and modifications stage.

By starting with the initial actual design of silver coin supplied by the Royal Canadian Mint, the whole process design solution based on the FEM tool was presented: Firstly, establishing the input data models for the FEM simulations from experimental and numerical approaches. Secondly, constructing the solid 2D and 3D engineering design models from the user inputs or the scanning of the coin design. Thirdly, performing the two-dimensional or three-dimensional coining process FEM simulations using the input models and initial engineering designs. Finally, from the simulations, one can obtain the effects of various design parameters, the material flow, load requirement, die cavity filling and other conditions like stress and strain in the coin and provide suggestions in the design modifications. 


\subsection{Recommendations}

In this research, fine silver was chosen as the only material used for the coining process simulation. It is well understood that the other materials like gold and other precious metals are also quite common materials for the coining process. Therefore, it is necessary to establish the input data pool of mechanical properties models and friction models for other materials used to produce coins or similar products at the Mint.

FEM simulations used in this research consider all the dies and side rings to be rigid bodies, which may be a reasonable assumption considering the large material strength difference between the very ductile fine silver and the hard tool steel. But this assumption may not be appropriate when other materials are used in the coining process. Therefore, it may be necessary to include the mechanical properties of the die and side ring in the simulations. That will not only benefit the investigation of the effects on the coin deformation but also contribute to study the effects on the life cycle of the expensive die. This will provides better and more useful results from the simulations.

The thermal and strain-rate effects were assumed to be negligible in the simulations carried out in this work. However, in the future, when higher speed coining processes (strain-rate is $10^{3} \mathrm{~s}^{-1}$ or higher) are to be analyzed, these effects should be included in the simulation model to investigate their effects on coin quality and die life.

Since no reference research or theory could be found for the silver dollar coining process, the FEM results from two-dimensional and three-dimensional simulations could be best verified if they were compared to experimental test results. A coining experiment with a simplified die set was proposed to compare the experimental results with the FEM results in this research. But unfortunately, the experiment couldn't be performed due to the fact that the fabrication of the simplified dies couldn't fit into the work schedule in the near future at the Royal Canadian Mint. Therefore experimental tests with the simple die geometries and idealized design parameters are recommended for the future work. The measurements on the actual loading patterns from the real coining processes will provide useful references to the numerical simulations. 
Finally, to establish the solid engineering model in the FEM simulations with ABAQUS using the scanning files, some conversion and simplification must be done to the original file which results in the loss of some details of profile design. This, in turn, will reduce the FEM simulation results from the actual coining situation at the Mint. Thus, a FEM code which can establish the solid model directly from the scanning files, would improve the design-simulation-modification cycles. Therefore, it will be able to simulate the exact coining model as designed. That will not only save time and effort in manipulating the scanning files, but also provide more realistic simulations to produce guidelines for further design modifications. 


\section{References}

ABAQUS theory manuals. Providence, RI: Hibbitt, Karlson and Sorenson, Inc.; 1989.

ABAQUS theory manuals. Providence, RI: Hibbitt, Karlson and Sorenson, Inc., 2000

ABAQUS user's manuals, 2006

Altan, T., Oh., S. I, and Gegel, H., "Metal forming: Fundamentals and Applications" ASM International, 1983, Metals Park, $\mathrm{OH}$

Altinbalik. T, Akata. H. E, Can. Y, "An approach for calculation of press loads in closed-die upsetting of gear blanks of gear pumps", Materials and Design Vol.28, 2007, p. 730-734.

Alves. M.L., Rodrigue. J.M.C, Martins. P.A.F, "Simulation of three-dimensional bulk forming processes by finite element flow formulation", Modeling Simul. Mater. Sci. Eng. Vol.11, 2003, p.803-821.

ASTM E8 / E8M - 08 Standard Test Methods for Tension Testing of Metallic Materials, ASTM Volume 03.01, ASTM

Bohm. J., Schubert. A., Otto. T., Burhhart. T., Microsystem Technologies, Vol. 7(4), p. 191-195. 2001.

Buffa. G, Fratini. L, Micari. F, "The Relevance of the Preform Design in Coining Processes of Cupronickel Alloy", AIP conference proceeding, p. 1005-1010, 2007

Chen, C and Kobayashi, S, Rigid plastic finite element analysis of ring compression, Appl. Numer. Methods Forming Process, AMD, (28), 1978, 163 (ASME)

Choi.H.H, Lee.J.H, Bijun.S.K, Kang.B.S, "Development of a three-dimensional finite-element program for metal forming and its application to precision coining", Journal of Materials Processing Technology Vol.72, p. 396-402, 1997

Christine Smith, "Evaluation of STL file, 3D modeling", January $16^{\text {th }}, 2009$

Clough, R. W., "The Finite Element Method in Plane Stress Analysis". J.Struct. Div., ASCE, Proc. $2^{\text {nd }}$ Conf. Electronic Computation, p.345, 1960. 
Courant, R., "Variational Methods for the Solution of Problems of Equilibrium and Vibrations". Bull.Amer. Math. Soc., Vol.49, p.1, 1943

Davey, K, Miller, B.C, Ward, M.J. Efficient strategies for the simulation of railway wheel forming, Journal of Material Processing Technology, Vol.188, p. 389-396, 2001

Delcam. Plc, ArtCAM developer, Italy, 2009

Edward M. Mielnik, Metalworking Science and Engineering, 1991 by McGraw-Hill

Engel. U., Eckstein. R., Journal of Material Processing Technology, Vol. 125-126. p. 35-44. 2002.

Fereshteh-Saniee. F, Hosseini. A.H., "The effects of flash allowance and bar size on forming load and metal flow in closed die forging", Journal of Materials Processing Technology, Vol. 177, 2006, p. 261-265.

Geiger. M., Kleiner. M., Eckstein. R., Tiesler. N., Engel. U., Annals of the CIRP, Vol. 50/2, p. 445-462, 2001.

Grill, R., Gnadenberger, A., "Niobium as mint metal: Production-properties-processing", International Journal of Refractory Metals \& Hard Materials Vol.24, p.275-282, 2006

Guo. K, Wang. X, Bell. R, Li. XY, "The experimental evaluation of friction conditions during a silver coining process. Steel Research International, Vol 79, 2008.

Haddad. Y. M, Mechanical behavior of engineering materials: static and quasi-static loading, Vol. 1, Kluwer Academic Publishers, 2000

Hayhurst. D.R, Chan. M.W, "Determination of friction models for metallic die-workpiece interfaces", International Journal of Mechanical Sciences Vol.47, p.1-25, 2005

Hawkyard.J.B, Johnson.W, "An analysis of the changes in geometry of a short hollow cylinder during axial compression”, Int. J. Mech. Sci. Vol. 9, p. 163-182, 1967

Ike. H., Plancak. J., Journal of Material Processing Technology, Vol. 80-81, p. 101-107, 1998.

Ike. H., Journal of Material Processing Technology, Vol. 138, p. 250-255, 2003.

Industrial Products Bulletin No. A-1, Handy \& Harman, New York, November, 1959 
Jain. V.K, Foster.B.J, "Investigation of friction behavior during non-isothermal deformation-processing”, ASME paper PED 54, Atlanta, 1991, S187-S201

Jorge M.C. Rodrigues, Paulo A.F. Martins, M.J.M. Barata Marques, "The PLAST3 system and its application to the simulation of an open die forging operation" Journal of Materials Processing Technology, Vol 47, 1994, p.111-125.

Journal of Research of the National Institute of Standards and Technology, Volume 100, Number 2, March-April 1995.

Kim. G.Y., Koc. M., Ni. J., “ Investigation on coining of micro-features using pure copper”, In ASME int. Conference on Manufacturing Science and Engineering, Ypsilanti, MI, Oct 8-11, 2006.

Kobayashi.S, Oh, S.I, Altan. T, Metal Forming and the Finite-Element Method, Oxford University Press, New York, 1989

Kudo.H, "Some analytical and experiemtal studies of axis-symmetric cold forging and extrusion". Int. J. Mech. Sci. 2. p. 102-127, 1960

Kunoqi.M, “A new method of cold extrusion”, J. Sci. Res. Inst. Tokyo, Vol. 50, p.215-246, 1956

Kwan. C.T., "An analysis of the closed-die forging of a general non-axisymmetric shape by the upper-bound elemental technique", Journal of Material Processing Technology. Vol.123, 2002, p.197-202. Elsevier Science.

Leitao. P.J, Teixeira. A.C, Rodrigues. J.M.C, Martins. P.A.F, "Development of an industrial process for minting a new type of bimetallic coin", Journal of Materials Processing Technology Vol.70, p. 178-184, 1997, Elsevier Science S.A

Male.A.T, Cockcroft. M.G, "A method for the determination of the coefficient of friction of metals under condition of bulk plastic deformation", J. Inst. Met. Vol. 93, p. 38-46, 1964

Marques. B.M, Martins. P, "A study of bi-metal coins by the finite element method", Journal of Materials Processing Technology, Vol. 26, p. 337-348, 1991

Mori, K, Osakada, K, Simulation of three-dimensional rolling by the finite-element method. Int. J. Mech. Sci. Vol.26, p.515-525, 1984 
Mote, C. D., Jr., Introduction to the Finite Element Method, Lecture Note, University of California at Berkeley, 1980

Nagtegaal, J. C., and Veldpaus, F. E., On the implementation of finite strain plasticity equations in a numerical model, "Numerical analysis of forming processes", edited by J. F. T. Pittman et al., Wiley, New York, pp.351, 1984

Oden. JT, Pires. EB, Nonlocal and nonlinear friction law and variational principles for contact problems in elasticity. Journal of Applied Mechanics. Vol 50, p.67-76,1983.

Park, J.J, Kobayashi, S, Three-dimensional finite element analysis of block compression. Int. J. Mech. Sci. Vol.26, p.165-176, 1984

Rowe, G.W., Sturgess, C.E.N., Hartley, P., and Pillinger, I., Finite Element Plasticity and Metalforming Analysis, Cambridge University Press, 1991

Royal Canadian Mint, Private conversation, 2007

Schey JA. Tribology in metalworking: friction, lubrication and wear. Metals Park (OH): American Society For Metals, 1983

Sedighi. M, Tokmechi. S, A new approach to preform design in forging process of complex parts, Journal of Materials processing Technology, 2007

Shiau. Y.C, Three-dimensional finite element analysis of open-die forging and plate rolling, $\mathrm{PhD}$ Dissertation, University of California, Berkeley, 1989

Siebel E. Resistance and deformation and the flow material during rolling. Stahl und Eisen Vol.50, p.1769-1775, 1930.

Standard Methods of Tension Testing of Metallic Materials, E8, Annual Book of ASTM standards, Volume 03.01, ASTM, Philadelphia, 1984, p. 130-150

Surdon. G, Chenot. J.L. Finite element calculation of three-dimensional hot forging. Int. J. Num. Mech. Eng. Vol. 24, p.2107-2117, 1987

Tekkaya. A.E, "A guide for validation of FE-simulations in bulk metal forming", The Arabian Journal for Science and Engineering, Vol 30, p.113-136, 2005

Tentative Specification for 999.0 Grade Refined Silver, ASTM B-413-64T, Philadelphia, 1964. 
Thomsen. E.G, Yang. C.T, Kobayashi. S, Mechanics of Plastic Deformation in Metal Processing, the Macmillan company, New York, 1965

Victor Vazquez, Taylan Altan, Die design for flashless forging of complex parts, Journal of Materials Processing Technology, Vol 98, p.81-89, 2000

Von Karman, T., "Contribution to the Theory of Rolling" (in German), Z Angew. Math. Mech. vol.5, p. 139-141, 1925

Wagoner.R.H., Chenot. J. L, Metal forming analysis. Cambridge University Press, Cambridge, New York, 2001

Wagoner. R.H., Chenot. J. L, Fundamentals of metal forming. Wiley, New York, 1997

Wang. C.J., Shan. D.B., Zhou. J., Guo. B., Sun. L.N., Journal of Material Processing Technology, 2007

Zhao Guoqun, Wang Guangchun, Grandhi. Ramana V. Die cavity design of near flashless forging process using FEM-based backward simulation. Journal of Material Processing Technology Vol 121, 2002, pp.173-181

Zienkiewicz OC. The finite element method. New York: Mcgraw-Hill; 1977

Zhong.ZH. "Contact problems with friction", Proceedings of Numiform 89, conference, Balkema, Rotterdam, p.599-606, 1989 


\section{Appendix}

In this section, the deformed mesh patterns and material flow velocity patterns of $2 \mathrm{D}$ axisymmetric coining are presented here.

Figure A-1 to A-5 show the deformed mesh patterns of $2 \mathrm{D}$ axisymmetric coining with various top die curvatures.

Figure A-6 to A-10 show the material flow patterns of $2 \mathrm{D}$ axisymmetric coining with various top die curvatures.

Figure A-11 to A-15 show the deformed mesh patterns of $2 \mathrm{D}$ axisymmetric coining with various bottom die curvatures.

Figure A-16 to A-20 show the material flow patterns of $2 \mathrm{D}$ axisymmetric coining with various bottom die curvatures.

Figure A-21 to A-26 show the deformed mesh patterns of $2 \mathrm{D}$ axisymmetric coining with various rim heights.

Figure A-27 to A-32 show the material flow patterns of 2D axisymmetric coining with various rim heights.

Figure A-33 to A-34 show the deformed mesh patterns of $2 \mathrm{D}$ axisymmetric coining with various relief heights.

Figure A-35 to A-36 show the material flow patterns of $2 \mathrm{D}$ axisymmetric coining with various relief heights.

Table A-1. Load against displacement results in ring test

Table A-2. Load Vs Displacement with varying top die curvature

Table A-3. Load Vs Displacement with varying bottom die curvature

Table A-4. Load Vs Displacement with varying rim height

Table A-5. Load Vs Displacement with varying relief height 
A). Material deforming patterns with varying top die curvatures

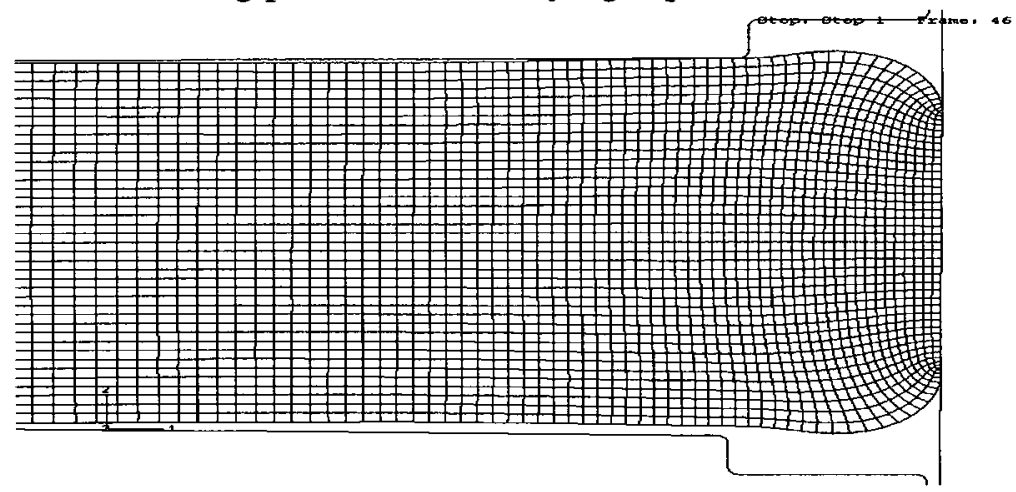

$$
\begin{gathered}
\text { (a) } 50 \% \text { axial } \\
\text { stroke }
\end{gathered}
$$

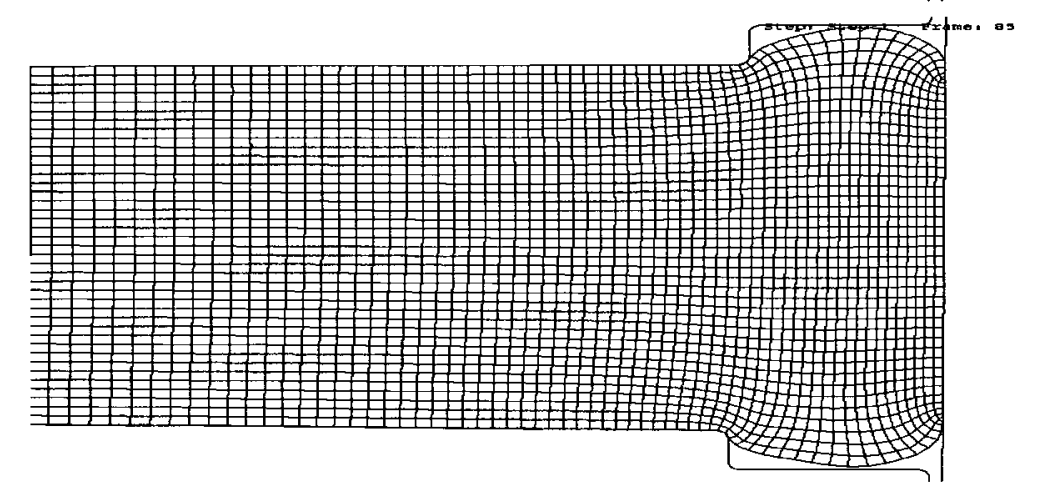

(b) $75 \%$ axial stroke

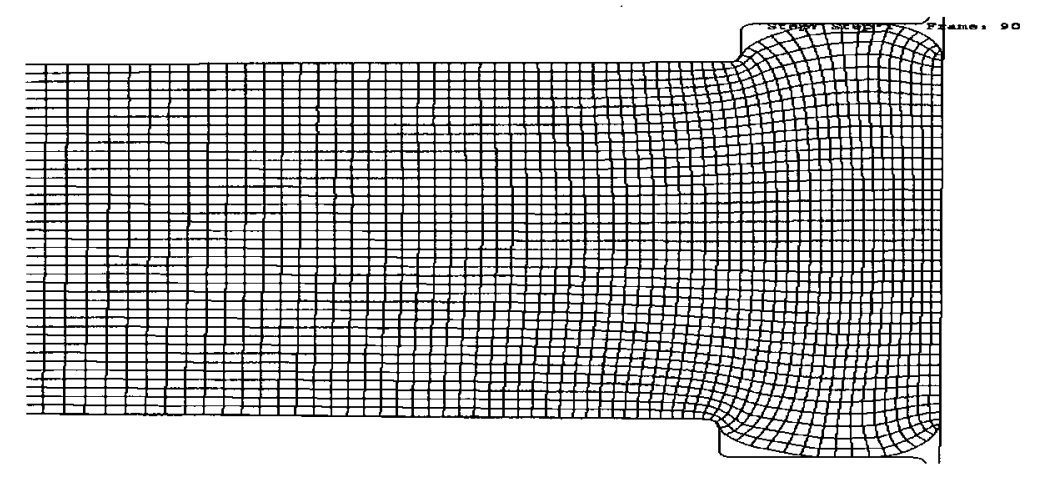
(c) $90 \%$ axial stroke

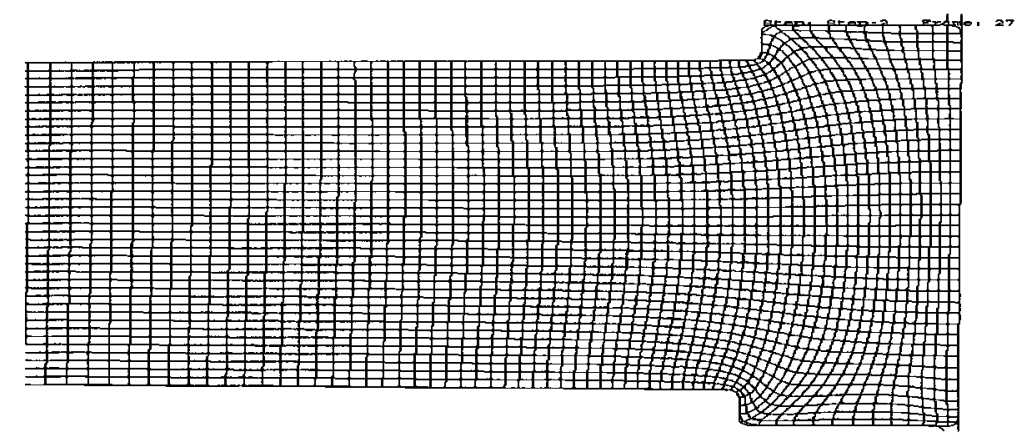
(d) $100 \%$ axial stroke

Figure A-1. Metal deformed mesh patterns showing outer $20 \%$ of blank (Top die curvature $=0.05 \mathrm{~mm}$, Bottom die curvature $=0.19 \mathrm{~mm}$, Rim Height $=0.271 \mathrm{~mm}$, no relief) 


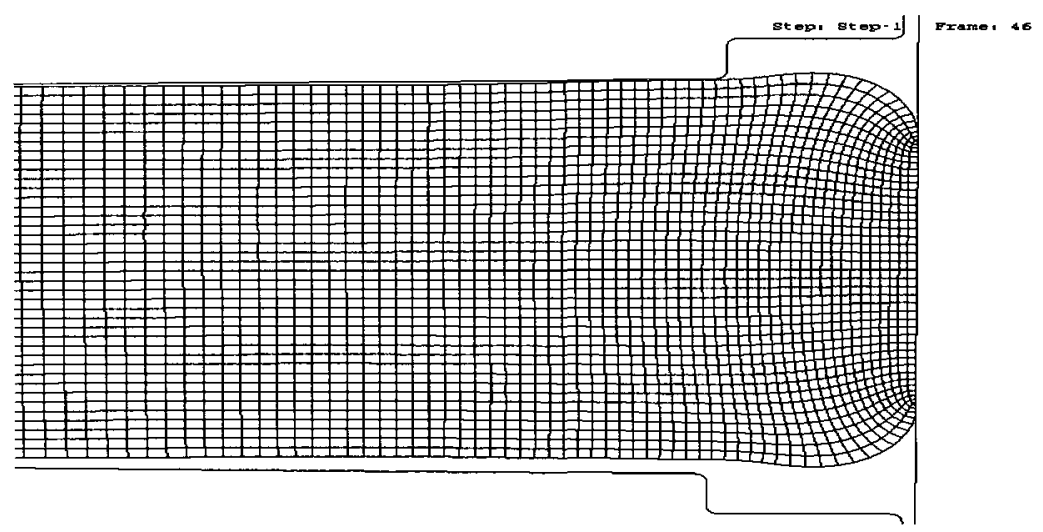

(a) $50 \%$ axial stroke

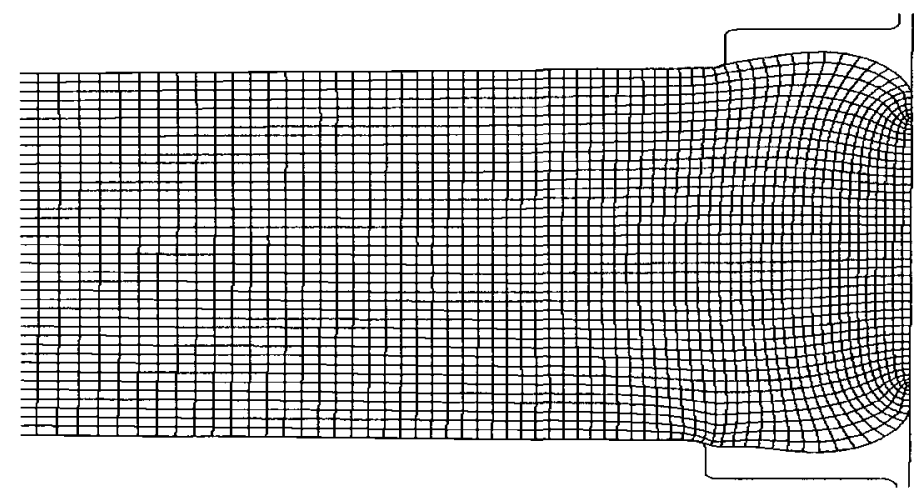

(b ) $75 \%$ axial stroke

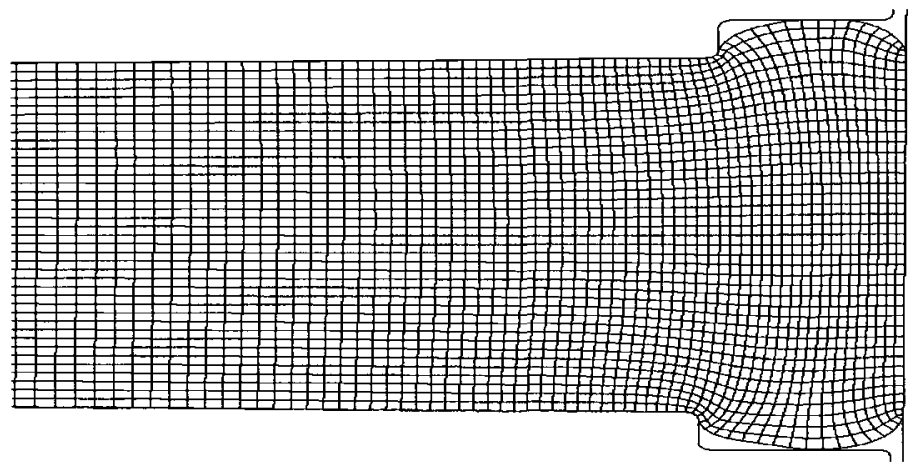

(c) $90 \%$ axial stroke

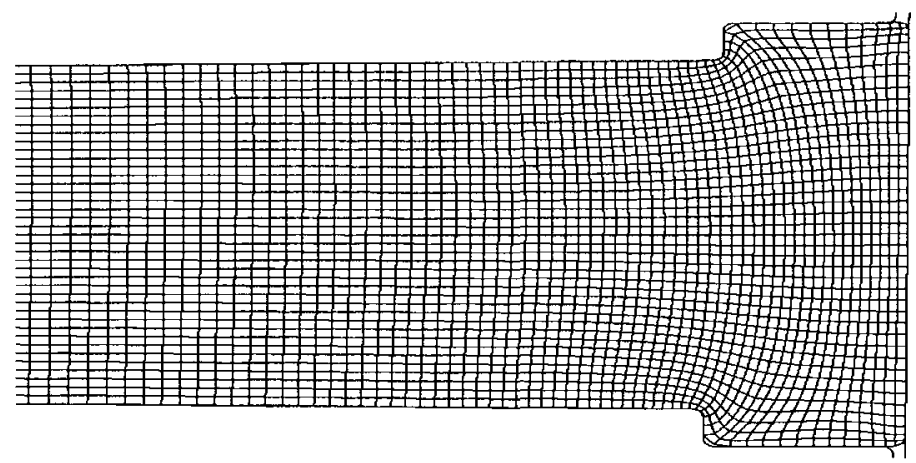

(d) $100 \%$ axial stroke

Figure A-2. Metal deformed mesh pattern showing outer $20 \%$ of blank

(Top die curvature $=0.1 \mathrm{~mm}$, Bottom die curvature $=0.19 \mathrm{~mm}$, Rim Height $=0.271 \mathrm{~mm}$, no relief) 


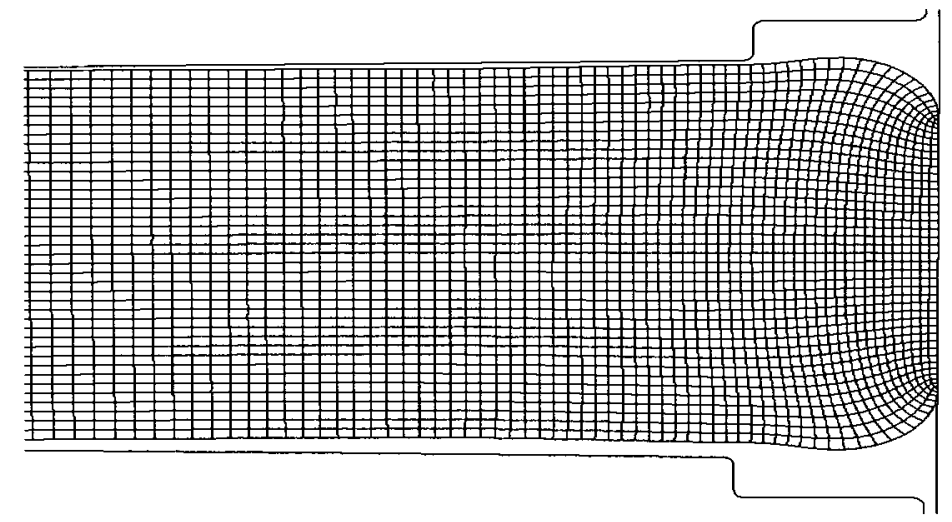

(a) $50 \%$ axial stroke

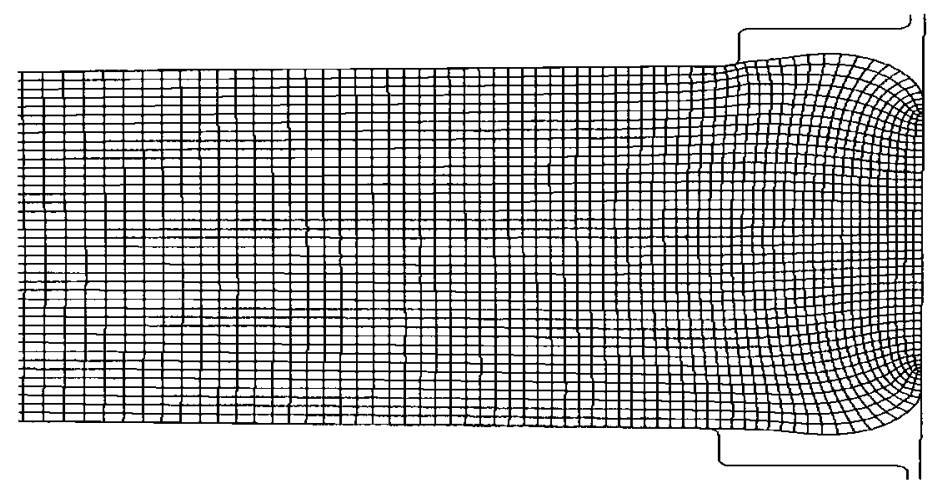

(b ) $75 \%$ axial stroke

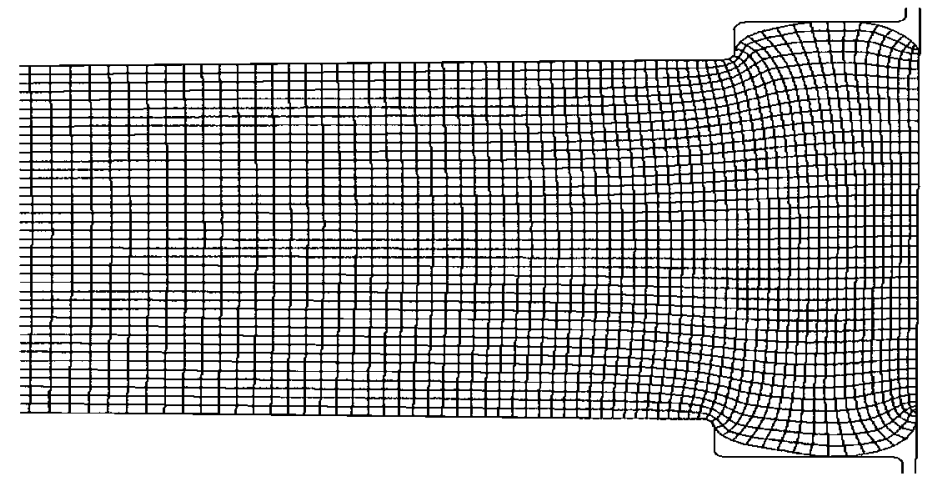

(c) $90 \%$ axial stroke

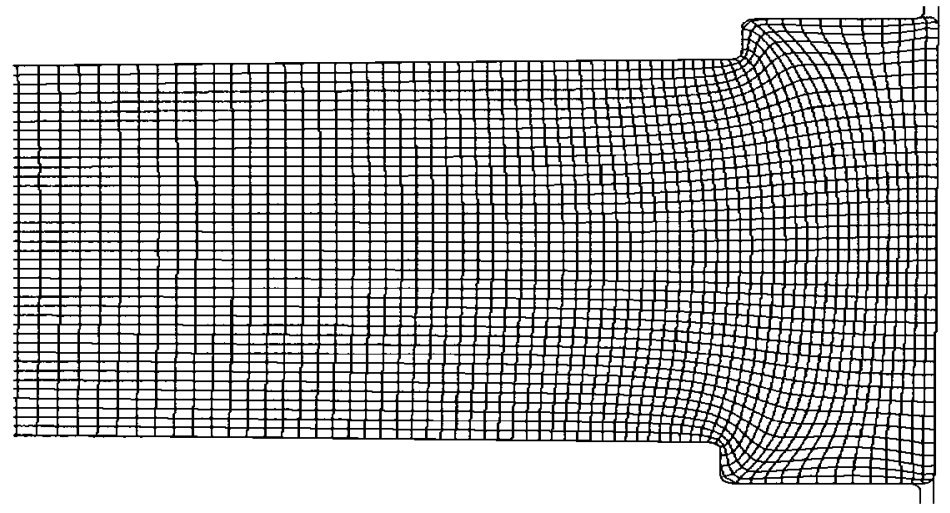

(d) $100 \%$ axial stroke

Figure A-3. Metal deformed mesh patterns showing outer 20\% of blank

(Top die curvature $=0.15 \mathrm{~mm}$, Bottom die curvature $=0.19 \mathrm{~mm}$, Rim Height $=0.271 \mathrm{~mm}$, no relief) 


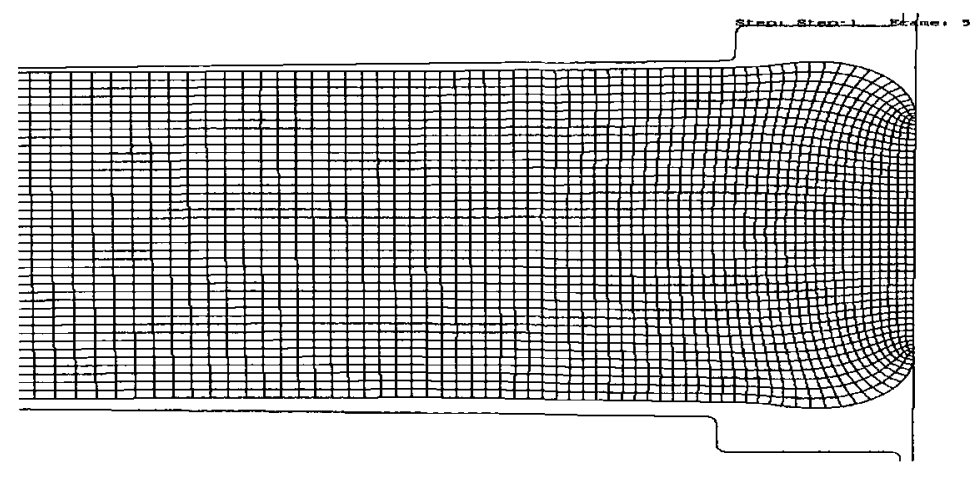

( a ) $50 \%$ axial stroke

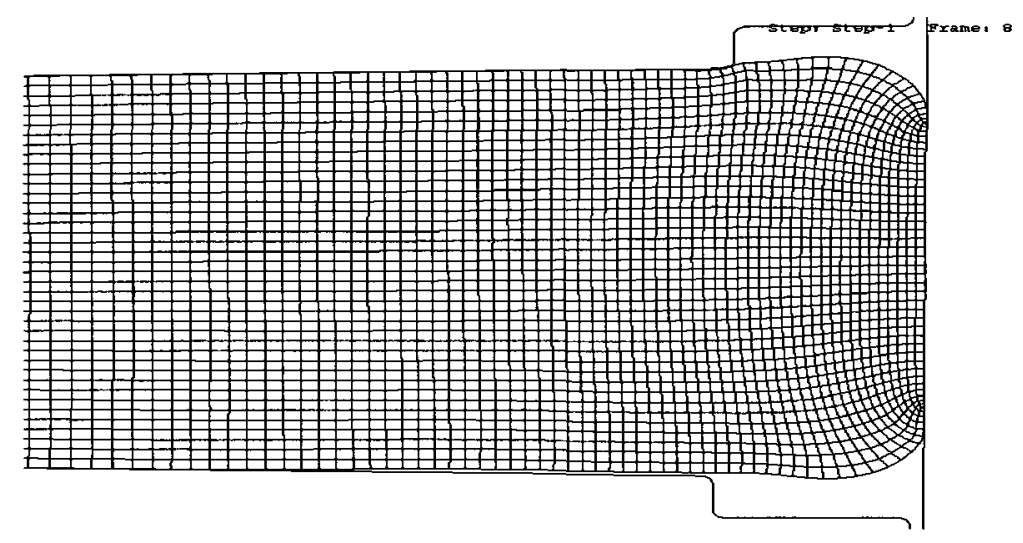

(b ) $75 \%$ axial stroke

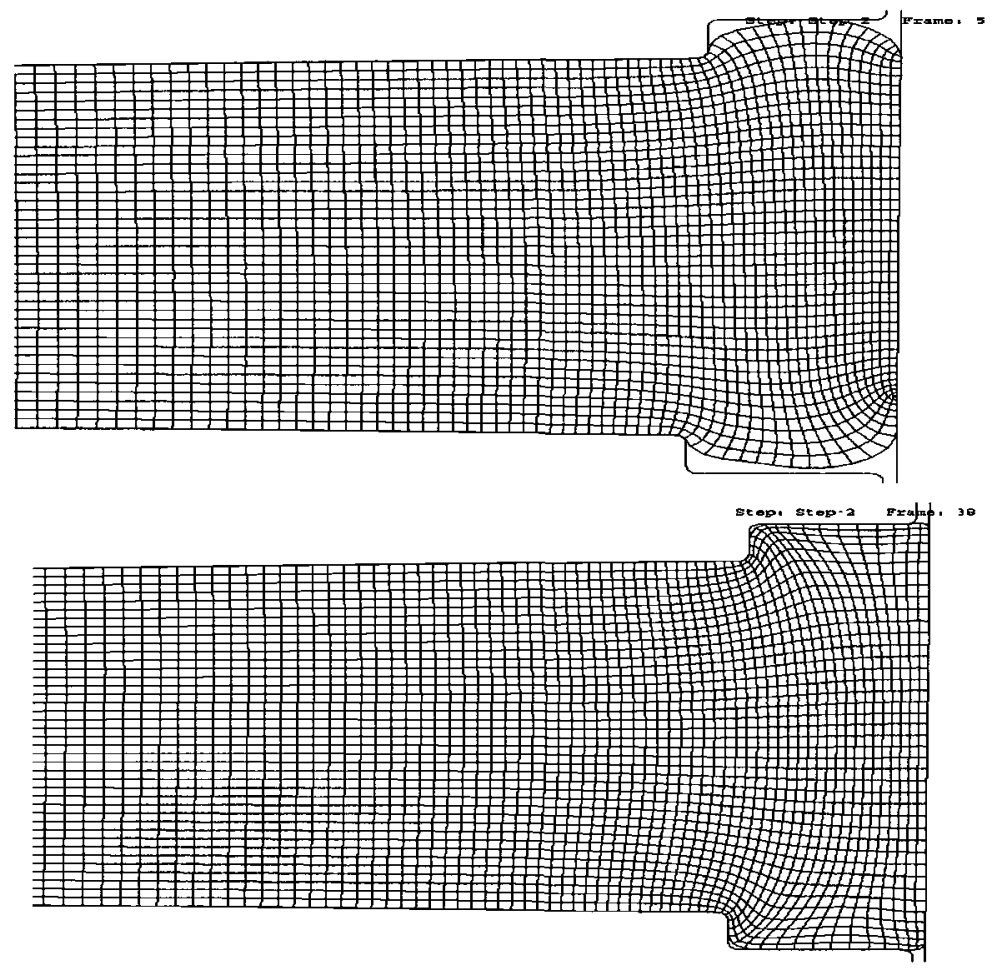

(c) $90 \%$ axial stroke

(d ) $100 \%$ axial stroke

Figure A-4. Metal deformed mesh patterns showing outer $20 \%$ of blank

(Top die curvature $=0.2 \mathrm{~mm}$, Bottom die curvature $=0.19 \mathrm{~mm}$, Rim Height $=0.271 \mathrm{~mm}$, no relief) 


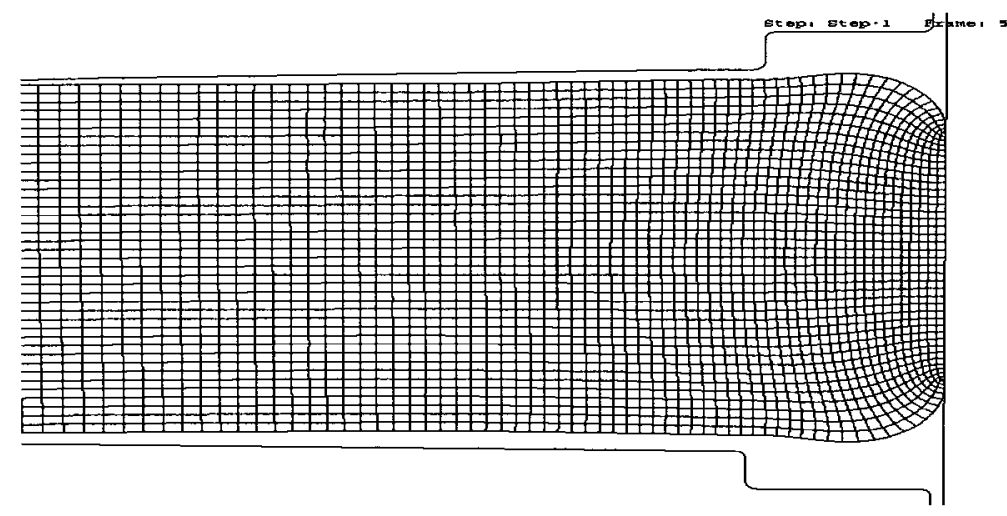

(a ) $50 \%$ axial stroke



(b ) $75 \%$ axial stroke

(c) $90 \%$ axial stroke

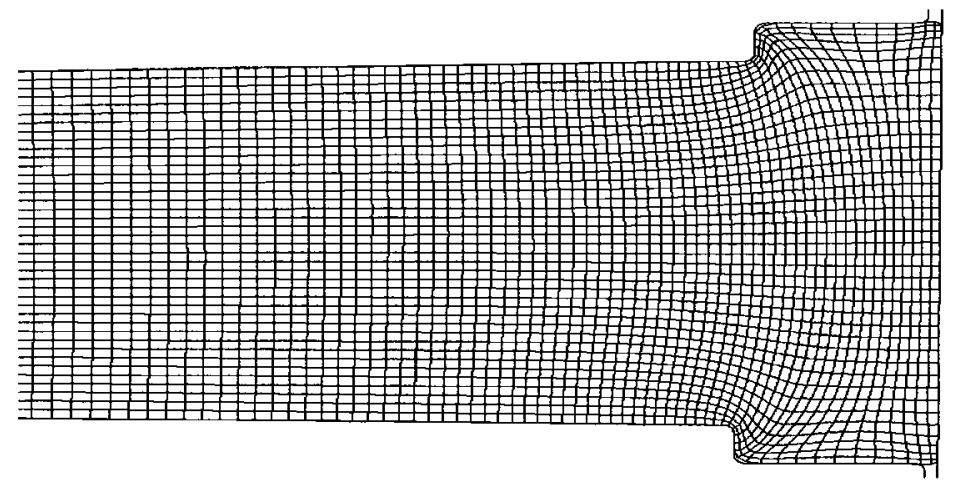

(d ) $100 \%$ axial stroke

Figure A-5. Metal deformed mesh patterns showing outer $20 \%$ of blank

(Top die curvature $=0.25 \mathrm{~mm}$, Bottom die curvature $=0.19 \mathrm{~mm}$, Rim Height $=0.271 \mathrm{~mm}$, no relief) 
B). Material flow velocities with varying top die curvatures

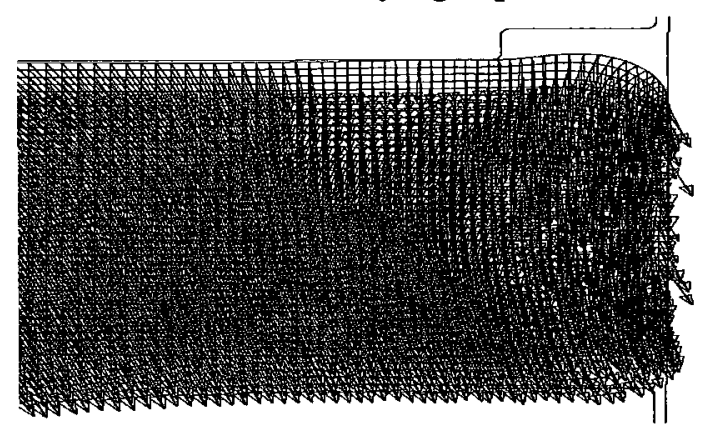

\author{
(a) $50 \%$ axial \\ stroke
}

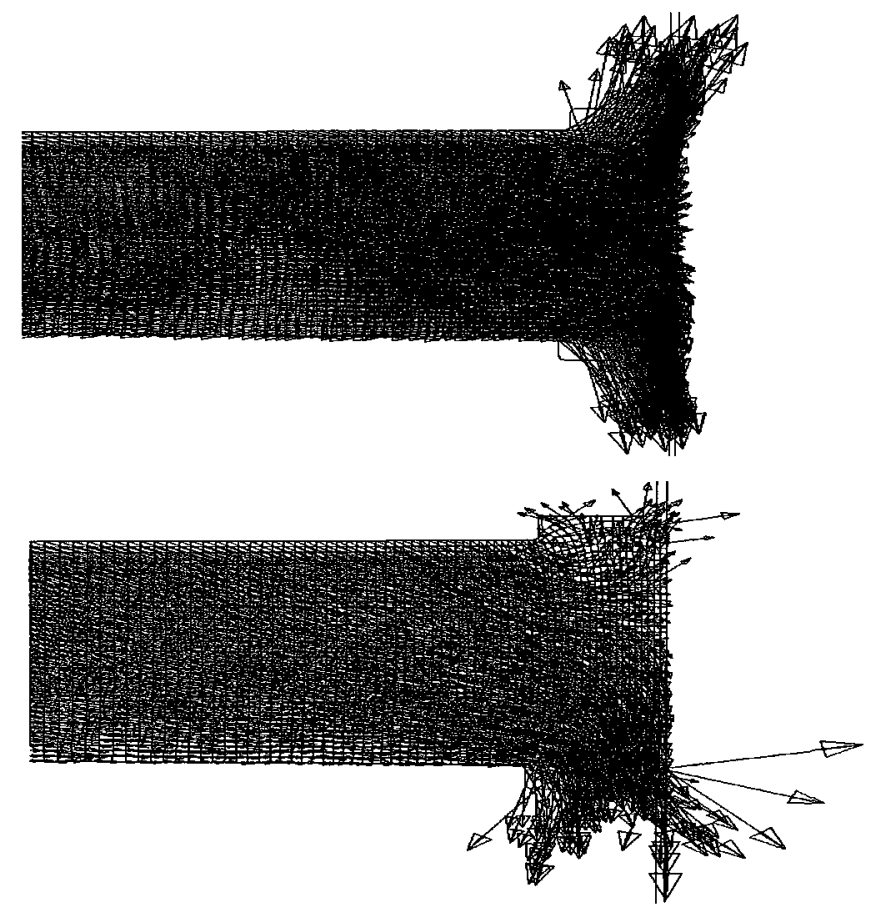

(b ) $75 \%$ axial stroke

(c) $90 \%$ axial stroke

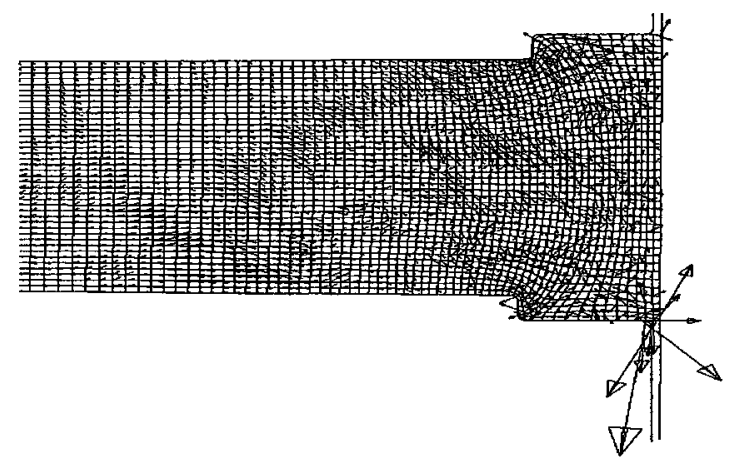

(d) $100 \%$ axial stroke

Figure A-6. Material flow velocity patterns showing outer $20 \%$ of blank (Top die curvature $=0.05 \mathrm{~mm}$, Bottom die curvature $=0.19 \mathrm{~mm}$, Rim Height $=0.271 \mathrm{~mm}$, no relief) 


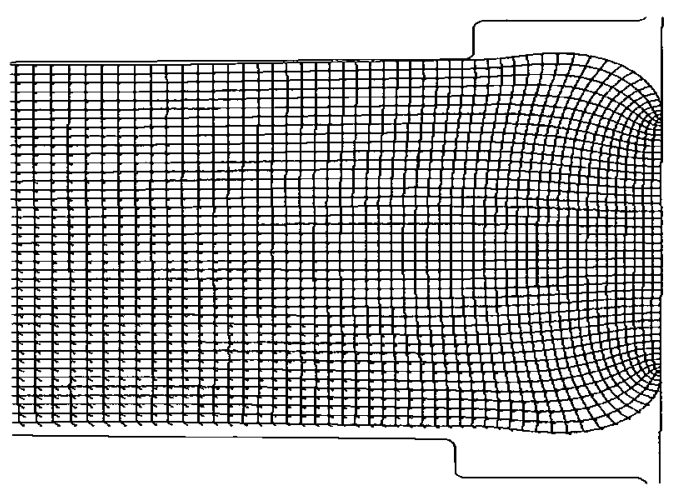
(a ) $50 \%$ axial stroke

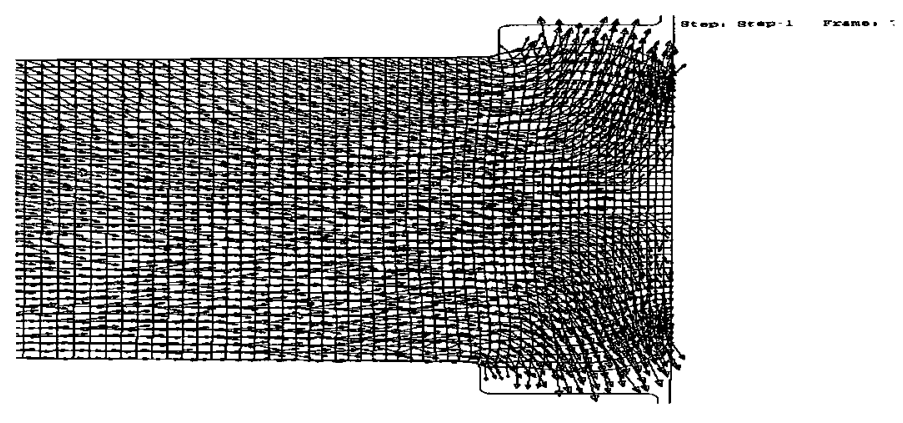

(b ) $75 \%$ axial stroke

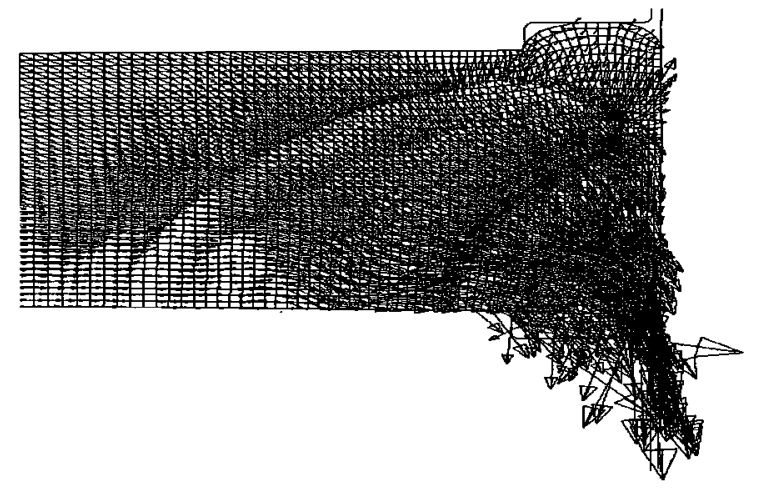

(c) $90 \%$ axial stroke

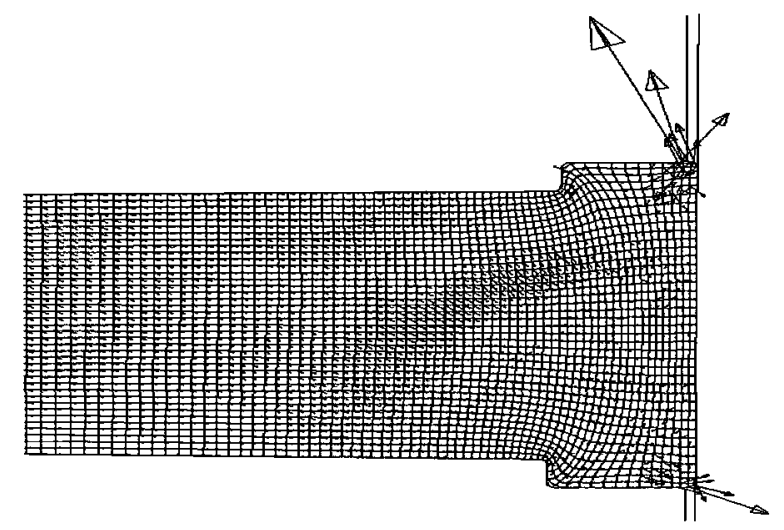

(d) $100 \%$ axial stroke

Figure A-7. Material flow velocity patterns showing outer $20 \%$ of blank

(Top die curvature $=0.1 \mathrm{~mm}$, Bottom die curvature $=0.19 \mathrm{~mm}$, Rim Height $=0.271 \mathrm{~mm}$, no relief) 


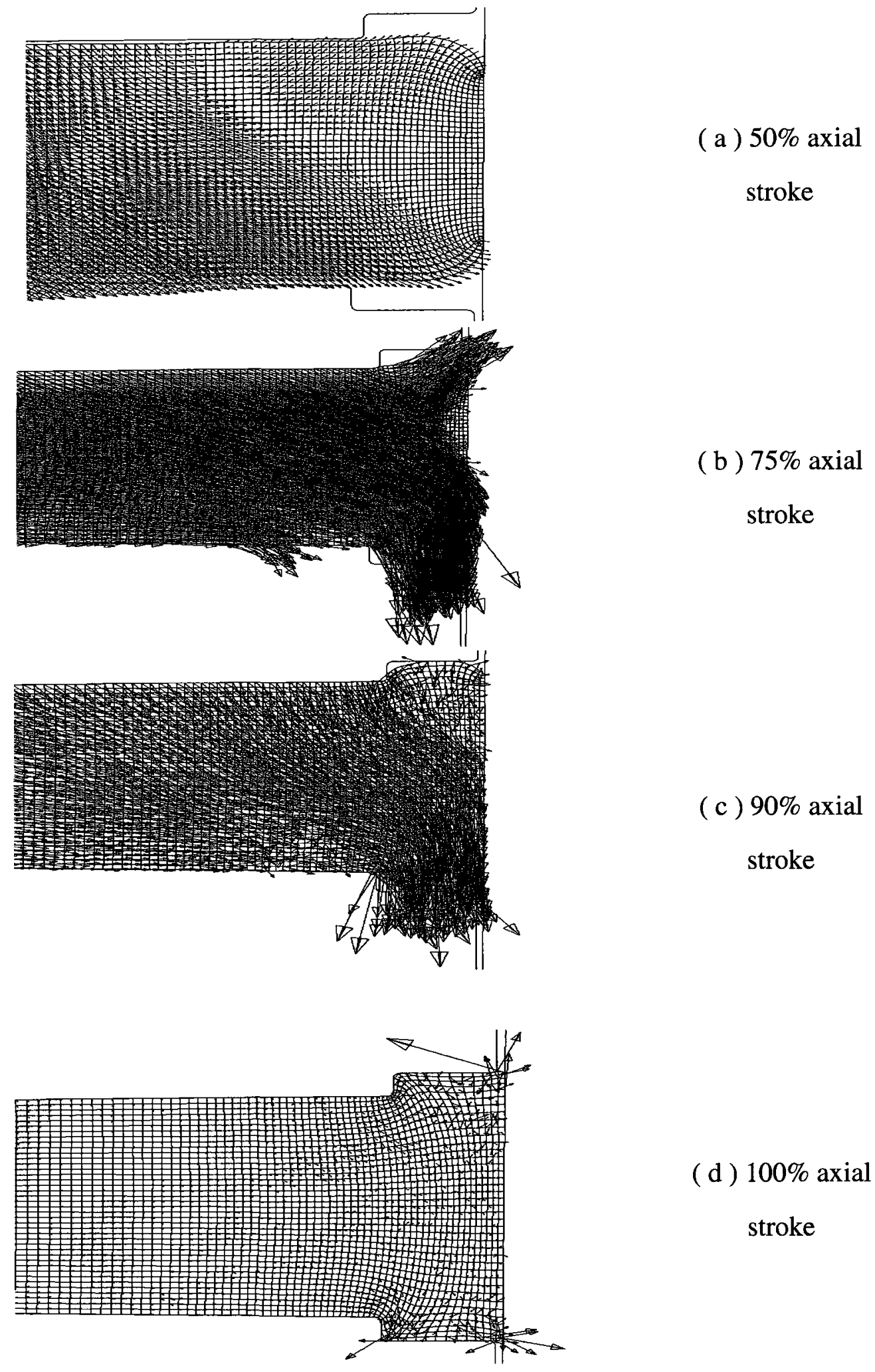

Figure A-8. Material flow velocity patterns showing outer $20 \%$ of blank

(Top die curvature $=0.15 \mathrm{~mm}$, Bottom die curvature $=0.19 \mathrm{~mm}$, Rim Height $=0.271 \mathrm{~mm}$, no relief) 


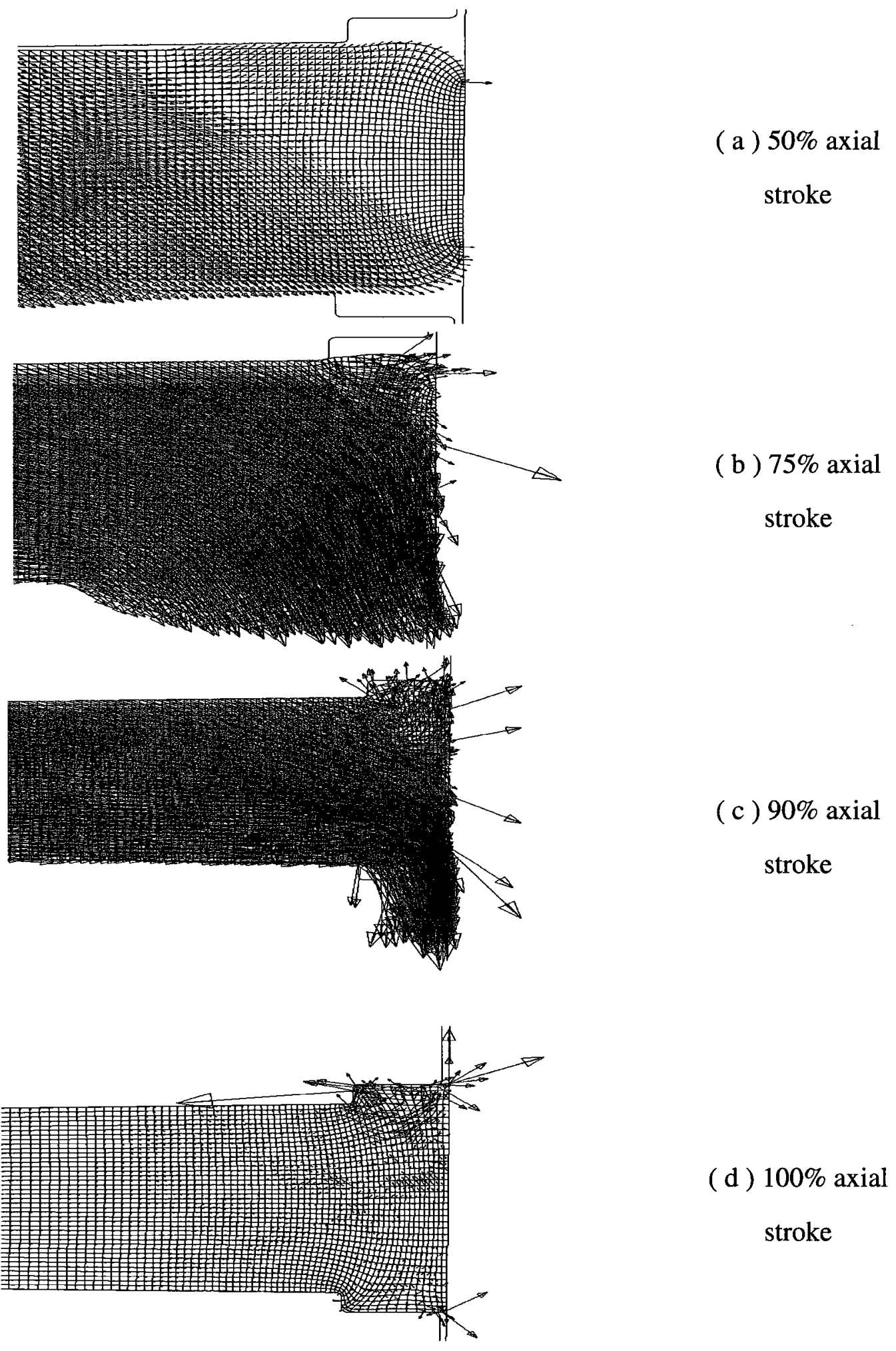

Figure A-9. Material flow velocity patterns showing outer $20 \%$ of blank (Top die curvature $=0.2 \mathrm{~mm}$, Bottom die curvature $=0.19 \mathrm{~mm}$, Rim Height $=0.271 \mathrm{~mm}$, no relief) 


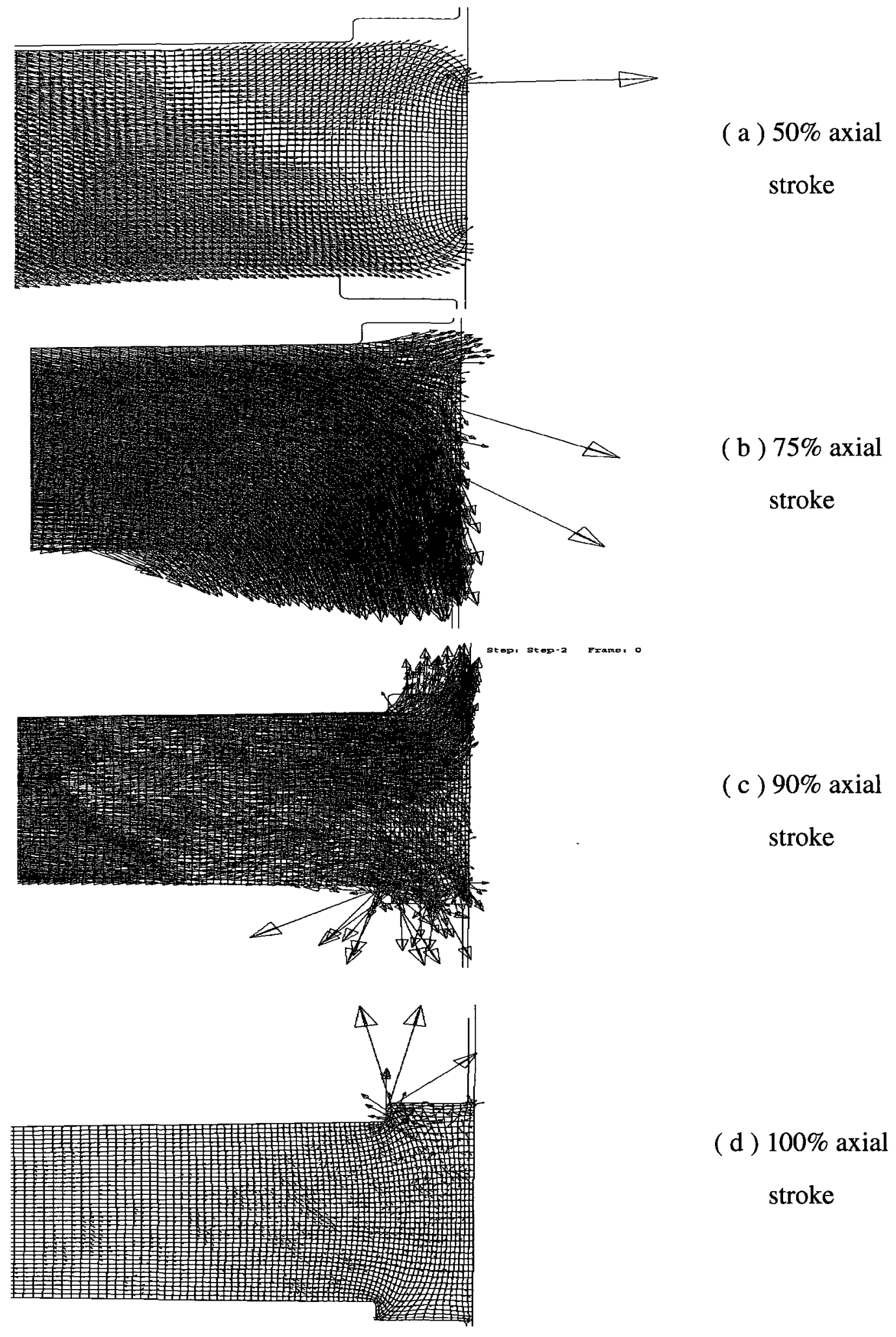

Figure A-10. Material flow velocity patterns showing outer $20 \%$ of blank

(Top die curvature $=0.25 \mathrm{~mm}$, Bottom die curvature $=0.19 \mathrm{~mm}$, Rim Height $=0.271 \mathrm{~mm}$, no relief) 
A). Material deforming patterns with varying bottom die curvatures
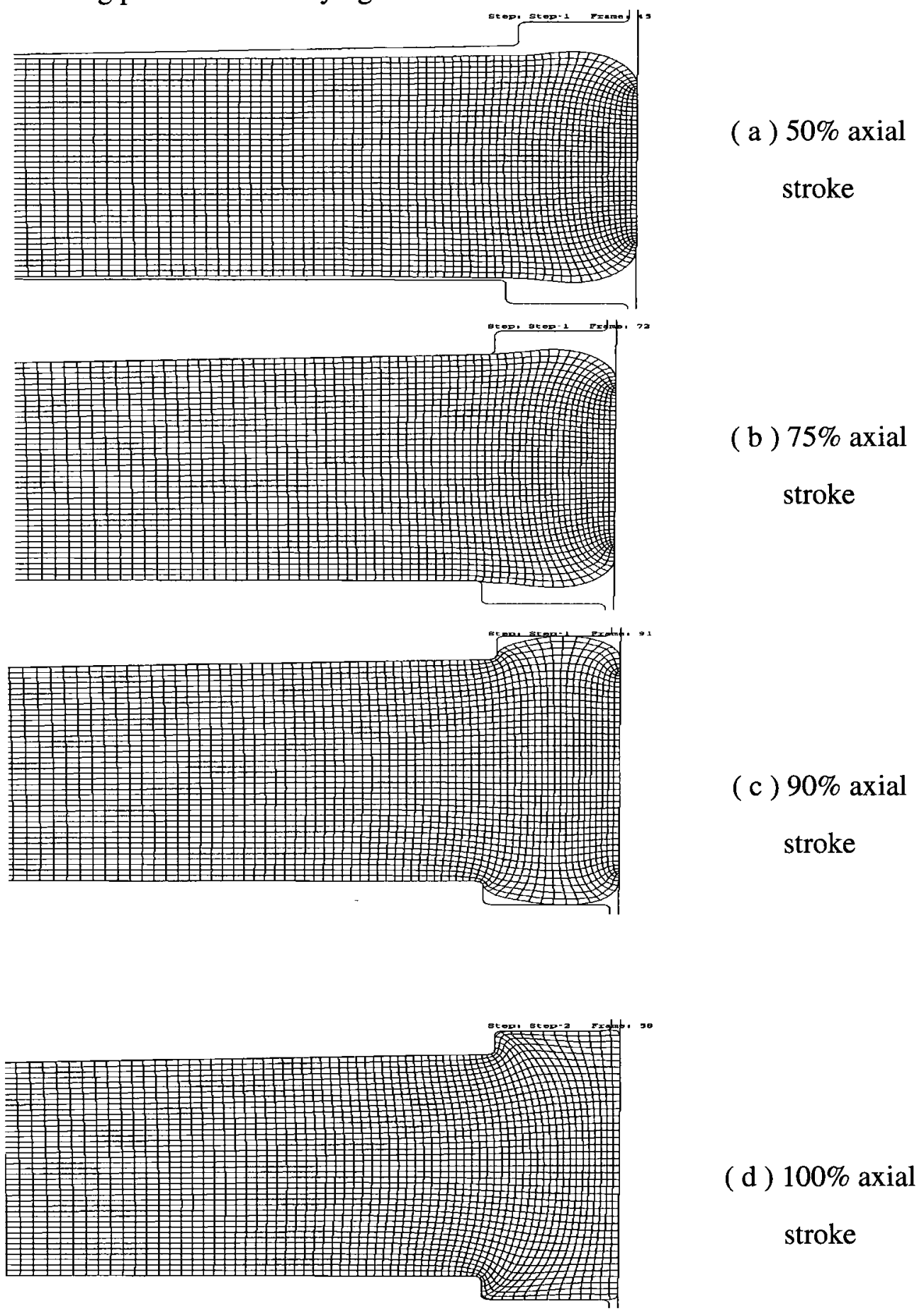

Figure A-11. Metal deformed mesh patterns showing outer $20 \%$ of blank (Top die curvature $=0.3 \mathrm{~mm}$, Bottom die curvature $=0.05 \mathrm{~mm}$, Rim Height $=0.271 \mathrm{~mm}$, no relief) 


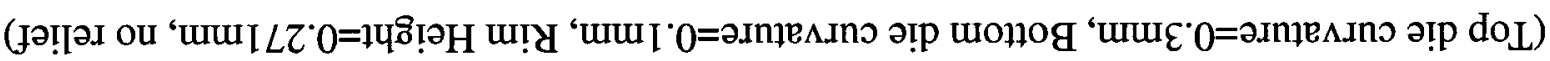

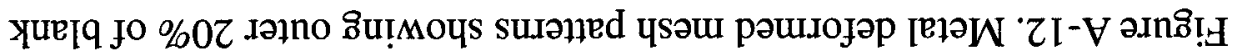

əxo.js
[EIXe \%00I ( p )

[e!xe \%00I ( p )

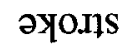

[e!xe \%06 ( o )

วYo.1?

$[\operatorname{PIXE} \% S L(q)$

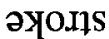

[E!XE \%0S ( e )
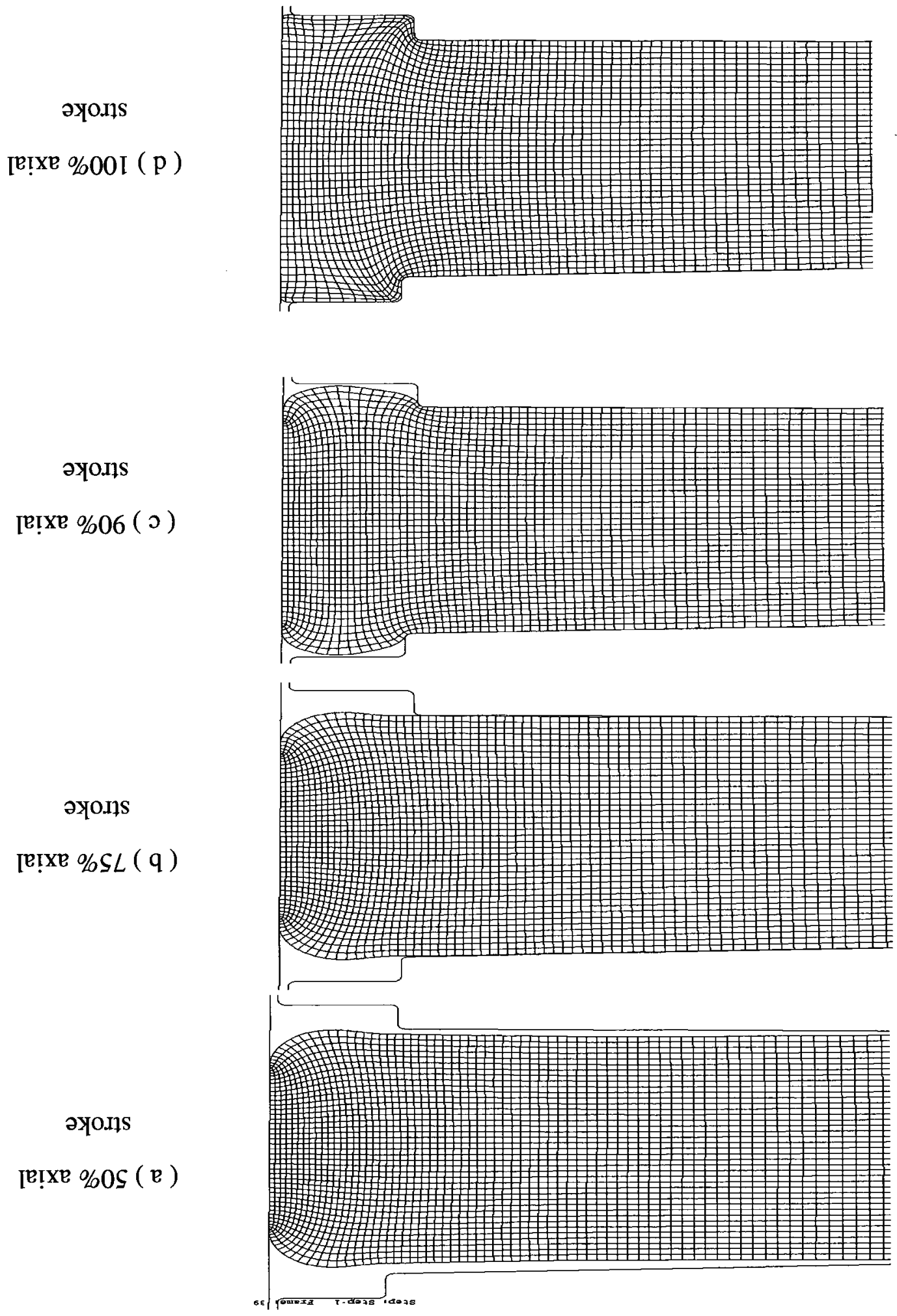


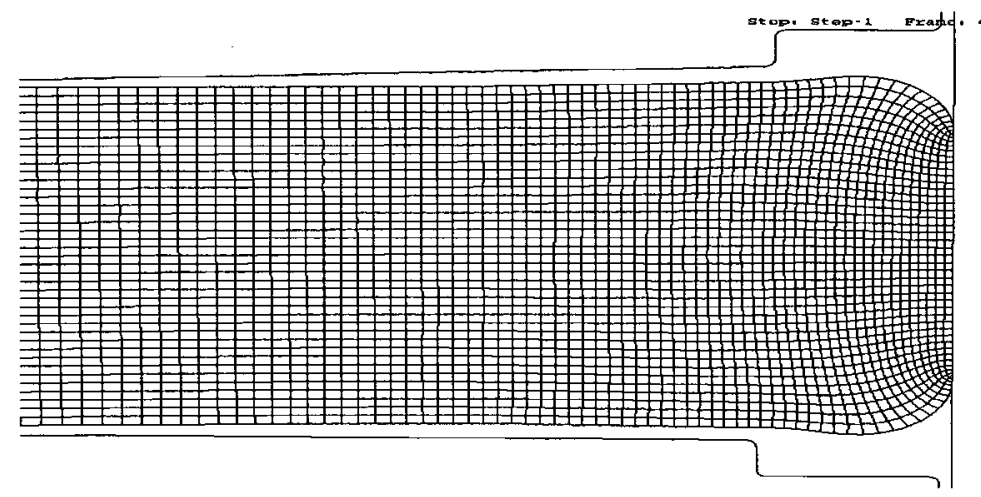

(a ) $50 \%$ axial

stroke

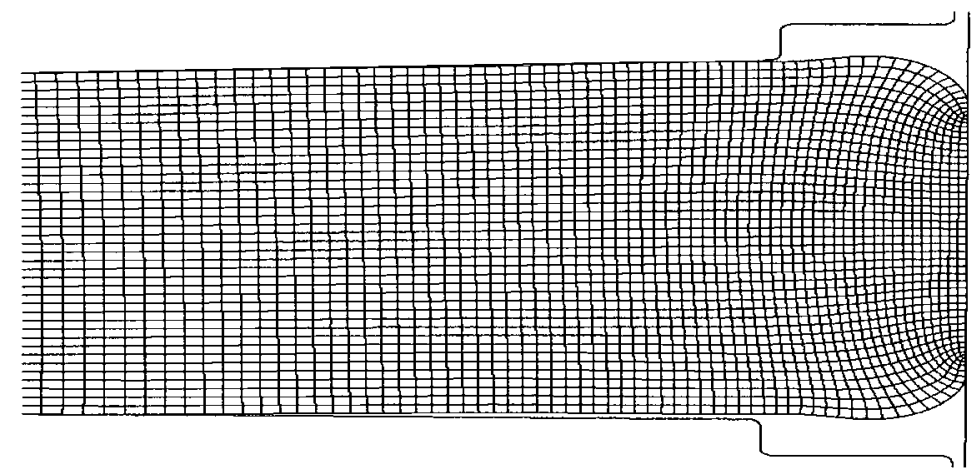

(b ) $75 \%$ axial

stroke

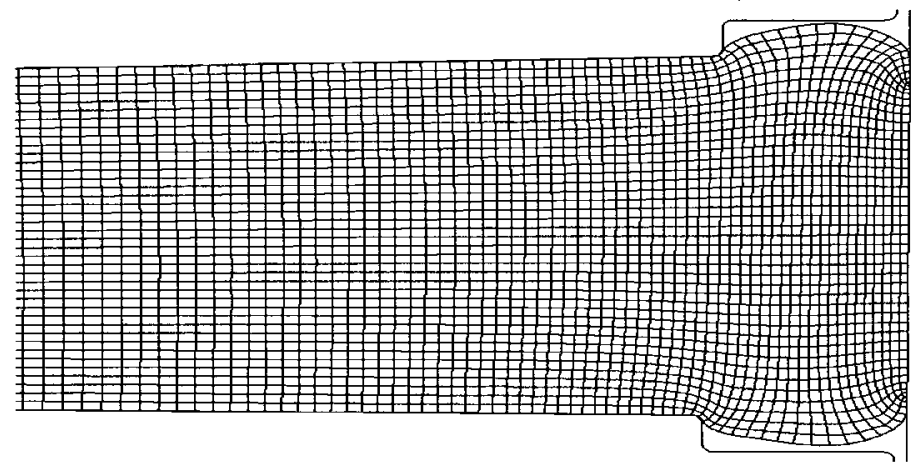

(c) $90 \%$ axial

stroke

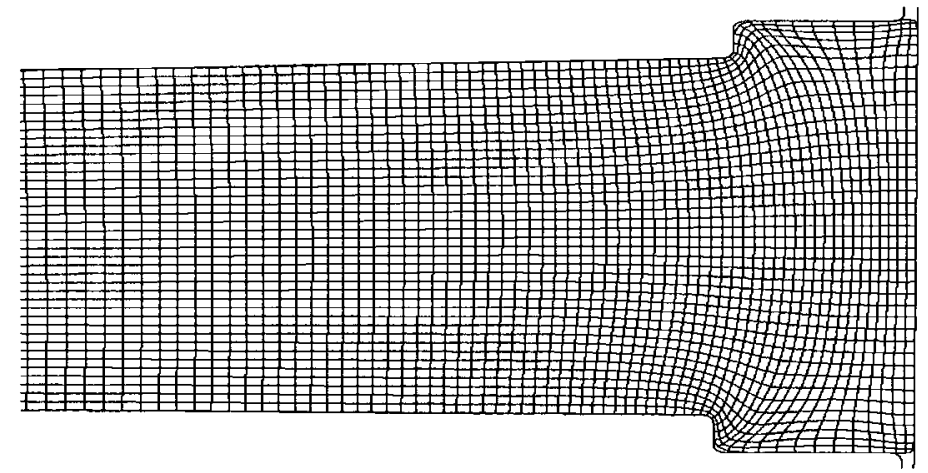

(d ) $100 \%$ axial

stroke

Figure A-13. Metal deformed mesh patterns showing outer $20 \%$ of blank

(Top die curvature $=0.3 \mathrm{~mm}$, Bottom die curvature $=0.15 \mathrm{~mm}$, Rim Height $=0.271 \mathrm{~mm}$, no relief) 


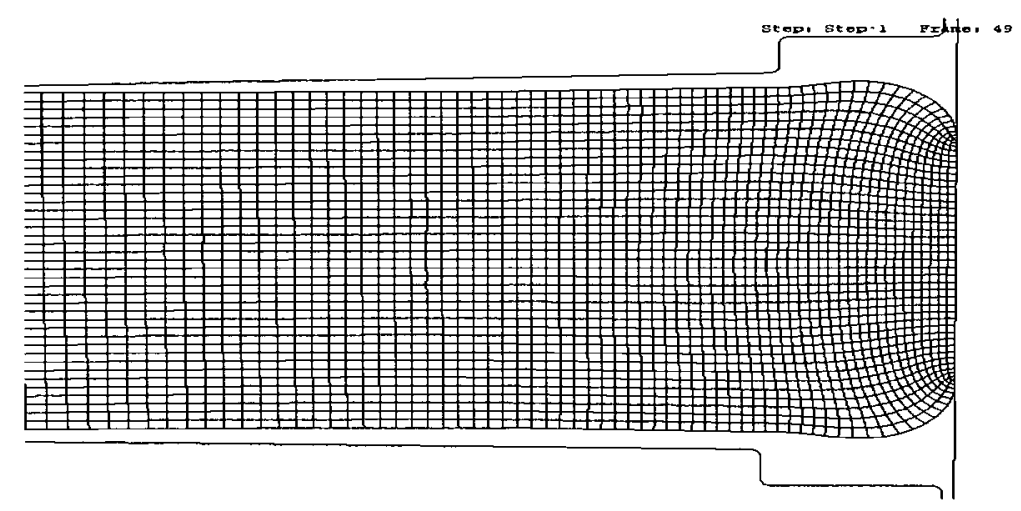
(a ) $50 \%$ axial
stroke

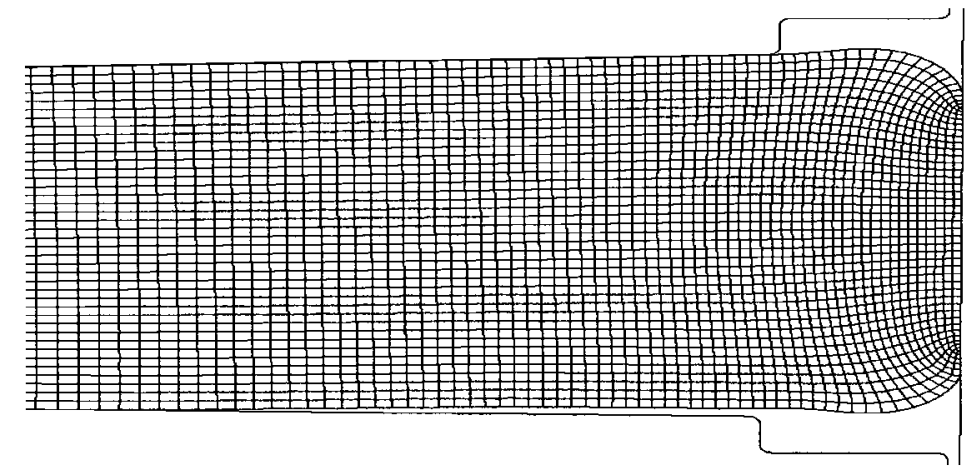

(b ) $75 \%$ axial

stroke

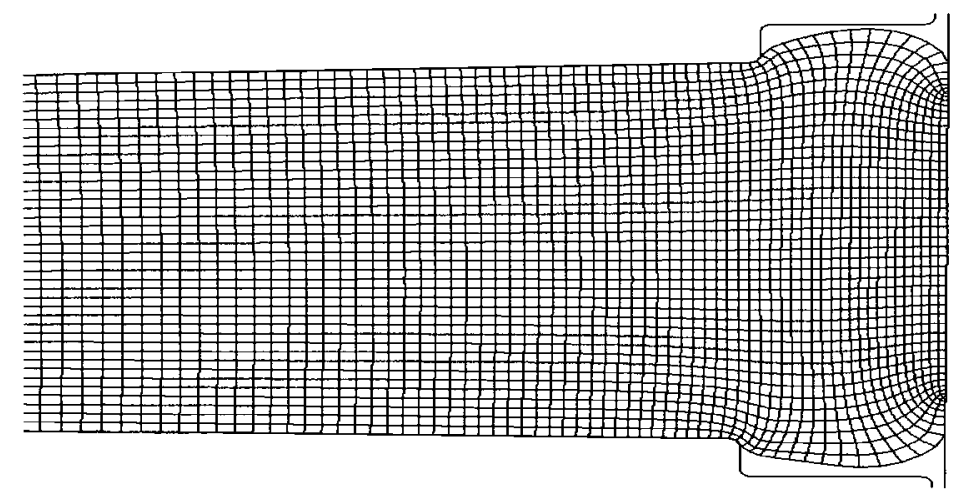
(c) $90 \%$ axial stroke

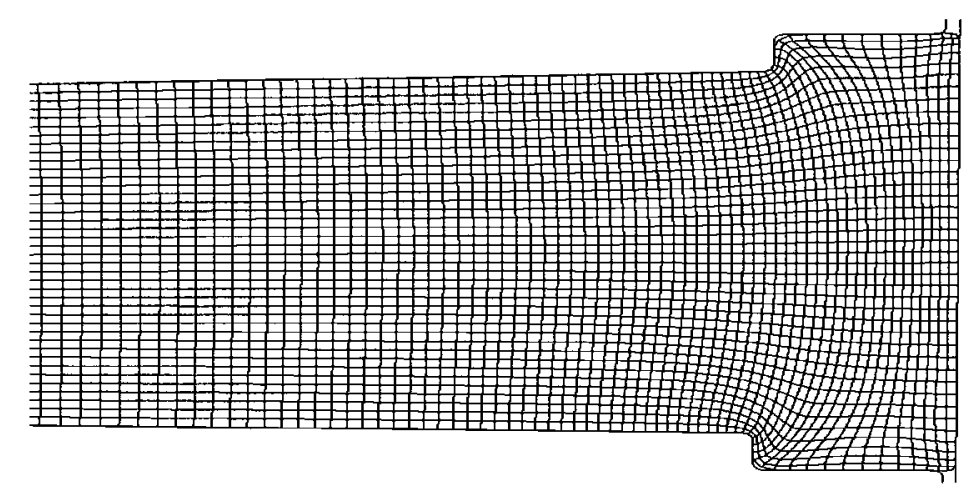

(d) $100 \%$ axial stroke

Figure A-14. Metal deformed mesh patterns showing outer $20 \%$ of blank (Top die curvature $=0.3 \mathrm{~mm}$, Bottom die curvature $=0.2 \mathrm{~mm}$, Rim Height $=0.271 \mathrm{~mm}$, no relief) 


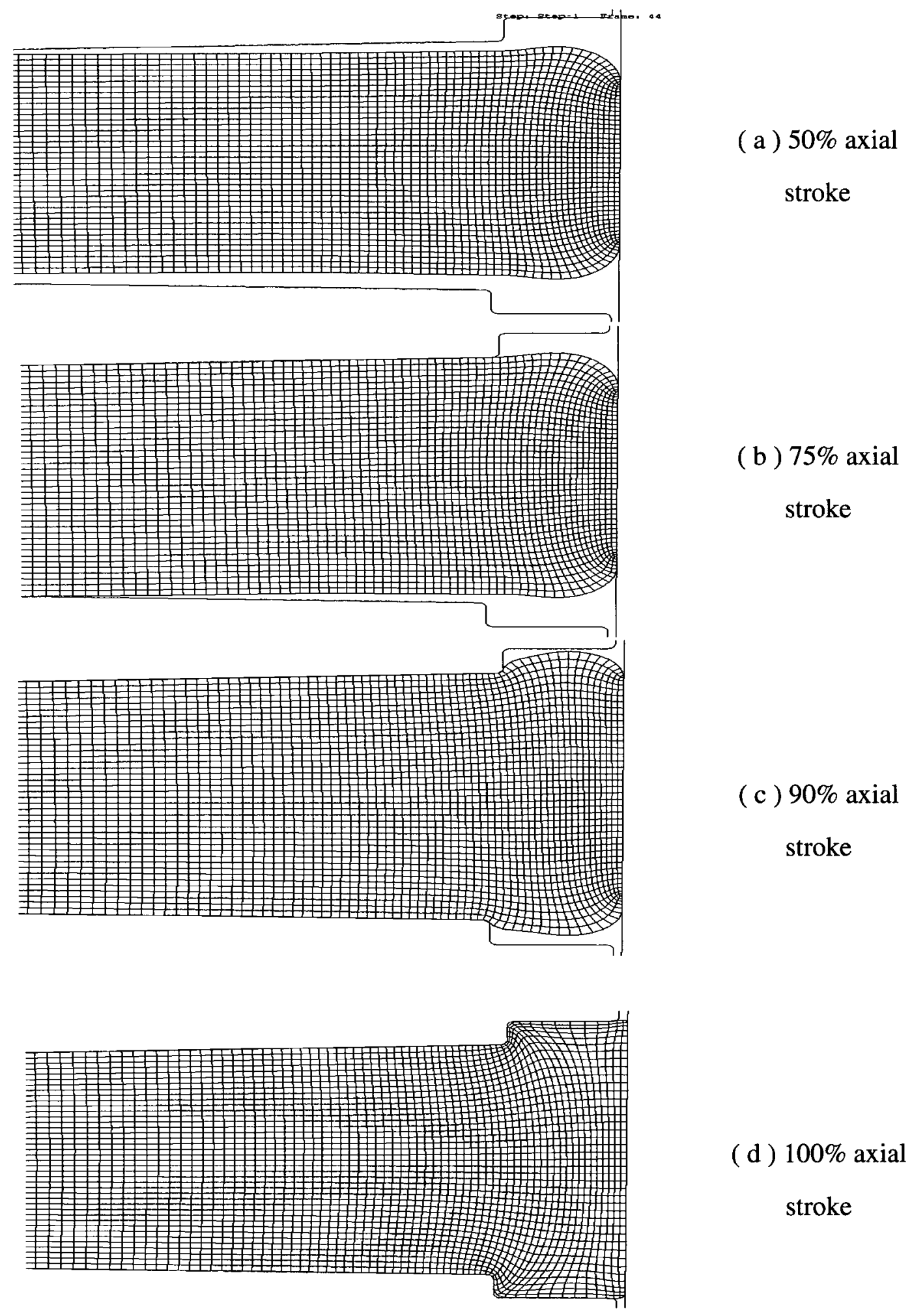

Figure A-15. Metal deformed mesh patterns showing outer $20 \%$ of blank (Top die curvature $=0.3 \mathrm{~mm}$, Bottom die curvature $=0.25 \mathrm{~mm}$, Rim Height $=0.271 \mathrm{~mm}$, no relief) 
B). Material flow velocities with varying bottom die curvatures

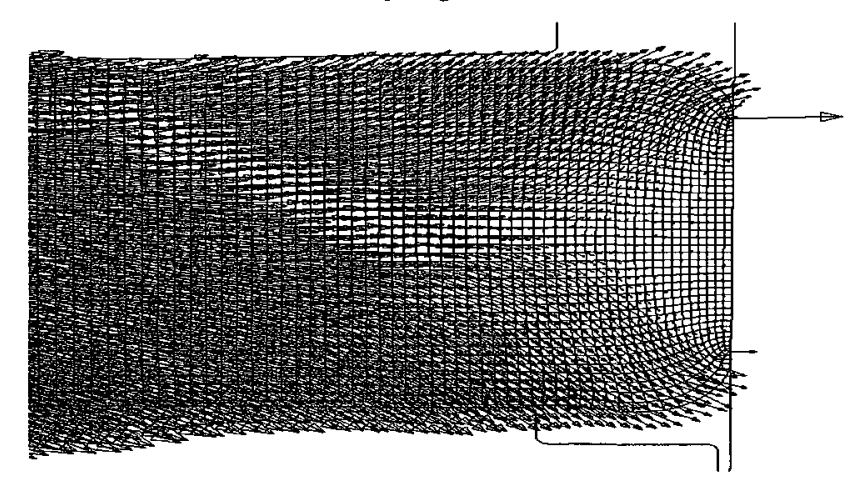

( a ) $50 \%$ axial stroke

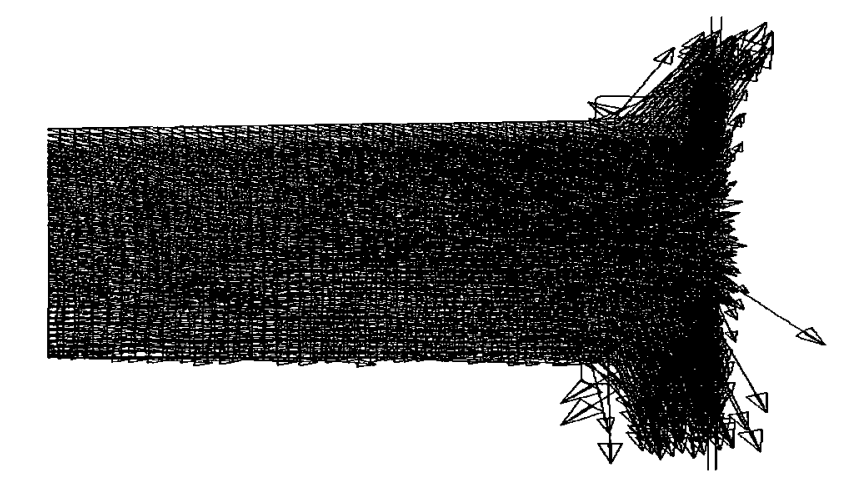

(b) $75 \%$ axial stroke

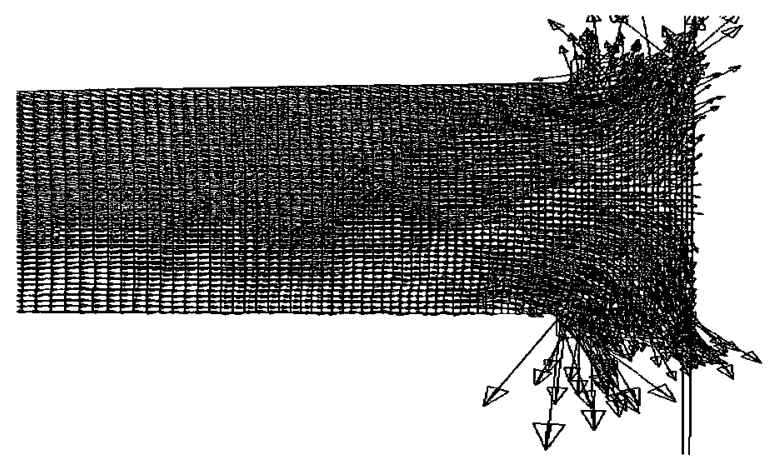

(c ) $90 \%$ axial stroke

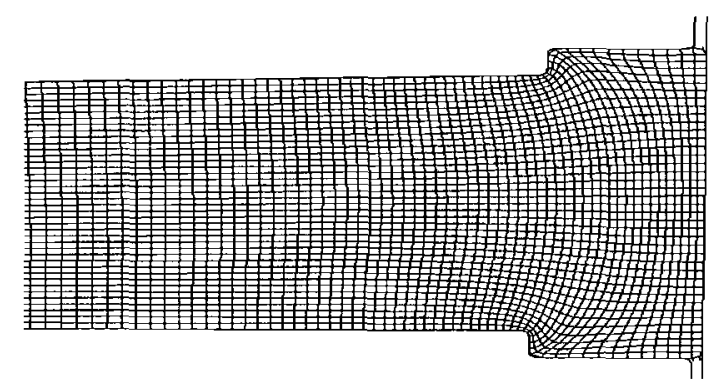

(d ) $100 \%$ axial stroke

Figure A-16. Material flow velocity patterns showing outer $20 \%$ of blank (Top die curvature $=0.3 \mathrm{~mm}$, Bottom die curvature $=0.05 \mathrm{~mm}$, Rim Height $=0.271 \mathrm{~mm}$, no relief) 


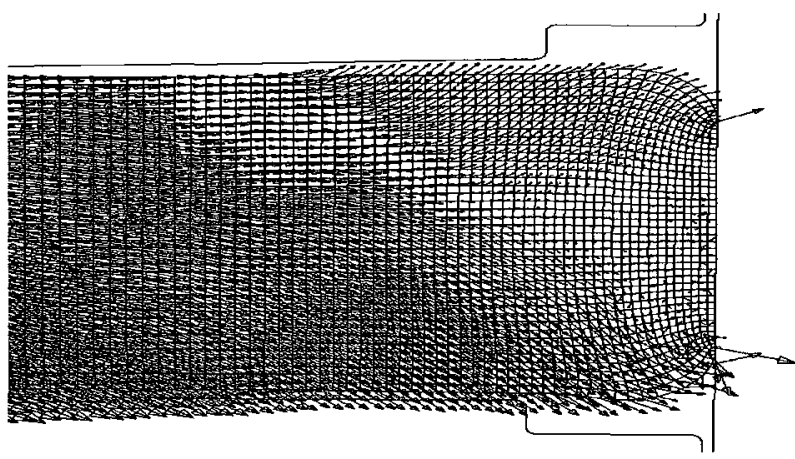

(a) $50 \%$ axial stroke

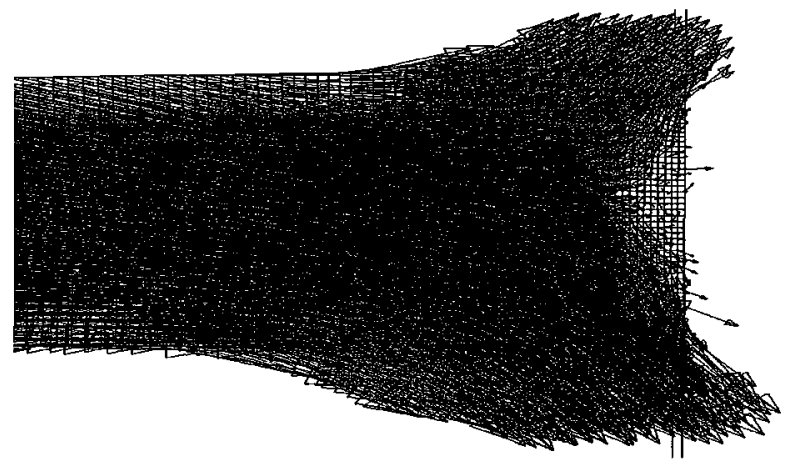

(b) $75 \%$ axial stroke

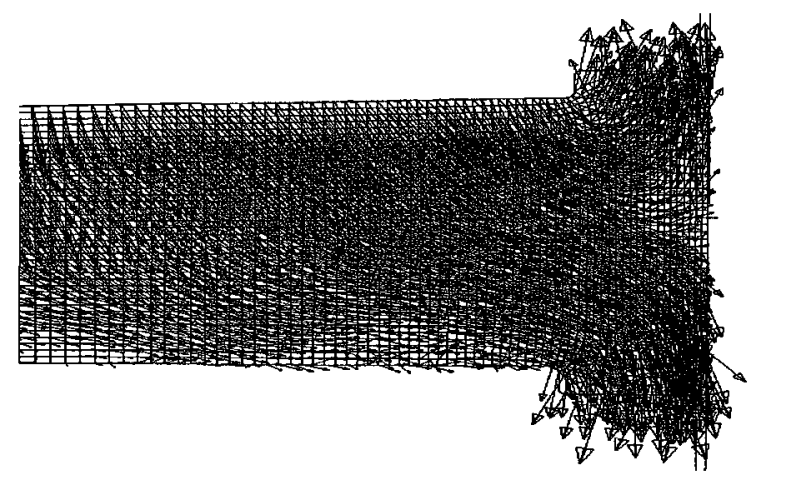

(c ) $90 \%$ axial stroke

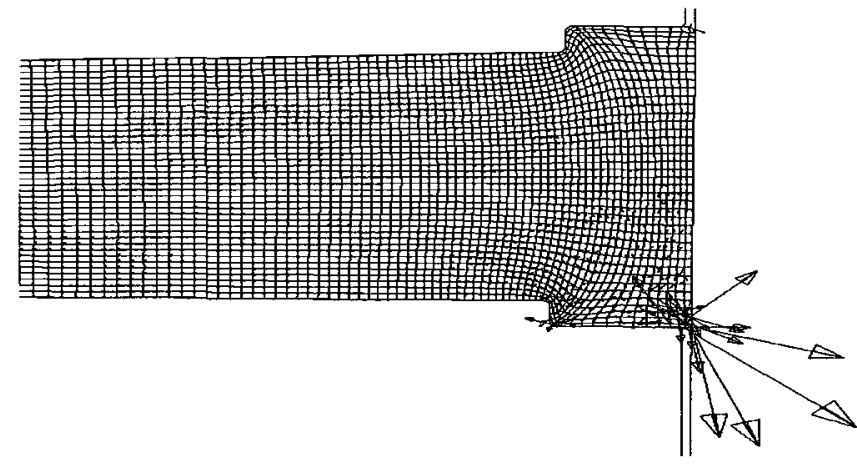

(d ) $100 \%$ axial stroke

Figure A-17. Material flow velocity patterns showing outer $20 \%$ of blank (Top die curvature $=0.3 \mathrm{~mm}$, Bottom die curvature $=0.1 \mathrm{~mm}$, Rim Height $=0.271 \mathrm{~mm}$, no relief) 


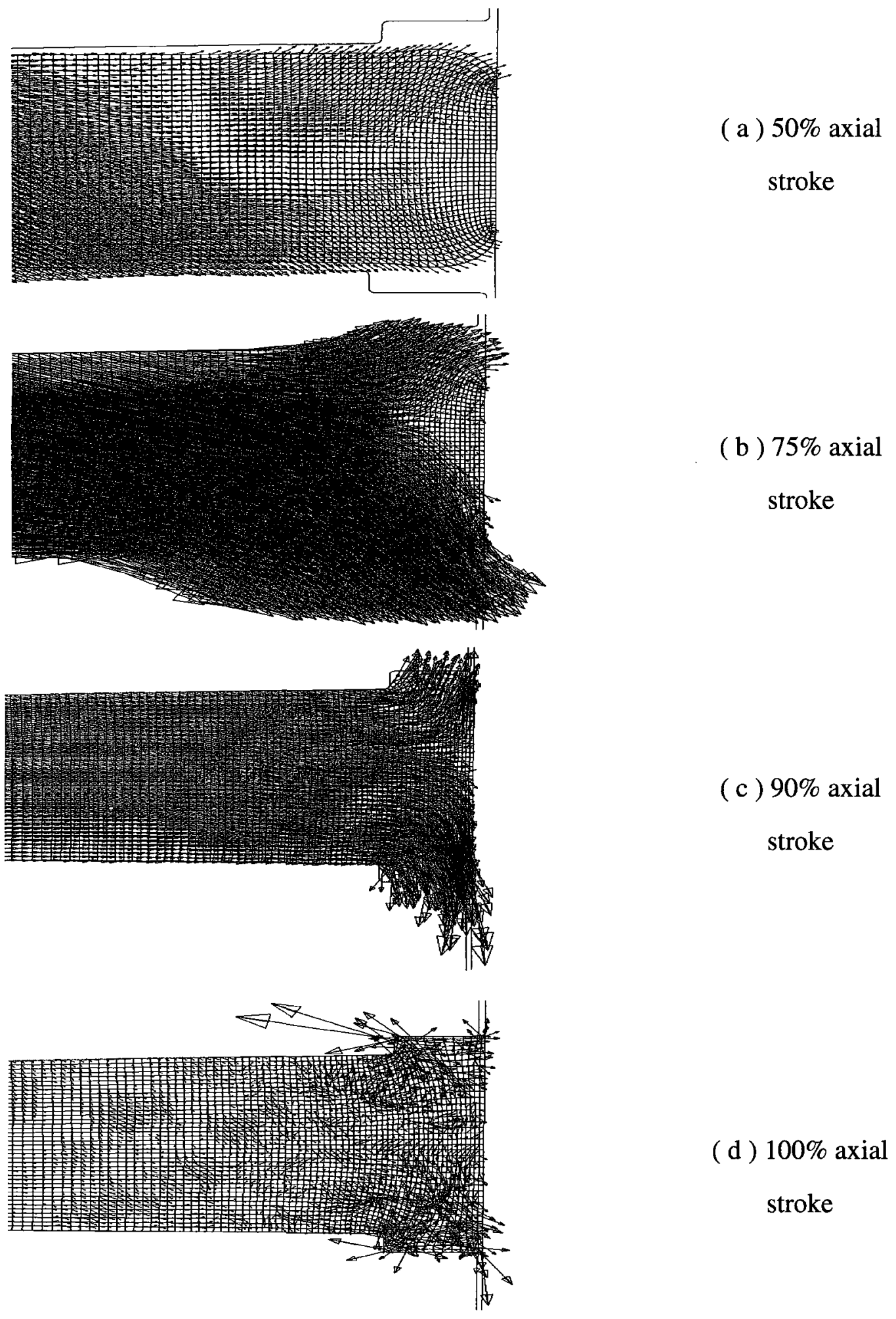

Figure A-18. Material flow velocity patterns showing outer $20 \%$ of blank (Top die curvature $=0.3 \mathrm{~mm}$, Bottom die curvature $=0.15 \mathrm{~mm}$, Rim Height $=0.271 \mathrm{~mm}$, no relief) 


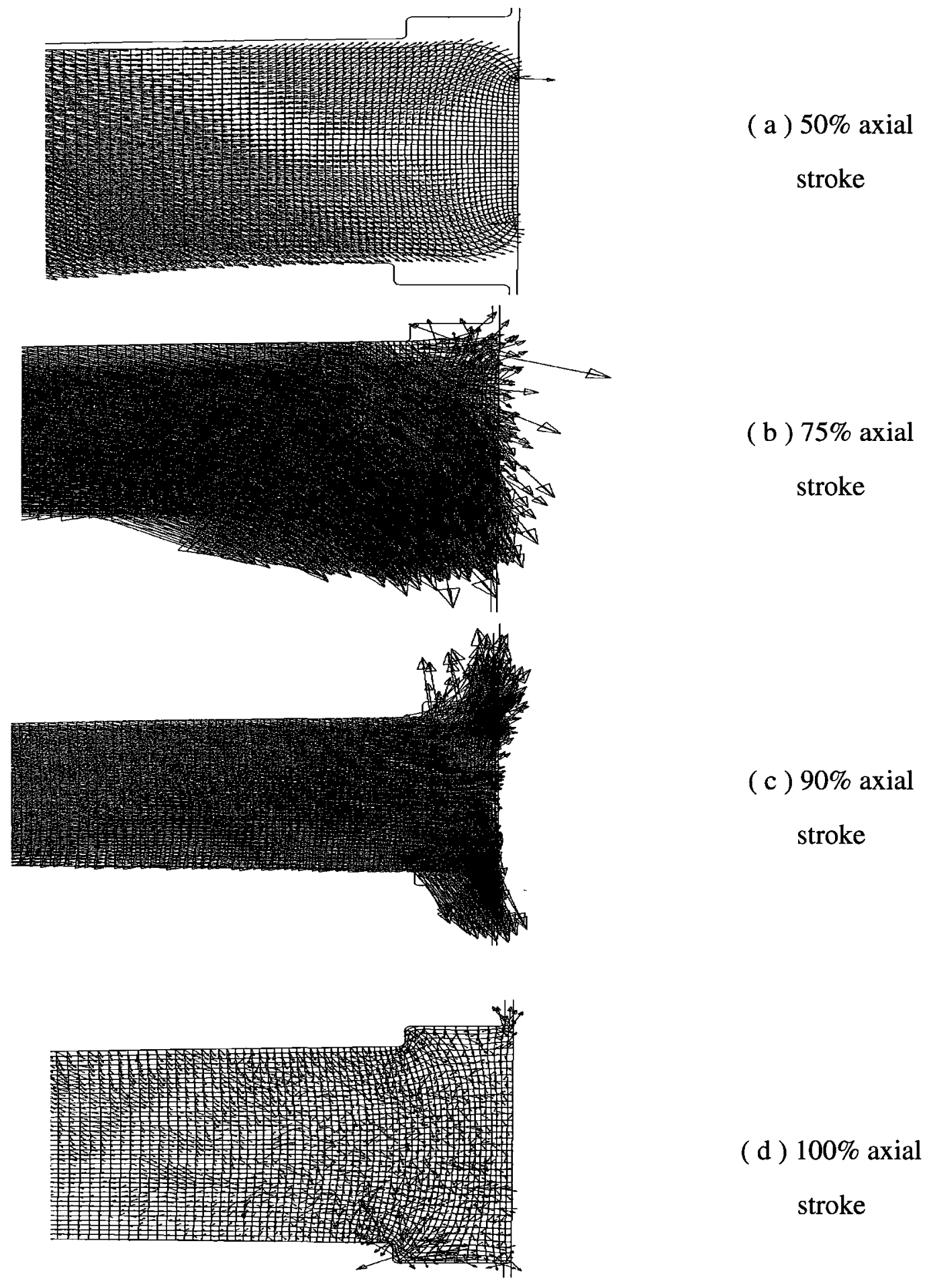

Figure A-19. Material flow velocity patterns showing outer $20 \%$ of blank (Top die curvature $=0.3 \mathrm{~mm}$, Bottom die curvature $=0.2 \mathrm{~mm}$, Rim Height $=0.271 \mathrm{~mm}$, no relief) 




Figure A-20. Material flow velocity patterns showing outer $20 \%$ of blank (Top die curvature $=0.3 \mathrm{~mm}$, Bottom die curvature $=0.25 \mathrm{~mm}$, Rim Height $=0.271 \mathrm{~mm}$, no relief) 
A). Material deforming patterns with varying rim height

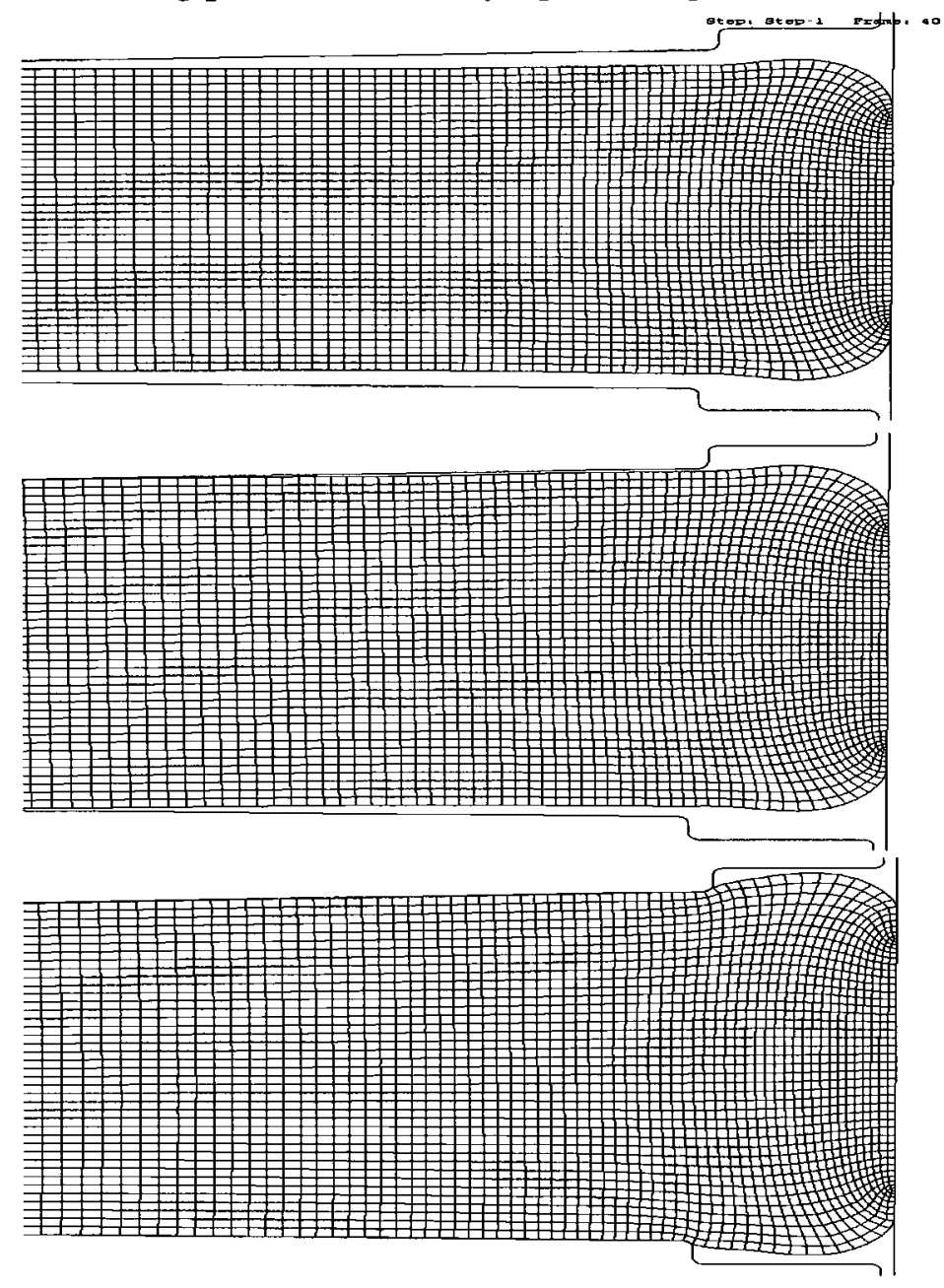
( a ) $50 \%$ axial stroke
(b) $75 \%$ axial stroke
(c) $90 \%$ axial stroke

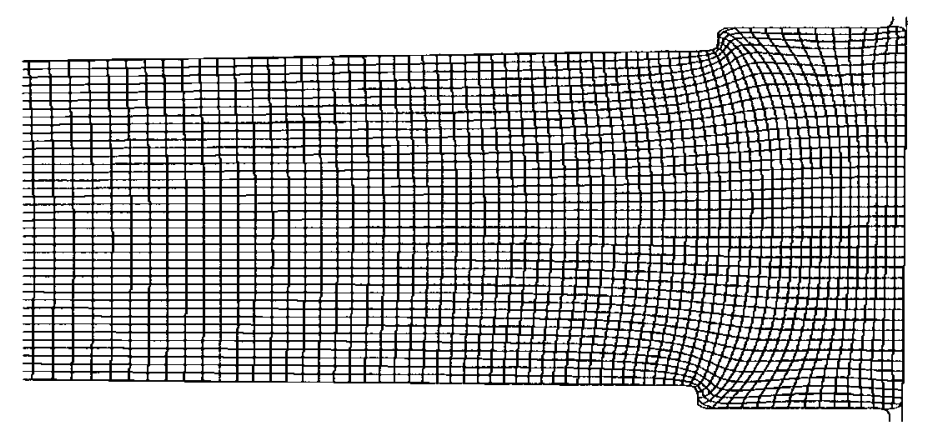
(d) $100 \%$ axial stroke

Figure A-21. Metal deformed mesh patterns showing outer $20 \%$ of blank (Top die curvature $=0.3 \mathrm{~mm}$, Bottom die curvature $=0.19 \mathrm{~mm}$, Rim Height $=0.18 \mathrm{~mm}$, no relief) 


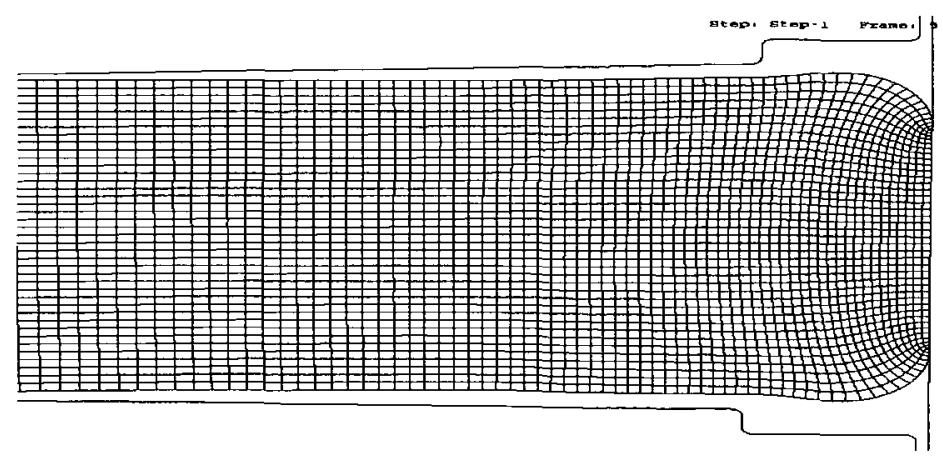

(a) $50 \%$ axial

stroke

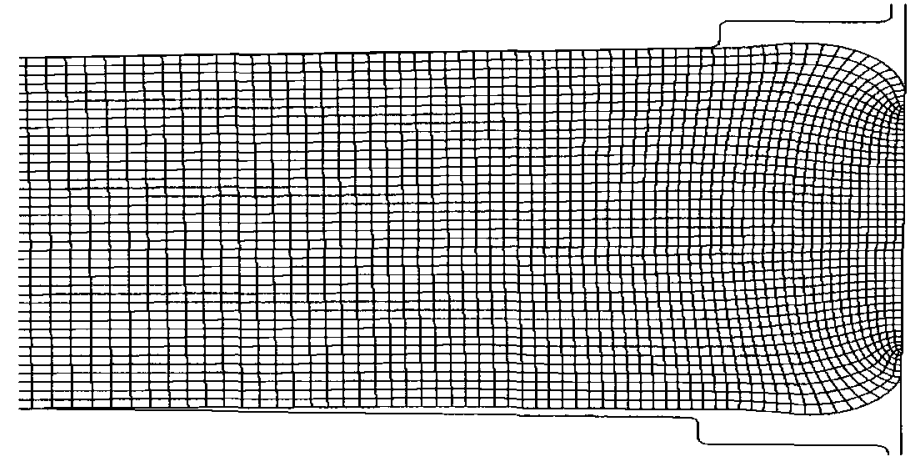

(b ) $75 \%$ axial stroke

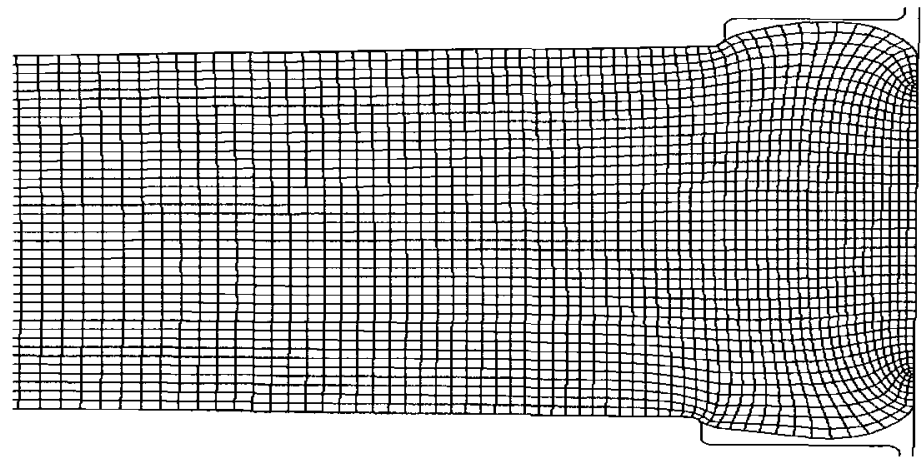

(c ) $90 \%$ axial stroke

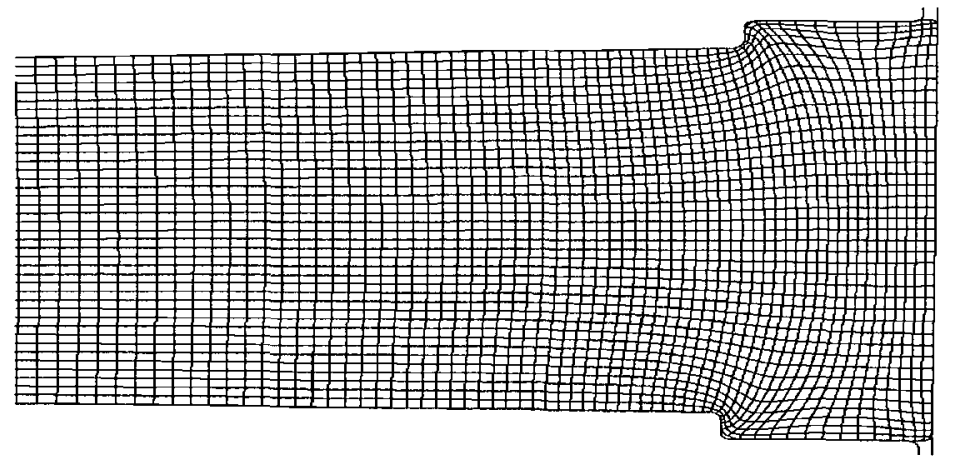

(d ) $100 \%$ axial stroke

Figure A-22. Metal deformed mesh patterns showing outer $20 \%$ of blank (Top die curvature $=0.3 \mathrm{~mm}$, Bottom die curvature $=0.19 \mathrm{~mm}$, Rim Height $=0.2 \mathrm{~mm}$, no relief) 


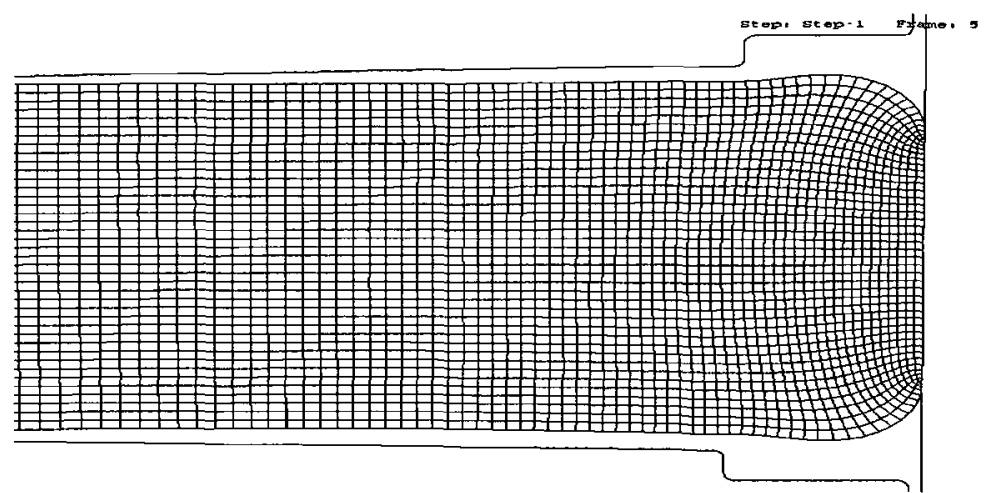
( a ) $50 \%$ axial stroke

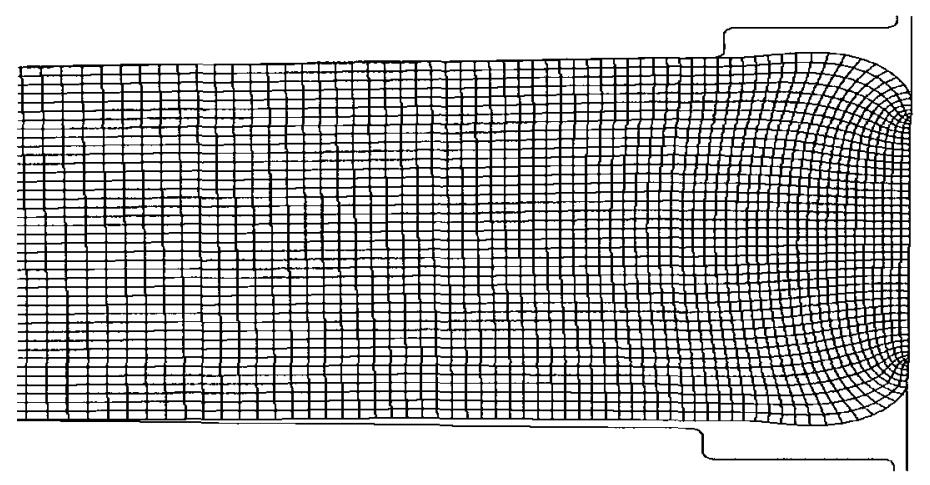

(b ) $75 \%$ axial stroke

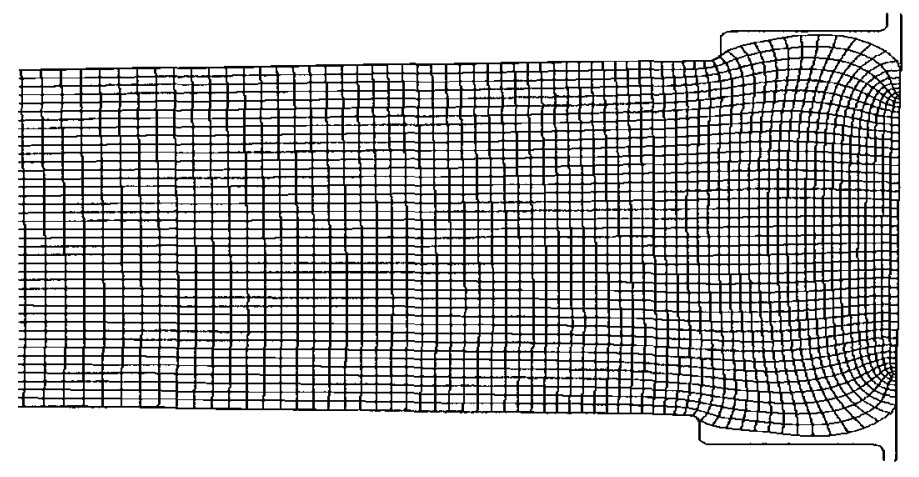

(c ) $90 \%$ axial stroke

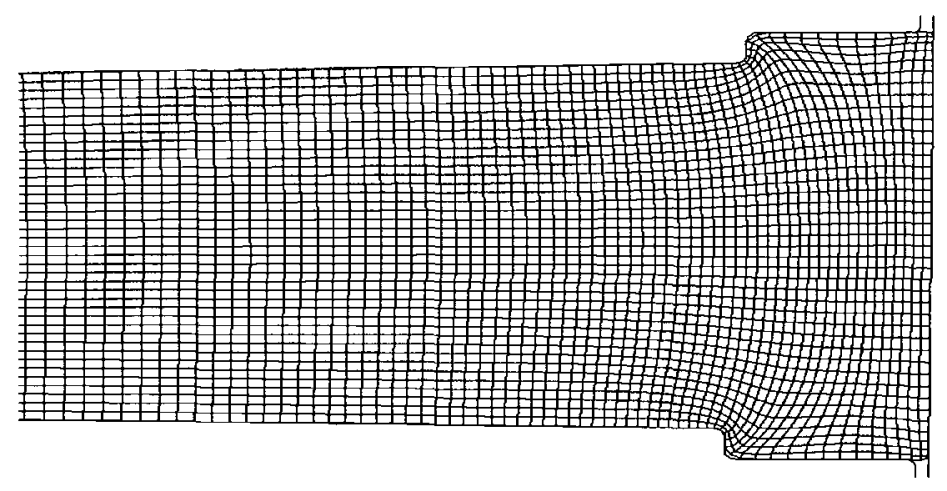

(d ) $100 \%$ axial stroke

Figure A-23. Metal deformed mesh patterns showing outer $20 \%$ of blank (Top die curvature $=0.3 \mathrm{~mm}$, Bottom die curvature $=0.19 \mathrm{~mm}$, Rim Height $=0.22 \mathrm{~mm}$, no relief) 


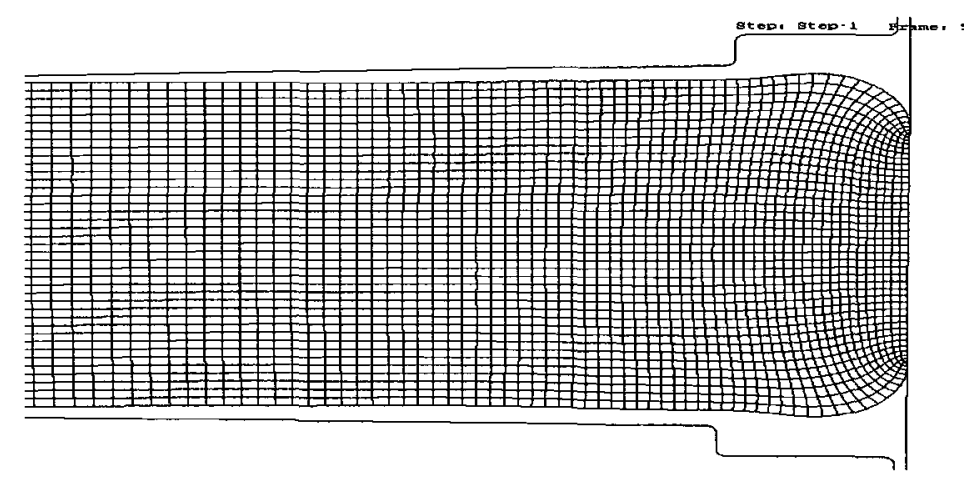
(a) $50 \%$ axial stroke

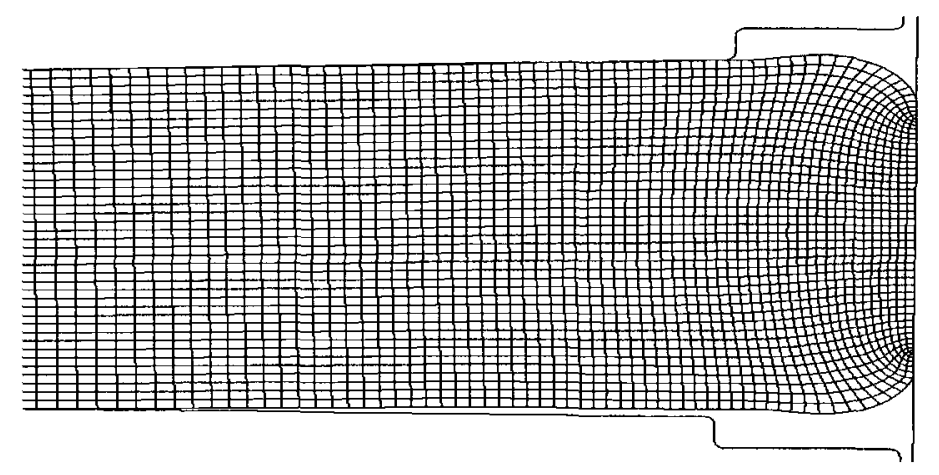

(b ) $75 \%$ axial stroke

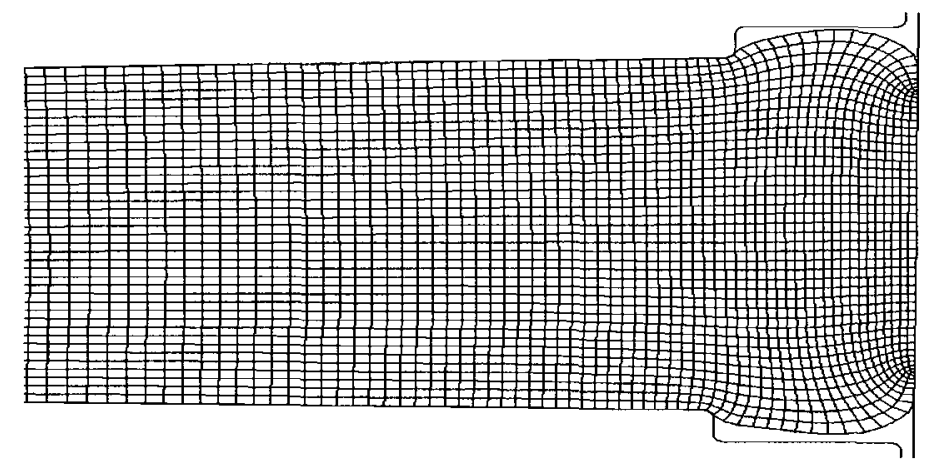
(c) $90 \%$ axial stroke

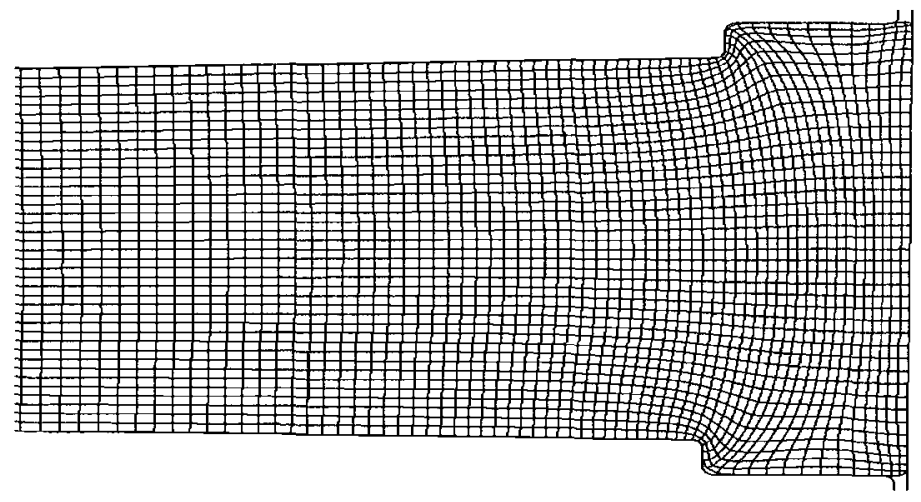
(d) $100 \%$ axial stroke

Figure A-24. Metal deformed mesh patterns showing outer $20 \%$ of blank (Top die curvature $=0.3 \mathrm{~mm}$, Bottom die curvature $=0.19 \mathrm{~mm}$, Rim Height $=0.24 \mathrm{~mm}$, no relief) 


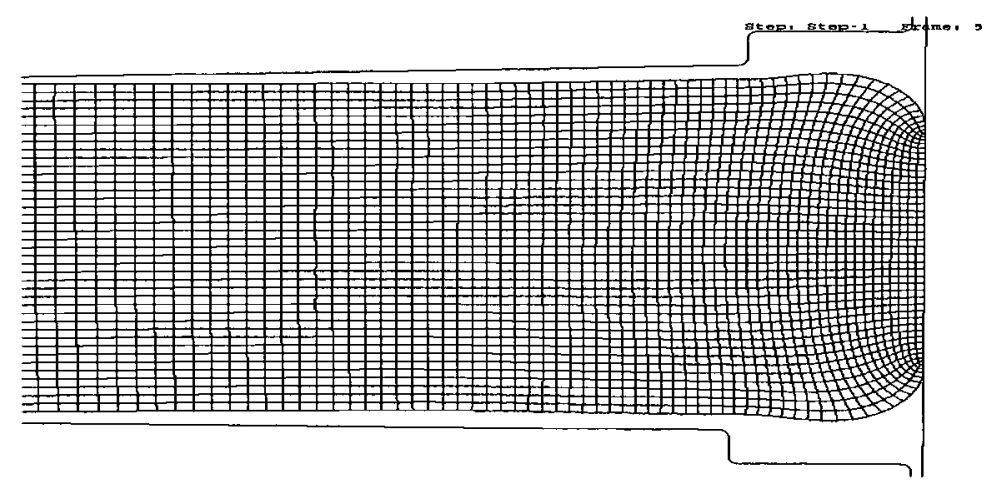
( a ) $50 \%$ axial stroke

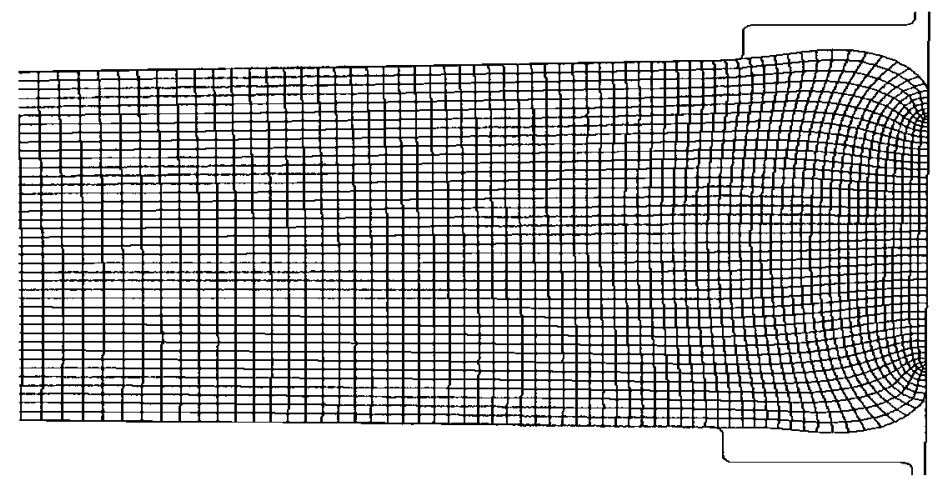

(b) $75 \%$ axial stroke

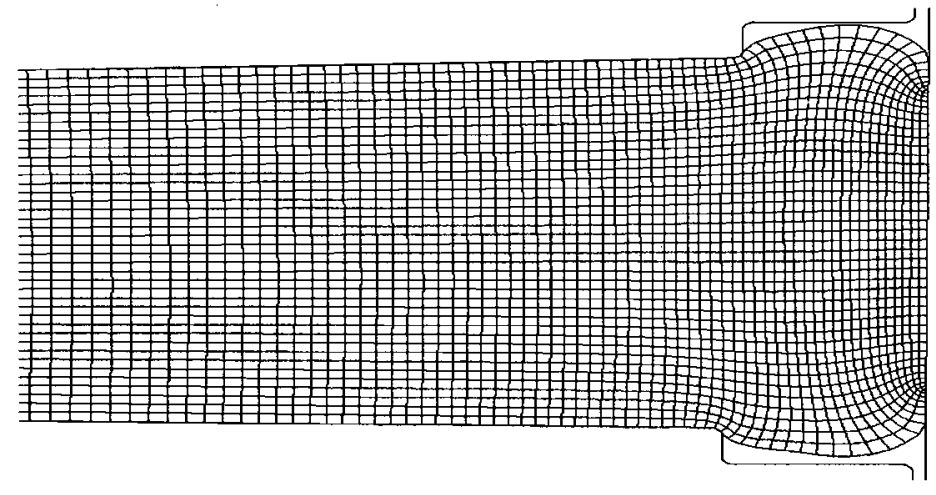

(c) $90 \%$ axial stroke

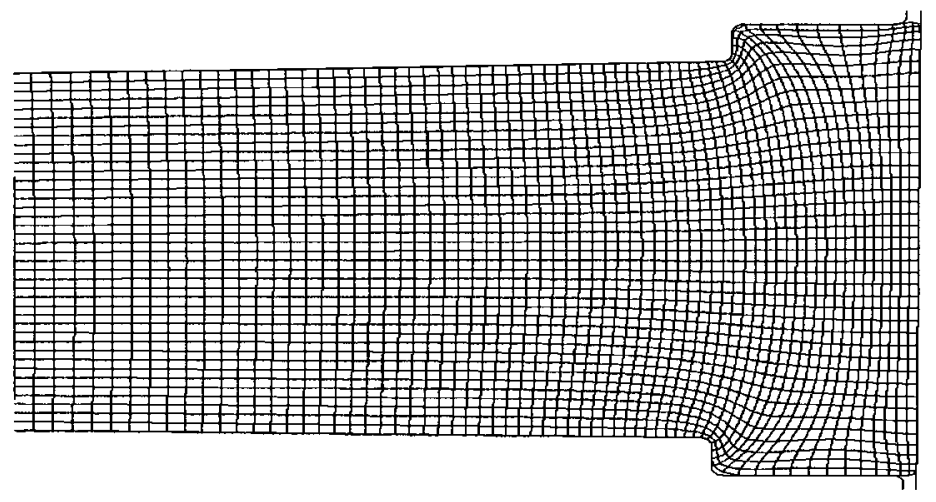

(d) $100 \%$ axial stroke

Figure A-25. Metal deformed mesh patterns showing outer $20 \%$ of blank (Top die curvature $=0.3 \mathrm{~mm}$, Bottom die curvature $=0.19 \mathrm{~mm}$, Rim Height $=0.26 \mathrm{~mm}$, no relief) 


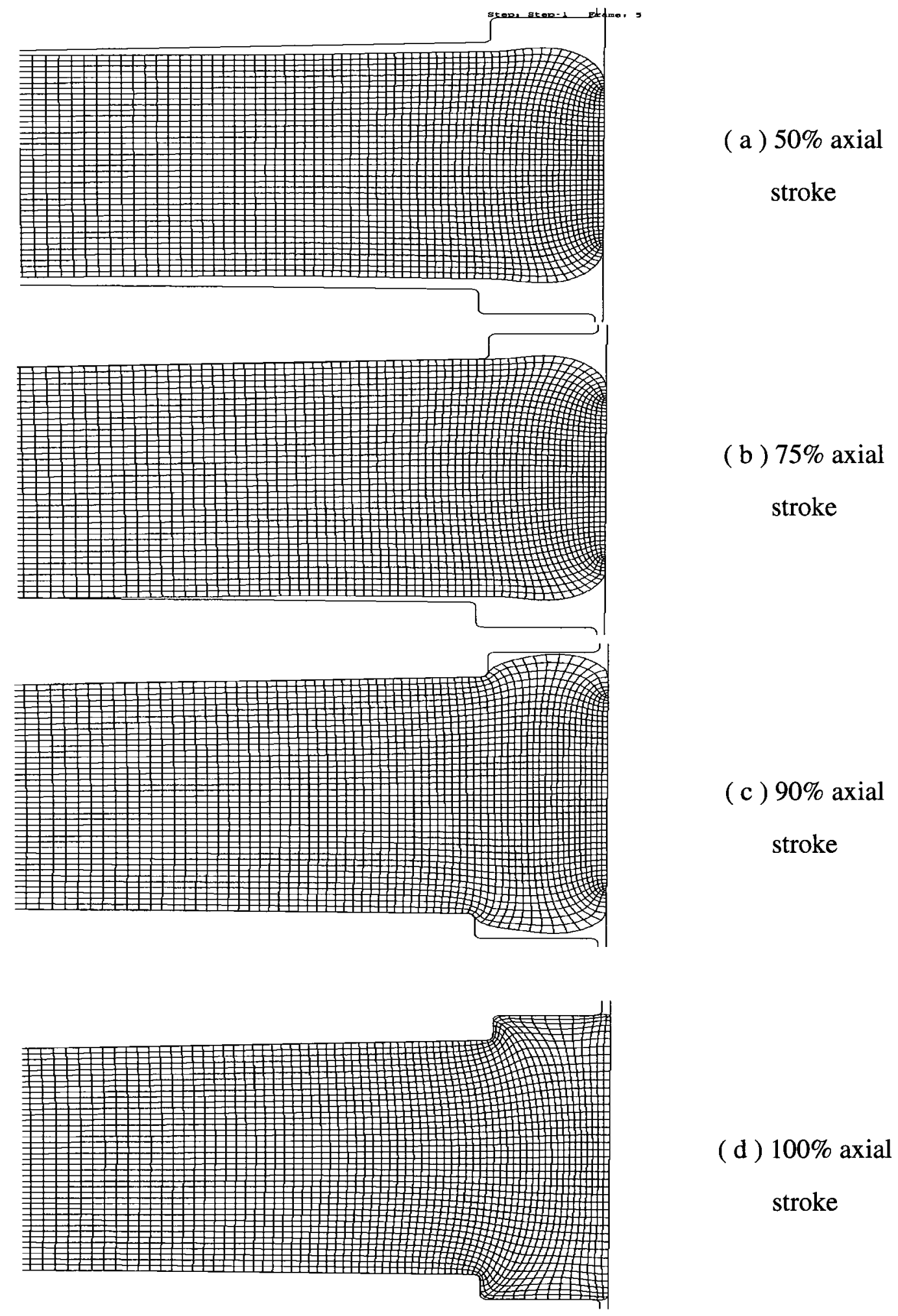

Figure A-26. Metal deformed mesh patterns showing outer $20 \%$ of blank (Top die curvature $=0.3 \mathrm{~mm}$, Bottom die curvature $=0.19 \mathrm{~mm}$, Rim Height $=0.28 \mathrm{~mm}$, no relief) 
B). Material flow velocities with varying rim height

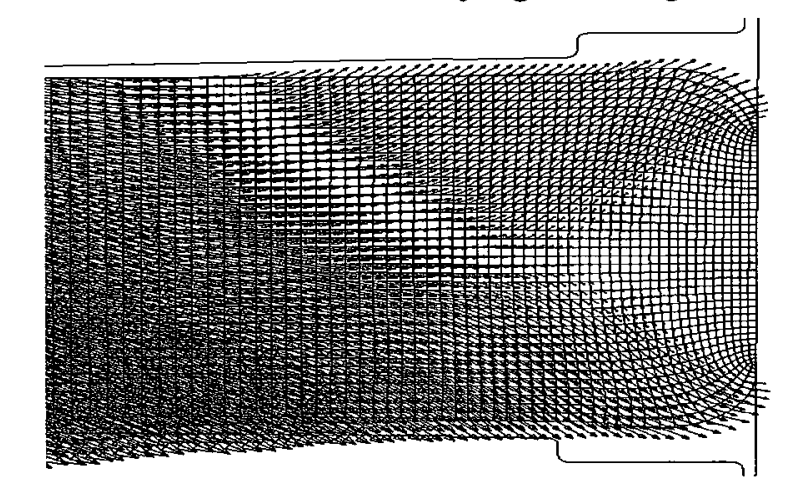

(a) $50 \%$ axial stroke

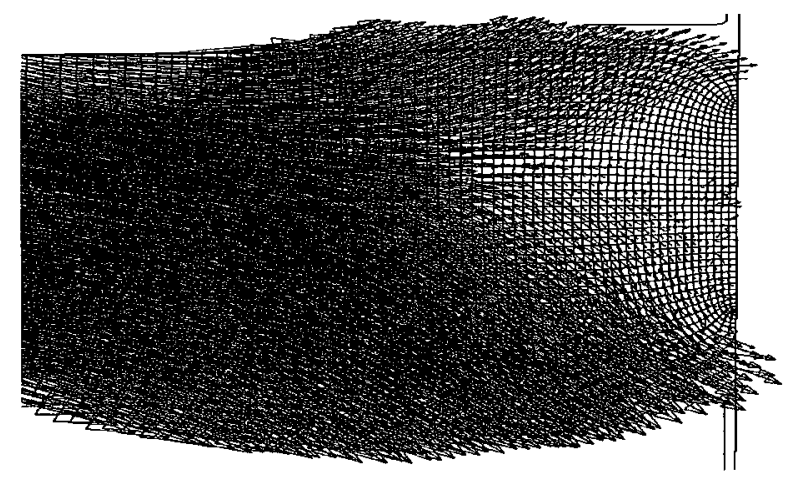

(b) $75 \%$ axial stroke

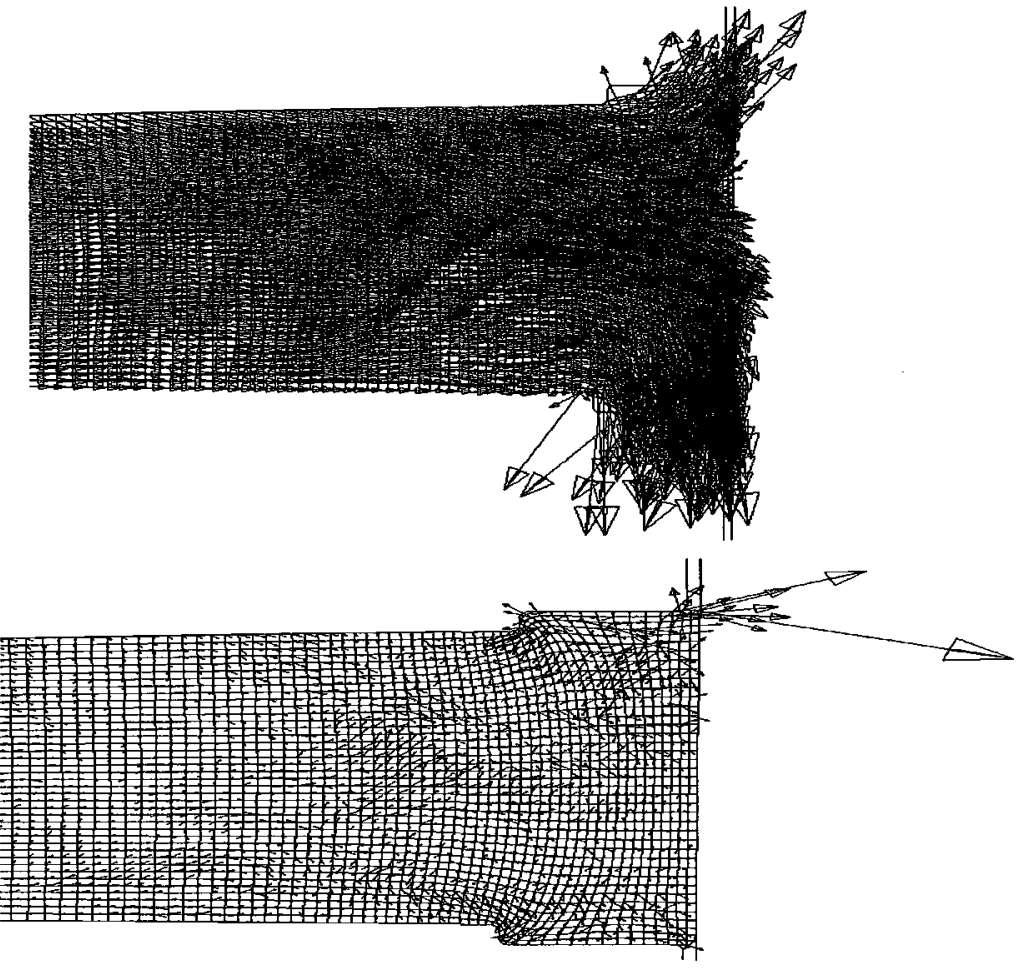

(c) $90 \%$ axial stroke

(d) $100 \%$ axial stroke

Figure A-27. Material flow velocity patterns showing outer $20 \%$ of blank (Top die curvature $=0.3 \mathrm{~mm}$, Bottom die curvature $=0.19 \mathrm{~mm}$, Rim Height $=0.18 \mathrm{~mm}$, no relief) 


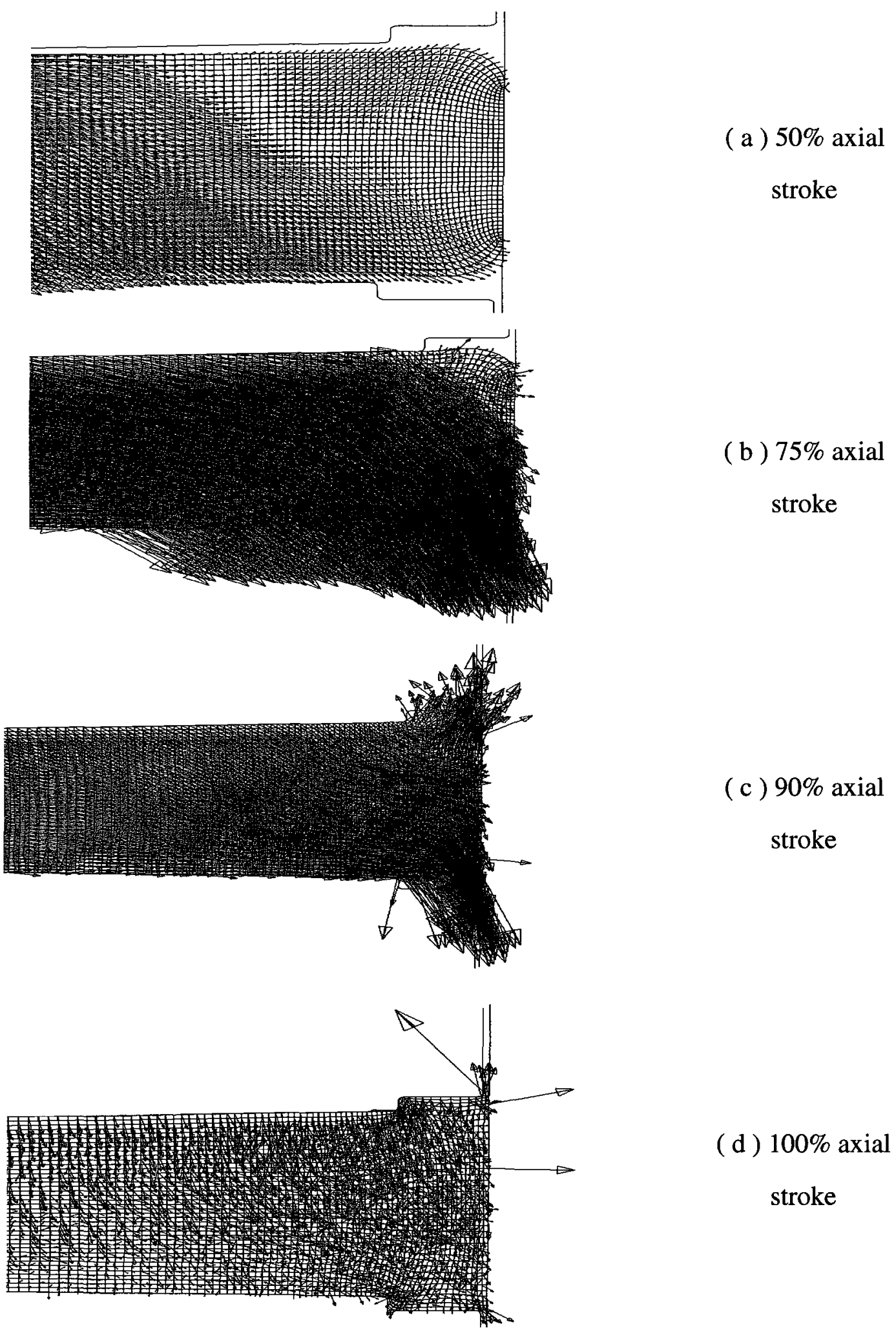

Figure A-28. Material flow velocity patterns showing outer $20 \%$ of blank

(Top die curvature $=0.3 \mathrm{~mm}$, Bottom die curvature $=0.19 \mathrm{~mm}$, Rim Height $=0.2 \mathrm{~mm}$, no relief) 


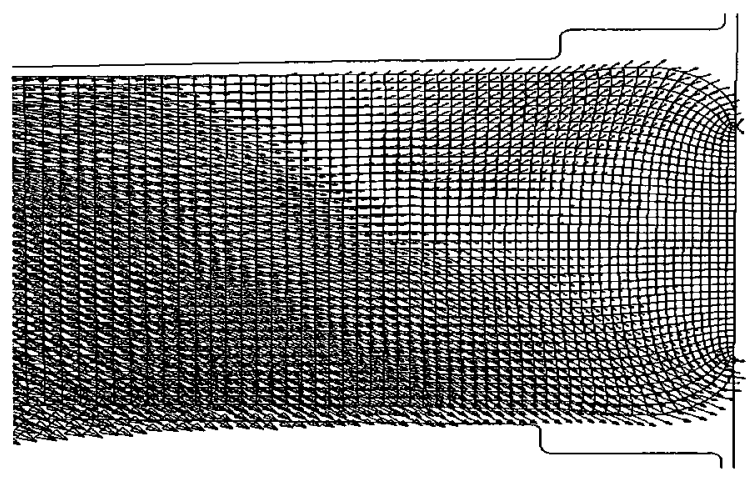
(a ) $50 \%$ axial stroke

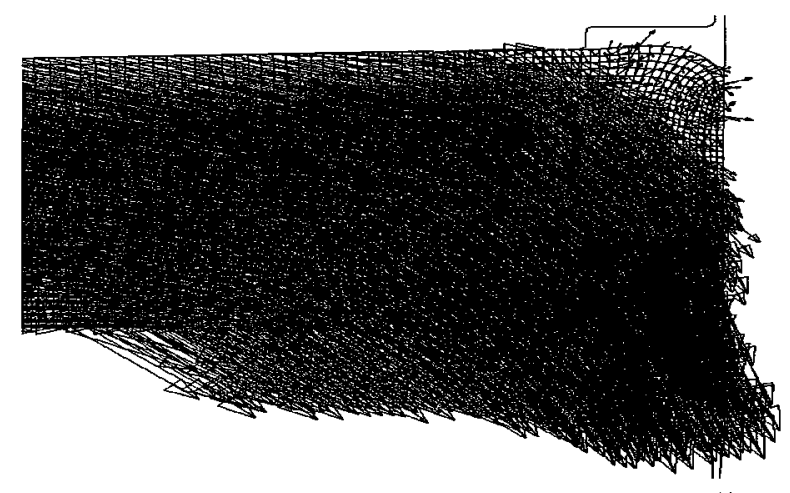

(b ) $75 \%$ axial stroke

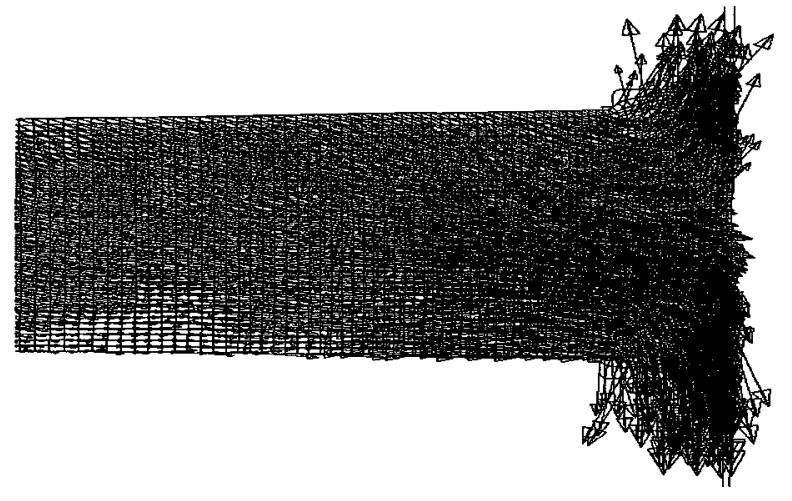

(c) $90 \%$ axial stroke

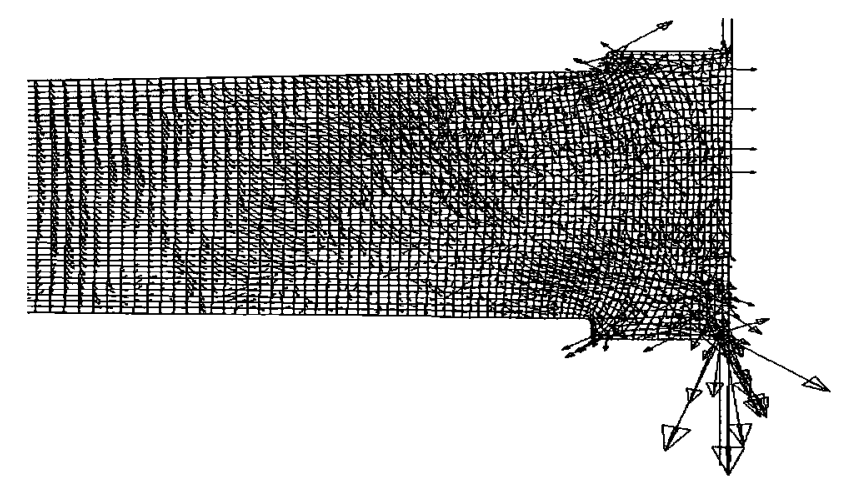

(d) $100 \%$ axial stroke

Figure A-29. Material flow velocity patterns showing outer $20 \%$ of blank (Top die curvature $=0.3 \mathrm{~mm}$, Bottom die curvature $=0.19 \mathrm{~mm}$, Rim Height $=0.22 \mathrm{~mm}$, no relief) 


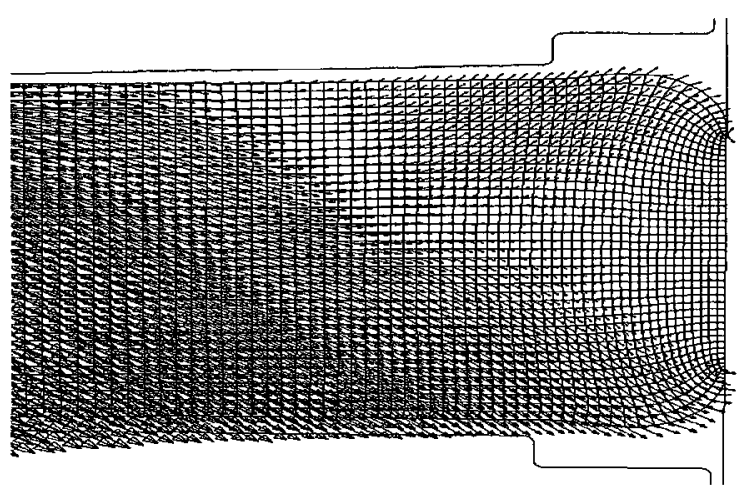
(a ) $50 \%$ axial stroke

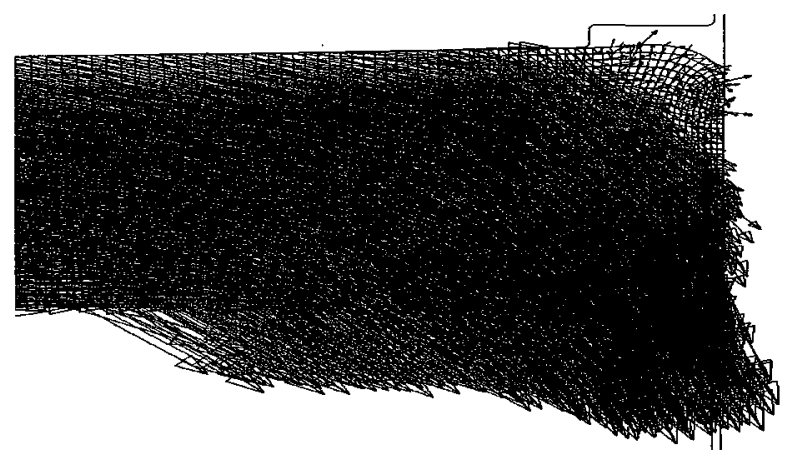

(b ) $75 \%$ axial stroke

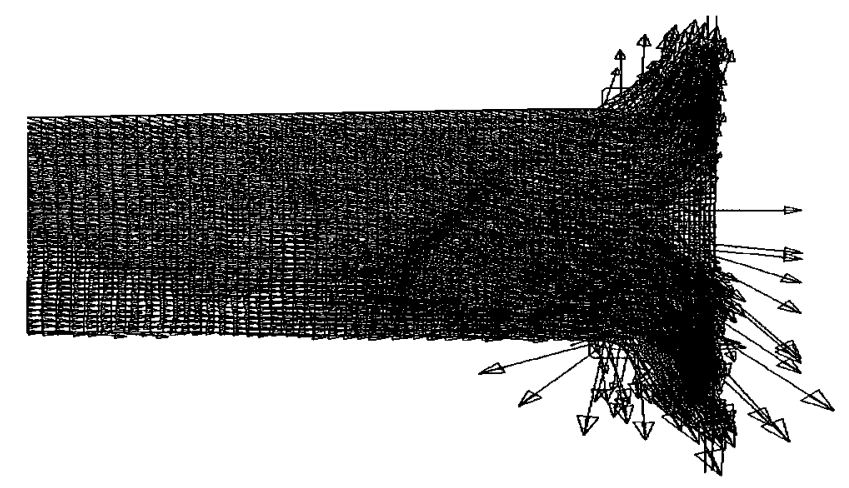

(c) $90 \%$ axial stroke

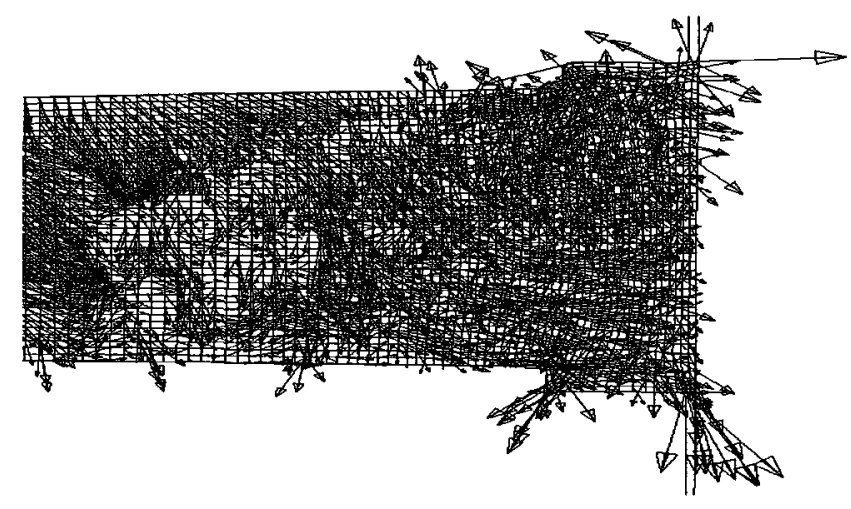

(d) $100 \%$ axial stroke

Figure A-30. Material flow velocity patterns showing outer $20 \%$ of blank (Top die curvature $=0.3 \mathrm{~mm}$, Bottom die curvature $=0.19 \mathrm{~mm}$, Rim Height $=0.24 \mathrm{~mm}$, no relief) 


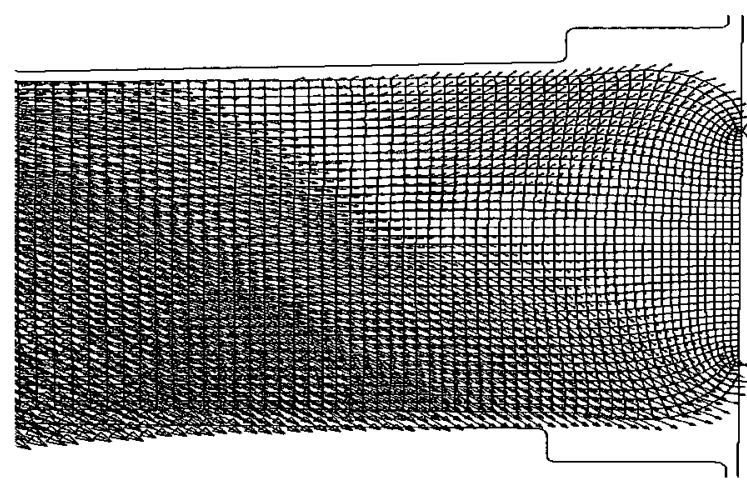

( a ) $50 \%$ axial stroke

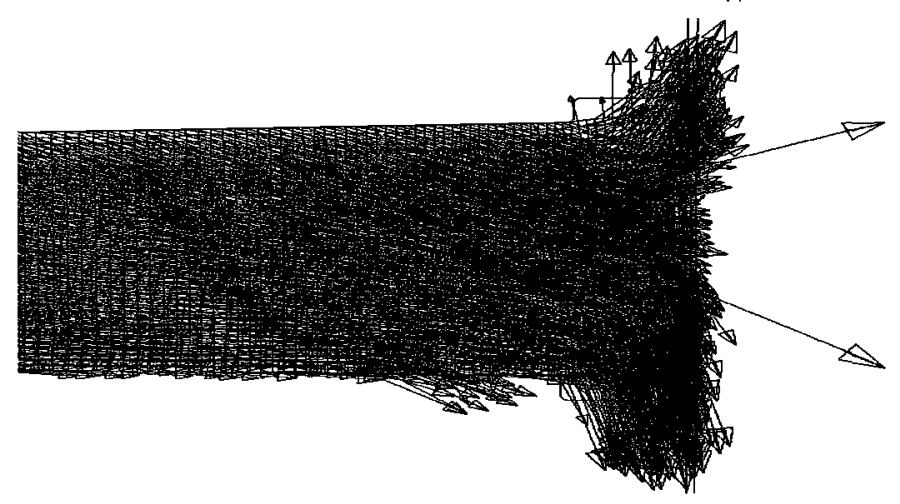

(b ) $75 \%$ axial stroke

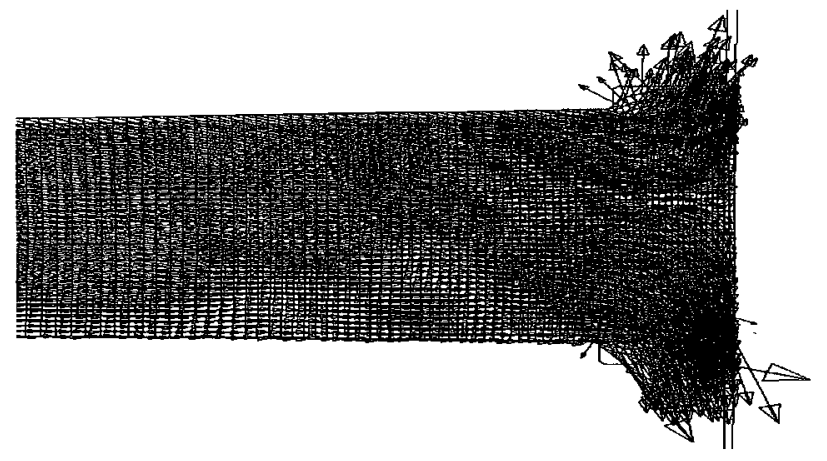

(c ) $90 \%$ axial stroke

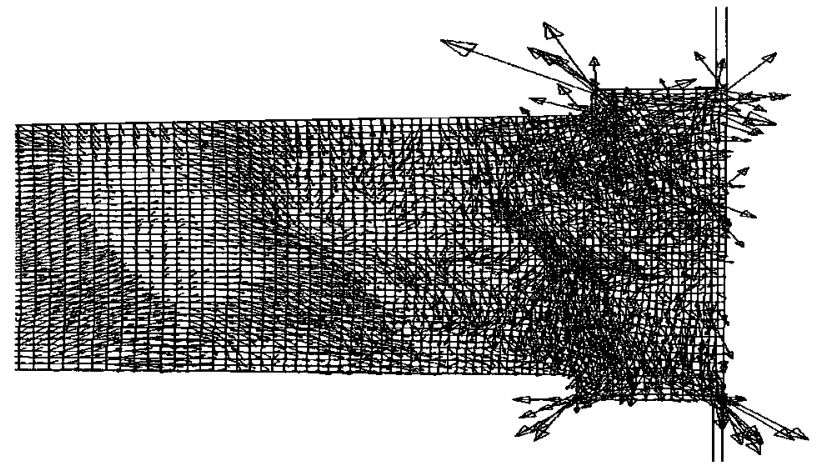

(d) $100 \%$ axial stroke

Figure A-31. Material flow velocity patterns showing outer $20 \%$ of blank (Top die curvature $=0.3 \mathrm{~mm}$, Bottom die curvature $=0.19 \mathrm{~mm}$, Rim Height $=0.26 \mathrm{~mm}$, no relief) 


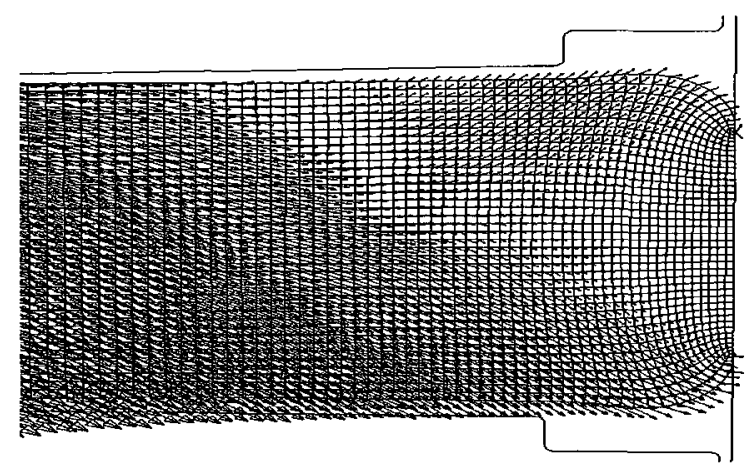

(a) $50 \%$ axial stroke

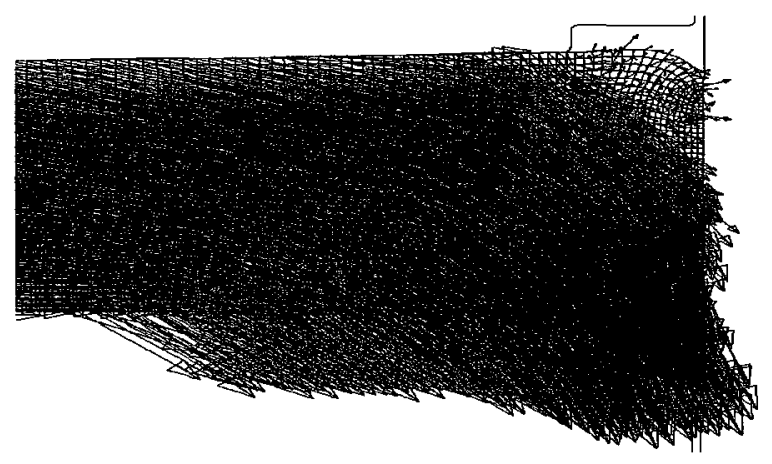

(b ) $75 \%$ axial stroke

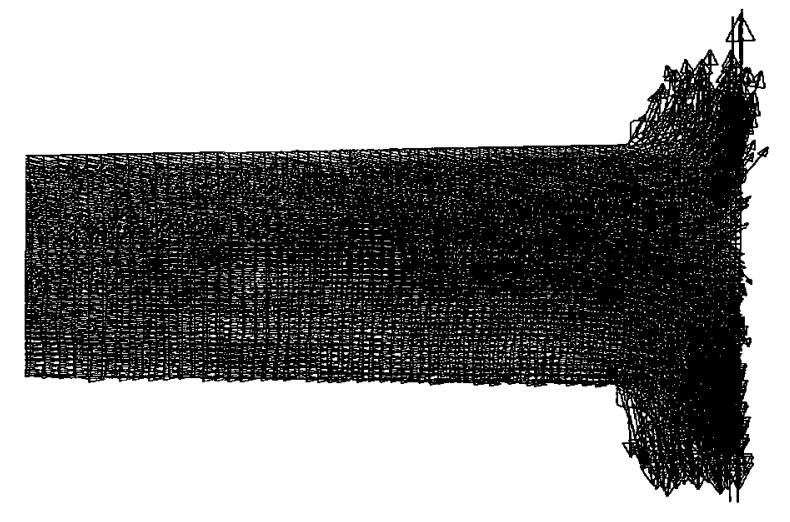

(c) $90 \%$ axial stroke

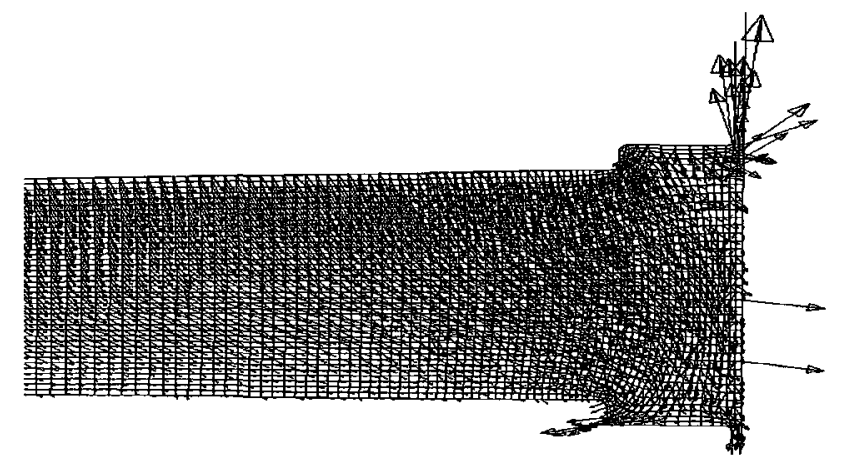

(d) $100 \%$ axial stroke

Figure A-32. Material flow velocity patterns showing outer $20 \%$ of blank (Top die curvature $=0.3 \mathrm{~mm}$, Bottom die curvature $=0.19 \mathrm{~mm}$, Rim Height $=0.28 \mathrm{~mm}$, no relief) 
A). Material deforming patterns with varying relief height

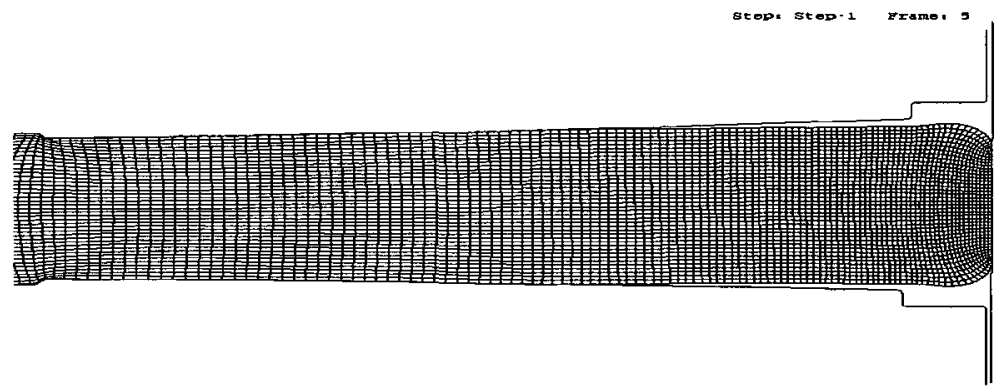

(a) $50 \%$ axial stroke

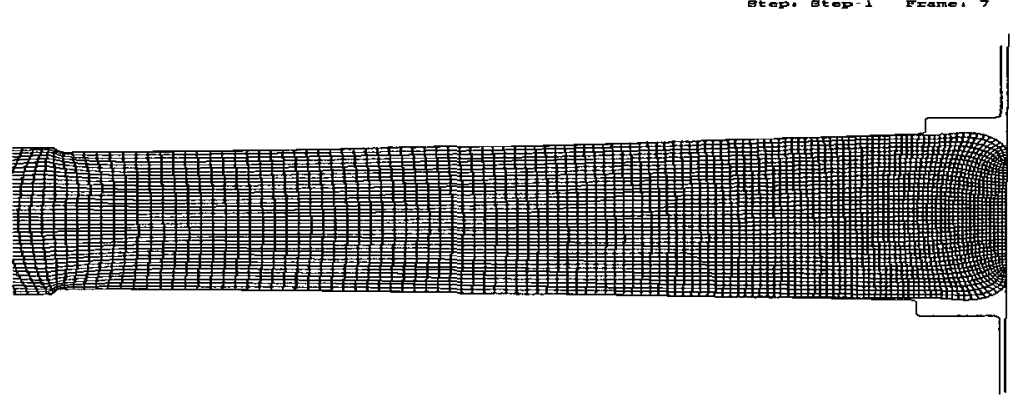

(b) $75 \%$ axial stroke

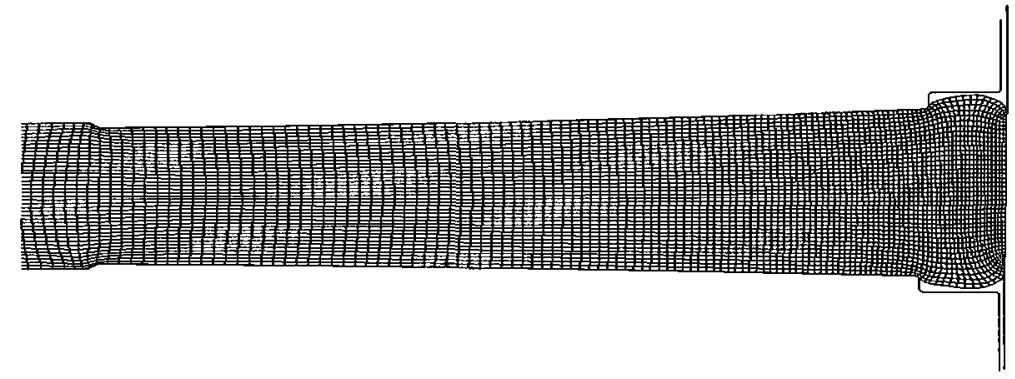

(c) $90 \%$ axial stroke

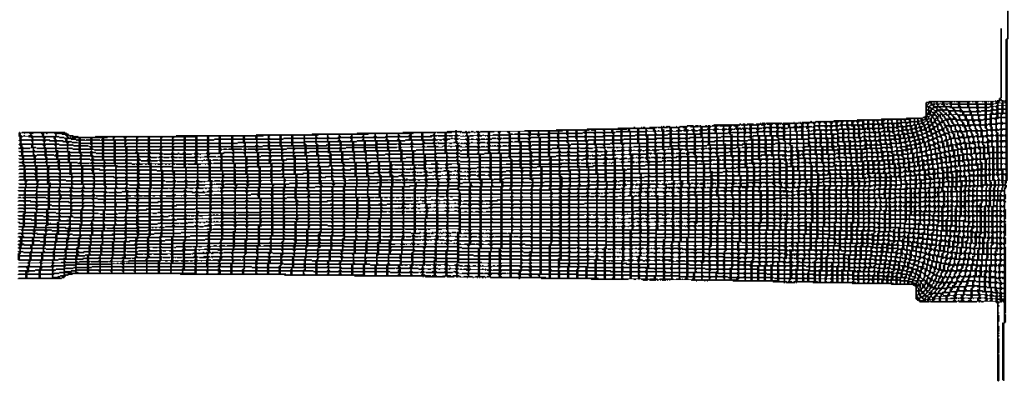

(d) $100 \%$ axial stroke

Figure A-33. Metal deformed mesh patterns showing outer 55\% of blank (Top die curvature $=0.3 \mathrm{~mm}$, Bottom die curvature $=0.19 \mathrm{~mm}$, Rim Height $=0.271 \mathrm{~mm}$, relief $=0.08 \mathrm{~mm}$ ) 


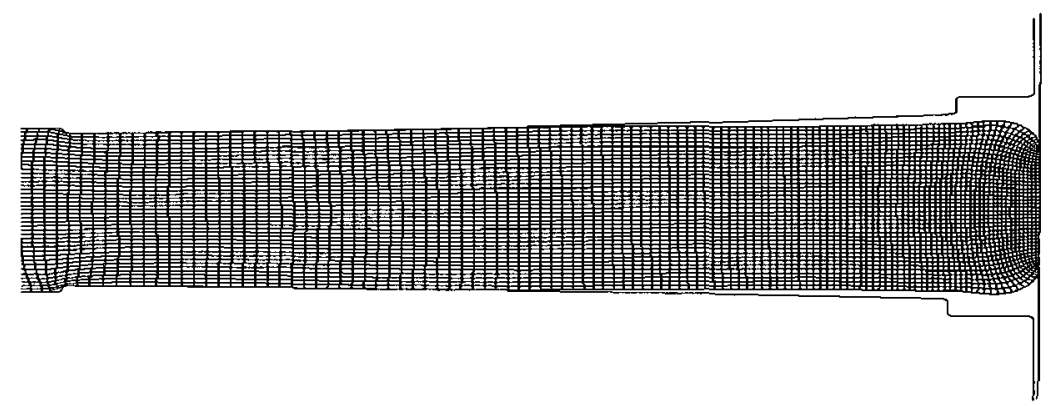

( a ) $50 \%$ axial stroke

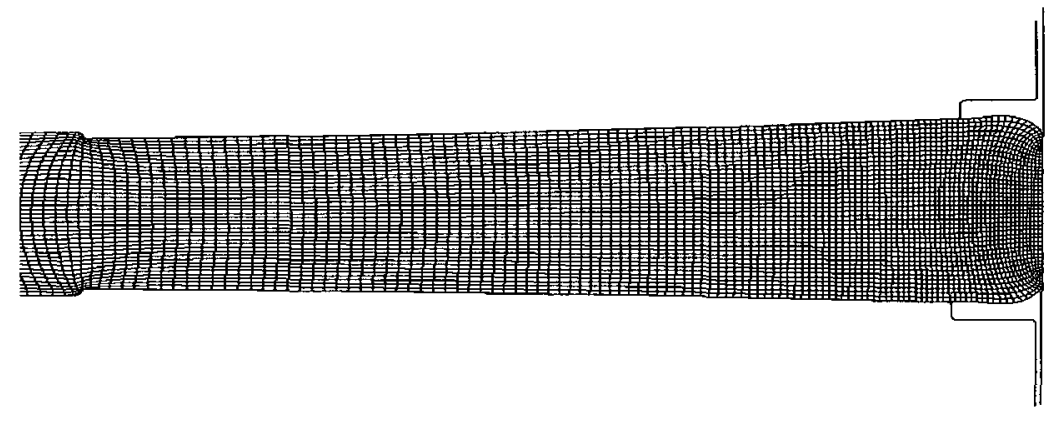

(b ) $75 \%$ axial stroke

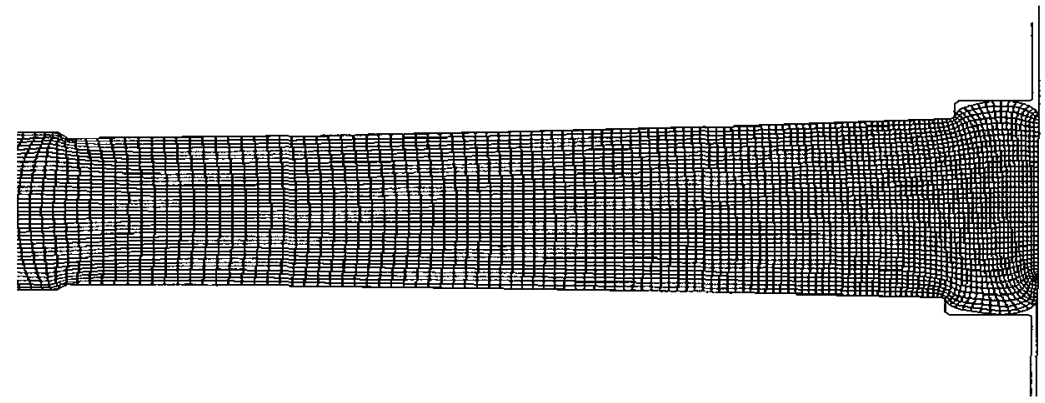

(c) $90 \%$ axial stroke

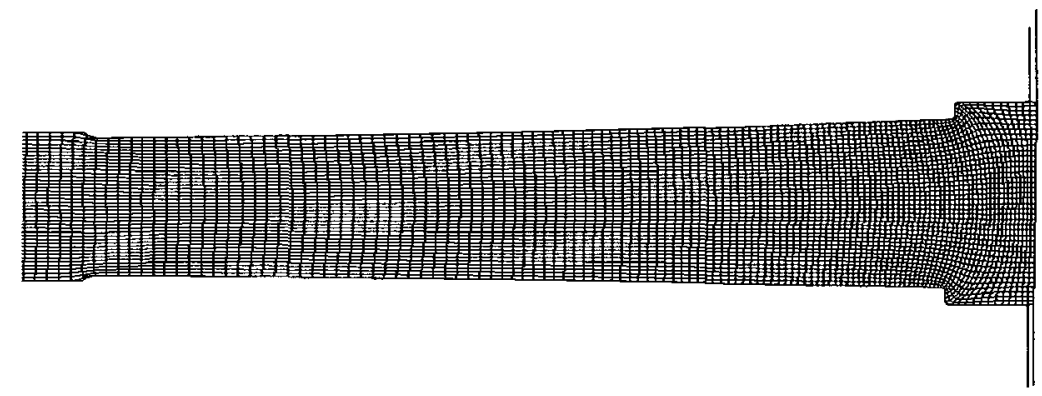

(d) $100 \%$ axial stroke

Figure A-34. Metal deformed mesh patterns showing outer $55 \%$ of blank (Top die curvature $=0.3 \mathrm{~mm}$, Bottom die curvature $=0.19 \mathrm{~mm}$, Rim Height $=0.271 \mathrm{~mm}$, relief $=0.09 \mathrm{~mm}$ ) 
B). Material flow velocity patterns with varying relief height

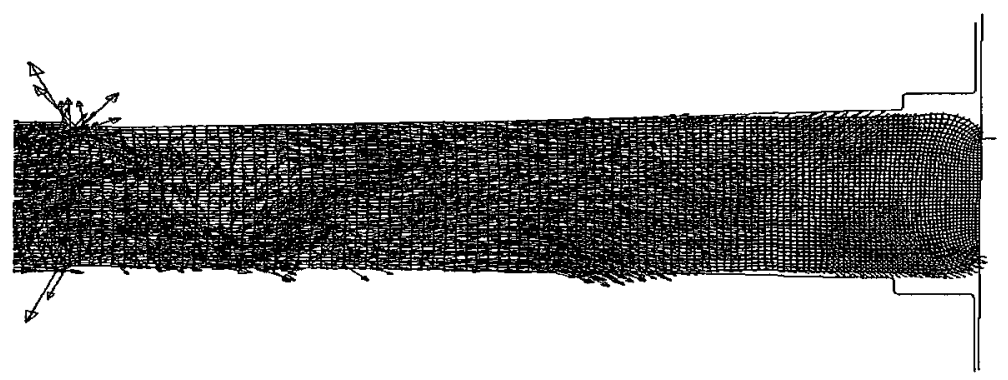

(a) $50 \%$ axial stroke

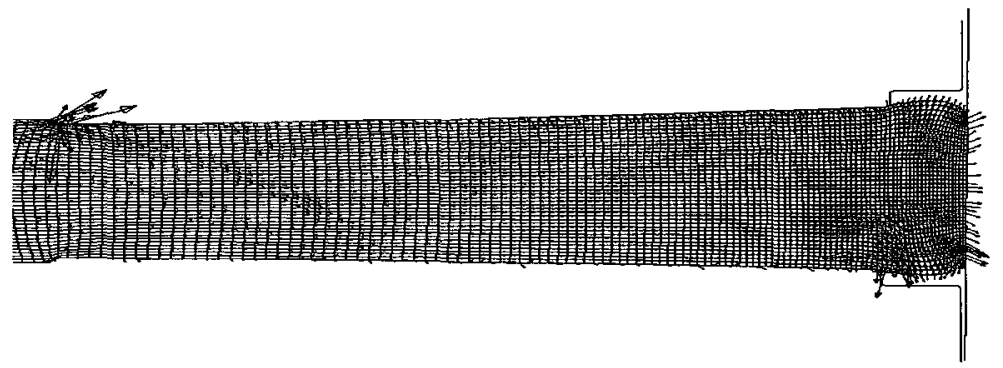

(b) $75 \%$ axial stroke

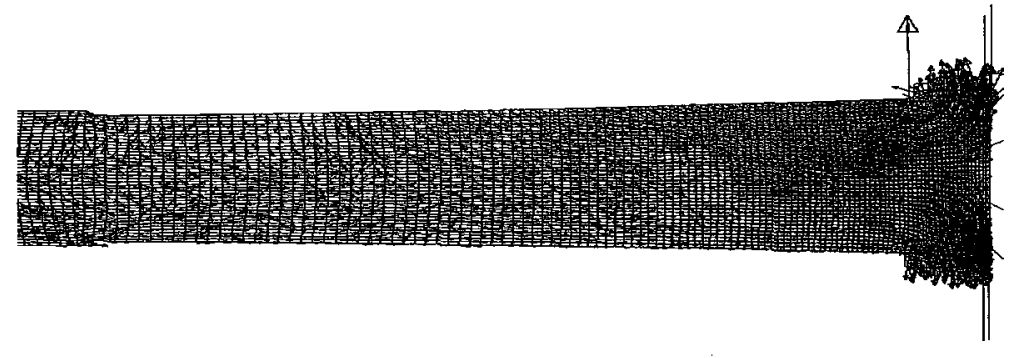

(c) $90 \%$ axial stroke

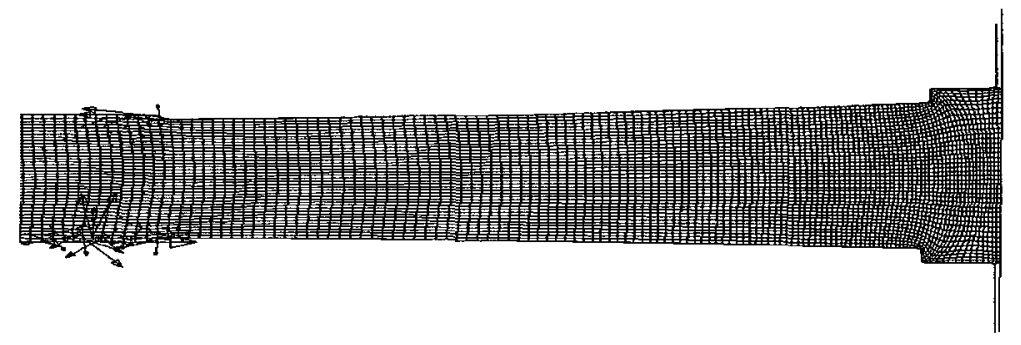

(d) $100 \%$ axial stroke

Figure A-35. Material flow velocity patterns showing outer 55\% of blank (Top die curvature $=0.3 \mathrm{~mm}$, Bottom die curvature $=0.19 \mathrm{~mm}$, Rim Height $=0.271 \mathrm{~mm}$, relief $=0.08 \mathrm{~mm}$ ) 


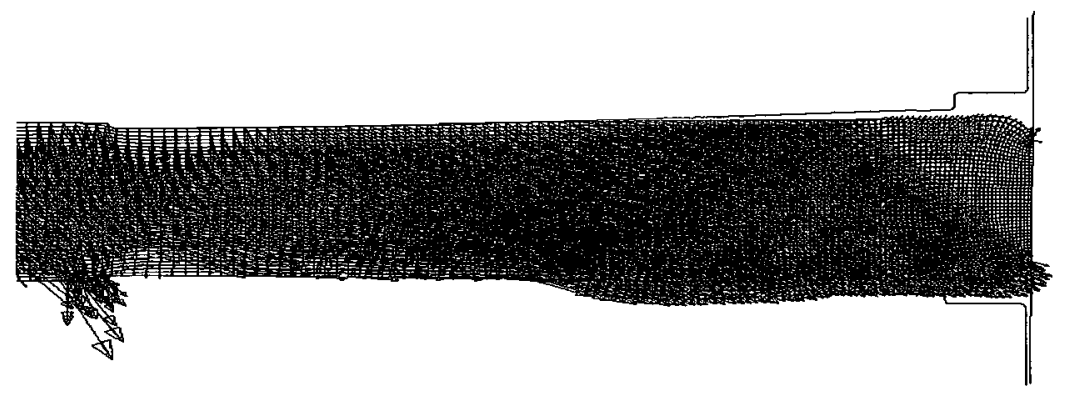

(a) $50 \%$ axial stroke

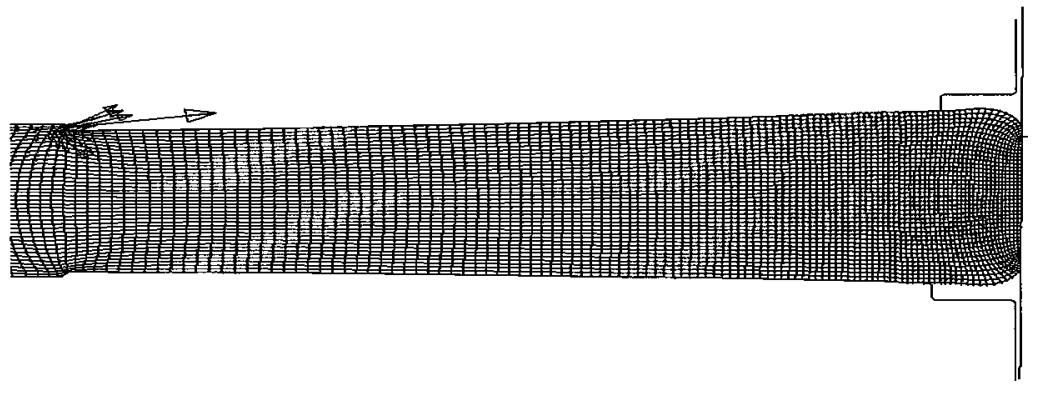

(b) $75 \%$ axial stroke

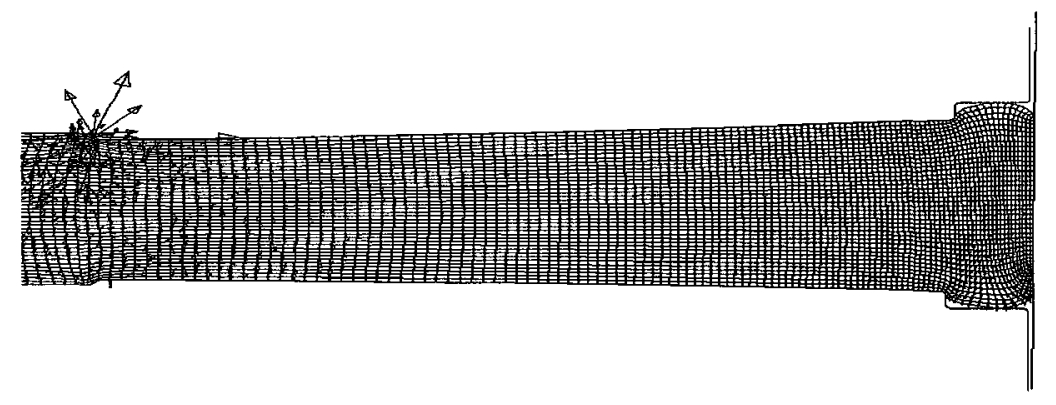

(c) $90 \%$ axial stroke

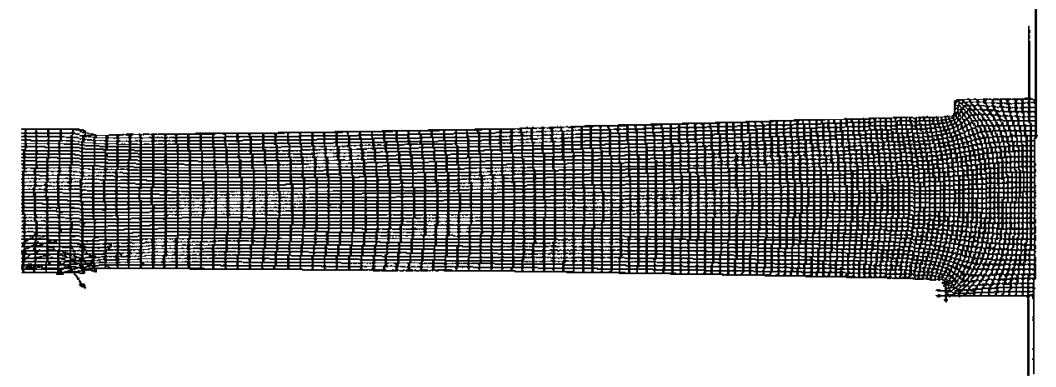

(d) $100 \%$ axial stroke

Figure A-36. Material flow velocity patterns showing outer 55\% of blank (Top die curvature $=0.3 \mathrm{~mm}$, Bottom die curvature $=0.19 \mathrm{~mm}$, Rim Height $=0.271 \mathrm{~mm}$, relief $=0.09 \mathrm{~mm}$ ) 


\begin{tabular}{|c|c|c|c|c|c|c|c|c|c|}
\hline \multicolumn{10}{|c|}{ Ring Compression Test (Load Vs. Displacement) } \\
\hline \multicolumn{2}{|c|}{ Experiment \#1 } & \multicolumn{2}{|c|}{ Experiment \#2 } & \multicolumn{2}{|c|}{ Experiment \#3 } & \multicolumn{2}{|c|}{ Experiment \#4 } & \multicolumn{2}{|c|}{ Experiment \#5 } \\
\hline $\begin{array}{c}\text { Displacement } \\
\qquad(\mathrm{mm})\end{array}$ & $\begin{array}{l}\text { Load } \\
(\mathrm{kN})\end{array}$ & $\begin{array}{c}\text { Displacement } \\
(\mathrm{mm})\end{array}$ & $\begin{array}{l}\text { Load } \\
(\mathrm{kN})\end{array}$ & $\begin{array}{c}\text { Displacement } \\
\text { (mm) }\end{array}$ & $\begin{array}{l}\text { Load } \\
(\mathrm{kN})\end{array}$ & $\begin{array}{c}\text { Displacement } \\
\text { (mm) }\end{array}$ & $\begin{array}{l}\text { Load } \\
(\mathrm{kN})\end{array}$ & $\begin{array}{c}\text { Displacement } \\
(\mathrm{mm})\end{array}$ & $\begin{array}{l}\text { Load } \\
(\mathrm{kN})\end{array}$ \\
\hline 0 & 0 & 0 & 0 & 0 & 0 & 0 & 0 & 0 & 0 \\
\hline 0.7 & 0.019 & 0.85 & 0.388 & 0.9 & 0.42 & 0.91 & 0.21 & 0.9 & 1.26 \\
\hline 0.86 & 1.063 & 0.9 & 4.36 & 1 & 11.14 & 1 & 7.96 & 1 & 13.3 \\
\hline 1.001 & 22.79 & 1 & 20.36 & 1.1 & 30.48 & 1.1 & 26.68 & 1.1 & 33.48 \\
\hline 1.151 & 59.6 & 1.1 & 41.75 & 1.201 & 54.64 & 1.2 & 49.28 & 1.201 & 58.13 \\
\hline 1.300 & 109.93 & 1.2 & 69.63 & 1.3 & 87.26 & 1.3 & 80.71 & 1.301 & 90.95 \\
\hline 1.450 & 165.14 & 1.3 & 104.94 & 1.401 & 122.88 & 1.401 & 116.72 & 1.401 & 128.72 \\
\hline 1.753 & 267.79 & 1.4 & 143.1 & 1.501 & 162.56 & 1.5 & 154.7 & 1.5 & 168.77 \\
\hline 1.860 & 305.71 & 1.501 & 184 & 1.6 & 202.71 & 1.601 & 194.61 & 1.6 & 210.53 \\
\hline 1.947 & 336.07 & 1.6 & 226.06 & 1.7 & 244.73 & 1.7 & 237.08 & 1.701 & 253.42 \\
\hline 2.050 & 359.02 & 1.7 & 267.52 & 1.801 & 288.64 & 1.8 & 279.77 & 1.8 & 295.03 \\
\hline 2.217 & 378.81 & 1.801 & 309.7 & 1.901 & 330.85 & 1.901 & 322.5 & 1.9 & 336.64 \\
\hline 2.393 & 491.94 & 1.9 & 352.47 & 2 & 371.7 & 2 & 357.96 & 2 & 377.72 \\
\hline 2.501 & 402.51 & 2 & 389.44 & 2.1 & 402.64 & 2.1 & 369 & 2.1 & 406.31 \\
\hline \multirow[t]{3}{*}{2.542} & 408.15 & 2.1 & 406.43 & 2.2 & 416.06 & 2.2 & 381.66 & 2.2 & 418.9 \\
\hline & & 2.200 & 422.53 & 2.384 & 442.47 & 2.3 & 394.89 & 2.3 & 432.32 \\
\hline & & 2.337 & 443.75 & & & 2.4 & 408.61 & 2.37 & 441.62 \\
\hline
\end{tabular}

Table A-1. Load against displacement results in ring test 


\begin{tabular}{|c|c|c|c|c|c|}
\hline \multicolumn{6}{|c|}{ Top Die Curvature Variation -1} \\
\hline \multicolumn{2}{|c|}{ Top curvature $=0.05 \mathrm{~mm}$} & \multicolumn{2}{|c|}{ Top curvature $=0.1 \mathrm{~mm}$} & \multicolumn{2}{|c|}{ Top curvature $=0.15 \mathrm{~mm}$} \\
\hline $\begin{array}{c}\text { Displacement } \\
(\mathrm{mm})\end{array}$ & $\begin{array}{l}\text { Load } \\
(\mathrm{kN})\end{array}$ & $\begin{array}{c}\text { Displacement } \\
(\mathrm{mm})\end{array}$ & $\begin{array}{l}\text { Load } \\
(\mathrm{kN})\end{array}$ & $\begin{array}{c}\text { Displacement } \\
(\mathrm{mm})\end{array}$ & $\begin{array}{l}\text { Load } \\
(\mathrm{kN})\end{array}$ \\
\hline 0.026 & 5.23 & 0.029 & 6.42 & 0.029 & 4.29 \\
\hline 0.052 & 16.25 & 0.057 & 21.91 & 0.057 & 18.58 \\
\hline 0.076 & 40.76 & 0.084 & 50.43 & 0.084 & 40.81 \\
\hline 0.1 & 100.38 & 0.11 & 72.03 & 0.11 & 69.32 \\
\hline 0.122 & 172.32 & 0.134 & 160.59 & 0.134 & 116.71 \\
\hline 0.144 & 266.54 & 0.158 & 246.88 & 0.158 & 169.12 \\
\hline 0.165 & 538.59 & 0.18 & 413.5 & 0.18 & 265.01 \\
\hline 0.184 & 721.46 & 0.201 & 683.62 & 0.201 & 539.07 \\
\hline 0.203 & 867.28 & 0.221 & 838.03 & 0.221 & 616.83 \\
\hline 0.221 & 1219.34 & 0.24 & 1087.43 & 0.24 & 771.56 \\
\hline 0.223 & 1263.81 & 0.243 & 1174.49 & 0.245 & 820.2 \\
\hline 0.224 & 1304.37 & 0.246 & 1244.85 & 0.249 & 854.91 \\
\hline 0.226 & 1360.85 & 0.249 & 1310.71 & 0.254 & 870.47 \\
\hline 0.228 & 1381.13 & 0.252 & 1390.08 & 0.258 & 923.51 \\
\hline 0.229 & 1424.94 & 0.255 & 1475.56 & 0.262 & 974.72 \\
\hline 0.231 & 1475.64 & 0.257 & 1544.38 & 0.267 & 1028.31 \\
\hline 0.232 & 1506.43 & 0.26 & 1641.59 & 0.271 & 1098.61 \\
\hline 0.234 & 1553.49 & 0.263 & 1741.39 & 0.275 & 1204.98 \\
\hline 0.235 & 1604.55 & 0.266 & 1827.65 & 0.279 & 1308.6 \\
\hline 0.237 & 1649.17 & 0.268 & 1913.88 & 0.283 & 1401.93 \\
\hline 0.238 & 1706.82 & 0.271 & 2012.95 & 0.287 & 1542.09 \\
\hline 0.24 & 1753.2 & 0.274 & 2108.81 & 0.291 & 1653.63 \\
\hline 0.241 & 1792.48 & 0.276 & 2218.69 & 0.295 & 1776.76 \\
\hline 0.242 & 1840.52 & 0.279 & 2311.45 & 0.299 & 1925.74 \\
\hline 0.244 & 1905.09 & 0.281 & 2396.5 & 0.303 & 2059.78 \\
\hline 0.245 & 1967.27 & 0.284 & 2498.86 & 0.306 & 2201.39 \\
\hline 0.247 & 2023.03 & 0.287 & 2457.11 & 0.31 & 2337.27 \\
\hline 0.248 & 2073.6 & 0.288 & 2490.02 & 0.314 & 2448.7 \\
\hline 0.25 & 2132.18 & & & 0.317 & 2615.91 \\
\hline 0.251 & 2186.4 & & & 0.319 & 2649.42 \\
\hline 0.252 & 2237.47 & & & & \\
\hline 0.254 & 2286.81 & & & & \\
\hline 0.257 & 2393.89 & & & & \\
\hline 0.258 & 2446.25 & & & & \\
\hline
\end{tabular}

Table A-2a. Load Vs Displacement with varying top curvature 


\begin{tabular}{|c|c|c|c|c|c|}
\hline \multicolumn{6}{|c|}{ Top Die Curvature Variation -2} \\
\hline \multicolumn{2}{|c|}{ Top curvature $=0.2 \mathrm{~mm}$} & \multicolumn{2}{|c|}{ Top curvature $=0.25 \mathrm{~mm}$} & \multicolumn{2}{|c|}{ Top curvature $=0.3 \mathrm{~mm}$} \\
\hline $\begin{array}{c}\text { Displacement } \\
(\mathrm{mm})\end{array}$ & $\begin{array}{l}\text { Load } \\
(\mathrm{kN})\end{array}$ & $\begin{array}{c}\text { Displacement } \\
(\mathrm{mm})\end{array}$ & $\begin{array}{l}\text { Load } \\
(\mathrm{kN})\end{array}$ & $\begin{array}{c}\text { Displacement } \\
(\mathrm{mm})\end{array}$ & $\begin{array}{l}\text { Load } \\
(\mathrm{kN})\end{array}$ \\
\hline 0.035 & 5.01 & 0.04 & 6.06 & 0.043 & 7.18 \\
\hline 0.068 & 17.58 & 0.079 & 20.32 & 0.084 & 21.96 \\
\hline 0.1 & 41.69 & 0.115 & 48.12 & 0.122 & 49.55 \\
\hline 0.129 & 86.28 & 0.149 & 90.45 & 0.158 & 92.39 \\
\hline 0.158 & 126.84 & 0.18 & 143.23 & 0.191 & 135.86 \\
\hline 0.184 & 190.77 & 0.209 & 214.68 & 0.221 & 196.53 \\
\hline 0.209 & 393.66 & 0.237 & 284.56 & 0.249 & 269.96 \\
\hline 0.255 & 657.38 & 0.262 & 438.91 & 0.275 & 358.8 \\
\hline 0.275 & 830.77 & 0.285 & 699.93 & 0.299 & 508.88 \\
\hline 0.279 & 860.22 & 0.306 & 837.79 & 0.321 & 794.12 \\
\hline 0.281 & 873.65 & 0.311 & 899.15 & 0.327 & 824.56 \\
\hline 0.283 & 885.98 & 0.313 & 922.97 & 0.33 & 848.44 \\
\hline 0.285 & 895.98 & 0.318 & 949.29 & 0.335 & 886 \\
\hline 0.287 & 914.77 & 0.32 & 972.29 & 0.34 & 912.93 \\
\hline 0.289 & 934.12 & 0.323 & 998.6 & 0.346 & 969.26 \\
\hline 0.29 & 953.41 & 0.325 & 1018.26 & 0.351 & 996.02 \\
\hline 0.292 & 980.83 & 0.327 & 1051.23 & 0.353 & 1021.75 \\
\hline 0.294 & 999.05 & 0.329 & 1132.47 & 0.356 & 1069.58 \\
\hline 0.296 & 1015.57 & 0.332 & 1159.6 & 0.358 & 1149.12 \\
\hline 0.298 & 1070.38 & 0.334 & 1215.04 & 0.361 & 1187.41 \\
\hline 0.3 & 1104.13 & 0.336 & 1232.42 & 0.363 & 1285.74 \\
\hline 0.302 & 1147.72 & 0.338 & 1300.72 & 0.365 & 1345.51 \\
\hline 0.303 & 1154.56 & 0.34 & 1358.85 & 0.368 & 1384.58 \\
\hline 0.305 & 1199.66 & 0.342 & 1414.83 & 0.37 & 1400.81 \\
\hline 0.307 & 1269.19 & 0.344 & 1482.43 & 0.372 & 1474.3 \\
\hline 0.309 & 1311.31 & 0.346 & 1562.95 & 0.375 & 1563.26 \\
\hline 0.311 & 1359.6 & 0.348 & 1603.35 & 0.377 & 1631.71 \\
\hline 0.312 & 1407.6 & 0.35 & 1679.83 & 0.379 & 1689.85 \\
\hline 0.314 & 1458.58 & 0.352 & 1744.46 & 0.381 & 1772.13 \\
\hline 0.316 & 1518.49 & 0.355 & 1800.72 & 0.383 & 1853.33 \\
\hline 0.318 & 1547.93 & 0.357 & 1885.45 & & \\
\hline 0.319 & 1620.94 & 0.358 & 1958.19 & & \\
\hline 0.321 & 1662.92 & 0.36 & 2031.08 & & \\
\hline
\end{tabular}

Table A-2b. Load Vs Displacement with varying top curvature 


\begin{tabular}{|c|c|c|c|c|c|}
\hline \multicolumn{6}{|c|}{ Bottom Die Curvature Variation -1 } \\
\hline \multicolumn{2}{|c|}{ Top curvature $=0.05 \mathrm{~mm}$} & \multicolumn{2}{|c|}{ Top curvature $=0.1 \mathrm{~mm}$} & \multicolumn{2}{|c|}{ Top curvature $=0.15 \mathrm{~mm}$} \\
\hline $\begin{array}{c}\text { Displacement } \\
(\mathrm{mm})\end{array}$ & $\begin{array}{l}\text { Load } \\
(\mathrm{kN})\end{array}$ & $\begin{array}{c}\text { Displacement } \\
(\mathrm{mm})\end{array}$ & $\begin{array}{l}\text { Load } \\
(\mathrm{kN}) \\
\end{array}$ & $\begin{array}{c}\text { Displacement } \\
(\mathrm{mm})\end{array}$ & $\begin{array}{l}\text { Load } \\
(\mathrm{kN})\end{array}$ \\
\hline 0.035 & 6.81 & 0.004 & 0.13 & 0.004 & 0.1 \\
\hline 0.1 & 59.23 & 0.047 & 9.87 & 0.043 & 8.15 \\
\hline 0.129 & 112.19 & 0.088 & 37.86 & 0.084 & 27.72 \\
\hline 0.158 & 179.35 & 0.126 & 82.73 & 0.122 & 62.5 \\
\hline 0.184 & 264.79 & 0.161 & 137.83 & 0.158 & 106.91 \\
\hline 0.209 & 408.57 & 0.194 & 229.58 & 0.191 & 166.16 \\
\hline 0.255 & 881.69 & 0.224 & 324 & 0.221 & 233.66 \\
\hline 0.275 & 1195.64 & 0.252 & 650.34 & 0.249 & 333.52 \\
\hline 0.276 & 1231.6 & 0.277 & 835.96 & 0.275 & 535.84 \\
\hline 0.278 & 1263.81 & 0.301 & 1112.8 & 0.299 & 822.77 \\
\hline 0.279 & 1288.53 & 0.312 & 1375.39 & 0.321 & 947.89 \\
\hline 0.28 & 1322.6 & 0.321 & 1631.53 & 0.323 & 974.32 \\
\hline 0.281 & 1351.27 & 0.322 & 1655.26 & 0.325 & 996.49 \\
\hline 0.283 & 1390.35 & 0.323 & 1681.82 & 0.327 & 1028.73 \\
\hline 0.284 & 1433.82 & 0.324 & 1734.73 & 0.33 & 1091.53 \\
\hline 0.285 & 1455.5 & 0.325 & 1787.33 & 0.332 & 1146.27 \\
\hline 0.287 & 1507.56 & 0.327 & 1830.39 & 0.334 & 1175.54 \\
\hline 0.288 & 1516.12 & 0.328 & 1880.1 & 0.336 & 1205.16 \\
\hline 0.289 & 1557.18 & 0.33 & 1931.79 & 0.338 & 1256 \\
\hline 0.29 & 1604.58 & 0.331 & 1982.64 & 0.34 & 1317.29 \\
\hline 0.292 & 1632.33 & 0.332 & 2035.06 & 0.342 & 1368.63 \\
\hline 0.2923 & 1652.48 & 0.334 & 2084 & 0.344 & 1407.7 \\
\hline 0.293 & 1662.66 & 0.335 & 2131.86 & 0.346 & 1452.9 \\
\hline 0.294 & 1715.07 & 0.336 & 2187.9 & 0.348 & 1517.68 \\
\hline 0.295 & 1767.13 & 0.338 & 2245.19 & 0.35 & 1567.14 \\
\hline 0.297 & 1823.23 & 0.339 & 2287.54 & 0.351 & 1638.49 \\
\hline 0.298 & 1857.24 & 0.34 & 2343 & 0.352 & 1669.75 \\
\hline 0.299 & 1899.48 & 0.342 & 2397.97 & 0.353 & 1695.66 \\
\hline 0.3 & 1948.23 & 0.343 & 2444.2 & 0.355 & 1767.29 \\
\hline 0.303 & 2050.72 & 0.344 & 2494.4 & 0.357 & 1829.51 \\
\hline 0.304 & 2094.05 & 0.346 & 2544.98 & 0.359 & 1900.59 \\
\hline 0.305 & 2135.49 & 0.347 & 2587.19 & 0.361 & 1964.07 \\
\hline 0.306 & 2187.56 & 0.348 & 2639.48 & & \\
\hline
\end{tabular}

Table A-3a. Load Vs Displacement with varying bottom curvature 


\begin{tabular}{|c|c|c|c|c|c|}
\hline \multicolumn{6}{|c|}{ Bottom Die Curvature Variation -2 } \\
\hline \multicolumn{2}{|c|}{ Top curvature $=0.2 \mathrm{~mm}$} & \multicolumn{2}{|c|}{ Top curvature $=0.25 \mathrm{~mm}$} & \multicolumn{2}{|c|}{ Top curvature $=0.3 \mathrm{~mm}$} \\
\hline $\begin{array}{c}\text { Displacement } \\
(\mathrm{mm})\end{array}$ & $\begin{array}{l}\text { Load } \\
(\mathrm{kN})\end{array}$ & $\begin{array}{c}\text { Displacement } \\
(\mathrm{mm})\end{array}$ & $\begin{array}{l}\text { Load } \\
(\mathrm{kN})\end{array}$ & $\begin{array}{c}\text { Displacement } \\
(\mathrm{mm})\end{array}$ & $\begin{array}{l}\text { Load } \\
(\mathrm{kN})\end{array}$ \\
\hline 0.005 & 0.11 & 0.006 & 0.11 & 0.065 & 10.32 \\
\hline 0.046 & 7.82 & 0.057 & 8.14 & 0.125 & 36.37 \\
\hline 0.089 & 25.62 & 0.11 & 33.55 & 0.178 & 87.31 \\
\hline 0.129 & 55.86 & 0.158 & 75.14 & 0.225 & 140.06 \\
\hline 0.167 & 100.34 & 0.201 & 126.46 & 0.267 & 208.67 \\
\hline 0.201 & 161.05 & 0.24 & 194.65 & 0.303 & 402.37 \\
\hline 0.233 & 220.84 & 0.275 & 287.81 & 0.365 & 586.76 \\
\hline 0.262 & 295.14 & 0.306 & 382.19 & 0.412 & 1024.01 \\
\hline 0.288 & 368.16 & 0.335 & 601.45 & 0.413 & 1035.12 \\
\hline 0.312 & 683.62 & 0.361 & 832.65 & 0.415 & 1057.36 \\
\hline 0.335 & 838.32 & 0.372 & 932.24 & 0.417 & 1060.98 \\
\hline 0.338 & 858.4 & 0.383 & 1024.78 & 0.418 & 1090 \\
\hline 0.342 & 875.38 & 0.385 & 1044.34 & 0.42 & 1114.04 \\
\hline 0.345 & 899.21 & 0.387 & 1056.55 & 0.422 & 1151.99 \\
\hline 0.348 & 927.12 & 0.389 & 1097.92 & 0.423 & 1187.16 \\
\hline 0.351 & 957.6 & 0.391 & 1147.95 & 0.425 & 1227.21 \\
\hline 0.354 & 1028.01 & 0.393 & 1181.78 & 0.426 & 1246.85 \\
\hline 0.358 & 1063.83 & 0.395 & 1234.31 & 0.428 & 1277.42 \\
\hline 0.361 & 1091.12 & 0.397 & 1278.14 & 0.43 & 1334.92 \\
\hline 0.364 & 1184.45 & 0.401 & 1348.64 & 0.431 & 1387.73 \\
\hline 0.367 & 1210.59 & 0.403 & 1418.72 & 0.433 & 1407.62 \\
\hline 0.369 & 1301.02 & 0.405 & 1469.9 & 0.434 & 1456.57 \\
\hline 0.372 & 1403.07 & 0.407 & 1529.76 & 0.436 & 1505.23 \\
\hline 0.375 & 1471.97 & 0.409 & 1569.64 & 0.437 & 1541.85 \\
\hline 0.378 & 1513.34 & 0.41 & 1592.75 & 0.439 & 1602.23 \\
\hline 0.381 & 1617.49 & 0.412 & 1670.6 & 0.442 & 1652.76 \\
\hline 0.383 & 1711.52 & 0.414 & 1731.9 & 0.442 & 1652.76 \\
\hline 0.386 & 1795.88 & 0.415 & 1799.98 & 0.443 & 1708.74 \\
\hline 0.389 & 1904.6 & 0.417 & 1856.14 & 0.445 & 1762.76 \\
\hline 0.391 & 1995.84 & 0.419 & 1915.23 & 0.446 & 1818.28 \\
\hline \multirow[t]{3}{*}{0.394} & 2092.66 & 0.42 & 1982.67 & 0.447 & 1863.06 \\
\hline & & & & 0.449 & 1922.36 \\
\hline & & & & 0.45 & 1971.77 \\
\hline
\end{tabular}

Table A-3b. Load Vs Displacement with varying bottom curvature 


\begin{tabular}{|c|c|c|c|c|c|}
\hline \multicolumn{6}{|c|}{ Rim Height Variation -1 } \\
\hline \multicolumn{2}{|c|}{ Rim height $=0.18 \mathrm{~mm}$} & \multicolumn{2}{|c|}{ Rim height $=0.2 \mathrm{~mm}$} & \multicolumn{2}{|c|}{ Rim height $=0.22 \mathrm{~mm}$} \\
\hline $\begin{array}{c}\text { Displacement } \\
(\mathrm{mm})\end{array}$ & $\begin{array}{l}\text { Load } \\
(\mathrm{kN})\end{array}$ & $\begin{array}{c}\text { Displacement } \\
(\mathrm{mm})\end{array}$ & $\begin{array}{l}\text { Load } \\
(\mathrm{kN})\end{array}$ & $\begin{array}{c}\text { Displacement } \\
(\mathrm{mm})\end{array}$ & $\begin{array}{l}\text { Load } \\
(\mathrm{kN})\end{array}$ \\
\hline 0.043 & 7.18 & 0.043 & 7.18 & 0.046 & 6.1 \\
\hline 0.084 & 21.96 & 0.084 & 21.96 & 0.089 & 23.85 \\
\hline 0.122 & 49.55 & 0.122 & 49.56 & 0.129 & 56.44 \\
\hline 0.158 & 92.39 & 0.191 & 147.57 & 0.167 & 102.74 \\
\hline 0.191 & 135.86 & 0.221 & 197.05 & 0.201 & 157.69 \\
\hline 0.221 & 196.53 & 0.249 & 266.6 & 0.233 & 227.32 \\
\hline 0.249 & 269.96 & 0.275 & 341.81 & 0.261 & 322.23 \\
\hline 0.275 & 358.8 & 0.299 & 510.54 & 0.288 & 427.55 \\
\hline 0.299 & 508.88 & 0.321 & 762.69 & 0.312 & 739.03 \\
\hline 0.321 & 794.12 & 0.328 & 809.25 & 0.335 & 869.05 \\
\hline 0.326 & 818.45 & 0.332 & 860.29 & 0.337 & 921.62 \\
\hline 0.328 & 834.69 & 0.334 & 892.92 & 0.339 & 959.52 \\
\hline 0.33 & 879.02 & 0.337 & 927.71 & 0.343 & 983.1 \\
\hline 0.332 & 894.31 & 0.339 & 968.63 & 0.345 & 1045.48 \\
\hline 0.334 & 936.65 & 0.343 & 1017.7 & 0.347 & 1071.3 \\
\hline 0.337 & 967.25 & 0.345 & 1082.64 & 0.349 & 1143.25 \\
\hline 0.339 & 1040.53 & 0.349 & 1172.95 & 0.351 & 1184.57 \\
\hline 0.341 & 1086.41 & 0.351 & 1222.06 & 0.356 & 1289.82 \\
\hline 0.343 & 1135.53 & 0.353 & 1289.43 & 0.357 & 1305.91 \\
\hline 0.345 & 1161.88 & 0.355 & 1338.17 & 0.359 & 1349.07 \\
\hline 0.349 & 1263.74 & 0.357 & 1416.17 & 0.361 & 1446.38 \\
\hline 0.353 & 1366.24 & 0.359 & 1442.41 & 0.363 & 1475.49 \\
\hline 0.355 & 1438.62 & 0.361 & 1521.55 & 0.367 & 1568.87 \\
\hline 0.357 & 1514.44 & 0.363 & 1581.45 & 0.369 & 1633.54 \\
\hline 0.358 & 1530.66 & 0.365 & 1657.04 & 0.371 & 1703.44 \\
\hline 0.359 & 1573.44 & 0.367 & 1711.94 & 0.373 & 1779.57 \\
\hline 0.361 & 1633.36 & 0.369 & 1786.62 & 0.374 & 1839.8 \\
\hline 0.363 & 1702.22 & 0.37 & 1856.56 & 0.378 & 1989.89 \\
\hline 0.365 & 1792.97 & 0.372 & 1929.19 & 0.381 & 2111.2 \\
\hline 0.367 & 1845.75 & & & 0.383 & 2182.22 \\
\hline 0.369 & 1927.16 & & & & \\
\hline 0.371 & 1992.09 & & & & \\
\hline 0.373 & 2066.63 & & & & \\
\hline
\end{tabular}

Table A-4a. Load Vs Displacement with varying rim height 


\begin{tabular}{|c|c|c|c|c|c|c|c|}
\hline \multicolumn{8}{|c|}{ Rim Height Variation -2} \\
\hline \multicolumn{2}{|c|}{ Rim height $=0.24 \mathrm{~mm}$} & \multicolumn{2}{|c|}{ Rim height $=0.26 \mathrm{~mm}$} & \multicolumn{2}{|c|}{ Rim height $=0.28 \mathrm{~mm}$} & \multicolumn{2}{|c|}{ Rim height $=0.3 \mathrm{~mm}$} \\
\hline $\begin{array}{c}\text { Displacement } \\
(\mathrm{mm})\end{array}$ & $\begin{array}{l}\text { Load } \\
(\mathrm{kN})\end{array}$ & $\begin{array}{c}\text { Displacement } \\
(\mathrm{mm})\end{array}$ & $\begin{array}{l}\text { Load } \\
(\mathrm{kN})\end{array}$ & $\begin{array}{c}\text { Displacement } \\
(\mathrm{mm})\end{array}$ & $\begin{array}{l}\text { Load } \\
(\mathrm{kN})\end{array}$ & $\begin{array}{c}\text { Displacement } \\
(\mathrm{mm})\end{array}$ & $\begin{array}{l}\text { Load } \\
(\mathrm{kN})\end{array}$ \\
\hline 0.046 & 6.1 & 0.046 & 6.1 & 0.046 & 6.1 & 0.046 & 6.1 \\
\hline 0.089 & 23.85 & 0.089 & 23.85 & 0.089 & 23.85 & 0.089 & 23.85 \\
\hline 0.129 & 56.44 & 0.129 & 56.44 & 0.129 & 56.44 & 0.129 & 56.44 \\
\hline 0.167 & 102.75 & 0.167 & 102.75 & 0.167 & 102.75 & 0.167 & 102.63 \\
\hline 0.201 & 157.69 & 0.201 & 157.59 & 0.201 & 157.69 & 0.201 & 153.79 \\
\hline 0.233 & 227.32 & 0.233 & 227.32 & 0.233 & 227.32 & 0.233 & 223.19 \\
\hline 0.261 & 322.23 & 0.261 & 322.23 & 0.261 & 322.23 & 0.262 & 300.89 \\
\hline 0.288 & 427.55 & 0.288 & 427.55 & 0.288 & 427.55 & 0.288 & 423.85 \\
\hline 0.312 & 739.03 & 0.312 & 739.03 & 0.312 & 739.03 & 0.312 & 701.49 \\
\hline 0.335 & 869.04 & 0.335 & 869.04 & 0.335 & 869.04 & 0.335 & 863.95 \\
\hline 0.34 & 932 & 0.34 & 929.64 & 0.344 & 910.26 & 0.341 & 897.21 \\
\hline 0.343 & 970.39 & 0.345 & 945.04 & 0.347 & 969.5 & 0.345 & 953.07 \\
\hline 0.348 & 1002.24 & 0.348 & 984.99 & 0.35 & 1002.36 & 0.351 & 967.49 \\
\hline 0.35 & 1065.01 & 0.35 & 1044.93 & 0.353 & 1026.57 & 0.354 & 1025.85 \\
\hline 0.353 & 1124.19 & 0.353 & 1062.78 & 0.357 & 1061.43 & 0.36 & 1098.51 \\
\hline 0.355 & 1182.11 & 0.355 & 1151.79 & 0.359 & 1137.43 & 0.363 & 1165.89 \\
\hline 0.358 & 1240.08 & 0.358 & 1210.69 & 0.362 & 1183.79 & 0.366 & 1239.94 \\
\hline 0.363 & 1336.69 & 0.363 & 1279.17 & 0.365 & 1287.51 & 0.368 & 1301.8 \\
\hline 0.365 & 1439.84 & 0.365 & 1322.76 & 0.368 & 1312.74 & 0.374 & 1429.74 \\
\hline 0.367 & 1465.77 & 0.367 & 1395.8 & 0.373 & 1440.1 & 0.376 & 1452.7 \\
\hline 0.37 & 1553.1 & 0.37 & 1483.8 & 0.376 & 1516.11 & 0.379 & 1517.15 \\
\hline 0.371 & 1577.42 & 0.372 & 1544.13 & 0.379 & 1626.83 & 0.382 & 1615.99 \\
\hline 0.372 & 1624.8 & 0.374 & 1583.2 & 0.381 & 1723.54 & 0.384 & 1703.04 \\
\hline 0.374 & 1704.03 & 0.376 & 1663.38 & 0.384 & 1804.84 & 0.389 & 1892.07 \\
\hline 0.376 & 1785.17 & 0.379 & 1742.81 & 0.386 & 1897.09 & 0.392 & 1981.01 \\
\hline 0.379 & 1863.48 & 0.381 & 1818.51 & 0.389 & 1991.38 & 0.394 & 2065.98 \\
\hline 0.381 & 1952.78 & 0.383 & 1891.55 & 0.391 & 2071.51 & 0.399 & 2242.86 \\
\hline 0.385 & 2098.36 & 0.387 & 2045.92 & 0.394 & 2154.11 & 0.403 & 2411.22 \\
\hline 0.387 & 2188 & 0.391 & 2219.33 & 0.398 & 2323.47 & & \\
\hline 0.39 & 2303.04 & 0.393 & 2290.55 & 0.403 & 2493.82 & & \\
\hline 0.393 & 2419.99 & & & & & & \\
\hline
\end{tabular}

Table A-4b. Load Vs Displacement with varying rim height 


\begin{tabular}{|c|c|c|c|c|c|}
\hline \multicolumn{6}{|c|}{ Relief Height Variation } \\
\hline \multicolumn{2}{|c|}{ Relief height $=0.08 \mathrm{~mm}$} & \multicolumn{2}{|c|}{ Relief height $=0.09 \mathrm{~mm}$} & \multicolumn{2}{|c|}{ Relief height $=0.1 \mathrm{~mm}$} \\
\hline $\begin{array}{c}\text { Displacement } \\
(\mathrm{mm})\end{array}$ & $\begin{array}{l}\text { Load } \\
(\mathrm{kN})\end{array}$ & $\begin{array}{c}\begin{array}{c}\text { Displacement } \\
\text { (mm) }\end{array} \\
\end{array}$ & $\begin{array}{l}\text { Load } \\
(\mathrm{kN})\end{array}$ & $\begin{array}{c}\text { Displacement } \\
(\mathrm{mm})\end{array}$ & $\begin{array}{l}\text { Load } \\
(\mathrm{kN})\end{array}$ \\
\hline 0.038 & 11.94 & 0.04 & 10.29 & 0.04 & 10.29 \\
\hline 0.073 & 26.56 & 0.079 & 25.52 & 0.079 & 25.3 \\
\hline 0.107 & 43.3 & 0.115 & 47.63 & 0.115 & 46.4 \\
\hline 0.139 & 146.09 & 0.149 & 111.34 & 0.149 & 70.8 \\
\hline 0.169 & 236.96 & 0.18 & 234.7 & 0.18 & 238.55 \\
\hline 0.197 & 329.52 & 0.209 & 338.87 & 0.209 & 337.27 \\
\hline 0.223 & 441.8 & 0.237 & 498.96 & 0.237 & 458.13 \\
\hline 0.248 & 697.75 & 0.261 & 760.01 & 0.261 & 750.88 \\
\hline 0.27 & 796.01 & 0.284 & 885.88 & 0.285 & 879.26 \\
\hline 0.29 & 940.98 & 0.306 & 1121.7 & 0.306 & 1037.27 \\
\hline 0.3 & 1078.05 & 0.31 & 1211.13 & 0.311 & 1169.64 \\
\hline 0.315 & 1330.54 & 0.314 & 1244.83 & 0.316 & 1310.02 \\
\hline 0.324 & 1591.73 & 0.323 & 1280.37 & 0.321 & 1401.7 \\
\hline 0.327 & 1699.38 & 0.326 & 1365.46 & 0.325 & 1525.22 \\
\hline 0.329 & 1786.48 & 0.33 & 1478.4 & 0.327 & 1641.68 \\
\hline 0.332 & 1892.46 & 0.334 & 1680 & 0.33 & 1715.57 \\
\hline 0.338 & 2081.53 & 0.338 & 1837.92 & 0.334 & 1784.91 \\
\hline 0.343 & 2288.19 & 0.342 & 1978.78 & 0.338 & 1963.98 \\
\hline 0.345 & 2385.73 & 0.345 & 2112.1 & 0.343 & 2123.34 \\
\hline 0.348 & 2483.25 & 0.349 & 2219.9 & 0.347 & 2229.46 \\
\hline 0.351 & 2585.95 & 0.352 & 2321.63 & 0.351 & 2336.84 \\
\hline 0.356 & 2803.5 & 0.354 & 3746.41 & 0.355 & 2428.13 \\
\hline
\end{tabular}

Table A-5. Load Vs Displacement with varying relief height 